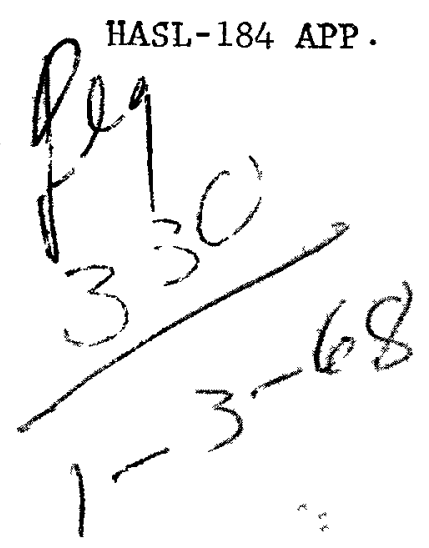

This document is

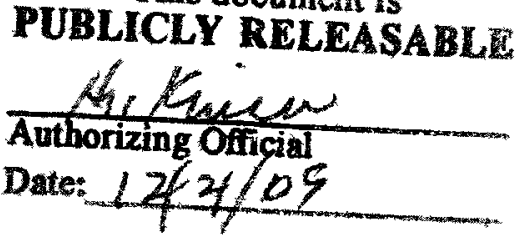

health and safety laboratory

A P P E N D I X

FALLOUT PROGRAM

QUARTERLY SUMMARY REPORT

January 1, 1968

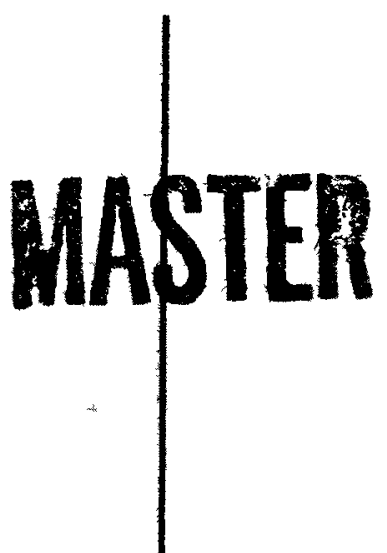


This report was prepared as an account of Government sponsored work. Neither the United States, nor the Commission, nor any person acting on behalf of the Commission:

A. Makes any warranty or representation, expressed or implied, with respect to the accuracy, completeness, or usefulness of the information contained in this report, or that the use of any information, apparatus, method, or process disclosed in this report may not infringe privately owned rights; or

B. Assumes any liabilities with respect to the use of, or for damages resulting from the use of any information, apparatus, method, or process disclosed in this report.

As used in the above, "person acting on behalf of the Commission" includes any employee or contractor of the Conmission, or employee of such contractor, to the extent that such employee or contractor of the Commission, or employee of such contractor prepares, disseminates, or provides access to, any information pursuant to his employment or contract with the Commission, or his employment with such contractor.

\section{Printed in the United States of America} Available from

Clearinghouse for Federal Scientific and Technical Information Nationa1 Bureau of Standards, U. S. Department of Commerce Springfield, Virginia 22151

Price: Printed Copy $\$ 3.00$; Microfiche $\$ 0.65$ 


\section{HASL -184}

APPENDIX TO

Hea1th and Safety Laboratory

FALLOUT PROGRAM

QUARTERLY SUMMARY REPORT

(September 1, 1967 through December 1, 1967)

Page

A. $\mathrm{Sr}^{90}$ and $\mathrm{Sr}^{89}$ in Monthly Deposition at World Land Sites $\mathrm{A}-1$

B. Fission Product and Activation Product Radionuclides in

B -1 Month1y Deposition at Selected Sites

C. Radiostrontium Deposition at At lantic Ocean Weather Stations

C -1

D. Radiostrontium in Milk and Tap Water

$\mathrm{D}-1$

E. Table of Conversion Factors

$E-1$

F. Table of Radionuclides

$F=1$

\section{LEGAL NOTICE}

This report was prepared as an account of Government sponsored work. Neither

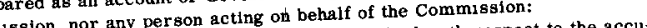
States, nor the Commission, nor any person, expressed or implied, with respect to the accu-
A. Makes any warranty or representation, export, or that the use racy, completeness, or usefulness of the information contalned in this report, or that the unge of any information, apparatus, method, or process declosed in this privately owned rights; or

B. Assumes any liabilities with respect to the use of, or for damages resulth

B. Assumes any lion, apparatus, method, or process disclosed in this report. ase of any information above, "person acting on behalf of the Commission" includes any employee or contractor of the Commission, or employee of such con such contractor prepares, such employee or contractor of the Commission, or employee to his employment or contract

dissemmates, or provides access to, any information pursuant 


\section{DISCLAIMER}

This report was prepared as an account of work sponsored by an agency of the United States Government. Neither the United States Government nor any agency Thereof, nor any of their employees, makes any warranty, express or implied, or assumes any legal liability or responsibility for the accuracy, completeness, or usefulness of any information, apparatus, product, or process disclosed, or represents that its use would not infringe privately owned rights. Reference herein to any specific commercial product, process, or service by trade name, trademark, manufacturer, or otherwise does not necessarily constitute or imply its endorsement, recommendation, or favoring by the United States Government or any agency thereof. The views and opinions of authors expressed herein do not necessarily state or reflect those of the United States Government or any agency thereof. 


\section{DISCLAIMER}

Portions of this document may be illegible in electronic image products. Images are produced from the best available original document. 
Appendix A

\section{$\mathrm{Sr}^{90}$ and $\mathrm{Sr}^{89}$ in Month1y Deposition at World Land Sites}

At present, there are 49 monthly monitoring sites in the United states and 107 in other countries. A map showing the sites is presented on page A-2. The collections are made using either stainless steel pots with exposed areas of 0.076 square meters, or plastic funnels with exposed areas of 0.072 square meters to which are attached ion-exchange columns.

In late 1958 and 1959 , the monthly samples were analyzed for $\mathrm{sr} 90$ and $\mathrm{sr}^{89}$. The $\mathrm{Sr}^{89}$ measurements were discontinued in 1960 at most sites and resumed starting with the September 1961 collections. Starting with the 1960 May and June collections, the monthly samples were combined on a two month basis since Sr90 levels had dropped considerably. Analyses of individual monthly collections were resumed in September 1961.

To facilitate the accurate storage, retrieval and handing of the data generated from the monthly fallout collection network, all data have been transcribed to punched cards. To accomplish this transcription several rigid criteria were applied to the data. One condition was that only monthly data were punched onto the cards. In the few cases where collections were incomplete or where co1lection times overlapped calendar months, the data were corrected to yield monthly values by interpolation using rainfall and observed concentrations in rainfall as guides. Where any corrections to the data have been made they are so indicated. In every case the best estimate of the true $\mathrm{Sr}^{90}$ deposition, rainfall and $\mathrm{Sr}^{89} / \mathrm{Sr}^{90}$ ratios, have been 1 isted.

The data printed out from the punched cards are presented in the tables beginning on page $\mathrm{A}-4$. A11 ratios of $\mathrm{Sr}-89$ to $\mathrm{Sr}-90$ have been extrapolated to the midpoint of the sampling month. Calculated values of the concentration of $\mathrm{Sr}-90$ in precipitation are given in units of $\mathrm{pCi} \mathrm{Sr}-90$ per liter. The total precipita.tion in centimeters and the $\mathrm{Sr}-90$ deposition in $\mathrm{mCi}$ per square kilometer for data available during a calendar year are listed in the extreme right hand column. The groups or organizations responsible for the sampling are also identifled on the Individual site data sheets. Monthly $\mathrm{Sr}-90$ deposition values for New York City since 1954 are shown in graph form on page A-3.

\section{Corrected New York City Sr $^{90}$ Data}

In HASL-146, pp 2-3, it was necessary to correct the previously reported strontium-90 deposition data for New York City during 1962 and 1963 . The fallout collector used during this period was found to be less efficient than the highwalled stalnless steel pot and funne1-ion exchange column collections. The tentatively corrected data were based upon deposition measurements made at Westwood, N.J. using the pot collector. Recently, resin samples from funnel-ion exchange column collectors that were exposed in New York during 1962 and for five months in 1963 were analyzed for strontium-90. These new data have now replaced the tentative values previously listed for New York City, on page A-35 of this report. 
WUTIHLY FALLCUI SAMELING INETWOKK

- POE

- Column

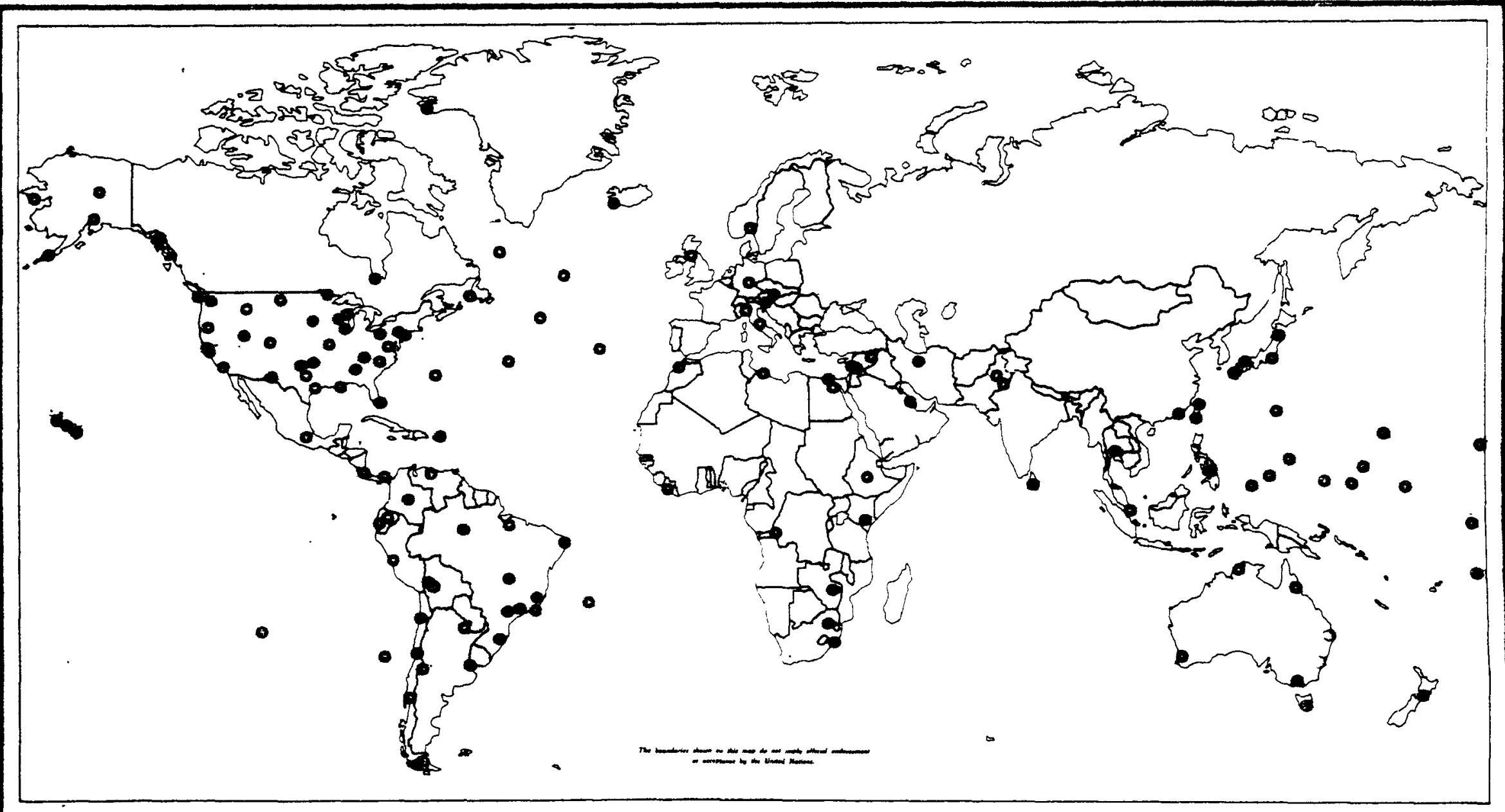




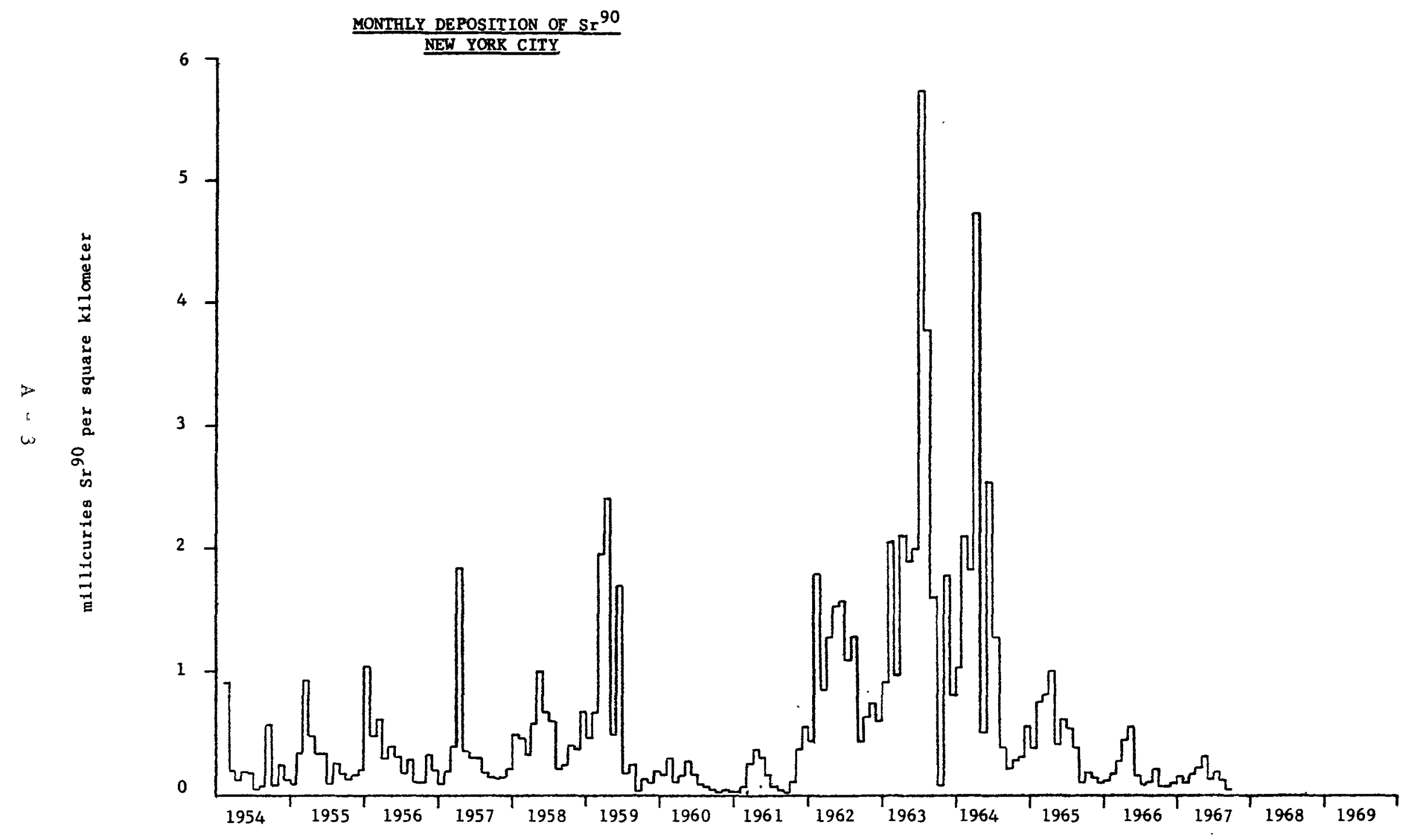




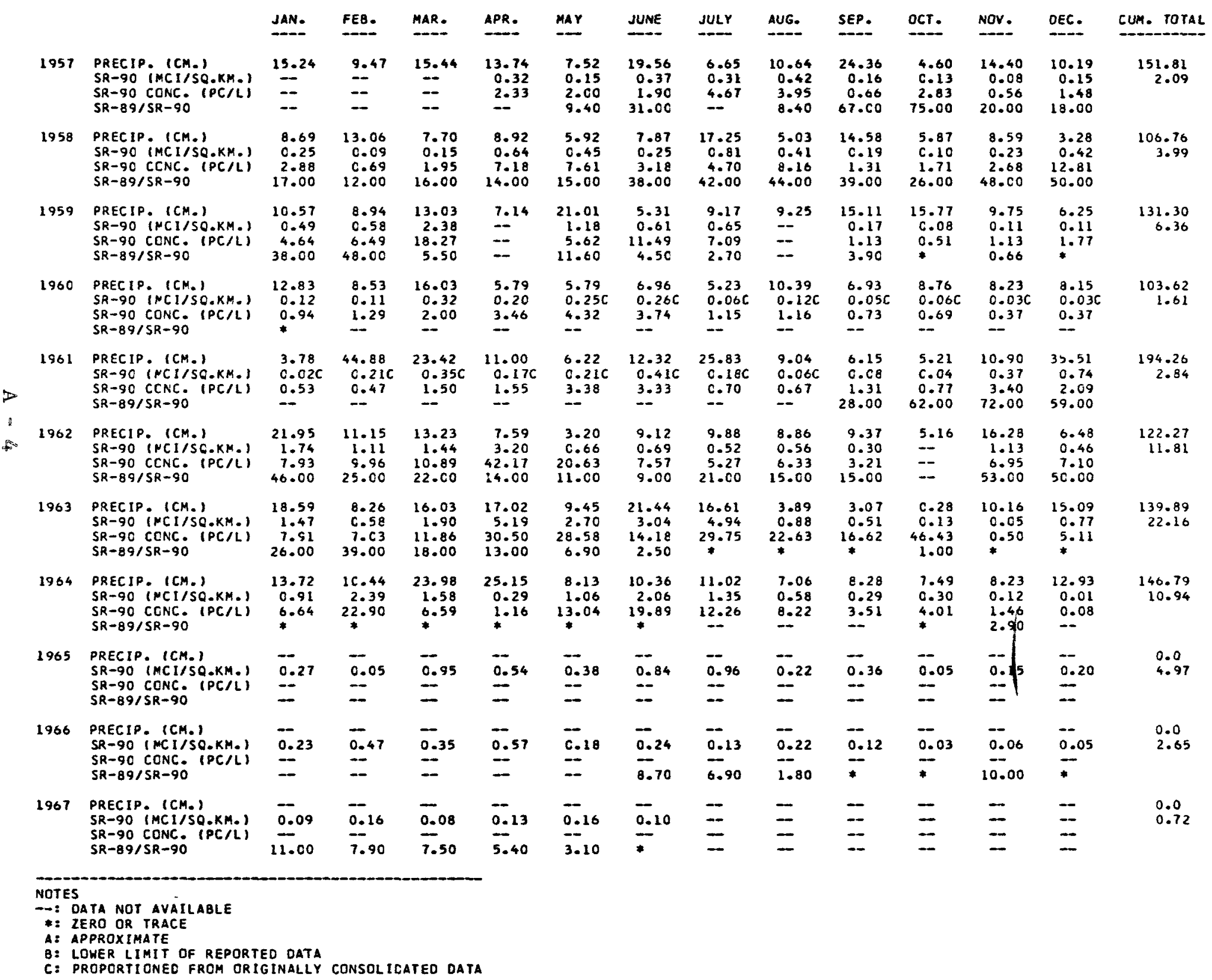




\begin{tabular}{|c|c|c|c|c|c|c|c|c|c|c|c|c|c|c|}
\hline & & JAN. & FEB. & MAR. & APR. & MAY & JUNE & Jutr & AUG. & SEP. & oct. & NOY. & DEC. & CUM. TOTAL \\
\hline 1959 & $\begin{array}{l}\text { PRECIP, (CM.) } \\
\text { SR-90 IMCI/SQ.KM-) } \\
\text { SR-90 CONC. (PC/L) } \\
\text { SR-89/SR-90 }\end{array}$ & $\begin{array}{l}-- \\
-- \\
--\end{array}$ & $\begin{array}{l}2.41 \\
-- \\
-- \\
--\end{array}$ & $\begin{array}{l}2.16 \\
-- \\
-\end{array}$ & $\begin{array}{r}3.35 \\
0.46 \\
13.74 \\
13.00\end{array}$ & $\begin{array}{r}1.85 \\
0.37 \\
20.01 \\
6.80\end{array}$ & $\begin{array}{r}0.05 \\
0.09 \\
180.00 \\
6.30\end{array}$ & $\begin{array}{r}11.25 \\
0.71 \\
6.32 \\
2.90\end{array}$ & $\begin{array}{l}9.53 \\
0.23 \\
2.42 \\
1.30\end{array}$ & $\begin{array}{l}4.27 \\
0.10 \\
2.35 \\
0.80\end{array}$ & $\begin{array}{l}2.18 \\
0.04 \\
1.04 \\
1.40\end{array}$ & $\begin{array}{l}1.75 \\
0.02 \\
1.15 \\
1.90\end{array}$ & $\begin{array}{l}3.53 \\
0.05 \\
1.42 \\
0\end{array}$ & $\begin{array}{r}42.33 \\
2.07\end{array}$ \\
\hline 1960 & $\begin{array}{l}\text { PRECIP. (CM.) } \\
\text { SR-90 (MCI/SQ.KM.) } \\
\text { SR-90 CONC. (PC/L) } \\
\text { SR-89/SR-90 }\end{array}$ & $\begin{array}{l}1.83 \\
0.08 \\
4.38 \\
-.\end{array}$ & $\begin{array}{l}1.14 \\
0.08 \\
7.02 \\
-.\end{array}$ & $\begin{array}{r}0.56 \\
0.07 \\
12.51 \\
-.-\end{array}$ & $\begin{array}{r}0.84 \\
0.10 \\
11.91 \\
-.\end{array}$ & $\begin{array}{l}1.07 \\
* \\
-- \\
=-\end{array}$ & $\begin{array}{l}0.61 \\
* \\
-- \\
--\end{array}$ & $\begin{array}{l}7.01 \\
* \\
-- \\
-\end{array}$ & $\begin{array}{l}9.40 \\
* \\
--\end{array}$ & $\begin{array}{l}12.17 \\
0.12 \\
0.99 \\
-.\end{array}$ & $\begin{array}{l}0.99 \\
= \\
=\end{array}$ & $\begin{array}{l}0.99 \\
0.026 \\
2.03 \\
\end{array}$ & $\begin{array}{l}2.64 \\
0.04 \mathrm{C} \\
1.52 \\
--\end{array}$ & $\begin{array}{r}39.25 \\
0.51\end{array}$ \\
\hline 1961 & $\begin{array}{l}\text { PRECIP. (CM.) } \\
\text { SR-9O IMCI/SO.KM.) } \\
\text { SR-9O CCNC. (PC/L) } \\
\text { SR-89/SR-90 }\end{array}$ & $\begin{array}{l}3.99 \\
0.13 \mathrm{C} \\
3.26 \\
--\end{array}$ & $\begin{array}{l}1.17 \\
0.04 C \\
3.42 \\
-.\end{array}$ & $\begin{array}{l}0.89 \\
0.016 \\
1.13 \\
=-\end{array}$ & $\begin{array}{l}3.48 \\
0.056 \\
1.44 \\
.-\end{array}$ & $\begin{array}{l}1.19 \\
0.026 \\
1.69 \\
-0\end{array}$ & $\begin{array}{l}3.76 \\
0.04 C \\
1.07 \\
-.\end{array}$ & $\begin{array}{l}5.59 \\
0.08 \mathrm{C} \\
1.44 \\
--\end{array}$ & $\begin{array}{l}4.90 \\
0.07 \mathrm{C} \\
1.43 \\
-.\end{array}$ & $\begin{array}{r}13.97 \\
0.11 \\
0.79 \\
63.00\end{array}$ & $\begin{array}{r}7.14 \\
0.13 \\
1.83 \\
53.00\end{array}$ & $\begin{array}{r}1.96 \\
0.07 \\
3.58 \\
83.00\end{array}$ & $\begin{array}{r}2.69 \\
0.32 \\
11.90 \\
59.00\end{array}$ & $\begin{array}{r}50.73 \\
1.07\end{array}$ \\
\hline 1962 & $\begin{array}{l}\text { PRECIP. (CM.) } \\
\text { SR-9O IMCI/SO.KM.) } \\
\text { SR-90 CONC. }(P C / L) \\
\text { SR-89/SR-90 }\end{array}$ & $\begin{array}{l}2.24 \\
0.10 \\
4.47 \\
--\end{array}$ & $\begin{array}{r}1.88 \\
0.25 \\
13.30 \\
32.00\end{array}$ & $\begin{array}{r}1.47 \\
0.22 \\
14.97 \\
19.00\end{array}$ & $\begin{array}{r}0.64 \\
0.35 \\
54.69 \\
19.00\end{array}$ & $\begin{array}{l}3.99 \\
0.33 \\
8.28 \\
9.00\end{array}$ & $\begin{array}{r}8.64 \\
0.88 \\
10.19 \\
6.00\end{array}$ & $\begin{array}{r}1.83 \\
0.42 \\
22.96 \\
4.00\end{array}$ & $\begin{array}{c}4.88 \\
0.44 C \\
9.02 \\
16.00 \mathrm{C}\end{array}$ & $\begin{array}{l}3.68 \\
0.33 \mathrm{C} \\
8.97 \\
16.00 \mathrm{C}\end{array}$ & $\begin{array}{r}3.96 \\
0.20 \\
5.08 \\
28.00\end{array}$ & $\begin{array}{r}1.24 \\
0.12 \\
9.68 \\
29.00\end{array}$ & $\begin{array}{r}3.00 \\
0.21 \\
7.01 \\
44.00\end{array}$ & $\begin{array}{r}37.45 \\
3.85\end{array}$ \\
\hline 1963 & $\begin{array}{l}\text { PRECIP. (CM.) } \\
\text { SR-90 (MCI/SQ.KM.) } \\
\text { SR-90 CONC. (PC/L) } \\
\text { SR-89/SR-90 }\end{array}$ & $\begin{array}{r}5.51 \\
0.32 \\
5.81 \\
33.00\end{array}$ & $\begin{array}{r}3.43 \\
0.87 \\
25.37 \\
26.00\end{array}$ & $\begin{array}{r}3.76 \\
0.42 \\
11.18 \\
14.00\end{array}$ & $\begin{array}{r}4.52 \\
0.78 \\
17.26 \\
10.00\end{array}$ & $\begin{array}{r}1.12 \\
0.65 \\
58.04 \\
7.20\end{array}$ & $\begin{array}{r}4.62 \\
1.38 \\
29.88 \\
3.10\end{array}$ & $\begin{array}{l}-- \\
-- \\
--\end{array}$ & $\begin{array}{r}7.24 \\
4.00 \\
55.25 \\
1.00\end{array}$ & $\begin{array}{r}2.49 \\
0.63 \\
25.31 \\
*\end{array}$ & $\begin{array}{r}2.57 \\
0.32 \\
12.46 \\
*\end{array}$ & $\begin{array}{r}0.30 \\
0.12 \\
40.01 \\
*\end{array}$ & $\begin{array}{l}4.19 \\
0.19 \\
4.54 \\
*\end{array}$ & $\begin{array}{r}39.75 \\
9.68\end{array}$ \\
\hline 1964 & $\begin{array}{l}\text { PRECIP. }\left(C M_{0}\right) \\
\text { SR-90 (MCI/SQ.KM ) } \\
\text { SR-90 CONC. (PC/L) } \\
\text { SR-89/SR-90 }\end{array}$ & $\begin{array}{r}0.89 \\
0.16 \\
17.98 \\
*\end{array}$ & $\begin{array}{l}2.92 \\
0.22 \\
7.54 \\
+\end{array}$ & $\begin{array}{r}2.92 \\
0.39 \\
13.36 \\
*\end{array}$ & $\begin{array}{l}1.78 B \\
0.47 B \\
26.41 \\
*\end{array}$ & $\begin{array}{l}2.46 \\
0.74 \\
30.09 \\
*\end{array}$ & $\begin{array}{r}4.39 \\
0.57 \\
12.99 \\
--\end{array}$ & $\begin{array}{l}-- \\
-- \\
-\end{array}$ & $\begin{array}{r}5.49 \\
0.62 \\
11.30 \\
-0\end{array}$ & $\begin{array}{r}2.06 \\
0.31 \\
15.05 \\
-\end{array}$ & $\begin{array}{l}5.82 \\
0.47 \\
8.08 \\
0.90\end{array}$ & $\begin{array}{l}6.88 \\
0.21 \\
3.06 \\
1.50\end{array}$ & $\begin{array}{l}1.60 \\
0.07 \\
4.38 \\
--\end{array}$ & $\begin{array}{r}37.22 \\
4.23\end{array}$ \\
\hline 1965 & $\begin{array}{l}\text { PREC IP. (CM.) } \\
\text { SR-90 IMCI/SQ.KM.) } \\
\text { SR-90 CONC. (PC/L) } \\
\text { SR-89/SR-90 }\end{array}$ & $\begin{array}{l}1.45 \\
0.06 \\
4.14 \\
-.\end{array}$ & $\begin{array}{l}1.70 \\
0.03 \\
1.77 \\
-.\end{array}$ & $\begin{array}{l}2.11 \\
0.19 \\
9.01 \\
-\end{array}$ & $\begin{array}{l}0.76 \\
0.05 \\
6.58 \\
-.\end{array}$ & $\begin{array}{l}1.30 \\
0.02 \\
1.54 \\
-.\end{array}$ & $\begin{array}{l}2.44 \\
0.24 \\
9.84 \\
-\end{array}$ & $\begin{array}{l}4.42 \\
0.35 \\
7.92 \\
-.\end{array}$ & $\begin{array}{l}4.01 \\
0.40 \\
9.98 \\
-.\end{array}$ & $\begin{array}{l}11.68 \\
0.29 \\
2.49 \\
--\end{array}$ & $\begin{array}{l}3.66 \\
0.05 \\
1.37 \\
-.\end{array}$ & $\begin{array}{l}4.70 \\
0.12 \\
2.56 \\
--\end{array}$ & $\begin{array}{l}3.48 \\
=- \\
=-\end{array}$ & $\begin{array}{r}41.71 \\
1.80\end{array}$ \\
\hline 1966 & $\begin{array}{l}\text { PRECIP. }(C M .) \\
\text { SR-9O (MCI/SQ.KM.) } \\
S R-90 \text { CONC }(P C / L) \\
\text { SR-89/SR-90 }\end{array}$ & $\begin{array}{l}1.60 \\
0.04 \\
2.51 \\
\end{array}$ & $\begin{array}{l}2.03 \\
0.05 \\
2.47 \\
-.\end{array}$ & $\begin{array}{l}1.12 \\
0.01 \\
0.90 \\
-\end{array}$ & $\begin{array}{l}1.78 \\
0.09 \\
5.06 \\
-0\end{array}$ & $\begin{array}{l}1.90 \\
0.12 \\
6.32 \\
-.\end{array}$ & $\begin{array}{r}0.68 \\
0.07 \\
10.30 \\
3.80\end{array}$ & $\begin{array}{l}1.80 \\
0.04 \\
2.23 \\
*\end{array}$ & $\begin{array}{l}6.30 \\
0.10 \\
1.59 \\
*\end{array}$ & $\begin{array}{l}6.22 \\
0.06 \\
0.97 \\
*\end{array}$ & $\begin{array}{l}2.18 \\
0.01 \\
0.46 \\
*\end{array}$ & $\begin{array}{r}2.82 \\
0.02 \\
0.71 \\
14.00\end{array}$ & $\begin{array}{l}2.69 \\
0.02 \\
0.75 \\
*\end{array}$ & $\begin{array}{r}31.12 \\
0.63\end{array}$ \\
\hline 1967 & $\begin{array}{l}\text { PRECIP. (CM.) } \\
\text { SR-90 (MCI/SQ.KM.) } \\
S R-90 \text { CONC. }(P C / L) \\
S R-89 / S R-90\end{array}$ & $\begin{array}{l}3.18 \\
0.02 \\
0.63 \\
\end{array}$ & $\begin{array}{l}2.56 \\
0.03 \\
1.18 \\
5.80\end{array}$ & $\begin{array}{l}2.49 \\
0.04 \\
1.61 \\
16.00\end{array}$ & $\begin{array}{l}1.24 \\
0.04 \\
3.23 \\
5.80\end{array}$ & $\begin{array}{l}2.72 \\
0.07 \\
2.58 \\
3.60\end{array}$ & $\begin{array}{l}3.66 \\
0.08 \\
2.19 \\
*\end{array}$ & $\begin{array}{l}6.27 \\
0.08 \\
1.28 \\
*\end{array}$ & $\overline{-}$ & $\begin{array}{l}-- \\
-- \\
--\end{array}$ & 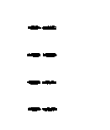 & $\begin{array}{l}-- \\
--\end{array}$ & $=$ & $\begin{array}{r}22.12 \\
0.36\end{array}$ \\
\hline $\begin{array}{c}\text { NOTE } \\
-A: \\
\text { A: } \\
\text { A: } \\
\text { C: }\end{array}$ & $\begin{array}{l}\text { AIA NOT AVAILABLE } \\
\text { ERO OR TRACE } \\
\text { PPROXIMATE } \\
\text { OWER LIMIT OF REPORT } \\
\text { ROPORTIONEO FROM OR I }\end{array}$ & $\begin{array}{l}\text { ED DATA } \\
\text { GINALLY }\end{array}$ & $c$ & TED DATA & & & & & & & & & & \\
\hline
\end{tabular}




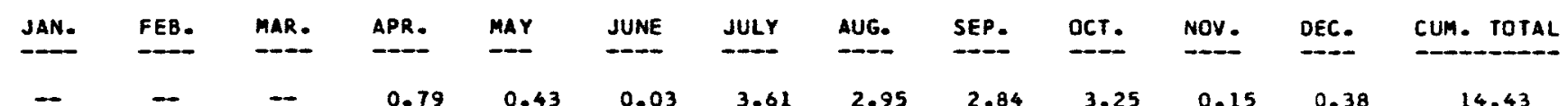

\begin{tabular}{|c|c|c|c|c|c|c|c|c|c|c|c|c|c|c|}
\hline 1959 & $\begin{array}{l}\text { PRECIP. ICM-1 } \\
\text { SR-90 (PCI/SO.KM-) } \\
\text { SR-90 CONC. (PC/L) } \\
\text { SR-89/SR-90 }\end{array}$ & $\bar{z}$ & $\begin{array}{l}\bar{z} \\
\bar{z}\end{array}$ & $\bar{z}$ & $\begin{array}{r}0.79 \\
0.01 \\
1.27 \\
12.00\end{array}$ & $\begin{array}{r}0.43 \\
0.15 \\
34.89 \\
8.00\end{array}$ & $\begin{array}{r}0.03 \\
0.07 \\
233.33 \\
5.70\end{array}$ & $\begin{array}{l}3.61 \\
0.32 \\
8.87 \\
3.60\end{array}$ & $\begin{array}{l}2.95 \\
0.07 \\
2.38 \\
2.40\end{array}$ & $\begin{array}{l}2.84 \\
0.01 \\
0.36 \\
*\end{array}$ & $\begin{array}{l}3.25 \\
0.01 \\
0.31 \\
\end{array}$ & $\begin{array}{l}0.15 \\
0.01 \\
6.67 \\
*\end{array}$ & $\begin{array}{l}0.38 \\
*- \\
*\end{array}$ & $\begin{array}{r}14.43 \\
0.65\end{array}$ \\
\hline 1960 & $\begin{array}{l}\text { PRECIP. (CM-) } \\
\text { SR-90 (NCI/SQ.KM.) } \\
\text { SR-90 CONC. (PC/L) } \\
\text { SR-89/SR-9O }\end{array}$ & $\begin{array}{r}0.36 \\
0.19 \\
52.78 \\
--\end{array}$ & $\begin{array}{l}3.30 \\
0.07 \\
2.13 \\
-.\end{array}$ & $\begin{array}{r}0.18 \\
0.07 \\
38.89 \\
--\end{array}$ & $\begin{array}{l}1.12 \\
0.05 \\
4.47 \\
-.\end{array}$ & $\begin{array}{l}0.51 \\
0.03 C \\
5.89 \\
--\end{array}$ & $\begin{array}{l}0.58 \\
0.03 \mathrm{C} \\
5.18 \\
--\end{array}$ & $\begin{array}{l}0.51 \\
-- \\
=\end{array}$ & $\begin{array}{l}3.00 \\
=- \\
=-\end{array}$ & $\begin{array}{l}1.35 \\
0.036 \\
2.23 \\
-\end{array}$ & $\begin{array}{l}0.94 \\
0.02 C \\
2.13 \\
--\end{array}$ & $\begin{array}{l}0.05 \\
0.00 C \\
0.01 \\
--\end{array}$ & $\begin{array}{l}0.99 \\
0.03 \mathrm{C} \\
3.04 \\
-0\end{array}$ & $\begin{array}{r}12.89 \\
0.52\end{array}$ \\
\hline 1961 & $\begin{array}{l}\text { PRECIP. (CM-) } \\
\text { SR-90 (NCI/SO.KM-) } \\
\text { SR-90 CCNC. (PC/L) } \\
\text { SR-89/SR-9O }\end{array}$ & $\begin{array}{c}0.69 \\
0.20 C \\
28.99 \\
--\end{array}$ & $\begin{array}{l}0.18 \\
0.050 \\
27.78 \\
--\end{array}$ & $\begin{array}{l}0.56 \\
0.04 c \\
7.15 \\
--\end{array}$ & $\begin{array}{l}0.38 \\
0.02 C \\
5.27 \\
--\end{array}$ & $\begin{array}{l}0.03 \\
0.00 \mathrm{C} \\
0.01 \\
--\end{array}$ & $\begin{array}{l}0.20 \\
0.03 \mathrm{C} \\
15.01 \\
-.\end{array}$ & $\begin{array}{l}4.57 \\
0.06 C \\
1.32 \\
--\end{array}$ & $\begin{array}{l}3.33 \\
0.04 C \\
1.21 \\
--\end{array}$ & $\begin{array}{r}0.79 \\
0.05 \\
6.33 \\
33.00\end{array}$ & $\begin{array}{r}1.12 \\
0.01 \\
c .90 \\
32.00\end{array}$ & $\begin{array}{l}0.48 \\
0.00 \\
0.01 \\
*\end{array}$ & $\begin{array}{l}0.28 \\
* \\
--\end{array}$ & $\begin{array}{r}12.61 \\
0.50\end{array}$ \\
\hline 1962 & $\begin{array}{l}\text { PRECIP. (CM.1 } \\
\text { SR-90 (MCI/SQ.KM.) } \\
\text { SR-90 CCNC. (PC/L) } \\
\text { SR-89/SR-90 }\end{array}$ & $\begin{array}{l}2.24 \\
* \\
*\end{array}$ & $\begin{array}{l}1.68 \\
* \\
*\end{array}$ & $\begin{array}{l}0.61 \\
\pm \\
=-\end{array}$ & $\begin{array}{r}0.66 \\
0.01 \\
1.52 \\
18.00\end{array}$ & $\begin{array}{r}14.22 \\
0.77 \\
5.42 \\
10.00\end{array}$ & $\begin{array}{l}-- \\
0.46 \\
\overline{5.00}\end{array}$ & $\begin{array}{l}\overline{-} \\
\bar{z}\end{array}$ & $\begin{array}{l}=- \\
=\end{array}$ & $\begin{array}{r}2.49 \\
0.59 \\
23.70 \\
18.00\end{array}$ & $\begin{array}{r}1.35 \\
0.00 \\
0.01 \\
16.00\end{array}$ & $\begin{array}{l}\bar{z} \\
\overline{=}\end{array}$ & $\begin{array}{l}0.15 \\
* \\
--\end{array}$ & $\begin{array}{r}23.40 \\
1.83\end{array}$ \\
\hline 1963 & $\begin{array}{l}\text { PRECIP. (CM-) } \\
\text { SR-90 IMCI/SQ.KM.) } \\
\text { SR-90 CONC. (PC/L) } \\
\text { SR-89/SR-9O }\end{array}$ & $\begin{array}{l}1.32 \\
0.06 \\
4.55 \\
40.00\end{array}$ & $\begin{array}{r}0.69 \\
0.12 \\
17.40 \\
19.00\end{array}$ & $\begin{array}{l}=- \\
\bar{z}\end{array}$ & $\begin{array}{l}\overline{-} \\
\overline{-}\end{array}$ & $\begin{array}{l}=- \\
=-\end{array}$ & $\begin{array}{l}=- \\
=- \\
=-\end{array}$ & $\begin{array}{l}= \\
=\end{array}$ & $\begin{array}{l}\bar{z} \\
\bar{z}\end{array}$ & $\begin{array}{r}0.48 \\
0.22 \\
45.84 \\
*\end{array}$ & $\begin{array}{l}2.34 \\
0.03 \\
1.29 \\
*\end{array}$ & $\begin{array}{r}0.33 \\
0.09 \\
27.28\end{array}$ & $\begin{array}{r}0.08 \\
0.09 \\
112.50 \\
--.\end{array}$ & $\begin{array}{l}5.24 \\
0.61\end{array}$ \\
\hline 1964 & $\begin{array}{l}\text { PRECIP. (CM.) } \\
\text { SR-90 IMCI/SQ.KM-) } \\
\text { SR-90 CONC. (PC/L) } \\
\text { SR-89/SR-SO }\end{array}$ & $\begin{array}{l}\bar{z} \\
\bar{z}\end{array}$ & $\bar{z}$ & $\bar{z}$ & $\bar{z}=$ & $\begin{array}{l}=- \\
=\end{array}$ & $\begin{array}{r}0.91 \\
0.25 \\
27.48 \\
-\end{array}$ & $\begin{array}{r}0.20 \\
0.10 \\
50.01 \\
--\end{array}$ & $\begin{array}{r}0.91 \\
0.21 \\
23.08 \\
--\end{array}$ & $\begin{array}{l}0.25 \\
0.02 \\
8.01 \\
-.\end{array}$ & $\begin{array}{l}2.06 \\
0.03 \\
1.46 \\
*\end{array}$ & $\begin{array}{l}0.74 \\
0.01 \\
1.36 \\
*\end{array}$ & $\begin{array}{l}0.43 \\
* \\
= \\
-\end{array}$ & $\begin{array}{l}5.50 \\
0.62\end{array}$ \\
\hline 1965 & $\begin{array}{l}\text { PRECIP_ (CM-) } \\
\text { SR-90 (MCI/SQ.KM-) } \\
\text { SR-90 CCNC. (PC/L) } \\
\text { SR-89/SR-90 }\end{array}$ & $\begin{array}{l}0.10 \\
* \\
=\end{array}$ & $\begin{array}{l}* \\
* \\
--\end{array}$ & $\begin{array}{l}0.74 \\
=\end{array}$ & $\begin{array}{l}1.68 \\
0.01 \\
0.60 \\
--\end{array}$ & $\begin{array}{l}0.94 \\
0.02 \\
2.13 \\
--\end{array}$ & $\begin{array}{r}0.13 \\
0.03 \\
23.08 \\
--\end{array}$ & $\begin{array}{l}2.136 \\
0.066 \\
2.82 \\
-.\end{array}$ & $\begin{array}{l}2.13 C \\
0.076 \\
3.29 \\
-\end{array}$ & $\begin{array}{l}1.09 \\
=- \\
=-\end{array}$ & $\begin{array}{l}1.73 \\
0.02 \\
1.16 \\
-\end{array}$ & $\begin{array}{l}2.92 \\
0.07 \\
2.40 \\
-\end{array}$ & $\begin{array}{l}1.30 \\
0.02 \\
1.54 \\
--\end{array}$ & $\begin{array}{r}14.89 \\
0.30\end{array}$ \\
\hline 1966 & $\begin{array}{l}\text { PRECIP. ICM-) } \\
\text { SR-90 (MCI/SQ-KM.) } \\
\text { SR-90 EONC. (PC/L) } \\
\text { SR-89/SR-90 }\end{array}$ & $\begin{array}{r}0.30 \\
0.05 \\
16.67 \\
--\end{array}$ & $\begin{array}{r}0.15 \\
0.08 \\
53.34 \\
--\end{array}$ & $\begin{array}{l}0.51 \\
0.01 \\
1.97 \\
-.\end{array}$ & $\begin{array}{l}0.71 \\
0.02 \\
2.82 \\
--\end{array}$ & $\begin{array}{l}0.38 \\
0.01 \\
2.64 \\
--\end{array}$ & $\begin{array}{l}0.94 \\
0.05 \\
5.32 \\
*\end{array}$ & $\begin{array}{l}5.33 \\
0.18 \\
3.38 \\
2.80\end{array}$ & $\begin{array}{l}1.45 \\
0.04 \\
2.76 \\
*\end{array}$ & $\begin{array}{l}1.22 \\
0.02 \\
1.64 \\
*\end{array}$ & $\begin{array}{l}1.24 \\
0.01 \\
0.81 \\
*\end{array}$ & $\begin{array}{l}1.30 \\
0.03 \\
2.31 \\
9.30\end{array}$ & $\begin{array}{l}0.58 \\
0.01 \\
1.73 \\
*\end{array}$ & $\begin{array}{r}14.11 \\
0.51\end{array}$ \\
\hline 1967 & $\begin{array}{l}\text { PRECIP. (CM-) } \\
\text { SR-90 (MCI/SO-KM-) } \\
\text { SR-90 CONC. (PC/L) } \\
\text { SR-89/SR-90 }\end{array}$ & $\begin{array}{l}0.48 \\
0.01 \\
2.09 \\
*\end{array}$ & $\begin{array}{r}0.05 \\
0.01 \\
20.01\end{array}$ & $\begin{array}{l}0.96 \\
0.02 \\
2.09 \\
*\end{array}$ & $\begin{array}{l}0.33 \\
0.01 \\
3.04 \\
*\end{array}$ & $\begin{array}{l}0.64 \\
0.01 \\
1.57 \\
*\end{array}$ & $\begin{array}{l}0.74 \\
0.02 \\
2.71 \\
*\end{array}$ & $\bar{z}$ & $\bar{z}$ & $\bar{z}$ & $\begin{array}{l}\overline{-} \\
\bar{z}\end{array}$ & $\begin{array}{l}\overline{-} \\
=\end{array}$ & $\begin{array}{l}\bar{z} \\
\bar{z}\end{array}$ & $\begin{array}{l}3.20 \\
0.08\end{array}$ \\
\hline
\end{tabular}

NOTES

- : DATA NOT AVAILABLE

*: ZERO OR TRACE

A: APPROXIMATE

8: LOWER LIMIT OF REPORTED DATA

C: PROPORTIONEC FROM ORTGINALLY CONSOLTDATED DATA 


\begin{tabular}{|c|c|c|c|c|c|c|c|c|c|c|c|c|c|c|}
\hline & & JAN. & FEB. & MAR. & APR. & MAY & JUNE & JULY & AUG. & SEP. & OCT. & NOV. & DEC. & CUM. TOTAL \\
\hline 1959 & $\begin{array}{l}\text { PRECIP. (CM.) } \\
\text { SR-9O IMCI/SO-KM-) } \\
\text { SR-9O CONC. (PC/L) } \\
\text { SR-89/SR-90 }\end{array}$ & $=$ & $\overline{-}$ & $=-$ & $\begin{array}{r}5.94 \\
0.80 \\
13.47 \\
9.00\end{array}$ & $\begin{array}{r}6.50 \\
0.72 \\
11.08 \\
11.00\end{array}$ & $\begin{array}{r}5.28 \\
1.37 \\
25.95 \\
5.00\end{array}$ & $\begin{array}{l}8.20 \\
0.68 \\
8.30 \\
3.10\end{array}$ & $\begin{array}{l}5.72 \\
0.22 \\
3.85 \\
1.40\end{array}$ & $\begin{array}{r}13.39 \\
0.15 \\
1.13 \\
0.46\end{array}$ & $\begin{array}{r}11.05 \\
0.06 \\
0.55 \\
1.20\end{array}$ & $\begin{array}{r}15.06 \\
0.23 \\
1.53 \\
0.40\end{array}$ & $\begin{array}{l}3.48 \\
0.17 \\
4.89 \\
0.60\end{array}$ & $\begin{array}{r}74.62 \\
4.40\end{array}$ \\
\hline 1960 & $\begin{array}{l}\text { PRECIP. (CM.) } \\
\text { SR-9O (NCI/SQ.KM.) } \\
\text { SR-90 CONC. (PC/L) } \\
\text { SR-Q9/SR-90 }\end{array}$ & $\begin{array}{l}9.53 \\
-- \\
-\end{array}$ & $\begin{array}{l}6.15 \\
0.12 \\
1.96 \\
-.\end{array}$ & $\begin{array}{l}1.65 \\
0.08 \\
4.85 \\
-.\end{array}$ & $\begin{array}{l}3.91 \\
0.00 \\
0.01 \\
-\end{array}$ & $\begin{array}{l}5.00 \\
C .08 C \\
1.61 \\
. .\end{array}$ & $\begin{array}{l}3.99 \\
0.076 \\
1.76 \\
-.\end{array}$ & $\begin{array}{l}3.00 \\
0.03 C \\
1.01 \\
.0\end{array}$ & $\begin{array}{l}11.00 \\
0.10 \mathrm{C} \\
0.91 \\
--\end{array}$ & $\begin{array}{l}6.99 \\
0.04 C \\
0.58 \\
--\end{array}$ & $\begin{array}{l}10.16 \\
0.06 \mathrm{C} \\
0.60 \\
--\end{array}$ & $\begin{array}{l}22.71 \\
0.06 C \\
0.27 \\
--\end{array}$ & $\begin{array}{c}11.99 \\
0.03 C \\
0.26 \\
--\end{array}$ & $\begin{array}{r}96.08 \\
0.67\end{array}$ \\
\hline 1961 & $\begin{array}{l}\text { PRECIP. (CM.) } \\
\text { SR-9O (NCI/SC.KM.) } \\
\text { SR-90 CONC. (PCAL) } \\
S R-89 / S R-90\end{array}$ & $\begin{array}{l}4.95 \\
0.036 \\
0.01 \\
-.\end{array}$ & $\begin{array}{l}4.11 \\
C .03 C \\
C .73 \\
--\end{array}$ & $\begin{array}{l}4.39 \\
0.08 C \\
1.83 \\
--\end{array}$ & $\begin{array}{l}4.27 \\
0.08 C \\
1.88 \\
-.\end{array}$ & $\begin{array}{l}2.41 \\
C .01 C \\
0.42 \\
--\end{array}$ & $\begin{array}{l}0.30 \\
0.006 \\
0.01 \\
--\end{array}$ & $\begin{array}{l}2.74 \\
-- \\
--\end{array}$ & $\begin{array}{l}8.89 \\
-- \\
--\end{array}$ & $\begin{array}{r}4.90 \\
0.14 \\
2.86 \\
78.00\end{array}$ & $\begin{array}{r}4.85 \\
0.17 \\
3.51 \\
75.00\end{array}$ & $\begin{array}{r}10.41 \\
.0 .29 \\
2.79 \\
85.00\end{array}$ & $\begin{array}{l}7.09 \\
=- \\
=-\end{array}$ & $\begin{array}{r}59.31 \\
0.83\end{array}$ \\
\hline 1962 & $\begin{array}{l}\text { PRECIP. (CM.) } \\
\text { SR-9O (MCI/SO.KM.) } \\
\text { SR-9O CCNC. (PC/L) } \\
S R-89 / S R-90\end{array}$ & $\begin{array}{l}6.43 \\
-- \\
--\end{array}$ & $\begin{array}{l}3.89 \\
-- \\
--\end{array}$ & $=$ & $\begin{array}{r}1.93 \\
0.26 \\
13.48 \\
14.00\end{array}$ & $\begin{array}{r}6.02 \\
1.52 \\
25.25 \\
7.00\end{array}$ & $\begin{array}{r}0.30 \\
1.00 \\
333.34 \\
10.00\end{array}$ & $\begin{array}{r}8.69 \\
0.32 \\
3.69 \\
15.00\end{array}$ & $\begin{array}{r}5.03 \\
0.69 \\
13.72 \\
13.00\end{array}$ & $\begin{array}{r}9.75 \\
0.50 \\
5.13 \\
24.00\end{array}$ & $\begin{array}{r}10.57 \\
0.17 \\
1.61 \\
33.00\end{array}$ & $\begin{array}{r}6.53 \\
0.24 \\
3.68 \\
41.00\end{array}$ & $\begin{array}{r}2.59 \\
0.11 \\
4.25 \\
43.00\end{array}$ & $\begin{array}{r}61.73 \\
4.81\end{array}$ \\
\hline 1963 & $\begin{array}{l}\text { PRECIP. }(C M-) \\
\text { SR-9O } \text { (NCI/SQ.KM }) \\
S R-90 \text { CCNC. }(P C / L) \\
S R-89 / S R-90\end{array}$ & $\begin{array}{r}12.47 \\
0.30 \\
2.41 \\
30.00\end{array}$ & $\begin{array}{r}1.09 \\
0.24 \\
22.02 \\
29.00\end{array}$ & $\begin{array}{r}7.67 \\
0.72 \\
9.39 \\
13.00\end{array}$ & $\begin{array}{r}4.22 \\
1.20 \\
28.44 \\
4.00\end{array}$ & $\begin{array}{r}3.35 \\
1.04 \\
31.05 \\
5.90\end{array}$ & $\begin{array}{r}2.69 \\
1.06 \\
39.41 \\
1.20\end{array}$ & $\begin{array}{r}1 C .87 \\
2.82 \\
25.95 \\
2.00\end{array}$ & $\begin{array}{l}6.83 \\
2.31 \\
33.83 \\
*\end{array}$ & $\begin{array}{r}9.17 \\
1.46 \\
15.93 \\
*\end{array}$ & $\begin{array}{l}7.67 \\
C .37 \\
4.83 \\
*\end{array}$ & $\begin{array}{l}3.71 \\
0.30 \\
8.09 \\
*\end{array}$ & $\begin{array}{l}5.11 \\
0.25 \\
4.90 \\
+\end{array}$ & $\begin{array}{l}74.85 \\
12.07\end{array}$ \\
\hline 1964 & $\begin{array}{l}\text { PRECIP }(C M-) \\
S R-90 \text { (MCI/SO.KM.) } \\
S R-90 \text { CCNC. }(P C / L) \\
S R-89 / S R-90\end{array}$ & $\begin{array}{r}3.38 \\
0.60 \\
17.76 \\
*\end{array}$ & $\begin{array}{r}4.39 \\
0.46 \\
10.48 \\
0.20\end{array}$ & $\begin{array}{c}3.35 \\
0.51 \\
15.23 \\
*\end{array}$ & $\begin{array}{r}0.53 \\
0.87 \\
164.16 \\
*\end{array}$ & $\begin{array}{r}2.92 \\
1.15 \\
39.39 \\
*\end{array}$ & $\begin{array}{r}4.70 \\
1.03 \\
21.92 \\
--\end{array}$ & $\begin{array}{r}4.27 \\
1.14 \\
26.70 \\
-\end{array}$ & $\begin{array}{l}9.04 \\
0.90 \\
9.96 \\
--\end{array}$ & $\begin{array}{l}13.34 \\
0.60 \\
4.50 \\
-.\end{array}$ & $\begin{array}{r}8.56 \\
0.05 \\
0.59 \\
10.00\end{array}$ & $=$ & $\begin{array}{r}3.33 \\
0.43 \\
12.92 \\
--\end{array}$ & $\begin{array}{r}57.81 \\
7.74\end{array}$ \\
\hline 1965 & $\begin{array}{l}\text { PRECIP (CM.) } \\
\text { SR-9O IMCI/SQ.KM-) } \\
\text { SR-9O CONC. (PC/L) } \\
\text { SR-89/SR-90 }\end{array}$ & $\begin{array}{l}0.40 \\
0.02 \\
0.32 \\
-\end{array}$ & $\begin{array}{l}7.06 \\
0.19 \\
2.70 \\
--\end{array}$ & $\begin{array}{l}7.75 \\
0.64 \\
8.26 \\
-.\end{array}$ & $\begin{array}{l}2.11 \\
0.19 \\
9.01 \\
-.\end{array}$ & $\begin{array}{l}6.76 \\
0.42 \\
6.22 \\
--\end{array}$ & $\begin{array}{r}6.45 \\
0.79 \\
12.25 \\
--\end{array}$ & $\begin{array}{r}3.05 \\
0.46 \\
15.09 \\
-\end{array}$ & $\begin{array}{l}3.99 \\
0.29 \\
7.27 \\
-.\end{array}$ & $\begin{array}{l}24.87 \\
1.10 \\
4.43 \\
-.\end{array}$ & $\begin{array}{l}6.99 \\
C .15 \\
2.15 \\
-\end{array}$ & $\begin{array}{l}6.53 \\
-- \\
--\end{array}$ & $\begin{array}{l}4.50 \\
0.46 \\
10.23 \\
--\end{array}$ & $\begin{array}{r}86.46 \\
4.71\end{array}$ \\
\hline 1966 & $\begin{array}{l}\text { PRECIP ( }(C M .) \\
S R-90 \text { (MCI/SQ.KM.) } \\
S R-90 \text { CONC. }(P C / L) \\
S R-89 / S R-90\end{array}$ & $\begin{array}{l}5.60 \\
-- \\
--\end{array}$ & $\begin{array}{l}4.00 \\
-- \\
--\end{array}$ & $\begin{array}{l}3.60 \\
-\end{array}$ & $=$ & 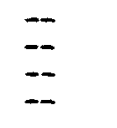 & $m$ & $\begin{array}{r}11.76 \\
0.09 \\
0.77 \\
*\end{array}$ & $\begin{array}{l}9.50 \\
0.18 \\
1.90 \\
*\end{array}$ & $\begin{array}{l}10.89 \\
0.07 \\
0.65 \\
*\end{array}$ & $\begin{array}{l}7.52 \\
0.04 \\
0.54 \\
*\end{array}$ & $\begin{array}{r}7.04 \\
0.08 \\
1.14 \\
17.00\end{array}$ & $\begin{array}{l}5.31 \\
0.10 \\
1.89 \\
*\end{array}$ & $\begin{array}{r}65.22 \\
0.56\end{array}$ \\
\hline 1967 & $\begin{array}{l}\text { PRECIP. (CM.) } \\
\text { SR-90 (MCI/SQ.KM.) } \\
\text { SR-90 CCNC. (PC/L) } \\
\text { SR-89/SR-90 }\end{array}$ & $\begin{array}{l}4.06 \\
0.07 \\
1.73 \\
9.30\end{array}$ & $\begin{array}{l}6.55 \\
0.09 \\
1.38 \\
9.50\end{array}$ & $\begin{array}{l}6.45 \\
0.04 \\
0.63 \\
9.90\end{array}$ & $\begin{array}{l}-- \\
=- \\
=-\end{array}$ & $\begin{array}{l}1.57 \\
0.14 \\
8.92 \\
3.90\end{array}$ & $\begin{array}{l}-\overline{0.16} \\
\overline{1.40}\end{array}$ & $\begin{array}{l}-- \\
0.04 \\
-\end{array}$ & $\begin{array}{l}= \\
-- \\
-\end{array}$ & $\begin{array}{l}=- \\
= \\
--\end{array}$ & $\begin{array}{l}-- \\
= \\
--\end{array}$ & $=$ & $\begin{array}{l}= \\
=- \\
--\end{array}$ & $\begin{array}{r}18.63 \\
0.54\end{array}$ \\
\hline \multicolumn{15}{|l|}{$\begin{array}{l}\text { NOTES } \\
--: D \\
A: Z E \\
A: A P \\
B: L \\
C: P\end{array}$} \\
\hline
\end{tabular}




\begin{tabular}{|c|c|c|c|c|c|c|c|c|c|c|c|c|c|c|}
\hline & & JAN. & FEB. & MAR. & APR. & MAY & JUNE & JULY & AUG. & SEP. & OCT. & NOV. & DEC. & CUM. TOIAL \\
\hline 1960 & $\begin{array}{l}\text { PRECIP. (CM.) } \\
\text { SR-9C (NCI/SQ.KM.) } \\
S R-90 \text { CONC. }(P C / L) \\
\text { SR-89/SR-9O }\end{array}$ & $\begin{array}{l}-- \\
--\end{array}$ & 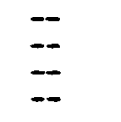 & $\begin{array}{l}0.51 \\
0.07 \\
13.73 \\
-.\end{array}$ & $\begin{array}{l}0.99 \\
0.08 \\
8.09 \\
--\end{array}$ & $\begin{array}{l}0.61 \\
0.63 c \\
4.92 \\
-.\end{array}$ & $\begin{array}{l}2.01 \\
0.08 C \\
3.99 \\
--\end{array}$ & $\begin{array}{l}3.51 \\
0.06 C \\
1.71 \\
-.\end{array}$ & $\begin{array}{l}3.99 \\
0.076 \\
1.76 \\
--\end{array}$ & $\begin{array}{l}7.75 \\
0.026 \\
0.26 \\
-.\end{array}$ & $\begin{array}{l}2.34 \\
0.01 C \\
0.43 \\
--\end{array}$ & $\begin{array}{l}1.04 \\
\pm \\
--\end{array}$ & $\begin{array}{l}0.58 \\
+- \\
--\end{array}$ & $\begin{array}{r}23.33 \\
0.42\end{array}$ \\
\hline 1961 & $\begin{array}{l}\text { PRECIP. (CM.) } \\
S R-9 C \text { (NCI/SQ-KM.) } \\
S R-90 \text { CCNC. }(P C / L) \\
S R-89 / S R-90\end{array}$ & $\begin{array}{l}0.69 \\
\div \\
=-\end{array}$ & $\begin{array}{l}0.43 \\
- \\
=-\end{array}$ & $\begin{array}{l}1.19 \\
0.04 C \\
3.37 \\
-.\end{array}$ & $\begin{array}{l}0.94 \\
0.03 C \\
3.20 \\
-.\end{array}$ & $\begin{array}{l}0.61 \\
0.04 C \\
6.56 \\
--\end{array}$ & $\begin{array}{l}2.26 \\
0.15 C \\
6.64 \\
--\end{array}$ & $\begin{array}{l}0.05 \\
0.036 \\
0.50 \\
-\end{array}$ & $\begin{array}{l}7.24 \\
0.03 C \\
0.42 \\
-.\end{array}$ & $\begin{array}{r}4.85 \\
0.15 \\
3.10 \\
32.00\end{array}$ & $\begin{array}{r}2.97 \\
0.02 \\
0.68 \\
70.00\end{array}$ & $\begin{array}{r}1.19 \\
0.03 \\
2.53 \\
58.00\end{array}$ & $\begin{array}{r}1.50 \\
0.05 \\
3.34 \\
51.00\end{array}$ & $\begin{array}{r}29.92 \\
0.57\end{array}$ \\
\hline 1962 & $\begin{array}{l}\text { PRECIP. (CM.) } \\
\text { SR-9O (NCI/SQ.KM.) } \\
\text { SR-9O CONC. (PC/L) } \\
\text { SR-89/SR-SO }\end{array}$ & $\begin{array}{r}1.75 \\
0.08 \\
4.58 \\
58.00\end{array}$ & $\begin{array}{r}3.20 \\
0.22 \\
6.88 \\
27.00\end{array}$ & $\begin{array}{r}1.93 \\
0.18 \\
9.33 \\
19.00\end{array}$ & $\begin{array}{r}0.69 \\
0.08 \\
11.60 \\
10.00\end{array}$ & $\begin{array}{r}1.57 \\
0.22 \\
14.02 \\
9.00\end{array}$ & $\begin{array}{r}5.64 \\
0.99 \\
17.56 \\
7.00\end{array}$ & $\begin{array}{r}11.05 \\
0.76 \\
6.88 \\
6.00\end{array}$ & $\begin{array}{r}12.70 \\
0.66 \\
5.20 \\
33.00\end{array}$ & $\begin{array}{r}1.09 \\
0.13 \\
11.93 \\
51.00\end{array}$ & $\begin{array}{r}0.71 \\
0.07 \\
9.86 \\
28.00\end{array}$ & $\begin{array}{r}0.43 \\
0.25 \\
58.14 \\
43.00\end{array}$ & $\begin{array}{r}1.42 \\
0.04 \\
2.82 \\
50.00\end{array}$ & $\begin{array}{r}42.18 \\
3.68\end{array}$ \\
\hline 1963 & $\begin{array}{l}\text { PRECIP. }(C M-) \\
\text { SR-9O INCI/SQ-KM.) } \\
\text { SR-90 CCNC. }(P C / L) \\
\text { SR-B9/SR-9O }\end{array}$ & $\begin{array}{r}4.52 \\
0.15 \\
3.32 \\
36.00\end{array}$ & $\begin{array}{r}0.69 \\
0.35 \\
50.73 \\
25.00\end{array}$ & $\begin{array}{r}5.33 \\
0.60 \\
11.26 \\
18.00\end{array}$ & $\begin{array}{r}1.24 \\
0.40 \\
32.26 \\
11.00\end{array}$ & $\begin{array}{r}0.28 \\
0.30 \\
107.15 \\
5.60\end{array}$ & $\begin{array}{r}5.11 \\
2.43 \\
47.56 \\
*\end{array}$ & $\begin{array}{r}6.22 \\
2.54 \\
40.84 \\
2.00\end{array}$ & $\begin{array}{l}6.38 \\
1.62 \\
25.40 \\
*\end{array}$ & $\begin{array}{r}0.48 \\
0.19 \\
39.59 \\
*\end{array}$ & $\begin{array}{l}4.14 \\
0.17 \\
4.11 \\
2.10\end{array}$ & $\begin{array}{l}0.46 \\
0.02 \\
4.35 \\
*\end{array}$ & $\begin{array}{l}0.97 \\
0.07 \\
7.22 \\
*\end{array}$ & $\begin{array}{r}35.82 \\
8.84\end{array}$ \\
\hline 1964 & $\begin{array}{l}\text { PRECIP. (CM.) } \\
\text { SR-90 IMCI/SQ.KM.) } \\
S R-90 \text { CCNC. }(P C / L) \\
S R-89 / S R-90\end{array}$ & $\begin{array}{l}1.754 \\
0.06 \\
3.43 \\
*\end{array}$ & $\begin{array}{l}3.684 \\
C .11 \\
2.99 \\
0.30\end{array}$ & $\begin{array}{l}1.85 A \\
0.07 \\
3.79 \\
*\end{array}$ & $\begin{array}{r}1.73 \\
0.22 \\
12.72 \\
0.61\end{array}$ & $\begin{array}{r}2.46 \\
0.44 \\
17.89 \\
2.00\end{array}$ & $\begin{array}{r}3.38 \\
1.37 \\
40.54 \\
--\end{array}$ & $\begin{array}{l}3.25 \\
0.01 \\
0.31 \\
-.\end{array}$ & $\begin{array}{l}0.02 \\
0.61 \\
10.14 \\
-.\end{array}$ & $\begin{array}{l}2.16 \\
0.14 \\
6.49 \\
-.\end{array}$ & $\begin{array}{l}1.35 \\
0.12 \\
8.89 \\
5.00\end{array}$ & $\begin{array}{l}2.18 \\
0.09 \\
4.13 \\
+\end{array}$ & $\begin{array}{l}0.86 \\
0.07 \\
8.14 \\
=-\end{array}$ & $\begin{array}{r}30.67 \\
3.31\end{array}$ \\
\hline 1965 & $\begin{array}{l}\text { PRECIP. (CM.) } \\
\text { SR-9O IMCI/SQ.KM.) } \\
\text { SR-9O CCNC. (PC/L) } \\
\text { SR-89/SR-SO }\end{array}$ & $\begin{array}{r}0.18 \\
0.04 \\
22.23 \\
--\end{array}$ & $\begin{array}{l}0.81 \\
0.02 \\
2.47 \\
+.\end{array}$ & $\begin{array}{r}0.66 \\
0.14 \\
21.22 \\
--\end{array}$ & $\begin{array}{l}1.19 \\
0.11 \\
9.25 \\
-.\end{array}$ & $\begin{array}{c}0.36 \\
0.07 \\
19.45 \\
-.\end{array}$ & $\begin{array}{r}2.95 \\
0.55 \\
18.65 \\
--\end{array}$ & $\begin{array}{l}3.53 \\
0.25 \\
7.09 \\
--\end{array}$ & $\begin{array}{l}3.76 \\
0.29 \\
7.72 \\
-.\end{array}$ & $\begin{array}{l}5.36 \\
0.14 \\
2.62 \\
--\end{array}$ & $\begin{array}{l}1.91 \\
0.28 \\
14.66 \\
--\end{array}$ & $\begin{array}{l}3.07 \\
0.06 \\
1.96 \\
-.\end{array}$ & $\begin{array}{l}4.88 \\
0.10 \\
2.05 \\
--\end{array}$ & $\begin{array}{r}28.66 \\
2.05\end{array}$ \\
\hline 1966 & $\begin{array}{l}\text { PRECIP. (CM.) } \\
\text { SR-9O INCI/SQ.KM.) } \\
\text { SR-SC CCNC. (PC/L) } \\
\text { SR-89/SR-90 }\end{array}$ & $\begin{array}{r}0.02 \\
0.03 \\
15 \mathrm{C} .00 \\
\end{array}$ & $\begin{array}{l}4.44 \\
* \\
-- \\
--\end{array}$ & $\begin{array}{l}0.86 \\
0.03 \\
3.49 \\
-.\end{array}$ & $\begin{array}{l}0.81 \\
* \\
-- \\
-\end{array}$ & $\begin{array}{r}0.96 \\
0.13 \\
13.55 \\
-.\end{array}$ & $\begin{array}{r}0.48 \\
0.12 \\
25.01 \\
6.40\end{array}$ & $\begin{array}{l}2.11 \\
0.09 \\
4.27 \\
*\end{array}$ & $\begin{array}{l}1.50 \\
0.06 \\
4.01 \\
*\end{array}$ & $\begin{array}{l}0.38 \\
+ \\
--\end{array}$ & $\begin{array}{l}1.57 \\
0.02 \\
1.28 \\
*\end{array}$ & $\begin{array}{l}4.37 \\
0.04 \\
0.92 \\
*\end{array}$ & $\begin{array}{l}0.41 \\
0.01 \\
2.44 \\
+\end{array}$ & $\begin{array}{r}17.91 \\
0.53\end{array}$ \\
\hline 1967 & $\begin{array}{l}\text { PRECIP }(C M)) \\
\text { SR-9O (MCI/SQ.KM.) } \\
\text { SR-9O CONC. (PC/L) } \\
\text { SR-89/SR-90 }\end{array}$ & $\begin{array}{l}1.02 \\
0.02 \\
1.97 \\
*\end{array}$ & $\begin{array}{l}2.21 \\
0.01 \\
0.46\end{array}$ & $\begin{array}{r}3.25 \\
0.02 \\
0.62 \\
11.00\end{array}$ & $\begin{array}{l}2.13 \\
0.01 \\
0.47 \\
*\end{array}$ & $\begin{array}{l}1.09 \\
0.05 \\
4.59 \\
4.00\end{array}$ & $\begin{array}{l}2.87 \\
0.07 \\
2.44 \\
+\end{array}$ & 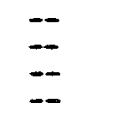 & $\overline{--}$ & $\begin{array}{l}-- \\
--\end{array}$ & $\begin{array}{l}-- \\
-- \\
--\end{array}$ & $\begin{array}{l}-- \\
-- \\
--\end{array}$ & $\begin{array}{l}-- \\
-- \\
--\end{array}$ & $\begin{array}{r}12.57 \\
0.18\end{array}$ \\
\hline \begin{tabular}{ll} 
NOTES \\
\hdashline$:$ & $D$ \\
$*:$ & $Z$ \\
$A:$ & $A$ \\
$B:$ & $L$ \\
$C:$ & $P$
\end{tabular} & $\begin{array}{l}\text { ATA NOT AVAILABLE } \\
\text { ERO OR TRACE } \\
\text { PPROXIMATE } \\
\text { OWER LIMIT OF REPOR } \\
\text { ROPORTIONED FROM OF }\end{array}$ & $\begin{array}{l}\text { OATA } \\
\text { NALY }\end{array}$ & & & & & & & & & & & & \\
\hline
\end{tabular}


JAN. FEB. MAR. APR.

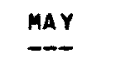

\section{JUNE}

JULY

AUG.

SEP.

OCT.

NOV.

DEC.

CUM. TOTAL

1959 PRECIP. (CM.) $S R-90$ (MCI/SO.KM.) $S R-90$ CONC.

$\overline{--}$

1960 PRECIP. (CM.)

SRSRTSO INCISSO.KM.) $S R-89 / S R-90$

1.7

1961 PRECIP. (CM.) 1 SR -90 CONC. (PCAL) $S R-90$ CONC.
$S R-89 / S R-90$

$\begin{array}{lll}-- & = \\ -- & = & =\end{array}$

9.63
2.17
22.54

\subsection{5}

18.77

14.61

14.83

15.34

17.32

22.54
7.50

15.95
5.00

3.6

2.47
1.80

1.22
0.90

0.59

$0.21 \quad 0.13$

$1.22 \quad 0.88$

0.88
0.80

$\begin{array}{lrrrlllll}9.80 & 5.21 & 13.36 & 3.99 & 3.99 & 8.99 & 11.00 & 12.12 & 22.00 \\ 0.17 & 0.10 & 0.15 & 0.11 & 0.10 C & 0.24 C & 0.22 C & 0.24 C & -\end{array}$

1.74

1.92

0.15

0.116

22.73

13.00

0.06
0.47

0.99

0.97

$-$

$5.55 \quad 10.34$

$6.78 \quad 9.96 \quad 12.07$

0.18

15.3431 .27

17.81

25.91

15.49
0.34

10.26

C.88

2.81

2.82

1.83

1.84

0.13

0.26

$--$

2.17

2.20

0.28
2.73

2.73
62.00

$1.93 \quad 12.70$

5.05

7.24

PRECIP. (CM.) 17.75 $S R-90$ CCNC. $(P C / L)$
$S R-89 / S R-90$

c.06

$\begin{array}{lrr}3.11 & 4.02 & 1.00 \\ * & 30.00 & 15.00\end{array}$

$\begin{array}{rr}7.24 & 12.07 \\ \text { C. } 52 & 1.39\end{array}$

12.0

13.23

24.77

18.77

10.24

20.73

$\begin{array}{ll}7.19 & 11.52 \\ 9.00 & 10.00\end{array}$

0.98
8.12

0.36
2.73

1.42
5.74

0.94
5.01

0.72

0.71

$8.00 \quad 8.00 \quad 23.00$

32.00

47.00

1963 PRECIP. (CM.)

16.64 SR-90 CONC. $(P C / L)$

9.53

9.78

5.13
1.13

11.51

$13.26 \quad 3.05 \quad=$

19.76

9.93

0.41

11.58

3.7

4.13

7.86

1964 PRECIP. (CM.) SR-9O CONC. (PC/L) $1 C^{\circ .66}$ $S R-89 / S R-90$

\subsection{4}

19.00

10.00

8.30

24.33
4.30

3.00
2.00

45.

11.05

17.40

17.63

8.84

6.58

18.67

$12.42 \quad 10.77$

.77
0.33 $2.02 \quad 3.07$

*.29 11

1965 PRECIP. (CM.) $\begin{array}{lll}S R-90 & 19.69 & 12.95\end{array}$

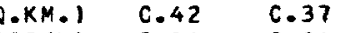
SR-B9/SR -90 (PC/L) 2.14

$\begin{array}{ll}2.95 & 4.22 \\ C .37 & 0.38 \\ 2.86 & 9.01\end{array}$

2.86

-.

*

15.52

6.87

9.73

0.31

7.10

10.82

$3.71 \quad 10.82$

$2.16 \quad 1.67$

122.30

$6.27 \quad 5.31$

0.56

$5.74 \quad 11.28 \quad 5.94$

4.7

2.7

26.10

$-$

1966 PRECIP. (CM.)

11.00

SR-GO (MCI/SQ.KM.) --

$S R-90$ CONC.
$S R-89 / S R-90$

$7.95 \quad 16.15$

$5.28 \quad 16.10$

4.42

$9.93 \quad 16.18$

20.83

17.70

11.15

11. 25

$0.03 \quad 0.10$

147.94

$6.17 \quad 2.42$

$\begin{array}{lll}5.12 & -- & 0.39 \\ 2.28 & -- & 0.83\end{array}$

8.83

$0.12 \quad 0.13$

0.2

0.13
0.74
$*$

0.27
22.00

0.89

2.16

1967 PRECIP. (CM.)

SR-GO (MCI/SO.KM.)

SR-90 CONC. (PC/L)

12.04

3.40

3.127 .47

7.29

--

$\rightarrow$

$=$

$=$

$\begin{array}{ll}-- & = \\ -- & = \\ -- & --\end{array}$

\section{NOIES}

- : DATA NOT AVAILABLE

*: ZERO OR TRACE

APPROXIMATE

OF REPORTED DATA

PROPORTIONEO FROM ORIGINALLY CONSOLIDATED DATA 
SOURCE: U. S. WEATHER BUREAU AIRPORT STATION

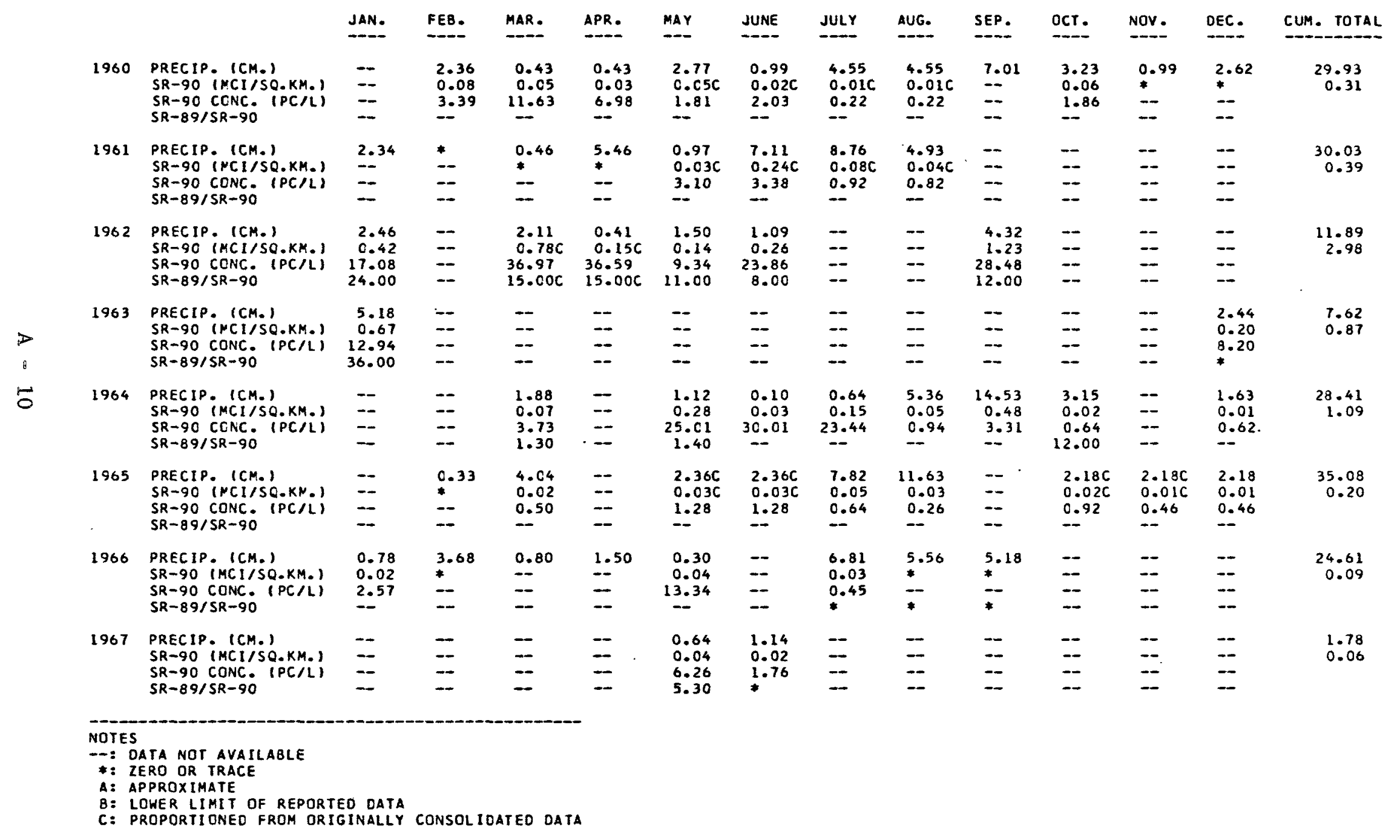




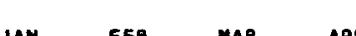

\begin{tabular}{|c|c|c|c|c|c|c|c|c|c|c|c|c|c|c|}
\hline & & JAN. & FEB. & MAR. & APR : & MAY & JUNE & JULY & AUG. & SEP. & OCT. & Nov. & DEC. & CUM. TOTAL \\
\hline 1956 & $\begin{array}{l}\text { PRECIP. (CM.) } \\
\text { SR-90 iMCI/SO-XM-) } \\
S R-90 \text { CONC. (PC/L) } \\
\text { SR-89/SR-90 }\end{array}$ & $\begin{array}{l}\bar{z} \\
\bar{z}\end{array}$ & $\begin{array}{l}= \\
=\end{array}$ & $\bar{z}$ & $\bar{z}$ & $\begin{array}{l}\bar{z} \\
\bar{z}\end{array}$ & $=$ & $\overline{=}$ & $\bar{z}$ & $\bar{z}$ & $\begin{array}{l}= \\
= \\
=\end{array}$ & $\begin{array}{l}= \\
=\end{array}$ & $\begin{array}{r}1.24 \\
0.06 \\
4.84 \\
44.00\end{array}$ & $\begin{array}{l}1.24 \\
0.06\end{array}$ \\
\hline 1957 & $\begin{array}{l}\text { PRECIP. }\left(C M_{-}\right) \\
\text {SR }-90 \text { (MCI/SO.KM) } \\
\text { SR }-90 \text { CCNC. (PC/L) } \\
\text { SR }-89 / S R-90\end{array}$ & $\begin{array}{r}9.86 \\
0.38 \\
3.86 \\
15.00\end{array}$ & $\begin{array}{l}4.93 \\
0.29 \\
5.89 \\
--\end{array}$ & $\begin{array}{l}2.41 \\
0.03 \\
1.25 \\
--\end{array}$ & $\begin{array}{l}3.38 \\
0.32 \\
9.47\end{array}$ & $\begin{array}{r}0.69 \\
0.69 \\
13.05 \\
15.00\end{array}$ & $\begin{array}{r}0.15 \\
0.05 \\
33.34 \\
13.00\end{array}$ & $\begin{array}{r}0.08 \\
0.36 \\
450.00 \\
0.90\end{array}$ & $\begin{array}{l}\frac{1}{c .02} \\
--.00\end{array}$ & $\begin{array}{l}* \\
0.02 \\
6.90\end{array}$ & $\begin{array}{r}3.84 \\
0.10 \\
2.61 \\
17.00\end{array}$ & $\begin{array}{r}1.30 \\
0.10 \\
7.70 \\
18.00\end{array}$ & $\begin{array}{r}5.33 \\
0.08 \\
1.51 \\
20.00\end{array}$ & $\begin{array}{r}31.97 \\
1.84\end{array}$ \\
\hline 1958 & $\begin{array}{l}\text { PRECIP. (CM.) } \\
\text { SR-9C iMCI/SQ.KM-) } \\
S R-90 \text { CONC. (PC/L) } \\
\text { SR-BG/SR-9O }\end{array}$ & $\begin{array}{r}3.78 \\
0.17 \\
4.50 \\
14.00\end{array}$ & $\begin{array}{r}15.90 \\
0.35 \\
2.21 \\
11.00\end{array}$ & $\begin{array}{r}13.34 \\
0.50 \\
3.75 \\
24.00\end{array}$ & $\begin{array}{r}5.18 \\
0.58 \\
11.20 \\
9.00\end{array}$ & $\begin{array}{l}0.03 \\
0.02 \\
86.67 \\
48.00\end{array}$ & $\begin{array}{l}* \\
0.04 \\
-- \\
4.00\end{array}$ & $\begin{array}{l}* \\
0.06 \\
-- \\
2.00\end{array}$ & $\begin{array}{r}0.05 \\
0.03 \\
60.01 \\
36.00\end{array}$ & $\begin{array}{r}0.08 \\
0.03 \\
37.51 \\
29.00\end{array}$ & $\begin{array}{r}0.81 \\
0.06 \\
7.41 \\
39.00\end{array}$ & $\begin{array}{l}* \\
0.04 \\
35.00\end{array}$ & $\begin{array}{r}0.03 \\
0.03 \\
100.00 \\
21.00\end{array}$ & $\begin{array}{r}39.20 \\
1.91\end{array}$ \\
\hline 1959 & $\begin{array}{l}\text { PRECIP. (CM.) } \\
\text { SR-90 (PCI/SQ.KM.) } \\
\text { SR-90 CONC. (PCAL) } \\
\text { SR-89/SR-90 }\end{array}$ & $\begin{array}{r}2.82 \\
0.70 \\
24.83 \\
41.00\end{array}$ & $\begin{array}{r}9.45 \\
1.28 \\
13.55 \\
28.00\end{array}$ & $\begin{array}{c}* \\
0.04 \\
15.00\end{array}$ & $\begin{array}{r}0.99 \\
0.24 \\
24.25 \\
12.00\end{array}$ & $\begin{array}{l}\text { C.08 } \\
-- \\
8.50\end{array}$ & $\frac{0.03}{3.40}$ & $\frac{0.03}{*}$ & $\begin{array}{l}* \\
0.02 \\
7.00\end{array}$ & $\begin{array}{c}0.10 \\
0.01 \\
10.01 \\
*\end{array}$ & $\begin{array}{r}0.03 \\
0.05 \\
166.67 \\
0.67\end{array}$ & $\begin{array}{l}0.15 \\
0.01 \\
6.67 \\
*\end{array}$ & $\begin{array}{l}2.82 \\
0.15 \\
5.32 \\
\end{array}$ & $\begin{array}{r}16.36 \\
2.64\end{array}$ \\
\hline 1960 & $\begin{array}{l}\text { PRECIP. (CM.) } \\
\text { SR-90 (NCI/SO-KM-) } \\
\text { SR-90 CONC. (PCCA) } \\
\text { SR-B9/SR } 90\end{array}$ & $\begin{array}{l}7.19 \\
0.10 \\
1.40 \\
-1\end{array}$ & $\begin{array}{l}7.37 \\
0.07 \\
0.95 \\
2.80\end{array}$ & $\begin{array}{l}0.53 \\
=- \\
=-\end{array}$ & $\begin{array}{l}5.08 \\
0.05 \\
0.99 \\
--\end{array}$ & $\begin{array}{l}* 0.02 c \\
--\end{array}$ & $\begin{array}{l}0.020 \\
=-\end{array}$ & $\begin{array}{l}* \\
0.02 c \\
--\end{array}$ & $\begin{array}{l}* 0.02 c \\
--\end{array}$ & $\begin{array}{l}0.02 c \\
--\end{array}$ & $\begin{array}{l}* .02 c \\
\therefore-\end{array}$ & $\begin{array}{l}0.78 \\
0.06 C \\
0.89 \\
-9\end{array}$ & $\begin{array}{l}0.15 \\
0.00 \mathrm{C} \\
0.01 \\
-0\end{array}$ & $\begin{array}{r}27.10 \\
0.40\end{array}$ \\
\hline 1961 & $\begin{array}{l}\text { PRECIP. (CM.) } \\
\text { SR-90 (KCI/SQ.KM-) } \\
\text { SR-90 CCNC. (PCIL) } \\
\text { SR-89/SR-90 }\end{array}$ & $\begin{array}{l}3.25 \\
0.076 \\
2.16 \\
-\end{array}$ & $\begin{array}{l}* \\
0.00 C \\
--\end{array}$ & $\begin{array}{l}1.17 \\
0.056 \\
4.28 \\
-.\end{array}$ & $\begin{array}{l}0.74 \\
0.036 \\
4.06 \\
--\end{array}$ & $\begin{array}{l}* \\
c .02 c \\
--\end{array}$ & $\begin{array}{l}0.016 \\
=-\end{array}$ & $\begin{array}{l}0.03 \\
c .016 \\
33.34 \\
--\end{array}$ & $\begin{array}{l}0.76 \\
0.226 \\
28.95 \\
--.\end{array}$ & $\begin{array}{r}0.10 \\
C .06 \\
60.01 \\
5.80\end{array}$ & $\frac{0.03}{43.00}$ & $\begin{array}{r}5.13 \\
0.64 \\
12.48 \\
74.00\end{array}$ & $\begin{array}{r}3.66 \\
0.35 \\
9.57 \\
51.00\end{array}$ & $\begin{array}{r}14.84 \\
1.48\end{array}$ \\
\hline 1962 & $\begin{array}{l}\text { PRECIP I (CM-) } \\
\text { SR-90 irCI/SO-KM-) } \\
\text { SR-9C CONC. (PC/L) } \\
\text { SR-89/SR-90 }\end{array}$ & $\begin{array}{r}6.81 \\
0.70 \\
10.28 \\
40.00\end{array}$ & $\begin{array}{r}39.78 \\
3.28 \\
8.25 \\
34.00\end{array}$ & $\begin{array}{r}3.12 \\
0.53 \\
16.99 \\
20.00\end{array}$ & $\begin{array}{c}* 0.07 \\
10.00\end{array}$ & $\begin{array}{r}0.13 \\
0.07 \\
53.85 \\
5.00\end{array}$ & $\begin{array}{l}* 0.05 \\
-- \\
4.00\end{array}$ & $\begin{array}{l}0.04 \\
0 .- \\
6.00\end{array}$ & $\begin{array}{l}* 0.06 \\
11.00\end{array}$ & $\begin{array}{l}0.02 \\
0 .-00 \\
18.00\end{array}$ & $\begin{array}{r}0.84 \\
0.10 \\
11.91 \\
4 C .00\end{array}$ & $\begin{array}{r}1.19 \\
0.04 \\
3.37 \\
24.00\end{array}$ & $\begin{array}{r}0.51 \\
0.08 \\
15.69 \\
35.00\end{array}$ & $\begin{array}{r}52.38 \\
5.04\end{array}$ \\
\hline 1963 & $\begin{array}{l}\text { PRECIP. (CM.) } \\
\text { SR-90 (NCI/SO.KM.) } \\
\text { SR-9C CONC. (PCAL) } \\
\text { SR-89/SR-90 }\end{array}$ & $\begin{array}{r}0.99 \\
0.19 \\
19.20 \\
27.00\end{array}$ & $\begin{array}{r}8.38 \\
1.00 \\
11.94 \\
35.00\end{array}$ & $\begin{array}{r}8.53 \\
3.23 \\
37.87 \\
9.20\end{array}$ & $\begin{array}{r}4.52 \\
1.58 \\
34.96 \\
9.00\end{array}$ & $\begin{array}{r}0.03 \\
0.23 \\
766.65 \\
8.40\end{array}$ & $\begin{array}{r}0.38 \\
0.59 \\
155.27 \\
3.40\end{array}$ & \begin{tabular}{l}
$*$ \\
0.05 \\
\hdashline
\end{tabular} & $\begin{array}{r}0.74 \\
0.11 \\
14.87 \\
*\end{array}$ & $\begin{array}{r}2.16 \\
0.45 \\
20.84 \\
*\end{array}$ & $\begin{array}{r}1.65 \\
0.27 \\
16.37 \\
0.50\end{array}$ & $\begin{array}{l}8.51 \\
0.42 \\
4.94 \\
*\end{array}$ & $\begin{array}{l}0.76 \\
0.03 \\
3.95 \\
*\end{array}$ & $\begin{array}{r}36.65 \\
8.15\end{array}$ \\
\hline 1964 & $\begin{array}{l}\text { PRECTP. (CH.) } \\
\text { SR-9C irC1/SQ.KM.) } \\
\text { SR-90 CONC. (PC/L) } \\
\text { SR-89/SR-90 }\end{array}$ & $\begin{array}{l}4.01 \\
0.20 \\
4.99 \\
*\end{array}$ & $\begin{array}{r}0.03 \\
c .04 \\
133.34 \\
*\end{array}$ & $\begin{array}{r}4.88 \\
C .78 \\
15.99 \\
*\end{array}$ & $\begin{array}{l}1.78 \\
0.04 \\
2.25 \\
--\end{array}$ & $\begin{array}{r}0.03 \\
0.03 \\
100.00 \\
*\end{array}$ & $\begin{array}{l}0.86 \\
=- \\
=-\end{array}$ & $\begin{array}{l}* \\
0.20 \\
--\end{array}$ & $\begin{array}{l}* \\
0.06 \\
-\infty\end{array}$ & $\begin{array}{l}0.10 \\
0 .\end{array}$ & $\begin{array}{l}1.27 \\
0.07 \\
5.52 \\
.\end{array}$ & $\begin{array}{l}6.35 \\
0.48 \\
7.56 \\
7\end{array}$ & $\begin{array}{r}11.43 \\
0.10 \\
0.88 \\
--\end{array}$ & $\begin{array}{r}30.64 \\
2.10\end{array}$ \\
\hline 1965 & $\begin{array}{l}\text { PRECIP. (CM.) } \\
\text { SR-90 iNCI/SO.KM-) } \\
\text { SR-SO CONC. (PC/L) } \\
\text { SR-BS/SR-9O }\end{array}$ & $\begin{array}{r}1.27 \\
0.76 \\
59.85 \\
--\end{array}$ & $\begin{array}{l}1.27 \\
0.08 \\
6.30 \\
-\end{array}$ & $\begin{array}{l}4.60 \\
0.35 \\
7.61 \\
-\end{array}$ & $\begin{array}{c}14.86 \\
1.21 \\
8.15 \\
--\end{array}$ & $\begin{array}{l}* \\
0.15 \\
-- \\
--\end{array}$ & $\begin{array}{r}0.23 \\
0.63 \\
273.92 \\
-0\end{array}$ & $\begin{array}{r}0.05 \\
0.05 \\
106.00 \\
-\end{array}$ & $\begin{array}{r}0.41 \\
0.05 \\
12.20 \\
\end{array}$ & $\begin{array}{r}0.58 \\
0.11 \\
18.97 \\
--\end{array}$ & $\begin{array}{l}-- \\
0.01 \\
--\end{array}$ & $\begin{array}{r}28.70 \\
0.54 \\
1.89 \\
--\end{array}$ & $\begin{array}{c}12.24 \\
0.09 \\
C .74 \\
--\end{array}$ & $\begin{array}{r}64.21 \\
4.03\end{array}$ \\
\hline 1966 & $\begin{array}{l}\text { PRECIP. (CM-) } \\
\text { SR-90 IMCI/SO.KM-) } \\
\text { SR-90 CONC. (PCAL) } \\
\text { SR-89/SR-90 }\end{array}$ & $\begin{array}{l}2.64 \\
0.16 \\
6.07 \\
--\end{array}$ & $\begin{array}{l}4.27 \\
0.05 \\
1.18 \\
-\end{array}$ & $\begin{array}{r}0.08 \\
0.14 \\
175.00 \\
-\end{array}$ & $\begin{array}{l}* \\
0.03 \\
=-\end{array}$ & $\begin{array}{l}-- \\
= \\
-\end{array}$ & $\frac{0.06}{*}$ & $\begin{array}{l}0.05 \\
c .03 \\
60.01 \\
*\end{array}$ & $\frac{*}{*}$ & $\begin{array}{l}0.61 \\
0.01 \\
1.64 \\
*\end{array}$ & $\begin{array}{l}0.13 \\
* \\
*\end{array}$ & $\begin{array}{r}12.70 \\
0.05 \\
0.40 \\
21.00\end{array}$ & $\begin{array}{r}11.56 \\
0.06 \\
0.52 \\
7.30\end{array}$ & $\begin{array}{r}32.04 \\
0.59\end{array}$ \\
\hline 1967 & $\begin{array}{l}\text { PRECIP. }(C M-) \\
\text { SR-90 iMCI/SO.KM-) } \\
\text { SR-90 CONC. }(P C / L) \\
\text { SR-89/SR-90 }\end{array}$ & $\begin{array}{r}12.93 \\
0.15 \\
1.17 \\
9.20\end{array}$ & $\begin{array}{r}0.20 \\
0.03 \\
15.01 \\
7.20\end{array}$ & $\begin{array}{r}7.49 \\
0.13 \\
1.74 \\
12.00\end{array}$ & $\begin{array}{l}9.58 \\
0.16 \\
1.68 \\
8.50\end{array}$ & $\begin{array}{r}0.33 \\
0.02 \\
6.07 \\
12.20\end{array}$ & $\begin{array}{l}* \\
0.01 \\
--\end{array}$ & $\begin{array}{l}= \\
= \\
=\end{array}$ & $\begin{array}{l}= \\
= \\
=\end{array}$ & $\begin{array}{l}=- \\
=\end{array}$ & $\begin{array}{l}\overline{-} \\
= \\
=\end{array}$ & $\begin{array}{l}\overline{-} \\
\bar{z} \\
\bar{z}\end{array}$ & $\begin{array}{l}=- \\
=- \\
=\end{array}$ & $\begin{array}{r}30.53 \\
0.50\end{array}$ \\
\hline
\end{tabular}

: ZERO OR AVACLABL

A: APPROXIMATE

B: LOWER LIMIT OF REPORTED OATA

C: PROPORTIONED FROM ORIGINALLY CONSOLIOATED DATA 
MONTHLY FALLOUT DEPOSITION COLLECTIONS

SITE: CALIFORNIA, PALO ALTO

LAT. $3730 \mathrm{~N}$ LONG.122 $15 \mathrm{~W}$ ALT.

6M. (POT)

SURFACE AIR SAMPLING STATION

SOURCE: HAZELTON-NUCLEAR SCIENCE CORP

SAMPLING TERMINATED IN DECEMBER 1965

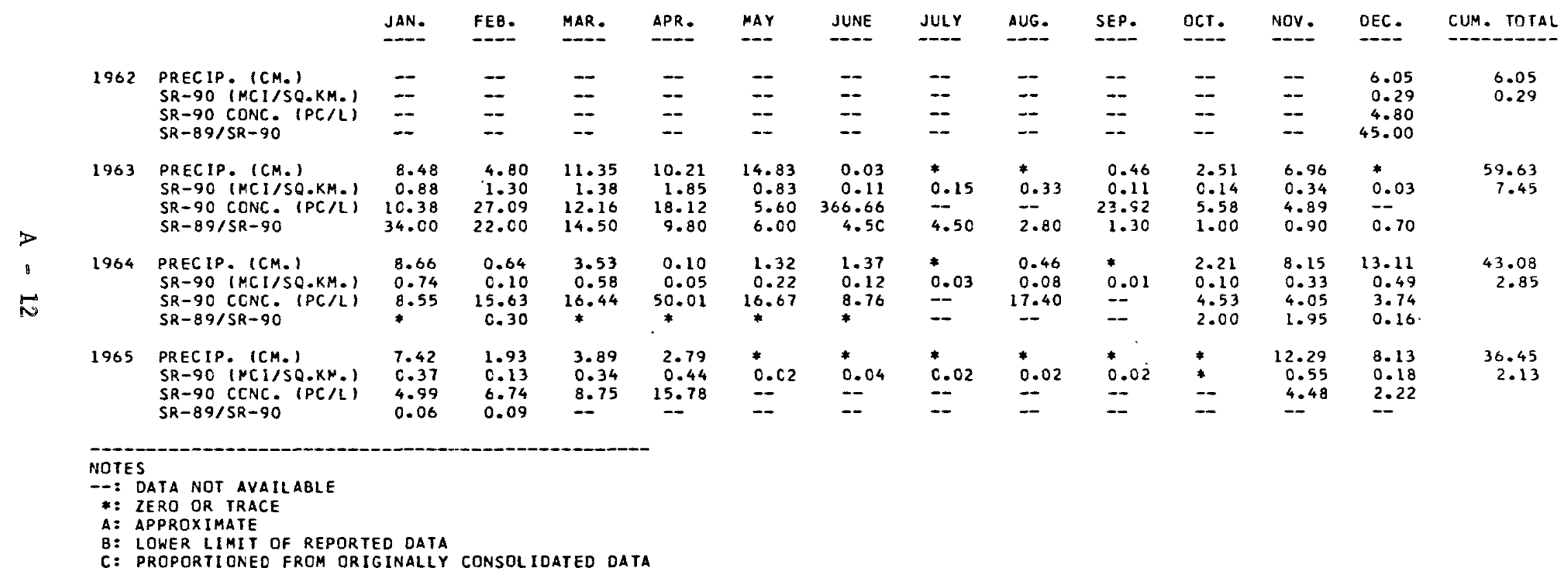


MONTHLY FALLOUT DEPOSITION COLLECTIONS

SITE: CALIFORNIA, PALO ALTO

LAT. 37 3ON LCNG.122 15 H ALT.

6M. (COLUMN)

SOURCE: HAZELTON-NUCLEAR SCIENCE CORP.

COLLECTIONS TERMINATED AFTER JUNE 1964

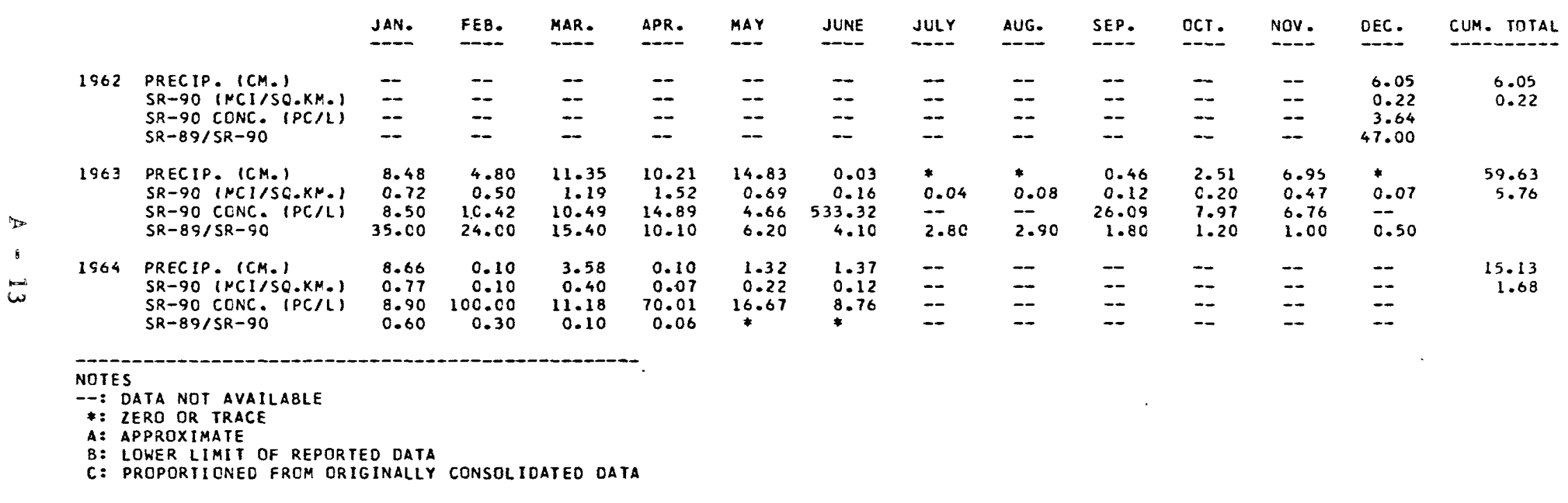


MONTHLY FALLOUT OEPOSITION COLLECTIONS

SITE: CALIFORNIA, RICHMONO

LAT. $3756 \mathrm{~N}$ LONG.108 $38 \mathrm{H}$ ALT.

20M. (POT)

SOURCE: TRACERLAB, INC.

COLLECTIONS TERMINATED AFTER JUNE 1963

\begin{tabular}{|c|c|c|c|c|c|c|c|c|c|c|c|c|}
\hline JAN. & FEB. & MAR . & APR . & MAY & JUNE & JULY & AUG. & SEP. & OCT. & Nov. & DEC. & CUM. TOTAL \\
\hline $\begin{array}{l}-- \\
= \\
-\end{array}$ & $\begin{array}{l}-- \\
\overline{-}\end{array}$ & $\begin{array}{r}16.87 \\
1.44 \\
8.54 \\
22.00\end{array}$ & $\begin{array}{c}\overline{0.22} \\
\overline{11.00}\end{array}$ & $\begin{array}{l}0.23 \\
18.60\end{array}$ & $\begin{array}{l}-- \\
0.10 \\
9.00\end{array}$ & $\begin{array}{c}* \\
0.03 \\
17.00\end{array}$ & $\begin{array}{c}* \\
0.01 \\
26.00\end{array}$ & $\begin{array}{r}0.13 \\
0.03 \\
23.08 \\
15.00\end{array}$ & $\begin{array}{r}0.53 \\
0.03 \\
5.67 \\
30.00\end{array}$ & $\begin{array}{c}* 000 \\
0.00\end{array}$ & $\begin{array}{r}4.50 \\
0.15 \\
3.34 \\
36.00\end{array}$ & $\begin{array}{r}22.03 \\
2.24\end{array}$ \\
\hline $\begin{array}{r}11.23 \\
0.73 \\
6.51 \\
30.00\end{array}$ & $\begin{array}{r}15.54 \\
1.03 \\
6.63 \\
28.00\end{array}$ & $\begin{array}{r}2.06 \\
0.30 \\
14.57 \\
15.00\end{array}$ & $\begin{array}{r}1.12 \\
0.19 \\
16.97 \\
10.00\end{array}$ & $\begin{array}{l}* \\
0.08 \\
6.60\end{array}$ & $\begin{array}{l}* \\
0.01 \\
=- \\
6.40\end{array}$ & $\begin{array}{l}* \\
* \\
*-\end{array}$ & $\begin{array}{l}* \\
0.01 \\
2.70\end{array}$ & $\begin{array}{l}7.39 \\
C .03 \\
0.41 \\
1.40\end{array}$ & $\begin{array}{l}\text { C.01 } \\
C .01 \\
c .90\end{array}$ & $\begin{array}{l}* \\
* \\
2.50\end{array}$ & $\begin{array}{l}4.11 \\
0.05 \\
1.22 \\
0.60\end{array}$ & $\begin{array}{r}41.45 \\
2.44\end{array}$ \\
\hline $\begin{array}{r}13.97 \\
0.14 \\
1.01 \\
1.00\end{array}$ & $\begin{array}{r}11.76 \\
C .14 \\
1.20 \\
0.90\end{array}$ & $\begin{array}{l}6.22 \\
0.17 \\
2.74 \\
5.70\end{array}$ & $\begin{array}{l}2.67 \\
0.03 \\
1.13 \\
0.60\end{array}$ & $\begin{array}{l}0.84 \\
0.63 \\
3.58 \\
0.30\end{array}$ & $\begin{array}{l}* \\
0.00 \\
-- \\
*\end{array}$ & $\begin{array}{l}0.01 \\
0 \\
-\end{array}$ & $\begin{array}{l}0.01 \\
0 \\
--\end{array}$ & $\begin{array}{l}* \\
0.00 \\
-- \\
--\end{array}$ & $\begin{array}{l}0.71 \\
0.01 \\
1.41 \\
--\end{array}$ & $\begin{array}{r}12.85 \\
0.05 \\
0.39 \\
--\end{array}$ & $\begin{array}{l}1.96 \\
0.03 \\
1.54 \\
--\end{array}$ & $\begin{array}{r}50.98 \\
0.62\end{array}$ \\
\hline $\begin{array}{l}7.57 \\
0.05 \\
0.67 \\
-\end{array}$ & $\begin{array}{l}3.00 \\
0.02 \\
0.67 \\
--\end{array}$ & $\begin{array}{l}7.09 \\
0.07 \\
0.99 \\
\end{array}$ & $\begin{array}{l}2.51 \\
0.05 \\
2.00 \\
--\end{array}$ & $\begin{array}{c}0.99 \\
0.10 \\
10.11 \\
--\end{array}$ & $\begin{array}{l}* \\
0.01 \\
=- \\
=-\end{array}$ & $\begin{array}{l}* \\
0.01 \\
-- \\
--\end{array}$ & $\begin{array}{c}0.20 \\
0.02 \\
10.01 \\
--\end{array}$ & $\begin{array}{r}0.76 \\
0.01 \\
1.32 \\
19.00\end{array}$ & $\begin{array}{r}0.23 \\
0.03 \\
13.05 \\
91.00\end{array}$ & $\begin{array}{r}12.65 \\
0.51 \\
4.04 \\
75.00\end{array}$ & $\begin{array}{r}3.05 \\
0.25 \\
8.20 \\
44.00\end{array}$ & $\begin{array}{r}38.05 \\
1.13\end{array}$ \\
\hline $\begin{array}{r}3.02 \\
0.32 \\
10.60 \\
39.00\end{array}$ & $\begin{array}{r}22.56 \\
1.64 \\
7.27 \\
26.00\end{array}$ & $\begin{array}{r}2.16 \\
0.27 \\
12.51 \\
21.00\end{array}$ & $\begin{array}{r}0.79 \\
0.30 \\
37.98 \\
11.00\end{array}$ & $\begin{array}{l}* \\
0.02 \\
7.60\end{array}$ & $\begin{array}{l}* \\
0.04 \\
-- \\
4.70\end{array}$ & $\begin{array}{l}* \\
0.03 \\
3.60\end{array}$ & $\begin{array}{l}0.25 \\
0.02 \\
8.01 \\
3.60\end{array}$ & $\begin{array}{r}0.25 \\
0.03 \\
12.01 \\
14.00\end{array}$ & $\begin{array}{r}16.59 \\
0.41 \\
2.48 \\
17.00\end{array}$ & $\begin{array}{r}2.29 \\
0.12 \\
5.25 \\
49.00\end{array}$ & $\begin{array}{r}8.38 \\
0.39 \\
4.66 \\
37.00\end{array}$ & $\begin{array}{r}56.29 \\
3.59\end{array}$ \\
\hline $\begin{array}{r}11.30 \\
0.98 \\
8.68 \\
29.00\end{array}$ & $\begin{array}{r}5.38 \\
0.93 \\
17.29 \\
22.00\end{array}$ & $\begin{array}{r}10.08 \\
2.23 \\
22.13 \\
16.00\end{array}$ & $\begin{array}{r}10.92 \\
2.61 \\
23.91 \\
9.00\end{array}$ & $\begin{array}{r}1.14 \\
0.41 \\
35.97 \\
6.30\end{array}$ & $\begin{array}{l}* \\
0.10 \\
2.20\end{array}$ & $\begin{array}{l}=- \\
=- \\
=-\end{array}$ & 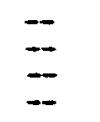 & $\begin{array}{l}-- \\
-- \\
--\end{array}$ & $\begin{array}{l}=- \\
-- \\
--\end{array}$ & $\begin{array}{l}= \\
=- \\
--\end{array}$ & $\begin{array}{l}-- \\
-- \\
--\end{array}$ & $\begin{array}{r}38.82 \\
7.26\end{array}$ \\
\hline
\end{tabular}

NOTES

--: DATA NOT AVAILABLE

*: ZERo OR TRACE

A: APPROXIMATE

B: LOHER LIMIT OF REPORTED DATA

$C$ : PROPORTIONED FROM ORIGINALLY CONSOLIOATED DATA 
MONTHLY FALLOUT DEPOSITION COLLECTIONS
SITE: CALIFORNIA, RICHMOND
LAT. 37 56N LONG.108 38W
ALT.
20M. (COLUMN)

SOURCE: TRACERLAB, INC.

COLLECTIONS TERMINATED AFTER JUNE 1963

\begin{tabular}{|c|c|c|c|c|c|c|c|c|c|c|c|c|}
\hline JAN. & FEB. & MAR. & APR. & MAY & JUNE & JULY & AUG. & SEP. & OCT. & NOV. & DEC. & CUM. TOTAL \\
\hline $\begin{array}{l}-- \\
\overline{--} \\
-\end{array}$ & $\begin{array}{l}-- \\
-- \\
--\end{array}$ & $\begin{array}{l}-- \\
-- \\
-\end{array}$ & $\begin{array}{l}-\infty \\
- \\
--\end{array}$ & $\begin{array}{l}=- \\
=- \\
=-\end{array}$ & $\begin{array}{l}-- \\
-- \\
-- \\
--\end{array}$ & $\begin{array}{l}* \\
0.00 \\
-- \\
-\end{array}$ & $\begin{array}{r}0.20 \\
0.02 \\
10.01 \\
-\end{array}$ & $\begin{array}{l}0.76 \\
0.02 \\
2.64 \\
7.20\end{array}$ & $\begin{array}{l}0.23 \\
0.02 \\
8.70 \\
9.20\end{array}$ & $\begin{array}{r}14.73 \\
0.33 \\
2.25 \\
93.06\end{array}$ & $\begin{array}{r}0.97 \\
0.10 \\
10.31 \\
49.00\end{array}$ & $\begin{array}{r}16.89 \\
0.49\end{array}$ \\
\hline $\begin{array}{r}3.02 \\
0.28 \\
9.28 \\
38.00\end{array}$ & $\begin{array}{r}15.90 \\
1.24 \\
7.80 \\
25.00\end{array}$ & $\begin{array}{r}8.05 \\
0.86 \\
10.69 \\
16.00\end{array}$ & $\begin{array}{r}0.79 \\
0.27 \\
34.18 \\
12.00\end{array}$ & \begin{tabular}{l}
$*$ \\
0.02 \\
\hdashline .50
\end{tabular} & $\begin{array}{l}* \\
0.05 \\
4.9 C\end{array}$ & $\begin{array}{l}* \\
0.04 \\
2.90\end{array}$ & $\begin{array}{r}0.25 \\
0.05 \\
20.01 \\
7.60\end{array}$ & $\begin{array}{r}0.25 \\
0.07 \\
28.01 \\
8.10\end{array}$ & $\begin{array}{r}16.51 \\
0.38 \\
2.31 \\
16.00\end{array}$ & $\begin{array}{r}2.29 \\
0.24 \\
10.49 \\
30.00\end{array}$ & $\begin{array}{r}8.38 \\
0.39 \\
4.66 \\
37.00\end{array}$ & $\begin{array}{r}55.44 \\
3.89\end{array}$ \\
\hline $\begin{array}{r}11.30 \\
1.00 \\
8.85 \\
36.00\end{array}$ & $\begin{array}{r}5.38 \\
C .79 \\
14.69 \\
22.00\end{array}$ & $\begin{array}{r}10.08 \\
1.52 \\
15.08 \\
20.00\end{array}$ & $\begin{array}{r}10.92 \\
2.44 \\
22.35 \\
10.00\end{array}$ & $\begin{array}{r}1.14 \\
0.74 \\
64.92 \\
2.80\end{array}$ & $\begin{array}{l}\frac{*}{0.12} \\
3.80\end{array}$ & $\begin{array}{l}=- \\
--\end{array}$ & $\begin{array}{l}=- \\
z- \\
z\end{array}$ & $\begin{array}{l}=- \\
-- \\
--\end{array}$ & $\begin{array}{l}-- \\
-- \\
--\end{array}$ & $\begin{array}{l}=- \\
\overline{-}\end{array}$ & $\begin{array}{l}-- \\
-- \\
--\end{array}$ & $\begin{array}{r}38.82 \\
6.61\end{array}$ \\
\hline
\end{tabular}

NOTES

--: DATA NOT AVAILABLE

*: ZERO OR TRACE

A: APPROXIMATE

8: LOWER LIMII OF REPORTED DATA

C: PROPORTIONEL FROM ORIGINALLY CONSOL IDATED DATA 


\begin{tabular}{|c|c|c|c|c|c|c|c|c|c|c|c|c|}
\hline JAN. & FEB. & MAR : & APR. & MAY & JUNE & JULY & AUG. & SEP. & OCT. & NOV. & DEC. & CUM. TOTAL \\
\hline $\begin{array}{l}-- \\
--\end{array}$ & $\begin{array}{r}11.84 \\
0.19 \\
1.61 \\
26.00\end{array}$ & $\begin{array}{l}0.76 \\
-- \\
--\end{array}$ & $\begin{array}{r}0.94 \\
0.15 \\
15.96 \\
14.00\end{array}$ & $\begin{array}{r}0.10 \\
0.14 \\
140.00 \\
8.10\end{array}$ & $\begin{array}{l}* \\
-- \\
--\end{array}$ & $\begin{array}{l}* \\
0.02 \\
1.00\end{array}$ & $\begin{array}{l}0.03 \\
*\end{array}$ & $\begin{array}{l}5.84 \\
0.04 \\
0.69 \\
*\end{array}$ & $\begin{array}{l}0.00 \\
0 \\
*\end{array}$ & $\begin{array}{l}* \\
0.07 \\
- \\
*\end{array}$ & $\begin{array}{l}5.00 \\
-- \\
-- \\
--\end{array}$ & $\begin{array}{r}24.48 \\
0.64\end{array}$ \\
\hline $\begin{array}{r}11.40 \\
0.19 \\
1.67 \\
--\end{array}$ & $\begin{array}{l}8.84 \\
C .09 \\
1.02 \\
-.\end{array}$ & $\begin{array}{l}2.97 \\
0.09 \\
3.04 \\
-\end{array}$ & $\begin{array}{l}1.85 \\
0.08 \\
4.33 \\
-.\end{array}$ & $\begin{array}{l}1.37 \\
0.056 \\
3.65 \\
-.\end{array}$ & $\begin{array}{l}* \\
0.000 \\
--\end{array}$ & $\begin{array}{l}* \\
* \\
-\end{array}$ & $\begin{array}{l}* \\
* \\
--\end{array}$ & $\begin{array}{l}* \\
0.000 \\
=-\end{array}$ & $\begin{array}{l}C .71 \\
0.026 \\
2.82 \\
--\end{array}$ & $\begin{array}{l}11.00 \\
0.016 \\
0.10 \\
=\end{array}$ & $\begin{array}{c}13.23 \\
0.02 \mathrm{C} \\
0.16 \\
--\end{array}$ & $\begin{array}{r}51.37 \\
0.55\end{array}$ \\
\hline $\begin{array}{l}6.71 \\
-- \\
--\end{array}$ & $\begin{array}{l}3.00 \\
0.13 \\
4.34 \\
--\end{array}$ & $\begin{array}{l}8.61 \\
0.14 \mathrm{C} \\
1.63 \\
-\end{array}$ & $\begin{array}{l}3.18 \\
0.05 C \\
1.58 \\
-.\end{array}$ & $\begin{array}{l}1.52 \\
0.10 C \\
6.58 \\
--\end{array}$ & $\begin{array}{l}0.25 \\
0.02 \\
8.01 \\
--\end{array}$ & $\begin{array}{l}* \\
0.000 \\
-\therefore \\
--\end{array}$ & $\begin{array}{l}0.10 \\
0.01 \\
10.01 \\
--\end{array}$ & $\begin{array}{l}1.04 \\
0.01 \\
0.97 \\
7.80\end{array}$ & $\begin{array}{r}0.08 \\
0.02 \\
25.01 \\
60.00\end{array}$ & $\begin{array}{r}11.10 \\
0.34 \\
3.07 \\
74.00\end{array}$ & $\begin{array}{r}4.62 \\
0.11 \\
2.39 \\
60.00\end{array}$ & $\begin{array}{r}40.21 \\
0.93\end{array}$ \\
\hline $\begin{array}{r}4.32 \\
0.29 \\
6.72 \\
41.00\end{array}$ & $\begin{array}{r}21.54 \\
1.32 \\
6.13 \\
27.00\end{array}$ & $\begin{array}{r}7.57 \\
0.43 \\
5.69 \\
19.00\end{array}$ & $\begin{array}{r}0.86 \\
0.21 \\
24.42 \\
11.00\end{array}$ & $\begin{array}{l}* 0.05 \\
7.00\end{array}$ & $\begin{array}{c}* \\
0.03 \\
10.00\end{array}$ & $\begin{array}{l}2.03 \\
6.00\end{array}$ & $\begin{array}{r}0.08 \\
0.02 \\
25.01 \\
6.00\end{array}$ & $\begin{array}{r}0.23 \\
0.24 \\
104.35 \\
2 C .00\end{array}$ & $\begin{array}{r}1 E .54 \\
0.20 \\
1.08 \\
31.00\end{array}$ & $\begin{array}{r}0.91 \\
0.07 \\
7.70 \\
35.00\end{array}$ & $\begin{array}{r}7.54 \\
0.21 \\
2.79 \\
61.00\end{array}$ & $\begin{array}{r}61.59 \\
5.10\end{array}$ \\
\hline $\begin{array}{r}11.35 \\
0.72 \\
6.35 \\
25.00\end{array}$ & $\begin{array}{r}5.16 \\
0.64 \\
12.41 \\
31.00\end{array}$ & $\begin{array}{r}10.01 \\
1.20 \\
11.99 \\
21.00\end{array}$ & $\begin{array}{r}9.40 \\
1.50 \\
15.96 \\
11.90\end{array}$ & $\begin{array}{r}1.27 \\
0.34 \\
26.78 \\
9.30\end{array}$ & $\frac{1.18}{1.00}$ & $\begin{array}{l}\frac{1}{0.02} \\
--00\end{array}$ & $\begin{array}{l}* \\
0.03 \\
*\end{array}$ & $\begin{array}{r}0.18 \\
0.07 \\
38.89 \\
*\end{array}$ & $\begin{array}{l}3.40 \\
0.29 \\
8.53 \\
*\end{array}$ & $\begin{array}{l}8.36 \\
0.41 \\
4.91 \\
*\end{array}$ & $\begin{array}{l}1.47 \\
0.36 \\
24.49 \\
*\end{array}$ & $\begin{array}{r}50.60 \\
6.76\end{array}$ \\
\hline $\begin{array}{r}11.05 \\
0.72 \\
6.52 \\
1.00\end{array}$ & $\begin{array}{r}0.69 \\
0.14 \\
20.29 \\
*\end{array}$ & $\begin{array}{l}4.95 \\
0.54 \\
10.91 \\
*\end{array}$ & $\begin{array}{r}0.03 \\
0.12 \\
399.99 \\
*\end{array}$ & $\begin{array}{c}0.81 \\
0.21 \\
25.93 \\
*\end{array}$ & $\begin{array}{r}0.15 \\
0.17 \\
113.34 \\
-\end{array}$ & $\begin{array}{l}* \\
0.05 \\
--\end{array}$ & $\begin{array}{r}0.03 \\
0.04 \\
133.34 \\
\end{array}$ & $\begin{array}{l}* \\
* \\
--\end{array}$ & $\begin{array}{l}3.89 \\
0.15 \\
3.86 \\
3.70\end{array}$ & $\begin{array}{l}7.95 \\
0.23 \\
2.90\end{array}$ & $\begin{array}{r}13.77 \\
0.52 \\
3.78 \\
--\end{array}$ & $\begin{array}{r}43.32 \\
2.89\end{array}$ \\
\hline $\begin{array}{r}11.10 \\
0.42 \\
3.79 \\
\end{array}$ & $\begin{array}{l}2.31 \\
0.10 \\
4.33 \\
--\end{array}$ & $\begin{array}{l}4.47 \\
0.37 \\
8.28 \\
--\end{array}$ & $\begin{array}{l}8.81 \\
0.58 \\
6.59 \\
--\end{array}$ & $\begin{array}{l}* \\
0.01 \\
-- \\
--\end{array}$ & $\begin{array}{l}* \\
0.01 \\
-\infty\end{array}$ & $\begin{array}{l}* \\
0.01 \\
--\end{array}$ & $\begin{array}{l}0.74 \\
* \\
-- \\
-\end{array}$ & $\begin{array}{l}* \\
0.01 \\
--\end{array}$ & $\begin{array}{l}* \\
* \\
--\end{array}$ & $\begin{array}{l}7.06 \\
0.25 \\
3.55 \\
-.\end{array}$ & $\begin{array}{c}12.75 \\
0.41 \\
3.22 \\
-.\end{array}$ & $\begin{array}{r}47.24 \\
2.17\end{array}$ \\
\hline $\begin{array}{l}6.86 \\
* \\
--\end{array}$ & $\begin{array}{l}8.08 \\
\$ \\
-- \\
--\end{array}$ & $\begin{array}{l}1.50 \\
0.07 \\
4.67 \\
-.\end{array}$ & $\begin{array}{l}1.02 \\
0.07 \\
6.87 \\
--\end{array}$ & $\begin{array}{l}+ \\
0.06 \\
--\end{array}$ & $\begin{array}{c}0.10 \\
0.07 \\
70.01 \\
*\end{array}$ & $\begin{array}{l}0.18 \\
0.01 \\
5.56 \\
*\end{array}$ & $\begin{array}{l}0.23 \\
\star \\
-- \\
*\end{array}$ & $\begin{array}{l}0.20 \\
* \\
-- \\
*\end{array}$ & $\begin{array}{l}* \\
C .01 \\
*\end{array}$ & $\begin{array}{r}12.17 \\
0.01 \\
0.09 \\
*\end{array}$ & $\begin{array}{r}10.06 \\
0.06 \\
0.60 \\
10.00\end{array}$ & $\begin{array}{r}40.40 \\
0.30\end{array}$ \\
\hline $\begin{array}{r}26.49 \\
0.22 \\
0.84 \\
7.20\end{array}$ & $\begin{array}{l}0.23 \\
0.02 \\
8.70 \\
7.80\end{array}$ & $\begin{array}{r}12.80 \\
0.16 \\
1.26 \\
8.10\end{array}$ & $\begin{array}{r}13.49 \\
0.14 \\
1.04 \\
8.80\end{array}$ & $\begin{array}{l}0.66 \\
0.02 \\
3.04 \\
*\end{array}$ & $\begin{array}{l}2.18 \\
0.04 \\
1.84 \\
+\end{array}$ & $\begin{array}{l}* \\
0.01 \\
--\end{array}$ & $\begin{array}{l}-- \\
-- \\
--\end{array}$ & $\begin{array}{l}-- \\
-- \\
--\end{array}$ & $\begin{array}{l}m \\
-- \\
--\end{array}$ & $\begin{array}{l}- \\
- \\
-\end{array}$ & $\begin{array}{l}-- \\
--\end{array}$ & $\begin{array}{r}55.85 \\
0.61\end{array}$ \\
\hline
\end{tabular}




\begin{tabular}{|c|c|c|c|c|c|c|c|c|c|c|c|c|c|c|}
\hline & & JAN. & FES. & Man. & Apr. & mar & JunE & JULY & wa. & SEP. & act. & NOV. & DEC. & CUM. TOTAL \\
\hline 1957 & $\begin{array}{l}\text { PREC1P. ICH.' } \\
\text { SR-90 IMCI/SC.KM.) } \\
\text { SR-90 CONC. (PCAL) } \\
\text { SR-89/SR-90 }\end{array}$ & $=$ & $\bar{z}$ & $=$ & $\begin{array}{r}12.80 \\
0.20 \\
1.57 \\
\end{array}$ & $\begin{array}{r}25.68 \\
0.19 \\
c .74 \\
19.00\end{array}$ & $\begin{array}{r}14.78 \\
0.22 \\
1.49 \\
36.00\end{array}$ & $\begin{array}{r}21.69 \\
0.58 \\
2.66 \\
-\end{array}$ & $\begin{array}{r}34.59 \\
0.29 \\
0.84 \\
58.00\end{array}$ & $\begin{array}{r}15.93 \\
0.20 \\
1.26 \\
40.00\end{array}$ & $\begin{array}{r}10.11 \\
0.16 \\
1.59 \\
40.00\end{array}$ & $\begin{array}{r}6.45 \\
0.11 \\
1.71 \\
16.00\end{array}$ & $\begin{array}{r}7.21 \\
0.24 \\
3.33 \\
15.00\end{array}$ & $\begin{array}{r}149.24 \\
2.19\end{array}$ \\
\hline 1958 & $\begin{array}{l}\text { PRECIP. }(C M-) \\
\text { SR-90 (YCI/SQ-KM-) } \\
S R-90 \text { CONC. }(P C / L) \\
S R-99 / S R-90\end{array}$ & $\begin{array}{r}6.68 \\
0.11 \\
1.65 \\
17.00\end{array}$ & $\begin{array}{r}4.47 \\
0.08 \\
1.79 \\
12.00\end{array}$ & $\begin{array}{r}12.90 \\
0.23 \\
1.79 \\
9.00\end{array}$ & $\begin{array}{r}4.32 \\
0.19 \\
4.40 \\
21.00\end{array}$ & $\begin{array}{r}41.83 \\
0.66 \\
1.58 \\
28.50\end{array}$ & $\begin{array}{r}23.65 \\
0.54 \\
2.29 \\
16.00\end{array}$ & $\begin{array}{r}10.92 \\
0.30 \\
2.75 \\
54.00\end{array}$ & $\begin{array}{r}24.94 \\
2.39 \\
9.59 \\
9.60\end{array}$ & $\begin{array}{r}9.55 \\
0.23 \\
2.41 \\
44.00\end{array}$ & $\begin{array}{r}0.31 \\
0.29 \\
3.49 \\
36.00\end{array}$ & $\begin{array}{r}10.80 \\
0.24 \\
2.23 \\
46.00\end{array}$ & $\begin{array}{r}15.01 \\
0.72 \\
4.80 \\
29.00\end{array}$ & $\begin{array}{r}173.30 \\
5.98\end{array}$ \\
\hline 1959 & $\begin{array}{l}\text { PRECIP. }(C M-) \\
S R-90 \text { INCI/SQ-KM-) } \\
S R-90 \text { CONC. (PC/L) } \\
S R-89 / S R-9 C\end{array}$ & $\begin{array}{r}6.22 \\
.0 .45 \\
7.24 \\
36.00\end{array}$ & $\begin{array}{r}5.97 \\
0.35 \\
5.87 \\
24.00\end{array}$ & $\begin{array}{r}11.46 \\
2.32 \\
20.25 \\
17.00\end{array}$ & $\begin{array}{r}5.00 \\
0.59 \\
11.81 \\
11.00\end{array}$ & $\begin{array}{r}30.12 \\
1.36 \\
4.52 \\
7.30\end{array}$ & $\begin{array}{l}38.58 \\
=\end{array}$ & $\begin{array}{r}24.89 \\
0.66 \\
2.66 \\
2.60\end{array}$ & $\begin{array}{r}25.70 \\
0.19 \\
0.74 \\
2.80\end{array}$ & $\begin{array}{r}23.32 \\
C .18 \\
C .18 \\
C .97\end{array}$ & $\begin{array}{r}20.07 \\
C .07 \\
C .35 \\
.\end{array}$ & $\begin{array}{r}31.90 \\
0.08 \\
0.26 \\
*\end{array}$ & $\begin{array}{l}2.87 \\
0.01 \\
0.35 \\
4\end{array}$ & $\begin{array}{r}220.10 \\
0.26\end{array}$ \\
\hline $196 \mathrm{C}$ & $\begin{array}{l}\text { PRECIP. }(C M, 1 \\
\text { SR-9C INCI/SO.KM-) } \\
\text { SR-90 CONC. }(P C / L) \\
\text { SR-89/SR-90 }\end{array}$ & $\begin{array}{l}1.78 \\
0.63 \\
1.69 \\
-9\end{array}$ & $\begin{array}{l}4.34 \\
0.04 \\
0.93 \\
--\end{array}$ & $\begin{array}{l}2.21 \\
0.03 \\
1.36 \\
\end{array}$ & $\begin{array}{l}27.66 \\
0.51 \\
1.85 \\
\end{array}$ & $\begin{array}{l}8.99 \\
= \\
=\end{array}$ & $\begin{array}{l}16.00 \\
= \\
=\end{array}$ & $\begin{array}{r}13.60 \\
0.05 \\
0.39 \\
\end{array}$ & $\begin{array}{r}11.60 \\
0.17 \\
1.55 \\
\end{array}$ & $\begin{array}{l}62 . c c \\
c . c 7 c \\
c .12 \\
--\end{array}$ & $\begin{array}{l}27.00 \\
0.036 \\
0.12 \\
-0\end{array}$ & $\begin{array}{l}5.99 \\
0.086 \\
1.01\end{array}$ & $\begin{array}{l}0.99 \\
0.016 \\
1.02 \\
--\end{array}$ & $\begin{array}{r}180.90 \\
1.00\end{array}$ \\
\hline 1961 & $\begin{array}{l}\text { PRECIP. (CM.) } \\
\text { SR-9O IFCI/SO.KM.) } \\
5 R-90 \text { CGNC. (PCIL) } \\
\text { SR-89/SR-90 }\end{array}$ & $\begin{array}{l}12.70 \\
0.07 \\
0.56 \\
-\end{array}$ & $\begin{array}{l}1.60 \\
0.01 \\
c .63 \\
--\end{array}$ & $\begin{array}{l}4.93 \\
0.05 \\
1.02 \\
-\infty\end{array}$ & $\begin{array}{l}1.40 \\
9.09 \\
6.43 \\
-\end{array}$ & $\frac{17.30}{-}$ & $\begin{array}{l}26.62 \\
= \\
=\end{array}$ & $\begin{array}{l}4.85 \\
0.026 \\
0.022 \\
\end{array}$ & $\begin{array}{c}11.89 \\
0.05 C \\
0.43 \\
--\end{array}$ & $\begin{array}{r}8.64 \\
0.03 \\
0.35 \\
45.60\end{array}$ & $\begin{array}{r}9.96 \\
0.11 \\
1.11 \\
84.00\end{array}$ & $\begin{array}{r}5.46 \\
0.14 \\
2.57 \\
89.00\end{array}$ & $\begin{array}{r}0.33 \\
0.10 \\
30.31 \\
63.00\end{array}$ & $\begin{array}{r}105.68 \\
0.67\end{array}$ \\
\hline 1962 & $\begin{array}{l}\text { PRECIP. ICH.) } \\
S R-90 \text { INCI/SC.KM.) } \\
S R-90 \text { CONC. (PC/L) } \\
S R-9 S / S R-90\end{array}$ & $\begin{array}{r}3.72 \\
0.24 \\
6.47 \\
41.00\end{array}$ & $\begin{array}{r}0.30 \\
0.24 \\
80.01 \\
35.00\end{array}$ & $\begin{array}{r}7.21 \\
0.62 \\
8.73 \\
15.00\end{array}$ & $\begin{array}{r}3.00 \\
1.35 \\
45.00 \\
11.00\end{array}$ & $\begin{array}{r}2.31 \\
0.37 \\
16.02 \\
17.00\end{array}$ & $\begin{array}{r}26.31 \\
0.80 \\
3.05 \\
13.00\end{array}$ & $\begin{array}{r}9.50 \\
0.50 \\
5.27 \\
17.00\end{array}$ & $\begin{array}{r}20.37 \\
0.37 \\
1.82 \\
8.00\end{array}$ & $\begin{array}{r}19.86 \\
0.47 \\
2.37 \\
13.00\end{array}$ & $\begin{array}{r}3.82 \\
0.51 \\
13.39 \\
41.00\end{array}$ & $\begin{array}{r}10.54 \\
0.15 \\
1.43 \\
47.00\end{array}$ & $\begin{array}{r}0.51 \\
0.15 \\
29.42 \\
33.00\end{array}$ & $\begin{array}{r}107.33 \\
5.77\end{array}$ \\
\hline 196.3 & $\begin{array}{l}P R E C[P \text {. ICM-) } \\
S R-90 \text { (MCI/SQ.KM-) } \\
S R-90 \text { CCAC, }(P C / L) \\
S R-89 / S R-90\end{array}$ & $\begin{array}{r}1.65 \\
0.45 \\
27.28 \\
41.00\end{array}$ & $\begin{array}{r}0.76 \\
6.54 \\
6.17 \\
32.00\end{array}$ & $\begin{array}{r}1.85 \\
1.49 \\
80.55 \\
13.00\end{array}$ & $\begin{array}{r}0.84 \\
3.63 \\
432.15 \\
13.00\end{array}$ & $\begin{array}{r}16.10 \\
2.35 \\
14.60 \\
2.30\end{array}$ & $\begin{array}{r}17.27 \\
2.36 \\
13.79 \\
0.60\end{array}$ & $\begin{array}{r}4.50 \\
0.64 \\
14.23 \\
5.70\end{array}$ & $\begin{array}{r}12.12 \\
0.59 \\
4.87 \\
\$\end{array}$ & $\begin{array}{r}28.24 \\
1.47 \\
5.21 \\
+\end{array}$ & $\begin{array}{r}11.25 \\
1.85 \\
16.45 \\
*\end{array}$ & $\begin{array}{l}3.63 \\
0.35 \\
9.65 \\
.\end{array}$ & $\begin{array}{r}10.82 \\
0.43 \\
3.98 \\
+\end{array}$ & $\begin{array}{r}117.03 \\
10.11\end{array}$ \\
\hline 1964 & $\begin{array}{l}\text { PRECIP. (CMA) } \\
\text { SR-SC IMCLISSO-KM-1) } \\
S R-90 \text { CONC. IPCAL) } \\
S R-B 9 / S R-90\end{array}$ & $\begin{array}{l}1.14 \\
0.75 \\
65.79 \\
*\end{array}$ & $\begin{array}{l}5.61 \\
0.39 \\
6.96 \\
*\end{array}$ & $\begin{array}{l}1.27 \\
0.12 \\
9.45 \\
*\end{array}$ & 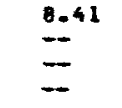 & $\begin{array}{l}11.06 \\
3.04 \\
25.64 \\
*\end{array}$ & $\begin{array}{r}26.62 \\
2.15 \\
8.08 \\
\end{array}$ & $\begin{array}{l}14.00 \\
0.97 \\
6.93 \\
-\end{array}$ & $\begin{array}{r}24.99 \\
0.53 \\
2.13 \\
\end{array}$ & $\begin{array}{r}10.72 \\
0.25 \\
2.34 \\
-\end{array}$ & $\begin{array}{l}24.82 \\
0.07 \\
c .29 \\
-\end{array}$ & $\begin{array}{l}7.62 \\
0.05 \\
0.66 \\
-\end{array}$ & $\begin{array}{r}15.85 \\
0.05 \\
0.32 \\
-\end{array}$ & $\begin{array}{r}152.91 \\
8.37\end{array}$ \\
\hline 1965 & $\begin{array}{l}\text { PRECIP. ICM-I } \\
\text { SR-90 IPCI/SQ-KM-) } \\
\text { SR-90 CONC. IPCILI } \\
\text { SR-89/SR-90 }\end{array}$ & $\begin{array}{l}5.03 \\
0.18 \\
3.58 \\
-\end{array}$ & $\begin{array}{l}7.57 \\
0.28 \\
3.70 \\
-\end{array}$ & $\begin{array}{l}10.08 \\
0.276 \\
2.68 \\
-\end{array}$ & $\begin{array}{l}3.05 \\
0.27 \mathrm{C} \\
8.86 \\
-\end{array}$ & $\begin{array}{r}1.12 \\
0.15 \\
13.40 \\
-\end{array}$ & $\begin{array}{l}* \\
0.19 \\
-\end{array}$ & $\begin{array}{l}-- \\
0.36 \\
=\end{array}$ & $\overline{0.43}$ & $\begin{array}{l}-\overline{0.29} \\
-\end{array}$ & $\begin{array}{l}-18 \\
-\end{array}$ & $\begin{array}{l}\overline{0.04} \\
=-\end{array}$ & $\begin{array}{l}\overline{0.08} \\
=-\end{array}$ & $\begin{array}{r}26.85 \\
2.72\end{array}$ \\
\hline 1966 & $\begin{array}{l}\text { PRECIP. (CM-) } \\
\text { SR-90 IMC1/SQ-KM-) } \\
5 R-90 \text { CONC. }(P C / L) \\
\text { SR-89/SR-90 }\end{array}$ & $\begin{array}{l}\overline{0 . c 6} \\
=\end{array}$ & $\bar{z}$ & $\overline{0.11}$ & $\overline{0.08}$ & $\overline{0.72}$ & $\overline{0.12}$ & $=$ & $\overline{0.07}$ & $\begin{array}{l}-\infty \\
0.04 C\end{array}$ & $\frac{\pi}{0.04 c}$ & $\frac{\overline{0.04 C}}{16.00}$ & $\frac{-\overline{0.03}}{*}$ & $\begin{array}{l}0.0 \\
1.42\end{array}$ \\
\hline 1967 & $\begin{array}{l}\text { PRECIP }(\text { ICM.) } \\
\text { SR-90 (MCI/SQ-KM.) } \\
\text { SR-90 CONC. (PC/L) } \\
5 R-89 / S R-90\end{array}$ & $\frac{\overline{0.05}}{7.80}$ & $\overline{0.03}$ & $\overline{0.16}$ & $\frac{\overline{0.01}}{*}$ & $\begin{array}{l}\overline{0.09} \\
\overline{2.20}\end{array}$ & $\bar{z}$ & $\bar{\Xi}$ & $\bar{z}$ & $\bar{z}$ & $\bar{z}$ & $\bar{z}$ & $\bar{z}$ & $\begin{array}{l}0.0 \\
0.34\end{array}$ \\
\hline 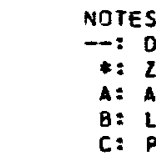 & $\begin{array}{l}\text { OATA NOT AUAILABLE } \\
\text { ZERO OR TRACE } \\
\text { APPROXIMATE } \\
\text { LOWER LIIIT OF REPO } \\
\text { PROPORTI ONED FROM O }\end{array}$ & $\begin{array}{l}\text { OATA } \\
\text { ALLY }\end{array}$ & & & & & & & & & & & & \\
\hline
\end{tabular}


MONTHLY FALLOUT DEPOSITION COLLECTIONS

SITE: FLORIOA, MIAMI

SOURCE: U. S. WEATHER BUREAU AIRPORT STATION
LAT. 25 G9M LOMG. 80 ITM ALT.

4M. (COLUMn) SURface AIR SAMPling Station

\begin{tabular}{|c|c|c|c|c|c|c|c|c|c|c|c|c|c|c|}
\hline & & JAN. & FEB. & MAR. & APR. & $\operatorname{maY}$ & JUNE & JULY & NUG. & SEP. & OCT. & NOV. & DEC. & CUM. TOTAL \\
\hline 1963 & $\begin{array}{l}\text { PRECIP }\left(C M_{*}\right) \\
\text { SR-90 } \text { (MCI/SC.KM.) } \\
S R-90 \text { CCNC. }(P C / L) \\
S R-89 / S R-S O\end{array}$ & $\begin{array}{l}1.65 \\
-- \\
--\end{array}$ & $\begin{array}{r}0.76 \\
0.75 \\
8.57 \\
28.00\end{array}$ & $\begin{array}{r}1.85 \\
1.05 \\
56.76 \\
19.00\end{array}$ & $\begin{array}{r}0.84 \\
0.67 \\
79.77 \\
11.00\end{array}$ & $\begin{array}{r}16.10 \\
2.64 \\
16.40 \\
6.5 C\end{array}$ & $\begin{array}{r}17.27 \\
3.42 \\
19.81 \\
1.4 \mathrm{C}\end{array}$ & $\begin{array}{r}4.50 \\
0.90 \\
2 C .01 \\
2.00\end{array}$ & $\begin{array}{r}12.12 \\
0.76 \\
6.28 \\
\end{array}$ & $\begin{array}{r}28.24 \\
0.01 \\
2.17 \\
1.40\end{array}$ & $\begin{array}{r}11.25 \\
1.24 \\
11.03 \\
1.40\end{array}$ & $\begin{array}{l}3.63 \\
0.32 \\
8.82 \\
*\end{array}$ & $\begin{array}{r}10.82 \\
0.31 \\
2.87 \\
*\end{array}$ & $\begin{array}{r}118.68 \\
12.67\end{array}$ \\
\hline 1964 & $\begin{array}{l}\text { PRECIP. (CM.) } \\
S R-9 C \text { (PCI/SQ.KM.) } \\
S R-90 \text { CONC. }(P C / L) \\
S R-89 / S R-90\end{array}$ & $\begin{array}{r}1.14 \\
6.19 \\
16.67 \\
4\end{array}$ & $\begin{array}{l}5.61 \\
C .47 \\
8.38 \\
+\end{array}$ & $\begin{array}{r}1.27 \\
0.19 \\
14.97 \\
*\end{array}$ & $\begin{array}{r}8.41 \\
0.96 \\
11.42 \\
\end{array}$ & $\begin{array}{l}12.91 \\
2.04 \\
17.13 \\
*\end{array}$ & $\begin{array}{r}26.62 \\
2.00 \\
7.52 \\
\end{array}$ & $\begin{array}{r}14.00 \\
1.16 \\
1.29 \\
-\end{array}$ & $\begin{array}{r}24.99 \\
0.46 \\
1.85 \\
-\end{array}$ & $\begin{array}{r}10.72 \\
0.30 \\
2.80 \\
-\end{array}$ & $\begin{array}{r}24.82 \\
0.20 \\
0.81 \\
19.00\end{array}$ & $\begin{array}{l}7.62 \\
0.20 \\
2.63 \\
2.10\end{array}$ & $\begin{array}{r}15.85 \\
0.24 \\
1.52 \\
--\end{array}$ & $\begin{array}{r}152.96 \\
8.41\end{array}$ \\
\hline 1965 & $\begin{array}{l}\text { PRECIP. }\left(C M_{-}\right) \\
\text {SR-90 (MCI/SQ.KM, } \\
\text { SR-90 CONC. }(P C / L) \\
\text { SR-89/SR-90 }\end{array}$ & $\begin{array}{l}5.03 \\
0.15 \\
2.99 \\
--\end{array}$ & $\begin{array}{l}7.57 \\
0.10 \\
1.33 \\
-.\end{array}$ & $\begin{array}{r}10.08 \\
0.32 \\
3.18 \\
--\end{array}$ & $\begin{array}{l}3.05 \\
0.05 \\
1.64 \\
--\end{array}$ & $\begin{array}{r}1.12 \\
0.21 \\
18.76 \\
-\end{array}$ & $\begin{array}{l}16.64 \\
0.64 \\
3.85 \\
--\end{array}$ & $\begin{array}{r}16.66 \\
0.48 \\
2.89 \\
-.\end{array}$ & $\begin{array}{r}12.62 \\
0.22 \\
1.75 \\
-.\end{array}$ & $\begin{array}{l}28.91 \\
0.12 \\
0.42 \\
-.\end{array}$ & $\begin{array}{r}42.65 \\
C .17 \\
C .40 \\
--\end{array}$ & $\begin{array}{l}2.44 \\
* \\
--\end{array}$ & $\begin{array}{r}1.80 \\
1.12 \\
62.23 \\
--\end{array}$ & $\begin{array}{r}148.57 \\
3.58\end{array}$ \\
\hline $196 t$ & $\begin{array}{l}\text { PRECIP. (CM.) } \\
\text { SR-90 (MCI/SQ.KM.) } \\
\text { SR-90 CCNC. (PC/L) } \\
S R-89 / S R-90\end{array}$ & $\begin{array}{r}1 C . C 8 \\
0.28 \\
2.78 \\
--\end{array}$ & $\begin{array}{l}16.66 \\
0.09 \\
0.55 \\
--\end{array}$ & $\begin{array}{l}8.26 \\
0.15 \\
1.82 \\
--\end{array}$ & $\begin{array}{l}4.57 \\
0.17 \\
3.72 \\
--\end{array}$ & $\begin{array}{l}14.05 \\
0.26 \\
1.86 \\
--\end{array}$ & $\begin{array}{r}55.96 \\
0.69 \\
1.24 \\
0.90\end{array}$ & $\begin{array}{r}21.59 \\
0.23 \\
1.07 \\
5.80\end{array}$ & $\begin{array}{r}19.35 \\
0.16 \\
0.83 \\
2.60\end{array}$ & $\begin{array}{r}20.32 \\
0.05 \\
0.25 \\
*\end{array}$ & $\begin{array}{r}27.64 \\
0.05 \\
0.19 \\
14.00\end{array}$ & $\begin{array}{r}9.75 \\
0.07 \\
0.72 \\
18.00\end{array}$ & $\begin{array}{l}1.88 \\
0.05 \\
2.06 \\
*\end{array}$ & $\begin{array}{r}210.11 \\
2.25\end{array}$ \\
\hline 1967 & $\begin{array}{l}\text { PRECIP. (CM.) } \\
S R-90 \text { (NCI/SQ.KM.) } \\
S R-90 \text { CONC. }(P C / L) \\
S R-89 / S R \rightarrow 90\end{array}$ & $\begin{array}{l}6.98 \\
0.07 \\
1.01 \\
14.00\end{array}$ & $\begin{array}{l}2.90 \\
0.03 \\
1.04 \\
*\end{array}$ & $\begin{array}{l}9.14 \\
0.09 \\
0.99 \\
9.70\end{array}$ & $\begin{array}{r}.0 .38 \\
0.04 \\
10.53 \\
*\end{array}$ & $\begin{array}{l}4.27 \\
0.04 \\
0.94\end{array}$ & $\begin{array}{l}40.59 \\
0.13 \\
0.33 \\
*\end{array}$ & $=$ & $\overline{-}$ & $\begin{array}{l}-- \\
-- \\
-\end{array}$ & $\begin{array}{l}-- \\
--\end{array}$ & $=$ & $\begin{array}{l}-- \\
-- \\
--\end{array}$ & $\begin{array}{r}64.26 \\
0.40\end{array}$ \\
\hline \begin{tabular}{c} 
NOTES \\
\hdashline$: D$ \\
$A: 2$ \\
$A:$ \\
$B:$ \\
$C:$
\end{tabular} & $\begin{array}{l}\text { ATA NOT AVAILABLE } \\
\text { ERO OR TRACE } \\
\text { PPROXIMATE } \\
\text { OHER LIIMI OF REPOR } \\
\text { ROPORTI ONED FRCM OR }\end{array}$ & DATA & & & & & & & & & & & & \\
\hline
\end{tabular}


SOURCE: U. S. HEATHER BUREAU AIRPORT STATION (ISLAND OF HAHAII)

\begin{tabular}{|c|c|c|c|c|c|c|c|c|c|c|c|c|c|c|}
\hline & & JAN. & FEB - & MAR . & APR. & $\operatorname{MaY}$ & JUNE & JULY & AUG. & SEP. & OCT & NOV. & DEC. & CUM. TOTAL \\
\hline 1959 & $\begin{array}{l}\text { PRECIP, (CM.) } \\
\text { SR-90 (MCI/SO.KM.) } \\
\text { SR-90 CONC. (PC/L) } \\
\text { SR-89/SR-90 }\end{array}$ & $=$ & $\begin{array}{r}33.35 \\
2.32 \\
6.96 \\
8.00\end{array}$ & $\begin{array}{l}19.28 \\
= \\
=\end{array}$ & $\begin{array}{r}20.12 \\
0.67 \\
3.34 \\
11.00\end{array}$ & $\begin{array}{r}17.81 \\
0.99 \\
5.56 \\
9.00\end{array}$ & $\begin{array}{l}7.44 \\
0.19 \\
2.56 \\
5.50\end{array}$ & $\begin{array}{r}14.68 \\
0.39 \\
2.66 \\
3.50\end{array}$ & $\begin{array}{r}18.80 \\
0.35 \\
1.87 \\
1.70\end{array}$ & $\begin{array}{l}3.35 \\
0.01 \\
0.30 \\
*\end{array}$ & $\begin{array}{l}14.94 \\
0.01 \\
0.07 \\
*\end{array}$ & $\begin{array}{l}76.33 \\
0.10 \\
0.14 \\
*\end{array}$ & $\begin{array}{l}36.50 \\
0.12 \\
0.33 \\
*\end{array}$ & $\begin{array}{r}262.60 \\
5.15\end{array}$ \\
\hline 1960 & $\begin{array}{l}\text { PRECIP. (CM.) } \\
\text { SR } 90 \text { IMCI/SQ.KM.) } \\
\text { SR-90 CCNC. (PC/L) } \\
S R-89 / S R-90\end{array}$ & $\begin{array}{l}65.91 \\
0.22 \\
0.34 \\
--\end{array}$ & $\begin{array}{l}40.56 \\
0.32 \\
0.79 \\
--\end{array}$ & $\begin{array}{r}18.11 \\
0.21 \\
1.16 \\
-.\end{array}$ & $\begin{array}{r}38.20 \\
0.56 \\
1.47 \\
-\end{array}$ & $\begin{array}{l}29.72 \\
0.61 \\
2.06 \\
-\end{array}$ & $\begin{array}{l}17.09 \\
=- \\
=-\end{array}$ & $\begin{array}{c}24.59 \\
0.16 \mathrm{C} \\
0.66 \\
.0\end{array}$ & $\begin{array}{l}32.72 \\
0.21 \mathrm{C} \\
0.65 \\
-\end{array}$ & $\begin{array}{c}30.48 \\
0.036 \\
0.10 \\
\end{array}$ & $\begin{array}{l}27.00 \\
0.03 \mathrm{C} \\
0.12 \\
-.\end{array}$ & $\begin{array}{c}37.92 \\
0.25 \mathrm{C} \\
0.66 \\
-.\end{array}$ & $\begin{array}{c}10.49 \\
0.07 C \\
0.67 \\
--\end{array}$ & $\begin{array}{r}372.79 \\
2.67\end{array}$ \\
\hline 1961 & $\begin{array}{l}\text { PRECIP. (CM.) } \\
\text { SR-90 IMCI/SQ.KM.) } \\
\text { SR-90 CONC. (PC/L) } \\
\text { SR-89/SR-90 }\end{array}$ & $\begin{array}{l}5.94 \\
0.126 \\
2.03 \\
-\end{array}$ & $\begin{array}{l}52.07 \\
1.07 C \\
2.06 \\
\end{array}$ & $\begin{array}{c}14.61 \\
0.18 \mathrm{C} \\
1.24 \\
-\end{array}$ & $\begin{array}{c}14.02 \\
0.18 \mathrm{C} \\
1.29 \\
\end{array}$ & $\begin{array}{c}20.62 \\
0.34 \mathrm{C} \\
1.65 \\
\end{array}$ & $\begin{array}{c}14.68 \\
0.28 \mathrm{C} \\
1.91 \\
--\end{array}$ & $\begin{array}{l}13.89 \\
0.08 C \\
0.58 \\
-\end{array}$ & $\begin{array}{l}19.76 \\
0.116 \\
0.56 \\
-\end{array}$ & $\begin{array}{r}17.17 \\
0.03 \\
0.18 \\
55.00\end{array}$ & $\begin{array}{r}58.29 \\
6.28 \\
0.49 \\
91.00\end{array}$ & $\begin{array}{l}32.61 \\
-- \\
-\end{array}$ & $\begin{array}{r}40.74 \\
0.55 \\
1.36 \\
58.00\end{array}$ & $\begin{array}{r}304.40 \\
3.22\end{array}$ \\
\hline 1962 & $\begin{array}{l}\text { PRECIP. (CM.) } \\
\text { SR-90 IMCI/SQ.KM.) } \\
\text { SR-90 CONC. }(P C / L) \\
\text { SR-89/SR } 90\end{array}$ & $\begin{array}{r}6.38 \\
0.31 \\
4.86 \\
42.00\end{array}$ & $\begin{array}{r}13.49 \\
1.43 \\
10.61 \\
31.00\end{array}$ & $\begin{array}{r}27.64 \\
2.20 \\
7.96 \\
22.00\end{array}$ & $\begin{array}{r}7.44 \\
0.32 \\
4.31 \\
13.00\end{array}$ & $\begin{array}{r}34.49 \\
3.38 \\
9.80 \\
9.00\end{array}$ & $\begin{array}{r}8.61 \\
0.84 \\
9.76 \\
10.00\end{array}$ & $\begin{array}{r}20.35 \\
1.06 \\
5.21 \\
12.00\end{array}$ & $\begin{array}{r}10.54 \\
0.48 \\
4.56 \\
8.00\end{array}$ & $\begin{array}{r}24.10 \\
1.01 \\
4.20 \\
26.00\end{array}$ & $\frac{6.10}{-}$ & $\begin{array}{r}16.84 \\
1.07 \\
6.36 \\
49.00\end{array}$ & $\begin{array}{r}5.87 \\
0.32 \\
5.46 \\
47.00\end{array}$ & $\begin{array}{r}181.85 \\
12.42\end{array}$ \\
\hline 1963 & $\begin{array}{l}\text { PRECIP. ICM.) } \\
\text { SR-9O (MCI/SQ.KM. }) \\
S R-90 \text { CONC. (PC/L) } \\
\text { SR-89/SR-90 }\end{array}$ & $\begin{array}{r}2.90 \\
0.41 \\
14.14 \\
35.00\end{array}$ & $\begin{array}{r}4.32 \\
0.86 \\
19.91 \\
23.00\end{array}$ & $\begin{array}{r}40.26 \\
4.55 \\
11.31 \\
15.00\end{array}$ & $\begin{array}{r}78.11 \\
0.33 \\
0.43 \\
11.00\end{array}$ & $\begin{array}{r}32.00 \\
6.17 \\
19.29 \\
7.50\end{array}$ & $\begin{array}{r}27.71 \\
4.83 \\
17.44 \\
1.70\end{array}$ & $\begin{array}{r}31.50 \\
2.86 \\
9.08 \\
2.00\end{array}$ & $\begin{array}{r}19.46 \\
2.76 \\
14.19 \\
1.00\end{array}$ & $\begin{array}{l}25.86 \\
1.50 \\
5.81 \\
1.70\end{array}$ & $\begin{array}{l}28.85 \\
1.80 \\
6.24 \\
*\end{array}$ & $\begin{array}{r}4.85 \\
1.06 \\
21.86 \\
*\end{array}$ & $\begin{array}{l}1.96 \\
0.24 \\
12.25 \\
*\end{array}$ & $\begin{array}{r}297.78 \\
27.37\end{array}$ \\
\hline 1964 & $\begin{array}{l}\text { PRECIP, (CM.) } \\
\text { SR-90 (MCI/SQ.KM.) } \\
S R-90 \text { CONC. (PC/L) } \\
S R-89 / 5 R-90\end{array}$ & $\begin{array}{l}37.21 \\
3.09 \\
8.31 \\
*\end{array}$ & $\begin{array}{r}46.28 \\
3.16 \\
6.83 \\
0.10\end{array}$ & $\begin{array}{l}49.73 \\
5.22 \\
10.50 \\
*\end{array}$ & $\begin{array}{l}28.02 \\
1.88 \\
6.71 \\
*\end{array}$ & $\begin{array}{r}63.53 \\
6.84 \\
10.77 \\
*\end{array}$ & $\begin{array}{r}17.81 \\
0.25 \\
1.41 \\
\end{array}$ & $\begin{array}{r}16.23 \\
1.85 \\
11.40 \\
-\end{array}$ & $\begin{array}{l}18.69 \\
0.94 \\
5.03 \\
--\end{array}$ & $\begin{array}{l}32.05 \\
0.81 \\
2.53 \\
-.\end{array}$ & $\begin{array}{r}25.36 \\
1.34 \\
4.57 \\
9.80\end{array}$ & $\begin{array}{l}59.39 \\
0.41 \\
0.70 \\
*\end{array}$ & $\begin{array}{l}24.51 \\
0.38 \\
1.56 \\
-\end{array}$ & $\begin{array}{r}422.81 \\
26.17\end{array}$ \\
\hline 1965 & $\begin{array}{l}\text { PRECIP. (CM.) } \\
S R-90 \text { (MCI/SQ.KM.) } \\
S R-90 \text { CONC. }(P C / L) \\
S R-89 / S R-90\end{array}$ & $\begin{array}{l}23.57 \\
1.24 \\
5.27 \\
--\end{array}$ & $\begin{array}{l}9.42 \\
0.72 \\
7.65 \\
-.\end{array}$ & $\begin{array}{l}21.16 \\
1.44 \\
6.81 \\
--\end{array}$ & $\begin{array}{l}46.96 \\
1.72 \\
3.67 \\
--\end{array}$ & $=$ & $\begin{array}{r}22.48 \\
2.68 \\
11.93 \\
\end{array}$ & $\begin{array}{l}-- \\
-- \\
--\end{array}$ & $\begin{array}{r}12.17 \\
0.70 \\
5.76 \\
-\end{array}$ & $\begin{array}{l}14.53 \\
0.23 \\
1.59 \\
-.\end{array}$ & $\begin{array}{l}14.73 \\
0.26 \\
1.77 \\
-\end{array}$ & $\begin{array}{l}48.72 \\
0.73 \\
1.50 \\
-\end{array}$ & $\begin{array}{l}37.95 \\
0.13 \\
0.35 \\
-.\end{array}$ & $\begin{array}{r}251.69 \\
9.85\end{array}$ \\
\hline 1966 & $\begin{array}{l}\text { PRECIP. (CM.) } \\
\text { SR-90 IMCI/SO.KM.) } \\
\text { SR-90 CONC. (PC/L) } \\
\text { SR-89/SR-90 }\end{array}$ & $\begin{array}{l}31.90 \\
0.11 \\
0.35 \\
--\end{array}$ & $\begin{array}{r}19.38 \\
0.45 \\
2.33 \\
=-\end{array}$ & $\begin{array}{l}14.20 \\
0.46 \\
3.24 \\
--\end{array}$ & $\begin{array}{l}13.30 \\
0.47 \\
3.54 \\
--\end{array}$ & $\begin{array}{r}12.80 \\
0.32 \\
2.51 \\
-.\end{array}$ & $\begin{array}{r}19.02 \\
0.54 \\
2.84 \\
5.00\end{array}$ & $\begin{array}{r}33.68 \\
0.46 \\
1.37 \\
3.40\end{array}$ & $\begin{array}{r}18.34 \\
0.15 \\
0.82 \\
3.50\end{array}$ & $\begin{array}{l}21.34 \\
0.09 \\
0.43 \\
*\end{array}$ & $\begin{array}{l}35.85 \\
0.18 \\
0.46 \\
*\end{array}$ & $\begin{array}{r}52.91 \\
0.13 \\
0.25 \\
25.00\end{array}$ & $\begin{array}{l}38.33 \\
0.25 \\
0.66 \\
*\end{array}$ & $\begin{array}{r}346.95 \\
4.28\end{array}$ \\
\hline 1967 & $\begin{array}{l}\text { PRECIP. (CM.) } \\
\text { SR-90 (MCI/SQ.KM.) } \\
\text { SR-90 CONC. }(P C / L) \\
\text { SR-89/SR-90 }\end{array}$ & $\begin{array}{r}20.42 \\
0.28 \\
1.38 \\
32.00\end{array}$ & $\begin{array}{r}26.29 \\
0.34 \\
1.30 \\
10.60\end{array}$ & $\begin{array}{r}24.03 \\
0.31 \\
1.30 \\
9.80\end{array}$ & $\begin{array}{r}54.00 \\
0.53 \\
0.99 \\
7.30\end{array}$ & $\begin{array}{r}24.99 \\
0.20 \\
0.81 \\
2.80\end{array}$ & $\begin{array}{r}15.90 \\
0.19 \\
1.20 \\
\$\end{array}$ & $\begin{array}{l}= \\
= \\
=\end{array}$ & $\begin{array}{l}- \\
- \\
-\end{array}$ & $=$ & $=$ & $=$ & $=$ & $\begin{array}{r}165.63 \\
1.85\end{array}$ \\
\hline
\end{tabular}

NOTES

- : DATA NOT AVAILABLE

*: zERO OR TRACE

A: APPROXIMATE

OF REPORTED DATA

C: PROPORTIONEC FROM ORIGINALLY CONSOLIDATED DATA 


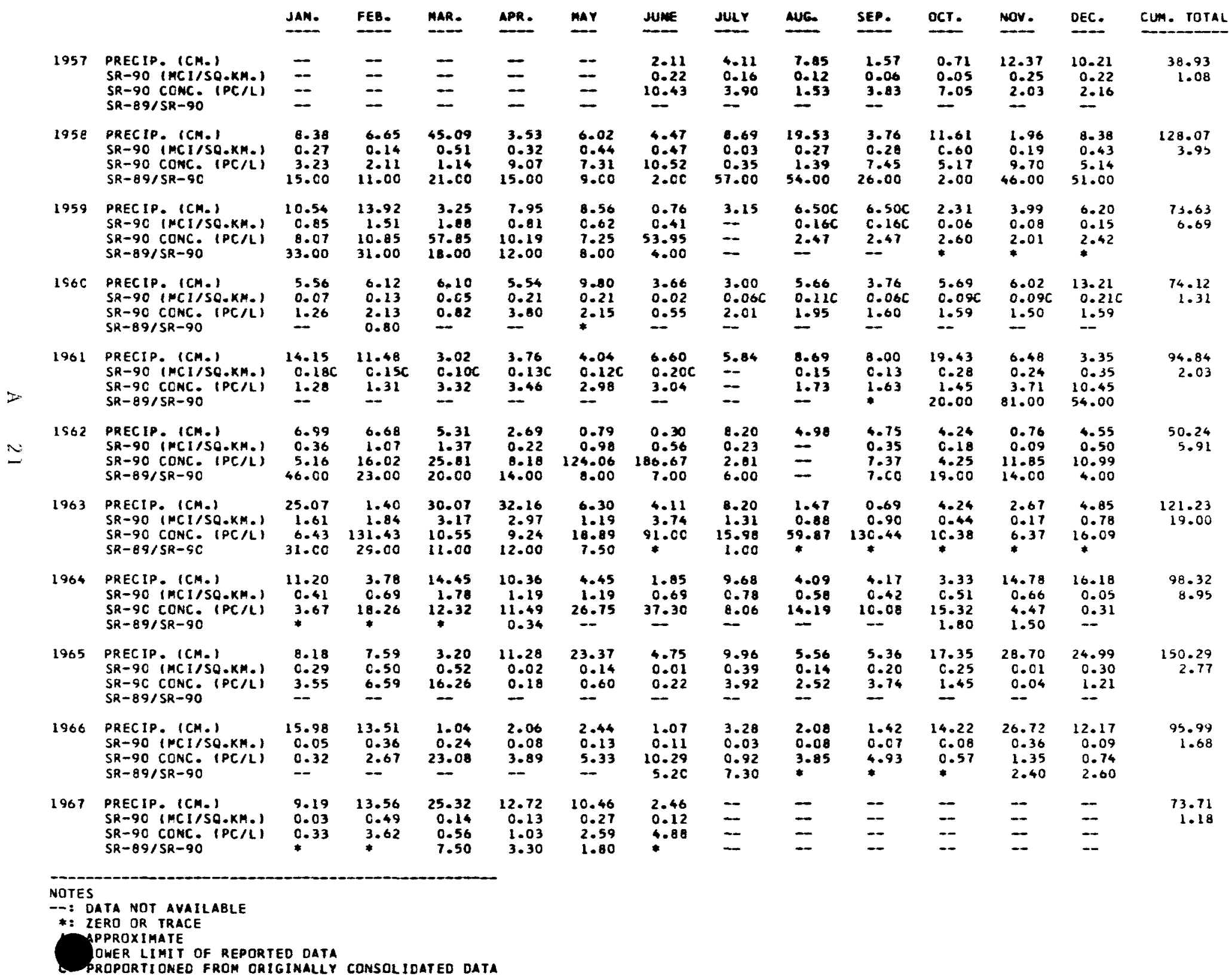




\section{MONTHY FALOUT DEPOSITION COLLETIONS}

\begin{tabular}{|c|c|c|c|c|c|c|c|c|c|c|c|c|c|c|}
\hline 1960 & $\begin{array}{l}\text { PRECIP. (CM.) } \\
\text { SR-90 (MCIISQ.KM) } \\
S R-90 \text { CONC. }(P C / L) \\
\text { SR-89/SR-90 }\end{array}$ & $=$ & $\begin{array}{l}10.52 \\
= \\
=\end{array}$ & $\begin{array}{l}24-69 \\
=\end{array}$ & $\begin{array}{l}2.95 \\
-\end{array}$ & $\begin{array}{l}6.86 \\
0.226 \\
3.21 \\
-\end{array}$ & $\begin{array}{l}3.71 \\
0.12 C \\
3.24 \\
-\end{array}$ & $\begin{array}{l}3.02 \\
0.036 \\
1.00 \\
-\end{array}$ & $\begin{array}{l}4.62 \\
0.056 \\
1.09 \\
-\end{array}$ & $\begin{array}{c}18.69 \\
0.066 \\
0.33 \\
-\end{array}$ & $\begin{array}{l}6.30 \\
0.026 \\
c .32 \\
-\end{array}$ & $\begin{array}{l}17.73 \\
0.05 C \\
0.29 \\
-.\end{array}$ & $\begin{array}{l}6.99 \\
0.02 C \\
0.29 \\
-\end{array}$ & $\begin{array}{r}108.54 \\
0.57\end{array}$ \\
\hline 1961 & $\begin{array}{l}\text { PRECIP. (CM-) } \\
\text { SR-90 (MCI/SO.KM.) } \\
\text { SR-90 CONC. }(P C / L) \\
S R-89 / 5 R-90\end{array}$ & $\begin{array}{l}3.18 \\
0.05 C \\
1.58 \\
-\end{array}$ & $\begin{array}{l}5.56 \\
0.096 \\
1.62 \\
-\end{array}$ & $\begin{array}{l}8.69 \\
0.076 \\
0.81 \\
\end{array}$ & $\begin{array}{c}12.67 \\
0.09 C \\
0.72 \\
\end{array}$ & $\begin{array}{l}2.59 \\
0.15 C \\
5.80 \\
-\end{array}$ & $\begin{array}{l}0.18 \\
0.016 \\
5.56 \\
-\end{array}$ & $\begin{array}{l}6.06 \\
= \\
-\end{array}$ & $\begin{array}{l}4.62 \\
- \\
-\end{array}$ & $\begin{array}{r}4.19 \\
0.07 \\
1.68 \\
123.00\end{array}$ & $\begin{array}{r}11.96 \\
0.13 \\
1.09 \\
111.00\end{array}$ & $\begin{array}{r}7.85 \\
0.39 \\
4.97 \\
103.00\end{array}$ & $\begin{array}{r}15.72 \\
0.25 \\
1.60 \\
65.00\end{array}$ & $\begin{array}{r}84.07 \\
1.30\end{array}$ \\
\hline 1962 & $\begin{array}{l}\text { PRECIP. }(C M-) \\
S R-90 \text { (NCI/SO.KM.) } \\
S R-90 \text { CONC. }(P C / L) \\
S R-89 / S R \rightarrow 90\end{array}$ & $\begin{array}{l}36.17 \\
1.354 \\
3.74 \\
-\end{array}$ & $\begin{array}{l}59.44 \\
1.354 \\
2.28 \\
\end{array}$ & $\begin{array}{l}33.22 \\
1.354 \\
4.07 \\
-\end{array}$ & $\begin{array}{c}6.02 \\
0.064 \\
14.29 \\
18.004\end{array}$ & $\begin{array}{l}27.64 \\
1.714 \\
6.19 \\
8.004\end{array}$ & $\begin{array}{c}17.45 \\
0.414 \\
2.35 \\
17.004\end{array}$ & $\begin{array}{c}4.19 \\
0.304 \\
7.16 \\
15.004\end{array}$ & $\underline{17.91}$ & $\begin{array}{r}3.33 \\
0.62 \\
18.62 \\
17.00\end{array}$ & $\begin{array}{l}6.83 C \\
6.31 C \\
4.54 \\
-.\end{array}$ & $\begin{array}{l}6.83 x \\
0.316 \\
4.54 \\
-\end{array}$ & $\begin{array}{r}2.90 \\
0.52 \\
17.94 \\
44.00\end{array}$ & $\begin{array}{r}221.93 \\
9.09\end{array}$ \\
\hline 1963 & $\begin{array}{l}\text { PRECIP. (CM-) } \\
\text { SR-90 IMCI/SO.KM-) } \\
\text { SR-90 CONC. (PC/R) } \\
\text { SR-B9/SR-90 }\end{array}$ & $\begin{array}{r}32.27 \\
0.89 \\
2.85 \\
65.00\end{array}$ & $\begin{array}{l}4.274 \\
0.681 \\
15.93 \\
29.004\end{array}$ & $\begin{array}{r}16.03 \\
2.14 \\
13.35 \\
10.00\end{array}$ & $\begin{array}{l}22.45 \\
2.44 \\
10.87 \\
10.00\end{array}$ & $\begin{array}{r}3.99 \\
1.20 \\
30.08 \\
11.10\end{array}$ & $\begin{array}{r}4.95 \\
1.81 \\
36.57 \\
2.20\end{array}$ & $\begin{array}{r}4.34 \\
0.56 \\
12.91 \\
2.00\end{array}$ & $\begin{array}{l}3.40 \\
0.29 \\
0.53 \\
.\end{array}$ & $\begin{array}{l}5.38 \\
0.22 \\
4.09 \\
0.50\end{array}$ & $\begin{array}{l}2.59 \\
0.23 \\
0.89 \\
0.50\end{array}$ & $\begin{array}{l}1.47 \\
0.10 \\
6.81 \\
*\end{array}$ & $\begin{array}{l}8.64 \\
0.35 \\
4.06 \\
1.00\end{array}$ & $\begin{array}{r}108.78 \\
10.91\end{array}$ \\
\hline 1964 & 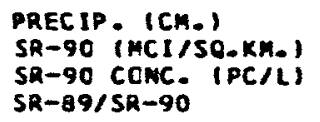 & $\begin{array}{r}17.81 \\
1.62 \\
9.10 \\
.\end{array}$ & $\begin{array}{l}4.39 \\
0.15 \\
3.42 \\
*\end{array}$ & $\begin{array}{r}25.81 \\
1.33 \\
5.16 \\
1.20\end{array}$ & $\begin{array}{r}7.32 \\
1.73 \\
23.64\end{array}$ & $\begin{array}{r}5.82 \\
0.84 \\
14.44 \\
+.44\end{array}$ & $\begin{array}{r}2.59 \\
4.40 \\
169.89 \\
\end{array}$ & $\begin{array}{r}7.62 \\
C .78 \\
10.24 \\
-.\end{array}$ & $\begin{array}{l}5.28 \\
0.35 \\
6.63 \\
-\end{array}$ & $\begin{array}{l}5.51 \\
0.19 \\
3.45 \\
\end{array}$ & $\begin{array}{l}8.28 \\
0.47 \\
5.68 \\
6.50\end{array}$ & $\begin{array}{r}24.03 \\
0.38 \\
1.59\end{array}$ & $\begin{array}{l}15.42 \\
0.10 \\
0.65 \\
-\end{array}$ & $\begin{array}{r}129.88 \\
12.34\end{array}$ \\
\hline 1965 & $\begin{array}{l}\text { PRECIP, (CM.) } \\
\text { SR-90 (MCI/SO-XM.) } \\
S R-90 \text { CONC. }(P C / L) \\
\text { SR-89/SR-90 }\end{array}$ & $\begin{array}{r}19.23 \\
0.30 \\
1.57 \\
\end{array}$ & $\begin{array}{l}0.40 \\
0.34 \\
5.32 \\
\end{array}$ & $\begin{array}{r}2.29 \\
0.32 \\
13.98 \\
-\end{array}$ & $\begin{array}{r}20.60 \\
0.45 \\
2.19 \\
-\end{array}$ & $\begin{array}{r}21.54 \\
0.57 \\
2.65 \\
-\end{array}$ & $\begin{array}{r}3.23 \\
0.33 \\
10.22 \\
-\end{array}$ & $\begin{array}{l}4.67 \\
C .16 \\
3.43 \\
-\end{array}$ & $\begin{array}{l}7.21 \\
0.14 \\
1.95 \\
\end{array}$ & $\begin{array}{l}9.27 \\
0.13 \\
1.41 \\
\end{array}$ & $\begin{array}{l}16.97 \\
= \\
=\end{array}$ & $\begin{array}{l}30.73 \\
0.25 \\
0.82 \\
\end{array}$ & $\begin{array}{l}7.72 \\
0.10 \\
1.30 \\
-\end{array}$ & $\begin{array}{r}149.86 \\
3.09\end{array}$ \\
\hline 1966 & $\begin{array}{l}\text { PRECIP. (CM-) } \\
S R-90 \text { IMCI/SO.KM.) } \\
\text { SR-90 CONC. (PC/L) } \\
S R-89 / S R-90\end{array}$ & $\begin{array}{l}4.11 \\
0.14 \\
3.41 \\
\end{array}$ & $\begin{array}{l}16.36 \\
0.56 \\
3.43 \\
-\end{array}$ & $\begin{array}{l}1.24 \\
0.09 \\
7.26 \\
-\end{array}$ & $\begin{array}{l}4.14 \\
0.31 \\
7.49 \\
-\end{array}$ & $\begin{array}{l}1.75 \\
0.09 \\
5.15 \\
\end{array}$ & $\begin{array}{l}2.74 \\
0.07 \\
2.56 \\
9.30\end{array}$ & $\begin{array}{l}5.00 \\
0.08 \\
1.61 \\
2.90\end{array}$ & $\begin{array}{l}5.18 \\
0.05 \\
0.97 \\
*\end{array}$ & $\begin{array}{l}3.84 \\
0.02 \\
0.53 \\
.\end{array}$ & $\begin{array}{r}28.98 \\
0.08 \\
0.28 \\
+\end{array}$ & $\begin{array}{r}20.65 \\
0.06 \\
0.30 \\
6.10\end{array}$ & $\begin{array}{l}9.37 \\
0.06 \\
0.65\end{array}$ & $\begin{array}{r}103.36 \\
1.61\end{array}$ \\
\hline 1967 & $\begin{array}{l}\text { PRECIP. (CM.) } \\
\text { SR-90 I HCI/SQ.KM.) } \\
\text { SR-90 CONC. (PC/L) } \\
\text { SR-89/SR-90 }\end{array}$ & $\begin{array}{r}14.20 \\
0.17 \\
1.20 \\
50.00\end{array}$ & $\begin{array}{r}16.74 \\
0.12 \\
0.72 \\
9.10\end{array}$ & $\begin{array}{r}22.66 \\
0.13 \\
0.58 \\
9.70\end{array}$ & $\begin{array}{l}4.06 \\
0.15 \\
3.70 \\
3.20\end{array}$ & $\begin{array}{r}10.29 \\
0.08 \\
0.78 \\
2.40\end{array}$ & $\begin{array}{l}5.31 \\
0.05 \\
0.95 \\
\end{array}$ & $=$ & $=$ & $\overline{-}$ & $\overline{-}$ & $\overline{-}$ & 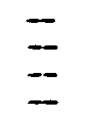 & $\begin{array}{r}73.26 \\
0.70\end{array}$ \\
\hline $\begin{array}{cc}\text { NOTES } \\
\text { : } \\
A: & Z \\
A: & A \\
B: & L \\
C: & P\end{array}$ & $\begin{array}{l}\text { DTA NOT AVAILABLE } \\
\text { ERO OR TRACE } \\
\text { PPROXIMATE } \\
\text { OWER LIMIT DF REPOR } \\
\text { ROPORTIONED FROM OS }\end{array}$ & DATA & & & & & & & & & & & & \\
\hline
\end{tabular}


MONTHLY FALLOUT DEPOSITION COLLECTIOMS

SITE: HaHAII, MAUMA LOA

\begin{tabular}{|c|c|c|c|c|c|c|c|c|c|c|c|c|c|c|}
\hline & & SAN. & FEB. & nAR. & APR . & may & JUNE & JULY & AUS. & SEP. & oCT. & nov. & DEC. & CUM, TOTAL \\
\hline 1959 & $\begin{array}{l}\text { PRECIP. (CM.) } \\
\text { SR-90 (MCI/SQ.KM.) } \\
\text { SR-90 CONC. (PC/L) } \\
S R-89 / 5 R-90\end{array}$ & $\bar{z}$ & $\begin{array}{r}0.92 \\
0.49 \\
5.50 \\
26.00\end{array}$ & $\begin{array}{l}0.28 \\
-\end{array}$ & $\begin{array}{r}2.24 \\
0.01 \\
0.45 \\
120.00\end{array}$ & $\frac{0.02}{6.70}$ & $\frac{0.00}{*}$ & $\begin{array}{l}14.63 \\
0.03 \\
0.21 \\
*\end{array}$ & $=$ & $\begin{array}{l}7.16 \\
-\end{array}$ & $\begin{array}{l}5.54 \\
0.01 \\
0.19\end{array}$ & $\begin{array}{l}7.16 \\
* \\
=\end{array}$ & $\begin{array}{l}0.71 \\
0.04 \\
5.64 \\
.\end{array}$ & $\begin{array}{r}61.27 \\
0.60\end{array}$ \\
\hline 1960 & $\begin{array}{l}\text { PRECIP. (CM.) } \\
S R-90 \text { IMCI/SQ.KMH) } \\
S R-90 \text { CONC. (PC/L) } \\
5 R-89 / S R-90\end{array}$ & $\begin{array}{l}0.76 \\
0.06 \\
7.90 \\
\end{array}$ & $\begin{array}{l}5.36 \\
0.07 \\
1.31 \\
-\end{array}$ & $\begin{array}{l}5.46 \\
0.07 \\
1.29 \\
-\end{array}$ & $\begin{array}{l}2.62 \\
0.05 \\
1.91 \\
\end{array}$ & $\begin{array}{l}2.84 \\
0.066 \\
2.12 \\
-\end{array}$ & $\begin{array}{l}5.87 \\
0.13 C \\
2.22 \\
-\end{array}$ & $\begin{array}{l}0.10 \\
- \\
-\end{array}$ & $\begin{array}{l}1.80 \\
+ \\
-\end{array}$ & $\begin{array}{l}0.84 \\
0.006 \\
0.01 \\
\end{array}$ & $\begin{array}{l}3.05 \\
0.006 \\
0.01 \\
-\end{array}$ & $\begin{array}{l}1.75 \\
- \\
-\end{array}$ & $\begin{array}{l}4.14 \\
-- \\
-\end{array}$ & $\begin{array}{r}34.59 \\
0.44\end{array}$ \\
\hline 1961 & $\begin{array}{l}\text { PRECIP. (CM.) } \\
\text { SR-9O IMCI/SQ.KM-) } \\
\text { SR-9O CONC. (PC/L) } \\
\text { SR-89/SR-90 }\end{array}$ & $\begin{array}{l}0.43 \\
0.01 \mathrm{C} \\
2.33 \\
-\end{array}$ & $\begin{array}{l}3.20 \\
0.056 \\
1.57 \\
-.\end{array}$ & $\begin{array}{l}1.60 \\
0.050 \\
3.13 \\
-\end{array}$ & $\begin{array}{l}1.96 \\
0.065 \\
3.07 \\
-\end{array}$ & $\begin{array}{l}2.59 \\
= \\
=\end{array}$ & $\begin{array}{l}0.10 \\
-\end{array}$ & $\frac{0.000}{-}$ & $\begin{array}{l}2.54 \\
0.016 \\
0.40 \\
-\end{array}$ & $\begin{array}{l}1.70 \\
+\end{array}$ & $\begin{array}{r}0.43 \\
0.02 \\
0.24 \\
60.00\end{array}$ & $\begin{array}{r}7.39 \\
0.02 \\
0.28 \\
88.00\end{array}$ & $\begin{array}{r}7.29 \\
0.03 \\
0.42 \\
86.00\end{array}$ & $\begin{array}{r}37.31 \\
0.25\end{array}$ \\
\hline 1962 & $\begin{array}{l}\text { PRECIP. (CM-) } \\
\left.\text { SR-90 I } M C I / S M_{-K M}\right) \\
S R-90 \text { CONC. (PC/L) } \\
S R-89 / 5 R-90\end{array}$ & $\begin{array}{r}3.15 \\
0.16 \\
5.08 \\
40.00\end{array}$ & $\begin{array}{l}0.33 \\
0.02 \\
6.07 \\
62.00\end{array}$ & $\begin{array}{r}5.44 \\
0.41 \\
7.54 \\
16.00\end{array}$ & $\begin{array}{r}4.52 \\
0.41 \\
9.08 \\
13.00\end{array}$ & $\begin{array}{r}5.46 \\
0.57 \\
10.44 \\
19.00\end{array}$ & $\begin{array}{l}1.24 \\
0.07 \\
5.65 \\
13.00\end{array}$ & $\begin{array}{l}0.08 \\
C .00 \\
0.01 \\
1.00\end{array}$ & $\begin{array}{l}1.63 \\
0.02 \\
1.23 \\
.\end{array}$ & $\begin{array}{l}3.45 \\
0.05 \\
1.45 \\
14.00\end{array}$ & $\begin{array}{l}0.13 \\
0.00 \\
0.01 \\
+\end{array}$ & $\begin{array}{r}0.10 \\
2.31 \\
2309.98 \\
36.00\end{array}$ & $\begin{array}{r}0.05 \\
0.02 \\
40.01 \\
17.00\end{array}$ & $\begin{array}{r}25.58 \\
4.04\end{array}$ \\
\hline 1963 & $\begin{array}{l}\text { PREC IP (CMH) } \\
\text { SR-SC (MCI/SQ.KM-) } \\
S R \rightarrow 90 \text { CONC }(P C / L) \\
S R-B 9 / S R-90\end{array}$ & $\begin{array}{r}8.89 \\
0.32 \\
3.60 \\
15.00\end{array}$ & $\begin{array}{r}1.50 \\
0.15 \\
10.01 \\
31.00\end{array}$ & $\begin{array}{r}11.07 \\
0.04 \\
7.59 \\
15.00\end{array}$ & $\begin{array}{r}16.43 \\
0.31 \\
1.89 \\
12.00\end{array}$ & $\begin{array}{r}10.52 \\
1.42 \\
13.50 \\
8.50\end{array}$ & $\begin{array}{r}2.21 \\
0.76 \\
34.39 \\
3.20\end{array}$ & $\begin{array}{l}5.03 \\
0.39 \\
7.76 \\
2.00\end{array}$ & $\begin{array}{l}4.60 \\
0.18 \\
3.92 \\
4\end{array}$ & $\begin{array}{l}4.27 \\
0.17 \\
3.99 \\
1.20\end{array}$ & $\begin{array}{l}0.46 \\
0.03 \\
6.53 \\
6\end{array}$ & $\begin{array}{l}0.43 \\
0.02 \\
4.66\end{array}$ & $\begin{array}{l}0.23 \\
\frac{0.00}{2.00}\end{array}$ & $\begin{array}{r}65.41 \\
4.82\end{array}$ \\
\hline 1964 & $\begin{array}{l}\text { PRECIP. }\left(C M_{-}\right) \\
S R-90 \text { IMCI/SQ.KM.) } \\
S R-90 \text { CONC. (PC/L) } \\
S R-Q 9 / S R-90\end{array}$ & $\begin{array}{r}0.81 \\
0.12 \\
14.82 \\
.\end{array}$ & $\begin{array}{r}0.71 \\
0.10 \\
14.09 \\
*\end{array}$ & $\begin{array}{l}6.35 \\
0.31 \\
4.89 \\
1.80\end{array}$ & $\begin{array}{r}2.08 \\
0.23 \\
11.06\end{array}$ & $\begin{array}{l}6.38 \\
0.23 \\
3.61 \\
*\end{array}$ & $\begin{array}{l}0.28 \\
0.01 \\
3.58 \\
-\end{array}$ & $\begin{array}{l}2.77 \\
0.20 \\
7.23 \\
-\end{array}$ & $\begin{array}{l}2.62 \\
0.06 \\
2.30 \\
\end{array}$ & $\begin{array}{l}3.15 \\
0.03 \\
0.56 \\
\end{array}$ & $\begin{array}{l}2.29 \\
C .05 \\
2.19 \\
*\end{array}$ & $\begin{array}{l}3.05 \\
0.06 \\
1.97 \\
3.90\end{array}$ & $\begin{array}{l}6.22 \\
0.09 \\
1.45 \\
-.\end{array}$ & $\begin{array}{r}36.71 \\
1.49\end{array}$ \\
\hline 1965 & $\begin{array}{l}\text { PRECIP. (CM.) } \\
S R-90 \text { (MCI/SQ.KM-I } \\
S R-90 \text { CONC. }(P C / L) \\
S R-99 / S R-90\end{array}$ & $\begin{array}{l}4.01 \\
0.16 \\
4.00 \\
-\end{array}$ & $\begin{array}{l}1.91 \\
0.06 \\
3.15 \\
\end{array}$ & $\begin{array}{l}2.62 \\
0.09 \\
3.44 \\
-\end{array}$ & $\begin{array}{l}7.04 \\
0.18 \\
2.56 \\
\end{array}$ & $\begin{array}{l}15.46 \\
0.27 \\
1.75 \\
\end{array}$ & $\begin{array}{l}2.18 \\
0.08 \\
3.67 \\
-\end{array}$ & $\begin{array}{l}4.78 \\
0.07 \\
1.47 \\
\end{array}$ & $\begin{array}{l}0.48 \\
-\end{array}$ & $\begin{array}{l}0.05 \\
0.07 \\
0.87 \\
-\end{array}$ & $\begin{array}{l}1.63 \\
0.04 \\
2.46 \\
-.\end{array}$ & $\begin{array}{l}7.01 \\
0.02 \\
0.29 \\
-.\end{array}$ & $\begin{array}{l}1.04 \\
0.03 \\
2.89 \\
-.\end{array}$ & $\begin{array}{r}56.19 \\
1.07\end{array}$ \\
\hline 1966 & $\begin{array}{l}\text { PRECIP. (CH) } \\
\text { SR-90 IMCI/SQ-KH-) } \\
S R-90 \text { CONC. }(P C / L) \\
S R-89 / S R-90\end{array}$ & $\begin{array}{l}0.50 \\
= \\
=\end{array}$ & $\begin{array}{l}10.44 \\
0.25 \\
2.40 \\
--\end{array}$ & $\begin{array}{l}1.55 \\
0.04 \\
2.59 \\
\end{array}$ & $\begin{array}{l}4.93 \\
0.04 \\
0.82 \\
-\end{array}$ & $\stackrel{*}{*}$ & $\frac{0}{0.01}$ & $\begin{array}{l}4.78 \\
0.02 \\
0.42\end{array}$ & $\frac{1.32}{-}$ & $\begin{array}{l}12.04 \\
0.01 \\
0.09 \\
.\end{array}$ & $\begin{array}{l}8.13 \\
0.06 \\
0.74 \\
.\end{array}$ & $\begin{array}{l}4.32 \\
0.02 \\
0.47 \\
.\end{array}$ & $\begin{array}{l}6.68 \\
0.04 \\
0.60 \\
*\end{array}$ & $\begin{array}{r}57.69 \\
0.49\end{array}$ \\
\hline 1967 & $\begin{array}{l}\text { PRECIP. (CM.) } \\
\text { SR-9O (MCI/SQ.KH.) } \\
S R-90 \text { CONC. }(P C / L) \\
S R-89 / S R-90\end{array}$ & $\begin{array}{r}8.81 \\
0.03 \\
0.35 \\
25.30\end{array}$ & $\begin{array}{l}0.86 \\
0.01 \\
1.17 \\
*\end{array}$ & $\begin{array}{r}10.49 \\
0.06 \\
0.58 \\
6.50\end{array}$ & $\begin{array}{l}5.23 \\
0.05 \\
0.96 \\
4.80\end{array}$ & $\begin{array}{l}6.98 \\
0.04 \\
0.58 \\
*\end{array}$ & $\begin{array}{l}5.10 \\
0.01 \\
0.20 \\
*\end{array}$ & $\begin{array}{l}9.30 \\
0.03 \\
0.33 \\
=\end{array}$ & $\bar{z}$ & $=$ & 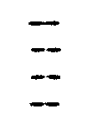 & 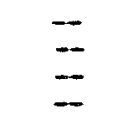 & $\begin{array}{l}-- \\
--\end{array}$ & $\begin{array}{r}46.77 \\
0.23\end{array}$ \\
\hline $\begin{array}{l}\text { NOTES } \\
: \\
*: \\
A: \\
B: \\
C:\end{array}$ & $\begin{array}{l}\text { ATA NOT AVAILABLE } \\
\text { ERO OR TRACE } \\
\text { PPROXIMATE } \\
\text { OWER LIMIT OF REPOR } \\
\text { PORTIONED FROM OR }\end{array}$ & $\begin{array}{l}\text { DATA } \\
\text { INALEY }\end{array}$ & yer & I & & & & & & & & & & \\
\hline
\end{tabular}




\begin{tabular}{|c|c|c|c|c|c|c|c|c|c|c|c|c|c|c|}
\hline & & دLN. & FEE. & man. & sm. & mar & ane & suLr & Nus & SEP. & 아. & nom. & DEC. & cum. TOTAL \\
\hline 1956 & 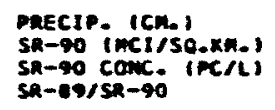 & $\underline{z}$ & $\bar{z}$ & $\bar{z}$ & $=$ & $\bar{z}$ & $=$ & $\bar{z}$ & $\bar{z}$ & $\bar{z}$ & $\bar{z}$ & $\bar{z}$ & $\begin{array}{l}3.20 \\
0.05 \\
1.57 \\
18.00\end{array}$ & $\begin{array}{l}3.20 \\
0.05\end{array}$ \\
\hline 1957 & 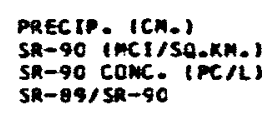 & $\begin{array}{r}5.23 \\
0.12 \\
2.30 \\
15.00\end{array}$ & $\begin{array}{l}4.50 \\
0.30 \\
2.23 \\
-\end{array}$ & $\begin{array}{l}5.03 \\
0.18 \\
3.50 \\
-.\end{array}$ & $\begin{array}{l}15.47 \\
0.46 \\
2.05 \\
-\end{array}$ & $\begin{array}{l}8.15 \\
0.10 \\
1.23 \\
8.30\end{array}$ & $\begin{aligned} 15.09 \\
0.19 \\
1.26 \\
37.00\end{aligned}$ & $\begin{array}{l}22.81- \\
0.61 \\
2.60\end{array}$ & $\begin{aligned} 13.61 \\
0.29 \\
2.14\end{aligned}$ & $\begin{array}{l}2.74 \\
0.05 \\
1.83 \\
62.00\end{array}$ & $\begin{aligned} 8.00 \\
0.00 \\
1.01 \\
20.00\end{aligned}$ & $\begin{array}{l}6.91 \\
8.08 \\
1.16 \\
16.00\end{array}$ & $\begin{array}{l}4.95 \\
=\end{array}$ & $\begin{array}{r}112.49 \\
2.24\end{array}$ \\
\hline 1958 & 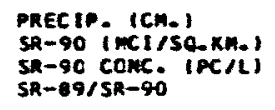 & $\bar{z}$ & $\bar{z}$ & 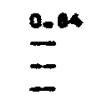 & $\begin{array}{l}4.17 \\
0.22 \\
5.28 \\
24.00\end{array}$ & $\begin{array}{l}8.25 \\
0.30 \\
3.69 \\
20.00\end{array}$ & $\begin{array}{l}16.33 \\
2.80 \\
11.52 \\
23.00\end{array}$ & $\begin{array}{r}12.04 \\
80.03 \\
0.25 \\
13.00\end{array}$ & $\begin{array}{r}6.36 \\
1.42 \\
22.26 \\
6.00\end{array}$ & $\begin{array}{l}4.72 \\
0.13 \\
2.76 \\
41.00\end{array}$ & $\begin{array}{r}5.38 \\
0.29 \\
5.40 \\
37.00\end{array}$ & $\begin{array}{l}4.01 \\
0.40 \\
9.98 \\
54.00\end{array}$ & $\begin{array}{l}1.45 \\
8.46 \\
11.04 \\
59.00\end{array}$ & $\begin{array}{r}63.47 \\
4.83\end{array}$ \\
\hline 1959 & $\begin{array}{l}P R E C I P \text { PR }\left(C M_{-}\right) \\
\text {SR-9C (NCI/SO-KM-) } \\
\text { SR-90 COMC. (RC/L) } \\
\text { SR-89/SR-90 }\end{array}$ & $\begin{array}{r}5.30 \\
0.39 \\
7.25 \\
37.00\end{array}$ & $\stackrel{6.07}{=}$ & $\underline{9.93}$ & $\begin{array}{l}10.16 \\
16.70 \\
160.74 \\
10.00\end{array}$ & $\begin{array}{r}9.63 \\
1.62 \\
16.69 \\
7.60\end{array}$ & $\begin{array}{l}15.09 \\
0.22 \\
1.46 \\
6.50\end{array}$ & $\begin{array}{l}12.12 \\
\text { c.13 } \\
1: .08 \\
:\end{array}$ & $\underline{\Xi}$ & $\begin{array}{l}5.51 \\
0.35 \\
6.36 \\
-\end{array}$ & $\underline{7.26}$ & $\begin{array}{l}7.65 \\
=\end{array}$ & $\begin{array}{l}5.08 \\
=\end{array}$ & $\begin{array}{r}98.22 \\
4.41\end{array}$ \\
\hline $196 \mathrm{c}$ & 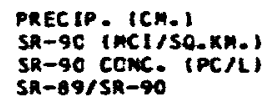 & $\stackrel{9.99}{=}$ & $\begin{array}{l}0.03 \\
0.08 \\
1.00 \\
-\end{array}$ & $\begin{array}{l}3.00 \\
0.42 \\
0.42 \\
-6=01\end{array}$ & $\begin{array}{l}2.00 \\
0.25 \\
3.13 \\
-\end{array}$ & $\underline{\underline{E}}=$ & $\frac{10.01}{=}$ & $\begin{array}{l}8.99 \\
0.156 \\
1.67 \\
-\end{array}$ & $\begin{array}{l}3.00 \\
3.05 c \\
0.056 \\
-6.07\end{array}$ & $\begin{array}{l}3.99 \\
3.056 \\
1.026 \\
-1.26\end{array}$ & $\begin{array}{l}3.99 \\
c .05 \mathrm{C} \\
3.26 \\
-\end{array}$ & $\begin{array}{l}3.00 \\
0.02 c \\
0.67\end{array}$ & $\begin{array}{l}3.00 \\
0.026 \\
0.67 \\
-\end{array}$ & $\begin{array}{r}71.02 \\
1.00\end{array}$ \\
\hline 1961 & 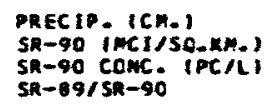 & $\begin{array}{l}\text { c.n1 } \\
0.016 \\
1.41\end{array}$ & $\begin{array}{l}2.31 \\
0.046 \\
1.74 \\
-\end{array}$ & $\begin{array}{l}7.49 \\
0.23 x \\
3.010\end{array}$ & $\begin{array}{l}8.31 \\
0.256 \\
3.02 \\
-\end{array}$ & $\begin{array}{l}3.69 \\
0.106 \\
6.63\end{array}$ & $\begin{array}{l}9.50 \\
0.436 \\
0.33\end{array}$ & $\begin{array}{l}7.58 \\
0.08 \\
1.00\end{array}$ & $\begin{array}{l}15.42 \\
0.11 \\
0.12 \\
.\end{array}$ & $\begin{array}{l}35.99 \\
0.06 \\
0.17 \\
63.00\end{array}$ & $\begin{array}{l}8.86 \\
6.17 \\
1.92 \\
83.00\end{array}$ & $\begin{array}{l}4.22 \\
0.35 \\
8.30 \\
83.00\end{array}$ & $\begin{aligned} 4.80 \\
0.27 \\
5.63 \\
64.00\end{aligned}$ & $\begin{array}{r}109.09 \\
2.18\end{array}$ \\
\hline 1962 & 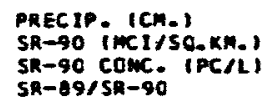 & $\begin{array}{l}6.40 \\
6.60 \\
9.36 \\
35.00\end{array}$ & $\begin{array}{r}4.40 \\
0.57 \\
12.40 \\
31.00\end{array}$ & $\begin{array}{l}4.00 \\
8.02 \\
0.44 \\
25.00\end{array}$ & $\begin{array}{l}4.01 \\
4.03 \\
0.63 \\
115.72 \\
11.00\end{array}$ & $\begin{array}{r}7.39 \\
2.69 \\
36.41 \\
10.00\end{array}$ & $\begin{array}{l}6.50 \\
0.64 \\
9.85 \\
8.00\end{array}$ & $\begin{aligned} 10.54 \\
0.72 \\
80.84 \\
17.00\end{aligned}$ & $\begin{array}{l}5.23 \\
0.09 \\
1.73 \\
20.00\end{array}$ & $\begin{array}{l}4.37 \\
0.32 \\
7.33 \\
33.00\end{array}$ & $\begin{array}{r}3.40 \\
0.40 \\
11.77 \\
28.00\end{array}$ & $\begin{array}{l}2.28 \\
0.12 \\
5.51 \\
56.00\end{array}$ & $\begin{array}{r}0.184 \\
0.09 \\
10.72 \\
49.00\end{array}$ & $\begin{array}{r}60.06 \\
6.89\end{array}$ \\
\hline 1963 & 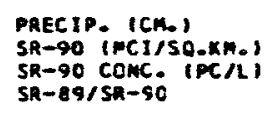 & $\begin{array}{r}2.49 \\
0.24 \\
9.64 \\
24.00\end{array}$ & $\begin{array}{r}1.91 \\
0.11 \\
5.76 \\
35.00\end{array}$ & $\begin{array}{l}4.67 \\
2.45 \\
32.47 \\
18.00\end{array}$ & $\begin{array}{r}7.96 \\
1.39 \\
17.42 \\
34.00\end{array}$ & $\begin{aligned} 5.89 \\
1.76 \\
29.89 \\
8.50\end{aligned}$ & $\begin{array}{r}6.10 \\
3.83 \\
62.79 \\
2.60\end{array}$ & $\begin{array}{r}17.91 \\
3.31 \\
18.69 \\
2.00\end{array}$ & $\begin{array}{r}3.07 \\
0.42 \\
13.69\end{array}$ & $\begin{array}{l}5.36 \\
0.37 \\
6.91 \\
6\end{array}$ & $\begin{aligned} 2.51 \\
0.51 \\
26.32 \\
*\end{aligned}$ & $\begin{array}{l}3.15 \\
0.25 \\
7.94 \\
2.40\end{array}$ & $\begin{array}{r}1.40 \\
0.18 \\
12.86\end{array}$ & $\begin{array}{l}62.44 \\
14.82\end{array}$ \\
\hline 1964 & $\begin{array}{l}\text { PRECIP. (CM-) } \\
\text { SR-SO iNCI/SQ-KMH) } \\
\text { SR-90 CONC. (PC/L) } \\
S R-89 / S R-90\end{array}$ & $\begin{array}{r}0.91 \\
0.29 \\
31.87 \\
*\end{array}$ & $\begin{array}{l}2.52 \\
0.13 \\
0.56 \\
: .56\end{array}$ & $\begin{array}{r}7.65 \\
1.28 \\
16.07 \\
-6\end{array}$ & 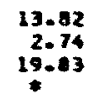 & $\begin{array}{r}6.62 \\
2.20 \\
25.98 \\
8.98\end{array}$ & $\begin{array}{r}7.57 \\
3.22 \\
42.54 \\
-5\end{array}$ & $\begin{array}{l}8.99 \\
8.96 \\
8.90 \\
-\end{array}$ & $\begin{array}{l}4.98 \\
0.53 \\
10.65 \\
-\end{array}$ & $\begin{array}{l}8.04 \\
8.39 \\
4.42 \\
-4\end{array}$ & $\begin{array}{r}0.61 \\
0.05 \\
8.20 \\
19.00\end{array}$ & $\begin{array}{l}4.24 \\
8.34 \\
8.02 \\
: 02\end{array}$ & $\begin{array}{l}2.69 \\
0.04 \\
10.49\end{array}$ & $\begin{array}{l}06.44 \\
11.02\end{array}$ \\
\hline 1965 & 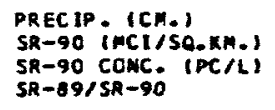 & $\begin{array}{l}8.66 \\
0.42 \\
0.65\end{array}$ & $\begin{array}{l}0.34 \\
0.17 \\
3.92\end{array}$ & $\begin{array}{l}6.40 \\
0.00 \\
1.26\end{array}$ & $\begin{array}{l}9.63 \\
0.78 \\
8.10 \\
0.40\end{array}$ & $\begin{array}{r}2.97 \\
0.38 \\
12.80\end{array}$ & $\begin{array}{r}5.31 \\
0.608 \\
22.81\end{array}$ & $\begin{array}{l}8.43 \\
0.36 \\
5.36 \\
-\end{array}$ & $\begin{array}{l}\begin{array}{l}21.41 \\
0.39 \\
1.83\end{array} \\
-\end{array}$ & $\begin{array}{r}15.21 \\
0.59 \\
3.86\end{array}$ & $\begin{array}{r}1.24 \\
0.15 \\
12.10\end{array}$ & $\begin{array}{l}2.26 \\
0.07 \\
3.13 \\
-\quad\end{array}$ & $\begin{array}{l}8.48 \\
0.17 \\
2.02 \\
-\end{array}$ & $\begin{array}{r}92.32 \\
4.24\end{array}$ \\
\hline 1906 & 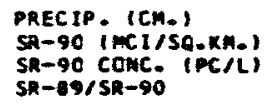 & $\begin{array}{l}1.93 \\
0.02 \\
1.04 \\
-\end{array}$ & $\begin{array}{l}3.35 \\
0.12 \\
3.59 \\
-\end{array}$ & $\begin{array}{l} \pm .74 \\
=\end{array}$ & $\begin{aligned} 10.84 \\
0.44 \\
6.06\end{aligned}$ & $\begin{array}{r}14.28 \\
0.26 \\
1.75 \\
-\end{array}$ & $\begin{array}{l}5.41 \\
0.19 \\
3.52 \\
9.30\end{array}$ & $\begin{array}{r}25.16 \\
6.06 \\
0.53 \\
0\end{array}$ & $\begin{array}{l}3.45 \\
0.06 \\
1.14 \\
.14\end{array}$ & $\begin{array}{l}2.46 \\
0.03 \\
1.22 \\
i\end{array}$ & $\begin{array}{l}5.08 \\
0.05 \\
0.99\end{array}$ & $\begin{aligned} 12.67 \\
0.10 \\
0.79 \\
31.00\end{aligned}$ & $\begin{array}{r}5.89 \\
0.07 \\
1.19 \\
13.00\end{array}$ & $\begin{array}{r}85.86 \\
1.42\end{array}$ \\
\hline 1967 & $\begin{array}{l}\text { PRECIP. (CM-3 } \\
\text { SR-90 inCI/SO-KM-) } \\
5 R-90 \text { CONC. }(P C / L) \\
\text { SR-09/SR-90 }\end{array}$ & $\begin{array}{l}7.80 \\
0.06 \\
0.77 \\
30.00\end{array}$ & $\begin{array}{l}2.92 \\
0.05 \\
1.72 \\
*\end{array}$ & $\begin{array}{l}4.44 \\
0.28 \\
6.31 \\
1.80\end{array}$ & $\begin{array}{l}13.44 \\
0.21 \\
1.57 \\
6.80\end{array}$ & $\begin{array}{l}3.35 \\
0.12 \\
3.59 \\
3.10\end{array}$ & $\begin{array}{r}12.09 \\
0.13 \\
1.08 \\
1.70\end{array}$ & $\bar{z}$ & $\bar{z}$ & $\bar{z}$ & $\bar{z}$ & $\bar{z}$ & $\bar{z}$ & $\begin{array}{r}44.04 \\
0.85\end{array}$ \\
\hline
\end{tabular}


MONTHLY FALLOUT OEPOSITION COLLECTIONS

SOURCE: RADIOCHEMISTRY, INC.

COLLECTIONS TERMINATED IN JULY 1963

\begin{tabular}{|c|c|c|c|c|c|c|c|c|c|c|c|c|c|c|}
\hline & & JAN. & FEQ. & man. & $\mathbf{M P R}$ & maY & June & suly & Aus. & SEP. & OCT. & nov. & DEC. & CUA TOTAL \\
\hline 1958 & $\begin{array}{l}\text { PRECIP. (CM) } \\
\text { SR-90 IMCI/SO.KM.) } \\
S R-90 \text { CONC. (PC/L) } \\
S R-89 / S R-90\end{array}$ & $\bar{z}$ & $\bar{z}$ & $=$ & $=$ & $=$ & $=$ & $=$ & $=$ & $\begin{array}{r}7.19 \\
0.10 \\
1.40 \\
15.00\end{array}$ & $\begin{array}{r}4.19 \\
0.18 \\
4.30 \\
23.00\end{array}$ & $\begin{array}{r}7.04 \\
0.25 \\
3.56 \\
19.00\end{array}$ & $\begin{array}{r}3.02 \\
0.23 \\
7.62 \\
41.00\end{array}$ & $\begin{array}{r}21.44 \\
0.76\end{array}$ \\
\hline 1959 & $\begin{array}{l}\text { PREC IP (CMA) } \\
\text { SR-9O INCI/SO.KM.) } \\
\text { SR-9O CONC. (PC/L) } \\
\text { SR-89/SR-9O }\end{array}$ & $\begin{array}{r}14.00 \\
0.85 \\
6.08 \\
45.00\end{array}$ & $\begin{array}{r}7.01 \\
0.61 \\
8.71 \\
32.00\end{array}$ & $\begin{array}{r}5.41 \\
1.22 \\
22.56 \\
23.00\end{array}$ & $\begin{array}{r}4.27 \\
1.29 \\
30.22 \\
13.00\end{array}$ & $\begin{array}{r}11.23 \\
1.15 \\
10.25 \\
12.00\end{array}$ & $\begin{array}{r}2.82 \\
0.36 \\
12.77 \\
7.00\end{array}$ & $\begin{array}{l}6.63 \\
0.26 \\
3.93 \\
4.00\end{array}$ & $\begin{array}{l}13.21 \\
0.11 \\
0.84 \\
0.70\end{array}$ & $\begin{array}{l}1.22 \\
0.03 \\
2.46 \\
2.00\end{array}$ & $\begin{array}{l}0.28 \\
0.14 \\
1.70 \\
1.10\end{array}$ & $\begin{array}{r}10.54 \\
0.09 \\
0.86 \\
1.40\end{array}$ & $\begin{array}{l}9.58 \\
0.17 \\
1.78 \\
2.40\end{array}$ & $\begin{array}{r}94.20 \\
6.28\end{array}$ \\
\hline 1960 & $\begin{array}{l}\text { PRECIP, (CM.) } \\
\text { SR-9O IMCI/SQ.KM.) } \\
S R-90 \text { CCNC. }(P C / L) \\
S R-89 / S R-90\end{array}$ & $\begin{array}{l}6.48 \\
0.35 \\
5.41 \\
2.40\end{array}$ & $\begin{array}{r}10.95 \\
0.47 \\
4.30 \\
1.40\end{array}$ & $\begin{array}{l}6.15 \\
0.15 \\
2.44 \\
4.10\end{array}$ & $\begin{array}{l}2.97 \\
0.22 \\
7.41 \\
0.40\end{array}$ & $\begin{array}{l}0.94 \\
0.46 \\
5.15 \\
0.20\end{array}$ & $\begin{array}{r}25.68 \\
0.50 \\
1.95 \\
0.20\end{array}$ & $\begin{array}{l}5.05 \\
0.07 \\
1.39 \\
0.50\end{array}$ & $\begin{array}{l}4.55 \\
0.06 \\
1.32 \\
-\end{array}$ & $\begin{array}{l}7.62 \\
0.04 \\
0.53 \\
\end{array}$ & $\begin{array}{l}4.01 \\
0.07 \\
1.75 \\
\end{array}$ & $\begin{array}{l}8.51 \\
0.05 \\
0.59 \\
\end{array}$ & $\begin{array}{l}5.18 \\
0.03 \\
0.58 \\
--\end{array}$ & $\begin{array}{r}96.09 \\
2.47\end{array}$ \\
\hline 1961 & $\begin{array}{l}\text { PREC IP }(C M)) \\
S R-90 \text { INCI/SQ.KM-) } \\
S R-90 \text { CONC. (PC/L) } \\
\text { SR-89/SR-90 }\end{array}$ & $\begin{array}{l}0.12 \\
0.05 \\
0.02 \\
-\end{array}$ & $\begin{array}{r}13.31 \\
0.13 \\
0.98 \\
\end{array}$ & $\begin{array}{r}19.38 \\
0.20 \\
1.04 \\
-\end{array}$ & $\begin{array}{l}12.27 \\
0.41 \\
.3 .35 \\
-.\end{array}$ & $\begin{array}{l}22.86 \\
0.47 \\
2.06 \\
-\end{array}$ & $\begin{array}{l}9.12 \\
0.17 \\
1.87 \\
\end{array}$ & $\begin{array}{r}14.73 \\
0.27 \\
1.26 \\
\end{array}$ & $\begin{array}{l}2.34 \\
0.07 \\
3.00 \\
-\end{array}$ & $\begin{array}{r}3.76 \\
0.04 \\
1.07 \\
32.00\end{array}$ & $\begin{array}{r}5.08 \\
0.10 \\
1.97 \\
70.00\end{array}$ & $\begin{array}{r}10.74 \\
0.29 \\
2.71 \\
83.00\end{array}$ & $\begin{array}{r}9.55 \\
0.34 \\
3.57 \\
63.00\end{array}$ & $\begin{array}{r}129.26 \\
2.44\end{array}$ \\
\hline 1962 & $\begin{array}{l}\text { PRECIP (CM.) } \\
\text { SR-9O IMCI/SQ.KH,) } \\
\text { SR-9O CONC. (PC/L) } \\
\text { SR-89/SR-SO }\end{array}$ & $\begin{array}{r}10.24 \\
0.80 \\
7.82 \\
46.00\end{array}$ & $\begin{array}{r}16.71 \\
0.92 \\
5.51 \\
27.00\end{array}$ & $\begin{array}{r}9.09 \\
1.16 \\
12.77 \\
25.00\end{array}$ & $\begin{array}{r}3.66 \\
0.51 \\
13.94 \\
15.00\end{array}$ & $\begin{array}{r}7.37 \\
1.52 \\
20.63 \\
17.00\end{array}$ & $\begin{array}{l}15.09 \\
1.55 \\
10.28 \\
10.00\end{array}$ & $\begin{array}{r}1.65 \\
0.40 \\
24.25 \\
14.00\end{array}$ & $\begin{array}{r}5.28 \\
0.33 \\
6.26 \\
15.00\end{array}$ & $\begin{array}{r}9.35 \\
0.21 \\
2.25 \\
33.00\end{array}$ & $\begin{array}{r}11.94 \\
0.64 \\
5.37 \\
17.00\end{array}$ & $\begin{array}{r}4.06 \\
1.07 \\
26.36 \\
23.00\end{array}$ & $\begin{array}{r}6.91 \\
0.46 \\
6.66 \\
27.00\end{array}$ & $\begin{array}{r}101.35 \\
9.57\end{array}$ \\
\hline 1963 & $\begin{array}{l}\text { PRECIP. (CM.) } \\
\text { SR-9O IMCI/SOEKM.) } \\
S R-90 \text { CONC. (PC/L) } \\
S R \rightarrow-99 / S R-90\end{array}$ & $\begin{array}{r}3.00 \\
0.66 \\
22.01 \\
26.00\end{array}$ & $\begin{array}{r}3.23 \\
0.85 \\
26.32 \\
20.00\end{array}$ & $\begin{array}{r}22.96 \\
2.70 \\
11.76 \\
13.00\end{array}$ & $\begin{array}{r}4.75 \\
1.53 \\
32.22 \\
15.00\end{array}$ & $\begin{array}{r}11.58 \\
5.80 \\
50.09 \\
7.00\end{array}$ & $\begin{array}{r}10.62 \\
4.05 \\
38.14 \\
6.00\end{array}$ & $\bar{z}$ & $\underline{-}$ & 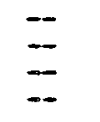 & $=$ & $=$ & $=$ & $\begin{array}{l}56.14 \\
15.59\end{array}$ \\
\hline $\begin{array}{l}\text { NOTES } \\
-:= \\
*: \\
A: \\
B: \\
C:\end{array}$ & $\begin{array}{l}\text { ATA NOT AVAILABLE } \\
\text { ERO OR TRACE } \\
\text { PPROXIMATE } \\
\text { OHER LIMIT OF REPOR } \\
\text { ROPORTIIONED FROM OR }\end{array}$ & DATA & & & & & & & & & & & & \\
\hline
\end{tabular}


nortin FMLOUT DEPOSITION COLECTIONS

SITE: KENTUCKY, LOUISVILLE

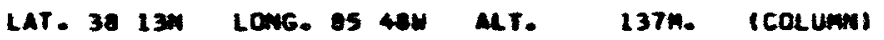

SOURCE: RAOIOCHEMISTRY, INC.

COLLECTIDNS TERMINATEO IN JULY 1961

$$
\begin{aligned}
& \text { JAN. FEQ. MAR. APR. MAY JUNE JUY AUG. SEP. OCT. NOV. OEC. CUM. TOTAL }
\end{aligned}
$$

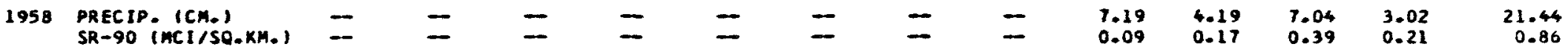

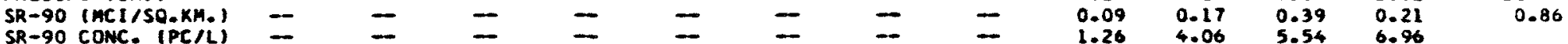

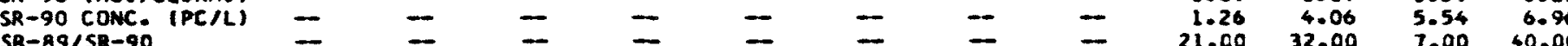

$$
\begin{aligned}
& 1959 \text { PRECIP. (CK.) } 14.00 \quad 7.02 \quad 5.41 \quad 4.2711 .23 \\
& \text { SR-90 CONC. }(P C / L) \quad 3.36
\end{aligned}
$$

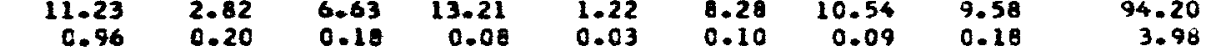

$$
\begin{aligned}
& 1.210 .06 \\
& \begin{array}{lllllll}
5.05 & 4.55 & 7.62 & 4.01 & 8.51 & 5.18 & 96.09
\end{array}
\end{aligned}
$$$$
\text { SR-9C (MCIISO.KM-) }
$$ 
SOURCE: U. S. MEATHER BUREAU AIRPORT STATIOA

\begin{tabular}{|c|c|c|c|c|c|c|c|c|c|c|c|c|c|c|}
\hline & & JAK. & FEB. & Mar. & $A P R$. & $\operatorname{mar}$ & JUME & JULY & Nut & SEP. & oct. & NON . & DEC. & CUM. TOTAL \\
\hline 1960 & $\begin{array}{l}\text { PRECIP, }(C M .) \\
\text { SR-90 (MCI/SQ.KM.) } \\
\text { SR-90 CONC. (PC/L) } \\
S R-89 / S R-90\end{array}$ & $\overline{-}$ & $\overline{=}$ & $=$ & $=$ & $\begin{array}{l}8.00 \\
0.076 \\
0.88 \\
--\end{array}$ & $\begin{array}{l}3.99 \\
0.036 \\
0.76 \\
-\end{array}$ & $\begin{array}{l}11.00 \\
=\end{array}$ & $\begin{array}{l}16.54 \\
=\end{array}$ & $\begin{array}{c}10.16 \\
- \\
-\end{array}$ & $\begin{array}{l}11.00 \\
=\end{array}$ & $\begin{array}{l}1.52 \\
0.026 \\
1.32 \\
-\end{array}$ & $\begin{array}{c}10.59 \\
0.13 C \\
1.23 \\
-\end{array}$ & $\begin{array}{r}72.80 \\
0.25\end{array}$ \\
\hline 1961 & $\begin{array}{l}\text { PRECIP. (CM.) } \\
\text { SR-9O IMCI/SQ.KM.) } \\
\text { SR-9O CONC. }(P C / 1) \\
S R-89 / S R-90\end{array}$ & $\begin{array}{l}17.63 \\
0.06 \\
0.35 \\
-\end{array}$ & $\begin{array}{c}22.86 \\
0.076 \\
0.31 \\
--\end{array}$ & $\begin{array}{c}21.67 \\
0.35 C \\
1.62 \\
\end{array}$ & $\begin{array}{l}7.32 \\
0.126 \\
1.64 \\
-\end{array}$ & $\begin{array}{r}18.47 \\
0.26 \mathrm{C} \\
1.41 \\
\end{array}$ & $\begin{array}{c}20.35 \\
0.29 C \\
1.43 \\
\end{array}$ & $\begin{array}{c}26.37 \\
0.136 \\
0.50 \\
\end{array}$ & $\begin{array}{c}18.44 \\
0.09 C \\
0.49 \\
\end{array}$ & $\begin{array}{r}22.61 \\
0.04 \\
0.18 \\
36.00\end{array}$ & $\begin{array}{r}1.30 \\
0.02 \\
1.54 \\
60.00\end{array}$ & $\begin{array}{r}22.00 \\
0.16 \\
0.73 \\
81.00\end{array}$ & $\begin{array}{r}15.21 \\
0.16 \\
1.06 \\
66.00\end{array}$ & $\begin{array}{r}214.23 \\
1.75\end{array}$ \\
\hline 1962 & $\begin{array}{l}\text { PRECIP. (CH.) } \\
\text { SR-90 (MCI/SO.KM.) } \\
\text { SR-90 CONC. (PCAL) } \\
\text { SR-89/SR-90 }\end{array}$ & $\begin{array}{r}10.64 \\
0.64 \\
6.02 \\
41.00\end{array}$ & $\begin{array}{r}2.97 \\
0.24 \\
8.09 \\
29.00\end{array}$ & $\begin{array}{r}4.06 \\
0.58 \\
14.29 \\
18.00\end{array}$ & $\begin{array}{r}6.76 \\
1.11 \\
16.43 \\
14.00\end{array}$ & $\begin{array}{r}3.33 \\
0.47 \\
14.12 \\
17.00\end{array}$ & $\begin{array}{r}22.53 \\
0.91 \\
4.04 \\
15.00\end{array}$ & $\begin{array}{r}11.94 \\
0.44 \\
3.69 \\
24.00\end{array}$ & $\frac{\overline{0.22}}{17.00}$ & $\begin{array}{l}0.40 \\
0.22 \\
3.44 \\
6.00\end{array}$ & $\begin{array}{r}6.36 \\
0.12 \\
1.44 \\
26.00\end{array}$ & $\begin{array}{r}4.90 \\
0.07 \\
1.41 \\
40.00\end{array}$ & $\begin{array}{r}11.35 \\
0.44 \\
3.88 \\
41.00\end{array}$ & $\begin{array}{r}93.32 \\
5.46\end{array}$ \\
\hline 1963 & $\begin{array}{l}\text { PRECIP }\left(C M_{-}\right) \\
\text {SR-9O IMCI/SQ.KM.) } \\
\text { SR-90 CONC. (PC/L) } \\
S R-89 / S R-90\end{array}$ & $\begin{array}{r}13.23 \\
0.60 \\
4.54 \\
30.00\end{array}$ & $\begin{array}{r}14.99 \\
0.62 \\
4.14 \\
26.00\end{array}$ & $\begin{array}{r}2.54 \\
0.25 \\
9.85 \\
18.00\end{array}$ & $\begin{array}{r}4.67 \\
0.88 \\
18.85 \\
14.60\end{array}$ & $\begin{array}{r}8.05 \\
1.05 \\
13.05 \\
15.70\end{array}$ & $\begin{array}{r}10.57 \\
1.18 \\
11.17 \\
3.70\end{array}$ & $\begin{array}{r}16.26 \\
1.92 \\
11.81 \\
3.00\end{array}$ & $\begin{array}{r}5.38 \\
1.01 \\
18.78 \\
*\end{array}$ & $\begin{array}{r}18.67 \\
0.29 \\
1.56 \\
*\end{array}$ & $\begin{array}{r}0.38 \\
0.10 \\
26.32\end{array}$ & $\begin{array}{l}19.94 \\
0.71 \\
3.57 \\
*\end{array}$ & $\begin{array}{r}13.34 \\
0.58 \\
4.35 \\
*\end{array}$ & $\begin{array}{r}128.02 \\
9.19\end{array}$ \\
\hline 1964 & $\begin{array}{l}\text { PRECIP. }\left(C H_{A}\right) \\
\text { SR-90 (MCI/SQ.KM ) } \\
\text { SR-90 CONC. }(P C / L) \\
\text { SR-Q9/SR-90 }\end{array}$ & $\begin{array}{r}24.38 \\
1.96 \\
8.04 \\
*\end{array}$ & $\begin{array}{r}13.59 \\
1.20 \\
8.84 \\
0.20\end{array}$ & $\begin{array}{l}13.84 \\
1.27 \\
9.18 \\
*\end{array}$ & $\begin{array}{l}14.38 \\
11.87 \\
13.01 \\
*\end{array}$ & $\begin{array}{r}4.29 \\
0.47 \\
10.96 \\
4\end{array}$ & $\begin{array}{r}14.02 \\
0.94 \\
6.71 \\
-.\end{array}$ & $\begin{array}{r}14.99 \\
0.91 \\
6.08 \\
-\end{array}$ & $\begin{array}{l}9.86 \\
0.44 \\
4.47 \\
-\end{array}$ & $\begin{array}{r}12.52 \\
0.16 \\
1.28 \\
--\end{array}$ & $\begin{array}{r}8.89 \\
c .10 \\
1.13 \\
10.00\end{array}$ & $\begin{array}{l}8.92 \\
0.13 \\
1.66 \\
3.80\end{array}$ & $\begin{array}{l}7.87 \\
0.17 \\
2.17 \\
--\end{array}$ & $\begin{array}{r}147.55 \\
9.62\end{array}$ \\
\hline 1965 & $\begin{array}{l}\text { PRECIP. }(C M .) \\
\text { SR-90 IMCI/SQ.KM.) } \\
\text { SR-90 CONC. }(P C / L) \\
\text { SR-89/SR-90 }\end{array}$ & $\begin{array}{r}11.38 \\
0.19 \\
1.67 \\
--\end{array}$ & $\begin{array}{r}13.34 \\
0.37 \\
2.78 \\
\end{array}$ & $\begin{array}{l}4.95 \\
0.19 \\
3.84 \\
\end{array}$ & $\begin{array}{r}0.84 \\
0.16 \\
16.67 \\
-\end{array}$ & $\begin{array}{l}9.19 \\
0.36 \\
3.92 \\
-\end{array}$ & $\begin{array}{l}5.61 \\
0.54 \\
9.63 \\
-\end{array}$ & $\begin{array}{r}13.36 \\
0.30 \\
2.25 \\
\end{array}$ & $\begin{array}{r}16.20 \\
0.27 \\
1.68 \\
\end{array}$ & $\begin{array}{l}25.48 \\
0.25 \\
0.99 \\
-\end{array}$ & $\begin{array}{l}2.62 \\
0.05 \\
1.91 \\
-\end{array}$ & $\begin{array}{l}3.78 \\
0.06 \\
1.59 \\
-\end{array}$ & $\begin{array}{l}18.67 \\
0.11 \\
0.59 \\
-\end{array}$ & $\begin{array}{r}125.32 \\
2.83\end{array}$ \\
\hline 1966 & $\begin{array}{l}\text { PRECIP. }\left(C M_{1}\right) \\
\text { SR-90 IACI/SQ.KM.) } \\
S R-90 \text { CONC. (PCAL) } \\
\text { SR-89/SR-90 }\end{array}$ & $\begin{array}{r}32.05 \\
0.26 \\
0.82 \\
-.\end{array}$ & $\begin{array}{r}25.68 \\
0.37 \\
1.45 \\
-\end{array}$ & $\begin{array}{l}4.83 \\
0.23 \\
4.77 \\
-\end{array}$ & $\begin{array}{r}12.50 \\
0.25 \\
2.01 \\
--\end{array}$ & $\begin{array}{l}23.65 \\
0.16 \\
0.68 \\
\end{array}$ & $\begin{array}{r}5.33 \\
0.04 \\
0.76 \\
05.00\end{array}$ & $\begin{array}{r}10.92 \\
C .20 \\
1.84 \\
4.10\end{array}$ & $\begin{array}{l}7.21 \\
0.08 \\
1.11 \\
+\end{array}$ & $\begin{array}{r}14.00 \\
0.07 \\
0.51 \\
*\end{array}$ & $\begin{array}{l}8.00 \\
0.03 \\
0.38 \\
+\end{array}$ & $\begin{array}{l}1.83 \\
0.03 \\
1.64 \\
+\end{array}$ & $\begin{array}{l}18.49 \\
0.10 \\
0.55 \\
.\end{array}$ & $\begin{array}{r}164.49 \\
1.82\end{array}$ \\
\hline 1967 & $\begin{array}{l}\text { PRECIP. }\left(C M_{\infty}\right) \\
\text { SR-90 (MC1/SQ.KM) } \\
S R-90 \text { CQNC. }(P C / L) \\
S R-89 / S R-90\end{array}$ & $\begin{array}{r}10.72 \\
0.06 \\
0.56 \\
6.70\end{array}$ & $\begin{array}{r}17.27 \\
0.06 \\
0.35 \\
5.50\end{array}$ & $\begin{array}{l}4.06 \\
0.07 \\
1.73 \\
5.00\end{array}$ & $\begin{array}{l}5.54 \\
0.04 \\
0.73 \\
3.50\end{array}$ & $\begin{array}{l}9.04 \\
0.10 \\
1.11 \\
2.20\end{array}$ & $\begin{array}{l}6.10 \\
0.05 \\
0.82 \\
*\end{array}$ & $=$ & $\overline{-}$ & $=$ & $=$ & $\overline{-}$ & $\overline{-}$ & $\begin{array}{r}52.73 \\
0.38\end{array}$ \\
\hline $\begin{array}{l}\text { NOTES } \\
-:: \\
*: \\
A: \\
B: \\
C:\end{array}$ & $\begin{array}{l}\text { ATA NOT AYAILABLE } \\
\text { ERO OR TRACE } \\
\text { PPROXIMATE } \\
\text { OWER LIMIT OF REPOR } \\
\text { ROPORTIONED FROM OR }\end{array}$ & $\begin{array}{l}\text { DATA } \\
\text { IALLY }\end{array}$ & & & & & & & & & & & & \\
\hline
\end{tabular}


MONTHLY FALLOUT DEPOSITION COLLECTIONS

SITE: MARYLANO, SILVER HILL

LAT. 38 59M LONG. T7 28H ALT. B2\%. (COLUMMI

SOURCE: U. S. MEATHER BUREAU

SITE DISCONTINUED DCTOBER 1960

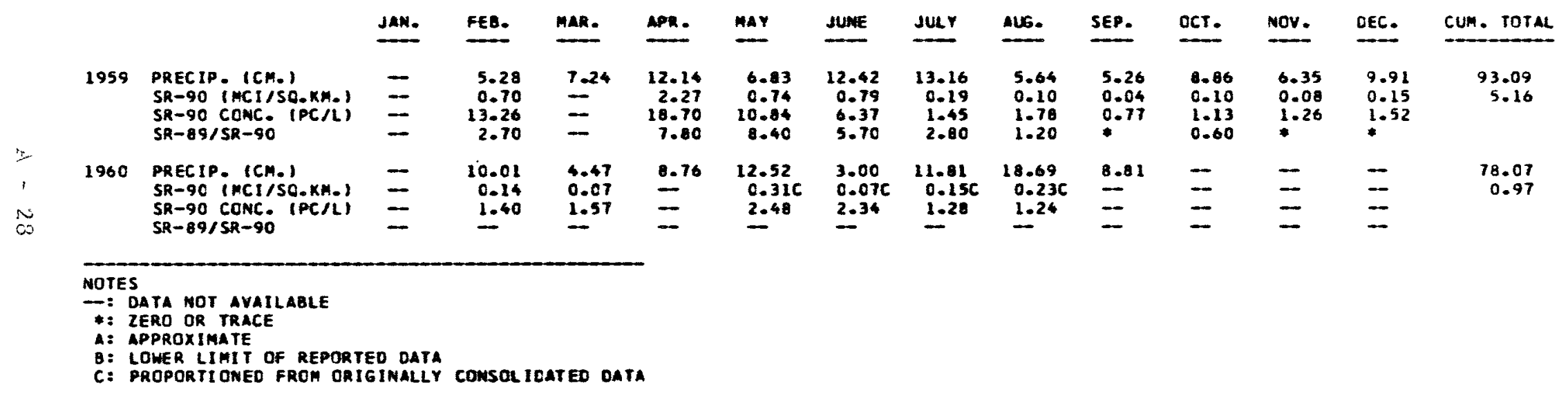




\begin{tabular}{|c|c|c|c|c|c|c|c|c|c|c|c|c|c|c|}
\hline & & JMA. & FEB. & MAR. & APR. & Mat & JUNE & sulv & Auc. & SEP. & oct. & MOV. & DEC. & CUM. TOTAL \\
\hline 1959 & $\begin{array}{l}\text { PRECIP. (CM-) } \\
\text { SA-90 IMCI/SO.KM-) } \\
S A-90 \text { CDMC. (PC/L) } \\
S A-99 / S R-90\end{array}$ & $\bar{z}$ & $\begin{array}{r}0.97 \\
0.04 \\
4.13 \\
60.00\end{array}$ & $\begin{array}{l}1.07 \\
-\end{array}$ & $\begin{array}{r}0.99 \\
0.42 \\
42.43 \\
11.00\end{array}$ & $\begin{array}{r}11.38 \\
1.93 \\
16.96 \\
7.40\end{array}$ & $\begin{array}{l}4.22 \\
-\end{array}$ & $\begin{array}{r}9.17 \\
1.23 \\
12.33 \\
3.10\end{array}$ & $\begin{array}{r}13.00 \\
0.37 \\
2.85 \\
1.40\end{array}$ & $\begin{array}{l}6.78 \\
0.07 \\
1.04 \\
0.66\end{array}$ & $\begin{array}{l}5.31 \\
0.07 \\
1.32 \\
1.20\end{array}$ & $\begin{array}{l}3.05 \\
0.02 \\
0.66\end{array}$ & $\begin{array}{l}2.36 \\
0.03 \\
1.28 \\
4.30\end{array}$ & $\begin{array}{r}58.30 \\
4.08\end{array}$ \\
\hline 1960 & $\begin{array}{l}\text { PRECIP. (CM.) } \\
\text { SR-9O (HCI/SQ.KM.) } \\
S R-90 \text { CONC. }(P C / L) \\
S R-99 / S R-90\end{array}$ & $\begin{array}{l}1.45 \\
0.04 \\
2.76 \\
\end{array}$ & $\begin{array}{l}0.84 \\
0.00 \\
0.01 \\
0\end{array}$ & $\begin{array}{r}1.02 \\
0.39 \\
38.24 \\
-\end{array}$ & $\begin{array}{l}5.16 \\
0.29 \\
5.63 \\
\end{array}$ & $\begin{array}{l}5.13 \\
= \\
=\end{array}$ & $\begin{array}{l}5.99 \\
-\end{array}$ & $\begin{array}{l}7.77 \\
0.036 \\
0.39 \\
-\end{array}$ & $\begin{array}{l}0.32 \\
0.030 \\
0.48 \\
\end{array}$ & $\begin{array}{l}4.75 \\
0.060 \\
1.27 \\
-\end{array}$ & $\begin{array}{l}4.93 \\
0.060 \\
1.22 \\
-\end{array}$ & $\begin{array}{l}3.58 \\
- \\
--\end{array}$ & $\begin{array}{l}4.24 \\
-- \\
--\end{array}$ & $\begin{array}{r}51.18 \\
0.90\end{array}$ \\
\hline 1961 & $\begin{array}{l}\text { PRECIP. (CM.) } \\
\text { SR-90 (MCI/SO-KM-) } \\
S R-90 \text { CONC. }(P C / L) \\
S R-89 / S R-90\end{array}$ & $\begin{array}{l}1.17 \\
0.056 \\
4.28 \\
\end{array}$ & $\begin{array}{l}2.16 \\
0.056 \\
4.17 \\
-.\end{array}$ & $\begin{array}{l}1.73 \\
0.096 \\
5.21 \\
-\end{array}$ & $\begin{array}{l}6.07 \\
0.30 \mathrm{C} \\
4.95 \\
-\end{array}$ & $\begin{array}{l}4.47 \\
0.326 \\
7.16 \\
-\end{array}$ & $\begin{array}{l}1.78 \\
0.136 \\
7.31 \\
-\end{array}$ & $\begin{array}{r}12.45 \\
0.22 C \\
1.77 \\
\end{array}$ & $\begin{array}{l}7.09 \\
0.126 \\
1.70 \\
\end{array}$ & $\begin{array}{r}18.69 \\
0.24 \\
1.29 \\
132.00\end{array}$ & $\begin{array}{r}2.26 \\
0.09 \\
3.99 \\
66.00\end{array}$ & $\begin{array}{r}2.46 \\
0.12 \\
4.88 \\
70.00\end{array}$ & $\begin{array}{r}2.36 \\
0.08 \\
3.39 \\
61.00\end{array}$ & $\begin{array}{r}62.69 \\
1.85\end{array}$ \\
\hline 1962 & $\begin{array}{l}\text { PREC IP. ICH.) } \\
S R-90 \text { (TCI/SO-KM.) } \\
S R-90 \text { CONC. (PC/L) } \\
S R-89 / S R-90\end{array}$ & $\begin{array}{r}2.39 \\
0.07 \\
2.93 \\
42.00\end{array}$ & $\begin{array}{r}3.23 \\
0.15 \\
4.65 \\
31.00\end{array}$ & $\begin{array}{r}1.93 \\
0.28 \\
14.51 \\
22.00\end{array}$ & $\begin{array}{r}4.57 \\
0.05 \\
1.20 \\
15.00\end{array}$ & $\begin{array}{r}15.62 \\
3.45 \\
22.09 \\
11.00\end{array}$ & $\begin{array}{r}8.76 \\
2.06 \\
23.52 \\
10.00\end{array}$ & $\begin{array}{r}16.79 \\
0.75 \\
4.47 \\
7.00\end{array}$ & $\begin{array}{l}6.65 \\
0.49 \\
7.37 \\
7.00\end{array}$ & $\begin{array}{r}9.07 \\
0.49 \\
5.41 \\
32.00\end{array}$ & $\begin{array}{r}0.58 \\
0.07 \\
12.07 \\
37.00\end{array}$ & $\begin{array}{r}1.24 \\
0.18 \\
14.52 \\
46.00\end{array}$ & $=$ & $\begin{array}{r}70.83 \\
8.04\end{array}$ \\
\hline 1963 & $\begin{array}{l}\text { PRECIP. }\left(C M_{-}\right) \\
\text {SR-90 INCI/SO.KK.) } \\
\text { SR-90 COKC. }(P C / L) \\
S R-89 / S R-90\end{array}$ & $\begin{array}{r}0.56 \\
0.20 \\
35.72 \\
26.00\end{array}$ & $\begin{array}{r}2.62 \\
0.15 \\
5.73 \\
21.00\end{array}$ & $\begin{array}{r}1.50 \\
0.26 \\
17.34 \\
16.00\end{array}$ & $\begin{array}{r}7.39 \\
5.10 \\
69.02 \\
9.00\end{array}$ & $\begin{array}{r}11.96 \\
5.04 \\
42.15 \\
7.40\end{array}$ & $\begin{array}{r}5.28 \\
1.88 \\
35.61 \\
0.30\end{array}$ & $\begin{array}{r}12.67 \\
5.09 \\
40.18 \\
2.00\end{array}$ & $\begin{array}{r}7.95 \\
2.79 \\
35.10 \\
*\end{array}$ & $\begin{array}{r}6.40 \\
0.89 \\
13.91 \\
\end{array}$ & $\begin{array}{l}0.86 \\
0.25 \\
29.07 \\
*\end{array}$ & $\begin{array}{r}2.84 \\
0.37 \\
13.03 \\
*\end{array}$ & $\begin{array}{l}3.02 \\
0.20 \\
6.63 \\
.\end{array}$ & $\begin{array}{l}63.05 \\
22.22\end{array}$ \\
\hline 1964 & $\begin{array}{l}\text { PRECIP. (CM.) } \\
S R-90 \text { IMCI/SO.KH.) } \\
S R-90 \text { CONC. }(P C / L) \\
S R-B 9 / S R \rightarrow 90\end{array}$ & $\begin{array}{l}1.88 \\
0.07 \\
3.73 \\
*\end{array}$ & $\begin{array}{r}1.60 \\
0.17 \\
10.63 \\
*\end{array}$ & $\begin{array}{r}1.65 \\
0.18 \\
10.91 \\
+\end{array}$ & $\begin{array}{r}7.42 \\
1.84 \\
24.80 \\
*\end{array}$ & $\begin{array}{r}5.38 \\
3.05 \\
56.70 \\
.\end{array}$ & $\begin{array}{l}19.00 \\
0.49 \\
2.58 \\
\end{array}$ & $\begin{array}{r}4.98 \\
1.58 \\
31.73 \\
\end{array}$ & $\begin{array}{r}16.61 \\
0.81 \\
4.88 \\
\end{array}$ & $\begin{array}{l}0.43 \\
0.29 \\
3.45 \\
\end{array}$ & $\begin{array}{r}1.07 \\
0.22 \\
20.57 \\
11.00\end{array}$ & $\begin{array}{l}1.85 \\
0.11 \\
5.95 \\
5.78\end{array}$ & $\begin{array}{l}2.13 \\
0.07 \\
3.29 \\
\end{array}$ & $\begin{array}{r}72.00 \\
8.88\end{array}$ \\
\hline 1965 & $\begin{array}{l}\text { PRECIP. }\left(C M_{-}\right) \\
S R-90 \text { (MCI/SO.KM.) } \\
S R=90 \text { COMC. }(P C / L) \\
S R-89 / S R-90\end{array}$ & $\begin{array}{l}0.56 \\
- \\
--\end{array}$ & $\begin{array}{l}1.09 \\
0.05 \\
4.59 \\
\end{array}$ & $\begin{array}{l}3.40 \\
0.04 \\
1.18 \\
\end{array}$ & $\begin{array}{r}3.48 \\
0.85 \\
24.43 \\
+.43\end{array}$ & $\begin{array}{l}8.99 \\
0.65 \\
7.24 \\
\end{array}$ & $\begin{array}{r}10.24 \\
1.56 \\
15.24 \\
-\end{array}$ & $\begin{array}{r}5.41 \\
0.56 \\
10.36 \\
\end{array}$ & $\begin{array}{l}1.34 \\
0.25 \\
3.41 \\
\end{array}$ & $\begin{array}{l}15.60 \\
0.44 \\
2.83 \\
-\end{array}$ & $\begin{array}{l}8.43 \\
0.07 \\
0.84 \\
\end{array}$ & $\begin{array}{l}6.68 \\
0.05 \\
0.75 \\
\end{array}$ & $\begin{array}{l}4.06 \\
0.04 \\
0.99 \\
--\end{array}$ & $\begin{array}{r}75.28 \\
4.56\end{array}$ \\
\hline 1966 & $\begin{array}{l}\text { PRECIP. (CM.) } \\
S R-90 \text { (PCI/SO.KHA) } \\
S R-90 \text { CONC. }(P C / L) \\
S R-89 / S R-90\end{array}$ & $\begin{array}{l}1.88 \\
0.03 \\
1.60 \\
-\end{array}$ & $\begin{array}{l}1.88 \\
0.11 \\
5.86 \\
-\end{array}$ & $\begin{array}{l}7.80 \\
0.24 \\
3.08 \\
-\end{array}$ & $\begin{array}{l}3.30 \\
0.19 \\
5.76 \\
\end{array}$ & $\begin{array}{l}3.05 \\
0.15 \\
4.92 \\
-.\end{array}$ & $\begin{array}{l}4.14 \\
0.25 \\
6.04 \\
5.00\end{array}$ & $\begin{array}{l}24.18 \\
0.08 \\
0.34 \\
4.70\end{array}$ & $\begin{array}{l}7.24 \\
0.18 \\
2.49 \\
2.60\end{array}$ & $\begin{array}{l}3.10 \\
0.05 \\
1.62\end{array}$ & $\begin{array}{l}3.99 \\
0.03 \\
0.76 \\
4\end{array}$ & $\begin{array}{l}2.03 \\
0.03 \\
1.48 \\
.\end{array}$ & $\begin{array}{l}2.34 \\
0.01 \\
0.43\end{array}$ & $\begin{array}{r}64.93 \\
1.35\end{array}$ \\
\hline 1967 & $\begin{array}{l}\text { PRECIP, (CM-) } \\
\text { SR-9O (MCI/SQ.KM.) } \\
\text { SR-9O COKC. (PC/L) } \\
\text { SR-89/SR-9O }\end{array}$ & $\begin{array}{r}2.41 \\
0.04 \\
1.66 \\
16.00\end{array}$ & $\begin{array}{l}1.83 \\
0.02 \\
1.10 \\
9.30\end{array}$ & $\begin{array}{l}2.51 \\
0.00 \\
3.19 \\
9.80\end{array}$ & $\begin{array}{l}7.92 \\
0.18 \\
2.28 \\
6.60\end{array}$ & $\begin{array}{l}2.84 \\
0.05 \\
1.77 \\
\end{array}$ & $\begin{array}{r}10.59 \\
0.15 \\
1.42 \\
1.40\end{array}$ & $\begin{array}{l}6.64 \\
0.18 \\
2.09\end{array}$ & $=$ & $=$ & $\begin{array}{l}= \\
=\end{array}$ & $=$ & $=$ & $\begin{array}{r}36.74 \\
0.70\end{array}$ \\
\hline
\end{tabular}

NOTES

-: DATA NOT AYAILABLE

* : ZERO OR TRACE

A: APPROXIMATE

8: LOWER LIMIT OF REPORTED DATA 


\begin{tabular}{|c|c|c|c|c|c|c|c|c|c|c|c|c|c|c|}
\hline & & JAM. & FEB. & MAR. & APR. & MAY & JUME & JULY & AUE. & SEP. & oCt. & NOV. & DEC. & CUM. TOTAL \\
\hline 1959 & $\begin{array}{l}\text { PRECIP. (CM-) } \\
\text { SR-9O IMCI/SO.KM-) } \\
\text { SR-9O CONC. (PC/L) } \\
\text { SR-QS/SR-SC }\end{array}$ & $=$ & $\begin{array}{r}5.44 \\
1.41 \\
25.92 \\
2.40\end{array}$ & $\begin{array}{r}9.61 \\
1.52 \\
17.66 \\
27.00\end{array}$ & $\begin{array}{r}6.15 \\
1.13 \\
18.38 \\
11.00\end{array}$ & $\begin{array}{r}11.05 \\
1.51 \\
13.67 \\
7.60\end{array}$ & $\begin{array}{r}0.30 \\
0.15 \\
50.01 \\
4.20\end{array}$ & $\begin{array}{r}10.08 \\
0.22 \\
2.19 \\
2.50\end{array}$ & $\begin{array}{l}2.79 \\
0.04 \\
1.44 \\
+\end{array}$ & $\begin{array}{r}13.74 \\
0.09 \\
0.66 \\
1.50\end{array}$ & $\begin{array}{r}10.64 \\
0.10 \\
0.94 \\
c .68\end{array}$ & $\begin{array}{l}1.42 \\
0.07 \\
4.93 \\
\end{array}$ & $\begin{array}{l}4.85 \\
0.11 \\
2.27 \\
0.90\end{array}$ & $\begin{array}{r}75.07 \\
6.35\end{array}$ \\
\hline 1960 & $\begin{array}{l}\text { PRECIP. (CM.) } \\
\text { SR-9O IMCI/SO.KM.) } \\
\text { SR-90 CONC. (PC/L) } \\
\text { SR-9Q/SR-9O }\end{array}$ & $\begin{array}{l}3.94 \\
0.06 \\
1.53 \\
-\end{array}$ & $\begin{array}{l}4.70 \\
0.14 \\
2.98 \\
-.\end{array}$ & $\begin{array}{l}5.56 \\
0.12 \\
2.16 \\
--\end{array}$ & $\begin{array}{l}13.00 \\
= \\
=\end{array}$ & $\begin{array}{l}10.64 \\
0.270 \\
2.54 \\
-.\end{array}$ & $\begin{array}{l}8.51 \\
0.22 C \\
2.59 \\
-\end{array}$ & $\frac{6.73}{-}$ & $\frac{0.69}{=}$ & $\begin{array}{l}6.73 \\
0.04 c \\
0.60 \\
-\end{array}$ & $\begin{array}{c}11.00 \\
0.076 \\
c .64 \\
-=\end{array}$ & $\begin{array}{l}2.13 \\
\pm \\
--\end{array}$ & $\frac{4.29}{-}$ & $\begin{array}{r}77.92 \\
0.92\end{array}$ \\
\hline 1961 & $\begin{array}{l}\text { PRECIP. (CM-) } \\
\text { SR-90 IMCI/SO.KM-) } \\
\text { SR COO CONC. (PC/L) } \\
\text { SR-Q9/SR-90 }\end{array}$ & $\begin{array}{l}1.75 \\
= \\
=-\end{array}$ & $\begin{array}{l}5.18 \\
=-\end{array}$ & $\begin{array}{c}11.89 \\
0.276 \\
2.28 \\
-\end{array}$ & $\begin{array}{l}11.94 \\
0.27 \mathrm{C} \\
2.27 \\
-\end{array}$ & $\begin{array}{l}16.08 \\
0.38 \mathrm{C} \\
2.37 \\
--\end{array}$ & $\begin{array}{l}13.36 \\
0.32 \mathrm{C} \\
2.40\end{array}$ & $\begin{array}{c}14.38 \\
6.190 \\
1.33 \\
-\end{array}$ & $\begin{array}{l}5.00 \\
0.07 c \\
1.41 \\
-\end{array}$ & $\begin{array}{r}20.83 \\
0.11 \\
0.53 \\
15.00\end{array}$ & $\begin{array}{r}7.26 \\
0.15 \\
2.07 \\
100.00\end{array}$ & $\begin{array}{r}8.53 \\
0.22 \\
2.58 \\
81.00\end{array}$ & $\begin{array}{r}4.14 \\
0.19 \\
4.59 \\
72.00\end{array}$ & $\begin{array}{r}120.34 \\
2.17\end{array}$ \\
\hline 1962 & 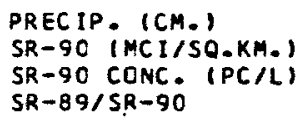 & $\begin{array}{r}4.22 \\
0.26 \\
6.17 \\
43.00\end{array}$ & $\begin{array}{r}5.08 \\
0.88 \\
17.33 \\
40.00\end{array}$ & $\begin{array}{r}8.64 \\
1.10 \\
12.74 \\
20.00\end{array}$ & $\begin{array}{r}3.38 \\
1.07 \\
31.66 \\
14.00\end{array}$ & $\begin{array}{r}6.58 \\
2.25 \\
34.20 \\
16.00\end{array}$ & $\begin{array}{r}3.58 \\
0.69 \\
19.28 \\
11.00\end{array}$ & $\begin{array}{r}9.09 \\
0.93 \\
10.24 \\
14.00\end{array}$ & $\begin{array}{r}10.52 \\
0.32 \\
3.05 \\
21.00\end{array}$ & $\begin{array}{r}17.09 \\
0.04 \\
6.24 \\
9.00\end{array}$ & $\begin{array}{r}6.30 \\
0.05 \\
0.80 \\
29.00\end{array}$ & $\begin{array}{r}1.88 \\
0.28 \\
14.90 \\
38.00\end{array}$ & $\begin{array}{r}1.96 \\
0.30 \\
15.31 \\
36.00\end{array}$ & $\begin{array}{r}78.32 \\
8.17\end{array}$ \\
\hline 1963 & $\begin{array}{l}\text { PREC IP. (CM-) } \\
\text { SR } \quad \text { SC (KCI/SO-KM.) } \\
\text { SR-90 CONC. (PCAL) } \\
S R-89 / S R-90\end{array}$ & $\begin{array}{r}1.02 \\
0.15 \\
14.71 \\
22.00\end{array}$ & $\begin{array}{r}0.46 \\
0.18 \\
39.14 \\
24.00\end{array}$ & $\begin{array}{r}8.71 \\
0.76 \\
8.73 \\
25.00\end{array}$ & $\begin{array}{r}5.46 \\
1.97 \\
36.09 \\
10.90\end{array}$ & $\begin{array}{r}11.51 \\
1.14 \\
9.91 \\
8.10\end{array}$ & $\begin{array}{r}3.26 \\
1.93 \\
60.32 \\
0.50\end{array}$ & $\begin{array}{r}14.48 \\
2.56 \\
17.68 \\
1.00\end{array}$ & $\begin{array}{r}10.97 \\
2.00 \\
18.24 \\
+\end{array}$ & $\begin{array}{r}5.26 \\
0.59 \\
11.22 \\
0.60\end{array}$ & $\begin{array}{r}1.98 \\
0.51 \\
25.76 \\
1.20\end{array}$ & $\begin{array}{l}4.47 \\
0.33 \\
7.39 \\
*\end{array}$ & $\begin{array}{l}1.52 \\
0.14 \\
9.22 \\
*\end{array}$ & $\begin{array}{l}69.04 \\
12.26\end{array}$ \\
\hline 1964 & $\begin{array}{l}\text { PRECIP. }\left(C M_{-}\right) \\
\text {SR-90 IMCI/SO.KM.) } \\
S R-90 \text { CONC. (PC/L) } \\
\text { SR-89/SR-9O }\end{array}$ & $\begin{array}{l}1.91 \\
0.47 \\
24.61 \\
*\end{array}$ & $\begin{array}{l}4.17 \\
0.41 \\
9.84 \\
*\end{array}$ & $\begin{array}{r}9.30 \\
1.30 \\
13.98\end{array}$ & $\begin{array}{r}17.81 \\
1.68 \\
9.44 \\
0.84\end{array}$ & $\begin{array}{r}17.09 \\
3.74 \\
21.89 \\
*\end{array}$ & $\begin{array}{r}7.57 \\
1.87 \\
24.71\end{array}$ & $\begin{array}{l}8.36 \\
1.24 \\
14.84\end{array}$ & $\begin{array}{r}1.96 \\
0.30 \\
15.31\end{array}$ & $\begin{array}{l}7.52 \\
0.29 \\
3.86 \\
-=\end{array}$ & $\begin{array}{r}0.91 \\
0.12 \\
13.19 \\
4.70\end{array}$ & $\begin{array}{l}7.92 \\
0.13 \\
1.65 \\
4\end{array}$ & $\begin{array}{l}3.30 \\
0.14 \\
4.25\end{array}$ & $\begin{array}{l}87.82 \\
11.69\end{array}$ \\
\hline 1965 & 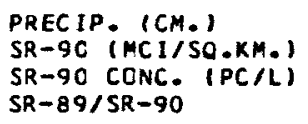 & $\begin{array}{l}6.40 \\
0.43 \\
6.72 \\
-\end{array}$ & $\begin{array}{l}2.92 \\
0.20 \\
6.85 \\
-\end{array}$ & $\begin{array}{l}8.64 \\
0.83 \\
9.61 \\
--\end{array}$ & $\begin{array}{r}11.40 \\
0.23 \\
2.02 \\
\end{array}$ & $\begin{array}{l}5.38 \\
0.42 \\
7.81 \\
--\end{array}$ & $\begin{array}{r}19.18 \\
1.51 \\
7.88 \\
--\end{array}$ & $\begin{array}{l}8.10 \\
0.32 \\
3.96 \\
-\end{array}$ & $\begin{array}{l}9.19 \\
0.28 \\
3.05 \\
-\end{array}$ & $\begin{array}{r}22.66 \\
0.58 \\
2.56 \\
-\end{array}$ & $\begin{array}{l}5.79 \\
0.18 \\
3.11 \\
-\end{array}$ & $\begin{array}{r}1.50 \\
0.20 \\
13.34 \\
--\end{array}$ & $\begin{array}{l}6.15 \\
0.08 \\
1.31 \\
-\end{array}$ & $\begin{array}{r}107.31 \\
5.26\end{array}$ \\
\hline 1966 & $\begin{array}{l}\text { PRECIP. (CM.) } \\
\text { SR-90 (HCI/SQ.KM.) } \\
\text { SR-90 CONC. (PC/L) } \\
\text { SR-89/SR-9O }\end{array}$ & $\begin{array}{l}0.53 \\
0.03 \\
5.67\end{array}$ & $\begin{array}{l}5.36 \\
\pm \\
=-\end{array}$ & $\begin{array}{l}2.13 \\
0.18 \\
8.46 \\
--\end{array}$ & $\begin{array}{r}10.62 \\
0.47 \\
4.43\end{array}$ & $\begin{array}{l}5.64 \\
0.32 \\
5.68\end{array}$ & $\begin{array}{l}8.33 \\
0.40 \\
4.81 \\
8.4 C\end{array}$ & $\begin{array}{r}10.36 \\
0.07 \\
0.68 \\
4.90\end{array}$ & $\begin{array}{l}3.71 \\
0.03 \\
0.81 \\
*\end{array}$ & $\begin{array}{l}8.43 \\
0.09 \\
1.07 \\
*\end{array}$ & $\begin{array}{l}5.21 \\
C .06 \\
1.16 \\
*\end{array}$ & $\begin{array}{l}3.50 \\
0.06 \\
1.72 \\
6.70\end{array}$ & $\begin{array}{l}4.98 \\
0.04 \\
0.81 \\
*\end{array}$ & $\begin{array}{r}68.80 \\
1.75\end{array}$ \\
\hline 1967 & $\begin{array}{l}\text { PREC IP. ICM ) } \\
\text { SR-90 (MCI/SQ.KM.) } \\
\text { SR-90 CONC. (PC/L) } \\
\text { SR-89/SR-90 }\end{array}$ & $\begin{array}{r}4.88 \\
0.05 \\
1.03 \\
12.00\end{array}$ & $\begin{array}{l}1.80 \\
0.04 \\
2.23 \\
7.40\end{array}$ & $\begin{array}{l}7.94 \\
0.19 \\
2.40 \\
7.30\end{array}$ & $\begin{array}{l}6.35 \\
0.21 \\
3.31 \\
4.50\end{array}$ & $\begin{array}{r}13.51 \\
0.13 \\
0.97 \\
3.00\end{array}$ & $\begin{array}{l}12.62 \\
0.11 \\
0.88 \\
*\end{array}$ & $\bar{z}$ & $\bar{z}$ & $=$ & $\begin{array}{l}= \\
=\end{array}$ & $\bar{z}$ & $\begin{array}{l}\bar{z} \\
=\end{array}$ & $\begin{array}{r}47.10 \\
0.73\end{array}$ \\
\hline
\end{tabular}

NOTES

--: DATA NOT AVAILABLE

*: ZERO OR TRACE

A: APPROXIMATE

C:- PROPORTIONEO FROM ORIGINALIF CONSOLTOATEO DATA 


\begin{tabular}{|c|c|c|c|c|c|c|c|c|c|c|c|c|c|c|}
\hline & & JAN. & FEB. & MAR. & APR. & MAY & JUAE & JULY & AU6. & SEP. & OCT. & MOY. & DEC. & CLM. TOTAL \\
\hline 1959 & $\begin{array}{l}\text { PRECIP. (CM.) } \\
S R-9 C \text { (NCI/SO.KM.) } \\
S R-90 \text { CONC. (PC/L) } \\
S R-89 / S R-90\end{array}$ & $=$ & $=$ & $\begin{array}{r}0.05 \\
0.22 \\
440.00 \\
25.00\end{array}$ & $\begin{array}{r}1.83 \\
0.78 \\
42.63 \\
*\end{array}$ & $\begin{array}{r}3.96 \\
0.59 \\
14.90 \\
8.70\end{array}$ & $\begin{array}{r}4.83 \\
0.90 \\
18.64 \\
5.30\end{array}$ & $\begin{array}{r}0.28 \\
0.15 \\
53.58 \\
2.80\end{array}$ & $\begin{array}{l}0.91 \\
0.03 \\
3.30 \\
2.50\end{array}$ & $\begin{array}{l}1.17 \\
0.07 \\
5.99 \\
+\end{array}$ & $\begin{array}{l}2.41 \\
-\end{array}$ & $\begin{array}{l}3.68 \\
0.01 \\
0.28 \\
+\end{array}$ & $\begin{array}{r}0.36 \\
0.07 \\
19.45 \\
1.20\end{array}$ & $\begin{array}{r}19.48 \\
2.82\end{array}$ \\
\hline $196 \mathrm{C}$ & $\begin{array}{l}\text { PREC IP ( }\left(C M_{-}\right) \\
S R-9 O\left(N C I / S Q . K M_{-}\right) \\
S R-90 \text { CONC. }(P C / L) \\
S R-89 / S R-90\end{array}$ & $\begin{array}{l}0.61 \\
0.02 \\
3.28 \\
+\end{array}$ & $\begin{array}{l}0.64 \\
0.04 \\
6.26 \\
0.80\end{array}$ & $\begin{array}{l}0.53 \\
0.04 \\
7.55 \\
\end{array}$ & $\begin{array}{l}3.96 \\
0.20 \\
5.06 \\
-\end{array}$ & $\begin{array}{l}2.39 \\
0.036 \\
1.26 \\
-\end{array}$ & $\begin{array}{l}0.58 \\
0.016 \\
1.73 \\
-\end{array}$ & $\begin{array}{l}2.59 \\
0.076 \\
2.71 \\
-\end{array}$ & $\begin{array}{l}5.33 \\
0.14 C \\
2.63 \\
-\end{array}$ & $\begin{array}{l}0.33 \\
0.026 \\
6.07 \\
-\end{array}$ & $\begin{array}{l}0.66 \\
0.036 \\
4.55 \\
-\end{array}$ & $\begin{array}{l}0.48 \\
- \\
=\end{array}$ & $\begin{array}{l}0.86 \\
* \\
--\end{array}$ & $\begin{array}{r}18.96 \\
0.60\end{array}$ \\
\hline 1961 & $\begin{array}{l}\text { PRECIP. (CM.) } \\
S R-90 \text { IMCI/SQ.KM.) } \\
S R-90 \text { CONC. (PC/L) } \\
S R-89 / S R-90\end{array}$ & $\begin{array}{l}0.23 \\
0.03 C \\
13.05 \\
\end{array}$ & $\begin{array}{l}0.15 \\
0.02 \mathrm{C} \\
13.34 \\
-\end{array}$ & $\begin{array}{l}2.62 \\
0.10 \mathrm{c} \\
3.82 \\
-\end{array}$ & $\begin{array}{l}2.21 \\
0.08 \mathrm{C} \\
3.62 \\
-\end{array}$ & $\begin{array}{l}3.53 \\
0.076 \\
1.99 \\
-\end{array}$ & $\begin{array}{l}1.98 \\
0.04 C \\
2.03 \\
-\end{array}$ & $\begin{array}{l}2.67 \\
0.096 \\
3.38 \\
-\end{array}$ & $\begin{array}{l}1.65 \\
0.06 C \\
3.64 \\
-\end{array}$ & $\begin{array}{r}2.95 \\
0.04 \\
1.36 \\
50.00\end{array}$ & $\begin{array}{r}0.41 \\
0.04 \\
9.76 \\
47.00\end{array}$ & $\begin{array}{r}0.94 \\
0.10 \\
10.64 \\
22.00\end{array}$ & $\begin{array}{r}1.40 \\
0.04 \\
2.86 \\
68.00\end{array}$ & $\begin{array}{r}20.74 \\
0.71\end{array}$ \\
\hline 1962 & $\begin{array}{l}\text { PRECIP. (CM.) } \\
S R-90 \text { IMCI/SO.KM-) } \\
S R-90 \text { CONC. }(P C / L) \\
S R-B 9 / S R-S O\end{array}$ & $\begin{array}{r}1.70 \\
0.04 \\
2.36 \\
54.00\end{array}$ & $\begin{array}{r}1.68 \\
0.14 \\
8.34 \\
30.00\end{array}$ & $\begin{array}{r}1.37 \\
0.16 \\
11.68 \\
19.00\end{array}$ & $\begin{array}{r}2.29 \\
0.06 \\
2.63 \\
18.00\end{array}$ & $\begin{array}{r}9.70 \\
1.66 \\
17.12 \\
8.00\end{array}$ & $\begin{array}{r}5.31 \\
1.33 \\
25.05 \\
10.00\end{array}$ & $\begin{array}{r}4.09 \\
1.34 \\
32.77 \\
25.00\end{array}$ & $\begin{array}{l}4.57 \\
1.04 \\
22.76 \\
10.00\end{array}$ & $\begin{array}{r}0.79 \\
0.20 \\
25.32 \\
13.00\end{array}$ & $\begin{array}{r}2.41 \\
0.22 \\
9.13 \\
20.00\end{array}$ & $\begin{array}{r}1.45 \\
0.11 \\
7.59 \\
40.00\end{array}$ & $\begin{array}{r}0.36 \\
0.08 \\
22.23 \\
19.00\end{array}$ & $\begin{array}{r}35.72 \\
6.38\end{array}$ \\
\hline 1963 & $\begin{array}{l}\text { PREC IP. (CM.) } \\
S R-90 \text { IMCIISQ.KM) } \\
S R-90 \text { CONC. }(P C / L) \\
S R \rightarrow 89 / S R-90\end{array}$ & $\begin{array}{r}1.12 \\
0.12 \\
10.72 \\
24.00\end{array}$ & $\begin{array}{r}0.64 \\
0.14 \\
21.88 \\
22.00\end{array}$ & $\begin{array}{r}0.58 \\
0.14 \\
24.14 \\
10.00\end{array}$ & $\begin{array}{r}2.57 \\
1.23 \\
47.86 \\
7.00\end{array}$ & $\begin{array}{r}3.38 \\
1.52 \\
44.98 \\
11.60\end{array}$ & $\begin{array}{r}6.27 \\
4.36 \\
69.54 \\
2.20\end{array}$ & $\begin{array}{l}2.34 \\
0.18 \\
7.70 \\
-.\end{array}$ & $\begin{array}{r}1.52 \\
1.21 \\
79.61 \\
-\end{array}$ & $\begin{array}{r}3.30 \\
0.67 \\
20.31 \\
0.30\end{array}$ & $\begin{array}{r}3.53 \\
0.72 \\
2 C .40 \\
*\end{array}$ & $\begin{array}{l}0.74 \\
0.12 \\
16.22 \\
*\end{array}$ & $\begin{array}{l}3.28 \\
0.16 \\
4.88 \\
+\end{array}$ & $\begin{array}{l}29.27 \\
10.57\end{array}$ \\
\hline 1964 & $\begin{array}{l}\text { PRECIP. }(C M-) \\
\text { SR-9O IMCI/SQ.KM.) } \\
S R-90 \text { CONC. }(P C / L) \\
S R-89 / S R-90\end{array}$ & $\begin{array}{r}0.79 \\
0.15 \\
18.99 \\
*\end{array}$ & $\begin{array}{r}0.66 \\
0.17 \\
25.76 \\
*\end{array}$ & $\begin{array}{l}1.32 \\
0.05 \\
3.79 \\
*\end{array}$ & $\begin{array}{r}1.73 \\
0.46 \\
26.59\end{array}$ & $\begin{array}{r}8.46 \\
1.43 \\
16.91 \\
*\end{array}$ & $\begin{array}{r}6.25 \\
2.76 \\
44.16 \\
\end{array}$ & $\begin{array}{r}2.11 \\
1.09 \\
51.66 \\
-\end{array}$ & $\begin{array}{r}4.75 \\
0.59 \\
12.43 \\
\end{array}$ & $\begin{array}{r}0.51 \\
0.17 \\
33.34 \\
-.\end{array}$ & $\begin{array}{l}0.10 \\
0.07 \\
70.01 \\
*\end{array}$ & $\begin{array}{l}1.35 \\
0.06 \\
4.45 \\
3.70\end{array}$ & $\begin{array}{l}2.49 \\
0.10 \\
4.02 \\
-.\end{array}$ & $\begin{array}{r}30.52 \\
7.10\end{array}$ \\
\hline 1965 & $\begin{array}{l}\text { PRECIP. (CM.) } \\
\text { SR-9O IMCI/SQ.KM.) } \\
S R-9 C \text { CONC. }(P C / L) \\
S R-89 / S R-90\end{array}$ & $\begin{array}{l}0.91 \\
0.05 \\
5.50 \\
-\end{array}$ & $\begin{array}{l}1.24 \\
0.05 \\
4.04 \\
-.\end{array}$ & $\begin{array}{l}2.16 \\
0.15 \\
6.95 \\
-\end{array}$ & $\begin{array}{l}2.49 \\
0.22 \\
8.84 \\
5.30\end{array}$ & $\begin{array}{r}5.59 \\
0.71 \\
12.71 \\
-\end{array}$ & $\overline{1.13}$ & $\begin{array}{r}1.52 \\
0.53 \\
34.87 \\
-\end{array}$ & $\begin{array}{l}4.88 \\
0.29 \\
5.95 \\
-.\end{array}$ & $\begin{array}{l}8.56 \\
0.25 \\
2.93 \\
-.\end{array}$ & $\begin{array}{l}0.33 \\
0.10 \\
30.31 \\
--\end{array}$ & $\begin{array}{l}1.57 \\
0.05 \\
3.19 \\
-.\end{array}$ & $\begin{array}{r}0.38 \\
0.05 \\
13.16 \\
-.\end{array}$ & $\begin{array}{r}29.63 \\
3.58\end{array}$ \\
\hline 1966 & $\begin{array}{l}\text { PRECIP }(C M=) \\
\text { SR-90 (MCI/SQ.KH.) } \\
\text { SR-90 CONC. }(P C / L) \\
\text { SR-89/SR-90 }\end{array}$ & $\begin{array}{l}1.17 \\
- \\
-\end{array}$ & $\begin{array}{l}0.84 \\
0.07 \\
8.34 \\
-\end{array}$ & $\begin{array}{l}0.81 \\
- \\
-\end{array}$ & $\begin{array}{r}1.32 \\
0.16 \\
12.13 \\
\end{array}$ & $\begin{array}{r}1.09 \\
0.17 \\
15.60 \\
-\end{array}$ & $\begin{array}{l}2.44 \\
0.22 \\
9.02 \\
8.90\end{array}$ & $\begin{array}{r}0.81 \\
C .11 \\
13.59 \\
3.30\end{array}$ & $\begin{array}{l}1.07 \\
0.08 \\
7.48 \\
+\end{array}$ & $\begin{array}{l}0.86 \\
0.05 \\
5.82 \\
+\end{array}$ & $\begin{array}{l}1.90 \\
0.04 \\
2.11 \\
\end{array}$ & $\begin{array}{l}2.69 \\
0.05 \\
1.86 \\
8.20\end{array}$ & $\begin{array}{l}1.85 \\
0.04 \\
2.17 \\
8.40\end{array}$ & $\begin{array}{r}16.85 \\
0.99\end{array}$ \\
\hline 1967 & $\begin{array}{l}\text { PRECIP }(C M-) \\
\text { SR-90 IMCI/SQ.KM.) } \\
\text { SR-90 CONC. (PC/L) } \\
\text { SR-89/SR-90 }\end{array}$ & $\begin{array}{r}1.24 \\
0.02 \\
1.62 \\
11.00\end{array}$ & $\begin{array}{l}1.57 \\
0.06 \\
3.83 \\
8.30\end{array}$ & $\begin{array}{r}3.63 \\
0.06 \\
1.66 \\
11.80\end{array}$ & $\begin{array}{l}6.07 \\
0.09 \\
1.49 \\
6.60\end{array}$ & $\begin{array}{l}5.13 \\
0.04 \\
0.78 \\
0\end{array}$ & $\begin{array}{l}5.84 \\
0.18 \\
3.09 \\
\end{array}$ & $=$ & $=$ & $=$ & $=$ & $=$ & $\begin{array}{l}= \\
=- \\
--\end{array}$ & $\begin{array}{r}23.48 \\
0.45\end{array}$ \\
\hline NC & $\begin{array}{l}\text { A NOT AVAILABLE } \\
\text { O OR TRACE } \\
\text { ROXIMATE } \\
\text { ER LIIMIT OF REPO } \\
\text { PORTI ONEO FROM }\end{array}$ & TA & & & & & & & & & & & & \\
\hline
\end{tabular}




\begin{tabular}{|c|c|c|c|c|c|c|c|c|c|c|c|c|c|c|}
\hline & & JAM. & FES. & MaR. & APR. & $\operatorname{mar}$ & sume & JULY & MUS. & SEP. & ocr. & mov. & DEC. & CUM. TOTAL \\
\hline 1957 & $\begin{array}{l}\text { PRECIP_ (CM.) } \\
\text { SR-90 IMCI/SQ.KN.) } \\
\text { SR-90 CONC. (PC/L) } \\
\text { SR-89/SR-90 }\end{array}$ & $\bar{z}$ & $\bar{z}$ & $\bar{z}$ & $\bar{z}$ & $\bar{z}$ & $\bar{z}$ & $\bar{z}$ & $\overline{0.63}$ & $\underline{\overline{0.46}}$ & $\overline{0.31}$ & $\overline{0}$ & $\begin{array}{l}\overrightarrow{0.44} \\
=\end{array}$ & $\begin{array}{l}0.0 \\
1.85\end{array}$ \\
\hline 1958 & 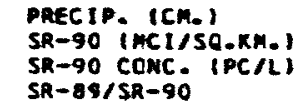 & $\begin{array}{l}16.31 \\
0.59 \\
3.62 \\
-\end{array}$ & $\begin{array}{l}\overline{0.20} \\
\overline{-}\end{array}$ & $\begin{array}{l}12.50 \\
0.39 \\
3.13 \\
-\end{array}$ & $\begin{array}{r}16.21 \\
0.67 \\
4.14 \\
23.00\end{array}$ & $\begin{array}{l}10.11 \\
1.08 \\
10.69 \\
12.00\end{array}$ & $\begin{array}{r}4.83 \\
0.41 \\
8.49 \\
13.00\end{array}$ & $\begin{array}{r}7.06 \\
6.43 \\
6.10 \\
40.60\end{array}$ & $\begin{array}{r}10.95 \\
0.43 \\
3.93 \\
23.00\end{array}$ & $\begin{array}{r}6.76 \\
0.27 \\
4.00 \\
27.00\end{array}$ & $\begin{array}{r}11.99 \\
0.63 \\
5.26 \\
48.00\end{array}$ & $\begin{array}{r}8.79 \\
0.53 \\
6.03 \\
39.00\end{array}$ & $\begin{array}{r}2.74 \\
0.30 \\
10.95 \\
32.00\end{array}$ & $\begin{array}{r}108.25 \\
6.01\end{array}$ \\
\hline 1959 & $\begin{array}{l}\text { PRECIP. }\left(C M_{-}\right) \\
S R-90 \text { iFCI/SO.KM.) } \\
S R-90 \text { CONC. }(P C / L) \\
S R-99 / S R-90\end{array}$ & $\begin{array}{r}6.76 \\
0.54 \\
7.99 \\
29.00\end{array}$ & $\begin{array}{r}5.56 \\
0.56 \\
10.08 \\
25.00\end{array}$ & $\begin{array}{r}9.55 \\
1.81 \\
19.96 \\
14.00\end{array}$ & $\begin{array}{r}8.20 \\
2.45 \\
29.88 \\
10.00\end{array}$ & $\begin{array}{r}2.74 \\
1.02 \\
37.23 \\
6.00\end{array}$ & $\begin{array}{r}13.87 \\
1.46 \\
10.53 \\
4.00\end{array}$ & $\begin{array}{r}15.77 \\
0.68 \\
4.32 \\
1.20\end{array}$ & $\begin{array}{r}14.73 \\
0.39 \\
2.65 \\
0.40\end{array}$ & $\begin{array}{l}6.15 \\
0.03 \\
0.49 \\
0\end{array}$ & $\begin{array}{r}16.15 \\
0.16 \\
1.00 \\
0.94\end{array}$ & $\begin{array}{r}10.80 \\
0.10 \\
0.93 \\
1.10\end{array}$ & $\begin{array}{r}11.76 \\
0.13 \\
1.11 \\
1.81\end{array}$ & $\begin{array}{r}122.04 \\
9.33\end{array}$ \\
\hline 1960 & $\begin{array}{l}\text { PRECIP, (CM-) } \\
\text { SR-9O (WCI/SO.KMH) } \\
\text { SR-9O CONC, (PC/L) } \\
\text { SR-89/SR-90 }\end{array}$ & $\begin{array}{l}7.95 \\
0.10 \\
1.26 \\
.\end{array}$ & $\begin{array}{r}12.42 \\
0.28 \\
2.26 \\
1.09\end{array}$ & $\begin{array}{l}5.44 \\
0.14 \\
2.58 \\
0.40\end{array}$ & $\begin{array}{l}10.13 \\
0.30 \\
2.97 \\
*\end{array}$ & $\begin{array}{l}0.86 \\
0.31 \\
4.52 \\
*\end{array}$ & $\begin{array}{l}3.81 \\
0.19 \\
4.99\end{array}$ & $\begin{array}{l}21.16 \\
0.22 \\
1.04\end{array}$ & $\begin{array}{l}17.09 \\
0.13 \\
0.17 \\
\end{array}$ & $\begin{array}{l}17.65 \\
0.11 \\
0.63 \\
\end{array}$ & $\begin{array}{l}0.58 \\
0.05 \\
0.76 \\
-\infty\end{array}$ & $\begin{array}{l}7.52 \\
0.05 \\
0.67 \\
-\end{array}$ & $\begin{array}{l}5.23 \\
0.03 \\
0.58 \\
--\end{array}$ & $\begin{array}{r}121.84 \\
1.91\end{array}$ \\
\hline 1961 & 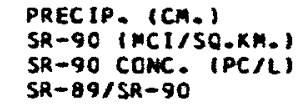 & $\begin{array}{l}7.521 \\
0.07 \\
0.94 \\
-\end{array}$ & $\begin{array}{l}0.971 \\
0.10 \\
1.12\end{array}$ & $\begin{aligned} 12.34 \\
0.31 \\
2.52 \\
-\end{aligned}$ & $\begin{array}{r}15.67 \\
0.61 \\
3.90 \\
\end{array}$ & $\begin{array}{l}7.09 \\
0.26 \\
3.67 \\
-.0\end{array}$ & $\begin{array}{l}4.95 \\
0.18 \\
3.64 \\
\end{array}$ & $\begin{array}{l}17.35 \\
0.15 \\
0.87 \\
-\end{array}$ & $\begin{array}{l}13.09 \\
0.08 \\
0.58 \\
-\end{array}$ & $\begin{array}{r}9.35 \\
0.06 \\
0.65 \\
30.00\end{array}$ & $\begin{array}{r}5.23 \\
0.12 \\
2.30 \\
72.00\end{array}$ & $\begin{array}{r}7.49 \\
0.45 \\
6.01 \\
84.00\end{array}$ & $\begin{array}{r}8.79 \\
0.56 \\
6.38 \\
49.00\end{array}$ & $\begin{array}{r}118.64 \\
2.95\end{array}$ \\
\hline 1962 & 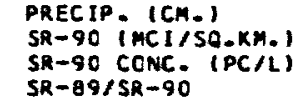 & $\begin{array}{r}7.49 \\
0.57 \\
7.62 \\
46.00\end{array}$ & $\begin{array}{r}11.28 \\
1.18 \\
10.47 \\
31.00\end{array}$ & $\begin{array}{r}11.61 \\
0.81 \\
6.98 \\
19.00\end{array}$ & $\begin{array}{r}8.56 \\
2.13 \\
24.89 \\
12.00\end{array}$ & $\begin{array}{r}3.20 \\
1.62 \\
31.88 \\
9.70\end{array}$ & $\begin{array}{r}15.98 \\
1.85 \\
11.58 \\
5.40\end{array}$ & $\begin{array}{r}3.28 \\
1.01 \\
30.80 \\
6.50\end{array}$ & $\begin{array}{r}18.97 \\
1.33 \\
7.02 \\
5.10\end{array}$ & $\begin{array}{r}8.76 \\
6.48 \\
5.48 \\
15.00\end{array}$ & $\begin{array}{l}11.94 \\
1.20 \\
10.06 \\
26.00\end{array}$ & $\begin{array}{r}11.71 \\
0.96 \\
8.20 \\
34.00\end{array}$ & $\begin{array}{r}7.24 \\
1.14 \\
15.75 \\
29.00\end{array}$ & $\begin{array}{r}120.02 \\
13.68\end{array}$ \\
\hline 1963 & $\begin{array}{l}\text { PRECIP, ICH-) } \\
\text { SR-9O INCI/SQ.KM-) } \\
\text { SR-9O CONC. (PC/L) } \\
\text { SR-89/SR } 90\end{array}$ & $\begin{array}{r}5.31 \\
1.01 \\
19.03 \\
29.00\end{array}$ & $\begin{array}{r}3.05 \\
1.04 \\
34.10 \\
19.00\end{array}$ & $\begin{array}{l}10.21 \\
2.43 \\
23.81 \\
12.00\end{array}$ & $\begin{array}{r}2.41 \\
3.00 \\
124.49 \\
7.60\end{array}$ & $\begin{array}{r}6.38 \\
3.79 \\
59.41 \\
5.30\end{array}$ & $\begin{array}{r}9.02 \\
2.44 \\
27.06 \\
3.60\end{array}$ & $\begin{array}{r}12.09 \\
5.17 \\
42.77 \\
2.40\end{array}$ & 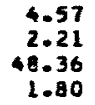 & $\begin{array}{r}11.91 \\
2.06 \\
17.30 \\
1.20\end{array}$ & $\begin{array}{r}2.34 \\
c .60 \\
25.65 \\
1.00\end{array}$ & $\begin{array}{r}11.38 \\
0.96 \\
8.62 \\
0.60\end{array}$ & $\begin{array}{r}3.84 \\
0.55 \\
14.33 \\
0.30\end{array}$ & $\begin{array}{l}22.51 \\
25.28\end{array}$ \\
\hline 1964 & $\begin{array}{l}\text { PREC IP } \text { (CM-I } \\
\text { SR-90 INCISSO.KM-) } \\
\text { SR-90 CONC. (PCAL) } \\
\text { SR-89/SR-90 }\end{array}$ & $\begin{array}{r}8.84 \\
1.04 \\
11.77 \\
0.20\end{array}$ & $\begin{array}{l}4.80 \\
0.42 \\
8.76 \\
0.20\end{array}$ & $\begin{array}{r}5.69 \\
1.35 \\
23.73\end{array}$ & $\begin{array}{l}15.60 \\
2.58 \\
16.54 \\
\end{array}$ & $\begin{array}{r}2.67 \\
0.86 \\
32.21 \\
--\end{array}$ & $\begin{array}{r}6.25 \\
2.39 \\
38.24 \\
--.\end{array}$ & $\begin{array}{l}12.93 \\
1.07 \\
8.28 \\
-\end{array}$ & $\begin{array}{r}2.54 \\
0.43 \\
16.93 \\
\end{array}$ & $\begin{array}{l}3.25 \\
0.11 \\
3.39\end{array}$ & $\begin{array}{l}3.45 \\
0.31 \\
8.99 \\
*\end{array}$ & $\begin{array}{l}0.10 \\
0.34 \\
5.58 \\
0.74\end{array}$ & $\begin{array}{l}10.97 \\
0.08 \\
0.73 \\
\end{array}$ & $\begin{array}{l}83.09 \\
10.98\end{array}$ \\
\hline 1965 & $\begin{array}{l}\text { PRECIP. }\left(C H_{-}\right) \\
S R-9 C \text { (PCI/SO.KM.) } \\
S R-90 \text { CONC. }(P C / L) \\
S R-89 / S R-90^{\circ}\end{array}$ & $\begin{array}{l}6.40 \\
0.42 \\
6.57 \\
-\end{array}$ & $\begin{array}{l}7.72 \\
0.66 \\
8.55 \\
-.\end{array}$ & $\begin{array}{r}3.96 \\
0.54 \\
13.64 \\
-\end{array}$ & $\begin{array}{r}4.60 \\
0.87 \\
18.92 \\
\end{array}$ & $\begin{array}{l}3.40 \\
0.51 \\
15.01 \\
-\end{array}$ & $\begin{array}{r}4.98 \\
1.10 \\
22.09 \\
-\end{array}$ & $\begin{array}{l}8.00 \\
0.62 \\
7.76 \\
-\end{array}$ & $\begin{array}{r}11.61 \\
0.34 \\
2.93 \\
\end{array}$ & $\begin{array}{l}7.72 \\
0.15 \\
1.95 \\
-\end{array}$ & $\begin{array}{l}6.12 \\
6.18 \\
2.95 \\
-\end{array}$ & $\begin{array}{l}4.77 \\
0.18 \\
3.78 \\
-0\end{array}$ & $\begin{array}{l}4.34 \\
0.13 \\
3.00 \\
-\end{array}$ & $\begin{array}{r}73.62 \\
5.70\end{array}$ \\
\hline 1960 & 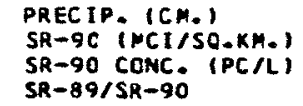 & $\begin{array}{l}5.26 \\
0.14 \\
2.67\end{array}$ & $\begin{array}{l}9.65 \\
0.11 \\
1.14 \\
-.\end{array}$ & $\begin{array}{r}3.30 \\
0.40 \\
11.84\end{array}$ & $\begin{array}{l}6.90 \\
0.43 \\
6.17 \\
-\end{array}$ & $\begin{array}{r}10.29 \\
0.69 \\
6.71 \\
\end{array}$ & $\begin{array}{l}2.11 \\
0.19 \\
9.01 \\
-\end{array}$ & $\begin{array}{l}4.34 \\
0.15 \\
3.46\end{array}$ & $\begin{array}{l}3.89 \\
0.05 \\
1.29 \\
-\end{array}$ & $\begin{array}{l}23.93 \\
0.12 \\
0.51 \\
\end{array}$ & $\begin{array}{l}6.83 \\
0.03 \\
0.44 \\
-\end{array}$ & $\begin{array}{r}11.00 \\
0.08 \\
0.73 \\
-\end{array}$ & $\begin{array}{l}7.32 \\
0.06 \\
0.82 \\
-\end{array}$ & $\begin{array}{r}94.98 \\
2.45\end{array}$ \\
\hline 1967 & $\begin{array}{l}P R E C 1 P \text {; }\left(C M_{-}\right) \\
\text {SR-90 i MCI/SO,KM.) } \\
\text { SR-90 CONC. (PC/L) } \\
S R-89 / S R-90\end{array}$ & $\begin{array}{l}4.29 \\
0.12 \\
2.80 \\
-\end{array}$ & $\begin{array}{l}6.48 \\
0.06 \\
0.93 \\
\end{array}$ & $\bar{z}$ & $\bar{z}$ & $=$ & $\begin{array}{l}= \\
=\end{array}$ & $\bar{z}$ & $\bar{z}$ & $=$ & $\bar{z}$ & $\bar{z}$ & $\bar{z}$ & $\begin{array}{r}10.77 \\
0.18\end{array}$ \\
\hline 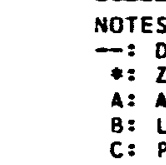 & $\begin{array}{l}\text { LATA NOT AVAILABLE } \\
\text { LERO OA TRACE } \\
\text { APPROXIMATE } \\
\text { OWWER LIMIT OF REPOA } \\
\text { PROPORTIONED FROM OF }\end{array}$ & $\begin{array}{l}\text { DATA } \\
\text { NALLY }\end{array}$ & & & & & & & & & & & & \\
\hline
\end{tabular}


MONTHLY FALLOUT DEPOSITION COLECTIONS

SITE: NEW JERSEY, WESTWOOD

LAT. 41 ON LONG. 74 2W MT.

38\%. (CDLUMm)

SOURCE: ISOTOPES, INC.

COLLECTIONS TERMINATED IN JULY 1964

\begin{tabular}{|c|c|c|c|c|c|c|c|c|c|c|c|c|c|c|}
\hline & & JAN. & FEB. & MAR. & APR. & mar & JURE & JULY & Auc. & SEP. & oct. & NOV. & DEC. & CUA. TOTAL \\
\hline 1959 & $\begin{array}{l}\text { PRECIP. (CM-) } \\
S R-90 \text { (PCI/SQ.KM) } \\
S R-90 \text { CONC. (PCIL) } \\
S R-89 / S R-90\end{array}$ & $=$ & $\overline{-}$ & 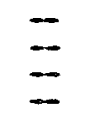 & $\begin{array}{r}8.20 \\
1.78 \\
21.71 \\
8.80\end{array}$ & $\begin{array}{r}2.74 \\
0.57 \\
20.81 \\
5.60\end{array}$ & $\begin{array}{r}13.87 \\
1.68 \\
12.12 \\
3.40\end{array}$ & $\begin{array}{r}15.77 \\
0.44 \\
2.80 \\
1.90\end{array}$ & $\begin{array}{r}14.73 \\
0.24 \\
1.63 \\
1.20\end{array}$ & $\begin{array}{l}6.15 \\
0.05 \\
0.82 \\
*\end{array}$ & $\begin{array}{r}16.15 \\
0.14 \\
0.87 \\
0.90\end{array}$ & $\begin{array}{r}10.80 \\
0.09 \\
0.84 \\
1.70\end{array}$ & $\begin{array}{r}11.76 \\
0.10 \\
0.86 \\
1.50\end{array}$ & $\begin{array}{r}100.17 \\
5.09\end{array}$ \\
\hline 1960 & $\begin{array}{l}\text { PRECIP. }\left(C H_{-}\right) \\
S R-9 C \text { (PCI/SO.KM) } \\
S R-90 \text { CONC= (PC/L) } \\
S R-89 / S R-90\end{array}$ & $\begin{array}{l}7.95 \\
0.13 \\
1.64 \\
0.30\end{array}$ & $\begin{array}{r}12.42 \\
0.19 \\
1.53 \\
1.60\end{array}$ & $\begin{array}{l}5.44 \\
0.15 \\
2.76 \\
0.10\end{array}$ & $\begin{array}{r}10.13 \\
0.27 \\
2.67 \\
.\end{array}$ & $\begin{array}{l}6.86 \\
0.29 \\
4.23 \\
+\end{array}$ & $\begin{array}{l}3.81 \\
0.23 \\
6.04 \\
+\end{array}$ & $\begin{array}{l}21.16 \\
0.16 \\
0.76 \\
\end{array}$ & $\begin{array}{l}17.09 \\
0.07 \\
0.41 \\
\end{array}$ & $\begin{array}{l}17.65 \\
0.08 \\
0.46 \\
-\end{array}$ & $\begin{array}{l}6.58 \\
0.07 \\
1.07 \\
-.\end{array}$ & $\begin{array}{l}7.52 \\
0.04 \\
0.54 \\
-.\end{array}$ & $\begin{array}{l}5.23 \\
* \\
=-\end{array}$ & $\begin{array}{r}121.84 \\
1.68\end{array}$ \\
\hline 1961 & $\begin{array}{l}\text { PRECIP. }(C M-) \\
S R-90 \text { (NCI/SQ-KM.) } \\
S R-90 \text { CONC. }(P C / L) \\
S R-89 / 5 R-90\end{array}$ & $\begin{array}{l}7.524 \\
0.20 \\
2.66 \\
-.\end{array}$ & $\begin{array}{l}0.974 \\
0.17 \\
1.90 \\
-.\end{array}$ & $\begin{array}{r}12.34 \\
0.40 \\
3.25 \\
--\end{array}$ & $\begin{array}{l}15.67 \\
0.41 \\
2.62 \\
-\end{array}$ & $\begin{array}{l}7.09 \\
0.23 \\
3.25 \\
-.\end{array}$ & $\begin{array}{l}4.95 \\
0.17 \\
3.44 \\
-\end{array}$ & $\begin{array}{r}17.35 \\
0.17 \\
0.98 \\
\end{array}$ & $\begin{array}{r}13.89 \\
0.18 \\
1.30 \\
-\end{array}$ & $\begin{array}{r}9.35 \\
0.06 \\
0.65 \\
16.00\end{array}$ & $\begin{array}{r}5.23 \\
6.10 \\
1.92 \\
65.00\end{array}$ & $\begin{array}{r}7.49 \\
0.42 \\
5.61 \\
12.00\end{array}$ & $\begin{array}{r}8.79 \\
0.49 \\
5.58 \\
57.00\end{array}$ & $\begin{array}{r}118.64 \\
3.00\end{array}$ \\
\hline 1962 & $\begin{array}{l}\text { PRECIP. }(C M+) \\
S R-90 \text { (MCI/SQ.KM.) } \\
S R-90 \text { CONC. (PC/L) } \\
S R-89 / S R-9 O\end{array}$ & $\begin{array}{r}7.49 \\
0.52 \\
6.95 \\
40.00\end{array}$ & $\begin{array}{r}11.28 \\
1.10 \\
9.76 \\
30.00\end{array}$ & $\begin{array}{r}11.61 \\
0.72 \\
6.21 \\
18.00\end{array}$ & $\begin{array}{r}8.56 \\
1.89 \\
22.08 \\
14.00\end{array}$ & $\begin{array}{r}3.20 \\
1.15 \\
35.94 \\
9.10\end{array}$ & $\begin{array}{r}15.98 \\
2.09 \\
13.08 \\
4.80\end{array}$ & $\begin{array}{r}3.20 \\
0.91 \\
27.75 \\
6.10\end{array}$ & $\begin{array}{r}18.97 \\
1.10 \\
5.80 \\
6.10\end{array}$ & $\begin{array}{r}8.76 \\
0.52 \\
5.94 \\
14.00\end{array}$ & $\begin{array}{r}11.94 \\
1.15 \\
5.64 \\
25.00\end{array}$ & $\begin{array}{r}11.71 \\
0.48 \\
4.10 \\
38.00\end{array}$ & $\begin{array}{r}7.24 \\
1.50 \\
20.72 \\
19.00\end{array}$ & $\begin{array}{r}120.02 \\
13.13\end{array}$ \\
\hline 1963 & $\begin{array}{l}\text { PRECIP. (CM.) } \\
\text { SR-90 IMCI/SQ.KM.) } \\
S R-90 \text { CONC. (PC/L) } \\
S R-89 / S R-90\end{array}$ & $\begin{array}{r}5.31 \\
1.00 \\
18.84 \\
27.00\end{array}$ & $\begin{array}{r}3.05 \\
0.90 \\
29.51 \\
18.00\end{array}$ & $\begin{array}{r}10.21 \\
1.47 \\
14.40 \\
13.00\end{array}$ & $\begin{array}{r}2.41 \\
2.87 \\
119.09 \\
5.90\end{array}$ & $\begin{array}{r}7.39 \\
3.73 \\
50.48 \\
5.10\end{array}$ & $\begin{array}{r}9.02 \\
1.82 \\
20.18 \\
4.10\end{array}$ & $\begin{array}{r}12.09 \\
4.63 \\
38.30 \\
2.80\end{array}$ & $\begin{array}{r}4.57 \\
2.03 \\
44.43 \\
2.50\end{array}$ & $\begin{array}{r}11.91 \\
1.79 \\
15.03 \\
1.30\end{array}$ & $\begin{array}{r}2.34 \\
6.58 \\
24.79 \\
1.00\end{array}$ & $\begin{array}{r}15.80 \\
0.97 \\
6.14 \\
0.60\end{array}$ & $\begin{array}{r}3.84 \\
0.54 \\
14.07 \\
*\end{array}$ & $\begin{array}{l}87.94 \\
22.33\end{array}$ \\
\hline 1964 & $\begin{array}{l}\text { PRECIP. (CM.) } \\
\text { SR-90 (MCI/SQ.KM.) } \\
\text { SR-90 CONC. (PCIL) } \\
\text { SR-89/SR-90 }\end{array}$ & $\begin{array}{l}8.84 \\
0.53 \\
6.00 \\
0.20\end{array}$ & $\begin{array}{r}4.80 \\
0.53 \\
11.05 \\
*\end{array}$ & $\begin{array}{r}10.77 \\
1.35 \\
12.54 \\
0.20\end{array}$ & $\begin{array}{l}15.60 \\
=\end{array}$ & $\begin{array}{r}2.67 \\
0.80 \\
29.97 \\
-\end{array}$ & $\begin{array}{r}6.25 \\
1.84 \\
29.44 \\
-\end{array}$ & $\overline{-}$ & $\overline{-}$ & $\overline{-}$ & $\overline{-}$ & $\bar{z}$ & $\bar{m}$ & $\begin{array}{r}48.93 \\
5.05\end{array}$ \\
\hline $\begin{array}{c}\text { NOTES } \\
--:= \\
\text { A: } \\
\text { A: } \\
\text { B: } \\
\text { C: }\end{array}$ & $\begin{array}{l}\text { ATA NOT AVAILABLE } \\
\text { ERO OR TRACE } \\
\text { PPROXIMATE } \\
\text { OWER LIIIT OF REPOR } \\
\text { ROPORTIONED FROM OR }\end{array}$ & $\begin{array}{l}\text { DATA } \\
\text { NALLY }\end{array}$ & $N 5$ & & & & & & & & & & & \\
\hline
\end{tabular}


MONTHY FALLOUT DEPOSITION COLECTIONS

SITE: NEW YORK, MEW YORK

LAT. 40 4ON LONG. 73 SON ALT.

17\%. (MOT)

SURFACE AIR SAMPLIMG STATION SOURCE: HEALTH AMO SAFETY LABORATORY, U. S. ATOMIC ENERGY COMMISSION

\begin{tabular}{|c|c|c|c|c|c|c|c|c|c|c|c|c|c|c|c|}
\hline & & & JAN. & FEB. & MAR. & APR. & Mar & JUAEE & Juer & NUG. & SEP. & OCT. & MOV. & DEC. & CUN. TOTAL \\
\hline & 1954 & $\begin{array}{l}\text { PRECIP. (CM.) } \\
S R-90 \text { (FCI/SQ.KM.) } \\
S R-90 \text { CONC. }(P C / L) \\
S R-89 / S R-90\end{array}$ & $\overline{=}$ & $\begin{array}{r}4.60 \\
0.91 \\
19.79 \\
-\end{array}$ & $\begin{array}{l}0.26 \\
0.20 \\
2.43 \\
-\end{array}$ & $\begin{array}{l}6.86 \\
0.13 \\
1.90 \\
.0\end{array}$ & $\begin{array}{l}7.87 \\
0.19 \\
2.42 \\
-\end{array}$ & $\begin{array}{l}3.35 \\
0.18 \\
5.38 \\
-\end{array}$ & $\begin{array}{l}2.44 \\
0.05 \\
2.05 \\
-\end{array}$ & $\begin{array}{l}15.47 \\
0.07 \\
0.46 \\
-\end{array}$ & $\begin{array}{r}12.07 \\
0.57 \\
4.73 \\
-\end{array}$ & $\begin{array}{l}5.05 \\
0.08 \\
1.59 \\
-.0\end{array}$ & $\begin{array}{r}12.52 \\
0.25 \\
2.00 \\
-\end{array}$ & $\begin{array}{l}7.70 \\
0.13 \\
1.69 \\
-0\end{array}$ & $\begin{array}{r}86.19 \\
2.76\end{array}$ \\
\hline & 1955 & $\begin{array}{l}\text { PRECIP. (CM-) } \\
S R-90 \text { (MCI/SO.KM-) } \\
S R-90 \text { CONC. }(P C / L) \\
S R-99 / S R-90\end{array}$ & $\begin{array}{l}1.96 \\
0.09 \\
4.60 \\
-\end{array}$ & $\begin{array}{l}7.65 \\
0.34 \\
4.45 \\
-\end{array}$ & $\begin{array}{l}9.42 \\
0.94 \\
9.98 \\
-\end{array}$ & $\begin{array}{l}5.00 \\
0.48 \\
9.61 \\
.\end{array}$ & $\begin{array}{l}6.71 \\
0.34 \\
5.07 \\
-\end{array}$ & $\begin{array}{l}6.40 \\
0.34 \\
5.32 \\
-\end{array}$ & $\begin{array}{l}1.30 \\
0.10 \\
7.70 \\
-\end{array}$ & $\begin{array}{r}27.58 \\
0.26 \\
0.95 \\
-\end{array}$ & $\begin{array}{l}6.78 \\
0.17 \\
2.51 \\
-.\end{array}$ & $\begin{array}{l}17.45 \\
0.14 \\
0.81 \\
-\end{array}$ & $\begin{array}{r}10.46 \\
0.16 \\
1.53 \\
-.\end{array}$ & $\begin{array}{c}0.64 \\
0.21 \\
32.82 \\
--\end{array}$ & $\begin{array}{r}101.35 \\
3.57\end{array}$ \\
\hline$\neq$ & 1956 & $\begin{array}{l}P R E C I P \text {. }(C M-) \\
S R-90 \text { i } M C I / S Q . K M-) \\
S R-90 \text { CONC. }(P C / L) \\
S R-B 9 / S R-90\end{array}$ & $\begin{array}{r}3.91 \\
1.05 \\
26.86 \\
-\end{array}$ & $\begin{array}{r}10.62 \\
0.49 \\
4.62 \\
--\end{array}$ & $\begin{array}{r}12.78 \\
0.62 \\
4.86 \\
-\end{array}$ & $\begin{array}{l}7.06 \\
0.30 \\
4.25 \\
-.\end{array}$ & $\begin{array}{l}5.59 \\
0.40 \\
7.16 \\
\end{array}$ & $\begin{array}{l}7.59 \\
0.31 \\
4.09 \\
-\end{array}$ & $\begin{array}{l}7.95 \\
0.18 \\
2.27 \\
-\end{array}$ & $\begin{array}{l}6.50 \\
0.29 \\
4.47 \\
-.\end{array}$ & $\begin{array}{l}5.89 \\
0.12 \\
2.04 \\
-\end{array}$ & $\begin{array}{l}9.17 \\
0.12 \\
1.31 \\
-.\end{array}$ & $\begin{array}{l}6.65 \\
0.34 \\
5.12 \\
--\end{array}$ & $\begin{array}{l}8.36 \\
0.21 \\
2.52 \\
--\end{array}$ & $\begin{array}{r}92.07 \\
4.43\end{array}$ \\
\hline & 1957 & $\begin{array}{l}\text { PRECIP }\left(C M_{-}\right) \\
\left.S R-9 C \text { I } M C I / S Q_{-K M}\right) \\
S R-9 C \text { CONC. }(P C / L) \\
S R-89 / S R-90\end{array}$ & $\begin{array}{r}4.32 \\
0.10 \\
2.32 \\
24.00\end{array}$ & $\begin{array}{r}0.17 \\
6.20 \\
3.25 \\
21.00\end{array}$ & $\begin{array}{l}5.05 \\
0.40 \\
7.93 \\
-\end{array}$ & $\begin{array}{l}11.46 \\
1.85 \\
16.15 \\
-\end{array}$ & $\begin{array}{r}9.32 \\
0.36 \\
3.87 \\
12.00\end{array}$ & $\begin{array}{r}4.70 \\
0.32 \\
6.81 \\
28.00\end{array}$ & $\begin{array}{r}3.73 \\
C .32 \\
8.58 \\
10.00\end{array}$ & $\begin{array}{r}7.29 \\
0.19 \\
2.61 \\
59.00\end{array}$ & $\begin{array}{r}7.65 \\
0.16 \\
2.10 \\
47.00\end{array}$ & $\begin{array}{r}8.31 \\
C .15 \\
1.81 \\
61.00\end{array}$ & $\begin{array}{r}11.33 \\
0.16 \\
1.42 \\
21.00\end{array}$ & $\begin{array}{r}13.36 \\
0.23 \\
1.73 \\
20.00\end{array}$ & $\begin{array}{r}92.69 \\
4.44\end{array}$ \\
\hline & 1958 & $\begin{array}{l}\text { PRECIP, }\left(C H_{-}\right) \\
S R-90 \text { IPCI/SQ.KM- }) \\
\text { SR-90 CONC. }(P C / L) \\
S R-89 / S R-90\end{array}$ & $\begin{array}{r}9.63 \\
0.50 \\
5.20 \\
21.00\end{array}$ & $\begin{array}{r}7.57 \\
0.47 \\
6.21 \\
16.00\end{array}$ & $\begin{array}{r}6.10 \\
0.34 \\
4.20 \\
17.00\end{array}$ & $\begin{array}{r}15.60 \\
0.59 \\
3.79 \\
12.00\end{array}$ & $\begin{array}{r}8.26 \\
1.02 \\
12.35 \\
11.00\end{array}$ & $\begin{array}{r}6.48 \\
0.68 \\
10.50 \\
12.00\end{array}$ & $\begin{array}{r}9.35 \\
0.61 \\
6.53 \\
11.00\end{array}$ & $\begin{array}{r}5.99 \\
0.23 \\
3.84 \\
20.00\end{array}$ & $\begin{array}{r}11.28 \\
0.25 \\
2.22 \\
28.00\end{array}$ & $\begin{array}{r}13.87 \\
6.41 \\
2.96 \\
53.00\end{array}$ & $\begin{array}{r}4.70 \\
0.38 \\
8.09 \\
29.00\end{array}$ & $\begin{array}{r}3.18 \\
0.68 \\
21.39 \\
46.00\end{array}$ & $\begin{array}{r}104.01 \\
6.16\end{array}$ \\
\hline & 1955 & $\begin{array}{l}\text { PRECIP. (CM.) } \\
\text { SR-9O IMCI/SQ.KM-) } \\
S R-90 \text { CONC. }(P C / L) \\
S R-89 / S R-90\end{array}$ & $\begin{array}{r}5.94 \\
0.46 \\
7.75 \\
32.00\end{array}$ & $\begin{array}{r}4.29 \\
0.67 \\
15.62 \\
23.00\end{array}$ & $\begin{array}{r}9.58 \\
1.96 \\
20.46 \\
13.00\end{array}$ & $\begin{array}{r}4.85 \\
2.42 \\
49.90 \\
12.00\end{array}$ & $\begin{array}{r}3.30 \\
0.50 \\
14.80 \\
5.20\end{array}$ & $\begin{array}{r}10.67 \\
1.71 \\
16.03 \\
5.04\end{array}$ & $\begin{array}{r}10.87 \\
0.19 \\
1.75 \\
3.33\end{array}$ & $\begin{array}{r}11.30 \\
0.26 \\
2.31 \\
1.00\end{array}$ & $\begin{array}{l}2.82 \\
0.05 \\
1.78 \\
1.65\end{array}$ & $\begin{array}{r}12.27 \\
0.14 \\
1.15 \\
6.33\end{array}$ & $\begin{array}{r}10.72 \\
0.12 \\
1.12 \\
0.32\end{array}$ & $\begin{array}{r}11.79 \\
0.20 \\
1.70 \\
*\end{array}$ & $\begin{array}{r}98.48 \\
8.68\end{array}$ \\
\hline & 1960 & $\begin{array}{l}\text { PRECIP. (CM.) } \\
\text { SR-9O IMCI/SQ.KM.) } \\
S R-90 \text { CONC. }(P C / L) \\
S R-Q 9 / S R-90\end{array}$ & $\begin{array}{l}6.10 \\
0.17 \\
2.79 \\
0.30\end{array}$ & $\begin{array}{r}11.25 \\
0.31 \\
2.76 \\
1.50\end{array}$ & $\begin{array}{l}7.52 \\
0.12 \\
1.60 \\
1.00\end{array}$ & $\begin{array}{l}7.75 \\
0.16 \\
2.07 \\
0.90\end{array}$ & $\begin{array}{l}7.54 \\
0.28 \\
3.72 \\
0.20\end{array}$ & $\begin{array}{l}4.42 \\
0.17 \\
3.85 \\
0.40\end{array}$ & $\begin{array}{r}21.06 \\
0.10 \\
0.48 \\
\end{array}$ & $\begin{array}{l}15.90 \\
0.08 \\
0.51 \\
-\end{array}$ & $\begin{array}{r}13.67 \\
0.06 \\
C .44 \\
\end{array}$ & $\begin{array}{l}7.16 \\
C .04 \\
C .56 \\
-\end{array}$ & $\begin{array}{l}7.75 \\
0.05 \\
0.65 \\
0\end{array}$ & $\begin{array}{l}7.72 \\
0.04 \\
0.52 \\
\ldots\end{array}$ & $\begin{array}{r}117.84 \\
1.58\end{array}$ \\
\hline
\end{tabular}


O 
MONTHLY FALLOUT DEPOSITION COLLECTIONS

SITE: OHIO, WOOSTER

LAT. $4047 \mathrm{~N}$ LONG. 82 SOW ALT.

367M. (POT)

SOURCE: OHIO STATE UNI VERSITY AGRICULTURAL EXPERIMENT STATION, DEPT. OF agRONOMY

\begin{tabular}{|c|c|c|c|c|c|c|c|c|c|c|c|c|c|c|}
\hline & & JAN. & FEB. & MAR. & APR. & MAY & JUNE & JULY & AUG. & SEP. & OCT. & NOV. & DEC. & CUM. TOTAL \\
\hline 1963 & $\begin{array}{l}\text { PRECIP. (CM-) } \\
\text { SR-90 (MCI/SQ.KM.) } \\
5 R-90 \text { CONC. }(P C / L) \\
S R-89 / S R-90\end{array}$ & $=$ & $\begin{array}{l}= \\
=- \\
=-\end{array}$ & $=$ & $\overline{-}$ & $=$ & $=$ & $=$ & $\begin{array}{r}6.12 \\
1.32 \\
21.57\end{array}$ & $\begin{array}{r}4.06 \\
0.54 \\
13.31 \\
*\end{array}$ & $\begin{array}{r}1.32 \\
0.21 \\
15.91 \\
0.50\end{array}$ & $\begin{array}{r}3.94 \\
0.40 \\
10.16\end{array}$ & $\begin{array}{l}1.85 \\
0.12 \\
6.49 \\
+\end{array}$ & $\begin{array}{r}17.29 \\
2.59\end{array}$ \\
\hline 1964 & $\begin{array}{l}\text { PRECIP. }\left(C M_{.}\right) \\
\text {SR-90 (PCI/SQ.KM.) } \\
S R-90 \text { CGNC. }(P C / L) \\
S R-89 / S R-90\end{array}$ & $\begin{array}{r}3.86 \\
1.11 \\
28.76 \\
1.20\end{array}$ & $\begin{array}{r}3.68 \\
0.54 \\
14.68 \\
*\end{array}$ & $\begin{array}{r}19.25 \\
1.54 \\
8.01 \\
1.30\end{array}$ & $\begin{array}{l}15.32 \\
3.14 \\
20.50 \\
*\end{array}$ & $\begin{array}{r}14.58 \\
3.03 \\
20.79 \\
*\end{array}$ & $\begin{array}{r}10.26 \\
3.04 \\
29.63 \\
-\end{array}$ & $\begin{array}{r}6.48 \\
1.35 \\
20.84 \\
-\end{array}$ & $\begin{array}{r}13.06 \\
0.75 \\
5.75 \\
\end{array}$ & $\begin{array}{l}1.70 \\
0.12 \\
7.06 \\
-\end{array}$ & $\begin{array}{r}2.69 \\
0.09 \\
3.35 \\
17.00\end{array}$ & $\begin{array}{l}3.30 \\
-- \\
=\end{array}$ & $\begin{array}{l}8.89 \\
0.09 \\
1.02 \\
--\end{array}$ & $\begin{array}{r}103.07 \\
14.80\end{array}$ \\
\hline 1965 & $\begin{array}{l}\text { PRECIP. }\left(C M_{-}\right) \\
\text {SR-90 IPCI/SO.KM.) } \\
5 R-90 \text { CONC. }(P C / L) \\
S R-89 / S R-90\end{array}$ & $\begin{array}{l}7.80 \\
0.51 \\
6.54 \\
-.-\end{array}$ & $\begin{array}{l}4.85 \\
0.09 \\
1.86 \\
-.\end{array}$ & $\begin{array}{l}5.89 \\
0.08 \\
1.36 \\
--\end{array}$ & $\begin{array}{r}5.03 \\
0.80 \\
15.91 \\
*\end{array}$ & $\begin{array}{l}9.78 \\
0.08 \\
0.82 \\
--\end{array}$ & $\begin{array}{l}4.55 \\
0.41 \\
9.02 \\
-0\end{array}$ & $\begin{array}{l}4.32 \\
0.18 \\
4.17 \\
--1\end{array}$ & $\begin{array}{r}12.32 \\
0.30 \\
2.44 \\
\end{array}$ & $\begin{array}{l}7.54 \\
0.54 \\
7.17 \\
-.0\end{array}$ & $\begin{array}{r}11.20 \\
0.03 \\
0.27 \\
--\end{array}$ & $\begin{array}{l}6.83 \\
0.11 \\
1.62 \\
\end{array}$ & $\begin{array}{l}2.18 \\
0.06 \\
2.76 \\
-\end{array}$ & $\begin{array}{r}82.29 \\
3.19\end{array}$ \\
\hline 1966 & $\begin{array}{l}\text { PRECIP. (CM-) } \\
\text { SR-90 (MCI/SO.KM.) } \\
S R-90 \text { CCNC. (PCIL) } \\
S R-89 / S R-90\end{array}$ & $\begin{array}{l}6.38 \\
0.15 \\
2.36 \\
-.\end{array}$ & $\begin{array}{l}4.52 \\
0.07 \\
1.55 \\
-.-\end{array}$ & $\begin{array}{l}4.06 \\
0.31 \\
7.64 \\
-.\end{array}$ & $\begin{array}{l}7.77 \\
0.29 \\
3.74 \\
--\end{array}$ & $\begin{array}{l}7.80 \\
0.20 \\
2.57 \\
--\end{array}$ & $\begin{array}{l}4.32 \\
0.42 \\
9.73 \\
3.40\end{array}$ & $\begin{array}{l}4.88 \\
0.14 \\
2.87 \\
3.50\end{array}$ & $\begin{array}{r}10.11 \\
0.09 \\
0.90 \\
4.20\end{array}$ & $\begin{array}{l}4.65 \\
0.04 \\
0.87 \\
+\end{array}$ & $\begin{array}{l}3.40 \\
0.04 \\
1.18 \\
+\end{array}$ & $\begin{array}{r}13.46 \\
0.32 \\
2.38 \\
26.00\end{array}$ & $\begin{array}{l}6.55 \\
0.03 \\
0.46 \\
+\end{array}$ & $\begin{array}{r}77.90 \\
2.10\end{array}$ \\
\hline 1967 & $\begin{array}{l}\text { PRECIP. (CM.) } \\
\text { SR-90 (MCIISO.KM.) } \\
\text { SR-90 CONC. (PC/L) } \\
\text { SR-89/SR-90 }\end{array}$ & $\begin{array}{r}2.11 \\
0.04 \\
1.90 \\
13.00\end{array}$ & $\begin{array}{r}5.05 \\
0.04 \\
0.80 \\
10.30\end{array}$ & $\begin{array}{l}9.17 \\
0.09 \\
0.99 \\
6.80\end{array}$ & $\begin{array}{l}8.30 \\
0.01 \\
0.13 \\
*\end{array}$ & $\begin{array}{l}11.86 \\
0.01 \\
0.09 \\
*\end{array}$ & $\begin{array}{l}3.56 \\
0.22 \\
6.18 \\
0\end{array}$ & $=$ & $=$ & $=$ & $\bar{z}$ & $m=$ & $\begin{array}{l}-- \\
- \\
-\end{array}$ & $\begin{array}{r}40.05 \\
0.41\end{array}$ \\
\hline \begin{tabular}{l} 
NOTE: \\
\hdashline$:$ \\
$*:$ \\
$A:$ \\
$B:$ \\
$C:$
\end{tabular} & $\begin{array}{l}5 \\
\text { DATA NOT AVAILABLE } \\
\text { ZERO OR TRACE } \\
\text { APPROXIMATE } \\
\text { LOWER LIMIT OF REPOR } \\
\text { PROPORTIONED FROM OR }\end{array}$ & $\begin{array}{l}\text { DATA } \\
\text { NALLLY }\end{array}$ & $y$ & ED & & & & & & & & & & \\
\hline
\end{tabular}


MONTHLY FALLOUT DEPOSITION COLLECTIONS

SITE: OKLAHOMA, MIOWEST CITY LAT. 35 25N LUNG. 97 3OW MLT. 364M. (POT) SURFACE AIR SAMPLING STATION

SOURCE : HAZELTON-NUCLEAR SCIENCE CORP.. THRU MR. E. COLLET COLLECTIONS TERMINATED IN DECEMBER 1965

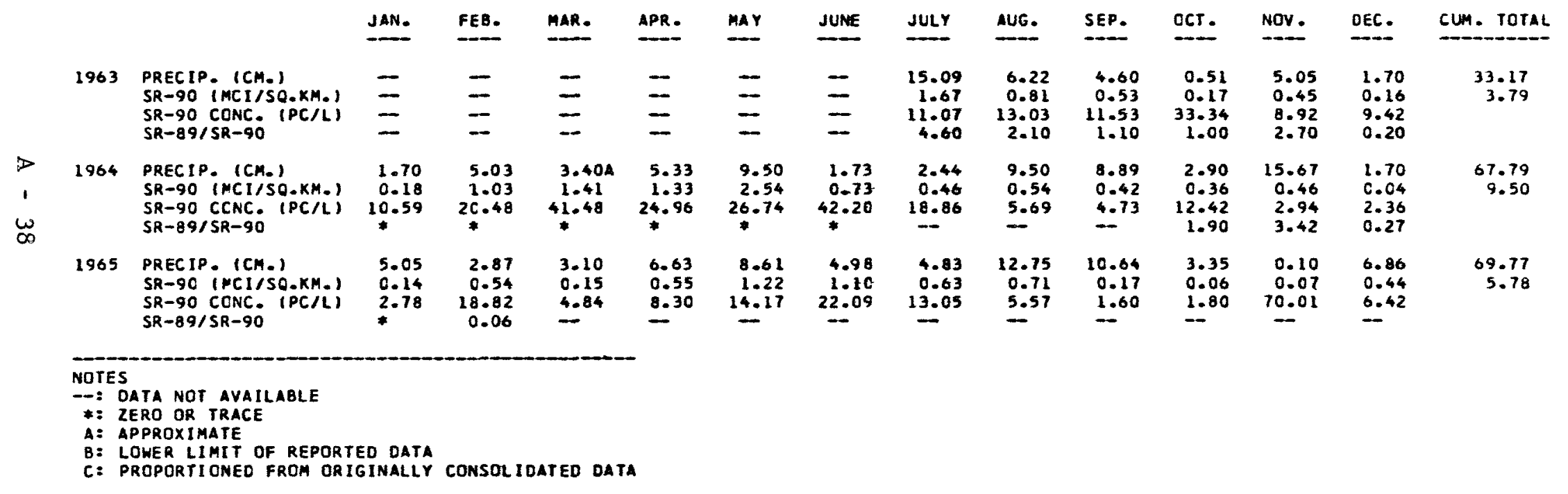




\begin{tabular}{|c|c|c|c|c|c|c|c|c|c|c|c|c|c|c|}
\hline & & JAN. & FEB. & MAR. & APR. & MAY & JWHE & suLY & AUS. & SEP. & ocr. & NOV. & DEC. & CUM. TOTAL \\
\hline 1958 & $\begin{array}{l}\text { PRECIP. }(C M-) \\
S R-90 \text { (MCI/SQ.KM.) } \\
S R-90 \text { CONC. (PC/L) } \\
S R-89 / S R-90\end{array}$ & $\begin{array}{r}4.52 \\
0.14 \\
3.10 \\
15.00\end{array}$ & $\begin{array}{l}2.18 \\
0.15 \\
6.89 \\
8.00\end{array}$ & $\begin{array}{r}15.60 \\
0.88 \\
5.65 \\
10.00\end{array}$ & $\begin{array}{r}11.15 \\
1.10 \\
9.87 \\
12.00\end{array}$ & $\begin{array}{l}9.37 \\
0.86 \\
9.18 \\
9.00\end{array}$ & $\begin{array}{r}7.82 \\
0.57 \\
7.29 \\
15.00\end{array}$ & $\begin{array}{r}8.46 \\
0.74 \\
8.75 \\
88.00\end{array}$ & $\begin{array}{r}9.09 \\
0.41 \\
4.52 \\
53.00\end{array}$ & $\begin{array}{r}9.17 \\
0.35 \\
3.82 \\
36.00\end{array}$ & $\begin{array}{r}0.53 \\
0.08 \\
15.10 \\
23.00\end{array}$ & $\begin{array}{l}3.53 \\
0.18 \\
5.10 \\
0.00\end{array}$ & $\begin{array}{r}2.08 \\
0.22 \\
10.58 \\
46.00\end{array}$ & $\begin{array}{r}83.50 \\
5.68\end{array}$ \\
\hline 1959 & $\begin{array}{l}\text { PRECIP. (CM-) } \\
S R-90 \text { (HCI/SQ-KM-) } \\
S R-90 \text { CONC. (PC/LL) } \\
S R-89 / 5 R-90\end{array}$ & $\begin{array}{r}3.12 C \\
0.76 C \\
24.36 \\
-\end{array}$ & $\begin{array}{l}3.126 \\
0.76 C \\
24.36 \\
-\end{array}$ & $\begin{array}{r}7.67 \\
2.49 \\
32.47 \\
13.00\end{array}$ & $\begin{array}{r}3.71 \\
1.23 \\
33.16 \\
8.60\end{array}$ & $\begin{array}{r}16.92 \\
2.30 \\
13.60 \\
8.30\end{array}$ & $\begin{array}{l}8.38 \\
0.62 \\
7.40 \\
4.20\end{array}$ & $\begin{array}{l}25.02 \\
0.56 \\
2.24 \\
2.30\end{array}$ & $\begin{array}{l}5.41 \\
0.10 \\
1.85 \\
1.70\end{array}$ & $\begin{array}{r}18.85 \\
0.22 \\
1.17 \\
0.38\end{array}$ & $\begin{array}{r}23.06 \\
0.03 \\
0.14 \\
.\end{array}$ & $\begin{array}{l}3.76 \\
0.02 \\
0.54 \\
\end{array}$ & $\begin{array}{l}0.43 \\
0.21 \\
3.27 \\
+\end{array}$ & $\begin{array}{r}125.45 \\
9.30\end{array}$ \\
\hline 1960 & $\begin{array}{l}\text { PRECIP (CM.) } \\
\text { SR-90 IMCI/SO.KM-) } \\
S R-90 \text { CONC. (PC/L) } \\
\text { SR-B9/SR-90 }\end{array}$ & $\begin{array}{l}3.10 \\
0.07 \\
2.26 \\
\end{array}$ & $\begin{array}{l}6.73 \\
-- \\
-\end{array}$ & $\begin{array}{l}2.69 \\
0.15 \\
5.58 \\
\end{array}$ & $\begin{array}{l}9.22 \\
0.37 \\
4.02 \\
-\end{array}$ & $\begin{array}{l}22.63 \\
0.65 C \\
2.88 \\
\end{array}$ & $\begin{array}{l}4.19 \\
0.126 \\
2.87 \\
-.\end{array}$ & $\begin{array}{c}22.89 \\
0.396 \\
1.71\end{array}$ & $\begin{array}{l}4.75 \\
0.080 \\
1.69\end{array}$ & $\begin{array}{l}2.26 \\
0.11 \\
4.87\end{array}$ & $\begin{array}{l}0.97 \\
= \\
=\end{array}$ & $\begin{array}{l}1.60 \\
0.016 \\
0.63\end{array}$ & $\begin{array}{l}7.65 \\
0.056 \\
0.66\end{array}$ & $\begin{array}{r}96.68 \\
2.00\end{array}$ \\
\hline 1961 & $\begin{array}{l}\text { PRECIP. (CM.) } \\
\text { SR-90 (NCI/SO.KM.) } \\
\text { SR-90 CONC. (PC/L) } \\
\text { SR-89/SR-90 }\end{array}$ & $\begin{array}{l}1.68 \\
0.05 C \\
2.98 \\
\end{array}$ & $\begin{array}{l}7.26 \\
0.276 \\
2.35 \\
=\end{array}$ & $\begin{array}{l}8.38 \\
0.38 C \\
4.54 \\
\end{array}$ & $\begin{array}{l}3.78 \\
0.176 \\
4.50 \\
-.\end{array}$ & $\begin{array}{c}11.56 C \\
0.50 C \\
4.33 \\
-\end{array}$ & $\begin{array}{l}11.56 C \\
-0.50 C \\
4.33 \\
-\end{array}$ & $\begin{array}{l}27.64 \\
0.27 \mathrm{C} \\
0.98 \\
\end{array}$ & $\begin{array}{l}0.03 \\
0.08 C \\
1.00 \\
\end{array}$ & $\begin{array}{r}18.72 \\
0.10 \\
0.54 \\
84.00\end{array}$ & $\begin{array}{r}2.18 \\
0.14 \\
0.43 \\
113.00\end{array}$ & $\begin{array}{r}8.08 \\
0.19 \\
2.36 \\
99.00\end{array}$ & $\begin{array}{l}4.19 C \\
0.71 C \\
16.95 \\
--\end{array}$ & $\begin{array}{r}113.06 \\
3.26\end{array}$ \\
\hline 1962 & $\begin{array}{l}\text { PRECIP. (CM.) } \\
\text { SR-9O IMCI/SQ.KM-) } \\
\text { SR-SO CONC. (PC/L) } \\
\text { SR-Q9/SR-90 }\end{array}$ & $\begin{array}{r}4.19 C \\
0.71 C \\
16.95 \\
\end{array}$ & $\begin{array}{l}4.196 \\
0.716 \\
16.95 \\
--\end{array}$ & $=$ & $\begin{array}{r}8.64 \\
0.88 \\
10.19 \\
19.00\end{array}$ & $\begin{array}{r}4.29 \\
2.17 \\
50.59 \\
20.00\end{array}$ & $\begin{array}{r}14.02 \\
1.11 \\
7.92 \\
15.00\end{array}$ & ${ }^{12.27}$ & $\begin{array}{l}7.87 \\
=\end{array}$ & $\begin{array}{r}26.67 \\
0.75 \\
2.82 \\
12.00\end{array}$ & $\begin{array}{l}9.96 \\
0.40 \\
4.02 \\
32.00\end{array}$ & $\begin{array}{l}3.12 C \\
0.450 \\
14.43 \\
-\end{array}$ & $\begin{array}{l}3.12 C \\
0.45 C \\
14.43 \\
-\end{array}$ & $\begin{array}{r}106.57 \\
7.63\end{array}$ \\
\hline 1963 & $\begin{array}{l}\text { PRECIP. (CM.) } \\
\text { SR-90 (MCI/SO.KM.) } \\
S R-90 \text { CCNC. (PC/LL) } \\
\text { SR-89/SR-90. }\end{array}$ & $\begin{array}{r}2.49 \\
0.10 \\
4.02 \\
22.00\end{array}$ & $\begin{array}{r}1.07 \\
0.60 \\
56.08 \\
27.00\end{array}$ & $\begin{array}{r}7.21 \\
0.79 \\
10.96 \\
25.00\end{array}$ & $\begin{array}{r}5.61 \\
1.81 \\
32.27 \\
12.00\end{array}$ & $\begin{array}{r}6.32 \\
2.56 \\
40.51 \\
\end{array}$ & $\begin{array}{r}1.35 \\
0.69 \\
51.12 \\
+\end{array}$ & $\begin{array}{r}26.92 \\
2.73 \\
10.15 \\
*\end{array}$ & $\begin{array}{r}8.33 \\
1.05 \\
12.61 \\
*\end{array}$ & $\begin{array}{l}5.11 \\
0.20 \mathrm{C} \\
3.92 \\
\end{array}$ & $\begin{array}{l}0.46 \\
0.20 C \\
43.48 \\
-.\end{array}$ & $\begin{array}{l}5.79 \\
0.20 \mathrm{C} \\
3.46 \\
+.\end{array}$ & $\begin{array}{l}2.69 \\
0.17 \\
6.83 \\
6\end{array}$ & $\begin{array}{l}73.15 \\
11.10\end{array}$ \\
\hline 1964 & $\begin{array}{l}\text { PRECIP. (CM.) } \\
\text { SR-90 IMCI/SQ.KM.) } \\
\text { SR-90 CQNC. (PC/L) } \\
\text { SR-89/SR-90 }\end{array}$ & $\begin{array}{r}1.60 \\
0.22 \\
13.76 \\
1.00\end{array}$ & $\begin{array}{l}5.51 \\
\overline{-}\end{array}$ & $\begin{array}{r}10.06 \\
2.35 \\
23.36 \\
\end{array}$ & $\begin{array}{l}14.91 \\
1.30 C \\
8.72 \\
-\end{array}$ & $\begin{array}{r}12.12 \\
1.600 \\
13.21 \\
-\end{array}$ & $\begin{array}{r}14.71 \\
1.69 \\
11.69 \\
-\end{array}$ & $\begin{array}{r}4.57 \\
1.21 \\
26.48 \\
-\end{array}$ & $\begin{array}{l}15.60 \\
\overline{-}\end{array}$ & $\begin{array}{l}1.46 \\
= \\
=\end{array}$ & $\begin{array}{r}3.15 \\
0.47 \\
14.93 \\
*\end{array}$ & $\begin{array}{l}17.53 \\
= \\
=\end{array}$ & $\begin{array}{l}4.24 \\
-- \\
=-\end{array}$ & $\begin{array}{r}112.46 \\
8.84\end{array}$ \\
\hline 1965 & $\begin{array}{l}\text { PRECIP. ICM.) } \\
S R-90 \text { (MCI/SQ.KM.) } \\
S R-90 \text { CONC. (PC/L) } \\
\text { SR-89/SR-90 }\end{array}$ & $=$ & $\bar{z}$ & $\bar{z}$ & $=$ & $\begin{array}{r}2.13 \\
3.80 \\
178.41 \\
\end{array}$ & $\begin{array}{l}4.55 \\
0.41 \\
9.02 \\
-\end{array}$ & $\bar{z}$ & $\begin{array}{l}9.45 \\
0.27 \\
2.86\end{array}$ & $\begin{array}{l}11.66 \\
0.31 \\
2.66 \\
-\end{array}$ & $\begin{array}{l}0.66 \\
0.05 \\
7.58\end{array}$ & $\begin{array}{l}0.08 \\
0.04 \\
50.01 \\
-\end{array}$ & $\begin{array}{l}10.90 \\
0.24 \\
2.21 \\
-.\end{array}$ & $\begin{array}{r}39.43 \\
5.12\end{array}$ \\
\hline 1966 & $\begin{array}{l}\text { PRECIP. }(C M .) \\
S R-90 \text { (KCI/SO.KM.) } \\
S R-90 \text { CONC. (PC/L) } \\
S R-89 / S R-90\end{array}$ & $\bar{m}=$ & $\begin{array}{l}5.97 \\
0.22 \\
3.69 \\
--\end{array}$ & $\begin{array}{l}2.18 \\
0.10 \\
4.59 \\
-\end{array}$ & $\begin{array}{r}12.29 \\
0.34 \\
2.77 \\
\end{array}$ & $\begin{array}{r}4.72 \\
0.55 \\
11.66 \\
-\end{array}$ & $\begin{array}{l}6.50 \\
0.20 \\
3.08 \\
+\end{array}$ & $\begin{array}{l}5.08 \\
0.07 \\
1.38 \\
6.00\end{array}$ & $\begin{array}{r}16.69 \\
0.14 \\
0.84 \\
1.60\end{array}$ & $\begin{array}{l}6.81 \\
0.06 \\
0.89\end{array}$ & $\begin{array}{l}3.53 \\
0.03 \\
0.85 \\
*\end{array}$ & $\begin{array}{l}1.30 \\
0.01 \\
0.77 \\
*\end{array}$ & $\begin{array}{l}6.43 \\
0.07 \\
1.09 \\
6.60\end{array}$ & $\begin{array}{r}71.50 \\
1.79\end{array}$ \\
\hline 1967 & $\begin{array}{l}\text { PRECIP. (CM.) } \\
S R-90 \text { (PCI/SQ.KM.) } \\
S R-90 \text { CONC. (PC/LL) } \\
\text { SR-89/SR-90 }\end{array}$ & $\begin{array}{r}3.84 \\
0.04 \\
1.05 \\
13.00\end{array}$ & $\begin{array}{l}1.65 \\
0.07 \\
4.25 \\
7.40\end{array}$ & $\begin{array}{l}3.61 \\
0.11 \\
3.05 \\
8.00\end{array}$ & $\begin{array}{r}12.93 \\
0.17 \\
1.32 \\
4.70\end{array}$ & $\begin{array}{r}13.56 \\
0.25 \\
1.85 \\
3.00\end{array}$ & $\begin{array}{r}11.68 \\
0.13 \\
1.12 \\
*\end{array}$ & $\bar{E}$ & $\bar{z}$ & $\bar{\Xi}$ & $\bar{z}$ & $\bar{z}$ & $\bar{m}=$ & $\begin{array}{r}47.27 \\
0.77\end{array}$ \\
\hline \begin{tabular}{l} 
Not: \\
\hdashline$::$ \\
$A:$ \\
$B:$ \\
$C:$
\end{tabular} & $\begin{array}{l}\text { ATA NOT AVAILABLE } \\
\text { ERO OR TRACE } \\
\text { PPROXIMATE } \\
\text { OWER LIMIY OF REPO } \\
\text { ROPORTIONED FROM O }\end{array}$ & $\begin{array}{l}\text { DATA } \\
\text { VALLLY }\end{array}$ & & ED & & & & & & & & & & \\
\hline
\end{tabular}


MONTHLY FALLOUT DEPOSITION COLLECTIONS

SITE: PENNSYLVANIA, PITTSBURGH

LAT. $4026 \mathrm{~N}$ LONG. 80 OW ALT. 227M. (POT)

SOURCE: NUCLEAR SCIENCE AND ENGINEERING CORP.

COLLECTIONS TERMINATED IN JUNE 1963

\begin{tabular}{|c|c|c|c|c|c|c|c|c|c|c|c|c|c|c|}
\hline & & JAM_ & FEB. & HAR . & APR . & MAY & JUNE & Jue $Y$ & AUG. & SEP. & OCT. & NOY. & DEC. & CUM. TOTAL \\
\hline 1957 & $\begin{array}{l}\text { PRECIP. (CM.) } \\
\text { SR-90 (KCI/SO.KM.) } \\
\text { SR-90 CONC. (PC/L) } \\
\text { SR-89/SR-90 }\end{array}$ & $=$ & $\underline{z}$ & $\bar{z}$ & $=$ & $=$ & $=$ & $\begin{array}{r}11.46 \\
0.33 \\
2.88 \\
-\end{array}$ & $\begin{array}{l}1.24 \\
0.06 \\
4.84 \\
\end{array}$ & $\begin{array}{r}11.73 \\
0.06 \\
0.52 \\
39.00\end{array}$ & $\begin{array}{r}4.93 \\
0.12 \\
2.44 \\
27.00\end{array}$ & $\begin{array}{r}5.51 \\
0.11 \\
2.00 \\
14.00\end{array}$ & $\begin{array}{r}12.52 \\
0.24 \\
1.92 \\
13.00\end{array}$ & $\begin{array}{r}47.39 \\
0.92\end{array}$ \\
\hline 1958 & $\begin{array}{l}\text { PRECIP. (CM-) } \\
\text { SR-90 (MCI/SO.KM.) } \\
S R-90 \text { CONC. (PC/L) } \\
S R-89 / S R-90\end{array}$ & $\begin{array}{l}8.64 \\
0.25 \\
2.90 \\
7.00\end{array}$ & $\begin{array}{r}2.54 \\
0.14 \\
5.52 \\
11.00\end{array}$ & $\begin{array}{r}8.53 \\
0.19 \\
2.23 \\
11.00\end{array}$ & $\begin{array}{r}9.83 \\
0.39 \\
3.97 \\
13.00\end{array}$ & $\begin{array}{r}9.83 \\
0.33 \\
3.36 \\
11.00\end{array}$ & $\begin{array}{r}7.62 \\
0.98 \\
12.87 \\
14.00\end{array}$ & $\begin{array}{r}21.51 \\
0.91 \\
4.24 \\
41.00\end{array}$ & $\begin{array}{r}13.54 \\
0.65 \\
4.81 \\
25.00\end{array}$ & $\begin{array}{r}11.02 \\
0.26 \\
2.36 \\
25.60\end{array}$ & $\begin{array}{r}2.13 \\
0.22 \\
10.33 \\
28.00\end{array}$ & $\begin{array}{r}7.95 \\
0.29 \\
3.65 \\
34.00\end{array}$ & $\begin{array}{r}3.00 \\
0.30 \\
10.01 \\
26.00\end{array}$ & $\begin{array}{r}106.14 \\
4.91\end{array}$ \\
\hline 1959 & $\begin{array}{l}\text { PRECIP. ICM.) } \\
\text { SR-90 INCI/SQ.KM-) } \\
5 R-90 \text { CCNC. (PC/L) } \\
5 R-89 / S R-90\end{array}$ & $\begin{array}{r}7.11 \\
0.56 \\
7.88 \\
24.00\end{array}$ & $\begin{array}{r}10.19 \\
0.77 \\
7.56 \\
21.00\end{array}$ & $\begin{array}{r}3.35 \\
1.33 \\
20.95 \\
18.00\end{array}$ & $\begin{array}{r}9.27 \\
2.08 \\
22.44 \\
10.00\end{array}$ & $\begin{array}{r}7.42 \\
1.12 \\
15.10 \\
7.00\end{array}$ & $\begin{array}{l}9.14 \\
0.80 \\
8.76 \\
5.00\end{array}$ & $\begin{array}{l}8.43 \\
0.26 \\
3.09 \\
3.00\end{array}$ & $\begin{array}{l}8.94 \\
0.17 \\
1.91 \\
1.80\end{array}$ & $\begin{array}{l}3.58 \\
0.02 \\
0.56 \\
0.30\end{array}$ & $\begin{array}{r}11.66 \\
0.14 \\
1.21 \\
0.20\end{array}$ & $\begin{array}{l}7.87 \\
0.14 \\
1.78 \\
0.50\end{array}$ & $\begin{array}{l}7.29 \\
0.14 \\
1.93 \\
0.40\end{array}$ & $\begin{array}{r}97.25 \\
7.53\end{array}$ \\
\hline 1900 & $\begin{array}{l}\text { PRECIP. }\left(C M_{-}\right) \\
S R-90 \text { (NCI/SQ.KM.) } \\
S R-90 \text { CONC. }(P C / L) \\
S R-89 / S R-90\end{array}$ & $\begin{array}{l}7.34 \\
0.10 \\
1.37 \\
0.26\end{array}$ & $\begin{array}{l}5.26 \\
0.15 \\
2.86 \\
1.30\end{array}$ & $\begin{array}{l}4.09 \\
0.13 \\
3.18 \\
1.80\end{array}$ & $\begin{array}{l}4.04 \\
0.20 \\
4.96 \\
0.45\end{array}$ & $\begin{array}{r}11.96 \\
0.31 \\
2.60 \\
0.13\end{array}$ & $\begin{array}{l}8.92 \\
0.31 \\
3.48 \\
0.24\end{array}$ & $\begin{array}{l}7.34 \\
0.18 \\
2.46 \\
-.\end{array}$ & $\begin{array}{l}6.35 \\
0.09 \\
1.42 \\
-.\end{array}$ & $\begin{array}{l}7.82 \\
0.07 \\
0.90 \\
\end{array}$ & $\begin{array}{l}4.90 \\
0.06 \\
1.23 \\
--\end{array}$ & $\begin{array}{l}3.51 \\
0.05 \\
1.43 \\
-\end{array}$ & $\begin{array}{l}7.01 \\
0.08 \\
1.15 \\
=-\end{array}$ & $\begin{array}{r}78.54 \\
1.73\end{array}$ \\
\hline 1961 & $\begin{array}{l}\text { PRECIP. (CM-) } \\
\text { SR-90 (FCI/SQ-KM.) } \\
S R-90 \text { CONC. (PC/L) } \\
S R-89 / S R-90\end{array}$ & $\begin{array}{l}2.29 \\
0.05 \\
2.19 \\
-\end{array}$ & $\begin{array}{l}7.65 \\
0.23 \\
3.01 \\
\end{array}$ & $\begin{array}{l}9.35 \\
0.30 \\
3.21 \\
-\end{array}$ & $\begin{array}{l}8.74 \\
0.43 \\
4.92 \\
-\end{array}$ & $\begin{array}{l}8.61 \\
C .37 \\
4.30 \\
-.\end{array}$ & $\begin{array}{l}10.74 \\
0.33 \\
3.08 \\
--\end{array}$ & $\begin{array}{r}10.36 \\
0.17 \\
1.65 \\
\end{array}$ & $\begin{array}{l}8.33 \\
0.08 \\
0.97 \\
\end{array}$ & $\begin{array}{r}4.27 \\
0.03 \\
0.71 \\
19.00\end{array}$ & $\begin{array}{r}7.85 \\
0.16 \\
2.04 \\
66.00\end{array}$ & $\begin{array}{r}8.18 \\
0.27 \\
3.31 \\
78.00\end{array}$ & $\begin{array}{r}5.18 \\
0.38 \\
7.34 \\
63.00\end{array}$ & $\begin{array}{r}91.55 \\
2.80\end{array}$ \\
\hline 1962 & $\begin{array}{l}\text { PREC 1P. (CM.) } \\
\text { SR-90 (MCI/SQ.KH.) } \\
\text { SR-90 CCNC. (PC/L) } \\
S R-89 / 5 R-90\end{array}$ & $\begin{array}{r}5.16 \\
0.51 \\
9.89 \\
49.00\end{array}$ & $\begin{array}{r}9.04 \\
0.80 \\
6.85 \\
29.00\end{array}$ & $\begin{array}{r}7.67 \\
0.88 \\
11.48 \\
21.00\end{array}$ & $\begin{array}{r}11.58 \\
1.49 \\
12.87 \\
15.00\end{array}$ & $\begin{array}{r}0.60 \\
1.35 \\
20.46 \\
9.60\end{array}$ & $\begin{array}{r}4.09 \\
0.60 \\
14.67 \\
6.40\end{array}$ & $\begin{array}{r}8.05 \\
1.46 \\
18.14 \\
6.00\end{array}$ & $\begin{array}{r}6.55 \\
1.24 \\
18.94 \\
5.00\end{array}$ & $\begin{array}{r}17.42 \\
0.59 \\
3.39 \\
19.00\end{array}$ & $\begin{array}{r}5.46 \\
0.53 \\
9.71 \\
24.00\end{array}$ & $\begin{array}{r}3.53 \\
0.62 \\
17.57 \\
43.00\end{array}$ & $\begin{array}{r}5.94 \\
0.73 \\
12.29 \\
32.00\end{array}$ & $\begin{array}{l}91.09 \\
10.80\end{array}$ \\
\hline 1963 & $\begin{array}{l}\text { PRECIP. ICM.) } \\
\text { SR-90 IHCI/SQ-KM.) } \\
S R-90 \text { CONC. (PC/L) } \\
\text { SR-89/SR-90 }\end{array}$ & $\begin{array}{r}5.00 \\
0.79 \\
15.81 \\
29.00\end{array}$ & $\begin{array}{r}6.48 \\
0.90 \\
13.89 \\
23.00\end{array}$ & $\begin{array}{r}17.40 \\
2.83 \\
16.27 \\
14.00\end{array}$ & $\begin{array}{r}7.72 \\
3.05 \\
39.51 \\
9.00\end{array}$ & $\begin{array}{r}4.22 \\
2.20 \\
52.14 \\
7.00\end{array}$ & $=$ & $=$ & - & $\overline{-}$ & $\bar{z}$ & $\overline{-}$ & $\begin{array}{l}-- \\
-\end{array}$ & $\begin{array}{r}40.82 \\
9.77\end{array}$ \\
\hline 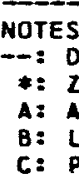 & $\begin{array}{l}\text { ATA NOT AVAILABLE } \\
\text { ERO OR TRACE } \\
\text { PPROXIMATE } \\
\text { OWER LIMIT OF REPOR } \\
\text { ROPORTIONED FROM OR }\end{array}$ & $\begin{array}{l}\text { OATA } \\
\text { NALLY }\end{array}$ & & & & & & & & & & & & \\
\hline
\end{tabular}


MONTHLY FALLOUT DEPOSITION COLLECTIONS

SITE: PENNSYlvaNia, PITTSBurgh

LAT. $4026 \mathrm{~N}$

LONG. 80 OH ALT.

227\%. (COLUnN)

SOURCE: NUCLEAR SCIENCE AND ENGINEERIMG CORP. COLLECTIONS TERMINATED IN JUNE 1963

\begin{tabular}{|c|c|c|c|c|c|c|c|c|c|c|c|c|c|c|}
\hline & & JAN. & FEB. & MAR. & APR. & MAY & JUNE & JuL $Y$ & Aut. & SEP. & OCT. & HOY. & DEC. & CUH. TOTAL \\
\hline 1960 & $\begin{array}{l}\text { PRECIP. ICM-1 } \\
S R-90 \text { i } Y C 1 / S Q . K M-) \\
S R-90 \text { CONC. }(P C / L) \\
S R-89 / S R-90\end{array}$ & $\overline{\bar{z}}$ & $\begin{array}{l}5.23 \\
0.07 \\
1.34 \\
3.30\end{array}$ & $\begin{array}{l}4.09 \\
0.07 \\
1.72 \\
1.90\end{array}$ & $\begin{array}{l}4.04 \\
0.13 \\
3.22 \\
1.00\end{array}$ & $\frac{11.96}{1.10}$ & $\begin{array}{l}9.83 \\
0.18 \\
1.84 \\
--\end{array}$ & $\begin{array}{l}7.34 \\
0.00 \\
0.01 \\
\end{array}$ & $\begin{array}{l}6.35 \\
0.23 \\
3.63 \\
-\end{array}$ & $\begin{array}{l}7.02 \\
0.10 \\
1.28 \\
\end{array}$ & $\begin{array}{l}4.90 \\
0.18 \\
3.68 \\
\end{array}$ & $\begin{array}{l}3.51 \\
0.14 \\
3.99 \\
\end{array}$ & $\begin{array}{l}7.01 \\
0.14 \\
2.00 \\
-.\end{array}$ & $\begin{array}{r}72.08 \\
1.24\end{array}$ \\
\hline 1961 & $\begin{array}{l}\text { PRECIP. }(C M-) \\
S R-90 \text { IMCI/SQ-KM-) } \\
S R-90 \text { CONC. }(P C / L) \\
S R-89 / S R-90\end{array}$ & $\begin{array}{l}2.29 \\
0.10 \\
4.37 \\
--\end{array}$ & $\begin{array}{l}7.65 \\
0.21 \\
2.75 \\
=-\end{array}$ & $\begin{array}{l}9.35 \\
0.17 \\
1.82 \\
--\end{array}$ & $\begin{array}{l}0.74 \\
0.14 \\
1.61 \\
-\end{array}$ & $\begin{array}{l}9.80 \\
0.15 \\
1.54 \\
\end{array}$ & $\begin{array}{l}9.55 \\
0.08 \\
0.84 \\
--\end{array}$ & $\begin{array}{r}10.36 \\
0.12 \\
1.16 \\
-.\end{array}$ & $\begin{array}{l}8.33 \\
0.02 \\
0.25 \\
\end{array}$ & $\begin{array}{r}4.27 \\
0.01 \\
0.24 \\
21.00\end{array}$ & $\begin{array}{r}7.85 \\
6.06 \\
0.77 \\
52.00\end{array}$ & $\begin{array}{r}0.28 \\
0.23 \\
2.82 \\
79.00\end{array}$ & $\begin{array}{r}5.18 \\
0.29 \\
5.60 \\
60.00\end{array}$ & $\begin{array}{r}91.55 \\
1.58\end{array}$ \\
\hline 1962 & $\begin{array}{l}\text { PRECIP. }\left(C M_{-}\right) \\
S R-90 \text { IMCI/SQ.KM-) } \\
S R-90 \text { CONC. }(P C / L) \\
S R-89 / S R-90\end{array}$ & $\begin{array}{r}5.16 \\
0.56 \\
10.86 \\
45.00\end{array}$ & $\begin{array}{r}9.04 \\
0.80 \\
8.85 \\
30.00\end{array}$ & $\begin{array}{r}7.67 \\
0.92 \\
12.00 \\
21.00\end{array}$ & $\begin{array}{r}11.58 \\
0.88 \\
7.60 \\
14.00\end{array}$ & $\begin{array}{r}6.60 \\
1.54 \\
23.34 \\
11.00\end{array}$ & $\begin{array}{r}4.09 \\
0.68 \\
16.63 \\
6.20\end{array}$ & $\begin{array}{r}8.05 \\
1.23 \\
15.28 \\
6.90\end{array}$ & $\begin{array}{r}6.55 \\
0.74 \\
11.30 \\
5.40\end{array}$ & $\begin{array}{r}17.42 \\
0.54 \\
3.10 \\
18.00\end{array}$ & $\begin{array}{r}5.46 \\
0.44 \\
8.06 \\
22.00\end{array}$ & $\begin{array}{r}3.53 \\
0.49 \\
13.89 \\
45.00\end{array}$ & $\begin{array}{r}5.94 \\
0.58 \\
9.77 \\
35.00\end{array}$ & $\begin{array}{r}91.09 \\
9.40\end{array}$ \\
\hline 1963 & $\begin{array}{l}\text { PRECIP. (CH.) } \\
\text { SR-90 IMCI/SQ.KM-) } \\
S R-90 \text { CONC. }(P C / L) \\
S R-89 / S R-90\end{array}$ & $\begin{array}{r}5.00 \\
0.70 \\
14.01 \\
29.00\end{array}$ & $\begin{array}{r}6.48 \\
0.86 \\
13.28 \\
21.00\end{array}$ & $\begin{array}{r}17.40 \\
2.62 \\
15.06 \\
13.00\end{array}$ & $\begin{array}{r}7.72 \\
2.72 \\
35.24 \\
10.00\end{array}$ & $\begin{array}{r}4.22 \\
2.18 \\
51.66 \\
6.00\end{array}$ & $=$ & $=$ & $\overline{-}$ & $\bar{z}$ & $=$ & $=$ & $=$ & $\begin{array}{r}40.82 \\
9.08\end{array}$ \\
\hline $\begin{array}{c}\text { NOTES } \\
--: \\
*: \\
A: \\
B: \\
C:\end{array}$ & $\begin{array}{l}\text { ATA NOT AVAILABLE } \\
\text { ERO OR TRACE } \\
\text { PPROXIMATE } \\
\text { OWER LIMIT OF REPOR } \\
\text { ROPORTI DNEO FRDM OR }\end{array}$ & DATA & & $=0$ & & & & & & & & & & \\
\hline
\end{tabular}




\begin{tabular}{|c|c|c|c|c|c|c|c|c|c|c|c|c|c|c|}
\hline & & JAN. & FEB - & MAR. & APR - & MaY & JUNE & JULY & AUG. & SEP. & OCT. & NOV. & DEC. & CUA. TOTAL \\
\hline 1959 & $\begin{array}{l}\text { PRECIP. }\left(C M_{-}\right) \\
\text {SR-90 }\left(M C I / S Q_{\left.-K M_{-}\right)}\right. \\
\text {SR-90 CONC. (PC/L) } \\
\text { SR-89/SR-90 }\end{array}$ & $\overline{--}$ & $=$ & $=$ & $\begin{array}{r}6.71 \\
0.97 \\
14.46 \\
12.20\end{array}$ & $\begin{array}{r}14.71 \\
0.5 .1 \\
3.47 \\
7.40\end{array}$ & $\begin{array}{l}6.78 \\
0.19 \\
2.81 \\
4.90\end{array}$ & $\begin{array}{c}35.23 \\
-- \\
--\end{array}$ & $\begin{array}{r}11.48 \\
0.12 \\
1.05 \\
1.60\end{array}$ & $\begin{array}{r}18.08 \\
0.05 \\
0.28 \\
0.91\end{array}$ & $\begin{array}{r}30.71 \\
0.20 \\
0.66 \\
0.65\end{array}$ & $\begin{array}{l}1.70 \\
0.08 \\
4.71 \\
4\end{array}$ & $\begin{array}{r}6.15 \\
0.05 \\
0.82 \\
14.00\end{array}$ & $\begin{array}{r}131.55 \\
2.17\end{array}$ \\
\hline 1960 & $\begin{array}{l}\text { PRECIP, (CM.) } \\
\text { SR-9O IMCI/SQ.KM-) } \\
\text { SR-90 CONC. }(P C / L) \\
\text { SR-89/SR-90 }\end{array}$ & $\begin{array}{l}18.16 \\
0.28 \\
1.55 \\
--\end{array}$ & $\begin{array}{r}14.05 \\
0.39 \\
2.78 \\
-\end{array}$ & $\begin{array}{l}15.67 \\
0.25 \\
1.60 \\
-\end{array}$ & $\begin{array}{l}9.93 \\
0.19 \\
1.92 \\
\end{array}$ & $\begin{array}{l}3.73 \\
0.12 \mathrm{C} \\
3.22 \\
-.\end{array}$ & $\begin{array}{l}6.02 \\
0.20 \mathrm{C} \\
3.33 \\
-\end{array}$ & $\begin{array}{c}12.17 \\
0.11 \mathrm{C} \\
0.91 \\
-\end{array}$ & $\begin{array}{l}0.69 \\
0.016 \\
1.45 \\
--\end{array}$ & $\begin{array}{l}10.01 \\
0.076 \\
0.70 \\
-.\end{array}$ & $\begin{array}{l}4.34 \\
0.03 C \\
0.70 \\
=-\end{array}$ & $\begin{array}{l}1.73 \\
0.03 C \\
1.74 \\
-.\end{array}$ & $\begin{array}{l}6.02 \\
0.09 C \\
1.50 \\
--\end{array}$ & $\begin{array}{r}102.52 \\
1.77\end{array}$ \\
\hline 1961 & $\begin{array}{l}\text { PRECIP. (CM.) } \\
\text { SR-9O INCI/SO.XM.) } \\
\text { SR-90 CONC. (PC/L) } \\
\text { SR-99/SR-90 }\end{array}$ & $\begin{array}{l}7.44 \\
0.15 \mathrm{C} \\
2.02 \\
--\end{array}$ & $\begin{array}{c}22.73 \\
0.45 \mathrm{C} \\
1.98 \\
-\end{array}$ & $\begin{array}{c}0.74 \\
0.19 \mathrm{C} \\
25.68 \\
--\end{array}$ & $\begin{array}{r}0.03 \\
0.016 \\
33.34 \\
\end{array}$ & $\begin{array}{l}7.57 \\
0.08 \mathrm{C} \\
1.06 \\
-.\end{array}$ & $\begin{array}{l}4.95 \\
0.05 \mathrm{C} \\
1.02 \\
-.\end{array}$ & $\begin{array}{c}14.48 \\
0.06 \mathrm{C} \\
0.42 \\
\end{array}$ & $\begin{array}{l}37.95 \\
0.15 \mathrm{C} \\
0.40 \\
\end{array}$ & $\begin{array}{r}3.71 \\
0.03 \\
0.81 \\
13.00\end{array}$ & $\begin{array}{r}2.08 \\
0.01 \\
0.49 \\
142.00\end{array}$ & $\begin{array}{r}2.57 \\
0.04 \\
1.56 \\
174.00\end{array}$ & $\begin{array}{r}12.04 \\
0.40 \\
3.33 \\
60.00\end{array}$ & $\begin{array}{r}116.29 \\
1.62\end{array}$ \\
\hline 1962 & $\begin{array}{l}\text { PRECIP. (CM.) } \\
\text { SR-90 INCI/SQ.KM.) } \\
\text { SR-90 CONC. (PC/LL) } \\
\text { SR-8S/SR-90 }\end{array}$ & $\begin{array}{r}16.48 \\
0.72 \\
4.37 \\
38.00\end{array}$ & $\begin{array}{r}13.31 \\
0.83 \\
6.24 \\
30.00\end{array}$ & $\begin{array}{l}13.46 \\
1.57 \\
11.67 \\
19.00\end{array}$ & $\begin{array}{r}8.15 \\
0.09 \\
1.12 \\
12.00\end{array}$ & $\begin{array}{r}10.44 \\
1.51 \\
14.47 \\
9.00\end{array}$ & $\begin{array}{r}7.59 \\
0.87 \\
11.47 \\
8.00\end{array}$ & $\begin{array}{r}6.78 \\
0.72 \\
10.62 \\
13.00\end{array}$ & $\begin{array}{r}10.52 \\
0.32 \\
3.05 \\
21.00\end{array}$ & $\begin{array}{r}7.24 \\
0.49 \\
6.77 \\
73.00\end{array}$ & $\begin{array}{r}2.13 \\
0.02 \\
C .94 \\
23.00\end{array}$ & $\begin{array}{r}11.51 \\
0.07 \\
0.61 \\
32.00\end{array}$ & $\begin{array}{r}5.77 \\
0.32 \\
5.55 \\
47.00\end{array}$ & $\begin{array}{r}113.38 \\
7.53\end{array}$ \\
\hline 1963 & $\begin{array}{l}\text { PREC IP }\left(C M_{-}\right) \\
\text {SR-90 (PCI/SQ.KM) } \\
\text { SR-90 CONC. (PC/L) } \\
S R-89 / S R-90\end{array}$ & $\begin{array}{l}18.974 \\
= \\
-\end{array}$ & $\begin{array}{r}10.01 \\
0.67 \\
6.70 \\
34.00\end{array}$ & $\begin{array}{r}8.33 \\
1.05 \\
12.61 \\
20.00\end{array}$ & $\begin{array}{r}20.62 \\
2.98 \\
28.07 \\
13.50\end{array}$ & $\begin{array}{r}7.29 \\
1.18 \\
16.19 \\
12.50\end{array}$ & $\begin{array}{r}12.29 \\
2.50 \\
20.35 \\
2.40\end{array}$ & $\begin{array}{r}6.30 \\
2.00 \\
31.75 \\
c .70\end{array}$ & $\begin{array}{r}4.85 \\
0.58 \\
11.96 \\
*\end{array}$ & $\begin{array}{r}10.11 \\
0.48 \\
4.75 \\
0.40\end{array}$ & $\frac{*}{0.03}$ & $\begin{array}{r}10.67 \\
0.56 \\
5.25 \\
+\end{array}$ & $\begin{array}{r}13.00 \\
0.68 \\
5.24 \\
*\end{array}$ & $\begin{array}{r}112.44 \\
12.71\end{array}$ \\
\hline 1964 & $\begin{array}{l}\text { PRECIP }(C M-) \\
S R-90 \text { IMCI/SQ.KM.) } \\
\text { SR-90 CONC. (PC/L) } \\
\text { SR-89/SR-90 }\end{array}$ & $\begin{array}{l}15.93 \\
0.34 \\
2.14 \\
--\end{array}$ & $\begin{array}{r}13.54 \\
1.47 \\
10.86 \\
c .30\end{array}$ & $\begin{array}{l}15.65 \\
1.70 \\
10.87 \\
*\end{array}$ & $\begin{array}{l}9.14 \\
0.74 \\
8.10 \\
1.80\end{array}$ & $\begin{array}{r}6.68 \\
1.14 \\
17.07 \\
*\end{array}$ & $\begin{array}{l}7.54 \\
1.30 \\
17.25 \\
--\end{array}$ & $\begin{array}{l}26.21 \\
1.54 \\
5.89 \\
--\end{array}$ & $\begin{array}{r}25.32 \\
0.48 \\
1.90 \\
-\end{array}$ & $\begin{array}{l}17.60 \\
0.28 \\
1.60 \\
-\end{array}$ & $\begin{array}{r}26.26 \\
0.36 \\
1.38 \\
1.20\end{array}$ & $\begin{array}{l}3.45 \\
0.09 \\
2.61 \\
6.20\end{array}$ & $\begin{array}{l}11.63 \\
0.32 \\
2.76 \\
--\end{array}$ & $\begin{array}{r}178.95 \\
9.76\end{array}$ \\
\hline 1965 & $\begin{array}{l}\text { PREC IP }(C M-) \\
S R-90 \text { i } M C I / S Q-K M-) \\
S R-90 \text { CONC. }(P C / L) \\
\text { SR-89/SR-90 }\end{array}$ & $\begin{array}{l}3.63 \\
0.19 \\
5.24 \\
-\end{array}$ & $\begin{array}{l}13.54 \\
\pm \\
=\end{array}$ & $\begin{array}{r}19.51 \\
0.93 \\
4.77 \\
--\end{array}$ & $\begin{array}{r}10.13 \\
0.02 \\
0.20 \\
-\end{array}$ & $\begin{array}{r}3.71 \\
0.39 \\
10.52 \\
--\end{array}$ & $\begin{array}{r}20.83 \\
0.78 \\
3.75 \\
-\end{array}$ & $\begin{array}{r}10.87 \\
0.48 \\
4.42 \\
-.\end{array}$ & $\begin{array}{r}23.85 \\
0.35 \\
1.47 \\
--\end{array}$ & $\begin{array}{l}15.21 \\
0.23 \\
1.52 \\
-\end{array}$ & $\begin{array}{l}5.94 \\
0.22 \\
3.71 \\
\end{array}$ & $\begin{array}{l}4.50 \\
0.10 \\
2.23 \\
--\end{array}$ & $\begin{array}{l}6.32 \\
0.01 \\
0.16 \\
-\end{array}$ & $\begin{array}{r}138.04 \\
3.70\end{array}$ \\
\hline 1966 & $\begin{array}{l}\text { PRECIP }(C M-) \\
\text { SR-90 (MCI/SO.KM.) } \\
\text { SR-90 CONC. (PC/L) } \\
\text { SR-89/SR-90 }\end{array}$ & $\begin{array}{c}18.95 \\
\div- \\
=\end{array}$ & $\begin{array}{r}11.53 \\
0.23 \\
2.00 \\
-\end{array}$ & $\begin{array}{l}5.66 \\
0.11 \\
1.95 \\
-\end{array}$ & $\begin{array}{l}9.09 \\
0.22 \\
2.43 \\
-\end{array}$ & $\begin{array}{r}15.60 \\
0.42 \\
2.70 \\
--\end{array}$ & $\begin{array}{l}9.30 \\
0.17 \\
1.83 \\
9.80\end{array}$ & $\begin{array}{l}7.29 \\
0.12 \\
1.65 \\
4.10\end{array}$ & $\begin{array}{l}8.18 \\
0.08 \\
0.98 \\
*\end{array}$ & $\begin{array}{l}5.13 \\
0.04 \\
0.78 \\
*\end{array}$ & $\begin{array}{l}6.27 \\
0.07 \\
1.12 \\
+\end{array}$ & $\begin{array}{l}2.67 \\
0.04 \\
1.50 \\
*\end{array}$ & $\begin{array}{l}9.47 \\
0.07 \\
0.74 \\
*\end{array}$ & $\begin{array}{r}109.14 \\
1.57\end{array}$ \\
\hline 1967 & $\begin{array}{l}\text { PRECIP. (CK-) } \\
\text { SR-90 IMCI/SQ.KM-) } \\
\text { SR-90 CONC. }(P C / L) \\
\text { SR-89/SR-90 }\end{array}$ & $\begin{array}{l}7.09 \\
0.11 \\
1.56 \\
8.10\end{array}$ & $\begin{array}{r}11.07 \\
0.14 \\
1.27 \\
5.60\end{array}$ & $\begin{array}{l}7.82 \\
0.08 \\
1.03 \\
9.00\end{array}$ & $\begin{array}{l}9.45 \\
0.15 \\
1.59 \\
7.00\end{array}$ & $\begin{array}{r}24.00 \\
0.20 \\
0.84 \\
4.00\end{array}$ & $\begin{array}{r}10.72 \\
0.09 \\
0.84 \\
*\end{array}$ & $\bar{z}=$ & $\begin{array}{l}= \\
=\end{array}$ & $=$ & $\overline{=}$ & $=$ & $=$ & $\begin{array}{r}70.15 \\
0.77\end{array}$ \\
\hline
\end{tabular}

NOTES

-: data not available

*: ZERO OR TRACE

A: APPROXIMATE

B: LOWER LIMIT OF REPORTED DATA CONSOLIDATEO DATA
C: PROPORTIONEO FROM ORIGINALLY CONSOL 


\begin{tabular}{|c|c|c|c|c|c|c|c|c|c|c|c|c|c|c|}
\hline & & JAN. & FEQ. & MAR. & APR: & MAY & JUNE & JULY & AUG. & SEP. & OCT. & Nov. & DEC. & CUM. TOTAL \\
\hline 1957 & $\begin{array}{l}\text { PRECIP. (CM-) } \\
\text { SR-90 iMCI/SQ.KM-) } \\
\text { SR-90 CONC. (PC/L) } \\
\text { SR-99/SR-90 }\end{array}$ & $\bar{z}$ & $\begin{array}{l}= \\
=\end{array}$ & $\begin{array}{l}\bar{z} \\
=\end{array}$ & $\begin{array}{l}3.43 \\
0.20 \\
5.84 \\
--\end{array}$ & $\begin{array}{r}10.59 \\
0.67 \\
6.33 \\
11.00\end{array}$ & $\begin{array}{r}6.02 \\
0.39 \\
6.48 \\
25.00\end{array}$ & $\begin{array}{r}10.90 \\
1.00 \\
9.91 \\
68.00\end{array}$ & $\begin{array}{r}4.11 \\
0.43 \\
10.47 \\
-.\end{array}$ & $\begin{array}{r}7.98 \\
0.34 \\
4.27 \\
33.00\end{array}$ & $\begin{array}{r}4.24 \\
0.36 \\
8.50 \\
51.00\end{array}$ & $\begin{array}{r}3.78 \\
0.05 \\
1.33 \\
15.00\end{array}$ & $\frac{0.02}{16.00}$ & $\begin{array}{r}51.05 \\
3.54\end{array}$ \\
\hline 1958 & $\begin{array}{l}\text { PRECIP. ICM-I } \\
S R-90 \text { iMC1/SO.KM,I) } \\
\text { SR-90 CONC. } P C / L) \\
S R-89 / S R-90\end{array}$ & $\begin{array}{r}0.56 \\
0.03 \\
5.36 \\
17.00\end{array}$ & $\begin{array}{r}5.41 \\
0.15 \\
2.78 \\
13.00\end{array}$ & $\begin{array}{r}1.32 \\
0.08 \\
6.07 \\
13.00\end{array}$ & $\begin{array}{r}0.00 \\
0.98 \\
12.26 \\
12.00\end{array}$ & $\begin{array}{r}4.70 \\
0.86 \\
18.73 \\
16.00\end{array}$ & $\begin{array}{l}2.77 \\
0.06 \\
2.17 \\
5.00\end{array}$ & $\begin{array}{r}11.35 \\
0.93 \\
8.20 \\
31.00\end{array}$ & $\begin{array}{r}0.48 \\
0.19 \\
39.59 \\
49.00\end{array}$ & $\begin{array}{r}2.24 \\
0.11 \\
4.92 \\
43.00\end{array}$ & $\begin{array}{l}* 0.02 \\
23.00\end{array}$ & $\begin{array}{r}2.39 \\
0.29 \\
12.14 \\
47.00\end{array}$ & $\begin{array}{r}0.18 \\
0.08 \\
44.45 \\
37.00\end{array}$ & $\begin{array}{r}39.40 \\
3.80\end{array}$ \\
\hline 1959 & 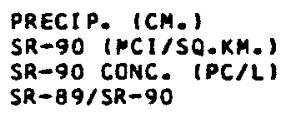 & $\begin{array}{r}0.69 \\
0.03 \\
4.35 \\
25.00\end{array}$ & $\begin{array}{r}2.13 \\
0.35 \\
16.44 \\
24.00\end{array}$ & $\begin{array}{r}2.59 \\
0.83 \\
32.05 \\
18.00\end{array}$ & $\begin{array}{r}3.38 \\
1.26 \\
37.28 \\
12.00\end{array}$ & $\begin{array}{l}19.99 \\
=\end{array}$ & $\begin{array}{l}6.93 \\
0.59 \\
8.52 \\
5.10\end{array}$ & $\begin{array}{l}3.35 \\
= \\
=\end{array}$ & $\begin{array}{l}8.00 \\
\overline{-}\end{array}$ & $\begin{array}{l}6.60 \\
0.16 \\
2.43 \\
0.37\end{array}$ & $\begin{array}{l}6.60 \\
0.14 \\
2.13 \\
0.31\end{array}$ & $\begin{array}{l}5.13 \\
0.09 \\
1.76 \\
*\end{array}$ & $\begin{array}{l}3.66 \\
0.06 \\
1.64 \\
1.30\end{array}$ & $\begin{array}{r}69.05 \\
3.51\end{array}$ \\
\hline 1960 & $\begin{array}{l}\text { PRECIP. }(C M-) \\
\text { SR-90 IVC1/SO.KM.) } \\
\text { SR-90 CONC. }(P C / L) \\
\text { SR-89/SR-90 }\end{array}$ & $\begin{array}{l}0.74 \\
0.01 \\
1.36\end{array}$ & $\begin{array}{l}0.25 \\
0.02 \\
8.02 \\
-\end{array}$ & $\begin{array}{l}2.77 \\
0.27 \\
9.75 \\
--\end{array}$ & $\begin{array}{l}8.99 \\
0.33 \\
3.68\end{array}$ & $\begin{array}{l}18.75 \\
0.96 \mathrm{C} \\
5.13 \\
--\end{array}$ & $\begin{array}{l}4.67 \\
0.24 c \\
5.14 \\
-9\end{array}$ & $\begin{array}{l}3.45 \\
0.07 \mathrm{C} \\
2.03 \\
--\end{array}$ & $\begin{array}{l}14.66 \\
0.28 \mathrm{C} \\
1.91 \\
-\end{array}$ & $\begin{array}{l}7.09 \\
0.099 \\
1.27 \\
-2\end{array}$ & $\begin{array}{l}1.05 \\
0.02 c \\
1.09 \\
--\end{array}$ & $\begin{array}{l}2.64 \\
0.04 C \\
1.52 \\
-\end{array}$ & $\begin{array}{l}1.07 \\
0.026 \\
1.87 \\
-1\end{array}$ & $\begin{array}{r}66.93 \\
2.35\end{array}$ \\
\hline 1961 & $\begin{array}{l}\text { PRECIP. }(C M-) \\
\text { SR-90 (KCI/SQ.KM-) } \\
\text { SR-90 CONC. (PC/L) } \\
\text { SR-89/SR-90 }\end{array}$ & $\begin{array}{l}0.43 \\
c . c 0 c \\
0.01 \\
--\end{array}$ & $\begin{array}{l}2.24 \\
0.026 \\
0.90 \\
-\end{array}$ & $\begin{array}{l}2.24 \\
0.11 \mathrm{C} \\
4.92 \\
--\end{array}$ & $\begin{array}{l}2.21 \\
0.11 \mathrm{c} \\
4.98\end{array}$ & $\begin{array}{l}9.98 \\
0.40 \mathrm{COC} \\
4.01 \\
-\end{array}$ & $\begin{array}{l}7.72 \\
0.31 \mathrm{C} \\
4.02 \\
--\end{array}$ & $\begin{array}{l}2.97 \\
0.056 \\
1.69 \\
-\end{array}$ & $\begin{array}{l}9.78 \\
0.15 \mathrm{C} \\
1.54 \\
--\end{array}$ & $\begin{array}{r}5.21 \\
0.29 \\
5.57 \\
141.00\end{array}$ & $\begin{array}{r}3.63 \\
0.07 \\
1.93 \\
65.00\end{array}$ & $\begin{array}{r}2.29 \\
0.35 \\
15.29 \\
62.00\end{array}$ & $\begin{array}{r}2.39 \\
0.10 \\
4.19 \\
53.00\end{array}$ & $\begin{array}{r}51.09 \\
1.96\end{array}$ \\
\hline 1962 & $\begin{array}{l}\text { PRECIP. }\left(C M_{-}\right) \\
\text {SR-90 iMCII/SQ.KM-) } \\
\text { SR-90 CONC. }(P C / L) \\
S R-B 9 / S R-90\end{array}$ & $\begin{array}{r}0.36 \\
0.06 \\
16.67 \\
38.00\end{array}$ & $\begin{array}{r}2.77 \\
0.48 \\
17.33 \\
60.00\end{array}$ & $\begin{array}{r}7.65 \\
1.27 \\
16.61 \\
19.00\end{array}$ & $\begin{array}{r}4.83 \\
1.95 \\
40.38 \\
10.00\end{array}$ & $\begin{array}{r}14.66 \\
5.98 \\
40.80 \\
9.00\end{array}$ & $\begin{array}{r}14.00 \\
1.56 \\
11.15 \\
4.00\end{array}$ & $\begin{array}{r}11.30 \\
0.99 \\
8.77 \\
26.00\end{array}$ & $\begin{array}{r}7.44 \\
1.29 \\
17.34 \\
11.00\end{array}$ & $\begin{array}{r}7.49 \\
1.13 \\
15.09 \\
13.00\end{array}$ & $\begin{array}{r}1.68 \\
0.36 \\
21.43 \\
27.00\end{array}$ & $\begin{array}{r}0.30 \\
0.03 \\
10.01 \\
29.00\end{array}$ & $\begin{array}{l}0.33 \\
-- \\
--\end{array}$ & $\begin{array}{l}72.81 \\
15.10\end{array}$ \\
\hline 1963 & $\begin{array}{l}\text { PRECIP. (CM.) } \\
\text { SR-90 INCI/SQ.KM.) } \\
\text { SR-90 CONC. (PC/L) } \\
\text { SR-89/SR-90 }\end{array}$ & $\begin{array}{r}2.24 \\
0.10 \\
4.47 \\
30.00\end{array}$ & $\begin{array}{r}c .64 \\
0.17 \\
26.57 \\
28.00\end{array}$ & $\begin{array}{r}1.98 \\
1.76 \\
88.89 \\
20.00\end{array}$ & $\begin{array}{r}4.19 \\
2.91 \\
69.46 \\
12.00\end{array}$ & $\begin{array}{r}4.98 \\
2.96 \\
59.44 \\
10.10\end{array}$ & $\begin{array}{r}11.40 \\
5.05 \\
44.30 \\
4.80\end{array}$ & $\begin{array}{r}7.21 \\
2.13 \\
29.55 \\
0.40\end{array}$ & $\begin{array}{l}6.81 \\
1.57 \\
23.06 \\
*\end{array}$ & $\begin{array}{l}5.61 \\
0.49 \\
8.74 \\
1.00\end{array}$ & $\begin{array}{r}1.19 \\
C .46 \\
38.66 \\
1.00\end{array}$ & $\begin{array}{r}0.25 \\
0.09 \\
36.01 \\
*\end{array}$ & $\begin{array}{l}1.30 \\
0.01 \\
0.77 \\
*\end{array}$ & $\begin{array}{l}47.80 \\
17.70\end{array}$ \\
\hline 1964 & $\begin{array}{l}\text { PREC 1P. (CM.) } \\
\text { SR-90 (MCCISPQ.KM.) } \\
\text { SR-90 CONC. (PCAL) } \\
\text { SR-89/SR-90 }\end{array}$ & $\begin{array}{c}0.41 \\
0.14 \\
34.15 \\
*\end{array}$ & $\begin{array}{r}0.28 \\
0.15 \\
53.58 \\
*\end{array}$ & $\begin{array}{r}4.62 \\
0.60 \\
12.59 \\
1.80\end{array}$ & $\begin{array}{r}9.50 \\
4.22 \\
44.43 \\
*\end{array}$ & $\begin{array}{r}8.79 \\
2.84 \\
32.31 \\
*\end{array}$ & $\begin{array}{r}10.69 \\
4.58 \\
42.85 \\
--\end{array}$ & $\begin{array}{l}10.36 \\
1.27 \\
12.26 \\
--\end{array}$ & $\begin{array}{r}10.06 \\
0.02 \\
0.20 \\
--\end{array}$ & $\begin{array}{l}7.11 \\
0.47 \\
6.62 \\
--\end{array}$ & $\begin{array}{r}0.18 \\
0.15 \\
83.34 \\
*\end{array}$ & $\begin{array}{r}0.36 \\
0.04 \\
11.12\end{array}$ & $\begin{array}{l}1.70 \\
0.03 \\
1.77 \\
--\end{array}$ & $\begin{array}{l}64.06 \\
14.51\end{array}$ \\
\hline 1965 & $\begin{array}{l}\text { PRECIP. ICM.) } \\
\text { SR-90 IMCI/SQ.KM.) } \\
\text { SR-90 CONC. }(P C / L) \\
\text { SR-89/SR-90 }\end{array}$ & $\begin{array}{r}0.30 \\
0.06 \\
20.01\end{array}$ & $\begin{array}{l}4.32 \\
0.03 \\
0.70 \\
--\end{array}$ & $\begin{array}{r}2.82 \\
0.61 \\
21.64 \\
-\end{array}$ & $\begin{array}{r}5.79 \\
0.83 \\
14.34 \\
*\end{array}$ & $\begin{array}{l}18.01 \\
2.61 \\
14.50 \\
--\end{array}$ & $\begin{array}{l}16.08 \\
1.79 \\
11.14 \\
-\end{array}$ & $\begin{array}{r}19.00 \\
1.21 \\
6.37 \\
--\end{array}$ & $\begin{array}{r}11.61 \\
0.36 \\
3.11 \\
--\end{array}$ & $\begin{array}{r}15.32 \\
0.62 \\
4.05 \\
--.\end{array}$ & $\begin{array}{l}1.57 \\
0.08 \\
5.10 \\
--\end{array}$ & $\begin{array}{r}1.27 \\
1.32 \\
103.94 \\
---\end{array}$ & $\begin{array}{r}0.51 \\
0.08 \\
15.69 \\
--\end{array}$ & $\begin{array}{r}96.60 \\
9.60\end{array}$ \\
\hline 1966 & $\begin{array}{l}\text { PRECIP. ICM-I } \\
\text { SR-90 IMCIISQ.KM-I } \\
\text { SR-90 CONC. IPCAL) } \\
\text { SR-89/SR-90 }\end{array}$ & $\begin{array}{l}1.22 \\
0.03 \\
2.46 \\
-2\end{array}$ & $\begin{array}{l}2.82 \\
0.04 \\
1.42 \\
--\end{array}$ & $\begin{array}{l}3.81 \\
0.10 \\
2.63 \\
--\end{array}$ & $\begin{array}{r}3.50 \\
0.39 \\
11.15\end{array}$ & $\begin{array}{r}2.40 \\
0.50 \\
20.84\end{array}$ & $\begin{array}{l}7.24 \\
0.59 \\
8.15 \\
8.30\end{array}$ & $\begin{array}{r}14.73 \\
0.35 \\
2.38 \\
4.50\end{array}$ & $\begin{array}{l}9.68 \\
0.20 \\
2.07 \\
2.20\end{array}$ & $\begin{array}{l}4.37 \\
0.16 \\
3.67 \\
*\end{array}$ & $\begin{array}{l}4.67 \\
0.10 \\
2.15 \\
*\end{array}$ & $\begin{array}{r}0.13 \\
0.02 \\
15.39 \\
*\end{array}$ & $\begin{array}{r}1.09 \\
0.03 \\
2.76 \\
16.00\end{array}$ & $\begin{array}{r}55.66 \\
2.51\end{array}$ \\
\hline 1967 & 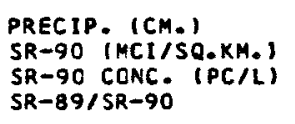 & $\begin{array}{r}2.06 \\
0.06 \\
2.92 \\
15.00\end{array}$ & $\begin{array}{l}2.29 \\
0.02 \\
0.88 \\
\end{array}$ & $\begin{array}{l}1.14 \\
0.07 \\
6.15 \\
7.600\end{array}$ & $\begin{array}{l}3.28 \\
0.06 \\
1.83 \\
3.60\end{array}$ & $\begin{array}{l}8.71 \\
0.25 \\
2.88 \\
3.20\end{array}$ & $\begin{array}{l}22.17 \\
0.41 \\
1.85 \\
*\end{array}$ & $\begin{array}{l}z \\
z\end{array}$ & $\begin{array}{l}\overline{-} \\
\bar{z}\end{array}$ & $\begin{array}{l}-= \\
\overline{z-} \\
--\end{array}$ & $\begin{array}{l}\bar{z} \\
\bar{z}\end{array}$ & $\begin{array}{l}\bar{z} \\
\bar{z}\end{array}$ & $\begin{array}{l}\bar{z} \\
\bar{z}\end{array}$ & $\begin{array}{r}39.65 \\
0.87\end{array}$ \\
\hline \multicolumn{15}{|c|}{$\begin{array}{l}\text { NOTES } \\
\text {--: DATA NOT AVAILABLE } \\
\text { *: 2ERO OR TRACE } \\
\text { A: APPROXIMATE } \\
\text { B: LOWER LIMIT OF REPC } \\
\text { C: PROPORTIONED FROM }\end{array}$} \\
\hline
\end{tabular}


MONTHLY FALLOUT DEPOSITION COLLECTIONS

SITE: TENMESSEE, ChATTANOOGA

LAT. 35 3N LONG. 85 2OH ALT.

206M. IPOT)

SURFACE AIR SAMPLING STATION

SOURCE: ISOTOPES, INC. THRU UNIVERSITY OF CHAT TANOOGA, DEPARTMENT OF GEOLOGY COLLECTIONS TERMINATED IN DECEMBER 1965

\begin{tabular}{|c|c|c|c|c|c|c|c|c|c|c|c|c|c|c|}
\hline & & JAN. & FEB. & MAR. & APR. & MAY & JUNE & JULY & MUG. & SEP. & OCT. & Nov. & DEC . & CUM. TOTAL \\
\hline 1963 & $\begin{array}{l}\text { PRECIP. (CM.) } \\
\text { SR-90 IMCI/SQ.KM.) } \\
\text { SR-90 CONC. }(P C / L) \\
S R-89 / S R-90\end{array}$ & $\overline{-}$ & $=$ & $\bar{m}$ & $\overline{-}$ & $\begin{array}{l}=- \\
--\end{array}$ & $\begin{array}{l}-- \\
- \\
-\end{array}$ & $=$ & $\begin{array}{r}2.11 \\
0.58 \\
27.49 \\
1.70\end{array}$ & $\begin{array}{r}2.39 \\
0.37 \\
15.49 \\
1.00\end{array}$ & $\frac{0.05}{*}$ & $\begin{array}{r}17.32 \\
0.78 \\
4.51 \\
0.60\end{array}$ & $\begin{array}{r}11.94 \\
0.61 \\
5.11 \\
0.60\end{array}$ & $\begin{array}{r}33.76 \\
2.39\end{array}$ \\
\hline 1964 & $\begin{array}{l}\text { PRECIP. }(C M .) \\
\text { SR-90 IMCI/SQ.KM.) } \\
\text { SR-90 CONC. (PC/L) } \\
\text { SR-89/SR-90 }\end{array}$ & $\begin{array}{r}14.30 \\
1.29 \\
9.03 \\
0.30\end{array}$ & $\begin{array}{r}13.97 \\
1.85 \\
13.25 \\
0.20\end{array}$ & $\begin{array}{r}28.96 \\
1.88 \\
6.50 \\
*\end{array}$ & $\begin{array}{r}24.97 \\
4.40 \\
17.63 \\
--\end{array}$ & $\begin{array}{l}12.55 \\
2.11 \\
16.82 \\
--\end{array}$ & $\begin{array}{r}8.43 \\
1.43 \\
16.97 \\
-.\end{array}$ & $\begin{array}{r}14.17 \\
0.86 \\
6.07 \\
-.\end{array}$ & $\begin{array}{r}15.72 \\
0.73 \\
4.65 \\
-\end{array}$ & $\begin{array}{l}7.57 \\
0.19 \\
2.51 \\
--\end{array}$ & $\begin{array}{l}9.73 \\
0.43 \\
4.42 \\
2.88\end{array}$ & $\begin{array}{l}8.66 \\
0.21 \\
2.43 \\
*\end{array}$ & $\begin{array}{l}7.57 \\
0.05 \\
0.67 \\
1.06\end{array}$ & $\begin{array}{r}166.60 \\
15.43\end{array}$ \\
\hline 1965 & $\begin{array}{l}\text { PRECIP. }\left(C M_{-}\right) \\
S R-90 \text { (MCI/SQ-KM-) } \\
S R-90 \text { CONC. }(P C / L) \\
S R-89 / S R-90\end{array}$ & $\begin{array}{l}8.28 \\
0.38 \\
4.59 \\
\end{array}$ & $\begin{array}{r}11.94 \\
0.54 \\
4.53 \\
-\end{array}$ & $\begin{array}{r}25.40 \\
0.82 \\
3.23 \\
-.\end{array}$ & $\begin{array}{l}9.86 \\
0.89 \\
9.03 \\
-0\end{array}$ & $\begin{array}{l}7.11 \\
0.66 \\
9.29 \\
--\end{array}$ & $\begin{array}{r}10.77 \\
0.64 \\
5.95 \\
--\end{array}$ & $\begin{array}{r}11.46 \\
C .39 \\
3.41 \\
-\end{array}$ & $\begin{array}{r}11.53 \\
0.16 \\
1.39 \\
\end{array}$ & $\begin{array}{r}11.76 \\
0.12 \\
1.03 \\
--\end{array}$ & $\begin{array}{l}2.26 \\
0.08 \\
3.54 \\
--\end{array}$ & $\begin{array}{l}10.13 \\
0.25 \\
2.47 \\
--\end{array}$ & $\begin{array}{l}2.13 \\
0.06 \\
2.82 \\
--\end{array}$ & $\begin{array}{r}122.63 \\
4.99\end{array}$ \\
\hline $\begin{array}{r}\text { NOTES } \\
-:: \\
*: 2 \\
A: \\
B: \\
C:\end{array}$ & $\begin{array}{l}\text { ATA NOT AVAILABLE } \\
\text { ERO OR TRACE } \\
\text { PPROXIMATE } \\
\text { OWER LIMIT OF REPOR } \\
\text { ROPORTI ONED FROM OR }\end{array}$ & NALLY & & & & & & & & & & & & \\
\hline
\end{tabular}




\begin{tabular}{|c|c|c|c|c|c|c|c|c|c|c|c|c|c|c|}
\hline & & JAN. & FEB. & MAR. & APR. & MAY & JUNE & JULY & AUG. & SEP. & OCT. & Nov. & DEC. & CUM. TOTAL \\
\hline 1959 & $\begin{array}{l}\text { PRECIP. (CM.) } \\
\text { SR-90 (MCI/SQ.KM.) } \\
S R-90 \text { CCNC. (PC/L) } \\
S R-89 / S R-90\end{array}$ & $\bar{m}$ & $\begin{array}{r}6.27 \\
0.82 \\
13.08 \\
33.00\end{array}$ & $\begin{array}{l}3.20 \\
-- \\
-\end{array}$ & $\begin{array}{r}1.83 \\
1.10 \\
60.11 \\
11.00\end{array}$ & $\begin{array}{r}8.08 \\
2.08 \\
25.75 \\
7.40\end{array}$ & $\begin{array}{l}8.03 \\
0.34 \\
4.24 \\
5.20\end{array}$ & $\begin{array}{l}8.26 \\
0.11 \\
1.34 \\
2.10\end{array}$ & $\begin{array}{l}4.50 \\
0.03 \\
0.67 \\
\end{array}$ & $\begin{array}{l}9.92 \\
0.08 \\
6.90 \\
1.30\end{array}$ & $\begin{array}{r}28.91 \\
0.00 \\
C .01 \\
*\end{array}$ & $\begin{array}{l}4.90 \\
0.05 \\
1.03 \\
*\end{array}$ & $\begin{array}{l}14.86 \\
0.12 \\
0.81 \\
*\end{array}$ & $\begin{array}{r}97.76 \\
4.73\end{array}$ \\
\hline 1960 & $\begin{array}{l}\text { PRECIP. (CM-) } \\
\text { SR-90 IMCI/SO.KM-) } \\
S R-90 \text { CONC. (PC/L) } \\
\text { SR-89/SR-90 }\end{array}$ & $\begin{array}{l}0.15 \\
0.21 \\
2.58 \\
-.\end{array}$ & $\begin{array}{l}7.19 \\
0.02 \\
0.28 \\
\end{array}$ & $\begin{array}{l}2.41 \\
0.12 \\
4.98 \\
-.\end{array}$ & $\begin{array}{l}7.57 \\
0.19 \\
2.51 \\
--\end{array}$ & $\begin{array}{l}5.66 \\
0.08 \mathrm{C} \\
1.42 \\
-.\end{array}$ & $\begin{array}{l}8.18 \\
0.116 \\
1.35 \\
-.\end{array}$ & $\begin{array}{l}18.64 \\
* \\
=\end{array}$ & $\begin{array}{l}8.41 \\
* \\
-\end{array}$ & $\begin{array}{l}6.63 \\
0.02 C \\
0.31 \\
-.\end{array}$ & $\begin{array}{l}9.50 \\
0.026 \\
0.22 \\
--\end{array}$ & $\begin{array}{l}2.08 \\
0.006 \\
0.01 \\
-\end{array}$ & $\begin{array}{c}21.59 \\
0.04 C \\
0.19 \\
=-\end{array}$ & $\begin{array}{r}106.01 \\
0.81\end{array}$ \\
\hline 1961 & 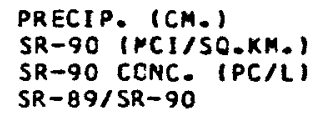 & $\begin{array}{l}0.61 \\
0.116 \\
1.28 \\
--\end{array}$ & $\begin{array}{l}8.15 \\
c .10 \mathrm{C} \\
1.23 \\
-\end{array}$ & $\begin{array}{c}12.45 \\
0.29 C \\
2.33 \\
--\end{array}$ & $\begin{array}{l}4.22 \\
0.10 C \\
2.37 \\
-\end{array}$ & $\begin{array}{l}3.33 \\
C . C 4 C \\
1.21 \\
-0\end{array}$ & $\begin{array}{l}19.94 \\
C .24 C \\
1.21 \\
-.\end{array}$ & $\begin{array}{l}3.30 \\
+ \\
-\end{array}$ & $\begin{array}{l}0.48 \\
=- \\
=-\end{array}$ & $\begin{array}{r}14.07 \\
0.03 \\
0.22 \\
87.00\end{array}$ & $\begin{array}{r}8.66 \\
0.03 \\
0.35 \\
101.00\end{array}$ & $\begin{array}{l}10.26 \\
0.04 \\
0.39 \\
-.\end{array}$ & $\begin{array}{r}8.10 \\
0.26 \\
3.21 \\
63.00\end{array}$ & $\begin{array}{r}101.57 \\
1.24\end{array}$ \\
\hline 1962 & $\begin{array}{l}\text { PRECIP. (CM.) } \\
\text { SR-90 (KCI/SO.KM.) } \\
\text { SR-90 CCNC. (PC/L) } \\
S R-89 / S R-90\end{array}$ & $\begin{array}{r}3.35 \\
0.32 \\
9.56 \\
38.00\end{array}$ & $\begin{array}{l}7.67 \\
0.40 \\
5.22 \\
--\end{array}$ & $\begin{array}{r}5.97 \\
0.98 \\
16.42 \\
18.00\end{array}$ & $\begin{array}{r}22.73 \\
2.86 \\
12.59 \\
12.00\end{array}$ & $\begin{array}{r}5.08 \\
0.36 \\
7.09 \\
19.00\end{array}$ & $\begin{array}{r}11.76 \\
1.38 \\
11.74 \\
8.00\end{array}$ & $\begin{array}{r}21.64 \\
0.29 \\
1.35 \\
18.00\end{array}$ & $\begin{array}{r}8.59 \\
0.13 \\
1.52 \\
20.00\end{array}$ & $\begin{array}{r}15.93 \\
0.03 \\
0.19 \\
14.00\end{array}$ & $\begin{array}{l}18.52 \\
=- \\
=\end{array}$ & $\begin{array}{r}14.53 \\
0.63 \\
4.34 \\
23.00\end{array}$ & $\begin{array}{r}3.28 \\
0.69 \\
21.04 \\
38.00\end{array}$ & $\begin{array}{r}139.05 \\
8.07\end{array}$ \\
\hline 1963 & $\begin{array}{l}\text { PRECIP. (CM-) } \\
S R-90 \text { (PCI/SO-KM-) } \\
S R-90 \text { CONC. (PC/L) } \\
S R-89 / S R-90\end{array}$ & $\begin{array}{r}1.24 \\
0.15 \\
12.10 \\
45.00\end{array}$ & $\begin{array}{r}0.76 \\
0.30 \\
39.48 \\
31.00\end{array}$ & $\begin{array}{r}1.68 \\
0.39 \\
23.22 \\
15.00\end{array}$ & $\begin{array}{r}16.15 \\
2.25 \\
13.94 \\
11.70\end{array}$ & $\begin{array}{r}4.53 \\
1.37 \\
27.79 \\
8.50\end{array}$ & $\begin{array}{l}3.76 \\
0.20 \\
5.32 \\
3.50\end{array}$ & $\begin{array}{r}4.34 \\
0.52 \\
11.99 \\
2.00\end{array}$ & $\begin{array}{r}0.05 \\
0.10 \\
200.00 \\
1.00\end{array}$ & $\begin{array}{l}2.39 \\
0.12 \\
5.03 \\
0.60\end{array}$ & $\begin{array}{l}0.03 \\
0.15 \\
499.99 \\
*\end{array}$ & $\begin{array}{r}4.57 \\
0.55 \\
12.04 \\
*\end{array}$ & $\begin{array}{l}4.60 \\
0.15 \\
3.27 \\
*\end{array}$ & $\begin{array}{r}44.50 \\
6.25\end{array}$ \\
\hline 1964 & $\begin{array}{l}\text { PRECIP. (CM.) } \\
\text { SR-9O ( } M C 1 / S Q-K M-) \\
S R-90 \text { CCNC. }(P C / L) \\
S R-89 / S R-90\end{array}$ & $\begin{array}{l}8.31 \\
0.51 \\
6.14 \\
.\end{array}$ & $\begin{array}{r}3.18 \\
1.08 \\
33.97 \\
\end{array}$ & $\begin{array}{r}11.51 \\
1.56 \\
13.56 \\
*\end{array}$ & $\begin{array}{r}8.20 \\
1.57 \\
19.25 \\
0.02\end{array}$ & $\begin{array}{r}8.61 \\
2.01 \\
23.35 \\
*\end{array}$ & $\begin{array}{l}0.76 \\
0.61 \\
80.27 \\
-\end{array}$ & $\begin{array}{l}0.48 \\
0.22 \\
45.84 \\
--\end{array}$ & $\begin{array}{l}9.22 \\
0.34 \\
3.69 \\
--\end{array}$ & $\begin{array}{l}27.10 \\
0.25 \\
0.93 \\
-.\end{array}$ & $\begin{array}{l}1.96 \\
6.10 \\
5.11 \\
3.70\end{array}$ & $\begin{array}{l}13.67 \\
0.02 \\
0.25 \\
-.\end{array}$ & $\begin{array}{l}2.18 \\
0.11 \\
5.05 \\
--\end{array}$ & $\begin{array}{r}95.18 \\
8.38\end{array}$ \\
\hline 1965 & $\begin{array}{l}\text { PRECIP. }(C M-) \\
S R-90 \text { (MCI/SO.KM.) } \\
S R-90 \text { CONC. (PC/L) } \\
S R-89 / S R-90\end{array}$ & $\begin{array}{l}4.75 \\
0.14 \\
2.95 \\
\end{array}$ & $\begin{array}{l}13.61 \\
0.35 \\
2.58 \\
-\end{array}$ & $\begin{array}{l}5.08 \\
0.23 \\
4.53 \\
-.\end{array}$ & $\begin{array}{l}5.16 \\
0.32 \\
6.21 \\
-\end{array}$ & $\begin{array}{l}23.57 \\
1.62 \\
6.88 \\
-\end{array}$ & $\begin{array}{l}7.06 \\
0.55 \\
7.80 \\
--\end{array}$ & $\begin{array}{l}0.41 \\
\neq \\
--\end{array}$ & $\begin{array}{l}4.32 \\
0.10 \\
2.32 \\
-.\end{array}$ & $\begin{array}{l}13.06 \\
0.91 \\
6.97 \\
-.\end{array}$ & $\begin{array}{l}4.09 \\
0.07 \\
1.72 \\
-.\end{array}$ & $\begin{array}{l}5.05 \\
0.06 \\
1.19 \\
--\end{array}$ & $\begin{array}{l}4.01 \\
0.07 \\
1.75 \\
-.\end{array}$ & $\begin{array}{r}90.17 \\
4.42\end{array}$ \\
\hline 1966 & $\begin{array}{l}\text { PRECIP. ICM.) } \\
\text { SR-90 IPCI/SQ.KM.) } \\
5 R-90 \text { CONC. (PC/L) } \\
S R-99 / S R-90\end{array}$ & $\begin{array}{l}4.52 \\
0.13 \\
2.88 \\
-\end{array}$ & $\begin{array}{l}0.38 \\
0.12 \\
1.44 \\
--\end{array}$ & $\begin{array}{l}6.05 \\
0.22 \\
3.64 \\
\end{array}$ & $\begin{array}{l}39.12 \\
0.46 \\
1.18 \\
-\end{array}$ & $\begin{array}{l}7.19 \\
0.13 \\
1.81 \\
--\end{array}$ & $\begin{array}{r}11.43 \\
0.12 \\
1.05 \\
14.00\end{array}$ & $\begin{array}{l}3.48 \\
0.09 \\
2.59 \\
4.90\end{array}$ & $\begin{array}{r}13.00 \\
0.10 \\
0.77 \\
*\end{array}$ & $\begin{array}{l}6.88 \\
0.04 \\
0.59 \\
+\end{array}$ & $\begin{array}{l}4.75 \\
0.02 \\
0.43 \\
.\end{array}$ & $\begin{array}{l}2.64 \\
0.02 \\
0.76 \\
7.80\end{array}$ & $\begin{array}{l}2.74 \\
0.04 \\
1.46\end{array}$ & $\begin{array}{r}110.18 \\
1.49\end{array}$ \\
\hline 1967 & $\begin{array}{l}\text { PRECIP. (CM.) } \\
\text { SR-90 IMCI/SO.KM.) } \\
\text { SR-90 CONC. (PC/L) } \\
\text { SR-89/SR-90 }\end{array}$ & $\begin{array}{l}0.86 \\
0.02 \\
2.33 \\
*\end{array}$ & $\begin{array}{l}1.90 \\
0.07 \\
3.69 \\
7.80\end{array}$ & $\begin{array}{l}4.57 \\
0.08 \\
1.76 \\
6.60\end{array}$ & $\begin{array}{r}10.29 \\
0.14 \\
1.37 \\
5.50\end{array}$ & $\begin{array}{l}9.22 \\
0.14 \\
1.52 \\
*\end{array}$ & $\begin{array}{l}7.39 \\
0.10 \\
1.36 \\
.\end{array}$ & $\begin{array}{l}-- \\
m \\
--\end{array}$ & $\bar{z}$ & 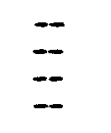 & $\begin{array}{l}= \\
=\end{array}$ & $=$ & $=-$ & $\begin{array}{r}34.23 \\
0.55\end{array}$ \\
\hline \begin{aligned} NOTES & \\
\hdashline$: & 0 \\
*: & 2 \\
A: & A \\
B: & L \\
C: & P\end{aligned}$ & $\begin{array}{l}\text { ATA NOT AVAILABLE } \\
\text { ERO DR TRACE } \\
\text { PPROXIMATE } \\
\text { OWER LIMI T OF REPOR } \\
\text { ROPORTI ONED FROM OR }\end{array}$ & DATA & & & & & & & & & & & & \\
\hline
\end{tabular}




\begin{tabular}{|c|c|c|c|c|c|c|c|c|c|c|c|c|c|c|c|}
\hline & & & JAN. & FEB. & MAR. & APR. & mar & JUNE & Jut $y$ & AU6. & SEP. & OCT. & NOV. & OEC. & CUM. TOYAL \\
\hline & 1959 & $\begin{array}{l}\text { PRECIP. (CM.) } \\
\text { SR-90 (KCI/SQ.KM.) } \\
\text { SR-90 CONC. (PC/L) } \\
\text { SR-89/SR-90 }\end{array}$ & $=$ & $\begin{array}{r}28.78 \\
0.08 \\
0.28 \\
1.80\end{array}$ & $\begin{array}{l}4.01 \\
=- \\
=\end{array}$ & $\begin{array}{l}19.71 \\
2.14 \\
10.86 \\
11.00\end{array}$ & $\begin{array}{r}15.75 \\
2.56 \\
16.26 \\
6.20\end{array}$ & $\begin{array}{l}7.06 \\
0.17 \\
2.41 \\
5.0 \mathrm{C}\end{array}$ & $\begin{array}{l}24.56 \\
=- \\
=-\end{array}$ & $\begin{array}{l}21.46 \\
0.10 \\
0.47 \\
0.68\end{array}$ & $\begin{array}{l}12.09 \\
0.08 \\
0.67 \\
0.90\end{array}$ & $\begin{array}{l}14.63 \\
0.03 \\
0.21 \\
*\end{array}$ & $\begin{array}{l}4.83 \\
0.04 \\
0.83 \\
0.59\end{array}$ & $\begin{array}{l}10.57 \\
0.12 \\
1.14 \\
*\end{array}$ & $\begin{array}{r}163.45 \\
5.32\end{array}$ \\
\hline & 1960 & $\begin{array}{l}\text { PREC IP. (CM.) } \\
S R-90 \text { (MCI/SQ-KM.) } \\
S R-90 \text { CONC. }(P C / L) \\
S R-89 / S R-90\end{array}$ & $\begin{array}{l}8.20 \\
0.14 \\
1.71 \\
\end{array}$ & $\begin{array}{l}9.98 \\
0.32 \\
3.21 \\
-\end{array}$ & $\begin{array}{r}0.97 \\
0.15 \\
15.47 \\
-\end{array}$ & $\begin{array}{l}4.67 \\
0.23 \\
4.93 \\
-\end{array}$ & $\begin{array}{l}2.29 \\
0.00 \mathrm{C} \\
0.01 \\
-\end{array}$ & $\begin{array}{l}37.24 \\
0.09 C \\
0.25 \\
-.\end{array}$ & $\begin{array}{l}5.94 \\
0.04 C \\
0.68 \\
-\end{array}$ & $\begin{array}{l}18.85 \\
0.136 \\
0.69 \\
--\end{array}$ & $\begin{array}{l}1.55 \\
0.016 \\
0.65 \\
-\end{array}$ & $\begin{array}{c}18.59 \\
C .08 C \\
0.44 \\
-.\end{array}$ & $\begin{array}{l}9.37 \\
0.02 \mathrm{C} \\
0.22 \\
-.-\end{array}$ & $\begin{array}{l}22.78 \\
0.06 C \\
0.27 \\
=-\end{array}$ & $\begin{array}{r}140.43 \\
1.27\end{array}$ \\
\hline & 1961 & $\begin{array}{l}\text { PRECIP, (CM.) } \\
\text { SR-9C IRCI/SO-KM.) } \\
\text { SR-9D CCNC. (PC/L) } \\
\text { SR-89/SR-90 }\end{array}$ & $\begin{array}{l}11.28 \\
0.36 C \\
3.20 \\
--\end{array}$ & $\begin{array}{l}9.86 \\
0.31 \mathrm{C} \\
3.15 \\
--\end{array}$ & $\begin{array}{l}4.67 \\
0.116 \\
2.36 \\
-.\end{array}$ & $\begin{array}{l}6.15 \\
0.15 C \\
2.44 \\
-\end{array}$ & $\begin{array}{l}9.12 \\
0.05 C \\
0.55 \\
-.\end{array}$ & $\begin{array}{l}28.22 \\
0.15 C \\
0.54 \\
--\end{array}$ & $\begin{array}{c}25.58 \\
C .14 C \\
0.55 \\
-.0\end{array}$ & $\begin{array}{l}10.59 \\
0.06 \mathrm{C} \\
0.57 \\
-.\end{array}$ & $\begin{array}{r}20.04 \\
0.02 \\
0.10 \\
4.90\end{array}$ & $\begin{array}{r}0.13 \\
C .01 \\
7.70 \\
40.00\end{array}$ & $\begin{array}{r}25.91 \\
0.13 \\
0.51 \\
67.00\end{array}$ & $\begin{array}{r}0.41 \\
0.13 \\
1.55 \\
64.00\end{array}$ & $\begin{array}{r}159.96 \\
1.62\end{array}$ \\
\hline$>$ & 1962 & $\begin{array}{l}\text { PRECIP, (CM.) } \\
\text { SR-9O (KCI/SO,KM.) } \\
\text { SR-9O CONC. (PC/L) } \\
\text { SR-89/SR-9O }\end{array}$ & $\begin{array}{r}4.39 \\
0.37 \\
8.43 \\
40.00\end{array}$ & $\begin{array}{r}1.80 \\
0.19 \\
10.56 \\
26.00\end{array}$ & $\begin{array}{r}2.39 \\
0.33 \\
13.81 \\
18.00\end{array}$ & $\begin{array}{r}12.22 \\
0.02 \\
0.17 \\
16.00\end{array}$ & $\begin{array}{r}2.92 \\
0.16 \\
5.48 \\
16.00\end{array}$ & $\begin{array}{r}18.80 \\
0.82 \\
4.37 \\
16.00\end{array}$ & $\begin{array}{r}0.18 \\
0.08 \\
33.34 \\
24.00\end{array}$ & $\begin{array}{r}7.04 \\
0.22 \\
3.13 \\
12.00\end{array}$ & $\begin{array}{r}10.08 \\
0.15 \\
1.49 \\
14.00\end{array}$ & $\begin{array}{r}7.92 \\
1.25 \\
15.79 \\
36.00\end{array}$ & $\begin{array}{r}14.43 \\
0.48 \\
3.33 \\
76.00\end{array}$ & $\begin{array}{r}12.14 \\
0.40 \\
3.30 \\
46.00\end{array}$ & $\begin{array}{r}94.31 \\
4.45\end{array}$ \\
\hline f & 1963 & $\begin{array}{l}\text { PRECIP. (CM-) } \\
\text { SR-9O (RCI/SC.KM.) } \\
\text { SR-90 CONC. (PCIL) } \\
\text { SR-89/SR-90 }\end{array}$ & $\begin{array}{l}7.85 \\
0.29 \\
3.70 \\
8.00\end{array}$ & $\begin{array}{r}6.60 \\
0.64 \\
9.70 \\
30.00\end{array}$ & $\begin{array}{r}1.40 \\
0.31 \\
22.15 \\
16.00\end{array}$ & $\begin{array}{l}2.34 \\
0.01 \\
0.43 \\
*\end{array}$ & $\begin{array}{r}1.57 \\
0.26 \\
16.57 \\
8.20\end{array}$ & $\begin{array}{r}19.79 \\
2.75 \\
13.90 \\
0.90\end{array}$ & $\begin{array}{r}5.28 \\
0.22 \\
13.64 \\
1.00\end{array}$ & $\begin{array}{l}4.70 \\
0.30 \\
6.39 \\
.\end{array}$ & $\begin{array}{l}4.93 \\
0.20 \\
4.06\end{array}$ & $\begin{array}{r}0.76 \\
0.14 \\
18.43 \\
*\end{array}$ & $\begin{array}{r}14.53 \\
0.60 \\
4.13 \\
+\end{array}$ & $\begin{array}{r}12.27 \\
0.96 \\
7.83 \\
*\end{array}$ & $\begin{array}{r}82.02 \\
7.18\end{array}$ \\
\hline & 1964 & $\begin{array}{l}\text { PRECIP, (CM-) } \\
S R-90 \text { (MCI/SQ.KM.) } \\
S R-90 \text { CCNC. }(P C / L) \\
S R-89 / S R-90\end{array}$ & $\begin{array}{l}7.34 \\
0.36 \\
4.91 \\
*\end{array}$ & $\begin{array}{r}12.62 \\
1.40 \\
11.10 \\
0.20\end{array}$ & $\begin{array}{l}5.69 \\
0.54 \\
9.50 \\
*\end{array}$ & $\begin{array}{l}4.14 \\
0.38 \\
9.18 \\
0.22\end{array}$ & $\begin{array}{l}5.72 \\
0.54 \\
9.45 \\
+\end{array}$ & $\begin{array}{r}4.80 \\
0.49 \\
10.21 \\
-\end{array}$ & $\begin{array}{r}4.27 \\
1.76 \\
41.22 \\
--\end{array}$ & $\begin{array}{l}6.63 \\
0.21 \\
3.17 \\
-\end{array}$ & $\begin{array}{l}17.17 \\
0.09 \\
0.53 \\
--\end{array}$ & $\begin{array}{r}5.97 \\
0.16 \\
2.69 \\
10.00\end{array}$ & $\begin{array}{r}10.87 \\
0.25 \\
2.30 \\
-\end{array}$ & $\begin{array}{r}14.15 \\
0.24 \\
1.70 \\
-.\end{array}$ & $\begin{array}{r}99.37 \\
6.42\end{array}$ \\
\hline & 1965 & $\begin{array}{l}\text { PRECIP (CM-) } \\
S R-90 \text { IMCI/SQ.KM, } \\
S R-90 \text { CONC. }(P C / L) \\
\text { SR-89/SR-90 }\end{array}$ & $\begin{array}{l}4.75 \\
0.09 \\
1.90 \\
-.\end{array}$ & $\begin{array}{l}8.31 \\
* \\
=-\end{array}$ & $\begin{array}{r}2.06 \\
0.39 \\
18.94 \\
--\end{array}$ & $\begin{array}{r}2.41 \\
0.30 \\
12.45 \\
-\end{array}$ & $\begin{array}{l}16.59 \\
0.83 \\
5.01 \\
-\end{array}$ & $\begin{array}{l}7.77 \\
0.63 \\
8.11 \\
-.\end{array}$ & $\begin{array}{l}3.99 \\
C .16 \\
4.02 \\
-.\end{array}$ & $\begin{array}{l}5.82 \\
0.12 \\
2.07 \\
\end{array}$ & $\begin{array}{l}9.04 \\
0.12 \\
1.33 \\
-.\end{array}$ & $\begin{array}{l}7.65 \\
0.22 \\
2.81 \\
--\end{array}$ & $\begin{array}{l}12.24 \\
0.12 \\
0.99 \\
-.\end{array}$ & $\begin{array}{l}15.62 \\
0.15 \\
0.97 \\
-.\end{array}$ & $\begin{array}{r}96.45 \\
3.13\end{array}$ \\
\hline & 1966 & 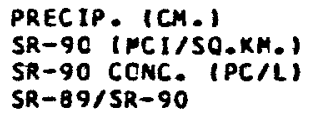 & $\begin{array}{l}11.32 \\
0.09 \\
0.80 \\
--\end{array}$ & $\begin{array}{l}19.68 \\
0.26 \\
1.33 \\
--\end{array}$ & $\begin{array}{l}5.59 \\
0.16 \\
2.87 \\
\end{array}$ & $\begin{array}{r}20.27 \\
0.30 \\
1.49 \\
-\end{array}$ & $\begin{array}{l}28.47 \\
0.12 \\
0.43 \\
\end{array}$ & $\begin{array}{r}11.23 \\
0.37 \\
3.30 \\
5.00\end{array}$ & $\begin{array}{l}3.68 \\
0.07 \\
1.91 \\
5.10\end{array}$ & $\begin{array}{l}18.06 \\
0.08 \\
0.45 \\
*\end{array}$ & $\begin{array}{l}10.18 \\
0.05 \\
0.50 \\
*\end{array}$ & $\begin{array}{l}13.84 \\
0.04 \\
0.29 \\
*\end{array}$ & $\begin{array}{l}3.96 \\
0.04 \\
1.02 \\
9.90\end{array}$ & $\begin{array}{l}3.87 \\
0.04 \\
1.04 \\
*\end{array}$ & $\begin{array}{r}150.15 \\
1.62\end{array}$ \\
\hline & 1967 & 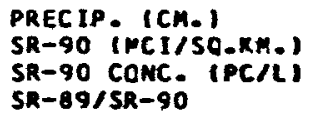 & $\begin{array}{l}6.12 \\
0.04 \\
0.66 \\
0.80\end{array}$ & $\begin{array}{r}5.51 \\
0.02 \\
0.37 \\
30.80\end{array}$ & $\begin{array}{l}4.65 \\
0.04 \\
0.87 \\
7.70\end{array}$ & $\begin{array}{r}11.23 \\
0.07 \\
0.63 \\
6.30\end{array}$ & $\begin{array}{l}0.45 \\
0.04 \\
0.63 \\
*\end{array}$ & $\begin{array}{l}0.43 \\
0.01 \\
2.33 \\
*\end{array}$ & $\begin{array}{l}- \\
- \\
-\end{array}$ & $=$ & $=$ & $=$ & $=$ & $=$ & $\begin{array}{r}34.39 \\
0.22\end{array}$ \\
\hline & \multicolumn{15}{|c|}{\begin{aligned} & NOTES \\
& \hdashline$-: D A \\
& *: 2 E \\
& A: A P \\
& B: L D \\
& C: P R\end{aligned}$} \\
\hline
\end{tabular}


MONTHLY FALLOUT OEPOSITION COLLEETIONS

SITE: TEXAS, HOUSTON

LAT. 29 45N LONG. $9525 \mathrm{~N}$ ALT.

12M. (POT)

SOURCE: TRACERLAB, INC.

COLLECTIONS DISCONTINUED IN JULY 1963

\begin{tabular}{|c|c|c|c|c|c|c|c|c|c|c|c|c|c|c|}
\hline & & JAN. & FEB. & MAR . & APR : & maY & JUNE & JULY & AUG. & SEP. & OCT. & NOY. & DEC. & CUM. TOTAL \\
\hline 1958 & $\begin{array}{l}\text { PRECIP. (CM.) } \\
\text { SR-90 (NCI/SO.KM.) } \\
\text { SR-90 CONC. (PC/L) } \\
\text { SR-89/SR-90 }\end{array}$ & $=$ & $\begin{array}{l}-- \\
-- \\
-\end{array}$ & $\begin{array}{l}-- \\
-- \\
--\end{array}$ & $\begin{array}{l}-- \\
-- \\
-\end{array}$ & $\begin{array}{r}3.94 \\
0.15 \\
3.81 \\
10.00\end{array}$ & $\begin{array}{r}5.33 \\
0.27 \\
5.07 \\
23.0 \mathrm{C}\end{array}$ & $\begin{array}{r}4.93 \\
0.21 \\
4.26 \\
66.00\end{array}$ & $\begin{array}{r}16.36 \\
0.24 \\
1.47 \\
57.00\end{array}$ & $\begin{array}{r}22.61 \\
0.20 \\
0.89 \\
30.00\end{array}$ & $\begin{array}{r}14.99 \\
0.25 \\
1.67 \\
34.00\end{array}$ & $\begin{array}{r}4.19 \\
0.10 \\
2.39 \\
32.00\end{array}$ & $\begin{array}{r}1.37 \\
0.19 \\
13.87 \\
41.00\end{array}$ & $\begin{array}{r}73.72 \\
1.61\end{array}$ \\
\hline 1959 & $\begin{array}{l}\text { PRECIP: (CM) } \\
\text { SR-9O INCI/SQ-KM) } \\
\text { SR-9OCCNC. (PC/L) } \\
\text { SR-89/SR-90 }\end{array}$ & $\begin{array}{r}14.17 \\
0.47 \\
3.32 \\
34.00\end{array}$ & $\begin{array}{r}15.52 \\
0.71 \\
4.58 \\
22.00\end{array}$ & $\begin{array}{r}2.13 \\
0.55 \\
25.83 \\
17.00\end{array}$ & $\begin{array}{r}17.58 \\
1.58 \\
8.99 \\
11.00\end{array}$ & $\begin{array}{r}15.65 \\
2.32 \\
12.18 \\
5.70\end{array}$ & $\begin{array}{r}16.56 \\
0.37 \\
2.24 \\
5.20\end{array}$ & $\begin{array}{r}20.75 \\
0.34 \\
1.64 \\
3.50\end{array}$ & $\begin{array}{r}17.75 \\
0.13 \\
0.74 \\
1.80\end{array}$ & $\begin{array}{r}10.77 \\
0.15 \\
1.40 \\
1.20\end{array}$ & $\begin{array}{r}12.42 \\
0.23 \\
1.86 \\
c .90\end{array}$ & $\begin{array}{l}3.68 \\
0.02 \\
0.55 \\
3.00\end{array}$ & $\begin{array}{l}14.45 \\
0.08 \\
0.56 \\
0.90\end{array}$ & $\begin{array}{r}164.83 \\
6.95\end{array}$ \\
\hline 1960 & $\begin{array}{l}\text { PRECIP. (CM.) } \\
\text { SR-9O (MCI/SO.KM.) } \\
\text { SR-9O CONC. (PC/L) } \\
\text { SR-89/SR-90 }\end{array}$ & $\begin{array}{l}4.95 \\
0.06 \\
1.22 \\
0.50\end{array}$ & $\begin{array}{r}10.13 \\
0.24 \\
2.37 \\
3.44\end{array}$ & $\begin{array}{l}m \\
--\end{array}$ & $\begin{array}{l}2.16 \\
0.10 \\
4.63 \\
2.30\end{array}$ & $\begin{array}{l}2.24 \\
0.03 \\
1.34 \\
0.30\end{array}$ & $\begin{array}{r}36.53 \\
0.22 \\
0.61 \\
0.10\end{array}$ & $\begin{array}{l}13.92 \\
0.04 \\
C .29 \\
--\end{array}$ & $\begin{array}{l}18.85 \\
0.12 \\
0.64 \\
--\end{array}$ & $\begin{array}{l}4.72 \\
0.03 \\
0.64 \\
-.\end{array}$ & $\begin{array}{l}27.56 \\
0.09 \\
0.33 \\
--\end{array}$ & $\begin{array}{l}12.01 \\
0.04 \\
0.34 \\
--\end{array}$ & $\begin{array}{l}17.07 \\
0.07 \\
0.42 \\
-.\end{array}$ & $\begin{array}{r}150.14 \\
1.04\end{array}$ \\
\hline 1961 & $\begin{array}{l}\text { PRECIP. (CM.) } \\
\text { SR-90 (NCI/SO.KM.) } \\
\text { SR-90 CONC. (PC/L) } \\
S R-89 / S R-90\end{array}$ & $\begin{array}{r}11.28 \\
0.10 \\
0.89 \\
--\end{array}$ & $\begin{array}{l}16.41 \\
0.18 \\
1.10 \\
--\end{array}$ & $\begin{array}{l}4.67 \\
0.09 \\
1.93 \\
-.\end{array}$ & $\begin{array}{l}0.15 \\
0.26 \\
4.23 \\
-.\end{array}$ & $\begin{array}{l}9.12 \\
0.06 \\
0.66 \\
0 .\end{array}$ & $\begin{array}{l}21.77 \\
0.16 \\
0.74 \\
--\end{array}$ & $\begin{array}{l}21.87 \\
0.10 \\
0.46 \\
-.\end{array}$ & $\begin{array}{l}8.20 \\
0.08 \\
0.98 \\
--\end{array}$ & $\begin{array}{r}20.04 \\
0.03 \\
0.15 \\
7.00\end{array}$ & $\begin{array}{r}0.53 \\
0.01 \\
1.89 \\
58.00\end{array}$ & $\begin{array}{r}14.48 \\
0.15 \\
1.04 \\
54.00\end{array}$ & $\begin{array}{r}2.24 \\
0.14 \\
6.26 \\
48.00\end{array}$ & $\begin{array}{r}136.76 \\
1.36\end{array}$ \\
\hline 1962 & $\begin{array}{l}\text { PRECIP }(C M-) \\
\text { SR-9C }\left(N C I / S Q_{-K M}\right) \\
S R-9 C \text { CONC. }(P C / L) \\
S R-89 / S R-90\end{array}$ & $\begin{array}{r}3.18 \\
0.31 \\
9.75 \\
59.00\end{array}$ & $\begin{array}{r}1.52 \\
0.26 \\
17.11 \\
27.00\end{array}$ & $\begin{array}{r}1.52 \\
0.39 \\
25.66 \\
18.00\end{array}$ & $\begin{array}{l}12.22 \\
1.86 \\
15.23 \\
13.00\end{array}$ & $\begin{array}{r}2.92 \\
0.17 \\
5.83 \\
20.00\end{array}$ & $\begin{array}{r}18.80 \\
1.59 \\
8.46 \\
8.70\end{array}$ & $\begin{array}{l}3.53 C \\
C .26 C \\
7.37 \\
--\end{array}$ & $\begin{array}{l}3.53 C \\
0.26 C \\
7.37 \\
-\end{array}$ & $\begin{array}{r}10.08 \\
0.2 C \\
1.99 \\
8.00\end{array}$ & $\begin{array}{r}7.92 \\
c .45 \\
5.69 \\
22.00\end{array}$ & $\begin{array}{r}14.43 \\
0.95 \\
6.59 \\
79.00\end{array}$ & $\begin{array}{r}12.01 \\
0.72 \\
6.00 \\
30.00\end{array}$ & $\begin{array}{r}91.66 \\
7.42\end{array}$ \\
\hline 1963 & $\begin{array}{l}\text { PRECIP. (CM.) } \\
\text { SR-90 (MCI/SQ.KM.) } \\
\text { SR-90 CONC. (PCAL) } \\
\text { SR-QS/SR-SO }\end{array}$ & $\begin{array}{r}7.85 \\
0.95 \\
12.11 \\
31.00\end{array}$ & $\begin{array}{r}4.45 \\
1.11 \\
24.95 \\
22.00\end{array}$ & $\begin{array}{r}1.40 \\
0.38 \\
27.15 \\
8.90\end{array}$ & $\begin{array}{r}2.34 \\
0.83 \\
35.48 \\
5.80\end{array}$ & $\begin{array}{r}1.57 \\
0.97 \\
61.79 \\
6.60\end{array}$ & $\begin{array}{l}\overline{2.17} \\
\overline{0.20}\end{array}$ & $\bar{z}=$ & $=$ & $=$ & $=$ & $m$ & $=$ & $\begin{array}{r}17.61 \\
6.41\end{array}$ \\
\hline $\begin{array}{cc}\text { NOTES } \\
--: & D \\
*: & Z \\
A: & A \\
B: & L \\
C: & P\end{array}$ & $\begin{array}{l}\text { ATA NOT AVAILABLE } \\
\text { ERO OR TRACE } \\
\text { PPROXIMATE } \\
\text { OHER LIMIT OF REPOR } \\
\text { ROPORTIONED FROM OR }\end{array}$ & $\begin{array}{l}\text { DATA } \\
\text { VALLY }\end{array}$ & & & & & & & & & & & & \\
\hline
\end{tabular}


MONTHLY FALLOUT DEPOS ITION COLLECTIONS

SITE: TEXAS, HOUSTON

LAT. 29 45N LONG. $9525 \mathrm{H}$ ALT.

12M. (COLUMN)

SOURCE: TRACERLAB, INC.

COLLECTIONS DISCONTINUED IN JULY 1963

\begin{tabular}{|c|c|c|c|c|c|c|c|c|c|c|c|c|c|c|}
\hline & & JAN. & FEB. & MAR: & APR: & MAY & JUNE & JULY & AUG. & SEP. & OCT: & NOV. & DEC. & CUM. rOTAL \\
\hline 1961 & $\begin{array}{l}\text { PRECIP. (CM.) } \\
\text { SR-9O }\left(M C I / S Q_{-} M_{0}\right) \\
\text { SR-9C CCNC. }(P C / L) \\
\text { SR-89/SR-90 }\end{array}$ & $\begin{array}{l}-- \\
-- \\
--\end{array}$ & $\begin{array}{l}=- \\
=\end{array}$ & $\bar{z}$ & $\begin{array}{l}\overline{-} \\
\overline{-}\end{array}$ & $=$ & $\begin{array}{l}-- \\
-- \\
--\end{array}$ & $\begin{array}{r}21.67 \\
0.03 \\
0.14 \\
-\end{array}$ & $\begin{array}{l}8.20 \\
0.07 \\
0.86 \\
-.\end{array}$ & $\begin{array}{r}20.04 \\
0.01 \\
0.05 \\
12.00\end{array}$ & $\begin{array}{r}0.53 \\
0.01 \\
1.89 \\
63.00\end{array}$ & $\begin{array}{r}14.48 \\
0.12 \\
0.83 \\
75.05\end{array}$ & $\begin{array}{r}2.24 \\
0.12 \\
5.36 \\
75.00\end{array}$ & $\begin{array}{r}67.36 \\
0.36\end{array}$ \\
\hline 1962 & $\begin{array}{l}\text { PRECIP. }(C M-) \\
\text { SR-90 IPCI/SQ.KM }) \\
\text { SR-9C CCNC. }(P C / L) \\
\text { SR-89/SR-90 }\end{array}$ & $\begin{array}{r}3.18 \\
0.45 \\
14.16 \\
40.00\end{array}$ & $\begin{array}{r}1.52 \\
0.39 \\
25.66 \\
28.00\end{array}$ & $\begin{array}{r}1.52 \\
0.70 \\
46.06 \\
19.00\end{array}$ & $\begin{array}{r}12.22 \\
1.90 \\
15.55 \\
14.00\end{array}$ & $\begin{array}{r}2.92 \\
0.18 \\
6.17 \\
17.00\end{array}$ & $\begin{array}{r}18.80 \\
1.04 \\
5.54 \\
8.50\end{array}$ & $\begin{array}{r}0.18 \\
0.16 \\
88.89 \\
13.00\end{array}$ & $\begin{array}{l}6.86 \\
0.34 \\
4.96 \\
9.20\end{array}$ & $\begin{array}{r}10.08 \\
0.10 \\
1.00 \\
9.00\end{array}$ & $\begin{array}{r}7.92 \\
0.48 \\
6.07 \\
23.00\end{array}$ & $\begin{array}{r}14.43 \\
0.63 \\
4.37 \\
0.66\end{array}$ & $\begin{array}{r}12.01 \\
0.64 \\
5.33 \\
40.00\end{array}$ & $\begin{array}{r}91.64 \\
7.01\end{array}$ \\
\hline 1963 & $\begin{array}{l}\text { PRECIP. (CM.) } \\
\text { SR-90 (KCI/SQ.KM.) } \\
\text { SR-90 CONC. (PC/L) } \\
\text { SR-89/SR-90 }\end{array}$ & $\begin{array}{r}7.85 \\
0.49 \\
6.25 \\
30.00\end{array}$ & $\begin{array}{r}4.45 \\
0.90 \\
20.23 \\
26.00\end{array}$ & $\begin{array}{r}1.40 \\
0.24 \\
17.15 \\
21.00\end{array}$ & $\begin{array}{r}2.34 \\
0.32 \\
13.68 \\
12.00\end{array}$ & $\begin{array}{r}1.57 \\
0.93 \\
59.24 \\
8.40\end{array}$ & $\begin{array}{l}-- \\
1.91 \\
0.70\end{array}$ & $=$ & $=$ & $\begin{array}{l}= \\
=- \\
--\end{array}$ & $\begin{array}{l}=- \\
=- \\
--\end{array}$ & $\begin{array}{l}= \\
=- \\
=-\end{array}$ & $\begin{array}{l}= \\
=- \\
--\end{array}$ & $\begin{array}{r}17.61 \\
4.79\end{array}$ \\
\hline
\end{tabular}

NOTES

--: data not availlable

*: 2ERO DR TRACE

A: APPROXIMATE

8: LOWER LIMIT OF REPORTEO DATA

C: PROPORTIOAED FROM ORIGINALLY CONSOLICATEO DATA 


\begin{tabular}{|c|c|c|c|c|c|c|c|c|c|c|c|c|c|c|}
\hline & & JAM: & EEB. & MAR: & APR: & mar & JUNE & JULY & AUG. & SEP: & oct: & Nov. & OEC. & CLM. TOTAL \\
\hline 1956 & 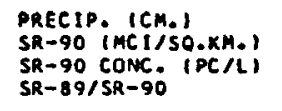 & $\ddot{z}$ & $\begin{array}{l}\ddot{z} \\
\bar{z}\end{array}$ & $\bar{z}$ & $\begin{array}{l}= \\
=\end{array}$ & $=$ & $=$ & $=$ & $\bar{z}$ & $\ddot{z}$ & $=$ & $=$ & $\begin{array}{l}4.24 \\
0.12 \\
2.84 \\
--\end{array}$ & $\begin{array}{l}6.24 \\
0.12\end{array}$ \\
\hline 1957 & $\begin{array}{l}\text { PRECIP. (CM.) } \\
\text { SR-90 iMCI/SO.KM.) } \\
S R-90 \text { CCNC. }(P C / L) \\
S R-O 9 / S R-9 C\end{array}$ & $\begin{array}{r}3.48 \\
0.31 \\
8.91 \\
16.00\end{array}$ & $\begin{aligned} 1.83 \\
0.32 \\
17.49 \\
16.00\end{aligned}$ & $\begin{array}{r}5.54 \\
0.92 \\
16.62 \\
9.30\end{array}$ & $\begin{array}{r}8.23 \\
8.89 \\
10.82 \\
-6\end{array}$ & $\begin{array}{l}8.56 \\
0.31 \\
3.63 \\
1.30\end{array}$ & $\begin{array}{r}3.73 \\
0.62 \\
16.63 \\
24.00\end{array}$ & $\begin{array}{r}0.79 \\
8.36 \\
45.57 \\
-0 .\end{array}$ & $\begin{array}{r}4.29 \\
0.49 \\
11.43 \\
-1\end{array}$ & $\begin{aligned} 0.84 \\
0.06 \\
77.15 \\
40.00\end{aligned}$ & $\begin{array}{r}1.98 \\
0.23 \\
11.62 \\
49.00\end{array}$ & $\begin{array}{r}3.48 \\
0.16 \\
4.60 \\
25.00\end{array}$ & $\begin{array}{l}4.09 \\
0.25 \\
6.12 \\
12.00\end{array}$ & $\begin{array}{r}46.84 \\
4.92\end{array}$ \\
\hline 1958 & 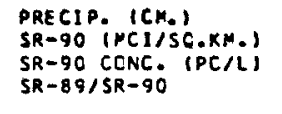 & $\begin{array}{r}2.21 \\
0.27 \\
12.22 \\
15.00\end{array}$ & $\begin{aligned} 5.59 \\
0.42 \\
7.52 \\
13.00\end{aligned}$ & $\begin{array}{r}5.56 \\
0.57 \\
10.26 \\
32.00\end{array}$ & $\begin{aligned} 7.42 \\
0.81 \\
10.92 \\
13.00\end{aligned}$ & $\begin{array}{r}0.76 \\
0.50 \\
65.79 \\
12.00\end{array}$ & $\begin{array}{r}0.10 \\
0.11 \\
110.00 \\
12.00\end{array}$ & $\begin{array}{r}0.13 \\
0.02 \\
15.39 \\
107.60\end{array}$ & $\begin{array}{l}0.58 \\
0.27 \\
0.27 \\
46.56 \\
44.00\end{array}$ & $\begin{array}{l}0.64 \\
\because- \\
--\end{array}$ & $\begin{array}{l}* .04 \\
47.00\end{array}$ & $\begin{array}{r}2.87 \\
0.10 \\
3.49 \\
16.00\end{array}$ & $\begin{array}{l}1.37 \\
1.19 \\
86.87 \\
43.00\end{array}$ & $\begin{array}{r}27.23 \\
4.30\end{array}$ \\
\hline 1959 & 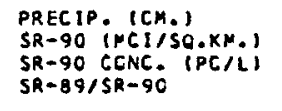 & $\begin{array}{r}4.00 \\
0.95 \\
23.40 \\
-.\end{array}$ & $\begin{array}{r}3.58 \\
2099 \\
27.66 \\
27.00\end{array}$ & $\begin{aligned} 2.01 \\
1.76 \\
87.57 \\
18.00\end{aligned}$ & $\begin{array}{r}4.09 \\
2.25 \\
55.02 \\
10.00\end{array}$ & $\begin{array}{l}5.21 \\
2.30 \\
4.35 \\
10.00\end{array}$ & $\begin{array}{r}3.51 \\
0.93 \\
26.56 \\
5.86\end{array}$ & $\begin{array}{r}0.48 \\
0.20 \\
41.87 \\
3.00\end{array}$ & $\begin{array}{l}4.47 \\
\because- \\
--\end{array}$ & $\begin{array}{l}4.22 \\
8.27 \\
60.40 \\
1.30\end{array}$ & $\begin{array}{r}0.56 \\
6.10 \\
17.86 \\
c .40\end{array}$ & $\begin{array}{r}0.25 \\
0.36 \\
14400 \\
0.11\end{array}$ & $\begin{array}{l}2.67 \\
0.10 \\
3.75 \\
=\end{array}$ & $\begin{array}{l}35.11 \\
10.21\end{array}$ \\
\hline 1980 & $\begin{array}{l}\text { PREC IP. (CM.) } \\
\text { SR-9C irC1/SQ.KM.) } \\
\text { SR-9C CONC. (PC/L) } \\
S R-99 / S R \rightarrow 90\end{array}$ & $\begin{array}{l}2.44 \\
0.07 \\
2.87 \\
--\end{array}$ & $\begin{array}{l}4.01 \\
0.15 \\
3.75 \\
-7\end{array}$ & $\begin{array}{l}6.22 \\
0.42 \\
0.76 \\
-.\end{array}$ & $\begin{array}{r}2.82 \\
0.36 \\
12.77\end{array}$ & $\begin{array}{l}2.02 \\
0.022 \\
1.00\end{array}$ & $\begin{array}{l}0.99 \\
0.016 \\
1.02 \\
0-1\end{array}$ & $\begin{array}{l}0.05 \\
0.000 \\
.0 .01 \\
-0\end{array}$ & $\begin{array}{l}3.00 \\
0.060 \\
2.01\end{array}$ & $\begin{array}{l}0.99 \\
0.000 \\
c .01 \\
-0\end{array}$ & $\begin{array}{l}3.00 \\
0.016 \\
0.34 \\
-\end{array}$ & $\begin{array}{l}3.99 \\
0.12 \\
3.01 \\
--\end{array}$ & $\begin{array}{l}0.99 \\
\because- \\
--\end{array}$ & $\begin{array}{r}30.51 \\
1.22\end{array}$ \\
\hline 1561 & $\begin{array}{l}\text { PRECIP. }\left(C M_{-}\right) \\
\text {SR-SC } \text { irCIISO-KM-) } \\
\text { SRR-90 CONC. (PC/L) } \\
\text { SR-S9/SR-90 }\end{array}$ & $\begin{array}{l}\text { c. } 18 \\
\text { c.c1c } \\
5.96 \\
-9\end{array}$ & $\begin{array}{l}5.21 \\
0.336 \\
6.34\end{array}$ & $\begin{array}{l}4.70 \\
0.296 \\
6.18\end{array}$ & $\begin{array}{l}2.39 \\
0.15 \mathrm{C} \\
6.28 \\
--\end{array}$ & $\begin{array}{l}0.61 \\
0.026 \\
3.28 \\
--\end{array}$ & $\begin{array}{l}0.18 \\
0.016 \\
5.56 \\
--5\end{array}$ & $\begin{array}{l}1.40 \\
0.03 c \\
2.15 \\
--\end{array}$ & $\begin{array}{l}3.00 \\
0.006 \\
2.01 \\
--\end{array}$ & $\begin{array}{r}2.79 \\
0.08 \\
2.87 \\
12.00\end{array}$ & $\begin{aligned} 4.06 \\
6.44 \\
10.84 \\
82.00\end{aligned}$ & $\begin{array}{r}2.92 \\
0.25 \\
8.57 \\
71.00\end{array}$ & $\begin{array}{r}2.24 \\
0.89 \\
39.84 \\
56.00\end{array}$ & $\begin{array}{r}29.68 \\
2.56\end{array}$ \\
\hline 1962 & $\begin{array}{l}\text { PRECIP. ICM.) } \\
\text { SR-90 IYCI/SOKKM-1) } \\
S R-90 \text { CCNC. }(P C / L I \\
S R-89 / S R-90\end{array}$ & $\begin{array}{r}2.13 \\
0.45 \\
21.13 \\
40.00\end{array}$ & $\begin{aligned} 3.61 \\
1: 74 \\
48.20 \\
25.00\end{aligned}$ & $\begin{array}{r}5.89 \\
3.13 \\
36.17 \\
18.00\end{array}$ & $\begin{array}{r}7.59 \\
2.61 \\
33.69 \\
13.00\end{array}$ & $\begin{array}{r}5.38 \\
4.11 \\
76.40 \\
8.60\end{array}$ & $\begin{array}{l}1.22 \\
0.13 \\
10.66 \\
20.06\end{array}$ & $\begin{array}{r}6.40 \\
0.68 \\
16.63 \\
25.00 \\
-6\end{array}$ & $\begin{array}{l}0.66 \\
0.20 \\
30.31 \\
14.00\end{array}$ & $\begin{array}{r}2.54 \\
0.19 \\
77.49 \\
27.00\end{array}$ & $\begin{array}{r}2.36 \\
0.57 \\
24.16 \\
3.00\end{array}$ & $\begin{array}{r}1.42 \\
0.35 \\
24.65 \\
42.00\end{array}$ & $\begin{aligned} 0.41 \\
6.08 \\
19.52 \\
55.00\end{aligned}$ & $\begin{array}{l}39.61 \\
13.24\end{array}$ \\
\hline 1963 & $\begin{array}{l}\text { PREC IP. (CH.) } \\
\text { SR-90 irC1/SO.KM-) } \\
\text { SR-9C CCAC. (PCA } \\
\text { SR-89/SR-90 }\end{array}$ & $\begin{array}{l}1.35 \\
0.42 C \\
31.12 \\
--\end{array}$ & $\begin{array}{l}1.70 \\
c .426 \\
24.71 \\
--\end{array}$ & $\begin{array}{r}5.36 \\
15.35 \\
25.19 \\
18.00\end{array}$ & $\begin{array}{l}10.31 \\
75.82 \\
75.85 \\
10.00\end{array}$ & $\begin{array}{r}0.58 \\
6.80 \\
137.94 \\
5.60\end{array}$ & $\begin{array}{r}4.24 \\
4.78 \\
112.74 \\
5.90\end{array}$ & $\frac{0.18}{0.70}$ & $\begin{array}{r}1.37 \\
0.73 \\
53.29 \\
8.29\end{array}$ & $\begin{array}{l}2.74 \\
6.93 \\
33.95 \\
*\end{array}$ & $\begin{array}{r}2.67 \\
6.88 \\
32.96 \\
0.40\end{array}$ & $\begin{array}{r}3.96 \\
0.56 \\
14.15 \\
*\end{array}$ & $\begin{array}{r}2.13 \\
0.28 \\
13.15 \\
--\end{array}$ & $\begin{array}{l}36.41 \\
19.15\end{array}$ \\
\hline 1964 & $\begin{array}{l}\text { PRECIP. (CM.) } \\
\text { SR-SC iNCI/SQ.KM.) } \\
S R-90 \text { CCNC. (PC/L) } \\
S R \rightarrow B 9 / S R \rightarrow 90\end{array}$ & $\begin{array}{r}2.57 \\
0.84 \\
32.69 \\
*\end{array}$ & $\begin{aligned} 0.89 \\
6.41 \\
46.07 \\
0.50\end{aligned}$ & $\begin{array}{r}5.77 \\
1.78 \\
30.85 \\
? .85\end{array}$ & $\begin{array}{r}9.02 \\
5.70 \\
63.20 \\
\$\end{array}$ & $\begin{array}{r}4.85 \\
2.31 \\
47.63 \\
7.63\end{array}$ & $\begin{array}{l}6.63 \\
6958 \\
99.25 \\
--9\end{array}$ & $\begin{array}{l}c .20 \\
0.26 \\
0.26 \\
0 .-01\end{array}$ & $\begin{array}{r}0.89 \\
0.31 \\
34.84 \\
-0\end{array}$ & $\begin{array}{l}\because- \\
= \\
=\end{array}$ & $\begin{array}{l}3.40 \\
8.53 \\
37.86 \\
* *\end{array}$ & $\begin{array}{r}4.39 \\
0.05 \\
14.81 \\
1.20\end{array}$ & $\begin{array}{l}8.92 \\
0.14 \\
1.57 \\
-0\end{array}$ & $\begin{array}{r}45.53 \\
29.41\end{array}$ \\
\hline 1965 & $\begin{array}{l}\text { PREC IP. }\left(C M_{-}\right) \\
\text {SR-9O imCI/SO-KM-) } \\
\text { SR-9C CCNC.S(PC/L) } \\
S R-89 / S R-90\end{array}$ & $\begin{array}{l}5.72 \\
0.52 \\
9.10 \\
--\end{array}$ & $=$ & $\begin{aligned} 1.91 \\
0.32 \\
10.76 \\
-.-\end{aligned}$ & $\begin{array}{r}5.36 \\
0.78 \\
14.56 \\
-.-56\end{array}$ & $\begin{array}{r}5.13 \\
1.56 \\
36.41 \\
-.9\end{array}$ & $\begin{array}{l}4.75 \\
0.30 \\
6.32 \\
-9\end{array}$ & $\begin{array}{l}3.02 \\
0.18 \\
3.97 \\
-.9\end{array}$ & $\begin{array}{l}7.34 \\
0.32 \\
4.36 \\
-\end{array}$ & $\begin{array}{l}2.84 \\
0.27 \\
9.51 \\
0 .\end{array}$ & $\begin{array}{l}0.99 \\
0.08 \\
8.09 \\
--\end{array}$ & $\begin{array}{l}2.87 \\
0.13 \\
4.53 \\
-.\end{array}$ & $\begin{array}{l}4.64 \\
0.15 \\
3.24 \\
--\end{array}$ & $\begin{array}{l}44.57 \\
4.61\end{array}$ \\
\hline 1966 & 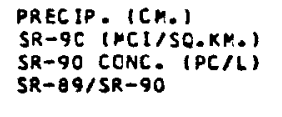 & $\begin{array}{r}0.56 \\
6.11 \\
19.65\end{array}$ & $\begin{array}{r}16.03 \\
0.22 \\
1.38\end{array}$ & $\begin{array}{l}2.02 \\
0.16 \\
7.97 \\
-9\end{array}$ & $\begin{array}{r}3.63 \\
0.39 \\
10.75 \\
--\end{array}$ & $\begin{array}{r}1.30 \\
6.16 \\
12.31\end{array}$ & $\begin{array}{l}0.18 \\
0.11 \\
01.12 \\
-0\end{array}$ & $\begin{array}{r}0.84 \\
0.09 \\
10.72 \\
-\end{array}$ & $\begin{array}{l}0.56 \\
0.05 \\
8.93 \\
--.\end{array}$ & $\begin{array}{l}2.12 \\
0.14 \\
5.15 \\
-15\end{array}$ & $\begin{array}{l}2.39 \\
0.06 \\
2.52 \\
--\end{array}$ & $\begin{array}{r}1.90 \\
0.07 \\
3.69 \\
16.00\end{array}$ & $\begin{array}{l}1.70 \\
0.00 \\
3.53 \\
.\end{array}$ & $\begin{array}{r}33.82 \\
1.62\end{array}$ \\
\hline 1967 & $\begin{array}{l}\text { PRECIP. (CM.) } \\
\text { SR-90 irCI/SOKM-) } \\
\text { SR-90 CONC. }(P C / L) \\
\text { SR-BQ/SR-90 }\end{array}$ & $\begin{array}{r}5.89 \\
0.28 \\
49.76 \\
27.00\end{array}$ & $\begin{array}{l}2.00 \\
0.13 \\
6.51 \\
9.80\end{array}$ & $\begin{array}{l}6.02 \\
0.206 \\
3.33 \\
8.20\end{array}$ & $\begin{array}{l}5.13 \\
0.200 \\
3.90 \\
8.20\end{array}$ & $\begin{array}{l}5.46 \\
0.22 \\
4.03 \\
3.40\end{array}$ & $\begin{array}{l}6.93 \\
0.22 \\
4.05 \\
*\end{array}$ & $\begin{array}{l}\bar{z} \\
\bar{z}\end{array}$ & $=$ & $\begin{array}{l}= \\
= \\
=\end{array}$ & $\begin{array}{l}= \\
= \\
=\end{array}$ & $=$ & $\begin{array}{l}= \\
=\end{array}$ & $\begin{array}{r}31.43 \\
1.31\end{array}$ \\
\hline
\end{tabular}


MONTHLY FALLOUT DEPOSITION COLLECTIONS

SITE: VIRGINIA, STERLING

LAT. $3859 \mathrm{~N}$ LONG. $7728 \mathrm{H}$ ALT.

82M. (COLUMN) SURFACE AIR SAMPLING STATION

SOURCE: U. S. WEATHER BUREAU, OBSERVATIONAL TEST aND DEVELOPMENT CENTER

\begin{tabular}{|c|c|c|c|c|c|c|c|c|c|c|c|c|c|c|}
\hline & & JAN. & FEB. & MAR. & $A P R$. & $\begin{array}{l}\text { MAY } \\
---\end{array}$ & JUNE & JULY & AUG. & SEP. & OCT. & NOV. & DEC. & CUM. TOTAL \\
\hline 1960 & $\begin{array}{l}\text { PRECIP. }\left(C M_{.}\right) \\
\text {SR-9O (MCI/SQ.KM.) } \\
S R-90 \text { CONC. (PC/L) } \\
S R-89 / S R-90\end{array}$ & $\begin{array}{l}-- \\
-- \\
--\end{array}$ & $\begin{array}{l}-- \\
-- \\
-\end{array}$ & $\begin{array}{l}-- \\
-- \\
--\end{array}$ & $=$ & $\begin{array}{l}-- \\
-- \\
--\end{array}$ & $\begin{array}{l}-- \\
-- \\
--\end{array}$ & $=$ & $\begin{array}{l}-- \\
-- \\
--\end{array}$ & $m-$ & $\begin{array}{l}3.68 \\
0.06 C \\
1.64 \\
-.\end{array}$ & $\begin{array}{l}4.85 \\
0.13 C \\
2.69 \\
-.\end{array}$ & $\begin{array}{l}4.52 \\
0.126 \\
2.66 \\
--\end{array}$ & $\begin{array}{r}13.05 \\
0.31\end{array}$ \\
\hline 1961 & $\begin{array}{l}\text { PRECIP. }(C M .) \\
S R-9 C \text { (NCI/SQ.KN.) } \\
S R-9 C \text { CCNC. }(P C / L) \\
S R-89 / S R-90\end{array}$ & $\begin{array}{l}8.13 \\
C . c 5 C \\
0.62 \\
--\end{array}$ & $\begin{array}{l}10.49 \\
C .07 C \\
0.67 \\
--\end{array}$ & $\begin{array}{l}10.29 \\
0.29 C \\
2.82 \\
-.\end{array}$ & $\begin{array}{l}8.18 \\
0.24 \mathrm{C} \\
2.94 \\
-\end{array}$ & $\begin{array}{l}8.89 \\
0.236 \\
2.59 \\
-\end{array}$ & $\begin{array}{l}8.79 \\
0.236 \\
2.62 \\
-.\end{array}$ & $\begin{array}{l}8.10 \\
0.096 \\
1.12 \\
-.\end{array}$ & $\begin{array}{l}9.04 \\
0.106 \\
1.11 \\
-=\end{array}$ & $\begin{array}{r}9.47 \\
0.04 \\
0.43 \\
20.00\end{array}$ & $\begin{array}{r}8.26 \\
0.12 \\
1.46 \\
90.00\end{array}$ & $\begin{array}{r}6.58 \\
0.30 \\
4.56 \\
57.00\end{array}$ & $\begin{array}{r}7.37 \\
0.32 \\
4.35 \\
48.00\end{array}$ & $\begin{array}{r}103.59 \\
2.08\end{array}$ \\
\hline 1962 & $\begin{array}{l}\text { PRECIP. (CM.) } \\
S R-9 C \text { (NCI/SQ.KM.) } \\
S R-S C \text { CCNC. (PC/L) } \\
S R-89 / S R-90\end{array}$ & $\begin{array}{r}4.85 \\
0.33 \\
6.81 \\
43.00\end{array}$ & $\begin{array}{r}9.91 \\
1.04 \\
10.50 \\
27.00\end{array}$ & $\begin{array}{r}15.01 \\
0.62 \\
4.14 \\
19.00\end{array}$ & $\begin{array}{r}6.15 \\
1.06 \\
17.24 \\
13.00\end{array}$ & $\begin{array}{r}10.26 \\
1.68 \\
16.38 \\
9.00\end{array}$ & $\begin{array}{r}10.46 \\
0.95 \\
9.09 \\
10.00\end{array}$ & $\begin{array}{r}3.48 \\
6.47 \\
13.51 \\
7.00\end{array}$ & $\begin{array}{r}0.43 \\
0.09 \\
26.94 \\
10.00\end{array}$ & $\begin{array}{r}5.77 \\
6.41 \\
7.11 \\
16.00\end{array}$ & $\begin{array}{r}3.43 \\
6.27 \\
7.88 \\
28.00\end{array}$ & $\begin{array}{r}12.75 \\
0.60 \\
4.71 \\
47.00\end{array}$ & $\begin{array}{r}7.39 \\
0.36 \\
4.88 \\
52.00\end{array}$ & $\begin{array}{r}89.99 \\
7.88\end{array}$ \\
\hline 1963 & $\begin{array}{l}\text { PRECIP. (CM.) } \\
\text { SR-9O I CCI/SQ.XM.) } \\
\text { SR-9OCCNC. (PC/L) } \\
\text { SR-89/SR-9O }\end{array}$ & $\begin{array}{r}3.61 \\
0.57 \\
15.79 \\
30.00\end{array}$ & $\begin{array}{r}4.14 \\
6.93 \\
22.47 \\
20.00\end{array}$ & $\begin{array}{r}12.42 \\
1.68 \\
13.53 \\
17.00\end{array}$ & $\begin{array}{r}2.69 \\
2.38 \\
88.48 \\
10.20\end{array}$ & $\begin{array}{r}3.94 \\
6.85 \\
21.58 \\
9.10\end{array}$ & $\begin{array}{r}15.32 \\
3.03 \\
19.78 \\
8.70\end{array}$ & $\begin{array}{r}2.51 \\
1.04 \\
41.44 \\
2.00\end{array}$ & $\begin{array}{r}13.13 \\
1.42 \\
10.82 \\
1.00\end{array}$ & $\begin{array}{l}7.80 \\
0.44 \\
5.65 \\
1.20\end{array}$ & $\begin{array}{r}0.30 \\
0.12 \\
40.01 \\
*\end{array}$ & $\begin{array}{l}17.86 \\
0.86 \\
4.82 \\
*\end{array}$ & $\begin{array}{l}6.93 \\
0.40 \\
5.78 \\
*\end{array}$ & $\begin{array}{l}90.65 \\
13.72\end{array}$ \\
\hline 1964 & 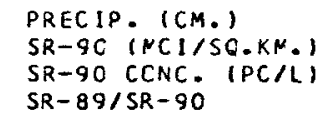 & $\begin{array}{l}8.51 \\
C .76 \\
8.94 \\
*\end{array}$ & $\begin{array}{r}6.63 \\
1.02 \\
15.39 \\
*\end{array}$ & $\begin{array}{r}5.77 \\
1.01 \\
17.51 \\
*\end{array}$ & $\begin{array}{r}9.45 \\
1.89 \\
20.00 \\
.\end{array}$ & $\begin{array}{r}1.63 \\
C .71 \\
43.56\end{array}$ & $\begin{array}{l}2.77 \\
0.81 \\
29.25 \\
-.\end{array}$ & $\begin{array}{r}5.51 \\
C .76 \\
13.80 \\
--\end{array}$ & $\begin{array}{l}7.21 \\
0.33 \\
4.58 \\
--\end{array}$ & $\begin{array}{l}8.86 \\
0.22 \\
2.49 \\
--\end{array}$ & $\begin{array}{l}4.32 \\
0.17 \\
3.94 \\
1.90\end{array}$ & $\begin{array}{l}4.34 \\
0.10 \\
2.31 \\
0.80\end{array}$ & $\begin{array}{l}10.08 \\
0.32 \\
3.18 \\
--\end{array}$ & $\begin{array}{r}75.08 \\
3.10\end{array}$ \\
\hline 1965 & $\begin{array}{l}\text { PRECIP. (CM-) } \\
\text { SR-90 (KCI/SQ.KM-) } \\
\text { SR-90 CONC. }(P C / L) \\
\text { SR-89/SR-SO }\end{array}$ & $\begin{array}{l}7.62 \\
C .20 \\
2.63 \\
-.\end{array}$ & $\begin{array}{l}7.29 \\
0.29 \\
3.98 \\
--\end{array}$ & $\begin{array}{l}10.87 \\
0.51 \\
4.70 \\
--\end{array}$ & $\begin{array}{r}6.22 \\
0.73 \\
11.74 \\
*\end{array}$ & $\begin{array}{r}3.33 \\
0.61 \\
18.32 \\
--\end{array}$ & $\begin{array}{r}4.95 \\
0.59 \\
11.92 \\
-.\end{array}$ & $\begin{array}{l}9.32 \\
0.68 \\
7.30 \\
--\end{array}$ & $\begin{array}{l}9.55 \\
0.24 \\
2.52 \\
--\end{array}$ & $\begin{array}{l}5.56 \\
0.10 \\
1.80 \\
--\end{array}$ & $\begin{array}{l}5.46 \\
0.08 \\
1.47 \\
--\end{array}$ & $\begin{array}{l}1.24 \\
0.10 \\
8 . C 7 \\
--\end{array}$ & $\begin{array}{l}1.14 \\
0.08 \\
7.02 \\
--\end{array}$ & $\begin{array}{r}72.55 \\
4.21\end{array}$ \\
\hline 1966 & $\begin{array}{l}\text { PRECIP. (CM.) } \\
S R-90 \text { (NCI/SQ.KM }) \\
S R-9 C \text { CCNC. }(P C / L) \\
S R-89 / S R-90\end{array}$ & $\begin{array}{l}10.72 \\
0.08 \\
0.75 \\
-.\end{array}$ & $\begin{array}{l}8.99 \\
0.18 \\
2.01 \\
--\end{array}$ & $\begin{array}{l}5.16 \\
0.18 \\
3.49 \\
-\end{array}$ & $\begin{array}{r}10.72 \\
0.29 \\
2.71 \\
--\end{array}$ & $\begin{array}{r}12.50 \\
0.25 \\
2.01 \\
--\end{array}$ & $\begin{array}{l}2.06 \\
0.06 \\
2.92 \\
9.0 C\end{array}$ & $\begin{array}{l}3.86 \\
0.09 \\
2.34 \\
\end{array}$ & $\begin{array}{l}4.34 \\
0.04 \\
0.93 \\
*\end{array}$ & $\begin{array}{l}23.11 \\
c .10 \\
0.44 \\
*\end{array}$ & $\begin{array}{l}7.98 \\
C .04 \\
0.51 \\
*\end{array}$ & $\begin{array}{l}3.81 \\
0.04 \\
1.05 \\
4.30\end{array}$ & $\begin{array}{l}0.07 \\
0.04 \\
0.66 \\
*\end{array}$ & $\begin{array}{r}99.32 \\
1.39\end{array}$ \\
\hline 1967 & $\begin{array}{l}\text { PRECIP. (CM.) } \\
\text { SR-9O IMCI/SQ.KM.) } \\
\text { SR-9C CCNC. (PC/L) } \\
S R-89 / S R-90\end{array}$ & $\begin{array}{l}3.48 \\
0.05 \\
1.44 \\
8.00\end{array}$ & $\begin{array}{r}3.56 \\
0.04 \\
1.13 \\
10.60\end{array}$ & $\begin{array}{r}12.37 \\
0.12 \\
0.98 \\
9.10\end{array}$ & $\begin{array}{l}2.54 \\
0.06 \\
2.37 \\
4.80\end{array}$ & $\begin{array}{r}10.92 \\
0.18 \\
1.65 \\
3.80\end{array}$ & $\begin{array}{l}5.72 \\
0.05 \\
C .88 \\
*\end{array}$ & $\begin{array}{l}8.18 \\
0.11 \\
1.35 \\
*\end{array}$ & $\overline{-}$ & $\begin{array}{l}-- \\
--\end{array}$ & $=$ & $\begin{array}{l}-- \\
-- \\
--\end{array}$ & $\overline{--}$ & $\begin{array}{r}46.77 \\
0.61\end{array}$ \\
\hline \begin{tabular}{c} 
NOTES \\
\hdashline$-:$ D \\
$*:$ Z \\
A: A \\
$B:$ L \\
$C:$
\end{tabular} & $\begin{array}{l}\text { DATA NOT AVAILABLE } \\
\text { LERO OR TRACE } \\
\text { APPROXIMATE } \\
\text { LOWER LIMIT OF REPOR } \\
\text { PROPORTI ONED FROM OR }\end{array}$ & DATA & & & & & & & & & & & & \\
\hline
\end{tabular}


SOURCE: U. S. WEATHER BUREAU AT QUILLAYUTE AIRPORT

(SAMPLER LOCATED ON TATOOSH ISLAND UNT IL AUGUST 1966)

\begin{tabular}{|c|c|c|c|c|c|c|c|c|c|c|c|c|c|c|}
\hline & & JAN. & FEB. & MAR. & APR: & MAY & JUNE & JULY & AUG. & SEP. & oct. & NOV. & DEC. & CUM. TOTAL \\
\hline 1959 & $\begin{array}{l}\text { PRECIP. (CM.) } \\
\text { SR-90 (NCI/SO.KM ) } \\
\text { SR-90 CCNC. (PC/L) } \\
\text { SR-89/SR-90 }\end{array}$ & $\begin{array}{l}30.68 \\
=- \\
=-\end{array}$ & $\begin{array}{l}19.33 \\
=- \\
=-\end{array}$ & $\begin{array}{l}26.67 \\
=- \\
=-\end{array}$ & $\begin{array}{l}20.73 \\
=- \\
=-\end{array}$ & $\begin{array}{l}5.99 \\
=- \\
=-\end{array}$ & $\begin{array}{l}1.06 \\
\therefore \\
\therefore\end{array}$ & $\begin{array}{l}3.56 \\
\pm \\
=\end{array}$ & $\begin{array}{l}4.09 \\
=- \\
=\end{array}$ & $\begin{array}{l}13.23 \\
\because- \\
=\end{array}$ & $\begin{array}{l}15.75 \\
\because- \\
-\therefore\end{array}$ & $\begin{array}{l}26.11 \\
\because \\
=\end{array}$ & $\begin{array}{l}23.83 \\
0.03 \\
0.13 \\
*\end{array}$ & $\begin{array}{r}197.03 \\
0.03\end{array}$ \\
\hline 1960 & $\begin{array}{l}\text { PRECIP. (CM.) } \\
\text { SR-9O (MCI/SQ.KM.) } \\
\text { SR-9C CONC. (PC/L) } \\
\text { SR-89/SR-SC }\end{array}$ & $\begin{array}{l}24.64 \\
0.03 \\
0.13 \\
--\end{array}$ & $\begin{array}{l}17.75 \\
0.27 \\
1.53 \\
--\end{array}$ & $\begin{array}{r}23.75 \\
0.45 \\
1.90 \\
--\end{array}$ & $\begin{array}{l}21.01 \\
0.13 \\
0.62 \\
--\end{array}$ & $\begin{array}{l}15.49 \\
0.076 \\
0.46 \\
--\end{array}$ & $\begin{array}{l}4.52 \\
0.026 \\
0.45 \\
--\end{array}$ & $\begin{array}{l}0.16 \\
0.006 \\
c .01 \\
--\end{array}$ & $\begin{array}{l}12.50 \\
0.076 \\
0.57 \\
-.\end{array}$ & $\begin{array}{l}7.21 \\
0.036 \\
c .42 \\
--\end{array}$ & $\begin{array}{c}25.27 \\
0.096 \\
C .36\end{array}$ & $\begin{array}{l}26.62 \\
0.10 \mathrm{C} \\
0.38\end{array}$ & $\begin{array}{c}19.84 \\
0.08 \mathrm{C} \\
0.41 \\
0-\end{array}$ & $\begin{array}{r}199.36 \\
1.34\end{array}$ \\
\hline 1961 & $\begin{array}{l}\text { PRECIP. (CM.) } \\
\text { SR-SC (NCI/SQ.KR.) } \\
S R-S C \text { CONC. }(P C / i) \\
\text { SR-89/SR-90 }\end{array}$ & $\begin{array}{l}38.07 \\
0.29 \mathrm{C} \\
0.77 \\
--\end{array}$ & $\begin{array}{l}53.75 \\
0.41 \mathrm{C} \\
0.77 \\
--\end{array}$ & $\begin{array}{l}22.10 \\
=- \\
=-\end{array}$ & $\begin{array}{l}13.61 \\
0.65 \\
4.78 \\
--\end{array}$ & $\begin{array}{l}8.76 \\
C .28 \mathrm{C} \\
3.20 \\
--\end{array}$ & $\begin{array}{l}4.47 \\
0.14 C \\
3.14 \\
-.\end{array}$ & $\begin{array}{l}2.06 \\
0.02 \\
0.98 \\
0.9\end{array}$ & $\begin{array}{l}6.07 \\
0.04 \\
0.86 \\
-0\end{array}$ & $\begin{array}{r}6.35 \\
6.18 \\
2.84 \\
32.00\end{array}$ & $\begin{array}{r}23.98 \\
6.47 \\
1.96 \\
106.00\end{array}$ & $\begin{array}{r}23.55 \\
0.47 \\
2.00 \\
90.00\end{array}$ & $\begin{array}{r}30.20 \\
0.93 \\
3.08 \\
62.00\end{array}$ & $\begin{array}{r}232.97 \\
3.88\end{array}$ \\
\hline 1962 & $\begin{array}{l}\text { PRECIP. }(C M \cdot) \\
\text { SR-90 (NCI/SQ.KM.) } \\
S R-90 \text { CCNC. }(P C / L) \\
S R-89 / S R-90\end{array}$ & $\begin{array}{r}18.06 \\
0.82 \\
4.55 \\
44.00\end{array}$ & $\begin{array}{r}9.32 \\
0.67 \\
7.19 \\
33.00\end{array}$ & $\begin{array}{r}15.90 \\
1.35 \\
8.50 \\
19.60\end{array}$ & $\begin{array}{r}25.91 \\
1.71 \\
6.60 \\
14.00\end{array}$ & $\begin{array}{r}5.38 \\
0.76 \\
14.13 \\
9.00\end{array}$ & $\begin{array}{l}4.50 \\
0.34 \\
7.56 \\
9.00\end{array}$ & $\begin{array}{r}2.26 \\
6.41 \\
18.15 \\
--\end{array}$ & $\begin{array}{r}12.14 \\
0.56 \\
4.02 \\
5.00\end{array}$ & $\begin{array}{l}9.30 \\
-- \\
--\end{array}$ & $\begin{array}{l}16.46 \\
-\therefore \\
-=\end{array}$ & $\begin{array}{r}41.40 \\
2.47 \\
5.97 \\
50.00\end{array}$ & $\begin{array}{r}32.82 \\
0.02 \\
0.07 \\
15.00\end{array}$ & $\begin{array}{r}193.45 \\
9.11\end{array}$ \\
\hline 1963 & $\begin{array}{l}\text { PRECIP. (CM-) } \\
\text { SR-9O iNCI/SO.KM.) } \\
\text { SR-9C CONC. (PC/L) } \\
\text { SR-B9/SR SC }\end{array}$ & $\begin{array}{r}7.26 \\
0.34 \\
4.69 \\
48.00\end{array}$ & $\begin{array}{r}22.78 \\
2.09 \\
5.18 \\
20.00\end{array}$ & $\begin{array}{l}16.26 \\
3.18 \\
19.56 \\
24.00\end{array}$ & $\begin{array}{r}14.63 \\
2.75 \\
18.80 \\
12.00\end{array}$ & $\begin{array}{r}8.46 \\
1.26 \\
14.90 \\
7.30\end{array}$ & $\begin{array}{r}4.83 \\
1.13 \\
23.40 \\
1.06\end{array}$ & $\begin{array}{r}11.86 \\
3.19 \\
26.90 \\
2.00\end{array}$ & $\begin{array}{l}\because \\
\therefore \\
\cdots\end{array}$ & $\begin{array}{r}3.12 \\
1.71 \\
54.81 \\
c .40\end{array}$ & $\begin{array}{r}34.59 \\
2.88 \\
8.33 \\
1.20\end{array}$ & $\begin{array}{l}37.57 \\
2.22 \\
5.91 \\
*\end{array}$ & $\begin{array}{l}38.43 \\
0.12 \\
0.32 \\
0.90\end{array}$ & $\begin{array}{r}199.79 \\
20.87\end{array}$ \\
\hline 1964 & $\begin{array}{l}\text { PRECIP. (CM-) } \\
\text { SR-90 (NCI/SQ.KM.) } \\
\text { SR-9O CCNC. (PC/L) } \\
\text { SR-B9/SR-9O }\end{array}$ & $\begin{array}{r}36.12 \\
2.69 \\
7.45 \\
*\end{array}$ & $\begin{array}{r}17.27 \\
1.32 \\
7.65 \\
*\end{array}$ & $\begin{aligned} 21.89 \\
2.17 \\
9.92 \\
*\end{aligned}$ & $\begin{array}{l}8.81 \\
0.15 \\
1.71 \\
0.07\end{array}$ & $\begin{array}{r}3.84 \\
0.91 \\
23.70 \\
*\end{array}$ & $\begin{array}{r}2.67 \\
1.59 \\
59.56\end{array}$ & $\begin{array}{r}9.47 \\
1.12 \\
11.83\end{array}$ & $\begin{array}{r}6.38 \\
0.66 \\
10.35 \\
--\end{array}$ & $\begin{array}{l}17.88 \\
0.47 \\
2.63 \\
-.\end{array}$ & $\begin{array}{l}11.58 \\
6.42 \\
3.60 \\
*\end{array}$ & $\begin{array}{r}20.02 \\
0.65 \\
3.25 \\
2.10\end{array}$ & $\begin{array}{r}18.42 \\
0.98 \\
5.33 \\
--\end{array}$ & $\begin{array}{r}174.45 \\
13.13\end{array}$ \\
\hline 1565 & 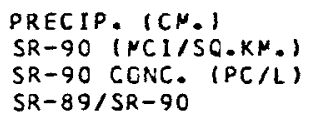 & $\begin{array}{l}32.44 \\
0.50 \\
1.55 \\
-\end{array}$ & $\begin{array}{l}33.15 \\
1.13 \\
3.41 \\
--\end{array}$ & $\begin{array}{l}7.47 \\
0.37 \\
4.96 \\
--\end{array}$ & $\begin{array}{r}12.78 \\
0.85 \\
6.66 \\
--\end{array}$ & $\begin{array}{l}2.53 \\
0.32 \\
3.76 \\
--\end{array}$ & $\begin{array}{l}2.16 \\
0.05 \\
2.32 \\
--\end{array}$ & $\begin{array}{l}1.78 \\
0.11 \\
6.18 \\
--\end{array}$ & $\begin{array}{l}6.05 \\
0.13 \\
2.15 \\
--\end{array}$ & $\begin{array}{r}3.00 \\
0.30 \\
10.01 \\
--\end{array}$ & $\begin{array}{l}27.89 \\
0.37 \\
1.33 \\
-.\end{array}$ & $\begin{array}{l}21.84 \\
0.27 \\
1.24 \\
--\end{array}$ & $\begin{array}{l}36.58 \\
0.51 \\
1.40 \\
-.\end{array}$ & $\begin{array}{r}193.67 \\
4.91\end{array}$ \\
\hline 1966 & $\begin{array}{l}\text { PRECIP. (CM-) } \\
\text { SR-90 iMCI/SQ.KMM) } \\
\text { SR-90 CONC. (PC/L) } \\
\text { SR-89/SR-90 }\end{array}$ & $\begin{array}{l}\bar{y} \\
\bar{z} \\
\overline{-}\end{array}$ & $\begin{array}{l}11.68 \\
* \\
--\end{array}$ & $\begin{array}{r}24.99 \\
0.99 \\
3.97 \\
--\end{array}$ & $\begin{array}{l}6.10 \\
0.60 \\
9.84 \\
--\end{array}$ & $\begin{array}{l}6.63 \\
0.02 \\
0.31 \\
--\end{array}$ & $\begin{array}{l}0.73 \\
0.21 \\
3.13 \\
0.90\end{array}$ & $\begin{array}{l}3.18 \\
0.10 \\
3.15 \\
6.00\end{array}$ & $\begin{array}{l}=- \\
=- \\
--\end{array}$ & $\begin{array}{l}= \\
= \\
--\end{array}$ & $\begin{array}{l}\because \\
\because \\
\because\end{array}$ & $\begin{array}{l}=- \\
\overline{-} \\
-\end{array}$ & $\begin{array}{l}=- \\
=- \\
=-\end{array}$ & $\begin{array}{r}59.31 \\
1.92\end{array}$ \\
\hline 1967 & $\begin{array}{l}\text { PRECIP. (CM.) } \\
\text { SR-90 (NCI/SQ.KM-) } \\
\text { SR-90 CCNC. (PC/L) } \\
\text { SR-89/SR-90 }\end{array}$ & $\begin{array}{l}= \\
= \\
=\end{array}$ & $\begin{array}{l}= \\
= \\
=\end{array}$ & $\begin{array}{r}29.79 \\
0.40 \\
1.35 \\
12.20\end{array}$ & $\begin{array}{r}14.99 \\
0.19 \\
1.27 \\
9.50\end{array}$ & $\begin{array}{l}6.35 \\
0.08 \\
1.26 \\
2.90\end{array}$ & $\begin{array}{l}1.04 \\
0.03 \\
2.89 \\
*\end{array}$ & $\begin{array}{l}2.79 \\
0.03 \\
1.08 \\
*\end{array}$ & $\begin{array}{l}=- \\
=- \\
--\end{array}$ & $\begin{array}{l}= \\
=- \\
=\end{array}$ & $\begin{array}{l}= \\
\because- \\
=\end{array}$ & $\begin{array}{l}=- \\
=- \\
=-\end{array}$ & $\begin{array}{l}=- \\
=- \\
=-\end{array}$ & $\begin{array}{r}54.96 \\
0.73\end{array}$ \\
\hline
\end{tabular}

NOTES

-: DATA NOT AVAILABLE

*: ZERO OR TRACE

A: APPROXIMATE

PROPORTIONED FROM ORIGINALlY CONSOL ICATED DaTA 
MONTHLY FALLOUT DEPOSITION COLLECTIONS

SITE: WASHINGTON, SEATTLE

LAT. 47 35N LONG.122 2OW ALT.

3r. (POT)

SURFACE AIR SAMPLING STATION

SOURCE: FOOD, CHEMICAL AND RESEARCH LABORATORIES, INC.

\begin{tabular}{|c|c|c|c|c|c|c|c|c|c|c|c|c|}
\hline JAN. & FEB. & MAR. & APR. & MAY & JUNE & JULY & AUG. & SEP. & OCI & NOV. & DEC. & CUM. TOTAL \\
\hline $\begin{array}{l}-- \\
-- \\
--\end{array}$ & $\begin{array}{l}-- \\
--\end{array}$ & $\begin{array}{l}-- \\
-- \\
--\end{array}$ & $\begin{array}{l}-- \\
-- \\
--\end{array}$ & $\begin{array}{l}-- \\
-- \\
--\end{array}$ & $\begin{array}{l}-- \\
=- \\
-- \\
--\end{array}$ & $\begin{array}{r}4.60 \\
0.49 \\
10.46 \\
2.80\end{array}$ & $\begin{array}{r}3.99 \\
0.69 \\
17.30 \\
2.20\end{array}$ & $\begin{array}{r}2.39 \\
0.93 \\
38.92 \\
0.20\end{array}$ & $\begin{array}{r}10.64 \\
1.04 \\
9.78 \\
1.00\end{array}$ & $\begin{array}{r}20.12 \\
1.49 \\
7.41 \\
0.10\end{array}$ & $\begin{array}{r}12.07 \\
1.07 \\
3.87 \\
0.40\end{array}$ & $\begin{array}{r}53.81 \\
5.71\end{array}$ \\
\hline $\begin{array}{r}18.77 \\
1.51 \\
8.05 \\
0.20\end{array}$ & $\begin{array}{r}3.18 \\
0.57 \\
17.93 \\
0.20\end{array}$ & $\begin{array}{c}7.54 \\
1.41 \\
18.71 \\
*\end{array}$ & $\begin{array}{r}3.38 \\
1.54 \\
45.57 \\
*\end{array}$ & $\begin{array}{r}3.53 \\
1.87 \\
52.98 \\
*\end{array}$ & $\begin{array}{r}7.80 \\
1.15 \\
14.75 \\
*\end{array}$ & $\begin{array}{l}-- \\
C .68 \\
*\end{array}$ & $\begin{array}{r}2.49 \\
0.33 \\
13.26 \\
*\end{array}$ & $\begin{array}{l}4.72 \\
0.30 \\
6.36 \\
*\end{array}$ & $\begin{array}{r}1.65 \\
0.18 \\
10.91 \\
0.80\end{array}$ & $\begin{array}{r}11.38 \\
0.80 \\
7.03 \\
1.80\end{array}$ & $\begin{array}{l}11.38 \\
0.63 \\
5.54 \\
*\end{array}$ & $\begin{array}{l}75.82 \\
10.97\end{array}$ \\
\hline $\begin{array}{l}14.81 \\
C .50 \\
3.38 \\
--\end{array}$ & $\begin{array}{r}11.05 \\
1.23 \\
11.14 \\
-.\end{array}$ & $\begin{array}{c}1.96 \\
0.32 \\
16.33 \\
-.\end{array}$ & $\begin{array}{l}9.04 \\
0.71 \\
7.86 \\
=-\end{array}$ & $\begin{array}{r}1.27 \\
0.43 \\
33.86 \\
+\end{array}$ & $\begin{array}{r}0.97 \\
0.21 \\
21.65 \\
2.09\end{array}$ & $\begin{array}{l}1.19 \\
0.10 \\
8.41 \\
0.69\end{array}$ & $\begin{array}{l}3.91 \\
0.25 \\
6.40 \\
0.27\end{array}$ & $\begin{array}{l}2.11 \\
0.09 \\
4.27 \\
-.-\end{array}$ & $\begin{array}{l}4.57 \\
0.11 \\
2.41 \\
--\end{array}$ & $\begin{array}{c}12.07 \\
0.22 \\
1.83 \\
-.\end{array}$ & $\begin{array}{c}14.42 \\
0.26 \\
1.81 \\
--\end{array}$ & $\begin{array}{r}77.37 \\
4.43\end{array}$ \\
\hline $\begin{array}{c}12.45 \\
0.22 \\
1.77 \\
-\end{array}$ & $\begin{array}{l}6.25 \\
0.24 \\
3.85 \\
-.\end{array}$ & $\begin{array}{c}12.09 \\
0.46 \\
3.81 \\
-\end{array}$ & $\begin{array}{l}5.13 \\
0.28 \\
5.46 \\
-.\end{array}$ & $\begin{array}{r}3.40 \\
0.38 \\
11.18 \\
-.\end{array}$ & $\begin{array}{l}1.90 \\
0.15 \\
7.90 \\
--\end{array}$ & $\begin{array}{l}2.77 \\
C .12 \\
4.34 \\
--\end{array}$ & $\begin{array}{l}0.25 \\
0.02 \\
8.01 \\
--\end{array}$ & $\begin{array}{l}3.66 \\
0.08 \\
2.19 \\
-.\end{array}$ & $\begin{array}{l}6.63 \\
0.05 \\
0.76 \\
--\end{array}$ & $\begin{array}{c}15.52 \\
0.16 \\
1.04 \\
-.\end{array}$ & $\begin{array}{l}20.17 \\
0.14 \\
0.70 \\
\ldots .\end{array}$ & $\begin{array}{r}90.22 \\
2.30\end{array}$ \\
\hline $\begin{array}{r}18.72 \\
0.11 \\
0.59\end{array}$ & $\begin{array}{l}4.62 \\
0.13 \\
2.82\end{array}$ & $\begin{array}{l}7.87 \\
0.18 \\
2.29\end{array}$ & $=$ & 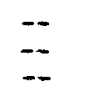 & $=$ & $=$ & $\begin{array}{l}-- \\
--\end{array}$ & $m$ & $=$ & $\begin{array}{l}-- \\
-- \\
--\end{array}$ & -- & $\begin{array}{r}31.21 \\
0.42\end{array}$ \\
\hline
\end{tabular}

NOTES

--: DATA NOT AVAILABLE

*: ZERO OR TRACE

8: LOWER LIMIT OF REPORTED DATA

C: PROPORTIONED FROM ORIGINALLY CONSOLICATED DATA 


\begin{tabular}{|c|c|c|c|c|c|c|c|c|c|c|c|c|c|c|c|}
\hline & & & JAN. & FEB. & mak. & Apk. & $\operatorname{mr} r$ & sune & NuLY & MUE. & SEP. & OCT. & mor. & DEC. & CUM. TOTAL \\
\hline & 1958 & $\begin{array}{l}\text { PRECIP. (CN.) } \\
\text { SR-90 inCI/SO.KN.) } \\
\text { SR-90 COMC. (PC/L) } \\
\text { SR-89/SR-90 }\end{array}$ & $=$ & $\overline{-}$ & $\bar{z}$ & $\begin{array}{r}3.40 \\
0.20 \\
5.89 \\
15.00\end{array}$ & $\begin{array}{r}2.34 \\
0.82 \\
35.05 \\
6.00\end{array}$ & $\begin{array}{r}1.03 \\
0.22 \\
12.03 \\
26.00\end{array}$ & $\frac{0.02}{4.00}$ & $\begin{array}{l}0.81 \\
0.06 \\
7.41 \\
8.00\end{array}$ & $\begin{array}{r}3.73 \\
0.19 \\
5.10 \\
49.00\end{array}$ & $\begin{array}{r}7.92 \\
0.25 \\
3.16 \\
48.00\end{array}$ & $\frac{16.59}{=}$ & $\begin{array}{r}14.00 \\
0.63 \\
4.51 \\
125.00\end{array}$ & $\begin{array}{r}50.62 \\
2.39\end{array}$ \\
\hline & 1959 & $\begin{array}{l}\text { PRECIP. (CM.) } \\
\text { SR-90 IACI/SO.KM-) } \\
\text { SR-90 CONC. (PC/L) } \\
\text { SR-89/SR-90 }\end{array}$ & $\begin{array}{l}20.27 \\
= \\
=\end{array}$ & $\begin{array}{r}9.25 \\
0.76 \\
8.22 \\
28.00\end{array}$ & $\begin{array}{r}10.46 \\
1.34 \\
12.82 \\
17.00\end{array}$ & $\begin{array}{r}9.12 \\
1.41 \\
15.47 \\
6.10\end{array}$ & $\begin{array}{r}4.06 \\
1.96 \\
48.28 \\
8.50\end{array}$ & $\begin{array}{r}4.62 \\
0.51 \\
11.04 \\
4.30\end{array}$ & $\begin{array}{r}2.36 \\
0.50 \\
21.19 \\
2.90\end{array}$ & $\begin{array}{l}1.52 \\
-\end{array}$ & $\begin{array}{r}11.68 \\
0.17 \\
1.46 \\
*\end{array}$ & $\begin{array}{l}6.78 \\
0.11 \\
1.63 \\
0\end{array}$ & $\begin{array}{l}20.68 \\
0.16 \\
0.78 \\
*\end{array}$ & $\begin{array}{l}2.92 \\
0.02 \\
0.69 \\
*\end{array}$ & $\begin{array}{r}103.72 \\
6.94\end{array}$ \\
\hline & 1960 & $\begin{array}{l}\text { PRECIP. (CNA) } \\
\text { SR-90 INCI/SQ.KH.) } \\
S R-90 \text { CDNC. (PC/L) } \\
\text { SR-89/SR-90 }\end{array}$ & $\begin{array}{r}13.92 \\
0.19 \\
1.37 \\
\end{array}$ & $\begin{array}{l}10.19 \\
= \\
=\end{array}$ & $\begin{array}{r}10.36 \\
0.14 \\
1.36 \\
-\end{array}$ & $\begin{array}{l}7.32 \\
0.18 \\
2.46 \\
\end{array}$ & $\begin{array}{l}6.81 \\
0.30 C \\
4.41 \\
-\end{array}$ & $\begin{array}{l}0.99 \\
0.05 C \\
5.06 \\
-.\end{array}$ & $\begin{array}{l}* 000 \\
-\end{array}$ & $\begin{array}{l}3.99 \\
0.09 C \\
2.26 \\
-\end{array}$ & $\begin{array}{l}2.46 \\
0.03 C \\
1.22 \\
-\end{array}$ & $\begin{array}{l}10.06 \\
0.13 \mathrm{C} \\
1.30 \\
=-\end{array}$ & $\begin{array}{l}6.83 \\
0.20 \mathrm{C} \\
2.93 \\
-.\end{array}$ & $\begin{array}{l}1.63 \\
0.05 C \\
3.07 \\
-.\end{array}$ & $\begin{array}{r}74.56 \\
1.36\end{array}$ \\
\hline & 1961 & $\begin{array}{l}\text { PRECIP. (CM.) } \\
\text { SR-90 iMCI/SO.KM.) } \\
\text { SR-90 CONC. (PC/L) } \\
\text { SR-89/SR-90 }\end{array}$ & $\begin{array}{c}19.58 \\
0.15 C \\
0.77 \\
-\end{array}$ & $\begin{array}{l}23.14 \\
0.17 \mathrm{C} \\
0.74 \\
\end{array}$ & $\begin{array}{c}11.30 \\
0.26 C \\
2.31 \\
-\end{array}$ & $\begin{array}{l}5.97 \\
0.14 C \\
2.35 \\
-\end{array}$ & $\begin{array}{l}7.80 \\
-- \\
-\end{array}$ & $\begin{array}{l}1.09 \\
-\infty \\
-\end{array}$ & $\begin{array}{l}1.91 \\
0.02 \mathrm{C} \\
1.05 \\
-\end{array}$ & $\begin{array}{l}2.08 \\
0.03 C \\
1.45 \\
-\end{array}$ & $\begin{array}{r}1.17 \\
0.02 \\
1.71 \\
115.00\end{array}$ & $\begin{array}{r}7.19 \\
0.23 \\
3.20 \\
78.00\end{array}$ & $\begin{array}{r}11.15 \\
0.46 \\
4.13 \\
70.00\end{array}$ & $\begin{array}{r}14.17 \\
0.98 \\
6.92 \\
45.00\end{array}$ & $\begin{array}{r}106.55 \\
2.46\end{array}$ \\
\hline$D$ & 1962 & $\begin{array}{l}\text { PRECIP. }\left(C M H_{-}\right) \\
\text {SR-90 IMCI/SO,KM.) } \\
S R-90 \text { CONC. }(P C / L) \\
S R-69 / S R-90\end{array}$ & $\begin{array}{r}6.17 \\
0.59 \\
9.57 \\
39.00\end{array}$ & $\frac{\overline{0.60}}{48.00}$ & $\frac{\overline{1.62}}{1 \overline{7 . c 0}}$ & $\frac{\overline{0.91}}{11.00}$ & $\begin{array}{l}\overline{1.02} \\
\overline{6.00}\end{array}$ & $\begin{array}{l}\overline{0.34} \\
\overline{8.0 C}\end{array}$ & $\begin{array}{l}2.82 \\
0.22 \\
7.81 \\
5.00\end{array}$ & $\begin{array}{l}4.98 \\
0.47 \\
9.44 \\
9.00\end{array}$ & $\begin{array}{r}5.13 \\
0.72 \\
14.04 \\
25.00\end{array}$ & $\begin{array}{r}8.66 \\
6.29 \\
3.35 \\
45.00\end{array}$ & $\begin{array}{l}0.86 \\
44.00\end{array}$ & $\begin{array}{r}9.73 \\
0.74 \\
7.61 \\
60.00\end{array}$ & $\begin{array}{r}37.49 \\
8.38\end{array}$ \\
\hline $\mathbb{G}$ & 1963 & $\begin{array}{l}\text { PREC IP (CM.) } \\
\text { SR-90 (MCI/SO-KM.) } \\
\text { SR-90 CONC. (PC/L) } \\
\text { SR-69/SR-90 }\end{array}$ & $\begin{array}{r}4.85 \\
0.74 \\
15.26 \\
46.00\end{array}$ & $\begin{array}{r}10.08 \\
1.17 \\
11.61 \\
41.00\end{array}$ & $\begin{array}{r}7.57 \\
1.52 \\
2 C .08 \\
18.00\end{array}$ & $\begin{array}{r}6.99 \\
2.06 \\
29.48 \\
13.00\end{array}$ & $\begin{array}{r}2.39 \\
0.52 \\
21.76 \\
6.10\end{array}$ & $\begin{array}{r}4.95 \\
2.11 \\
42.63 \\
*\end{array}$ & $\begin{array}{r}2.16 \\
1.48 \\
68.52 \\
2.00\end{array}$ & $\begin{array}{r}1.93 \\
0.66 \\
34.20 \\
*\end{array}$ & $\begin{array}{r}1.75 \\
0.81 \\
46.29 \\
*\end{array}$ & $\begin{array}{r}10.57 \\
1.42 \\
13.44 \\
*\end{array}$ & $\begin{array}{r}19.38 \\
1.64 \\
8.47 \\
\end{array}$ & $\begin{array}{r}12.52 \\
1.20 \\
9.59 \\
*\end{array}$ & $\begin{array}{l}85.14 \\
15.33\end{array}$ \\
\hline & 1964 & $\begin{array}{l}\text { PRECIP. }(C M-) \\
\text { SR-90 (MCI/SQ.KMH) } \\
\text { SR-90 CGNC. (PC/L) } \\
5 R-89 / S R-90\end{array}$ & $\begin{array}{r}20.73 \\
1.20 \\
5.79 \\
*\end{array}$ & $\begin{array}{r}3.94 \\
0.81 \\
2 C .56 \\
*\end{array}$ & $\begin{array}{r}8.13 \\
0.97 \\
11.94 \\
*\end{array}$ & $\begin{array}{r}3.28 \\
1.47 \\
44.82 \\
+\end{array}$ & $\begin{array}{r}2.72 \\
1.83 \\
67.28 \\
1.00\end{array}$ & $\begin{array}{r}9.35 \\
2.56 \\
27.38 \\
\end{array}$ & $\begin{array}{r}2.13 \\
0.85 \\
39.91 \\
-\end{array}$ & $\begin{array}{r}3.71 \\
0.48 \\
12.94 \\
-\end{array}$ & $\begin{array}{l}5.11 \\
0.35 \\
6.85 \\
--\end{array}$ & $\begin{array}{l}2.11 \\
0.13 \\
t .17 \\
*\end{array}$ & $\begin{array}{r}20.60 \\
0.63 \\
3.06 \\
1.70\end{array}$ & $\begin{array}{r}12.34 \\
0.12 \\
C .98 \\
-\end{array}$ & $\begin{array}{l}94.15 \\
11.40\end{array}$ \\
\hline & 1965 & $\begin{array}{l}\text { PRECIP. (CM.) } \\
\text { SR }-90 \text { (MCI/SQ-KM-) } \\
\text { SR-90 CONC. (PC/L) } \\
\text { SR-89/SR-90 }\end{array}$ & $\begin{array}{r}14.81 \\
0.56 \\
3.79 \\
\end{array}$ & $\begin{array}{r}10.87 \\
0.36 \\
3.32 \\
\end{array}$ & $\begin{array}{l}1.12 \\
0.01 \\
0.90 \\
-\end{array}$ & $\begin{array}{l}9.63 \\
0.45 \\
4.68 \\
-\end{array}$ & $\begin{array}{r}3.18 \\
0.44 \\
13.84 \\
--\end{array}$ & $\begin{array}{r}1.19 \\
0.29 \\
24.37 \\
-\end{array}$ & $\begin{array}{l}1.22 \\
0.10 \\
8.20 \\
-\end{array}$ & $\begin{array}{r}4.09 \\
0.51 \\
12.47 \\
\end{array}$ & $\begin{array}{r}1.91 \\
0.20 \\
10.48 \\
--\end{array}$ & $\begin{array}{l}5.16 \\
0.08 \\
1.56 \\
--\end{array}$ & $\begin{array}{r}12.72 \\
0.30 \\
2.36 \\
--\end{array}$ & $\begin{array}{r}17.93 \\
0.35 \\
1.96 \\
-\end{array}$ & $\begin{array}{r}83.83 \\
3.65\end{array}$ \\
\hline & 1966 & $\begin{array}{l}\text { PRECIP. }\left(C H_{-}\right) \\
\text {SR-90 (MCI/SO.KM.) } \\
\text { SR-90 CONC. (PC/L) } \\
\text { SR-89/SR-90 }\end{array}$ & $\begin{array}{r}16.26 \\
0.10 \\
0.62 \\
-\end{array}$ & $\begin{array}{l}5.99 \\
0.20 \\
3.34 \\
-\end{array}$ & $\begin{array}{r}12.09 \\
0.32 \\
2.65 \\
--\end{array}$ & $\begin{array}{l}5.13 \\
0.44 \\
8.58 \\
\end{array}$ & $\begin{array}{l}3.40 \\
0.24 \\
7.06 \\
--\end{array}$ & $\begin{array}{l}1.90 \\
0.13 \\
6.85 \\
2.80\end{array}$ & $\begin{array}{l}3.53 \\
0.15 \\
4.25 \\
1.10\end{array}$ & $\begin{array}{l}0.43 \\
0.03 \\
6.98 \\
+\end{array}$ & $\begin{array}{l}3.99 \\
0.08 \\
2.01 \\
*\end{array}$ & $\begin{array}{l}5.61 \\
0.06 \\
1.07\end{array}$ & $\begin{array}{r}18.24 \\
0.16 \\
0.88 \\
14.00\end{array}$ & $\begin{array}{r}19.63 \\
0.16 \\
0.82 \\
2.50\end{array}$ & $\begin{array}{r}96.20 \\
2.07\end{array}$ \\
\hline & 1967 & $\begin{array}{l}\text { PRECIP. (CM-) } \\
\text { SR-90 (MCI/SQ.KM-) } \\
\text { SR-90 CONC. }(P C / L) \\
\text { SR-89/SR-90 }\end{array}$ & $\begin{array}{r}22.91 \\
0.20 \\
0.88 \\
9.80\end{array}$ & $\begin{array}{r}5.54 \\
0.07 \\
1.27 \\
18.20\end{array}$ & $\begin{array}{r}9.17 \\
0.16 \\
1.75 \\
10.10\end{array}$ & $\begin{array}{l}7.01 \\
0.10 \\
1.43 \\
9.40\end{array}$ & $\begin{array}{l}1.32 \\
0.06 \\
4.55 \\
.\end{array}$ & $\begin{array}{l}3.43 \\
0.07 \\
2.05 \\
*\end{array}$ & $=$ & $\overline{-}$ & $=$ & $=$ & $\ddot{-}$ & 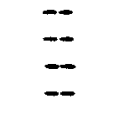 & $\begin{array}{r}49.38 \\
0.66\end{array}$ \\
\hline & & $\begin{array}{l}\text { TA NOT AVAILABLE } \\
\text { RO OR TRACE } \\
\text { PROXIMATE } \\
\text { WER LIMIT OF REPO } \\
\text { IOPORTIONEC FAOH }\end{array}$ & & & & & & & & & & & & & \\
\hline
\end{tabular}


MONTHLY FALLOUT DEPOSITION COLLECTIONS

SIIE: WISCONSIN, APPLETON

LAT. 44 15N LCNG. $8825 \mathrm{H}$ ALT.

229M. (POT)

SURFACE AIR SAMPLING STATION

SOURCE: ISOTOPES, INC., IHRU THE INSTITUTE OF PAPER CHEMISTRY

COLLECTIONS TERMINATED IN DECEMBER 1965

\begin{tabular}{|c|c|c|c|c|c|c|c|c|c|c|c|c|c|c|}
\hline & & JAN. & FEB. & MAR. & APR. & MAY & JUNE & $\begin{array}{l}\text { JLLY } \\
---\end{array}$ & AUG. & SEP. & CCT. & NOV. & LEC. & CUM. TOTAL \\
\hline 1963 & $\begin{array}{l}\text { PRECIP, (CM.) } \\
S R-9 O \text { INCI/SQ.KM.) } \\
S R-S C \text { CCNC. (PC/L) } \\
S R-89 / S R-90\end{array}$ & $\begin{array}{l}-- \\
-- \\
--\end{array}$ & $\begin{array}{l}-- \\
-- \\
--\end{array}$ & $\begin{array}{l}-- \\
=- \\
--\end{array}$ & $\begin{array}{l}-- \\
--\end{array}$ & $\begin{array}{l}-- \\
-- \\
-\end{array}$ & $\begin{array}{l}-- \\
-- \\
--\end{array}$ & $\begin{array}{l}-- \\
-- \\
--\end{array}$ & $\begin{array}{r}5.03 \\
1.50 \\
29.83 \\
1.90\end{array}$ & $\begin{array}{r}9.70 \\
1.54 \\
15.88 \\
1.40\end{array}$ & $\begin{array}{r}1.57 \\
0.67 \\
42.68 \\
1.00\end{array}$ & $\begin{array}{r}4.29 \\
0.94 \\
21.92 \\
0.60\end{array}$ & $\begin{array}{r}1.45 \\
0.20 \\
13.80 \\
0.50\end{array}$ & $\begin{array}{r}22.04 \\
4.85\end{array}$ \\
\hline 1964 & $\begin{array}{l}P R E C I P \text { (CM.) } \\
S R-9 O \text { INCI/SQ.KM.) } \\
S R-90 \text { CCNC. (PC/L) } \\
S R-89 / S R-90\end{array}$ & $\begin{array}{r}2.64 \\
0.59 \\
22.35 \\
0.20\end{array}$ & $\begin{array}{l}0.41 \\
0.12 \\
29.27 \\
*\end{array}$ & $\begin{array}{r}4.22 \\
0.82 \\
19.44 \\
0.20\end{array}$ & $\begin{array}{l}6.60 \\
1.35 \\
20.46 \\
-\end{array}$ & $\begin{array}{r}13.51 \\
2.46 \\
18.21 \\
--\end{array}$ & $\begin{array}{l}3.84 \\
0.77 \\
20.06 \\
--\end{array}$ & $\begin{array}{l}14.05 \\
1.19 \\
8.47 \\
--\end{array}$ & $\begin{array}{l}6.17 \\
0.71 \\
11.51 \\
--\end{array}$ & $\begin{array}{l}9.58 \\
0.65 \\
6.52 \\
-.\end{array}$ & $\begin{array}{r}0.79 \\
0.38 \\
48.11 \\
*\end{array}$ & $\begin{array}{l}5.41 \\
0.45 \\
8.32 \\
*\end{array}$ & $\begin{array}{l}1.40 \\
0.16 \\
11.43 \\
*\end{array}$ & $\begin{array}{r}69.02 \\
9.65\end{array}$ \\
\hline 1965 & $\begin{array}{l}\text { PRECIP. (CM.) } \\
\text { SR-9O (NCI/SQ.KMI) } \\
\text { SR-90 CCNC. (PC/L) } \\
\text { SR-89/SR-90 }\end{array}$ & $\begin{array}{l}1.73 \\
0.16 \\
9.25 \\
--\end{array}$ & $\begin{array}{l}1.93 \\
0.14 \\
7.26 \\
--\end{array}$ & $\begin{array}{l}8.00 \\
0.54 \\
6.76 \\
--\end{array}$ & $\begin{array}{c}9.55 \\
1.47 \\
15.40 \\
--\end{array}$ & $\begin{array}{c}5.99 \\
1.24 \\
20.71 \\
--\end{array}$ & $\begin{array}{l}8.15 \\
0.78 \\
9.58 \\
--\end{array}$ & $\begin{array}{l}6.35 \\
0.32 \\
5.04 \\
--\end{array}$ & $\begin{array}{l}9.42 \\
0.51 \\
5.42 \\
--\end{array}$ & $\begin{array}{r}19.59 \\
0.27 \\
1.36 \\
--\end{array}$ & $\begin{array}{l}4.75 \\
C .17 \\
3.58 \\
--\end{array}$ & $\begin{array}{l}4.72 \\
0.20 \\
4.24 \\
--\end{array}$ & $\begin{array}{l}6.70 \\
0.17 \\
2.54 \\
--\end{array}$ & $\begin{array}{r}87.28 \\
5.97\end{array}$ \\
\hline \begin{tabular}{l} 
NOIES \\
\hdashline$:$ : \\
$*:$ \\
$A: A$ \\
$B: L$ \\
$C: P$
\end{tabular} & $\begin{array}{l}\text { ATA NOT AVAILABLE } \\
\text { ERO OR TRACE } \\
\text { PPROXIMATE } \\
\text { OWER LIMIT OF REPORT } \\
\text { ROPORTIONED FROM CRI }\end{array}$ & $\begin{array}{l}\text { DOATA } \\
\text { INALLY }\end{array}$ & & ED & & & & & & & & & & \\
\hline
\end{tabular}




\begin{tabular}{|c|c|c|c|c|c|c|c|c|c|c|c|c|c|c|}
\hline & & JAN. & FEB. & MAR. & APR. & MAY & JUNE & JULY & AUG. & SEP. & OCT. & NOV. & DEC. & CUM. TOTAL \\
\hline 1959 & $\begin{array}{l}\text { PRECIP. }(C M .) \\
\text { SR-SO (HCI/SQ.KM.) } \\
\text { SR-SO CONC. (PC/L) } \\
\text { SR-8S/SR-SO }\end{array}$ & $m$ & $\begin{array}{r}5.03 \\
0.24 \\
4.78 \\
13.00\end{array}$ & $\begin{array}{l}4.75 \\
-- \\
--\end{array}$ & $\begin{array}{r}7.21 \\
2.06 \\
28.58 \\
15.00\end{array}$ & $\begin{array}{l}9.80 \\
0.81 \\
8.27 \\
*\end{array}$ & $\begin{array}{r}3.20 \\
0.55 \\
17.18 \\
4.7 C\end{array}$ & $\begin{array}{r}10.69 \\
0.36 \\
3.37 \\
2.40\end{array}$ & $\begin{array}{l}6.88 \\
0.25 \\
3.64 \\
1.40\end{array}$ & $\begin{array}{r}13.13 \\
0.08 \\
0.61 \\
1.3 \mathrm{C}\end{array}$ & $\begin{array}{l}8.31 \\
C .10 \\
1.21 \\
C .60\end{array}$ & $\begin{array}{l}4.09 \\
0.05 \\
1.23 \\
+\end{array}$ & $\begin{array}{l}7.24 \\
0.10 \\
1.39 \\
0.60\end{array}$ & $\begin{array}{r}80.33 \\
4.60\end{array}$ \\
\hline 1960 & $\begin{array}{l}\text { PRECIP. }(C M-) \\
\text { SR-9O (NCI/SQ.KM.) } \\
S R-9 O C C N C \text { (PC/L) } \\
S R-Q S / S R-S C\end{array}$ & $\begin{array}{l}2.64 \\
0.08 \\
3.04 \\
\ldots\end{array}$ & $\begin{array}{l}1.22 \\
0.00 \\
0.01 \\
*\end{array}$ & $\begin{array}{r}3.07 \\
0.36 \\
11.73 \\
\end{array}$ & $\begin{array}{l}7.95 \\
-- \\
=\end{array}$ & $\begin{array}{l}19.69 \\
0.11 \mathrm{C} \\
0.56 \\
-\end{array}$ & $\begin{array}{l}7.80 \\
0.04 C \\
0.52 \\
--\end{array}$ & $\begin{array}{l}4.75 \\
C .01 C \\
C .22 \\
-\end{array}$ & $\begin{array}{l}8.94 \\
0.026 \\
0.23 \\
-.\end{array}$ & $\begin{array}{l}7.85 \\
0.15 \mathrm{C} \\
1.52 \\
-.\end{array}$ & $\begin{array}{l}5.89 \\
0.11 C \\
1.87 \\
--\end{array}$ & $\begin{array}{l}1.75 \\
0.06 C \\
3.43 \\
-.\end{array}$ & $\begin{array}{l}0.25 \\
0.01 C \\
4.01 \\
-.\end{array}$ & $\begin{array}{r}71.80 \\
0.95\end{array}$ \\
\hline $15 \in 1$ & $\begin{array}{l}\text { PRECIP. (CK.) } \\
\text { SR-GC INCI/SO.KM.) } \\
S R-G O C O N C . \quad(P C / L) \\
S R-B 9 / S R-90\end{array}$ & $\begin{array}{l}0.79 \\
0.02 \mathrm{C} \\
2.54 \\
-.\end{array}$ & $\begin{array}{l}2.36 \\
0.06 C \\
2.55 \\
--\end{array}$ & $\begin{array}{l}5.38 \\
0.20 \mathrm{C} \\
3.72 \\
--\end{array}$ & $\begin{array}{l}4.24 \\
0.16 C \\
3.78 \\
--\end{array}$ & $\begin{array}{l}3.61 \\
0.20 \mathrm{C} \\
5.55 \\
-.\end{array}$ & $\begin{array}{l}10.95 \\
0.61 C \\
5.58 \\
--\end{array}$ & $\begin{array}{l}12.47 \\
-- \\
--\end{array}$ & $\begin{array}{l}6.30 \\
C .22 \\
3.50 \\
--\end{array}$ & $\begin{array}{r}12.75 \\
0.07 \\
0.55 \\
50.00\end{array}$ & $\begin{array}{r}8.48 \\
C .14 \\
1.66 \\
96.00\end{array}$ & $\begin{array}{r}0.60 \\
0.16 \\
2.43 \\
70.00\end{array}$ & $\begin{array}{r}3.23 \\
0.09 \\
2.79 \\
64.00\end{array}$ & $\frac{77.0 .16}{1.93}$ \\
\hline 1962 & $\begin{array}{l}\text { PQECIP. (CM.) } \\
S R-S C \text { INCI/SO-KM.) } \\
S R-S C \text { CONC. }(P C / L) \\
S R-89 / S R-90\end{array}$ & $\begin{array}{r}3.23 \\
C .10 \\
3.10 \\
39.00\end{array}$ & $\begin{array}{r}5.13 \\
C .66 \\
12.87 \\
28.00\end{array}$ & $\begin{array}{r}2.90 \\
0.72 \\
24.83 \\
18.00\end{array}$ & $\begin{array}{r}6.48 \\
1.02 \\
15.59 \\
13.00\end{array}$ & $\begin{array}{r}7.26 \\
0.21 \\
2.90 \\
112.00\end{array}$ & $\begin{array}{r}11.05 \\
1.83 \\
16.57 \\
8.06\end{array}$ & $\begin{array}{r}6.86 \\
C .81 \\
11.81 \\
1 C .00\end{array}$ & $\begin{array}{r}7.26 \\
1.00 \\
13.78 \\
15.00\end{array}$ & $\begin{array}{r}9.83 \\
C .03 \\
C .31 \\
21.00\end{array}$ & $\begin{array}{r}4.93 \\
C .50 \\
10.15 \\
23.00\end{array}$ & $\begin{array}{r}2.13 \\
0.23 \\
10.20 \\
39.00\end{array}$ & $\begin{array}{r}2.62 \\
0.09 \\
3.44 \\
30.00\end{array}$ & $\begin{array}{r}69.66 \\
7.19\end{array}$ \\
\hline 1963 & $\begin{array}{l}\text { PRECIP. }(C M,) \\
S R-9 C \text { INCI/SQ.KN.) } \\
S R-S C C C N C .(P C / L) \\
S R-89 / S R-90\end{array}$ & $\begin{array}{r}2.59 \\
C .21 \\
8.11 \\
28.00\end{array}$ & $\begin{array}{r}1.50 \\
0.06 \\
4.01 \\
33.00\end{array}$ & $\begin{array}{l}6.55 \\
1.27 \\
19.39 \\
19.00\end{array}$ & $\begin{array}{r}2.49 \\
1.32 \\
53.02 \\
10.20\end{array}$ & $\begin{array}{r}3.91 \\
1.68 \\
42.97 \\
7.90\end{array}$ & $\begin{array}{r}6.78 \\
2.64 \\
38.94 \\
*\end{array}$ & $\begin{array}{r}7.04 \\
2.22 \\
31.54 \\
1.00\end{array}$ & $\begin{array}{r}5.26 \\
1.21 \\
23.01 \\
1.00\end{array}$ & $\begin{array}{r}7.62 \\
1.08 \\
14.18 \\
*\end{array}$ & $\begin{array}{r}1.85 \\
0.50 \\
27.03 \\
1.50\end{array}$ & $\begin{array}{l}4.14 \\
0.42 \\
10.15 \\
+\end{array}$ & $\begin{array}{l}1.85 \\
0.17 \\
9.19 \\
*\end{array}$ & $\begin{array}{l}51.58 \\
12.78\end{array}$ \\
\hline 1964 & $\begin{array}{l}\text { PRECIP. (CM.) } \\
S R-90 \text { INCI/SC.KM.) } \\
S R-9 C \text { CCNC. }(P C / L) \\
S R-89 / S R-90\end{array}$ & $\begin{array}{r}2.50 \\
0.52 \\
17.94 \\
*\end{array}$ & $\begin{array}{r}0.66 \\
0.07 \\
10.61 \\
1.60\end{array}$ & $\begin{array}{r}4.47 \\
0.52 \\
11.64 \\
*\end{array}$ & $\begin{array}{r}6.48 \\
1.42 \\
21.92 \\
1.10\end{array}$ & $\begin{array}{l}10.52 \\
1.73 \\
16.45 \\
*\end{array}$ & $\begin{array}{l}2.67 \\
1.59 \\
59.56 \\
--\end{array}$ & $\begin{array}{l}11.56 \\
1.52 \\
13.15 \\
--\end{array}$ & $\begin{array}{l}6.91 \\
0.78 \\
11.29 \\
--\end{array}$ & $\begin{array}{r}17.12 \\
0.35 \\
2.05 \\
-.\end{array}$ & $\begin{array}{r}1.68 \\
0.22 \\
13.10 \\
1.70\end{array}$ & $\begin{array}{l}5.26 \\
0.24 \\
4.57 \\
4.20\end{array}$ & $\begin{array}{l}1.78 \\
0.15 \\
8.43 \\
--\end{array}$ & $\begin{array}{r}72.01 \\
9.11\end{array}$ \\
\hline 1965 & $\begin{array}{l}\text { PRECIP. (CN.) } \\
\text { SR-SC INCI/SQ.KM) } \\
\text { SR-9C CCAC. (PC/L) } \\
\text { SR-89/SR-9O }\end{array}$ & $\begin{array}{l}2.36 \\
0.13 \\
5.51 \\
-\end{array}$ & $\begin{array}{l}2.16 \\
0.13 \\
6.02 \\
--\end{array}$ & $\begin{array}{l}6.05 \\
0.32 \\
5.29 \\
--\end{array}$ & $\begin{array}{r}9.19 \\
1.07 \\
11.65 \\
+\end{array}$ & $\begin{array}{r}10.03 \\
1.04 \\
10.37 \\
-.\end{array}$ & $\begin{array}{l}4.8 C \\
0.27 \\
5.63 \\
--\end{array}$ & $\begin{array}{r}5.23 \\
C .54 \\
10.33 \\
--\end{array}$ & $\begin{array}{l}8.59 \\
0.46 \\
5.36 \\
--\end{array}$ & $\begin{array}{l}19.81 \\
0.46 \\
2.33 \\
--\end{array}$ & $\begin{array}{l}3.35 \\
0.04 \\
1.20 \\
-.\end{array}$ & $\begin{array}{l}5.56 \\
0.14 \\
2.52 \\
--\end{array}$ & $\begin{array}{l}5.87 \\
0.33 \\
5.63 \\
-\end{array}$ & $\begin{array}{r}83.00 \\
4.93\end{array}$ \\
\hline 1986 & $\begin{array}{l}\text { PRECIP. (CM.) } \\
S R-S C \text { (NCI/SC.KM.) } \\
S R-90 \text { CCNC. }(P C / L) \\
S R-89 / S R-90\end{array}$ & $\begin{array}{l}3.00 \\
0.01 \\
0.34 \\
--\end{array}$ & $\begin{array}{l}5.72 \\
0.14 \\
2.45 \\
--\end{array}$ & $\begin{array}{l}6.25 \\
0.45 \\
7.21 \\
-.\end{array}$ & $\begin{array}{l}3.50 \\
0.21 \\
6.01 \\
--\end{array}$ & $\begin{array}{l}3.25 \\
0.18 \\
5.54 \\
--\end{array}$ & $\begin{array}{l}2.77 \\
0.11 \\
3.98 \\
4.20\end{array}$ & $\begin{array}{r}10.64 \\
0.23 \\
2.17 \\
5.40\end{array}$ & $\begin{array}{l}6.73 \\
0.22 \\
3.27 \\
*\end{array}$ & $\begin{array}{l}3.07 \\
0.04 \\
1.31 \\
*\end{array}$ & $\begin{array}{l}1.83 \\
0.02 \\
1.10 \\
*\end{array}$ & $\begin{array}{r}4.01 \\
0.06 \\
1.50 \\
20.00\end{array}$ & $\begin{array}{l}4.19 \\
0.03 \\
0.72 \\
*\end{array}$ & $\begin{array}{r}54.96 \\
1.70\end{array}$ \\
\hline 1967 & $\begin{array}{l}\text { PRECIP. }(C M \cdot) \\
\text { SR-9C }(N C I / S Q . K M \cdot) \\
S R-90 C C N C . \quad(P C / L) \\
S R-89 / S R-90\end{array}$ & $\begin{array}{r}6.40 \\
0.04 \\
0.63 \\
20.00\end{array}$ & $\begin{array}{r}2.13 \\
0.01 \\
0.47 \\
26.90\end{array}$ & $\begin{array}{r}2.87 \\
0.09 \\
3.14 \\
10.70\end{array}$ & $\begin{array}{l}7.04 \\
0.23 \\
3.27 \\
6.70\end{array}$ & $\begin{array}{l}6.22 \\
0.12 \\
1.93 \\
3.10\end{array}$ & $\begin{array}{l}21.51 \\
0.20 \\
0.93 \\
*\end{array}$ & $\begin{array}{l}-- \\
-- \\
--\end{array}$ & $\begin{array}{l}-- \\
-- \\
--\end{array}$ & $\begin{array}{l}-- \\
-- \\
--\end{array}$ & $\begin{array}{l}-- \\
-- \\
--\end{array}$ & $\begin{array}{l}-- \\
-- \\
--\end{array}$ & $=$ & $\begin{array}{r}46.17 \\
0.69\end{array}$ \\
\hline
\end{tabular}


MONTHLY FALLCUT DEPOSITION COLLECTIONS

SITE: ANERICAN SAMOA, PAGO PAGO SOURCE: U. S. WEATHER BUREAU
LAT. 14 16S LCNG.170 $43 \mathrm{~W}$ ALT. OM. (COLUMN)

\begin{tabular}{|c|c|c|c|c|c|c|c|c|c|c|c|c|c|c|}
\hline & & JAN. & FEB. & MAR. & APR. & MAY & JUNE & $\begin{array}{l}\text { JULY } \\
---\end{array}$ & AUG. & SEP. & OCT. & NOV. & DEC. & CUM. TOTAL \\
\hline 1965 & $\begin{array}{l}\text { PRECIP. (CM.) } \\
5 R-90 \text { (MCI/SQ.KM.) } \\
5 R-9 C \text { CONC. (PC/L) } \\
S R-89 / S R-90\end{array}$ & $\begin{array}{l}-- \\
-- \\
--\end{array}$ & $\begin{array}{l}-- \\
-- \\
--\end{array}$ & $\begin{array}{l}-- \\
-- \\
--\end{array}$ & $\begin{array}{l}-- \\
-- \\
--\end{array}$ & $\begin{array}{l}-- \\
-- \\
--\end{array}$ & $\begin{array}{l}-- \\
-- \\
--\end{array}$ & $\begin{array}{l}-- \\
-- \\
--\end{array}$ & $\begin{array}{l}-- \\
--\end{array}$ & $\begin{array}{l}3.76 \\
0.05 \\
1.33 \\
--\end{array}$ & $\begin{array}{l}49.91 \\
0.18 \\
0.37 \\
-.\end{array}$ & $\begin{array}{l}3.48 \\
0.05 \\
1.44 \\
--\end{array}$ & $\begin{array}{l}31.88 \\
0.03 \\
0.10 \\
-.\end{array}$ & $\begin{array}{r}89.03 \\
0.31\end{array}$ \\
\hline 1SEt & $\begin{array}{l}\text { PRECIP. }(C M .) \\
S R-9 C \text { INCI/SG.KM }) \\
5 R-90 \text { CCNC. }(P C / L) \\
S R-89 / 5 R-9 O\end{array}$ & $\begin{array}{l}40.00 \\
0.07 \\
0.18 \\
--\end{array}$ & $\begin{array}{l}15.24 \\
0.02 \\
0.14 \\
--\end{array}$ & $\begin{array}{l}26.04 \\
0.06 \\
0.24 \\
--\end{array}$ & $\begin{array}{l}26.64 \\
0.06 \\
0.23 \\
--\end{array}$ & $\begin{array}{l}52.02 \\
0.04 \\
0.08 \\
--\end{array}$ & $\begin{array}{r}23.25 \\
0.01 \\
0.05 \\
*\end{array}$ & $\begin{array}{r}1 t .26 \\
c .02 \\
0.13 \\
30.00\end{array}$ & $\begin{array}{r}19.35 \\
0.04 \\
0.21 \\
18.00\end{array}$ & $\begin{array}{r}21.45 \\
0.67 \\
3.12 \\
96.00\end{array}$ & $\begin{array}{r}46.63 \\
0.09 \\
C .20 \\
56.00\end{array}$ & $\begin{array}{r}14.63 \\
0.06 \\
0.42 \\
35.00\end{array}$ & $\begin{array}{r}41.17 \\
0.12 \\
0.30 \\
13.00\end{array}$ & $\begin{array}{r}342.76 \\
1.26\end{array}$ \\
\hline 1967 & $\begin{array}{l}\text { PRECIP. }(C \mu \cdot) \\
S R-90 \text { (NCI/SQ.KN.) } \\
S R-90 \text { CCNC. }(P C / L) \\
S R-89 / S R-90\end{array}$ & $\begin{array}{r}17.70 \\
C .04 \\
0.23 \\
8.30\end{array}$ & $\begin{array}{r}21.84 \\
0.04 \\
0.19 \\
7.10\end{array}$ & $\begin{array}{r}12.57 \\
0.02 \\
0.16 \\
*\end{array}$ & $\begin{array}{l}62.13 \\
0.02 \\
0.04 \\
*\end{array}$ & $\begin{array}{l}25.32 \\
C . C 3 \\
0.12 \\
*\end{array}$ & $\begin{array}{r}29.82 \\
0.12 \\
0.41 \\
15.1 C\end{array}$ & $\begin{array}{r}2.86 \\
0.07 \\
2.45 \\
107.00\end{array}$ & $\begin{array}{l}-- \\
-- \\
--\end{array}$ & $\begin{array}{l}= \\
= \\
--\end{array}$ & $\begin{array}{l}-- \\
=- \\
--\end{array}$ & $\begin{array}{l}-- \\
-- \\
--\end{array}$ & $\begin{array}{l}-- \\
=- \\
--\end{array}$ & $\begin{array}{r}172.24 \\
0.34\end{array}$ \\
\hline 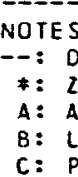 & $\begin{array}{l}\text { OATA NOT AVAILABLE } \\
\text { ZERO OR TRACE } \\
\text { QPPROXIMATE } \\
\text { LOWER LIMIT OF REPORT } \\
\text { PROPORTIONEO FRCM ORI }\end{array}$ & $\begin{array}{l}\text { D DATA } \\
\text { INALLY }\end{array}$ & NSOI & ED & & & & & & & & & & \\
\hline
\end{tabular}


MONTHLY FALLCUT DEPOSITION COLLECTIONS

SITE: ARGENTINA, FORMOSA

LAT. 26115 LONG. 58 LOW ALT. 58N. (COLUMN)

SOURCE: COMISION NACIONAL DE ENERGIA ATOMICA

\begin{tabular}{|c|c|c|c|c|c|c|c|c|c|c|c|c|c|c|}
\hline & & JAN. & FEB. & MAR. & APR : & MAY & JUNE & JULY & AUG. & SEP. & OCT: & NOV. & DEC. & CUM. FOTAL \\
\hline 1961 & $\begin{array}{l}\text { PRECIP. (CM.) } \\
S R-90 \text { (NCI/SO.KM.) } \\
S R-90 \text { CONC. (PC/L) } \\
S R-89 / S R-90\end{array}$ & $\begin{array}{l}-- \\
-- \\
--\end{array}$ & $\begin{array}{l}-- \\
-- \\
--\end{array}$ & 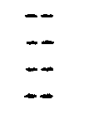 & $\begin{array}{l}=- \\
-- \\
--\end{array}$ & $\ddot{--}$ & $\begin{array}{l}7.11 \\
0.02 \\
0.29 \\
-.\end{array}$ & $\begin{array}{r}10.34 \\
0.18 \\
1.75 \\
*\end{array}$ & $\begin{array}{l}4.67 \\
-- \\
--\end{array}$ & $\begin{array}{l}15.34 \\
-- \\
-- \\
--\end{array}$ & $\begin{array}{l}16.46 \\
0.07 \\
0.43 \\
*\end{array}$ & $\begin{array}{l}17.35 \\
0.05 \\
0.29 \\
*\end{array}$ & $\begin{array}{l}13.06 \\
0.08 \\
0.45 \\
*\end{array}$ & $\begin{array}{r}87.33 \\
0.40\end{array}$ \\
\hline 1962 & $\begin{array}{l}\text { PRECIP. }(C M .) \\
S R-S C \text { INCI/SQ.KN) } \\
S R-90 \text { CCNC. }(P C / L) \\
S R-89 / S R-90\end{array}$ & $\begin{array}{l}23.22 \\
C .08 \\
0.35 \\
+\end{array}$ & $\begin{array}{r}12.37 \\
6.06 \\
0.49 \\
4.00\end{array}$ & $\begin{array}{r}15.88 \\
0.05 \\
0.32 \\
4.00\end{array}$ & $\begin{array}{r}19.51 \\
0.04 \\
0.21 \\
17.00\end{array}$ & $\begin{array}{r}16.18 \\
0.06 \\
0.38 \\
49.00\end{array}$ & $\begin{array}{r}0.23 \\
0.03 \\
13.05 \\
*\end{array}$ & $\begin{array}{l}2.39 \\
c .04 \\
1.68 \\
\end{array}$ & $\begin{array}{r}3.12 \\
0.08 \\
2.57 \\
30.00\end{array}$ & $\begin{array}{r}5.54 \\
0.09 \\
1.52 \\
27.00\end{array}$ & $\begin{array}{l}11.89 \\
-- \\
16.00\end{array}$ & $\begin{array}{r}C .03 \\
0.11 \\
366.66 \\
8.00\end{array}$ & $\begin{array}{r}10.06 \\
0.10 \\
1.00 \\
8.00\end{array}$ & $\begin{array}{r}120.82 \\
0.74\end{array}$ \\
\hline $19 \in 3$ & $\begin{array}{l}\text { PRECIP. (CM.) } \\
\text { SR-90 iNCI/SG.KN.) } \\
\text { SR-90 CCNC. (PC/L) } \\
\text { SR-89/SR-90 }\end{array}$ & $\begin{array}{r}22.68 \\
0.11 \\
0.49 \\
34.00\end{array}$ & $\begin{array}{l}10.59 \\
-- \\
--\end{array}$ & $\begin{array}{r}12.27 \\
0.04 \\
0.33 \\
*\end{array}$ & $\begin{array}{l}17.68 \\
0.06 \\
0.34 \\
\end{array}$ & $\begin{array}{l}9.17 \\
0.04 \\
0.44 \\
*\end{array}$ & $\begin{array}{l}9.58 \\
0.05 \\
0.53 \\
*\end{array}$ & $\begin{array}{l}8.28 \\
-- \\
--\end{array}$ & $\begin{array}{l}0.58 \\
0.03 \\
5.18 \\
*\end{array}$ & $\begin{array}{l}1.83 \\
0.08 \\
4.38 \\
*\end{array}$ & $\begin{array}{l}2.26 \\
0.02 \\
0.89 \\
2.00\end{array}$ & $\begin{array}{l}8.97 \\
0.07 \\
0.78 \\
*\end{array}$ & $\begin{array}{l}13.64 \\
0.06 \\
0.44 \\
*\end{array}$ & $\begin{array}{r}117.55 \\
0.56\end{array}$ \\
\hline 1964 & $\begin{array}{l}\text { PRECIP. }(C N-) \\
S R-90 \text { (NCI/SC-KM.) } \\
S R-G C C C A C .(P C / L) \\
S R-8 G / S R-9 O\end{array}$ & $\begin{array}{l}4.72 \\
0.03 \\
0.04 \\
*\end{array}$ & $\begin{array}{l}4.45 \\
0.03 \\
0.68 \\
*\end{array}$ & $\begin{array}{l}18.44 \\
0.02 \\
0.11 \\
--\end{array}$ & $\begin{array}{l}34.80 \\
0.02 \\
0.06 \\
-.\end{array}$ & $\begin{array}{l}10.16 \\
=- \\
=-\end{array}$ & $\begin{array}{l}2.16 \\
0.01 \\
0.47 \\
--\end{array}$ & $\begin{array}{l}4.06 \\
C .03 \\
C .74 \\
--\end{array}$ & $\begin{array}{l}-- \\
0.20 \\
--\end{array}$ & $\begin{array}{l}-- \\
0.31 \\
--\end{array}$ & $\begin{array}{l}2.03 \\
0.09 \\
4.44\end{array}$ & $\begin{array}{l}7.26 \\
* \\
-- \\
--\end{array}$ & $\begin{array}{r}20.40 \\
0.14 \\
0.69 \\
\ldots\end{array}$ & $\begin{array}{r}108.48 \\
0.88\end{array}$ \\
\hline 1965 & $\begin{array}{l}\text { PRECIP. ICM.) } \\
S R-9 C \text { INCI/SG.KN.) } \\
S R-9 C \text { CCNC. }(P C / L) \\
S R-89 / S R-90\end{array}$ & $\begin{array}{l}9.91 \\
0.11 \\
1.11 \\
\ldots\end{array}$ & $\begin{array}{r}19.05 \\
0.20 \\
1.05 \\
--\end{array}$ & $\begin{array}{l}6.30 \\
0.06 \\
0.96 \\
--\end{array}$ & $\begin{array}{l}14.91 \\
0.11 \\
0.74 \\
-.\end{array}$ & $\begin{array}{l}10.85 \\
C .06 \\
0.56 \\
--\end{array}$ & $\begin{array}{l}3.86 \\
0.03 \\
0.78 \\
--\end{array}$ & $\begin{array}{l}1.91 \\
C . c 2 \\
1.05 \\
--\end{array}$ & $\begin{array}{l}6.43 \\
0.12 \\
1.87 \\
-.\end{array}$ & $\begin{array}{l}6.86 \\
C .13 \\
1.90 \\
--\end{array}$ & $\begin{array}{l}24.05 \\
0.16 \\
0.67 \\
--\end{array}$ & $\begin{array}{r}13.75 \\
0.13 \\
0.95 \\
--\end{array}$ & $\begin{array}{r}28.74 \\
0.22 \\
0.77 \\
-.\end{array}$ & $\begin{array}{r}170.67 \\
1.35\end{array}$ \\
\hline 1966 & $\begin{array}{l}\text { PRECIP. }\{C M=) \\
S R-90 \text { INCI/SQ.KM.) } \\
S R-90 \text { CCNC. }(P C / L) \\
S R-85 / S R-9 C\end{array}$ & $\begin{array}{r}26.83 \\
0.06 \\
0.23 \\
-.\end{array}$ & $\begin{array}{r}64.47 \\
0.25 \\
0.39 \\
-.-\end{array}$ & $\begin{array}{l}21.58 \\
0.10 \\
0.47 \\
--\end{array}$ & $\begin{array}{l}9.19 \\
0.05 \\
0.55 \\
--\end{array}$ & $\begin{array}{l}9.85 \\
0.06 \\
0.61 \\
--\end{array}$ & $\begin{array}{l}7.50 \\
0.02 \\
0.27 \\
*\end{array}$ & $\begin{array}{l}1.84 \\
C .02 \\
1.09 \\
*\end{array}$ & $\begin{array}{l}1.46 \\
* \\
*\end{array}$ & $\begin{array}{r}2.19 \\
0.05 \\
2.29 \\
46.00\end{array}$ & $\begin{array}{l}-- \\
0.26 \\
-- \\
98.00\end{array}$ & $\begin{array}{l}-- \\
0.13 \\
31.00\end{array}$ & $\begin{array}{l}0.06 \\
16.00\end{array}$ & $\begin{array}{r}144.91 \\
1.06\end{array}$ \\
\hline 1967 & $\begin{array}{l}\text { PRECIP. }(C M \cdot) \\
\text { SR-9O INCI/SQ.KN.) } \\
S R-9 O C C N C \text {. }(P C / L) \\
S R-Q S / S R-S O\end{array}$ & $\begin{array}{r}34.01 \\
0.07 \\
0.21 \\
6.80\end{array}$ & $\begin{array}{l}13.63 \\
0.03 \\
0.23 \\
*\end{array}$ & $\begin{array}{l}23.62 \\
0.02 \\
0.09 \\
*\end{array}$ & $\begin{array}{l}6.45 \\
0.01 \\
0.16 \\
*\end{array}$ & $\begin{array}{l}9.47 \\
0.03 \\
0.32 \\
*\end{array}$ & $\begin{array}{r}1.33 \\
0.03 \\
2.26 \\
71.00\end{array}$ & $\begin{array}{l}-- \\
-- \\
--\end{array}$ & $\begin{array}{l}=- \\
-- \\
--\end{array}$ & $\begin{array}{l}-- \\
-- \\
--\end{array}$ & $\begin{array}{l}-- \\
-- \\
--\end{array}$ & $\begin{array}{l}-- \\
-- \\
--\end{array}$ & $\begin{array}{l}-- \\
-- \\
--\end{array}$ & $\begin{array}{r}83.51 \\
0.19\end{array}$ \\
\hline $\begin{array}{l}\text { NOTES } \\
-=: \\
*: 2 \\
A: 1 \\
B: 1 \\
C:\end{array}$ & $\begin{array}{l}\text { ATA NOT AVAILABLE } \\
\text { ERRO OR TRACE } \\
\text { PPROXIMATE } \\
\text { OWER LIMIT OF REPOR } \\
\text { OROPORTICNED FROM OR }\end{array}$ & $\begin{array}{l}\text { DAIA } \\
\text { VALLY }\end{array}$ & & & & & & & & & & & & \\
\hline
\end{tabular}




\begin{tabular}{|c|c|c|c|c|c|c|c|c|c|c|c|c|c|c|}
\hline & & JaN. & FES. & $\max$. & ant. & $\operatorname{mar}$ & sune & sut & AuG. & SEP. & OCT. & NOV. & DEC. & CUN. TOTAL \\
\hline 1958 & $\begin{array}{l}\text { PRECIP. ICM.) } \\
\text { SR-90 IFCI/SO-KR-) } \\
S R=90 \text { CONC. }(P C / L) \\
S R-9 / S R-90\end{array}$ & $=$ & $\bar{z}$ & $=$ & $\bar{z}$ & $=$ & $\begin{array}{r}0.58 \\
0.02 \\
3.45 \\
14.00\end{array}$ & $\begin{array}{r}0.08 \\
0.06 \\
C .75 \\
14.00\end{array}$ & $\begin{array}{r}5.97 \\
0.13 \\
2.18 \\
45.00\end{array}$ & $\begin{array}{r}7.62 \\
0.19 \\
2.50 \\
18.00\end{array}$ & $\begin{array}{r}6.22 \\
0.27 \\
4.35 \\
18.00\end{array}$ & $\begin{array}{r}0.86 \\
0.08 \\
9.53 \\
14.00\end{array}$ & $\begin{array}{r}0.64 \\
0.04 \\
6.26 \\
14.00\end{array}$ & $\begin{array}{r}29.95 \\
0.79\end{array}$ \\
\hline 1959 & $\begin{array}{l}\text { PRECIP. ICM-) } \\
\text { SR-90 (MCI/SQ.KM-) } \\
S R-90 \text { CONC. (PC/L) } \\
\text { SR-89/SR-9O }\end{array}$ & $\begin{array}{l}0.74 \\
0.03 \\
4.06 \\
\end{array}$ & $\begin{array}{l}2.11 \\
0.08 \\
3.80 \\
5.70\end{array}$ & $\begin{array}{l}2.36 \\
0.02 \\
0.85 \\
0.30\end{array}$ & $\begin{array}{l}0.86 \\
0.02 \\
2.33 \\
-\end{array}$ & $\begin{array}{l}0.38 \\
0.02 \\
5.27 \\
-\end{array}$ & $\begin{array}{l}0.91 \\
0.02 \\
2.20 \\
\end{array}$ & $\begin{array}{l}3.76 \\
0.04 \\
1.07 \\
5.40\end{array}$ & $\begin{array}{l}4.70 \\
0.08 \\
1.71 \\
6.00\end{array}$ & $\begin{array}{l}3.23 \\
0.08 \\
2.46 \\
0\end{array}$ & $\begin{array}{l}2.26 \\
C .11 \\
4.87 \\
-.\end{array}$ & $\begin{array}{l}2.36 \\
0.07 \\
2.97 \\
1.10\end{array}$ & $\begin{array}{l}5.08 \\
0.05 \\
0.99 \\
2.30\end{array}$ & $\begin{array}{r}28.75 \\
0.62\end{array}$ \\
\hline $196 \mathrm{C}$ & $\begin{array}{l}\text { PRECIP. (CM-) } \\
\text { SR-9O IPCI/SO-KM-) } \\
S R-90 \text { CQNC. }(P C / L) \\
S R-89 / S R-90\end{array}$ & $\begin{array}{l}0.97 \\
0.03 \\
3.10 \\
-\end{array}$ & $\begin{array}{l}4.78 \\
0.02 \\
0.21 \\
\end{array}$ & $\begin{array}{l}2.13 \\
0.04 \\
1.08 \\
-\end{array}$ & $\begin{array}{l}5.56 \\
0.05 \\
0.90 \\
\end{array}$ & $\begin{array}{l}12.12 \\
0.046 \\
0.34 \\
-\end{array}$ & $\begin{array}{l}4.06 \\
0.026 \\
0.5 \mathrm{C} \\
\end{array}$ & $\begin{array}{l}3.61 \\
0.046 \\
1.11 \\
-.\end{array}$ & $\begin{array}{l}4.01 \\
0.05 C \\
1.25 \\
-.\end{array}$ & $\begin{array}{l}9.25 \\
0.18 \mathrm{C} \\
1.95 \\
\end{array}$ & $\begin{array}{l}2.18 \\
C .04 C \\
1.84 \\
-.\end{array}$ & $\begin{array}{l}9.65 \\
0.216 \\
2.18 \\
--\end{array}$ & $\begin{array}{l}0.28 \\
0.016 \\
3.58 \\
--\end{array}$ & $\begin{array}{r}58.60 \\
0.72\end{array}$ \\
\hline 1961 & $\begin{array}{l}\text { PRECIP. }(C M-) \\
S R-90 \text { (FCI/SO.KM-) } \\
S R-90 \text { CONC. }(P C / L) \\
\text { SR-89/SR-90 }\end{array}$ & $\begin{array}{l}0.48 \\
0.02 C \\
4.27 \\
--\end{array}$ & $\begin{array}{l}0.61 \\
0.04 C \\
4.94 \\
-\end{array}$ & $\begin{array}{l}0.71 \\
0.000 \\
0.01 \\
-\end{array}$ & $\begin{array}{l}12.19 \\
0.076 \\
0.58 \\
-\end{array}$ & $\begin{array}{l}3.25 \\
0.046 \\
1.24 \\
-.\end{array}$ & $\begin{array}{l}6.50 \\
0.08 \mathrm{C} \\
1.24 \\
-\end{array}$ & $\begin{array}{l}5.21 \\
0.086 \\
1.56 \\
-\end{array}$ & $\begin{array}{l}3.84 \\
0.066 \\
1.57 \\
-\end{array}$ & $\begin{array}{l}2.39 \\
0.05 \\
2.10 \\
\end{array}$ & $\begin{array}{r}1.12 \\
C .16 \\
14.29 \\
*\end{array}$ & $\begin{array}{r}0.81 \\
0.12 \\
14.82\end{array}$ & $\begin{array}{l}0.56 \\
0.05 \\
8.93 \\
+\end{array}$ & $\begin{array}{r}37.87 \\
0.77\end{array}$ \\
\hline 1962 & $\begin{array}{l}P R E C I P \text { (CM-) } \\
S R-90 \text { (MCI/SO-KM) } \\
S R-9 C \text { CONC. }(P C / L) \\
S R-89 / S R-S C\end{array}$ & $\begin{array}{l}1.42 \\
0.06 \\
4.23 \\
4\end{array}$ & $\begin{array}{l}2.24 \\
0.13 \\
5.81 \\
+\end{array}$ & $\begin{array}{l}2.34 \\
0.06 \\
2.57 \\
*\end{array}$ & $\begin{array}{r}0.20 \\
0.02 \\
10.01 \\
5.00\end{array}$ & $\begin{array}{l}9.27 \\
0.09 \\
6.98 \\
5.00\end{array}$ & $\begin{array}{r}5.41 \\
0.10 \\
1.85 \\
36.0 \mathrm{C}\end{array}$ & $\begin{array}{r}3.38 \\
0.09 \\
2.67 \\
24.00\end{array}$ & $\begin{array}{r}5.87 \\
0.05 \\
0.86 \\
18.00\end{array}$ & $\begin{array}{r}1.47 \\
0.04 \\
2.73 \\
21.00\end{array}$ & $\begin{array}{r}6.55 \\
0.14 \\
2.14 \\
15.00\end{array}$ & $\begin{array}{l}1.35 \\
0.12 \\
0.89 \\
8.00\end{array}$ & $\begin{array}{l}4.29 \\
0.11 \\
2.57 \\
7.00\end{array}$ & $\begin{array}{r}43.79 \\
1.01\end{array}$ \\
\hline 1963 & 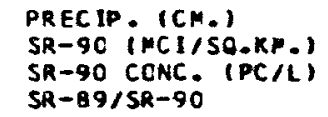 & $\begin{array}{r}8.00 \\
0.26 \\
3.26 \\
18.00\end{array}$ & $\begin{array}{r}0.23 \\
0.07 \\
30.44 \\
4.00\end{array}$ & $\begin{array}{l}0.08 \\
0.00 \\
0.01 \\
3.00\end{array}$ & $\begin{array}{l}4.67 \\
0.11 \\
2.36 \\
9.10\end{array}$ & $\begin{array}{r}12.90 \\
0.23 \\
1.79 \\
+\end{array}$ & $\begin{array}{r}10.29 \\
0.14 \\
1.37 \\
0.40\end{array}$ & $\begin{array}{r}10.13 \\
0.14 \\
1.39\end{array}$ & $\begin{array}{l}6.48 \\
C .02 \\
0.31 \\
+\end{array}$ & $\begin{array}{l}3.33 \\
0.07 \\
2.11 \\
\end{array}$ & $\begin{array}{l}4.70 \\
0.12 \\
2.56 \\
\end{array}$ & $\begin{array}{r}0.61 \\
0.07 \\
11.48\end{array}$ & $\begin{array}{r}0.28 \\
0.05 \\
17.86\end{array}$ & $\begin{array}{r}61.70 \\
1.28\end{array}$ \\
\hline 1964 & $\begin{array}{l}\text { PRECIP. }(C M-) \\
S R-90 \text { IMCIISO-KM-) } \\
S R-90 \text { CCNC. }(P C / L) \\
S R-89 / S R-90\end{array}$ & $\begin{array}{l}1.04 \\
0.07 \\
6.74 \\
.\end{array}$ & $\begin{array}{l}0.91 \\
0.07 \\
7.70 \\
.\end{array}$ & $\begin{array}{l}0.23 \\
0.02 \\
0.70 \\
0\end{array}$ & $\begin{array}{l}5.94 \\
0.12 \\
2.03 \\
\end{array}$ & $\begin{array}{l}4.93 \\
6.10 \\
2.03 \\
-.\end{array}$ & $\begin{array}{l}3.66 \\
0.19 \\
5.20 \\
-\end{array}$ & $\begin{array}{r}10.41 \\
c .35 \\
3.37 \\
-\end{array}$ & $\begin{array}{l}3.40 \\
6.17 \\
5.02 \\
\end{array}$ & $\begin{array}{l}7.99 \\
0.58 \\
7.30 \\
\end{array}$ & $\begin{array}{l}9.26 \\
0.30 \\
3.64 \\
-\end{array}$ & $\begin{array}{r}10.26 \\
0.27 \\
2.64 \\
\end{array}$ & $\begin{array}{l}2.06 \\
0.16 \\
7.77 \\
-.7\end{array}$ & $\begin{array}{r}59.05 \\
2.40\end{array}$ \\
\hline 1965 & $\begin{array}{l}\text { PRECIP. }\left(C M_{+}\right) \\
S R=90 \text { INCI/SO-KM.) } \\
S R-90 \text { CONC. (PC/L) } \\
S R-89 / S R-90\end{array}$ & $\begin{array}{r}0.23 \\
0.07 \\
30.44 \\
-\end{array}$ & $\stackrel{0.05}{-}$ & $\begin{array}{l}0.94 \\
0.06 \\
6.52 \\
\end{array}$ & $\begin{array}{l}2.67 \\
0.09 \\
3.36\end{array}$ & $\begin{array}{l}7.49 \\
0.20 \\
2.68 \\
\end{array}$ & $\begin{array}{l}3.00 \\
0.06 \\
2.01 \\
-.\end{array}$ & $\begin{array}{l}5.23 \\
0.19 \\
3.64 \\
-\end{array}$ & $\begin{array}{l}5.36 \\
0.20 \\
3.74 \\
-\end{array}$ & $\begin{array}{l}2.95 \\
0.16 \\
5.43 \\
-.\end{array}$ & $\begin{array}{r}0.53 \\
0.06 \\
11.33 \\
-\end{array}$ & $\begin{array}{l}2.59 \\
0.12 \\
4.64 \\
-\end{array}$ & $\begin{array}{l}3.15 \\
0.16 \\
5.08 \\
-.\end{array}$ & $\begin{array}{r}34.14 \\
1.44\end{array}$ \\
\hline 1966 & $\begin{array}{l}\text { PREC IP. (CM) } \\
\text { SR-9C (PCI/SQ-KM-) } \\
5 R-90 \text { CONC }(P C / L) \\
\text { SR-89/SR-90 }\end{array}$ & $\begin{array}{r}0.36 \\
0.10 \\
27.78 \\
\end{array}$ & $\begin{array}{l}1.37 \\
0.04 \\
2.92 \\
\end{array}$ & $\begin{array}{l}2.60 \\
0.07 \\
2.70 \\
\end{array}$ & $\begin{array}{l}0.71 \\
0.04 \\
5.64 \\
\end{array}$ & $\begin{array}{l}7.10 \\
0.09 \\
1.27 \\
-\end{array}$ & $\begin{array}{l}7.5 C \\
0.10 \\
1.34 \\
-0\end{array}$ & $\begin{array}{l}0.80 \\
0.11 \\
1.26 \\
\end{array}$ & $\begin{array}{l}3.70 \\
0.08 \\
2.17 \\
\end{array}$ & $\begin{array}{l}7.30 \\
0.17 \\
2.33 \\
\end{array}$ & $\begin{array}{l}3.60 \\
0.13 \\
3.62 \\
\end{array}$ & $\begin{array}{l}3.50 \\
0.12 \\
3.43 \\
\end{array}$ & $\begin{array}{l}6.80 \\
0.20 \\
2.95 \\
-\end{array}$ & $\begin{array}{r}53.34 \\
1.25\end{array}$ \\
\hline 1967 & $\begin{array}{l}\text { PRECIP. (CM.) } \\
\text { SR-90 (PCI/SO.KM.) } \\
\text { SR-90 CONC. (PC/L) } \\
\text { SR-89/SR-90 }\end{array}$ & $\begin{array}{l}1.60 \\
0.03 \\
1.88 \\
-\end{array}$ & $\begin{array}{l}3.60 \\
0.06 \\
1.67 \\
-\end{array}$ & $\begin{array}{r}0.30 \\
0.04 \\
13.34 \\
-\end{array}$ & $\begin{array}{l}1.00 \\
0.04 \\
4.01 \\
-\end{array}$ & $\begin{array}{l}2.60 \\
0.66 \\
2.31 \\
-\end{array}$ & $\begin{array}{l}0.91 \\
0.02 \\
2.20 \\
.\end{array}$ & $\begin{array}{l}5.00 \\
0.06 \\
1.21 \\
-\end{array}$ & $\bar{z}$ & $=$ & $=$ & $=$ & $\begin{array}{l}-- \\
--\end{array}$ & $\begin{array}{r}15.01 \\
0.31\end{array}$ \\
\hline 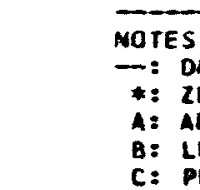 & $\begin{array}{l}\text { DATA NOT AVATLABLE } \\
\text { ERO OR TRACE } \\
\text { PPROXIMATE } \\
\text { OWER LIMIT OF REPO } \\
\text { PROPORTI ONEO FROM O }\end{array}$ & DATA & & & & & & & & & & & & \\
\hline
\end{tabular}


JAN. FEB. MAR. APR. MAY

1958 PRECIP. (CM.) SR-90 (NCI/SO.KM) $S R-90$ CONC. (PC/L) $S R-89 / S R-S O$

1959 PRECIP * (CM-) SR$S R-90$ CCNC. $(P C / L)$
$S R-89 / S R-90$

1960 PRECIP. (CM.) SR-GC INCI/SQ.KM.I $S R-9 C$ CONC. (PC/L)
$S R-89 / S R-90$

1961 PRECIP. (CM.) $S R-9 C$ (MCI/SQ.KM.) $S R-90$ CCNC. (PC/L) $S R-89 / S R-90$

1962 PRECIP. (CM.) 16.36 $S R-Q S / S R-S C$

1563 PRECIP. (CM.) SR-9O INCL/SC.KM, 14.73 $S R-9 C$ CCNC. (PC/L) $S R-89 / S R-90$

1964 PRECIP. (CM.) SR-90 (NCI ISO KM ) SR-SO TNCISSQ.KM. $S R-9 C$ CCNC.
$S R-89 / S R-90$ (CM.) 12.24 SR-90 (NCI/SC.KM.) 12.24 SR-9C CONC. (PC/L) 3.27 $S R-89 / S R-90$

1966 PRECIP. (CM.) SRETP ICM.) PRECIP. ICM. I $S R-89 / S R-90$

1967 PRECIP. (CM.) RECIP. ICM.) SR-9O CCNC. IPC/LI $S R-89 / 5 R-90$

$\begin{array}{llll}-- & -- & -- & = \\ -- & - & - & = \\ -- & - & - & -\end{array}$

$8.44 \quad 13.61 \quad 14.83$

0.00

$0.00 \quad 0.11$

C.

0.75
3.50

2.51
0.03

0.03
1.20

2.90

8.26

1.88

.1511 .56

C. 70

0.73

$-2$

1.60

$--$

17.68

C. $10 \mathrm{CC}$

4.11

$0.08 \mathrm{C}$

2.72

0.05
1.84

1.84

9.63

$9.63 \quad 11.00$

1.04

1.00
0.13
1.19

4.42
0.02

0.46

3.71

0.06

0.06
1.62

36.12

0.38

5.87

0.08
1.37

1.37
$*$

$15.65 \quad 29.51$

29.51

8.94

0.11

1. 24

*

4.60

0.81

$0.10 \quad 0.07$

2.18

8.6

5.66

0.11

1.95

11.30

10.80

12.30

12.30
0.07

0.07
0.57

1.

1.

5.70

0.06

1.06

0.42

$-$

NOTES

DATA NOT AVAILABLE

APPROXIMATE

: LOWER LIMIT OF REPORTED DATA

C: PROPORTIONED FROM ORIGINALLY CONSOLICATED OATA
JUNE

23.65

AUG.

SE P.

OCT.

Nov

DEC.

CUM. TOTAL

0.09

0.09
44.06

4.19

$4.95 \quad 3.51$

2.90

$0.10 \quad 0.09$

0.09
0.33

67.68

$46.04 \quad 2.23$

10.55
62.00

19.00

$--$

$\begin{array}{ll}2.95 & 1.17 \\ 0.05 & 0.07\end{array}$

$1.70 \quad 5.95$

6.58

0.46

10.34

11.91

20.27

13.36

$\begin{array}{lll}0.15 & 0.11 & 0.14\end{array}$

0.14
1.05

116.43
1.03

$\begin{array}{llllll}0.61 & 4.35 & 1.17 & 1.26 & 0.55 & 1.05 \\ 0.90 & * & * & 1.10 & * & *\end{array}$

$4.19 \quad 2.84 \quad 3.78$

1.17

3.05

3.05

11.89

11.58

71.40

$\begin{array}{ll}4.05 \mathrm{C} & 0.04 \mathrm{C} \\ 1.20 & 1.41\end{array}$

C. 53

0.86

1.64

1.64

$0.16 \mathrm{C} \quad 0.15 \mathrm{C}$

0.76

$\begin{array}{lll}3.33 & 3.10 & 7.39\end{array}$

$0.04 \mathrm{C}$

$\begin{array}{ll}0.21 & 0.03 \mathrm{C}\end{array}$

$0.06 \mathrm{C}$

2.62

0.0

$5.64 \quad 13.11$

1.30

107.58
1.02

3.23

$--$

\subsection{4}

1.4

1.6

$0.13 \quad 0.13$

1.12

105.14

1.50

0.93
38.00

$\begin{array}{ll}0.04 & 0.14 \\ 2.25 & 1.18\end{array}$

$41.0 \mathrm{C}$

22.00

0.07
18.79

$\begin{array}{ll}4.39 & 3.25 \\ 0.12 & 0.11\end{array}$

8.56

21.67

1.67

8.00

126.92

1.75

$0.120 .02 \quad 0.01$

6.96
0.12

1.70

8.66

13.18

17.07

1.82

118.79

$8.15 \quad 2.87 \quad 3.94$

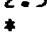

$+$

$\begin{array}{lll}0.06 & 0.10 & 0.05\end{array}$

2.16

9.04

5.61

5.11

19.76

0.44

$2 . \mathrm{CO}$

$-\infty$

1.27

6.95

4.7

$\begin{array}{lll}2.59 & 6.68 \quad 23.55\end{array}$

1.55

1.95

$3.10 \quad 20.90$

$0.03 \quad 0.03$

$0.97 \quad 0.15$

C.68

0.1

.25

0.24
2.05
--

7.34

11.56

6.83

0.08

1.18

101.65

$2.01 \quad 12.00$

2.32

1.30

3.60

$\begin{array}{rrr}5.30 & 16.90 & 10.60 \\ c .10 & 0.17 & 0.17\end{array}$

$\begin{array}{lll}1.89 & 1.01 & 1.61\end{array}$

$10.00 \quad 64.80$

$\begin{array}{ll}0.03 & 0.10 \\ c .31 & 0.16\end{array}$

1.00

0.5

0.56

--

$-$

$-$

4.70

$0.04=$

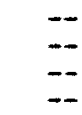

$=$

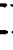

152.20
0.47

2.20
0.47

112.71

1.08 
JAN. FEB. MAR. APR. MAY

1958

PRECIP O (CMDI

SR-90 (MCI/SQ.KM-) SR-90 CONC. IPC

1959 PRECIP. (CM.) SR-90 (MCI/SQ.KM.) SR-90 CONC. $(P C / L)$ $S R-89 / S R \rightarrow 90$

1960 PRECIP. (CM.) $S R-89 / S R=90$

1961 PRECIP. (CM.) $S R=90(\mu \mathrm{CL} / S 0 . K M-126.31 \quad 36.03$ $S R-89 / S R-90$

1962 PRECIP. (CM.) SR-90 IMCI/SO.KM.I 0.02 $S R-89 / S R-90$

1963 PRECIP. (CM.) SRT-90 (MCI/SO.KM. $5 R-89 / S R-90$

1964 PRECIP. (CM.) 31.90 SR-90 (MCI/SQ.KM.) 0.25 $S R-90$ CONC. $(P C / L) \quad 0$.
$S R-89 / S R-90$

1965 PRECIP ICM, PRECIP ( $C M_{0}$ ) 13. SR-90 (MCI/SO.KM.) 0.24 SR -90 CONC. IPC/L) $S R-89 / S R-90$

1966 PRECIP. (CM.) $S R=90$ (MCI/SQ.KM.) $S R-90$ CONC
$S R-89 / S R-90$

1967 PRECIP. ICM. SR-90 INCI/SO.KM. $S R-90$ CONC. (PC/L)
$S R-89 / S R-90$

$=$
$=$
$=$
10.34
0.13
1.26
5.80
41.40
0.08
0.20
-
36.83
0.026
0.06
-

46.48 0.06 0.13

\subsection{2}

0.07
0.22

0.22
5.30

$=$

$=$

$14.07 \quad 33.12$

0.07

1.64

2.2

-

9.42

$0.04 C$
0.43

$0.42 \quad 100.00$

17.53

2.64
0.02
0.76

0.76
10.00

$*$

0.03

$+$

$22.73 \quad 12.52$

$*$

$\frac{0.05}{*}$

0.18

0.220 .64

\subsection{8}

\begin{tabular}{l}
0.18 \\
0.01 \\
5.56 \\
\hline
\end{tabular}

7.78

19.63

0.72

43.30

0.19

0.44

26.40
0.10

0.38

0.08

0.02

25.01

$\begin{array}{rrr}43.40 & 63.90 & 28.90 \\ 0.03 & 0.08 & 0.06\end{array}$

3.00

3.70

3.70
0.02
0.55

$-.07$

$-13$

0.2 $\overline{-}=$

JULY

LU6.

SEP

\subsection{17
51.52}

0.02

$\begin{array}{ll}51.52 & 66.67 \\ 13.00 \quad 22.00\end{array}$

43.00

*

1.22

0.01

0.00

7.00

$+$

$\begin{array}{ll}8.13 & 1.32 \quad \\ 0.03 & 0.036\end{array}$

$\begin{array}{lll}0.03 & 0.03 C & 0.00 C\end{array}$

0.016

$-$

0.016

$-$

$9.73 \quad 0.03$

$0.04 \mathrm{C} \quad 0.03 \mathrm{C}$$$
5.0
$$

$000 \quad *$

$* 01 \mathrm{0}$

0.016

$--$

$=$

$-$

$0.03 \quad 0.03$

33.34

0.02
66.67

0.0

18.00

$1.42 \quad 0.03$

$\begin{array}{lr}0.02 & 0.01 \\ 1.41 & 33.34\end{array}$

--

$*$
0.01
--

*
0.0

*

$-$

$*$

*.02

$-$

*

$*$

$\begin{array}{ll}* 0.02 \quad= \\ * & =\end{array}$ ocr.

NOV.

DEC.

NOTES

--: DATA NOT AVAILABLE

*: ZERO OR TRACE

A: APPROXIMATE

$B$ : LOWER LIMIT OF REPORTED DATA

C: PROPORTIONED FROM ORIGINALLY CONSOLIDATED DATA 
MONTHLY FALLOUT DEPOSITION COLLECTIONS

\begin{tabular}{|c|c|c|c|c|c|c|c|c|c|c|c|c|c|c|}
\hline & & JAN. & FEB. & MAR. & APR . & MAY & JUNE & JULY & AUS. & SEP. & CCT. & Nov. & LEC. & CUM. TOTAL \\
\hline 1958 & $\begin{array}{l}\text { PRECIP. (CM.) } \\
\text { SR-90 IMCI/SQ-KM_) } \\
\text { SR-90 CONC. (PC/L) } \\
\text { SR-89/SR-90 }\end{array}$ & $\bar{m}=$ & $\bar{z}$ & $=$ & $\overline{-}$ & $=$ & $\begin{array}{l}3.40 \\
0.03 \\
0.89 \\
2.10\end{array}$ & $\begin{array}{l}5.49 \\
0.02 \\
0.37 \\
\end{array}$ & $\begin{array}{r}13.82 \\
0.08 \\
0.58 \\
32.00\end{array}$ & $\begin{array}{r}2.21 \\
0.03 \\
1.36 \\
25.00\end{array}$ & $\begin{array}{r}12.24 \\
0.13 \\
1.07 \\
14.00\end{array}$ & $\begin{array}{r}3.25 \\
0.11 \\
3.39 \\
15.00\end{array}$ & $\begin{array}{r}13.06 \\
0.19 \\
1.46 \\
-\end{array}$ & $\begin{array}{r}53.47 \\
0.59\end{array}$ \\
\hline 1959 & $\begin{array}{l}\text { PRECIP. (CM.) } \\
\text { SR-9O (MCI/SQ-KM-) } \\
\text { SR-9C CCNC. (PC/L) } \\
S R-89 / S R-90\end{array}$ & $\begin{array}{l}3.45 \\
0.12 \\
3.48 \\
9.30\end{array}$ & $\begin{array}{l}4.24 \\
0.11 \\
2.60 \\
7.20\end{array}$ & $\begin{array}{l}1.12 \\
0.04 \\
3.58 \\
4.00\end{array}$ & $\begin{array}{l}5.23 \\
0.03 \\
0.58 \\
+\end{array}$ & $\begin{array}{l}1.80 \\
0.04 \\
2.23 \\
5.00\end{array}$ & $\begin{array}{l}3.51 \\
0.03 \\
0.86 \\
4.40\end{array}$ & $\begin{array}{l}5.46 \\
0.03 \\
0.55 \\
4.30\end{array}$ & $\begin{array}{l}1.60 \\
0.07 \\
4.30 \\
1.00\end{array}$ & $\begin{array}{l}7.82 \\
0.06 \\
0.77 \\
*\end{array}$ & $\begin{array}{l}2.54 \\
0.10 \\
3.94 \\
1.70\end{array}$ & $\begin{array}{l}2.11 \\
0.05 \\
2.37 \\
*\end{array}$ & $\begin{array}{l}10.01 \\
0.07 \\
0.70 \\
*\end{array}$ & $\begin{array}{r}48.89 \\
0.75\end{array}$ \\
\hline 1960 & $\begin{array}{l}\text { PRECIP, }\left(C M_{-}\right) \\
\text {SR-9C IMCI/SQ.KM.) } \\
\text { SR-9C CONC. }(P C / L) \\
\text { SR-89/SR- } 90\end{array}$ & $\begin{array}{l}6.25 \\
0.08 \\
1.29 \\
--\end{array}$ & $\begin{array}{l}1.24 \\
0.03 \\
2.42 \\
-.\end{array}$ & $\begin{array}{l}1.50 \\
0.02 \\
1.34 \\
-\end{array}$ & $\begin{array}{r}24.77 \\
0.12 \\
0.49 \\
-\end{array}$ & $\begin{array}{l}9.68 \\
0.044 \\
0.42 \\
-\end{array}$ & $\begin{array}{l}5.05 \\
0.02 \\
0.40 \\
-\end{array}$ & $\begin{array}{l}5.41 \\
0.036 \\
0.56 \\
-\end{array}$ & $\begin{array}{l}2.62 \\
0.02 \mathrm{C} \\
0.77 \\
-\end{array}$ & $\begin{array}{l}8.36 \\
0 . \csc 1 \\
c .60 \\
-\end{array}$ & $\begin{array}{l}6.53 \\
C .04 C \\
C .62 \\
\end{array}$ & $\begin{array}{l}2.95 \\
0.066 \\
2.04 \\
-\infty\end{array}$ & $\begin{array}{l}0.99 \\
0.02 C \\
2.03 \\
--\end{array}$ & $\begin{array}{r}75.35 \\
0.53\end{array}$ \\
\hline 1961 & $\begin{array}{l}\text { PRECIP. ICM-) } \\
S R-9 C \text { (PCI/SO.KM.) } \\
S R-90 \text { CONC. }(P C / L) \\
S R-89 / S R-90\end{array}$ & $\begin{array}{l}2.90 \\
0.06 C \\
2.07 \\
-\end{array}$ & $\begin{array}{l}3.05 \\
0.065 \\
1.97 \\
--\end{array}$ & $\begin{array}{l}1.098 \\
0.06 \\
3.04 \\
\end{array}$ & $=$ & $\begin{array}{l}3.12 \\
0.06 \\
1.9 .9 \\
\end{array}$ & $\begin{array}{l}5.996 \\
0.076 \\
1.17 \\
--\end{array}$ & $\begin{array}{l}5.996 \\
0.076 \\
1.17 \\
-\end{array}$ & $\begin{array}{l}7.21 \\
0.096 \\
1.25 \\
-.\end{array}$ & $\begin{array}{l}3.33 \\
\cos 107 \\
2.041\end{array}$ & $\begin{array}{l}2.95 \\
0.14 \\
4.75 \\
+\end{array}$ & $\begin{array}{l}1.35 \\
0.02 \\
1.49 \\
*\end{array}$ & $\begin{array}{l}4.14 \\
0.08 \\
1.94\end{array}$ & $\begin{array}{r}47.01 \\
0.79\end{array}$ \\
\hline 1962 & $\begin{array}{l}\text { PRECIP. }(C M .) \\
\text { SR-90 (MCI/SQ.KM.) } \\
S R-90 \text { CONC. (PC/L) } \\
\text { SR-B9/SR-90 }\end{array}$ & $\begin{array}{l}3.45 \\
0.08 \\
2.32 \\
*\end{array}$ & $\begin{array}{l}1.37 \\
0.05 \\
3.65 \\
*\end{array}$ & $\begin{array}{l}3.53 \\
0.05 \\
1.42 \\
*\end{array}$ & $\begin{array}{l}4.19 \\
0.03 \\
0.72 \\
+\end{array}$ & $\begin{array}{l}6.58 \\
0.033 \\
0.46 \\
*\end{array}$ & $\begin{array}{r}6.17 \\
0.06 \\
0.98 \\
20.00\end{array}$ & $\begin{array}{l}6.53 \\
0.06 \\
0.92 \\
5.00\end{array}$ & $\begin{array}{r}9.22 \\
0.04 \\
0.44 \\
20.00\end{array}$ & $\begin{array}{r}9.75 \\
0.07 \\
0.72 \\
19.00\end{array}$ & $\begin{array}{l}7.49 \\
0.07 \\
0.94 \\
\end{array}$ & $\begin{array}{r}2.31 \\
0.07 \\
3.04 \\
10.00\end{array}$ & $\begin{array}{l}4.39 \\
0.08 \\
1.83 \\
7.00\end{array}$ & $\begin{array}{r}64.98 \\
0.69\end{array}$ \\
\hline 1963 & $\begin{array}{l}\text { PRECIP, }\left(C M_{-}\right) \\
\text {SR-9C IMCI/SQ.KM.) } \\
\text { SR-90 CONC. }(P C / L) \\
\text { SR-89/SR-90 }\end{array}$ & $\begin{array}{l}2.24 \\
C .07 \\
3.13 \\
5.80\end{array}$ & $\begin{array}{l}2.64 \\
0.08 \\
3.04 \\
4.00\end{array}$ & $\begin{array}{l}2.92 \\
6.05 \\
1.72 \\
7.00\end{array}$ & $\begin{array}{l}1.02 \\
0.03 \\
2.95 \\
3.60\end{array}$ & $\begin{array}{l}3.05 \\
0.10 \\
3.28 \\
3.80\end{array}$ & $\begin{array}{l}2.82 \\
0.03 \\
1.07 \\
+\end{array}$ & $\begin{array}{l}8.30 \\
0.09 \\
0.97 \\
*\end{array}$ & $\begin{array}{l}3.78 \\
0.03 \\
0.80 \\
--\end{array}$ & $\begin{array}{l}7.1 .1 \\
0.06 \\
1.46\end{array}$ & $\begin{array}{l}1.10 \\
0.10 \\
3.23 \\
*\end{array}$ & $\begin{array}{l}3.20 \\
0.16 \\
5.01 \\
+\end{array}$ & $\begin{array}{l}1.40 \\
0.08 \\
5.72 \\
*\end{array}$ & $\begin{array}{r}39.58 \\
0.88\end{array}$ \\
\hline 1964 & $\begin{array}{l}\text { PRECIP. (CM.) } \\
\text { SR-9C I KCI/SQ.KM.) } \\
S R-90 \text { CONC. (PC/L) } \\
\text { SR-89/SR-90 }\end{array}$ & $\begin{array}{l}4.09 \\
0.10 \\
2.45 \\
*\end{array}$ & $\begin{array}{r}17.07 \\
0.28 \\
1.65 \\
*\end{array}$ & $\begin{array}{l}5.54 \\
0.04 \\
0.73 \\
*\end{array}$ & $\begin{array}{l}2.84 \\
0.07 \\
2.47 \\
*\end{array}$ & $\begin{array}{l}9.32 \\
0.12 \\
1.29 \\
--\end{array}$ & $\begin{array}{l}5.69 \\
0.06 \\
1.06 \\
-\end{array}$ & $\begin{array}{l}6.25 \\
0.13 \\
2.09 \\
\end{array}$ & $\begin{array}{l}4.90 \\
0.13 \\
2.66 \\
-\end{array}$ & $\begin{array}{l}5.56 \\
0.14 \\
2.52 \\
--\end{array}$ & $\begin{array}{l}3.07 \\
0.07 \\
2.29 \\
\end{array}$ & $\begin{array}{l}5.41 \\
0.20 \\
3.70 \\
-\end{array}$ & $\begin{array}{l}9.09 \\
0.39 \\
4.30 \\
-\end{array}$ & $\begin{array}{r}78.83 \\
1.73\end{array}$ \\
\hline 1965 & $\begin{array}{l}\text { PRECIP. }\left(C M_{-}\right) \\
\text {SR-90 (MCI/SQ.KM.) } \\
\text { SR-90 CONC. (PC/L) } \\
\text { SR-89/SR-90 }\end{array}$ & $\begin{array}{l}6.27 \\
0.36 \\
5.75 \\
--\end{array}$ & $\begin{array}{r}0.79 \\
0.10 \\
12.66 \\
--\end{array}$ & $\begin{array}{l}4.55 \\
0.20 \\
4.40 \\
-\end{array}$ & $\begin{array}{l}10.21 \\
0.20 \\
1.96 \\
--\end{array}$ & $\begin{array}{l}7.59 \\
0.13 \\
1.72 \\
\end{array}$ & $\begin{array}{l}1.35 \\
0.02 \\
1.49 \\
--\end{array}$ & $\begin{array}{l}0.84 \\
0.03 \\
3.58 \\
--\end{array}$ & $\begin{array}{l}-4.50 \\
0.08 \\
1.78 \\
--\end{array}$ & $\begin{array}{l}3.71 \\
0.07 \\
1.09 \\
\end{array}$ & $\begin{array}{l}4.002 \\
0.14 \\
3.449 \\
-\end{array}$ & $\begin{array}{l}\log 33 \\
0.12 \\
1.76\end{array}$ & $\begin{array}{l}3.18 \\
0.12 \\
3.78 \\
--\end{array}$ & $\begin{array}{r}53.84 \\
1.57\end{array}$ \\
\hline 1966 & $\begin{array}{l}\text { PRECIP, (CM.) } \\
\text { SR-9O (NCI/SQ.KM.) } \\
\text { SR-9O CONC. (PC/L) } \\
\text { SR-89/SR-90 }\end{array}$ & $\begin{array}{l}1.22 \\
0.06 \\
4.92 \\
--\end{array}$ & $\begin{array}{l}1.90 \\
0.05 \\
2.64 \\
-\end{array}$ & $\begin{array}{l}8.40 \\
0.11 \\
1.31 \\
-.\end{array}$ & $\begin{array}{l}8.10 \\
0.11 \\
1.36 \\
-.\end{array}$ & $\begin{array}{l}4.30 \\
0.06 \\
1.40 \\
-1\end{array}$ & $\begin{array}{l}0.97 \\
0.04 \\
4.13 \\
--\end{array}$ & $\begin{array}{l}9.50 \\
c .05 \\
0.53 \\
--\end{array}$ & $\begin{array}{l}5.80 \\
0.07 \\
1.21 \\
-\end{array}$ & $\begin{array}{r}13 . .30 \\
0.007 \\
0.53 \\
-\end{array}$ & $\begin{array}{r}10.60 \\
C .11 \\
1.04 \\
-\end{array}$ & $\begin{array}{l}2.90 \\
0.09 \\
3.11 \\
-\end{array}$ & $\begin{array}{l}1.70 \\
0.12 \\
7.06 \\
-.\end{array}$ & $\begin{array}{r}68.69 \\
0.94\end{array}$ \\
\hline 1967 & $\begin{array}{l}\text { PRECIP. }(C M .) \\
\text { SR-90 (MCI/SQ.KH.) } \\
\text { SR-90 CONC. }(P C / L) \\
S R-89 / S R-90\end{array}$ & $\begin{array}{l}1.60 \\
0.06 \\
3.76 \\
\end{array}$ & $\begin{array}{l}2.40 \\
0.03 \\
1.26 \\
-\end{array}$ & $\begin{array}{l}1.60 \\
0.04 \\
2.51 \\
--\end{array}$ & $\begin{array}{l}2.10 \\
0.05 \\
2.39 \\
--\end{array}$ & $\begin{array}{l}1.20 \\
0.03 \\
2.51 \\
\end{array}$ & $\begin{array}{l}6.20 \\
0.03 \\
0.49 \\
-.\end{array}$ & $\begin{array}{l}10.20 \\
0.04 \\
0.40 \\
--\end{array}$ & $\bar{m}$ & $\bar{m}$ & $\overline{-}$ & $\overline{-}$ & $=$ & $\begin{array}{r}25.30 \\
0.28\end{array}$ \\
\hline $\begin{array}{l}\text { NOTES } \\
*:=0 \\
*: 2 \\
B=A \\
B=L \\
C: D\end{array}$ & $\begin{array}{l}\text { ATA NOT AVAILABLE } \\
\text { ERO DR TRACE } \\
\text { PPROXIMATE } \\
\text { OWER LIMIT OF REPO } \\
\text { ROPORTI ONED FROM O }\end{array}$ & DAT & & & & & & & & & & & & \\
\hline
\end{tabular}


RCE: ATOMIC WEAPONS TESTS SAFETY COMMITTEE

(POT USED THRDUGH DECEMBER, 1963)

\begin{tabular}{|c|c|c|c|c|c|c|c|c|c|c|c|c|c|c|}
\hline & & JAN. & FEB. & MAR. & APR . & MAY & JUNE & JULY & ALG. & SEP. & OCT. & NOV. & DEC. & CUM. TOTAL \\
\hline 1958 & $\begin{array}{l}\text { PRECIP }(C M+) \\
\text { SR-90 }\left(M C I / S Q_{-K M-)}\right. \\
\text { SR-90 CONC. (PC/L) } \\
S R-89 / S R-90\end{array}$ & $\begin{array}{l}-- \\
--\end{array}$ & $=-$ & $=$ & $=-$ & $\overrightarrow{-}$ & $\begin{array}{l}1.83 \\
0.03 \\
1.64 \\
2.50\end{array}$ & $\begin{array}{r}5.03 \\
0.02 \\
0.40 \\
22.00\end{array}$ & $\begin{array}{r}8.10 \\
0.07 \\
0.87 \\
12.00\end{array}$ & $\begin{array}{r}7.70 \\
0.09 \\
1.17 \\
28.00\end{array}$ & $\begin{array}{r}10.26 \\
0.17 \\
1.66 \\
20.00\end{array}$ & $\begin{array}{l}7.04 \\
0.11 \\
1.57 \\
=-\end{array}$ & $\begin{array}{r}3.18 \\
0.09 \\
2.84 \\
11.00\end{array}$ & $\begin{array}{r}43.14 \\
0.58\end{array}$ \\
\hline 1959 & $\begin{array}{l}\text { PRECIP }\{C M=1 \\
S R=90 \text { (KCI/SQ.KM.) } \\
S R-90 \text { CONC. (PC/L) } \\
S R-99 / S R-90\end{array}$ & $\begin{array}{l}1.37 \\
0.07 \\
5.11 \\
*\end{array}$ & $\begin{array}{l}3.25 \\
0.12 \\
3.70 \\
4.40\end{array}$ & $\begin{array}{r}10.41 \\
0.09 \\
0.87 \\
4.60\end{array}$ & $\begin{array}{l}1.57 \\
0.04 \\
2.55 \\
*\end{array}$ & $\begin{array}{l}3.73 \\
0.04 \\
1.08 \\
3.60\end{array}$ & $\begin{array}{l}6.15 \\
0.08 \\
1.31 \\
+\end{array}$ & $\begin{array}{l}6.53 \\
0.05 \\
0.77 \\
*\end{array}$ & $\begin{array}{l}4.70 \\
0.06 \\
1.28 \\
-.\end{array}$ & $\begin{array}{l}10.41 \\
0.10 \\
0.97 \\
+\end{array}$ & $\begin{array}{l}8.28 \\
=\end{array}$ & $\begin{array}{l}2.34 \\
0.09 \\
3.05\end{array}$ & $\begin{array}{l}6.96 \\
0.09 \\
1.30 \\
+\end{array}$ & $\begin{array}{r}65.70 \\
0.83\end{array}$ \\
\hline 1960 & $\begin{array}{l}\text { PRECIP. (CM.) } \\
\text { SR-9O (MCI/SQ.KM-) } \\
\text { SR-9O CONC. (PC/L) } \\
\text { SR-BS/SR-SO }\end{array}$ & $\begin{array}{l}4.57 \\
0.07 \\
1.54 \\
--\end{array}$ & $\begin{array}{l}6.12 \\
0.05 \\
0.82 \\
--\end{array}$ & $\begin{array}{l}0.97 \\
0.06 \\
0.19 \\
-.\end{array}$ & $\begin{array}{r}19.48 \\
0.10 \\
0.52 \\
-. .\end{array}$ & $\begin{array}{l}9.53 \\
0.03 C \\
0.32 \\
-.\end{array}$ & $\begin{array}{l}1.98 \\
0.016 \\
0.51 \\
-\end{array}$ & $\begin{array}{l}5.18 \\
0.05 C \\
0.97 \\
--\end{array}$ & $\begin{array}{l}7.09 \\
0.076 \\
0.99 \\
\end{array}$ & $\begin{array}{l}9.25 \\
0.15 \mathrm{C} \\
1.63 \\
.\end{array}$ & $\begin{array}{l}3.89 \\
0.06 c \\
1.55 \\
-.\end{array}$ & $\begin{array}{l}13.79 \\
0.23 C \\
1.67 \\
-\infty\end{array}$ & $\begin{array}{l}0.40 \\
0.111 \\
2.72 \\
=.\end{array}$ & $\begin{array}{r}88.25 \\
0.99\end{array}$ \\
\hline 1961 & $\begin{array}{l}\text { PRECIP. }(C M+) \\
S R-90\left(M C I / S Q_{-K M}\right) \\
S R-90 \text { CONC. }(P C / L) \\
S R-B 9 / S R-90\end{array}$ & $\begin{array}{l}3.35 \\
0.05 \mathrm{C} \\
1.50 \\
=-\end{array}$ & $\begin{array}{l}3.76 \\
0.06 C, \\
1.60 \\
-.\end{array}$ & $\begin{array}{l}5.99 \\
0.08 C \\
1.34 \\
-.\end{array}$ & $\begin{array}{l}7.57 \\
0.106 \\
1.33 \\
=-\end{array}$ & $\begin{array}{l}7.80 \\
0.096 \\
1.16 \\
--\end{array}$ & $\begin{array}{l}5.13 \\
0.06 \mathrm{C} \\
1.17 \\
-.\end{array}$ & $\begin{array}{l}5.36 \\
0.056 \\
1.12 \\
-.\end{array}$ & $\begin{array}{l}5.59 \\
0.06 C \\
1.08 \\
--\end{array}$ & $\begin{array}{l}2.57 \\
0.05 \\
1.95 \\
*\end{array}$ & $\begin{array}{l}3.58 \\
0.11 \\
3.08 \\
+\end{array}$ & $\begin{array}{l}2.62 \\
0.15 \\
5.73 \\
\end{array}$ & $\begin{array}{l}4.72 \\
0.11 \\
2.34 \\
*\end{array}$ & $\begin{array}{r}58.04 \\
0.98\end{array}$ \\
\hline 1962 & $\begin{array}{l}\text { PREC IP. (CM.) } \\
\text { SR-9O IMCI/SQ.KM.) } \\
\text { SR-90 CONC. (PC/L) } \\
S R-89 / S R-S O\end{array}$ & $\begin{array}{l}6.02 \\
0.17 \\
2.83 \\
*\end{array}$ & $\begin{array}{l}3.07 \\
0.15 \\
4.89 \\
+\end{array}$ & $\begin{array}{l}0.97 \\
0.05 \\
5.16 \\
*\end{array}$ & $\begin{array}{l}2.69 \\
0.06 \\
2.24 \\
5.00\end{array}$ & $\begin{array}{r}11.63 \\
0.06 \\
0.52 \\
3.00\end{array}$ & $\begin{array}{l}4.19 \\
0.01 \\
0.24 \\
*\end{array}$ & $\begin{array}{r}4.32 \\
0.08 \\
1.86 \\
16.00\end{array}$ & $\begin{array}{l}5.33 \\
0.06 \\
1.13 \\
9.00\end{array}$ & $\begin{array}{l}3.10 \\
0.10 \\
3.23 \\
9.00\end{array}$ & $\begin{array}{r}11.10 \\
6.11 \\
1.00 \\
19.00\end{array}$ & $\begin{array}{l}2.67 \\
0.10 \\
3.75 \\
8.00\end{array}$ & $\begin{array}{l}3.53 \\
0.17 \\
4.82 \\
7.00\end{array}$ & $\begin{array}{r}58.62 \\
1.12\end{array}$ \\
\hline 1963 & $\begin{array}{l}\text { PRECIP. }(C M=) \\
\text { SR-90 (HCI/SQ.KM-) } \\
5 R-90 \text { CONC. (PC/L) } \\
S R-89 / S R-90\end{array}$ & $\begin{array}{l}17.58 \\
0.19 \\
1.09 \\
--\end{array}$ & $\begin{array}{l}4.88 \\
0.18 \\
3.69 \\
--\end{array}$ & $\begin{array}{l}4.88 \\
0.09 \\
1.85 \\
8.00\end{array}$ & $\begin{array}{l}1.09 \\
0.05 \\
4.59 \\
2.90\end{array}$ & $\begin{array}{l}7.77 \\
0.13 \\
1.68 \\
*\end{array}$ & $\begin{array}{l}4.27 \\
0.05 \\
1.18 \\
*\end{array}$ & $\begin{array}{l}0.36 \\
0.16 \\
1.92 \\
+\end{array}$ & $\begin{array}{l}4.39 \\
0.03 \\
0.09 \\
*\end{array}$ & $\begin{array}{l}9.17 \\
0.13 \\
1.42 \\
+\end{array}$ & $\begin{array}{l}0.79 \\
0.22 \\
2.51 \\
*\end{array}$ & $\begin{array}{l}2.49 \\
0.22 \\
8.84 \\
*\end{array}$ & $\begin{array}{l}1.96 \\
0.09 \\
4.60 \\
*\end{array}$ & $\begin{array}{r}75.63 \\
1.54\end{array}$ \\
\hline 1964 & $\begin{array}{l}\text { PRECIP. }(C M .) \\
S R-90 \quad\left(M C I / S Q_{-K M}\right) \\
S R-90 \text { CONC. (PC/L) } \\
S R-89 / S R-90\end{array}$ & $\begin{array}{r}0.23 \\
0.03 \\
13.05 \\
+\end{array}$ & $\begin{array}{r}11.94 \\
0.26 \\
2.18 \\
+\end{array}$ & $\begin{array}{l}2.79 \\
0.13 \\
4.66 \\
*\end{array}$ & $\begin{array}{r}10.01 \\
0.14 \\
1.40 \\
+\end{array}$ & $\begin{array}{l}7.57 \\
0.15 \\
1.99 \\
--\end{array}$ & $\begin{array}{l}2.9 C \\
0.12 \\
4.14 \\
0\end{array}$ & $\begin{array}{l}6.93 \\
0.27 \\
3.90 \\
-.\end{array}$ & $\begin{array}{l}5.18 \\
0.25 \\
4.83 \\
--\end{array}$ & $\begin{array}{l}7.75 \\
0.40 \\
5.17 \\
+\end{array}$ & $\begin{array}{l}6.81 \\
0.16 \\
2.35 \\
-.\end{array}$ & $\begin{array}{r}3.43 \\
0.36 \\
10.50 \\
--\end{array}$ & $\begin{array}{l}9.55 \\
0.60 \\
6.29 \\
--\end{array}$ & $\begin{array}{r}75.09 \\
2.87\end{array}$ \\
\hline 1965 & $\begin{array}{l}\text { PRECIP. (CM.) } \\
\text { SR-90 }(M C I / S Q . K M-) \\
S R-90 \text { CONC. (PC/L) } \\
S R-89 / S R-90\end{array}$ & $\begin{array}{l}1.32 \\
0.20 \\
15.16 \\
--\end{array}$ & $\begin{array}{l}0.05 \\
0.03 \\
60.01 \\
-\infty\end{array}$ & $\begin{array}{l}1.40 \\
0.11 \\
7.86 \\
--\end{array}$ & $\begin{array}{r}11.30 \\
0.17 \\
1.51 \\
--\end{array}$ & $\begin{array}{l}7.32 \\
0.14 \\
1.92 \\
--\end{array}$ & $\begin{array}{l}2.67 \\
0.12 \\
4.50 \\
-.\end{array}$ & $\begin{array}{l}6.27 \\
0.16 \\
2.56 \\
--\end{array}$ & $\begin{array}{r}10.08 \\
0.28 \\
2.78 \\
-\end{array}$ & $\begin{array}{l}4.85 \\
0.18 \\
3.72 \\
--\end{array}$ & $\begin{array}{l}1.27 \\
0.11 \\
8.67 \\
-.\end{array}$ & $\begin{array}{l}7.34 \\
0.20 \\
2.73 \\
--\end{array}$ & $\begin{array}{l}0.63 \\
0.09 \\
1.36 \\
-0\end{array}$ & $\begin{array}{r}60.50 \\
1.79\end{array}$ \\
\hline 1966 & $\begin{array}{l}\text { PRECIP (CM.) } \\
\text { SR-90 (MCI/SQ.KM.) } \\
5 R-90 \text { CONC. (PC/L) } \\
S R-89 / S R-90\end{array}$ & $\begin{array}{l}3.90 \\
0.09 \\
2.31 \\
--\end{array}$ & $\begin{array}{l}4.60 \\
0.07 \\
1.53 \\
--\end{array}$ & $\begin{array}{l}6.90 \\
0.12 \\
1.74 \\
-.\end{array}$ & $\begin{array}{l}0.20 \\
0.08 \\
1.30 \\
-=\end{array}$ & $\begin{array}{l}6.00 \\
0.05 \\
0.04 \\
-\end{array}$ & $\begin{array}{l}3.80 \\
0.03 \\
0.19 \\
\sim\end{array}$ & $\begin{array}{l}5.20 \\
0.08 \\
1.54 \\
--\end{array}$ & $\begin{array}{l}6.10 \\
0.06 \\
0.99 \\
-\end{array}$ & $\begin{array}{l}0.30 \\
0.06 \\
0.96 \\
--\end{array}$ & $\begin{array}{l}6.50 \\
0.14 \\
2.16 \\
=-\end{array}$ & $\begin{array}{l}4.00 \\
0.07 \\
1.76\end{array}$ & $\begin{array}{c}10.20 \\
0.21 \\
2.06 \\
--\end{array}$ & $\begin{array}{r}69.70 \\
1.06\end{array}$ \\
\hline 1967 & $\begin{array}{l}\text { PRECIP. (CM.) } \\
\text { SR-9D (MCI/SQ.KM.) } \\
\text { SR-9O CONC. (PC/L) } \\
S R-89 / S R-90\end{array}$ & $\begin{array}{l}2.10 \\
0.07 \\
3.34 \\
--\end{array}$ & $\begin{array}{l}0.36 \\
0.03 \\
8.34 \\
-\end{array}$ & $\begin{array}{l}1.40 \\
0.04 \\
2.86 \\
--\end{array}$ & $\begin{array}{l}6.00 \\
0.04 \\
0.67 \\
--\end{array}$ & $\begin{array}{l}4.20 \\
0.04 \\
0.96 \\
-0\end{array}$ & $\begin{array}{l}3.60 \\
0.02 \\
0.56 \\
--\end{array}$ & $\begin{array}{l}3.90 \\
0.04 \\
1.03 \\
-.\end{array}$ & $=-$ & 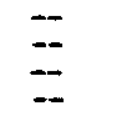 & $=$ & $\begin{array}{l}-- \\
--\end{array}$ & $=$ & $\begin{array}{r}21.56 \\
0.28\end{array}$ \\
\hline \multicolumn{15}{|c|}{$\begin{array}{l}\text { NOTES } \\
\text {-: DATA NOT AVAILABLE } \\
\text { *: LERO OR TRACE } \\
\text { A: APPROXIMATE } \\
\text { B: LOWER LIMIT OF REPORTED DATA } \\
\text { C: PROPORTIONED FROM ORIGINALLY }\end{array}$} \\
\hline
\end{tabular}




\begin{tabular}{|c|c|c|c|c|c|c|c|c|c|c|c|c|c|c|}
\hline & & JAN. & FEB. & MAR. & APR. & MAY & JLNE & JULY & AUG. & SEP. & OCT. & Nov. & LEC. & CUM. TOTAL \\
\hline 1958 & $\begin{array}{l}\text { PRECIP. (CMO) } \\
\text { SR-90 (MCLI/SQ.KMM-) } \\
\text { SR-90 CONC. (PC/L) } \\
\text { SR } \rightarrow-89 / S R-S O\end{array}$ & $\begin{array}{l}=- \\
= \\
=\end{array}$ & $\begin{array}{l}= \\
= \\
=\end{array}$ & $\begin{array}{l}=- \\
= \\
=\end{array}$ & $\begin{array}{l}\bar{z} \\
\overline{-}\end{array}$ & $\begin{array}{l}=- \\
\overline{-}\end{array}$ & $\begin{array}{l}7.01 \\
0.03 \\
0.43 \\
8.90\end{array}$ & $\begin{array}{r}42.49 \\
0.06 \\
0.15 \\
15.00\end{array}$ & $\begin{array}{r}6.76 \\
0.07 \\
1.04 \\
40.00\end{array}$ & $\begin{array}{r}3.38 \\
0.13 \\
3.85 \\
31.00\end{array}$ & $\begin{array}{r}6.50 \\
0.04 \\
0.62 \\
20.00\end{array}$ & $\begin{array}{r}1.17 \\
0.04 \\
3.42 \\
17.00\end{array}$ & $\begin{array}{r}1.24 \\
0.06 \\
4.84 \\
14.00\end{array}$ & $\begin{array}{r}68.55 \\
0.43\end{array}$ \\
\hline 1959 & $\begin{array}{l}\text { PRECIP. (CM.) } \\
\text { SR-9C IMCI/SQ.KM.) } \\
\text { SR-90 CCNC. (PC/L) } \\
\text { SR-89/SR-90 }\end{array}$ & $\begin{array}{r}0.58 \\
0.02 \\
3.45 \\
13.00\end{array}$ & $\begin{array}{l}0.86 \\
0.03 \\
3.49 \\
7.00\end{array}$ & $\begin{array}{l}0.51 \\
0.05 \\
9.81 \\
5.00\end{array}$ & $\begin{array}{l}3.12 \\
0.04 \\
1.29 \\
4.00\end{array}$ & $\begin{array}{l}7.52 \\
c .10 \\
1.33 \\
3.20\end{array}$ & $\begin{array}{l}16.15 \\
0.11 \\
0.69 \\
*\end{array}$ & $\begin{array}{l}7.77 \\
c .0 .7 \\
0.91 \\
4.70\end{array}$ & $\begin{array}{r}11.53 \\
0.09 \\
0.79 \\
*\end{array}$ & $\begin{array}{l}3.05 \\
0.08 \\
2.63 \\
*\end{array}$ & $\begin{array}{l}5.33 \\
0.07 \\
1.32 \\
.\end{array}$ & $\begin{array}{l}3.58 \\
0.02 \\
0.56 \\
*\end{array}$ & $\begin{array}{l}1.32 \\
0.01 \\
0.76 \\
*\end{array}$ & $\begin{array}{r}61.32 \\
0.69\end{array}$ \\
\hline 1961 & $\begin{array}{l}\text { PREC IP. (CM.) } \\
\text { SR-9C (NCI/SG-KM.) } \\
\text { SR-90 CONC. (PC/L }) \\
\text { SR-89/SR-90 }\end{array}$ & $\begin{array}{l}1.12 \\
0.056 \\
4.47\end{array}$ & $\begin{array}{l}0.81 \\
0.036 \\
3.71 \\
--\end{array}$ & $\begin{array}{l}4.17 \\
0.04 C \\
0.96 \\
--\end{array}$ & $\begin{array}{l}9.80 \\
0.086 \\
0.82\end{array}$ & $\begin{array}{l}8.66 \\
0.036 \\
0.35 \\
--\end{array}$ & $\begin{array}{l}16.26 \\
0.06 \mathrm{C} \\
0.37 \\
--\end{array}$ & $\begin{array}{l}16.38 \\
0.116 \\
0.68 \\
--\end{array}$ & $\begin{array}{l}15.32 \\
0.106 \\
0.66 \\
--\end{array}$ & $\begin{array}{l}4.57 \\
0.07 \\
1.54 \\
*\end{array}$ & $\begin{array}{l}2.44 \\
0.06 \\
2.46 \\
*\end{array}$ & $\begin{array}{l}0.41 \\
0.02 \\
4.88 \\
*\end{array}$ & $\begin{array}{l}2.29 \\
0.08 \\
3.50 \\
*\end{array}$ & $\begin{array}{r}82.23 \\
0.73\end{array}$ \\
\hline 1962 & $\begin{array}{l}\text { PRECIP. (CM-) } \\
\text { SR-9C iNCI/SQ.KM.) } \\
\text { SR-90 CONC. (PC/L) } \\
\text { SR-89/SR-90 }\end{array}$ & $\begin{array}{l}0.18 \\
0.03 \\
16.67 \\
*\end{array}$ & $\begin{array}{l}0.84 \\
0.03 \\
3.58 \\
*\end{array}$ & $\begin{array}{l}4.17 \\
0.01 \\
0.24 \\
*\end{array}$ & $\begin{array}{l}0.86 \\
0.02 \\
2.33 \\
*\end{array}$ & $\begin{array}{l}15.14 \\
=- \\
--\end{array}$ & $\begin{array}{r}14.43 \\
0.14 \\
0.98 \\
29.00\end{array}$ & $\begin{array}{r}15.42 \\
0.12 \\
0.78 \\
19.00\end{array}$ & $\begin{array}{r}8.61 \\
0.07 \\
0.82 \\
19.00\end{array}$ & $\begin{array}{r}7.19 \\
0.07 \\
0.98 \\
14.00\end{array}$ & $\begin{array}{l}2.36 \\
0.03 \\
1.28 \\
5.00\end{array}$ & $\begin{array}{l}3.84 \\
0.05 \\
1.31 \\
4.00\end{array}$ & $\begin{array}{l}1.35 \\
0.07 \\
5.19 \\
8.00\end{array}$ & $\begin{array}{r}74.39 \\
0.64\end{array}$ \\
\hline 1564 & $\begin{array}{l}\text { PRECIP. (CMM-) } \\
\text { SR-90 INCIISO.KM-) } \\
\text { SR-90 CONC. (PC/L) } \\
\text { SR-89/SR-90 }\end{array}$ & $\begin{array}{l}* \\
0.03 \\
*-\end{array}$ & $\begin{array}{l}0.36 \\
0.02 \\
5.56 \\
*\end{array}$ & $\begin{array}{l}3.78 \\
0.04 \\
1.06 \\
*\end{array}$ & $\begin{array}{l}7.16 \\
0.03 \\
0.42 \\
*\end{array}$ & $\begin{array}{l}1.40 \\
0.05 \\
3.58 \\
--\end{array}$ & $\begin{array}{l}29.46 \\
0.68 \\
2.31 \\
--\end{array}$ & $\begin{array}{r}24.18 \\
0.52 \\
2.16 \\
--\end{array}$ & $\begin{array}{r}14.50 \\
0.61 \\
4.21 \\
-.\end{array}$ & $\begin{array}{l}9.37 \\
0.16 \\
1.71 \\
-=\end{array}$ & $\begin{array}{l}6.53 \\
0.21 \\
3.22 \\
--\end{array}$ & $\begin{array}{l}0.71 \\
0.07 \\
9.86 \\
--\end{array}$ & $\begin{array}{l}2.90 \\
0.02 \\
0.69 \\
--\end{array}$ & $\begin{array}{r}100.35 \\
2.44\end{array}$ \\
\hline 1965 & $\begin{array}{l}\text { PRECIP. (CM.) } \\
\text { SR-90 (MCLI/SO.KM.) } \\
\text { SR-90 CONC. (PCLAL) } \\
\text { SR-89/SR-90 }\end{array}$ & $\begin{array}{l}1.24 \\
0.08 \\
6.46 \\
--\end{array}$ & $\begin{array}{r}0.10 \\
0.02 \\
20.01 \\
--\end{array}$ & $\begin{array}{l}0.99 \\
0.04 \\
4.05 \\
--\end{array}$ & $\begin{array}{l}4.32 \\
0.08 \\
1.86 \\
--\end{array}$ & $\begin{array}{r}16.69 \\
0.30 \\
1.80 \\
--\end{array}$ & $\begin{array}{r}22.99 \\
0.44 \\
1.92 \\
--\end{array}$ & $\begin{array}{r}17.70 \\
0.27 \\
1.53 \\
-\end{array}$ & $\begin{array}{r}18.49 \\
0.32 \\
1.74 \\
--\end{array}$ & $\begin{array}{l}6.50 \\
0.18 \\
2.77 \\
--\end{array}$ & $\begin{array}{r}14.02 \\
0.25 \\
1.79 \\
--\end{array}$ & $\begin{array}{l}5.16 \\
0.11 \\
2.14 \\
--\end{array}$ & $\begin{array}{l}1.17 \\
0.05 \\
4.28 \\
--\end{array}$ & $\begin{array}{r}109.37 \\
2.14\end{array}$ \\
\hline 1966 & $\begin{array}{l}\text { PRECIP. ICM-) } \\
\text { SR-90 (NCIISO.KM_) } \\
\text { SR-90 CONC. (PCIL) } \\
\text { SR-89/SR-90 }\end{array}$ & $\begin{array}{r}0.15 \\
0.05 \\
33.34 \\
--\end{array}$ & $\begin{array}{l}0.36 \\
0.03 \\
8.34 \\
--\end{array}$ & $\begin{array}{l}0.48 \\
0.03 \\
6.26 \\
--\end{array}$ & $\begin{array}{l}5.20 \\
0.06 \\
1.16 \\
--\end{array}$ & $\begin{array}{l}5.30 \\
0.06 \\
1.14 \\
--\end{array}$ & $\begin{array}{r}21.70 \\
0.15 \\
0.70 \\
--\end{array}$ & $\begin{array}{r}18.00 \\
0.24 \\
1.34 \\
-\end{array}$ & $\begin{array}{l}8.90 \\
0.16 \\
1.80 \\
--\end{array}$ & $\begin{aligned} 10.10 \\
-0.17 \\
1.69 \\
--\end{aligned}$ & $\begin{array}{l}4.80 \\
0.08 \\
1.67 \\
-\end{array}$ & $\begin{array}{l}0.71 \\
0.04 \\
5.64 \\
--\end{array}$ & $\begin{array}{l}1.60 \\
0.11 \\
6.88 \\
--\end{array}$ & $\begin{array}{r}77.30 \\
1.18\end{array}$ \\
\hline 1967 & $\begin{array}{l}\text { PRECIP. ICM.) } \\
\text { SR-90 IMCIISQ.KM_) } \\
\text { SR-90 CONC. (PC/L) } \\
\text { SR-89/SR-90 }\end{array}$ & $\begin{array}{r}0.28 \\
0.04 \\
14.29 \\
--\end{array}$ & $\begin{array}{l}0.86 \\
0.03 \\
3.49 \\
--\end{array}$ & $\begin{array}{l}1.20 \\
0.05 \\
4.17 \\
--\end{array}$ & $\begin{array}{l}5.80 \\
0.07 \\
1.21 \\
--\end{array}$ & $\begin{array}{r}22.10 \\
0.09 \\
0.41 \\
--\end{array}$ & $\begin{array}{r}28.90 \\
0.13 \\
0.45 \\
--\end{array}$ & $\begin{array}{r}17.50 \\
0.12 \\
0.69 \\
-\end{array}$ & $\begin{array}{l}= \\
\bar{z}\end{array}$ & $\begin{array}{l}=- \\
\bar{z}\end{array}$ & $\begin{array}{l}= \\
=- \\
=\end{array}$ & $\begin{array}{l}\bar{z} \\
\bar{z}\end{array}$ & $\begin{array}{l}=- \\
= \\
=\end{array}$ & $\begin{array}{r}76.64 \\
0.53\end{array}$ \\
\hline
\end{tabular}


MONTHLY FALLOUT OEPOSITION CULLEGIIUNS

TE: AUSTRALIA, SYONEY

LAT. 33525 LONG.15L I2E ALT.

42M. (COLUMN)

SOURCE: ATOMIC WEAPONS TESTS SAFETY COMMITTEE

(POT USED THROUGH DECEMBER, 1963)

\begin{tabular}{|c|c|c|c|c|c|c|c|c|c|c|c|c|c|c|}
\hline & & JAN. & FEB. & MAR. & $A P R$. & MAY & JUNE & JULY & AUG. & SEP. & OCT. & Nov. & DEC. & CUM. TOTAL \\
\hline 1958 & $\begin{array}{l}\text { PRECIP. (CM.) } \\
\text { SR-90 (MCI/SO.KM.) } \\
S R-90 \text { CONC. }(P C / L) \\
S R-89 / S R-90\end{array}$ & 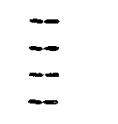 & $=-$ & $\ddot{m}$ & $\bar{m}$ & $=$ & $\begin{array}{r}22.12 \\
0.13 \\
0.59 \\
0.90\end{array}$ & $\begin{array}{r}2.87 \\
0.10 \\
3.49 \\
23.00\end{array}$ & $\begin{array}{l}9.42 \\
0.17 \\
1.81 \\
43.00\end{array}$ & $\begin{array}{l}3.02 \\
0.10 \\
3.32 \\
+\end{array}$ & $\begin{array}{r}5.56 \\
0.09 \\
1.62 \\
25.00\end{array}$ & $\begin{array}{r}1.70 \\
0.08 \\
4.71 \\
15.00\end{array}$ & $\begin{array}{r}14.83 \\
0.22 \\
1.49 \\
8.20\end{array}$ & $\begin{array}{r}39.52 \\
0.89\end{array}$ \\
\hline 1959 & $\begin{array}{l}\text { PRECIP. (CM.) } \\
S R-90 \text { (MCI/SO.KM.) } \\
S R-9 C \text { CCNC. }(P C / L) \\
S R-89 / S R-90\end{array}$ & $\begin{array}{r}16.36 \\
0.25 \\
1.53 \\
9.40\end{array}$ & $\begin{array}{r}20.22 \\
0.17 \\
0.85 \\
5.60\end{array}$ & $\begin{array}{l}14.30 \\
0.08 \\
0.56 \\
=.\end{array}$ & $\begin{array}{l}4.09 \\
0.02 \\
0.49\end{array}$ & $\begin{array}{l}5.79 \\
0.08 \\
1.39 \\
2.40\end{array}$ & $\begin{array}{r}10.97 \\
0.14 \\
1.28\end{array}$ & $\begin{array}{r}19.61 \\
0.10 \\
0.51 \\
2.30\end{array}$ & $\begin{array}{l}0.53 \\
0.05 \\
0.59 \\
*\end{array}$ & $\begin{array}{l}8.64 \\
0.12 \\
1.39 \\
*\end{array}$ & $\begin{array}{l}28.27 \\
0.26 \\
c .92 \\
*\end{array}$ & $\begin{array}{l}9.88 \\
0.13 \\
1.32 \\
+\end{array}$ & $\begin{array}{l}4.90 \\
0.08 \\
1.64 \\
+\end{array}$ & $\begin{array}{r}151.56 \\
1.48\end{array}$ \\
\hline 1960 & $\begin{array}{l}\text { PRECIP. (CM.) } \\
S R-9 C \text { (MCIISO.KM.) } \\
S R-90 C C N C .(P C / L) \\
S R-89 / S R-90\end{array}$ & $\begin{array}{l}5.77 \\
0.05 \\
0.87 \\
=-\end{array}$ & $\begin{array}{l}9.35 \\
0.08 \\
0.86 \\
0 .\end{array}$ & $\begin{array}{l}5.28 \\
0.05 \\
0.95 \\
-\end{array}$ & $\begin{array}{l}2.95 \\
0.07 \\
2.38 \\
--\end{array}$ & $\begin{array}{c}10.87 \\
0.056 \\
0.40 \\
-.\end{array}$ & $\begin{array}{l}9.04 \\
0.04 C \\
0.45 \\
-\end{array}$ & $\begin{array}{l}14.17 \\
6.05 C \\
0.36 \\
=-\end{array}$ & $\begin{array}{l}4.50 \\
C .01 C \\
0.23 \\
--\end{array}$ & $\begin{array}{l}6.81 \\
0.05 C \\
0.74 \\
-.\end{array}$ & $\begin{array}{l}28.02 \\
0.20 \mathrm{C} \\
0.72 \\
-.\end{array}$ & $\begin{array}{l}7.14 \\
0.086 \\
1.13 \\
--\end{array}$ & $\begin{array}{l}24.51 \\
0.276 \\
1.11 \\
-.\end{array}$ & $\begin{array}{r}128.41 \\
1.00\end{array}$ \\
\hline 1961 & $\begin{array}{l}\text { PRECIP. (CM.) } \\
\text { SR-9C IMCI/SQ.KM-) } \\
S R-90 \text { CCNC. }(P C / L) \\
S R-89 / S R-90\end{array}$ & $\begin{array}{l}6.25 \\
0.096 \\
1.45\end{array}$ & $\begin{array}{l}8.56 \\
0.12 \mathrm{C} \\
1.41 \\
-.\end{array}$ & $\begin{array}{l}3.02 \\
0.036 \\
1.00 \\
-.\end{array}$ & $\begin{array}{l}8.94 \\
0.08 \mathrm{C} \\
0.90 \\
-.\end{array}$ & $\begin{array}{l}3.12 \\
0.06 \mathrm{C} \\
1.93 \\
-. .\end{array}$ & $\begin{array}{l}5.64 \\
0.1 \mathrm{cC} \\
1.78 \\
-.-\end{array}$ & $\begin{array}{l}3.94 \\
C .02 C \\
0.51 \\
-. .\end{array}$ & $\begin{array}{c}23.95 \\
C .15 \mathrm{C} \\
0.63 \\
=-\end{array}$ & $\begin{array}{l}5.23 \\
C .06 \\
1.15 \\
*\end{array}$ & $\begin{array}{l}7.42 \\
0.19 \\
2.57 \\
+\end{array}$ & $\begin{array}{l}54.46 \\
0.46 \\
0.85 \\
*\end{array}$ & $\begin{array}{r}15.19 \\
0.18 \\
1.19 \\
*\end{array}$ & $\begin{array}{r}145.72 \\
1.54\end{array}$ \\
\hline 1962 & $\begin{array}{l}\text { PRECIP. (CM.) } \\
\text { SR-9O IMCI/SQ.KM-) } \\
S R-90 \text { CONC. }(P C / L) \\
S R-Q S / S R-S O\end{array}$ & $\begin{array}{r}13.26 \\
0.23 \\
1.74 \\
*\end{array}$ & $\begin{array}{l}15.16 \\
0.09 \\
0.60 \\
*\end{array}$ & $\begin{array}{l}6.35 \\
0.11 \\
1.74 \\
\end{array}$ & $\begin{array}{r}10.03 \\
0.05 \\
0.50 \\
*\end{array}$ & $\begin{array}{r}27.00 \\
0.06 \\
0.23 \\
6.00\end{array}$ & $\begin{array}{l}0.41 \\
0.06 \\
0.01 \\
.\end{array}$ & $\begin{array}{r}6.96 \\
0.06 \\
0.87 \\
28.00\end{array}$ & $\begin{array}{r}10.97 \\
0.04 \\
0.37 \\
19.00\end{array}$ & $\begin{array}{l}8.79 \\
0.05 \\
0.57 \\
7.00\end{array}$ & $\begin{array}{r}2.18 \\
0.09 \\
4.13 \\
14.00\end{array}$ & $\begin{array}{r}1.04 \\
0.13 \\
12.51 \\
11.00\end{array}$ & $\begin{array}{r}17.22 \\
0.12 \\
0.70 \\
50.00\end{array}$ & $\begin{array}{r}119.37 \\
1.03\end{array}$ \\
\hline 1963 & $\begin{array}{l}\text { PRECIP. }\left(C M_{-}\right) \\
\text {SR-9C IMCI/SQ.KM-) } \\
S R-90 \text { CONC. (PC/L) } \\
\text { SR-89/SR-90 }\end{array}$ & $\begin{array}{r}14.10 \\
C .23 \\
1.64 \\
5.00\end{array}$ & $\begin{array}{l}4.80 \\
0.06 \\
1.26 \\
5.10\end{array}$ & $\begin{array}{r}38.81 \\
0.46 \\
1.19 \\
8.50\end{array}$ & $\begin{array}{r}23.85 \\
0.24 \\
1.01 \\
2.40\end{array}$ & $\begin{array}{r}21.08 \\
0.10 \\
0.48 \\
*\end{array}$ & $\begin{array}{l}26.44 \\
0.22 \\
0.84 \\
*\end{array}$ & $\begin{array}{l}6.71 \\
0.11 \\
1.64 \\
*\end{array}$ & $\begin{array}{r}26.92 \\
0.28 \\
1.05 \\
*\end{array}$ & $\begin{array}{l}2.46 \\
0.14 \\
5.70 \\
\end{array}$ & $\begin{array}{l}6.22 \\
0.13 \\
2.10 \\
*\end{array}$ & $\begin{array}{l}3.23 \\
0.12 \\
3.72 \\
*\end{array}$ & $\begin{array}{l}27.89 \\
0.10 \\
0.36 \\
*\end{array}$ & $\begin{array}{r}202.51 \\
2.19\end{array}$ \\
\hline 1964 & $\begin{array}{l}\text { PRECIP. (CM+) } \\
\left.S R-90 \text { ( } P C I / S M_{-K M}\right) \\
S R-90 \text { CQNC. (PC/L) } \\
S R-89 / S R-90\end{array}$ & $\begin{array}{l}1.80 \\
0.08 \\
4.45\end{array}$ & $\begin{array}{l}5.03 \\
0.14 \\
2.79 \\
*\end{array}$ & $\begin{array}{r}17.15 \\
0.25 \\
1.46 \\
*\end{array}$ & $\begin{array}{r}13.94 \\
0.15 \\
1.08 \\
*\end{array}$ & $\begin{array}{l}7.80 \\
0.15 \\
1.93 \\
-.\end{array}$ & $\begin{array}{l}40.74 \\
0.32 \\
0.79 \\
--\end{array}$ & $\begin{array}{l}0.94 \\
C .06 \\
6.39 \\
--\end{array}$ & $\begin{array}{l}2.57 \\
0.12 \\
4.67 \\
--\end{array}$ & $\begin{array}{l}1.52 \\
0.12 \\
7.90 \\
--\end{array}$ & $\begin{array}{l}5.94 \\
0.25 \\
4.21 \\
--\end{array}$ & $\begin{array}{l}10.13 \\
0.62 \\
6.13 \\
-.\end{array}$ & $\begin{array}{l}3.78 \\
0.27 \\
7.15 \\
-.\end{array}$ & $\begin{array}{r}111.34 \\
2.53\end{array}$ \\
\hline 1965 & $\begin{array}{l}\text { PRECIP. }(C M-) \\
\text { SR-90 (MCI/SQ.KM-) } \\
S R-90 \text { CONC. }(P C / L) \\
S R-89 / S R-90\end{array}$ & $\begin{array}{l}3.63 \\
0.27 \\
7.44 \\
-.\end{array}$ & $\begin{array}{l}2.26 \\
0.20 \\
8.85 \\
-.\end{array}$ & $\begin{array}{l}0.94 \\
0.14 \\
14.90 \\
--\end{array}$ & $\begin{array}{l}8.51 \\
0.17 \\
2.00 \\
--\end{array}$ & $\begin{array}{l}4.27 \\
0.10 \\
2.35 \\
-=\end{array}$ & $\begin{array}{l}20.78 \\
0.24 \\
1.16 \\
-\end{array}$ & $\begin{array}{l}9.53 \\
0.20 \\
2.10 \\
-.\end{array}$ & $\begin{array}{l}2.01 \\
0.11 \\
5.48 \\
-.\end{array}$ & $\begin{array}{l}8.86 \\
0.18 \\
2.04 \\
--\end{array}$ & $\begin{array}{l}14.40 \\
0.19 \\
1.32 \\
--\end{array}$ & $\begin{array}{l}4.52 \\
0.14 \\
3.10 \\
--\end{array}$ & $\begin{array}{l}0.91 \\
0.18 \\
2.61 \\
-.\end{array}$ & $\begin{array}{r}86.62 \\
2.12\end{array}$ \\
\hline 1966 & $\begin{array}{l}\text { PRECIP, (CM-) } \\
\text { SR-9C IMCI/SQ.KM-) } \\
S R-90 \text { CONC. }(P C / L) \\
S R-89 / S R-90\end{array}$ & $\begin{array}{l}2.51 \\
0.12 \\
4.79 \\
--\end{array}$ & $\begin{array}{r}11.18 \\
0.20 \\
1.79 \\
-.\end{array}$ & $\begin{array}{l}17.20 \\
0.23 \\
1.34 \\
--\end{array}$ & $\begin{array}{l}17.80 \\
0.14 \\
0.79 \\
-\end{array}$ & $\begin{array}{l}6.30 \\
0.11 \\
1.75 \\
-\end{array}$ & $\begin{array}{l}9.70 \\
0.03 \\
0.31 \\
-\end{array}$ & $\begin{array}{l}1.73 \\
0.05 \\
2.90 \\
--\end{array}$ & $\begin{array}{l}9.60 \\
0.09 \\
0.94 \\
-.\end{array}$ & $\begin{array}{l}4.60 \\
0.14 \\
3.05 \\
-.\end{array}$ & $\begin{array}{l}3.80 \\
0.11 \\
2.90 \\
--\end{array}$ & $\begin{array}{l}15.10 \\
0.11 \\
0.73 \\
-.\end{array}$ & $\begin{array}{l}7.30 \\
0.17 \\
2.33 \\
-.\end{array}$ & $\begin{array}{r}106.82 \\
1.50\end{array}$ \\
\hline 1967 & $\begin{array}{l}\text { PRECIP. }(C M .) \\
\text { SR-90 IMCI/SO.KM.) } \\
S R-90 \text { CONC. }(P C / L) \\
S R-89 / S R-90\end{array}$ & $\begin{array}{l}14.80 \\
0.07 \\
0.48 \\
--\end{array}$ & $\begin{array}{l}15.80 \\
0.12 \\
0.76 \\
--\end{array}$ & $\begin{array}{r}11.00 \\
0.12 \\
1.10 \\
--\end{array}$ & $\begin{array}{l}4.20 \\
0.05 \\
1.20 \\
\end{array}$ & $\begin{array}{l}4.10 \\
0.02 \\
0.49 \\
--\end{array}$ & $\begin{array}{l}21.70 \\
0.05 \\
0.24 \\
--\end{array}$ & $\begin{array}{l}2.70 \\
0.03 \\
1.12 \\
-.\end{array}$ & $\begin{array}{l}-- \\
-- \\
--\end{array}$ & $\begin{array}{l}-- \\
-- \\
--\end{array}$ & $\begin{array}{l}-- \\
-- \\
--\end{array}$ & $\begin{array}{l}-- \\
-- \\
--\end{array}$ & $=-$ & $\begin{array}{r}74.30 \\
0.46\end{array}$ \\
\hline $\begin{array}{l}\text { NOTES } \\
--: D \\
*: 2 \\
A: A \\
B: L \\
C: A\end{array}$ & $\begin{array}{l}\text { S } \\
\text { DATA NOT AVAILABLE } \\
\text { ZERO OR TRACE } \\
\text { APPROXIMATE } \\
\text { LOWER LIMIT OF REPORT } \\
\text { PROPORTI ONEO FROM ORI }\end{array}$ & $\begin{array}{l}\text { DATA } \\
\text { NALLY }\end{array}$ & & & & & & & & & & & & \\
\hline
\end{tabular}




\begin{tabular}{|c|c|c|c|c|c|c|c|c|c|c|c|c|c|c|}
\hline & & JAN. & FEB. & MAR. & APR : & MAY & JUNE & JULY & AUG. & SEP. & OCT. & NOV. & DEC. & CUM. TOTAL \\
\hline 1958 & $\begin{array}{l}\text { PRECIP. ICM.) } \\
\text { SR-90 (MCI/SQ.KM.) } \\
\text { SR-90 CONC. (PC/L) } \\
\text { SR-89/SR-90 }\end{array}$ & $\begin{array}{l}-- \\
-- \\
--\end{array}$ & $\begin{array}{l}-- \\
--\end{array}$ & $\begin{array}{l}-- \\
--\end{array}$ & $\overline{-}$ & $=$ & $\begin{array}{r}2.46 \\
0.10 \\
4.07 \\
16.00\end{array}$ & $\frac{0.04}{4.00}$ & $\begin{array}{r}0.18 \\
0.03 \\
16.67 \\
13.00\end{array}$ & $\begin{array}{l}0.58 \\
0.01 \\
1.73 \\
-.\end{array}$ & $\begin{array}{r}0.28 \\
0.02 \\
7.15 \\
18.00\end{array}$ & $\begin{array}{r}2.54 \\
0.03 \\
1.19 \\
86.00\end{array}$ & $\begin{array}{r}8.86 \\
0.09 \\
1.02 \\
12.00\end{array}$ & $\begin{array}{r}14.90 \\
0.32\end{array}$ \\
\hline 1959 & $\begin{array}{l}\text { PRECIP. ICM-) } \\
\text { SR-9O (MCI/SO.KM.) } \\
\text { SR-90 CONC. }(P C / L) \\
\text { SR-89/SR-90 }\end{array}$ & $\begin{array}{r}37.67 \\
0.18 \\
0.48 \\
8.30\end{array}$ & $\begin{array}{l}7.01 \\
0.01 \\
0.15 \\
. .\end{array}$ & $\begin{array}{r}15.52 \\
0.14 \\
0.91 \\
5.00\end{array}$ & $\begin{array}{l}11.51 \\
0.02 \\
0.18 \\
*\end{array}$ & $\begin{array}{l}5.72 \\
0.02 \\
0.35 \\
+\end{array}$ & $\begin{array}{l}0.51 \\
0.01 \\
1.97 \\
+\end{array}$ & $\begin{array}{l}0.18 \\
0.00 \\
0.01 \\
-\end{array}$ & $\begin{array}{l}* \\
* \\
-\end{array}$ & $\begin{array}{l}* \\
0.00 \\
*\end{array}$ & $\begin{array}{l}0.03 \\
* \\
*\end{array}$ & $\begin{array}{l}5.23 \\
0.05 \\
0.96 \\
*\end{array}$ & $\begin{array}{r}40.82 \\
0.06 \\
0.15 \\
.\end{array}$ & $\begin{array}{r}124.20 \\
0.49\end{array}$ \\
\hline 1960 & $\begin{array}{l}\text { PRECIP. (CM-) } \\
\text { SR-90 (MCI/SQ.KM.) } \\
\text { SR-90 CONC. }(P C / L) \\
\text { SR-89/SR-90 }\end{array}$ & $\begin{array}{l}13.92 \\
0.10 \\
0.72 \\
--\end{array}$ & $\begin{array}{l}55.96 \\
0.07 \\
0.13 \\
--\end{array}$ & $\begin{array}{r}36.55 \\
0.02 \\
0.03 \\
=-\end{array}$ & $\begin{array}{l}0.86 \\
0.01 \\
1.17 \\
\end{array}$ & $\begin{array}{l}4.52 \\
0.02 C \\
0.45 \\
--.\end{array}$ & $\begin{array}{l}0.74 \\
0.00 \mathrm{C} \\
0.01 \\
--\end{array}$ & $\begin{array}{l}0.13 \\
0.000 \\
0.01 \\
--\end{array}$ & $\begin{array}{l}0.10 \\
0.000 \\
0.01 \\
=.\end{array}$ & $\begin{array}{l}0.36 \\
0.01 \mathrm{C} \\
2.78 \\
-.\end{array}$ & $\begin{array}{l}1.14 \\
0.036 \\
2.64 \\
-.\end{array}$ & $\begin{array}{l}13.51 \\
0.08 C \\
0.60 \\
--\end{array}$ & $\begin{array}{l}8.97 \\
0.056 \\
0.56 \\
--\end{array}$ & $\begin{array}{r}136.76 \\
0.39\end{array}$ \\
\hline 1961 & $\begin{array}{l}\text { PRECIP. (CM.) } \\
\text { SR-90 INCI/SQ.KM.) } \\
\text { SR-90 CONC. }(P C / L) \\
S R-89 / S R-90\end{array}$ & $\begin{array}{l}3.40 \\
0.000 \\
0.01 \\
--\end{array}$ & $\begin{array}{l}22.07 \\
0.04 \mathrm{C} \\
0.19 \\
=-\end{array}$ & $\begin{array}{l}3.02 \\
0.05 C \\
1.66 \\
-0\end{array}$ & $\begin{array}{l}0.89 \\
0.026 \\
2.25 \\
-.\end{array}$ & $\begin{array}{l}0.76 \\
* \\
--\end{array}$ & $\begin{array}{l}* \\
* \\
-\infty\end{array}$ & $\begin{array}{l}0.25 \\
0.00 \mathrm{C} \\
0.01 \\
-.\end{array}$ & $\begin{array}{l}0.86 \\
0.006 \\
0.01 \\
-\end{array}$ & $\begin{array}{l}* \\
0.03 \\
*\end{array}$ & $\begin{array}{l}0.41 \\
+ \\
=- \\
*\end{array}$ & $\begin{array}{l}12.98 \\
0.08 \\
0.62 \\
*\end{array}$ & $\begin{array}{l}6.45 \\
0.07 \\
1.09 \\
+\end{array}$ & $\begin{array}{r}51.09 \\
0.29\end{array}$ \\
\hline 1962 & $\begin{array}{l}\text { PRECIP. (CM-) } \\
\text { SR-9C IMCI/SQ-KM-) } \\
S R-9 C \text { CONC. }(P C / L) \\
S R-B S / S R-90\end{array}$ & $\begin{array}{r}19.69 \\
C .08 \\
C .41 \\
*\end{array}$ & $\begin{array}{r}39.55 \\
0.10 \\
0.26 \\
+\end{array}$ & $\begin{array}{l}9.12 \\
0.04 \\
0.44 \\
0\end{array}$ & $\begin{array}{l}3.18 \\
0.00 \\
0.01 \\
*\end{array}$ & $\begin{array}{r}0.43 \\
0.03 \\
6.98 \\
10.00\end{array}$ & $\begin{array}{r}1.60 \\
0.03 \\
1.88 \\
35.0 \mathrm{C}\end{array}$ & $\begin{array}{l}1.35 \\
0.02 \\
1.49 \\
*\end{array}$ & $\begin{array}{l}0.69 \\
0.00 \\
0.01 \\
*\end{array}$ & $\begin{array}{r}1.14 \\
C . C 4 \\
3.51 \\
14.00\end{array}$ & $\begin{array}{r}c .08 \\
C .01 \\
12.51 \\
*\end{array}$ & $\begin{array}{l}2.69 \\
0.03 \\
1.12 \\
-.\end{array}$ & $\begin{array}{r}10.08 \\
0.12 \\
1.20 \\
10.00\end{array}$ & $\begin{array}{r}89.60 \\
0.50\end{array}$ \\
\hline 1963 & $\begin{array}{l}\text { PRECIP. (CM.) } \\
\text { SR-9C IMCI/SQ.KM-) } \\
\text { SR-9C CDNC= (PC/L) } \\
S R-89 / S R-90\end{array}$ & $\begin{array}{r}41.05 \\
0.19 \\
0.47 \\
5.00\end{array}$ & $\begin{array}{r}14.35 \\
6.05 \\
6.35 \\
3.00\end{array}$ & $\begin{array}{r}36.09 \\
0.08 \\
0.23 \\
6.00\end{array}$ & $\begin{array}{l}5.99 \\
0.02 \\
0.34 \\
3.60\end{array}$ & $\begin{array}{l}0.51 \\
0.03 \\
5.89 \\
*\end{array}$ & $\begin{array}{l}0.25 \\
0.00 \\
0.01 \\
*\end{array}$ & $\begin{array}{l}* \\
0.02 \\
*\end{array}$ & $\begin{array}{l}3.20 \\
0.05 \\
1.57 \\
+\end{array}$ & $\begin{array}{l}* \\
0.02 \\
*\end{array}$ & $\begin{array}{r}0.15 \\
C .03 \\
20.01 \\
*\end{array}$ & $\begin{array}{l}1.19 \\
0.08 \\
6.73 \\
*\end{array}$ & $\begin{array}{l}3.94 \\
0.09 \\
2.29 \\
+\end{array}$ & $\begin{array}{r}106.72 \\
0.66\end{array}$ \\
\hline 1964 & $\begin{array}{l}\text { PRECIP. (CM.) } \\
\text { SR-9O (MCI/SO.KM-) } \\
S R-9 C \text { CCNC. }(P C / L) \\
S R-89 / S R-90\end{array}$ & $\begin{array}{l}17.15 \\
0.09 \\
0.53 \\
\$\end{array}$ & $\begin{array}{l}51.44 \\
0.12 \\
C .24 \\
*\end{array}$ & $\begin{array}{r}13.82 \\
0.02 \\
0.15 \\
*\end{array}$ & $\begin{array}{l}3.84 \\
0.01 \\
0.27 \\
*\end{array}$ & $\begin{array}{l}3.78 \\
0.04 \\
1.06\end{array}$ & $\begin{array}{l}5.38 \\
0.07 \\
1.31 \\
-.\end{array}$ & $\begin{array}{l}2.57 \\
0.02 \\
0.78 \\
--\end{array}$ & $\begin{array}{l}0.33 \\
0.01 \\
3.04 \\
=-\end{array}$ & $\begin{array}{c}0.03 \\
0.02 \\
66.67 \\
--\end{array}$ & $\begin{array}{l}5.11 \\
0.10 \\
1.96 \\
-.\end{array}$ & $\begin{array}{l}4.78 \\
0.12 \\
2.52 \\
-.\end{array}$ & $\begin{array}{l}17.81 \\
0.25 \\
1.41 \\
-.\end{array}$ & $\begin{array}{r}126.04 \\
0.87\end{array}$ \\
\hline 1965 & $\begin{array}{l}\text { PRECIP. (CM.) } \\
\text { SR-9C (MCI/SQ.KM.) } \\
\text { SR-9O CCNC. (PC/L) } \\
\text { SR-89/SR-90 }\end{array}$ & $\begin{array}{l}27.64 \\
0.11 \\
0.40 \\
--\end{array}$ & $\begin{array}{l}3.56 \\
0.10 \\
2.11 \\
==\end{array}$ & $\begin{array}{l}30.58 \\
0.06 \\
0.20 \\
--\end{array}$ & $\begin{array}{l}9.73 \\
0.02 \\
0.21 \\
-.\end{array}$ & $\begin{array}{l}2.46 \\
0.03 \\
1.22 \\
--\end{array}$ & $\begin{array}{l}0.81 \\
0.02 \\
2.47 \\
-.\end{array}$ & $\begin{array}{r}0.05 \\
0.02 \\
40.01 \\
-\end{array}$ & $\begin{array}{l}0.10 \\
0.02 \\
20.01 \\
-.\end{array}$ & $\begin{array}{l}0.02 \\
=- \\
=-\end{array}$ & $\begin{array}{l}0.76 \\
0.05 \\
6.58 \\
-.\end{array}$ & $\begin{array}{l}0.79 \\
0.03 \\
3.80 \\
--\end{array}$ & $\begin{array}{l}27.02 \\
0.19 \\
0.71 \\
--\end{array}$ & $\begin{array}{r}103.50 \\
0.67\end{array}$ \\
\hline 1966 & $\begin{array}{l}\text { PRECIP. (CM.) } \\
\text { SR-9O IMCI/SQ.KM.) } \\
\text { SR-9O CONC. (PC/L) } \\
S R-89 / S R-90\end{array}$ & $\begin{array}{l}24.00 \\
0.16 \\
0.67 \\
--\end{array}$ & $\begin{array}{l}10.00 \\
0.07 \\
0.71 \\
-\end{array}$ & $\begin{array}{l}3.10 \\
0.06 \\
1.94 \\
-\end{array}$ & $\begin{array}{l}2.60 \\
0.02 \\
0.77 \\
-.\end{array}$ & $\begin{array}{l}3.10 \\
0.04 \\
1.30 \\
-.\end{array}$ & $\begin{array}{l}1.04 \\
0.02 \\
1.93 \\
-\end{array}$ & $\begin{array}{l}* \\
0.01 \\
--\end{array}$ & $\begin{array}{l}1.57 \\
0.02 \\
1.28 \\
--\end{array}$ & $\begin{array}{l}0.02 \\
0.01 \\
50.01 \\
--\end{array}$ & $\begin{array}{l}0.89 \\
0.06 \\
6.75 \\
-\end{array}$ & $\begin{array}{l}5.20 \\
0.16 \\
3.08 \\
-.0\end{array}$ & $\begin{array}{l}1.80 \\
0.08 \\
4.45 \\
-.\end{array}$ & $\begin{array}{r}53.32 \\
0.71\end{array}$ \\
\hline 1967 & $\begin{array}{l}\text { PRECIP. ICM.) } \\
\text { SR-90 IMCI/SQ-KM_) } \\
S R-90 \text { CCNC. (PC/L) } \\
S R-89 / S R-90\end{array}$ & $\begin{array}{r}10.80 \\
0.18 \\
1.67 \\
-\end{array}$ & $\begin{array}{l}18.60 \\
0.03 \\
0.17 \\
--\end{array}$ & $\begin{array}{r}18.90 \\
0.04 \\
0.22 \\
-\end{array}$ & $\begin{array}{r}0.30 \\
0.03 \\
10.01 \\
-\infty\end{array}$ & $\begin{array}{l}0.58 \\
0.03 \\
5.18 \\
--\end{array}$ & $\begin{array}{l}10.70 \\
0.02 \\
0.19 \\
-\end{array}$ & $\begin{array}{r}0.08 \\
0.02 \\
25.01 \\
-\end{array}$ & $\begin{array}{l}-- \\
= \\
--\end{array}$ & $\begin{array}{l}=- \\
=- \\
--\end{array}$ & $\begin{array}{l}-- \\
--\end{array}$ & $\begin{array}{l}-- \\
--\end{array}$ & $\begin{array}{l}-- \\
-- \\
--\end{array}$ & $\begin{array}{r}59.96 \\
0.35\end{array}$ \\
\hline DTES & $\begin{array}{l}\text { A NOT AVAILABLE } \\
\text { O OR TRACE } \\
\text { ROXIMATE } \\
\text { ER LIMIT OF REPDI } \\
\text { PORTIONED FROM O }\end{array}$ & $1 \mathrm{~A}$ & & & & & & & & & & & & \\
\hline
\end{tabular}




\begin{tabular}{|c|c|c|c|c|c|c|c|c|c|c|c|c|c|c|}
\hline & & JAM. & FEA. & nak. & APR. & $\operatorname{mar}$ & SunE & sul $r$ & AUE. & SEP. & ocr. & nok. & DEC. & CUM. TORAL \\
\hline 1957 & 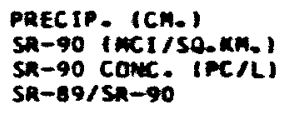 & $\bar{z}$ & $=$ & $\bar{z}$ & $=$ & $=$ & $=$ & $\bar{E}$ & $\begin{array}{l}0.92 \\
0.45 \\
5.05 \\
-\end{array}$ & $\begin{array}{l}9.91 \\
0.18 \\
1.82 \\
-\end{array}$ & $\begin{array}{l}2.97 \\
0.03 \\
1.02 \\
\end{array}$ & $\begin{array}{l}1.98 \\
0.03 \\
1.52 \\
-\end{array}$ & $\begin{array}{r}1.98 \\
0.03 \\
1.52 \\
27.00\end{array}$ & $\begin{array}{r}25.76 \\
0.72\end{array}$ \\
\hline 1958 & $\begin{array}{l}\text { PAECIP }(C M-) \\
\text { SR-9C }\left(M C I / S M_{-} \mathrm{KM}\right) \\
S R-90 \text { CONC. }(P C / L) \\
S R-99 / 5 R-90\end{array}$ & $\begin{array}{r}6.93 \\
0.05 \\
0.73 \\
25.00\end{array}$ & $\begin{array}{r}5.94 \\
0.07 \\
1.10 \\
10.00\end{array}$ & $\begin{array}{r}3.96 \\
0.44 \\
11.12 \\
22.000\end{array}$ & $\begin{array}{r}10.01 \\
0.49 \\
4.90 \\
18.00\end{array}$ & $\begin{array}{r}3.96 \\
0.19 \\
4.00 \\
18.00\end{array}$ & $\frac{20.00}{=}$ & $\begin{array}{r}12.88 \\
2.35 \\
10.49 \\
6.00\end{array}$ & $\begin{array}{r}16.04 \\
0.88 \\
5.23 \\
27.00\end{array}$ & $\begin{array}{r}6.93 \\
0.31 \\
-4.48 \\
26.00\end{array}$ & $\begin{array}{r}7.92 \\
0.25 \\
3.16 \\
22.00\end{array}$ & $\begin{array}{r}9.91 \\
0.58 \\
1.82 \\
52.00\end{array}$ & $\begin{array}{r}8.92 \\
0.40 \\
4.49 \\
45.00\end{array}$ & $\begin{array}{r}115.00 \\
4.61\end{array}$ \\
\hline 1959 & $\begin{array}{l}\text { PRECIP. }\left(C M_{-}\right) \\
5 R-90 \text { iFCI/SQ.KM.) } \\
5 R-90 \text { CONC. }(P C / L) \\
5 R-89 / 5 R-90\end{array}$ & $\begin{array}{r}0.99 \\
0.12 \\
12.13 \\
28.00\end{array}$ & \pm & $\begin{array}{r}6.100 \\
0.29 \\
4.76 \\
68.00\end{array}$ & $\begin{array}{r}15.01 \\
1.46 \\
8.73 \\
8.80\end{array}$ & $\begin{array}{l}5.99 \\
0.45 \\
7.52 \\
7.90\end{array}$ & $\begin{array}{r}16.03 \\
1.39 \\
8.68 \\
5.10\end{array}$ & $\begin{array}{l}7.57 \\
6.046 \\
6.08 \\
2.70\end{array}$ & $\begin{array}{r}12.55 \\
0.42 \\
3.35 \\
2.10\end{array}$ & $\begin{array}{l}2.49 \\
0.05 \\
2.01 \\
0.98\end{array}$ & $\begin{array}{l}5.64 \\
C .07 \\
1.25 \\
1.80\end{array}$ & $\begin{array}{l}7.87 \\
0.02 \\
0.26 \\
*\end{array}$ & $\begin{array}{l}9.60 \\
0.07 \\
0.73 \\
2.880\end{array}$ & $\begin{array}{r}89.84 \\
4.80\end{array}$ \\
\hline 1960 & $\begin{array}{l}\text { PRECIP (CH.) } \\
S R-90 \text { IFCI/SO.KM-) } \\
S R-90 \text { CONC. }(P C / L) \\
S R-89 / S R-90\end{array}$ & $\begin{array}{l}2.18 \\
0.15 \\
6.87 \\
-\end{array}$ & $\begin{array}{l}4.47 \\
0.06 \\
1.35 \\
-\end{array}$ & $\begin{array}{l}7.75 \\
0.71 \\
1.92 \\
-.-\end{array}$ & $\begin{array}{l}4.57 \\
0.12 \\
2.63 \\
-\end{array}$ & $\begin{array}{l}3.25 \\
0.24 \\
1.39 \\
-\end{array}$ & $\begin{array}{r}10.6 .2 \\
0.20 \\
1.89 \\
-. .\end{array}$ & $\begin{array}{l}15.70 \\
0.14 C \\
0.90 \\
--\end{array}$ & $\begin{array}{l}10.31 \\
0.096 \\
0.88 \\
-\end{array}$ & $\begin{array}{l}13.26 \\
0.066 \\
0.46 \\
-\end{array}$ & $\begin{array}{l}10.92 \\
0.05 c \\
0.066 \\
-\end{array}$ & $\begin{array}{l}10.01 \\
0.070 \\
0.70 \\
-.\end{array}$ & $\begin{array}{l}11.28 \\
0.08 C \\
0.71 \\
--\end{array}$ & $\begin{array}{r}104.32 \\
1.37\end{array}$ \\
\hline 1961 & $\begin{array}{l}\text { PREC IP }(C H-) \\
S R-9 C \text { INCIISO.KH.) } \\
S R-90 \text { CONC. }(P C / L) \\
S R-89 / S R-90\end{array}$ & $\begin{array}{l}5.18 \\
0.076 \\
1.36 \\
\end{array}$ & $\begin{array}{l}1.09 \\
0.016 \\
0.92 \\
-.\end{array}$ & $\begin{array}{l}3.05 \\
0.086 \\
2.693 \\
-\end{array}$ & $\begin{array}{l}2.67 \\
0.08 \mathrm{C} \\
3.00 \\
\end{array}$ & $\begin{array}{l}7.52 \\
0.276 \\
3.60 \\
=-\end{array}$ & $\begin{array}{l}6.60 \\
0.236 \\
3.49 \\
+\end{array}$ & $\begin{array}{l}12.85 \\
0.24 C \\
1.87 \\
--.\end{array}$ & $\begin{array}{l}2.82 \\
0.056 \\
2.70 \\
-\end{array}$ & $\begin{array}{l}4.32 \\
c .03 \\
c .70 \\
3.90\end{array}$ & $\begin{array}{r}10.41 \\
0.15 \\
1.45 \\
56.00\end{array}$ & $\begin{array}{r}11.38 \\
0.26 \\
2.29 \\
83.00\end{array}$ & $\begin{array}{r}7.62 \\
0.19 \\
2.50 \\
62.00\end{array}$ & $\begin{array}{r}75.51 \\
1.66\end{array}$ \\
\hline 1562 & $\begin{array}{l}\text { PRECIP. (CMA) } \\
S R-90 \text { (PCI/SO.KMA) } \\
S R-90 \text { CONC. }(P C / L) \\
S R-89 / S R-90\end{array}$ & $\begin{array}{r}-5.16 \\
0.16 \\
3.69 \\
41.00\end{array}$ & $\begin{array}{r}1.91 \\
0.12 \\
6.29 \\
26.00\end{array}$ & $\begin{array}{r}5.44 \\
0.29 \\
5.34 \\
1.9 .00\end{array}$ & $\begin{array}{r}5.31 \\
0.57 \\
10.74 \\
3.00\end{array}$ & $\begin{array}{r}21.46 \\
2.20 \\
10.26 \\
9.00\end{array}$ & $\begin{array}{r}11.38 \\
1.84 \\
16.17 \\
11.00\end{array}$ & $\begin{array}{r}13.39 \\
2.25 \\
16.21 \\
6.00\end{array}$ & $\begin{array}{r}2.34 \\
0.36 \\
15.39 \\
13.00\end{array}$ & $\begin{array}{r}10.64 \\
0.66 \\
6.21 \\
18.60\end{array}$ & $\begin{array}{r}10.49 \\
0.48 \\
1.72 \\
41.00\end{array}$ & $\begin{array}{r}10.977 \\
0.218 \\
3.47 \\
36.00\end{array}$ & $\begin{array}{r}3.43 \\
0.19 \\
5.54 \\
33.00\end{array}$ & $\begin{array}{r}101.92 \\
9.23\end{array}$ \\
\hline 1963 & $\begin{array}{l}\text { PRECIP. }\left(C M_{-}\right) \\
S R-90 \text { (MCI/SQ } \\
S R-90 \text { CCNC. }(P C / L) \\
S R-89 / S R-90\end{array}$ & $\begin{array}{r}4.52 \\
0.23 \\
5.09 \\
43.00\end{array}$ & $\begin{array}{r}5.183 \\
0.25 \\
4.08 \\
25.00\end{array}$ & $\begin{array}{r}6.55 \\
0.69 \\
10.54 \\
17.00\end{array}$ & $\begin{array}{r}4.47 \\
2.19 \\
49.00 \\
14.00\end{array}$ & $\begin{array}{r}15.24 \\
5.47 \\
35.90 \\
5.40\end{array}$ & $\begin{array}{r}6.68 \\
1.51 \\
22.61 \\
*\end{array}$ & $\begin{array}{r}6.25 \\
3.78 \\
60.48 \\
1.00\end{array}$ & $\begin{array}{r}15.04 \\
4.11 \\
27.33 \\
*\end{array}$ & $\begin{array}{r}9.22 \\
1.14 \\
12.37 \\
*\end{array}$ & $\begin{array}{r}2.39 \\
1.69 \\
70.72 \\
7\end{array}$ & $\begin{array}{l}14.07 \\
0.01 \\
4.034 \\
*\end{array}$ & $\begin{array}{r}5.41 \\
0.67 \\
12.39 \\
\end{array}$ & $\begin{array}{l}94.97 \\
22.34\end{array}$ \\
\hline 1964 & $\begin{array}{l}\text { PRECIP. (CH) } \\
\text { SR-9O (MCI/SQ.KM) } \\
\text { SR-9O CONC. }(P C / L) \\
\text { SR-89/SR-9O }\end{array}$ & $\begin{array}{r}0.03 \\
0.12 \\
399.99 \\
*\end{array}$ & $\begin{array}{r}0.99 \\
0.14 \\
14.15 \\
+\end{array}$ & $\begin{array}{r}4.05 \\
0.56 \\
12.05\end{array}$ & $\begin{array}{r}8.03 \\
1.47 \\
18.31 \\
*\end{array}$ & $\begin{array}{r}7.32 \\
1.78 \\
24.32 \\
-\end{array}$ & $\begin{array}{r}12.17 \\
1.65 \\
11.92 \\
\end{array}$ & $\begin{array}{r}10.21 \\
2.23 \\
22.06 \\
\end{array}$ & $\stackrel{1.12}{-}$ & $\begin{array}{l}5.84 \\
0.32 \\
5.48 \\
\end{array}$ & $\begin{array}{r}26.92 \\
0.45 \\
1.420 \\
8.90\end{array}$ & $\begin{array}{l}7.85 \\
0.03 \\
0.39 \\
\end{array}$ & $\begin{array}{l}6.60 \\
0.14 \\
2.13 \\
\end{array}$ & $\begin{array}{r}90.51 \\
8.81\end{array}$ \\
\hline 1965 & $\begin{array}{l}\text { PRECIP }(C M-) \\
S R-90 \text { (MCI/SQ-KM-) } \\
S R-90 \text { CONC. }(P C / L) \\
\text { SR-89/SR-90 }\end{array}$ & $\begin{array}{l}6.88 \\
0.28 \\
4.07 \\
--\end{array}$ & $\begin{array}{r}0.05 \\
0.02 \\
40.01 \\
-\end{array}$ & $\begin{array}{l}4.95 \\
0.02 \\
0.42 \\
-\end{array}$ & $\begin{array}{r}14.83 \\
0.59 \\
3.98 \\
\end{array}$ & $\bar{m}$ & $=$ & $\begin{array}{l}20.17 \\
1.61 \\
7.99 \\
-\end{array}$ & $\begin{array}{l}10.74 \\
0.89 \\
8.29 \\
-.\end{array}$ & $\begin{array}{r}20.27 \\
0.55 \\
2.72 \\
-\end{array}$ & $\begin{array}{r}0.05 \\
0.07 \\
140.00 \\
\end{array}$ & $\begin{array}{l}55.85 \\
0.19 \\
1.20 \\
-\end{array}$ & $\begin{array}{l}2.23 \\
0.09 \\
1.10 \\
\end{array}$ & $\begin{array}{r}101.92 \\
4.31\end{array}$ \\
\hline 1966 & $\begin{array}{l}\text { PRECIP. }(C M-) \\
\text { SR-9O (PCI/SO-KM-) } \\
\text { SR-9O CONC. (PC/L) } \\
\text { SR-89/SR-9O }\end{array}$ & $\begin{array}{l}2.34 \\
0.08 \\
3.42 \\
-.\end{array}$ & $\begin{array}{l}3.40 \\
0.02 \\
0.59 \\
-\end{array}$ & $\begin{array}{l}2.59 \\
0.18 \\
6.95 \\
-\end{array}$ & $\begin{array}{l}4.22 \\
0.16 \\
3.80 \\
-\end{array}$ & $\begin{array}{l}7.23 \\
0.09 \\
1.25 \\
--\end{array}$ & $\begin{array}{l}7.36 \\
0.18 \\
2.45 \\
8.90\end{array}$ & $\begin{array}{r}11.50 \\
0.11 \\
0.96 \\
5.00\end{array}$ & $\begin{array}{r}12.67 \\
0.28 \\
2.21 \\
1.30\end{array}$ & $\begin{array}{r}32.90 \\
0.08 \\
0.22 \\
.\end{array}$ & $\begin{array}{l}8.02 \\
0.05 \\
0.63 \\
.\end{array}$ & $\begin{array}{r}12.66 \\
0.07 \\
0.56 \\
8.10\end{array}$ & $\frac{--}{0.04}$ & $\begin{array}{r}109.89 \\
1.34\end{array}$ \\
\hline 1967 & $\begin{array}{l}\text { PRECIP. (CM.) } \\
\text { SR-90 IWCI/SG-KM.) } \\
\text { SR-90 COMC. (PC/L) } \\
\text { SR-89/SR-90 }\end{array}$ & $\begin{array}{l}1.17 \\
0.01 \\
0.86 \\
*\end{array}$ & $\begin{array}{l}2.62 \\
0.03 \\
1.15 \\
*\end{array}$ & $\begin{array}{r}8.99 \\
0.05 \\
0.56 \\
11.70\end{array}$ & $\begin{array}{l}7.01 \\
0.07 \\
1.00 \\
7.40\end{array}$ & $\begin{array}{l}6.85 \\
0.18 \\
2.63 \\
3.80\end{array}$ & $\begin{array}{l}= \\
=\end{array}$ & $\bar{z}$ & $\overline{-}$ & $\overline{-}$ & $\bar{z}$ & $\bar{z}$ & $\overline{-}$ & $\begin{array}{r}26.64 \\
0.34\end{array}$ \\
\hline \begin{tabular}{l} 
NOTES \\
\hdashline$:$ OA \\
$A: Z A$ \\
$A:$ Af \\
$B:$ LC \\
$C:$ PI
\end{tabular} & $\begin{array}{l}\text { A ROT AVAILABLE } \\
\text { O OR TRACE } \\
\text { ROXFATE } \\
\text { ER LIMIT OF REPO } \\
\text { PORTIONEQ FROH O }\end{array}$ & VALLY & & & & & & & & & & & & \\
\hline
\end{tabular}




\begin{tabular}{|c|c|c|c|c|c|c|c|c|c|c|c|c|c|c|}
\hline & & JAN. & FEB. & MAR. & APR. & MAY & JUNE & JULY & AUG. & SEP. & OCT. & NOV. & DEC.. & CUM. TOTAL \\
\hline 1957 & $\begin{array}{l}\text { PRECIP. ICH. } \\
\text { SR-90 IMCI/SQ.KH.) } \\
S R-90 \text { CONC. }(P C / L) \\
\text { SR-89/SR-90 }\end{array}$ & $\begin{array}{l}= \\
=\end{array}$ & $\begin{array}{l}-- \\
= \\
=\end{array}$ & $\begin{array}{l}-- \\
--\end{array}$ & $\bar{m}$ & $\begin{array}{l}-- \\
-- \\
--\end{array}$ & $\begin{array}{l}1.98 \\
0.17 \\
8.59 \\
--\end{array}$ & $\begin{array}{l}12.88 \\
0.61 \\
4.74 \\
--\end{array}$ & $\begin{array}{l}6.93 \\
0.30 \\
4.33 \\
\end{array}$ & $\begin{array}{l}5.94 \\
0.23 \\
3.88 \\
--\end{array}$ & $\begin{array}{l}* \\
0.01 \\
--\end{array}$ & $\begin{array}{l}4.95 \\
0.08 \\
1.62 \\
-\end{array}$ & $\begin{array}{r}3.96 \\
0.04 \\
1.02 \\
27.00\end{array}$ & $\begin{array}{r}36.64 \\
1.44\end{array}$ \\
\hline 1958 & $\begin{array}{l}\text { PRECIP, }\left(C M_{-}\right) \\
S R-90 \text { (MCI/SQ.KM.) } \\
S R-9 C \text { CONC. }(P C / L) \\
S R-B 9 / S R-90\end{array}$ & $\begin{array}{l}2.97 \\
0.06 \\
2.03 \\
25.00\end{array}$ & $\begin{array}{l}5.94 \\
0.10 \\
1.69 \\
14.00\end{array}$ & $\begin{array}{r}6.93 \\
0.14 \\
2.03 \\
15.00\end{array}$ & $\begin{array}{r}1.98 \\
0.27 \\
13.64 \\
18.00\end{array}$ & $\begin{array}{r}1.98 \\
0.27 \\
13.64 \\
17.00\end{array}$ & $\begin{array}{r}14.86 \\
1.21 \\
8.15 \\
1.00\end{array}$ & $\begin{array}{r}4.95 \\
0.41 \\
8.29 \\
12.00\end{array}$ & $\begin{array}{r}9.91 \\
0.71 \\
7.17 \\
20.00\end{array}$ & $\begin{array}{l}1.98 \\
C .01 \\
0.51 \\
--\end{array}$ & $\begin{array}{r}6.93 \\
\text { C.29 } \\
4.19 \\
34.00\end{array}$ & $\begin{array}{r}2.97 \\
0.19 \\
6.40 \\
42.00\end{array}$ & $\begin{array}{l}6.93 \\
0.47 \\
6.79 \\
46.00\end{array}$ & $\begin{array}{r}68.33 \\
4.13\end{array}$ \\
\hline 1959 & $\begin{array}{l}\text { PRECIP. ICM.) } \\
S R-9 C \text { IMCI/SO.KM.) } \\
S R-90 \text { CONC. }(P C / L) \\
S R-89 / S R-90\end{array}$ & $\begin{array}{r}2.54 \\
0.17 \\
6.70 \\
33.00\end{array}$ & $\begin{array}{l}2.01 \\
=- \\
=-\end{array}$ & $\begin{array}{r}3.53 \\
0.44 \\
12.47 \\
15.50\end{array}$ & $\begin{array}{r}9.02 \\
1.49 \\
16.52 \\
11.70\end{array}$ & $\begin{array}{r}5.00 \\
1.00 \\
20.00 \\
8.00\end{array}$ & $\begin{array}{r}15.01 \\
1.6 C \\
10.66 \\
5.00\end{array}$ & $\begin{array}{r}15.09 \\
0.90 \\
5.97 \\
2.90\end{array}$ & $\begin{array}{r}11.38 \\
0.36 \\
3.17 \\
1.40\end{array}$ & $\begin{array}{r}0.05 \\
0.20 \\
400.00 \\
*\end{array}$ & $\begin{array}{l}-- \\
-- \\
--\end{array}$ & $\begin{array}{l}5.92 \\
0.03 \\
0.51 \\
*\end{array}$ & $\begin{array}{l}-- \\
-\overline{-} \\
--\end{array}$ & $\begin{array}{r}69.55 \\
6.19\end{array}$ \\
\hline 1960 & $\begin{array}{l}\text { PRECIP. ICM.) } \\
\text { SR-9O (MCI/SQ.KM-I) } \\
\text { SR-90 CONC. (PC/L) } \\
\text { SR-89/SR-90 }\end{array}$ & $\begin{array}{l}4.70 \\
0.01 \\
0.22 \\
--\end{array}$ & $\begin{array}{l}C .84 \\
0.05 \\
5.96 \\
--\end{array}$ & $\begin{array}{l}4.06 \\
0.14 \\
3.45 \\
--\end{array}$ & $\begin{array}{l}2.46 \\
0.08 \\
3.26 \\
--\end{array}$ & $\begin{array}{l}5.41 \\
-- \\
--\end{array}$ & $\begin{array}{l}7.90 \\
-- \\
-- \\
--\end{array}$ & $\begin{array}{l}8.71 \\
0.04 C \\
0.46 \\
--\end{array}$ & $\begin{array}{l}7.09 \\
0.036 \\
0.43 \\
--\end{array}$ & $\begin{array}{l}5.00 \\
0.04 C \\
C .81 \\
--\end{array}$ & $\begin{array}{l}6.38 \\
0.05 C \\
0.79 \\
--\end{array}$ & $\begin{array}{l}1.80 \\
-- \\
--\end{array}$ & $\begin{array}{l}2.72 \\
-- \\
--\end{array}$ & $\begin{array}{r}57.07 \\
0.44\end{array}$ \\
\hline 1961 & $\begin{array}{l}\text { PRECIP. (CH-) } \\
S R-90 \text { (NCI/SQ-KM-) } \\
S R-9 C \text { CONC. (PC/L) } \\
S R-8 S / S R-S C\end{array}$ & $\begin{array}{l}0.89 \\
0.026 \\
2.25 \\
-.\end{array}$ & $\begin{array}{l}6.81 \\
0.14 C \\
2.06 \\
-.\end{array}$ & $\begin{array}{l}3.56 \\
* \\
--\end{array}$ & $\begin{array}{l}6.48 \\
* \\
--\end{array}$ & $\begin{array}{l}8.41 \\
0.12 C \\
1.43 \\
--\end{array}$ & $\begin{array}{l}4.80 \\
0.076 \\
1.46 \\
--\end{array}$ & $\begin{array}{l}8.28 \\
C .05 C \\
C .61 \\
--\end{array}$ & $\begin{array}{l}2.82 \\
0.026 \\
0.71 \\
--\end{array}$ & $\begin{array}{r}2.24 \\
0.03 \\
1.34 \\
37.00\end{array}$ & $\begin{array}{r}5.87 \\
0.18 \\
3.07 \\
62.00\end{array}$ & $\begin{array}{l}-- \\
\overline{-} \\
--\end{array}$ & $\begin{array}{l}5.21 \\
0.07 \\
1.35 \\
3.00\end{array}$ & $\begin{array}{r}55.37 \\
0.70\end{array}$ \\
\hline 1562 & $\begin{array}{l}\text { PRECIP. }(C M-) \\
S R-90 \text { INCI/SQ.KM-) } \\
S R-90 \text { CCNC. }(P C / L) \\
S R-89 / S R-S C\end{array}$ & $=$ & $\begin{array}{l}3.99 \\
=- \\
--\end{array}$ & $\begin{array}{r}5.36 \\
0.44 \\
8.21 \\
18.00\end{array}$ & $\begin{array}{r}6.43 \\
0.69 \\
10.74 \\
12.00\end{array}$ & $\begin{array}{r}5.18 \\
1.72 \\
33.21 \\
8.00\end{array}$ & $\begin{array}{r}2.49 \\
0.46 \\
18.48 \\
1.50\end{array}$ & $\begin{array}{r}5.18 \\
1.01 \\
19.50 \\
8.00\end{array}$ & $\begin{array}{r}2.54 \\
0.46 \\
18.12 \\
5.00\end{array}$ & $\begin{array}{r}2.51 \\
0.24 \\
9.57 \\
19.00\end{array}$ & $\begin{array}{r}4.93 \\
0.35 \\
7.10 \\
32.00\end{array}$ & $\begin{array}{r}10.01 \\
0.47 \\
4.70 \\
42.00\end{array}$ & $\begin{array}{r}4.27 \\
0.27 \\
6.33 \\
46.00\end{array}$ & $\begin{array}{r}52.89 \\
6.11\end{array}$ \\
\hline 1963 & $\begin{array}{l}\text { PREC IP. (CM+) } \\
\text { SR-90 (MCI/SQ.KM.) } \\
\text { SR-9O CCNC. (PC/L) } \\
S R-89 / S R-90\end{array}$ & $\begin{array}{r}3.96 \\
3.26 \\
82.33 \\
34.00\end{array}$ & $\begin{array}{r}2.21 \\
0.10 \\
4.53 \\
24.00\end{array}$ & $\begin{array}{r}4.75 \\
0.81 \\
17.06 \\
16.00\end{array}$ & $\begin{array}{l}2.84 \\
1.28 \\
45.08 \\
14.00\end{array}$ & $\begin{array}{r}6.99 \\
2.62 \\
37.49 \\
6.30\end{array}$ & $\begin{array}{r}5.28 \\
2.60 \\
49.25 \\
*\end{array}$ & $\begin{array}{r}1.07 \\
0.86 \\
80.38 \\
0.40\end{array}$ & $\begin{array}{r}8.51 \\
1.92 \\
22.57 \\
1.00\end{array}$ & $\begin{array}{r}4.85 \\
0.81 \\
12.58 \\
0.70\end{array}$ & $\begin{array}{l}2.44 \\
0.24 \\
9.84 \\
1.20\end{array}$ & $\begin{array}{r}3.02 \\
0.35 \\
11.59 \\
+\end{array}$ & $\begin{array}{r}0.58 \\
0.30 \\
51.73 \\
*\end{array}$ & $\begin{array}{l}46.50 \\
14.95\end{array}$ \\
\hline 1964 & $\begin{array}{l}\text { PRECIP I ICM.) } \\
\text { SR-9C IMCI/SQ-KM.) } \\
S R-90 \text { CONC. (PC/LI) } \\
\text { SR-89/SR-90 }\end{array}$ & $\begin{array}{r}C .74 \\
c .12 \\
16.22 \\
0.70\end{array}$ & $\begin{array}{r}2.92 \\
0.58 \\
19.87 \\
*\end{array}$ & $\begin{array}{r}7.21 \\
1.05 \\
14.57 \\
*\end{array}$ & $\begin{array}{r}6.10 \\
0.69 \\
11.32 \\
3.00\end{array}$ & $\begin{array}{l}6.81 \\
2.83 \\
41.56 \\
-0\end{array}$ & $\begin{array}{r}0.03 \\
0.08 \\
266.67 \\
--\end{array}$ & $\begin{array}{r}3.73 \\
0.94 \\
25.21 \\
--\end{array}$ & $\begin{array}{r}5.23 \\
1.03 \\
19.70 \\
--\end{array}$ & $\begin{array}{r}2.08 \\
0.24 \\
11.54 \\
-\end{array}$ & $\begin{array}{r}13.18 \\
C .17 \\
1.29 \\
1.80\end{array}$ & $\begin{array}{l}2.62 \\
0.18 \\
6.88 \\
--\end{array}$ & $\begin{array}{l}4.85 \\
0.15 \\
3.10 \\
--\end{array}$ & $\begin{array}{r}55.50 \\
8.06\end{array}$ \\
\hline 1965 & $\begin{array}{l}\text { PRECIP }(C M-) \\
\text { SR-90 INCI/SQ-KM-) } \\
\text { SR-90 CONC. }(P C / L) \\
\text { SR-89/SR-90 }\end{array}$ & $\begin{array}{r}4.24 \\
0.75 \\
17.69 \\
-0\end{array}$ & $\begin{array}{l}4.75 \\
0.07 \\
1.48 \\
--\end{array}$ & $\begin{array}{l}4.39 \\
0.02 \\
0.46 \\
-.\end{array}$ & $\begin{array}{r}13.16 \\
0.70 \\
5.32 \\
--\end{array}$ & $\begin{array}{l}-- \\
-- \\
--\end{array}$ & $\begin{array}{l}-- \\
-- \\
=-\end{array}$ & $\begin{array}{r}8.33 \\
1.83 \\
21.97 \\
--\end{array}$ & $\begin{array}{r}5.69 \\
0.62 \\
10.90 \\
--\end{array}$ & $\begin{array}{l}4.83 \\
0.05 \\
1.04 \\
-.\end{array}$ & $\begin{array}{r}C .03 \\
C .02 \\
66.67 \\
-.\end{array}$ & $\begin{array}{l}2.95 \\
-- \\
--\end{array}$ & $\begin{array}{l}6.78 \\
0.06 \\
0.89 \\
--\end{array}$ & $\begin{array}{r}55.15 \\
4.12\end{array}$ \\
\hline 1966 & $\begin{array}{l}\text { PRECIP }(C M-) \\
\text { SR-9O IMCI/SQ-KM.) } \\
\text { SR-9C CONC. }(P C / L) \\
\text { SR-89/SR-90 }\end{array}$ & $\begin{array}{l}3.37 \\
0.15 \\
4.46 \\
-.\end{array}$ & $\begin{array}{l}5.33 \\
0.04 \\
0.76 \\
--\end{array}$ & $\begin{array}{r}2.18 \\
0.25 \\
11.47 \\
--\end{array}$ & $\begin{array}{l}3.92 \\
0.18 \\
4.60 \\
-.\end{array}$ & $\begin{array}{l}6.89 \\
0.37 \\
5.38 \\
--\end{array}$ & $\begin{array}{l}8.43 \\
0.02 \\
0.24 \\
*\end{array}$ & $\begin{array}{r}12.34 \\
0.16 \\
1.30 \\
5.10\end{array}$ & $\begin{array}{l}13.30 \\
0.69 \\
0.68 \\
*\end{array}$ & $\begin{array}{l}1.86 \\
* \\
--\end{array}$ & $\begin{array}{l}5.37 \\
0.06 \\
1.12 \\
*\end{array}$ & $\begin{array}{l}8.37 \\
0.11 \\
1.32 \\
6.30\end{array}$ & $\begin{array}{r}6.67 \\
0.15 \\
2.25 \\
17.20\end{array}$ & $\begin{array}{r}78.03 \\
1.58\end{array}$ \\
\hline 1967 & $\begin{array}{l}\text { PRECIP. (CM.) } \\
\text { SR-9O IMCI/SU.KM-) } \\
\text { SR-90 CDNC. (PC/L) } \\
\text { SR-89/SR-90 }\end{array}$ & $\begin{array}{r}3.59 \\
0.06 \\
1.68 \\
10.20\end{array}$ & $\begin{array}{l}3.06 \\
0.05 \\
1.64 \\
8.90\end{array}$ & $\begin{array}{l}4.01 \\
0.15 \\
3.75 \\
6.60\end{array}$ & $\begin{array}{l}3.20 \\
0.05 \\
1.57 \\
+\end{array}$ & $\begin{array}{l}9.07 \\
0.12 \\
1.33 \\
4.70\end{array}$ & $\begin{array}{l}-- \\
-- \\
--\end{array}$ & $\begin{array}{l}-- \\
--\end{array}$ & $\begin{array}{l}-- \\
-- \\
--\end{array}$ & $\begin{array}{l}-- \\
-- \\
--\end{array}$ & $\begin{array}{l}-\overline{-} \\
\overline{-} \\
-\overline{-}\end{array}$ & $=$ & $\begin{array}{l}-- \\
--\end{array}$ & $\begin{array}{r}22.93 \\
0.43\end{array}$ \\
\hline \multicolumn{15}{|c|}{$\begin{array}{l}\text { NOTES } \\
-\because: \text { DATA NOT AVAILABLE } \\
\text { *: ZERO OR TRACE } \\
\text { A: APPROXIMATE } \\
\text { B: LOWER LIMIT OF REPORTED DATA }\end{array}$} \\
\hline
\end{tabular}




$$
\begin{aligned}
& \text { JAN. FEB. MAR. APR. MAY JUNE JULY AUG. SEP. OCT. NOV. OEC. CUM. TOTAL }
\end{aligned}
$$

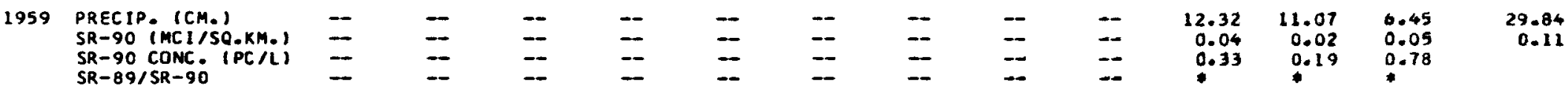

PRECIP. (CM.) 21.39

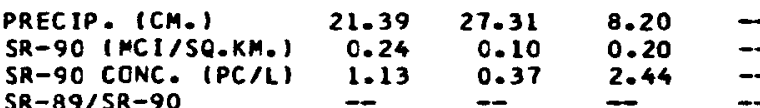

1961 PRECIP. (CM.)

$S R-89 / S R-90$
$S R-90$ C

SR-90 (MCI/SO-KM.) 0.30

$\begin{array}{rr}S R-90 & \text { CONC. } \\ S R-89 / S R-90 & 4.44\end{array}$

1963 PRECIP. (CM.) $\begin{array}{lr}S R-90 \text { CONC. }(P C / L) & 9.44 \\ S R-89 / S R-90 & 33.00\end{array}$

1964 PRECIP. (CM.)

SR-90 (MC $1 / S Q . K M$.) $S R-89 / S R-90$

1965 PRECIP, (CM,)

\begin{tabular}{lrrrr} 
& & & & \\
SR-90 (MCI/SO.KM.) & 0.45 & 21.59 & 12.88 & 2.21 \\
\hline & 0.39 & 0.42 & 0.30
\end{tabular}

SR-90 CONC. (PC/L) 2.64

1966 PRECIP. (CM.)

SR-90 (KCI/SO.KM.) 0.03

SR-90 CONC. (PC/L) 0.09

1967 PRECIP. (CM.)

SR-90 (MCIISO-KH) 19.25 SR-90 CCNC. (PC/L) 0.94 $S R-89 / S R-90$

0.94
5.00

0.49

0.01
0.10

0.01

$--$

*: ZERO OR TRACE

A: APPROXIMATE

OF REPORTED DATA

C: PROPURTIUIED FRON CRTGINALT' CONSOLICATEO DATA 
SITE: BAHAMAS, BIMINI

MONTHEY FALLOUT DEPOSITION COLLECTIONS

SOURCE： LERNER MARIIE LUORATORY

LAT. 25 4OM LONG. 79 15N ALT. OM. (COLUMN) SURFACE AIR SAMPLING STATIDN

\begin{tabular}{|c|c|c|c|c|c|c|c|c|c|c|c|c|c|c|}
\hline & & JAN. & FEB. & MAR. & APR. & MAY & JUNE & JULY & AUG. & SEP. & OCT. & NOV. & DEC. & CUM. TOTAL \\
\hline 1966 & $\begin{array}{l}\text { PRECIP. (CM-) } \\
\text { SR-90 IMCI/SQ-KM-) } \\
\text { SR-90 CONC. (PC/L) } \\
\text { SR-89/SR-90 }\end{array}$ & $\begin{array}{l}6.86 \\
-- \\
--\end{array}$ & $\begin{array}{l}7.52 \\
-- \\
--\end{array}$ & $\begin{array}{l}7.42 \\
-- \\
--\end{array}$ & $\begin{array}{l}2.44 \\
-- \\
-\end{array}$ & $\begin{array}{l}5.33 \\
0.14 \\
2.63 \\
-\end{array}$ & $\begin{array}{r}12.70 \\
0.08 \\
0.63 \\
18.00\end{array}$ & $\begin{array}{l}9.12 \\
0.06 \\
0.66 \\
*\end{array}$ & $\begin{array}{l}9.32 \\
0.12 \\
1.29 \\
*\end{array}$ & $\begin{array}{r}12.60 \\
0.52 \\
4.13 \\
*\end{array}$ & $\begin{array}{l}28.55 \\
=- \\
--\end{array}$ & $\begin{array}{l}1.75 \\
0.04 \\
2.29\end{array}$ & $\begin{array}{l}1.62 \\
0.03 \\
1.86 \\
*\end{array}$ & $\begin{array}{r}105.23 \\
0.99\end{array}$ \\
\hline 1967 & $\begin{array}{l}\text { PRECIP. (CM.) } \\
\text { SR-90 INCI/SQ.KM) } \\
\text { SR-90 CONC. }(P C / L) \\
\text { SR-89/SR-90 }\end{array}$ & $\begin{array}{r}3.48 \\
0.04 \\
1.15 \\
14-00\end{array}$ & $\begin{array}{l}0.96 \\
0.02 \\
2.09 \\
8.20\end{array}$ & $\begin{array}{r}0.13 \\
0.04 \\
30.77 \\
10-00\end{array}$ & $\frac{*}{0.01}$ & $\begin{array}{l}3.68 \\
0.04 \\
1.09 \\
6.30\end{array}$ & $\begin{array}{l}46.30 \\
0.17 \\
0.37 \\
*\end{array}$ & $\begin{array}{l}3-40 \\
=- \\
=-\end{array}$ & $\begin{array}{l}14.43 \\
=- \\
=-\end{array}$ & $\begin{array}{l}-- \\
--\end{array}$ & $\overline{-}$ & $\overline{-}$ & $\overline{-}$ & $\begin{array}{r}72.38 \\
0.32\end{array}$ \\
\hline
\end{tabular}

NOTES

-: DATA NOT AVAILABLE

*: ZERO OR TRACE

A: APPROXIMATE

B: LOWER LIMIT OF REPORTED DATA

$C$ : PROPORTIONED FROM ORIGINALLY CONSOLITANED EATA 


\begin{tabular}{|c|c|c|c|c|c|c|c|c|c|c|c|c|}
\hline JAN. & FEB. & MAR. & APR. & MAY & JUME & JULY & AUE. & SEEP - & $00 \pi$ & MDY & OEC. & CUM TOTAL \\
\hline $\begin{array}{l}13.64 \\
= \\
-\end{array}$ & $\frac{14.20}{=}$ & $\begin{array}{l}11.46 \\
=\end{array}$ & $\begin{array}{l}13.49 \\
=\end{array}$ & $\begin{array}{l}5.23 \\
-\end{array}$ & $\begin{array}{l}12.90 \\
= \\
=\end{array}$ & $=$ & $\begin{array}{l}16.38 \\
=\end{array}$ & $\begin{array}{r}14=76 \\
0-06 \\
D=41 \\
1-30\end{array}$ & $\begin{array}{r}16.411 \\
0.0 .5 \\
0.31 \\
1.00\end{array}$ & $\begin{array}{r}14.02 \\
0.13 \\
0.93 \\
0.50\end{array}$ & $\begin{array}{r}16.03 \\
0.16 \\
1.00 \\
*\end{array}$ & $\begin{array}{r}152,63 \\
0.040\end{array}$ \\
\hline $\begin{array}{l}10.13 \\
0.05 \\
0.50 \\
-.\end{array}$ & $\begin{array}{l}\mathrm{C}_{-12} \\
--\end{array}$ & $\begin{array}{l}10.31 \\
0.22 \\
2.24 \\
-\end{array}$ & $\begin{array}{l}3.02 \\
0.10 \\
3.32 \\
-\end{array}$ & $\begin{array}{c}18.34 \\
= \\
=\end{array}$ & $\begin{array}{c}10.59 \\
= \\
=\end{array}$ & $\begin{array}{l}11.33 \\
* \\
-\end{array}$ & $\begin{array}{l}9 . B 3 \\
- \\
-\end{array}$ & $\begin{array}{c}25.35 \\
0.050 \\
0.20 \\
-\end{array}$ & $\begin{array}{c}2 C .42 \\
C .04 C \\
D .20 \\
-\end{array}$ & $\begin{array}{l}5.08 \\
C .026 \\
0.40 \\
-\end{array}$ & $\begin{array}{l}29.79 \\
0.126 \\
0.41 \\
-\end{array}$ & $\begin{array}{r}154,17 \\
0.72\end{array}$ \\
\hline $\begin{array}{c}10.92 \\
0.10 \mathrm{C} \\
0.92 \\
-\end{array}$ & $\begin{array}{l}6.88 \\
0.06 C \\
0.88 \\
-\end{array}$ & $\begin{array}{l}4.34 \\
C .236 \\
5.30 \\
-\end{array}$ & $\begin{array}{c}16.87 \\
0.06 C \\
0.36 \\
-\end{array}$ & $\begin{array}{c}22.17 \\
C . C 2 C \\
0.10 \\
=\end{array}$ & $\begin{array}{c}15.24 \\
0.026 \\
0.14 \\
=\end{array}$ & $\begin{array}{l}8.48 \\
* \\
=\end{array}$ & $\begin{array}{c}10.67 \\
* \\
=-\end{array}$ & $\begin{array}{r}6.73 \\
0.04 \\
0.60 \\
47.00\end{array}$ & $\begin{array}{r}13.49 \\
0.05 \\
0.38 \\
60.00\end{array}$ & $\begin{array}{r}17.25 \\
0.01 \\
0.06 \\
43.00\end{array}$ & $\begin{array}{r}8.43 \\
0.26 \\
3.09 \\
60.00\end{array}$ & $\begin{array}{r}141.47 \\
0.85\end{array}$ \\
\hline $\begin{array}{r}10.39 \\
0.40 \\
3.85 \\
44.00\end{array}$ & $\begin{array}{r}5.92 \\
6.42 \\
7.10 \\
34.00\end{array}$ & $\begin{array}{r}22.94 \\
2.71 \\
11.82 \\
12.60\end{array}$ & $\begin{array}{r}13.03 \\
1.07 \\
8.22 \\
13.00\end{array}$ & $\begin{array}{l}8.43 \\
0.84 \\
9.97 \\
8.00\end{array}$ & $\begin{array}{r}36.27 \\
0.43 \\
1.19 \\
13.00\end{array}$ & $\begin{array}{r}10.46 \\
0.11 \\
1.06 \\
22.00\end{array}$ & $\begin{array}{r}14.22 \\
0.35 \\
2.47 \\
9.00\end{array}$ & $\begin{array}{r}26.54 \\
0.48 \\
1.81 \\
15.00\end{array}$ & $\begin{array}{r}6.10 \\
0.49 \\
8.04 \\
35.00\end{array}$ & $\begin{array}{r}26.77 \\
0.81 \\
3.03 \\
60.00\end{array}$ & $\begin{array}{r}11.79 \\
1.07 \\
9.08 \\
49.00\end{array}$ & $\begin{array}{r}192.86 \\
9.18\end{array}$ \\
\hline $\begin{array}{r}13.59 \\
0.18 \\
1.33 \\
30.00\end{array}$ & $\begin{array}{r}21.77 \\
0.13 \\
0.60 \\
20.00\end{array}$ & $\begin{array}{r}8.59 \\
0.78 \\
9.09 \\
23.00\end{array}$ & $\begin{array}{r}5.38 \\
0.33 \\
6.14 \\
11.00\end{array}$ & $\begin{array}{r}12.99 \\
0.09 \\
0.70 \\
7.20\end{array}$ & $\begin{array}{l}7.49 \\
0.27 \\
3.61 \\
*\end{array}$ & $\begin{array}{r}11.99 \\
0.97 \\
8.10 \\
5.00\end{array}$ & $\begin{array}{r}26.77 \\
1.06 \\
3.96 \\
*\end{array}$ & $\begin{array}{r}10.49 \\
0.49 \\
4.68 \\
1.40\end{array}$ & $\begin{array}{r}28.85 \\
0.78 \\
2.71 \\
0.80\end{array}$ & $\begin{array}{r}15.85 \\
0.43 \\
2.72 \\
*\end{array}$ & $\begin{array}{r}12.53 \\
1.40 \\
12.15 \\
*\end{array}$ & $\begin{array}{r}175.23 \\
6.91\end{array}$ \\
\hline $\begin{array}{l}16.69 \\
1.13 \\
6.78 \\
*\end{array}$ & $\begin{array}{r}14.17 \\
1.44 \\
10.17 \\
*\end{array}$ & $\begin{array}{r}4.85 \\
0.66 \\
13.61 \\
*\end{array}$ & $\begin{array}{r}2.01 \\
0.42 \\
20.90 \\
*\end{array}$ & $\begin{array}{l}8.46 \\
0.79 \\
9.34 \\
*\end{array}$ & $\begin{array}{c}10.95 \\
1.16 \\
10.60 \\
--\end{array}$ & $\begin{array}{r}4.27 \\
0.47 \\
11.01 \\
--\end{array}$ & $\begin{array}{c}20.65 \\
0.51 \\
2.50 \\
--\end{array}$ & $\begin{array}{r}27.48 \\
0.05 \\
0.19 \\
\end{array}$ & $\begin{array}{r}13.16 \\
0.29 \\
2.21 \\
6.90\end{array}$ & $\begin{array}{r}13.59 \\
0.32 \\
2.36 \\
*\end{array}$ & $\begin{array}{l}8.61 \\
0.37 \\
4.30 \\
-\end{array}$ & $\begin{array}{r}144.69 \\
7.61\end{array}$ \\
\hline $\begin{array}{l}7.87 \\
0.50 \\
6.36 \\
--\end{array}$ & $\begin{array}{l}6.32 \\
0.41 \\
6.49 \\
-\end{array}$ & $\begin{array}{r}12.88 \\
0.42 \\
3.27 \\
\end{array}$ & $\begin{array}{l}7.98 \\
0.69 \\
8.65 \\
-\end{array}$ & $\begin{array}{l}2.44 \\
0.22 \\
9.02 \\
\end{array}$ & $\begin{array}{l}9.19 \\
0.41 \\
6.47 \\
\end{array}$ & $\begin{array}{r}10.13 \\
0.03 \\
0.30 \\
\end{array}$ & $\begin{array}{l}9.88 \\
0.07 \\
0.71 \\
\end{array}$ & $\begin{array}{l}0.33 \\
0.16 \\
1.93 \\
\end{array}$ & $\begin{array}{l}10.29 \\
0.05 \\
0.49 \\
-\end{array}$ & $\begin{array}{c}10.54 \\
0.06 \\
0.57 \\
-\end{array}$ & $\begin{array}{c}20.93 \\
= \\
=\end{array}$ & $\begin{array}{r}11+78 \\
3.02\end{array}$ \\
\hline $\begin{array}{l}16.54 \\
0.06 \\
0.37 \\
-\end{array}$ & $\begin{array}{c}10.54 \\
0.38 \\
3.61 \\
--\end{array}$ & $\begin{array}{c}12.22 \\
0.42 \\
3.44 \\
--\end{array}$ & $\begin{array}{l}6.10 \\
0.21 \\
3.45 \\
\end{array}$ & $\begin{array}{l}3.66 \\
0.10 \\
2.74 \\
-\end{array}$ & $\begin{array}{r}11.51 \\
0.21 \\
1.83 \\
3.30\end{array}$ & $\begin{array}{r}15.27 \\
0.14 \\
0.92 \\
6.20\end{array}$ & $\begin{array}{l}8.48 \\
0.05 \\
0.59 \\
*\end{array}$ & $\begin{array}{r}12.72 \\
0.08 \\
0.63 \\
*\end{array}$ & $\begin{array}{r}28.07 \\
0.06 \\
0.22 \\
*\end{array}$ & $\begin{array}{r}13.87 \\
0.07 \\
0.51 \\
9.40\end{array}$ & $\begin{array}{l}10.57 \\
0.06 \\
0.57 \\
*\end{array}$ & $\begin{array}{r}149.55 \\
1.84\end{array}$ \\
\hline $\begin{array}{r}9.73 \\
0.05 \\
0.52 \\
10.00\end{array}$ & $\begin{array}{r}15.80 \\
0.11 \\
C .70 \\
9.90\end{array}$ & $\begin{array}{r}5.82 \\
0.10 \\
1.72 \\
10.50\end{array}$ & $\begin{array}{l}9.35 \\
0.17 \\
1.82 \\
7.70\end{array}$ & $\begin{array}{l}8.38 \\
0.10 \\
1.20 \\
3.00\end{array}$ & $\begin{array}{r}10.74 \\
0.07 \\
0.66 \\
2.70\end{array}$ & $\begin{array}{l}8.15 \\
0.06 \\
0.74 \\
*\end{array}$ & $=$ & 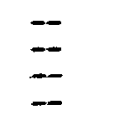 & $\begin{array}{l}= \\
=- \\
-\end{array}$ & $\begin{array}{l}-- \\
-- \\
--\end{array}$ & $\bar{z}$ & $\begin{array}{r}87.97 \\
0.66\end{array}$ \\
\hline
\end{tabular}


MONTHLY FALLCUT DEPOSITION COLLECTIONS

SITE: BOLIVIA, CHACALTAYA

LAT. 16215 LONG. 68 8W ALT.

5222M. (COLUMN) SURFACE AIR SAMPLING STATION SOURCE: UNIVERSIDAD MAYOR DE SAN ANDRES, LABORATORIO DE FISICA COSMICA

\begin{tabular}{|c|c|c|c|c|c|c|c|c|c|c|c|c|c|c|}
\hline & & JAN. & FEB. & MAR . & APR : & MAY & JUNE & JULY & AUG. & SEP: & OtT. & NOV. & DEC. & CUM. TOTAL \\
\hline 1963 & $\begin{array}{l}\text { PRECIP. }\left(C M_{-}\right) \\
\text {SR-90 (MCI/SQ.KM-) } \\
S R-90 \text { CCNC. }(P C / L) \\
S R-89 / S R-90\end{array}$ & $\begin{array}{l}-- \\
-- \\
--\end{array}$ & $\begin{array}{l}-- \\
-- \\
--\end{array}$ & $\begin{array}{l}-- \\
=- \\
--\end{array}$ & $\begin{array}{l}-\infty \\
-- \\
-\end{array}$ & $\begin{array}{l}3.94 \\
0.03 \\
0.77 \\
0.40\end{array}$ & $\begin{array}{l}9.026 \\
0.126 \\
1.34 \\
-.\end{array}$ & $\begin{array}{l}9.02 C \\
0.12 C \\
1.34 \\
-.\end{array}$ & $\begin{array}{l}1.45 A \\
0.05 A \\
3.45 \\
-.\end{array}$ & $\begin{array}{l}5.08 \\
0.08 \\
1.42 \\
+\end{array}$ & $\begin{array}{l}2.54 \\
0.05 \\
1.97 \\
\end{array}$ & $\begin{array}{l}5.13 \\
0.06 \\
1.17 \\
*\end{array}$ & $\begin{array}{l}8.99 \\
0.07 \\
0.78 \\
--\end{array}$ & $\begin{array}{r}45.73 \\
0.58\end{array}$ \\
\hline 1964 & $\begin{array}{l}\text { PRECIP. (CM.) } \\
\text { SR-90 (PCI/SQ.KM.) } \\
S R-90 \text { CCNC. }(P C / L) \\
S R-89 / S R-90\end{array}$ & $\begin{array}{l}9.58 \\
0.05 \\
0.53 \\
*\end{array}$ & $\begin{array}{l}6.65 \\
0.03 \\
0.46 \\
*\end{array}$ & $\begin{array}{l}13.54 \\
0.03 \\
0.23 \\
*\end{array}$ & $\begin{array}{r}0.12 \\
0.02 \\
16.67 \\
*\end{array}$ & $\begin{array}{r}0.07 \\
0.02 \\
28.58 \\
1.20\end{array}$ & $\begin{array}{r}0.01 \\
0.04 \\
399.97 \\
--\end{array}$ & $\begin{array}{r}0.09 \\
C .02 \\
22.23 \\
-.\end{array}$ & $\begin{array}{l}0.21 \\
0.01 \\
4.77 \\
-.\end{array}$ & $\begin{array}{c}0.27 \\
0.67 \\
25.93 \\
-.\end{array}$ & $\begin{array}{l}0.40 \\
c .03 \\
7.51 \\
+\end{array}$ & $\begin{array}{r}0.44 \\
0.14 \\
31.82 \\
*\end{array}$ & $\begin{array}{l}0.81 \\
0.05 \\
6.18 \\
-.\end{array}$ & $\begin{array}{r}32.19 \\
0.51\end{array}$ \\
\hline 1965 & $\begin{array}{l}\text { PRECIP. }(C M .) \\
\text { SR-90 (MCI/SO.KM-) } \\
S R-90 \text { CONC. }(P C / L) \\
\text { SR-89/SR-90 }\end{array}$ & $\begin{array}{l}2.53 \\
0.08 \\
3.17 \\
-.\end{array}$ & $\begin{array}{l}0.82 \\
0.06 \\
7.32 \\
-.\end{array}$ & $\begin{array}{l}0.06 \\
0.02 \\
33.34 \\
--\end{array}$ & $\begin{array}{r}0.15 \\
0.02 \\
13.34 \\
-\infty\end{array}$ & $\begin{array}{r}0.10 \\
0.01 \\
10.01 \\
-\end{array}$ & $\begin{array}{l}0.02 \\
* \\
--\end{array}$ & $\begin{array}{l}0.19 \\
C .01 \\
5.27 \\
--\end{array}$ & $\begin{array}{l}0.14 \\
0.01 \\
7.15 \\
--\end{array}$ & $\begin{array}{l}0.57 \\
0.03 \\
5.27 \\
-=\end{array}$ & $\begin{array}{r}0.14 \\
0.07 \\
\text { SC.01 } \\
=\end{array}$ & $\begin{array}{r}0.46 \\
0.06 \\
13.05 \\
--\end{array}$ & $\begin{array}{l}1.19 \\
+- \\
-\infty\end{array}$ & $\begin{array}{l}6.37 \\
0.37\end{array}$ \\
\hline 1966 & $\begin{array}{l}\text { PRECIP. (CM.) } \\
\text { SR-90 INCI/SQ.KM-) } \\
S R-90 \text { CONC. (PC/L) } \\
S R-89 / S R=90\end{array}$ & $\begin{array}{l}0.84 \\
0.07 \\
8.34 \\
-\end{array}$ & $\begin{array}{l}1.22 \\
0.01 \\
0.82 \\
--\end{array}$ & $\begin{array}{l}6.31 \\
* \\
--\end{array}$ & $\begin{array}{l}0.29 \\
0.02 \\
6.90 \\
--\end{array}$ & $\begin{array}{l}-- \\
- \\
-\end{array}$ & $\begin{array}{l}-- \\
-- \\
--\end{array}$ & $\begin{array}{l}-- \\
-- \\
--\end{array}$ & $\begin{array}{l}-- \\
--\end{array}$ & $\begin{array}{l}-- \\
-- \\
--\end{array}$ & $\begin{array}{l} \pm \\
-- \\
-2\end{array}$ & $=$ & $m+$ & $\begin{array}{l}8.60 \\
0.10\end{array}$ \\
\hline 1967 & $\begin{array}{l}\text { PRECIP. (CM.) } \\
\text { SR-90 IMCI/SQ.KM.) } \\
\text { SR-90 CONC. (PC/L) } \\
\text { SR-89/SR-90 }\end{array}$ & $\begin{array}{l}1.94 \\
0.02 \\
1.04 \\
*\end{array}$ & $\begin{array}{l}9.44 \\
0.02 \\
0.22 \\
*\end{array}$ & $\begin{array}{l}6.52 \\
0.02 \\
0.31 \\
*\end{array}$ & $\begin{array}{l}* \\
0.01 \\
*\end{array}$ & $\begin{array}{l}2.29 \\
0.01 \\
0.44 \\
*\end{array}$ & $\begin{array}{l}* \\
0.01 \\
- \\
*\end{array}$ & $\begin{array}{l}-- \\
--\end{array}$ & $=$ & $\begin{array}{l}-- \\
-- \\
-- \\
--\end{array}$ & $\begin{array}{l}-- \\
-- \\
--\end{array}$ & $\begin{array}{l}- \\
-- \\
--\end{array}$ & $\begin{array}{l}-- \\
-\end{array}$ & $\begin{array}{r}20.19 \\
0.09\end{array}$ \\
\hline 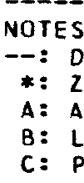 & $\begin{array}{l}\text { ATA NOT AVAILABLE } \\
\text { ERO OR TRACE } \\
\text { PPROXIMATE } \\
\text { OWER LIMIT OF REPOF } \\
\text { ROPORTIONED FROM OF }\end{array}$ & $\begin{array}{l}\text { DATA } \\
\text { NALLY }\end{array}$ & & & & & & & & & & & & \\
\hline
\end{tabular}


MONTHLY FALLOUT DEPOSITION COLLECTIONS

SITE: BOLIVIA, LA PAZ (CITY)

LAT. 16 30S LONG. 68 TK ALT. 3508M. (COLUMN)

SOURCE: UNIVERSIDAD MAYOR DE SAN ANDRES, LABORATORID DE FISICA COSMICA

\begin{tabular}{|c|c|c|c|c|c|c|c|c|c|c|c|c|c|c|}
\hline & & JAN. & FEB. & MAR. & APR. & MAY & JUNE & JULY & AUG. & SEP. & OCT. & NOV. & OEC. & CUM. TOTAL \\
\hline 1964 & $\begin{array}{l}P R E C I P \text {. }(C M-) \\
\text { SR-SC IMCI/SQ.KM-) } \\
\text { SR-9D CONC. }(P C / L) \\
S R-89 / S R-90\end{array}$ & $\begin{array}{l}-- \\
-- \\
-\end{array}$ & $\begin{array}{l}-- \\
--\end{array}$ & $=$ & $\begin{array}{l}1.50 \\
0.02 \\
1.34 \\
0.04\end{array}$ & $\begin{array}{r}0.16 \\
0.02 \\
12.51 \\
2.40\end{array}$ & $\begin{array}{l}* \\
0.01 \\
--\end{array}$ & $\begin{array}{l}0.32 \\
0.03 \\
9.38 \\
-\end{array}$ & $\begin{array}{l}0.81 \\
0.03 \\
3.71 \\
*\end{array}$ & $\begin{array}{l}1.31 \\
0.07 \\
5.35 \\
\end{array}$ & $\begin{array}{r}1.24 \\
0.14 \\
11.30 \\
*\end{array}$ & $\begin{array}{l}4.55 \\
0.08 \\
1.76 \\
*\end{array}$ & $\begin{array}{l}7.19 \\
0.08 \\
1.12 \\
--\end{array}$ & $\begin{array}{r}17.08 \\
0.48\end{array}$ \\
\hline 1965 & $\begin{array}{l}\text { PRECIP. (CM.) } \\
\text { SR-90 I MCI/SQ.KM.) } \\
S R-90 \text { CONC. (PC/L) } \\
\text { SR-89/SR-90 }\end{array}$ & $\begin{array}{l}17.75 \\
0.08 \\
0.46 \\
--\end{array}$ & $\begin{array}{l}9.93 \\
0.03 \\
0.31 \\
\ldots .\end{array}$ & $\begin{array}{l}10.29 \\
0.07 \\
0.69 \\
--\end{array}$ & $\begin{array}{l}0.40 \\
0.02 \\
5.01 \\
--\end{array}$ & $\begin{array}{l}* \\
0.01 \\
--\end{array}$ & $\begin{array}{l}0.01 \\
--\end{array}$ & $\begin{array}{l}0.37 \\
* \\
--\end{array}$ & $\begin{array}{r}0.13 \\
0.30 \\
230.77 \\
-5 .\end{array}$ & $\begin{array}{l}1.91 \\
0.04 \\
2.10 \\
=-\end{array}$ & $\begin{array}{l}* \\
0.05 \\
--\end{array}$ & $\begin{array}{l}1.73 \\
0.03 \\
1.74 \\
-.\end{array}$ & $\begin{array}{l}12.50 \\
0.05 \\
0.41 \\
--\end{array}$ & $\begin{array}{r}55.01 \\
0.69\end{array}$ \\
\hline 1966 & $\begin{array}{l}\text { PRECIP. (CM.) } \\
S R-90 \text { (MCI/SQ.KM.) } \\
S R-90 \text { CONC. }(P C / L) \\
S R-89 / S R-90\end{array}$ & $\begin{array}{l}2.08 \\
0.07 \\
3.37 \\
--\end{array}$ & $\begin{array}{l}13.62 \\
--\end{array}$ & $\begin{array}{l}7.40 \\
0.03 \\
0.41 \\
--\end{array}$ & $\begin{array}{l}1.17 \\
0.02 \\
1.71 \\
-.\end{array}$ & $\begin{array}{l}-- \\
-- \\
--\end{array}$ & $=$ & $=$ & $=$ & $\overline{-}$ & $m-$ & $=$ & $\begin{array}{r}3.33 \\
0.03 \\
0.91 \\
14.00\end{array}$ & $\begin{array}{r}27.60 \\
0.15\end{array}$ \\
\hline 1967 & $\begin{array}{l}\text { PRECIP. }\left(C M_{-}\right) \\
S R-90 \text { (MCI/SQ.KM.) } \\
\text { SR-90 CONC. }(P C / L) \\
S R-89 / S R-90\end{array}$ & $\begin{array}{l}2.57 \\
0.02 \\
0.78 \\
*\end{array}$ & $\begin{array}{l}6.67 \\
0.02 \\
0.30 \\
*\end{array}$ & $\begin{array}{l}6.32 \\
0.01 \\
0.16 \\
.\end{array}$ & $\begin{array}{l}0.23 \\
0.01 \\
4.35 \\
+\end{array}$ & $\begin{array}{l}0.89 \\
0.01 \\
1.13 \\
*\end{array}$ & $\begin{array}{l}0.11 \\
0.01 \\
9.10 \\
*\end{array}$ & $=$ & $\overline{-}$ & $=$ & $\overline{-}$ & $=-$ & $\begin{array}{l}-- \\
-- \\
--\end{array}$ & $\begin{array}{r}16.79 \\
0.08\end{array}$ \\
\hline 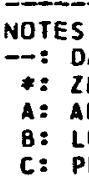 & $\begin{array}{l}\text { ATA NOT AVAILABLE } \\
\text { ERO OR TRACE } \\
\text { PPROXIMATE } \\
\text { OWER LIMIT OF REPOR } \\
\text { ROPORTIONED FROM ORI }\end{array}$ & $\begin{array}{l}\text { DATA } \\
\text { NALLYY }\end{array}$ & & & & & & & & & & & & \\
\hline
\end{tabular}




\begin{tabular}{|c|c|c|c|c|c|c|c|c|c|c|c|c|c|c|}
\hline & & JAN. & FEQ. & MAR. & APR. & MAY & JUNE & JULY & AUG. & SEP. & OCT. & NOV. & DEC. & CUM. TOTAL \\
\hline $196 \mathrm{C}$ & $\begin{array}{l}\text { PRECIP (CM.) } \\
\text { SR-90 (MCI/SQ-KH.) } \\
\text { SR-90 CONC. (PC/L) } \\
\text { SR-89/SR-90 }\end{array}$ & $\begin{array}{l}= \\
= \\
=\end{array}$ & $=$ & $\bar{z}$ & $\begin{array}{l}3.33 \\
0.07 \\
2.11 \\
-\end{array}$ & $\begin{array}{l}0.81 \\
0.05 \\
6.18 \\
--\end{array}$ & $\begin{array}{l}0.47 C \\
= \\
=-\end{array}$ & $\begin{array}{l}0.47 C \\
= \\
=\end{array}$ & $\begin{array}{l}0.47 \mathrm{C} \\
= \\
=\end{array}$ & $\begin{array}{l}0.01 \\
* \\
=-\end{array}$ & $\begin{array}{l}0.02 \\
+ \\
--\end{array}$ & $\begin{array}{c}14.66 \\
0.04 C \\
0.28 \\
--\end{array}$ & $\begin{array}{l}5.94 \\
0.02 C \\
0.34 \\
--\end{array}$ & $\begin{array}{r}26.18 \\
0.18\end{array}$ \\
\hline 1961 & $\begin{array}{l}\text { PRECIP. }\left(C M_{0}\right) \\
\text { SR-90 (MCI/SQ-KM.) } \\
S R-90 \text { CONC. }(P C / L) \\
S R-89 / S R-90\end{array}$ & $\begin{array}{l}12.14 \\
* \\
=-\end{array}$ & $\begin{array}{l}8.86 \\
* \\
--\end{array}$ & $\begin{array}{l}4.86 \\
0.026 \\
0.42 \\
\end{array}$ & $\begin{array}{l}6.39 \\
0.02 C \\
0.32 \\
--\end{array}$ & $\begin{array}{l}3.19 \\
0.04 \mathrm{C} \\
1.26 \\
-\end{array}$ & $\begin{array}{l}0.10 \\
0.006 \\
0.01 \\
-.\end{array}$ & $\begin{array}{l}0.02 \\
+ \\
=\end{array}$ & $\begin{array}{l}0.01 \\
* \\
--\end{array}$ & $\begin{array}{l}4.47 \\
* \\
-\end{array}$ & $\begin{array}{l}3.94 \\
0.03 \\
0.77 \\
0.00\end{array}$ & $\begin{array}{l}4.47 \\
0.03 \\
0.68 \\
6.00\end{array}$ & $\begin{array}{l}9.88 \\
0.02 \\
0.21 \\
+\end{array}$ & $\begin{array}{r}58.39 \\
0.16\end{array}$ \\
\hline 1962 & $\begin{array}{l}\text { PRECIP. (CM.) } \\
\text { SR-90 (MCI/SQ.KM.) } \\
S R-90 \text { CONC. }(P C / L) \\
S R-89 / S R-S O\end{array}$ & $\begin{array}{l}7.55 \\
0.34 \\
4.51 \\
*\end{array}$ & $\begin{array}{l}5.62 \\
0.02 \\
0.36 \\
7.00\end{array}$ & $\begin{array}{r}10.77 \\
0.01 \\
0.10 \\
14.00\end{array}$ & $\begin{array}{l}2.80 \\
0.02 \\
0.72 \\
*\end{array}$ & $\begin{array}{r}0.53 \\
0.06 \\
11.33 \\
45.00\end{array}$ & $=$ & $\ddot{-}$ & $=$ & $\begin{array}{l}3.94 \\
0.22 \\
5.59 \\
--\end{array}$ & $\begin{array}{r}2.55 \\
0.05 \\
1.97 \\
21.00\end{array}$ & $\begin{array}{r}3.00 \\
0.03 \\
1.01 \\
20.00\end{array}$ & $\begin{array}{r}11.41 \\
0.07 \\
0.62 \\
19.00\end{array}$ & $\begin{array}{r}48.17 \\
0.82\end{array}$ \\
\hline 1963 & $\begin{array}{l}\text { PRECIP. ICM.) } \\
\text { SR-90 IMCI/SQ.KM.) } \\
S R-90 \text { CDNC. }(P C / L) \\
S R-89 / S R-9 O\end{array}$ & $\begin{array}{r}13.77 \\
0.08 \\
0.59 \\
9.00\end{array}$ & $\begin{array}{l}9.30 \\
0.01 \\
0.11 \\
*\end{array}$ & $\begin{array}{l}6.72 \\
0.03 \\
0.45 \\
9.00\end{array}$ & $\begin{array}{l}4.14 \\
0.01 \\
0.25 \\
*\end{array}$ & $\begin{array}{l}0.94 \\
0.02 \\
2.13\end{array}$ & $\begin{array}{l}1.11 \\
0.03 \\
2.71\end{array}$ & $\frac{*}{0.01}$ & $\begin{array}{r}0.64 \\
0.07 \\
10.94 \\
1.00\end{array}$ & $\begin{array}{l}2.47 \\
0.12 \\
4.86 \\
+\end{array}$ & $\begin{array}{l}1.56 \\
C .05 \\
3.21 \\
*\end{array}$ & $\begin{array}{l}2.33 \\
0.05 \\
2.15 \\
1.00\end{array}$ & $\begin{array}{l}10.16 \\
0.05 \\
0.50 \\
*\end{array}$ & $\begin{array}{r}53.14 \\
0.53\end{array}$ \\
\hline 1964 & $\begin{array}{l}\text { PRECIP. (CM.) } \\
\text { SR-90 IMCI/SO.KM.) } \\
S R-90 \text { CCNC. }(P C / L) \\
S R-89 / S R-90\end{array}$ & $\begin{array}{l}6.70 \\
0.03 \\
0.45\end{array}$ & $\begin{array}{l}11.64 \\
0.03 \\
0.26 \\
+\end{array}$ & $\begin{array}{r}12.99 \\
0.03 \\
0.24 \\
*\end{array}$ & $\begin{array}{l}1.07 \\
0.03 \\
2.81 \\
*\end{array}$ & $\begin{array}{r}0.07 \\
0.03 \\
42.86 \\
*\end{array}$ & $\begin{array}{l}* \\
0.01 \\
--\end{array}$ & $\begin{array}{l}0.51 \\
0.07 \\
13.73 \\
-2\end{array}$ & $\begin{array}{l}0.25 \\
0.03 \\
12.01 \\
--\end{array}$ & $\begin{array}{l}1.09 \\
0.07 \\
6.43 \\
-0\end{array}$ & $\begin{array}{l}2.07 \\
0.10 \\
4.84 \\
+\end{array}$ & $\begin{array}{l}4.73 \\
0.14 \\
2.96 \\
=-\end{array}$ & $\begin{array}{l}9.92 \\
0.10 \\
1.01 \\
-.\end{array}$ & $\begin{array}{r}51.04 \\
0.67\end{array}$ \\
\hline 1965 & $\begin{array}{l}\text { PRECIP, (CM.) } \\
\text { SR-9O (MCI/SQ.KM.) } \\
S R-90 \text { CONC. }(P C / L) \\
S R=89 / S R=90\end{array}$ & $\begin{array}{l}17.62 \\
0.12 \\
0.69 \\
-\end{array}$ & $\begin{array}{l}7.01 \\
0.02 \\
0.29 \\
-0\end{array}$ & $\begin{array}{l}10.29 \\
0.05 \\
0.49 \\
-\end{array}$ & $\begin{array}{l}1.26 \\
0.02 \\
1.59 \\
\end{array}$ & $\begin{array}{c}0.06 \\
0.01 \\
16.67 \\
-0\end{array}$ & $\stackrel{*}{*}$ & $\begin{array}{l}0.48 \\
* \\
-- \\
--\end{array}$ & $\begin{array}{l}0.17 \\
0.03 \\
17.65 \\
--\end{array}$ & $\begin{array}{l}0.80 \\
0.03 \\
3.76 \\
-\end{array}$ & $\begin{array}{l}0.02 \\
--\end{array}$ & $\begin{array}{l}3.08 \\
0.10 \\
3.25 \\
-.\end{array}$ & $\begin{array}{l}8.78 \\
+ \\
--\end{array}$ & $\begin{array}{r}49.55 \\
0.40\end{array}$ \\
\hline 1966 & $\begin{array}{l}\text { PRECIP. }(C M=) \\
\text { SR-90 (MCI/SQ.KM.) } \\
\text { SR-90 CONC. }(P C / L) \\
S R-89 / S R-90\end{array}$ & $\begin{array}{l}3.36 \\
0.10 \\
2.98 \\
--\end{array}$ & $\begin{array}{r}10.78 \\
0.03 \\
0.28 \\
-0\end{array}$ & $\begin{array}{l}4.06 \\
0.02 \\
0.50 \\
--\end{array}$ & $\begin{array}{l}0.33 \\
0.02 \\
6.07 \\
-\infty\end{array}$ & $=$ & $=$ & $\begin{array}{l}- \\
- \\
-\end{array}$ & $\begin{array}{l}-- \\
--\end{array}$ & $\begin{array}{l}= \\
= \\
-\end{array}$ & $=$ & $=$ & $\begin{array}{r}2.84 \\
0.04 \\
1.41 \\
27.00\end{array}$ & $\begin{array}{r}21.37 \\
0.21\end{array}$ \\
\hline 1967 & $\begin{array}{l}\text { PRECIP }\left(C M_{*}\right) \\
\text { SR-90 }\left(M C I / S Q_{. K M}\right) \\
S R-90 \text { CONC. }(P C / L) \\
S R-89 / S R \rightarrow 90\end{array}$ & $\begin{array}{r}4.73 \\
0.03 \\
0.64 \\
13.10\end{array}$ & $\begin{array}{l}8.07 \\
0.02 \\
0.25 \\
*\end{array}$ & $\begin{array}{l}5.35 \\
0.01 \\
0.19 \\
*\end{array}$ & $\begin{array}{l}0.54 \\
0.01 \\
1.86 \\
*\end{array}$ & $\begin{array}{l}0.78 \\
0.02 \\
2.57 \\
*\end{array}$ & $\begin{array}{l}* \\
0.02 \\
*\end{array}$ & $=$ & $=$ & $m-$ & $m$ & $\begin{array}{l}-- \\
--\end{array}$ & $\begin{array}{l}-- \\
--\end{array}$ & $\begin{array}{r}19.47 \\
0.11\end{array}$ \\
\hline
\end{tabular}

NOTES

-: data not available

*: ZERO OR TRACE

A: APPROXIMATE

B: LOWER LIMIT OF REPORTED DATA

C: PROPORTI ONED FROM ORIGINALLY CONSOLIOATED DATA 


\begin{tabular}{|c|c|c|c|c|c|c|c|c|c|c|c|c|c|c|}
\hline & & JAN. & FEB. & MAR: & APR. & MAY & JUNE & JULY & AUG. & SEP. & OCT. & NOV. & DEC. & CUM. TOTAL \\
\hline 1959 & $\begin{array}{l}\text { PRECIP. (CM.) } \\
\text { SR-90 IMCI/SO.KM.) } \\
\text { SR-90 CONC. (PC/L) } \\
\text { SR-89/SR-90 }\end{array}$ & $=$ & $\begin{array}{l}\overline{-} \\
\overline{-}\end{array}$ & $\begin{array}{l}\overline{-} \\
\overline{-}\end{array}$ & $\begin{array}{l}\overline{-} \\
\bar{m}\end{array}$ & $=$ & $=$ & 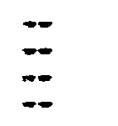 & $\begin{array}{l}\ddot{z} \\
\ddot{E}\end{array}$ & $\begin{array}{l}=- \\
= \\
--\end{array}$ & $=$ & $\begin{array}{l}17.504 \\
0.02 \mathrm{~A} \\
0.12 \\
+\end{array}$ & $\begin{array}{l}28.884 \\
0.034 \\
0.11 \\
*\end{array}$ & $\begin{array}{r}46.38 \\
0.05\end{array}$ \\
\hline 1960 & $\begin{array}{l}\text { PRECI P. (CM.) } \\
\text { SR-90 IMCI/SQ.KM.) } \\
\text { SR-90 CONC. (PCIL) } \\
\text { SR- } 89 / S R-90\end{array}$ & $\begin{array}{l}19.41 \mathrm{~A} \\
0.02 \mathrm{~A} \\
0.11 \\
-.\end{array}$ & $\begin{array}{c}26.39 A \\
0.15 A \\
0.57 \\
--\end{array}$ & $\begin{array}{c}35.994 \\
0.104 \\
0.28 \\
--\end{array}$ & $\begin{array}{l}24.694 \\
0.014 \\
0.05 \\
-\end{array}$ & $\begin{array}{l}14.994 \\
* \\
=\end{array}$ & $\begin{array}{l}20.624 \\
* \\
=-\end{array}$ & $\begin{array}{l}7.01 \mathrm{~A} \\
\pm \\
=-\end{array}$ & $\begin{array}{l}2.014 \\
= \\
=\end{array}$ & $\begin{array}{l}5.99 A \\
0.02 A \\
\text { C. } 34 \\
--\end{array}$ & $\begin{array}{l}9.864 \\
0.024 \\
0.21 \\
.-\end{array}$ & $\begin{array}{l}10.46 \mathrm{~A} \\
0.00 \mathrm{~A} \\
0.01 \\
=-\end{array}$ & $\begin{array}{l}43.744 \\
0.00 A \\
0.01 \\
=-\end{array}$ & $\begin{array}{r}221.16 \\
0.32\end{array}$ \\
\hline 1961 & $\begin{array}{l}\text { PREC IP. (CM.) } \\
S R-90 \text { INCI/SQ.KM.) } \\
S R-90 \text { CONC. }(P C / L) \\
S R-89 / S R-90\end{array}$ & $\begin{array}{l}35.994 \\
=- \\
=-\end{array}$ & $\begin{array}{l}38.61 \mathrm{~A} \\
-- \\
-- \\
--\end{array}$ & $\begin{array}{l}87.764 \\
0.13 A \\
0.15 \\
--\end{array}$ & $\begin{array}{l}3.564 \\
0.004 \\
0.01 \\
=\end{array}$ & $\begin{array}{l}20.554 \\
0.004 \\
0.01 \\
=\end{array}$ & $\begin{array}{l}12.67 A \\
0.004 \\
0.01 \\
-\infty\end{array}$ & $\begin{array}{l}14.814 \\
* \\
--\end{array}$ & $\begin{array}{l}19.511 \\
* \\
--\end{array}$ & $\begin{array}{l}11.944 \\
0.034 \\
0.26 \\
1.504\end{array}$ & $\begin{array}{l}8.00 \mathrm{~A} \\
0.01 \mathrm{~A} \\
\mathrm{C.} .13 \\
11.00 \mathrm{~A}\end{array}$ & $\begin{array}{l}18.03 A \\
0.05 A \\
0.28 \\
63.00 A\end{array}$ & $\begin{array}{r}20.094 \\
0.09 A \\
0.45 \\
46.00 A\end{array}$ & $\begin{array}{r}291.52 \\
0.31\end{array}$ \\
\hline 1962 & $\begin{array}{l}\text { PRECIP. ICM.) } \\
\text { SR-90 IMCI/SQ.KM.) } \\
\text { SR-90 CONC. (PC/L) } \\
\text { SR-89/SR-SC }\end{array}$ & $\begin{array}{l}31.75 \mathrm{~A} \\
= \\
=- \\
3.904\end{array}$ & $\begin{array}{l}38.10 A \\
=- \\
=\end{array}$ & $\begin{array}{r}38.86 A \\
0.09 A \\
0.24 \\
13.00 A\end{array}$ & $\begin{array}{l}43.944 \\
=- \\
3.004\end{array}$ & $\begin{array}{l}26.964 \\
0.054 \\
0.18 \\
=\end{array}$ & $\begin{array}{c}11.434 \\
0.05 A \\
0.44 \\
=-\end{array}$ & $\begin{array}{l}12.45 A \\
C .03 A \\
C .25 \\
=-\end{array}$ & $\begin{array}{l}6.354 \\
0.034 \\
0.48 \\
-.\end{array}$ & $\begin{array}{l}7.874 \\
0.044 \\
0.51 \\
15.004\end{array}$ & $\begin{array}{l}12.95 \\
\because- \\
=\end{array}$ & $\begin{array}{r}13.97 \\
0.05 \\
0.36 \\
33.00\end{array}$ & $\begin{array}{r}12.70 \\
0.10 \\
0.79 \\
45.00\end{array}$ & $\begin{array}{r}259.33 \\
0.44\end{array}$ \\
\hline 1963 & $\begin{array}{l}\text { PRECIP. (CM.) } \\
\text { SR-9C IMCI/SO.KM.) } \\
\text { SR-9O CCNC. (PC/L) } \\
S R-89 / S R-90\end{array}$ & $\begin{array}{l}23.77 A \\
C . C 5 A \\
0.22 \\
--\end{array}$ & $\begin{array}{l}23.77 A \\
0.05 A \\
0.22 \\
--\end{array}$ & $\begin{array}{r}57.33 \\
0.17 \\
0.30 \\
13.00\end{array}$ & $\begin{array}{r}52.83 \\
0.03 \\
0.06 \\
11.00\end{array}$ & $\begin{array}{r}21.08 \\
0.05 \\
0.24 \\
7.10\end{array}$ & $\begin{array}{r}25.65 \\
0.12 \\
0.47 \\
1.60\end{array}$ & $\begin{array}{l}6.86 \\
0.05 \\
0.73 \\
*\end{array}$ & $\begin{array}{l}10.41 \\
0.08 \\
0.77 \\
*\end{array}$ & $\begin{array}{l}4.32 \\
0.08 \\
1.86 \\
*\end{array}$ & $\begin{array}{r}18.29 \\
6.09 \\
C .50 \\
*\end{array}$ & $\begin{array}{r}11.46 \\
0.01 \\
0.09 \\
*\end{array}$ & $\begin{array}{l}39.70 \\
0.09 \\
0.23 \\
*\end{array}$ & $\begin{array}{r}295.47 \\
0.87\end{array}$ \\
\hline 1964 & $\begin{array}{l}\text { PRECIP }\left(C_{-}\right) \\
\text {SR-9O (MCI/SQ.KM.) } \\
\text { SR-9O CONC. }(P C / L) \\
\text { SR-89/SR-90 }\end{array}$ & $\begin{array}{l}48.64 \\
0.18 \\
0.38 \\
*\end{array}$ & $\begin{array}{r}51.94 \\
0.94 \\
1.81 \\
*\end{array}$ & $\begin{array}{r}60.33 \\
0.07 \\
0.12 \\
*\end{array}$ & $\begin{array}{l}23.75 \\
0.18 \\
0.76 \\
0.58\end{array}$ & $\begin{array}{l}51.31 \\
0.36 \\
0.71 \\
*\end{array}$ & $\begin{array}{l}9.65 \\
0.09 \\
0.94 \\
--\end{array}$ & $\begin{array}{l}6.20 \\
c .10 \\
1.62 \\
--\end{array}$ & $\begin{array}{l}9.60 \\
0.06 \\
0.63 \\
--\end{array}$ & $\begin{array}{r}16.08 \\
0.08 \\
0.50 \\
=-\end{array}$ & $\begin{array}{l}8.10 \\
C .07 \\
0.87 \\
--\end{array}$ & $\begin{array}{l}5.87 \\
0.02 \\
0.35 \\
\end{array}$ & $\begin{array}{l}16.05 \\
0.01 \\
0.07 \\
=-\end{array}$ & $\begin{array}{r}307.52 \\
2.16\end{array}$ \\
\hline 1965 & $\begin{array}{l}\text { PRECIP. }(C M-) \\
\text { SR-90 }\left(M C I / S M_{-K M-1}\right. \\
S R-9 C \text { CONC. }(P C / L) \\
S R-89 / S R-90\end{array}$ & $\begin{array}{l}30.35 \\
0.13 \\
0.43 \\
--\end{array}$ & $\begin{array}{l}27.43 \\
0.14 \\
0.52 \\
--\end{array}$ & $\begin{array}{l}46.30 \\
0.08 \\
0.18 \\
--\end{array}$ & $\begin{array}{l}44.42 \\
0.17 \\
0.39 \\
-.\end{array}$ & $\begin{array}{l}40.39 \\
0.06 \\
0.15 \\
-\end{array}$ & $\begin{array}{l}22.23 \\
0.08 \\
0.36 \\
--\end{array}$ & $\begin{array}{l}12.85 \\
0.03 \\
C .24 \\
--\end{array}$ & $\begin{array}{l}10.39 \\
0.03 \\
0.29 \\
--\end{array}$ & $\begin{array}{l}7.70 \\
0.07 \\
0.91 \\
--\end{array}$ & $\begin{array}{l}12.07 \\
6.07 \\
0.58 \\
--\end{array}$ & $\begin{array}{l}6.83 \\
0.14 \\
2.05 \\
-.\end{array}$ & $\begin{array}{r}17.88 \\
0.35 \\
1.96 \\
=-\end{array}$ & $\begin{array}{r}278.84 \\
1.35\end{array}$ \\
\hline 1966 & $\begin{array}{l}\text { PRECIP. (CM.) } \\
\text { SR-90 (MCI/SQ.KM.) } \\
\text { SR-90 CONC. (PC/L) } \\
\text { SR-89/SR-90 }\end{array}$ & $\begin{array}{l}46.53 \\
* \\
=-\end{array}$ & $\begin{array}{l}42.24 \\
0.16 \\
0.38 \\
-\end{array}$ & $\begin{array}{r}37.97 \\
0.02 \\
0.06 \\
--\end{array}$ & $\begin{array}{r}35.38 \\
0.06 \\
0.17 \\
--\end{array}$ & $\begin{array}{l}37.80 \\
0.05 \\
0.14 \\
--\end{array}$ & $\begin{array}{r}28.30 \\
0.03 \\
0.11 \\
7.10\end{array}$ & $\begin{array}{l}9.73 \\
c .63 \\
0.31 \\
+\end{array}$ & $\begin{array}{r}0.53 \\
0.02 \\
0.24 \\
34.00\end{array}$ & $\begin{array}{r}14.27 \\
0.03 \\
0.22 \\
9.60\end{array}$ & $\begin{array}{l}8.23 \\
0.24 \\
2.92 \\
7.50\end{array}$ & $\begin{array}{r}14.99 \\
0.06 \\
0.41 \\
41.00\end{array}$ & $\begin{array}{l}\ddot{-} \\
\ddot{-}\end{array}$ & $\begin{array}{r}283.97 \\
0.70\end{array}$ \\
\hline 1967 & $\begin{array}{l}\text { PRECIP. (CM-) } \\
\text { SR-90 I } C \text { CI/SQ.KM.) } \\
\text { SR-90 CONC. (PCIL) } \\
S R-89 / S R-90\end{array}$ & $\frac{--}{0.04}$ & $\begin{array}{l}-0.04 \\
8.40\end{array}$ & $\begin{array}{l}-\overline{0.05} \\
\overline{4.20}\end{array}$ & $=-$ & $\overline{--}$ & $\begin{array}{r}14.30 \\
0.02 \\
0.14 \\
8.30\end{array}$ & $\begin{array}{l}-- \\
-- \\
-\end{array}$ & $\begin{array}{l}-- \\
-- \\
--\end{array}$ & $\begin{array}{l}=- \\
=-\end{array}$ & $=-$ & $\begin{array}{l}-- \\
-- \\
--\end{array}$ & $=$ & $\begin{array}{r}14.30 \\
0.15\end{array}$ \\
\hline 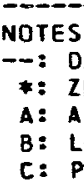 & 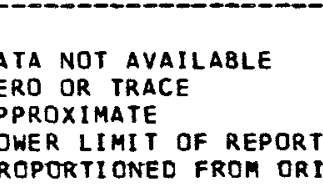 & 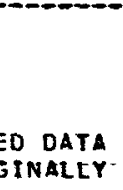 & & & & & & & & & & & & \\
\hline
\end{tabular}




\begin{tabular}{|c|c|c|c|c|c|c|c|c|c|c|c|c|c|c|}
\hline & & JAN. & FEB. & MAR. & APR : & MAY & JUNE & JULY & A UG. & SEP. & OCT. & Nov. & DEC. & CUM. TDTAL \\
\hline 1959 & $\begin{array}{l}\text { PRECIP. (CM.) } \\
\text { SR-90 (MCI/SQ.KM.) } \\
\text { SR-90 CONC. }(P C / L) \\
\text { SR-89/SR-90 }\end{array}$ & $\overline{--}$ & $\begin{array}{l}-- \\
-- \\
=-\end{array}$ & $=$ & $\overline{-}$ & $=$ & $=$ & $\ddot{m}=$ & $=$ & $\begin{array}{l}3.00 \\
* \\
*\end{array}$ & $\begin{array}{l}9.02 \\
0.03 \\
0.34 \\
*\end{array}$ & $\begin{array}{r}12.01 \\
0.00 \\
0.01 \\
*\end{array}$ & $\begin{array}{l}4.01 \\
0.01 \\
0.25 \\
*\end{array}$ & $\begin{array}{r}28.04 \\
0.04\end{array}$ \\
\hline 1960 & $\begin{array}{l}\text { PRECIP. (CM.) } \\
\text { SR-9O I } M C I / S Q . K M \cdot) \\
\text { SR-90 CONC. }(P C / L) \\
\text { SR-89/SR-90 }\end{array}$ & $\begin{array}{l}\bar{c} . c 0 \\
--\end{array}$ & $\begin{array}{l}-- \\
-\infty \\
-\end{array}$ & $\begin{array}{l}- \\
0.01 \\
--\end{array}$ & $\begin{array}{l}-- \\
--\end{array}$ & $=$ & $\begin{array}{l}-- \\
--\end{array}$ & $m$ & $\begin{array}{l}0.10 \\
= \\
=\end{array}$ & $\begin{array}{l}* \\
--\end{array}$ & $\begin{array}{l}2.21 \\
C .03 C \\
1.36 \\
--\end{array}$ & $\begin{array}{l}5.31 \\
0.016 \\
0.19 \\
-.\end{array}$ & $\begin{array}{l}6.40 \\
0.016 \\
0.16 \\
--\end{array}$ & $\begin{array}{r}14.02 \\
0.07\end{array}$ \\
\hline 1961 & $\begin{array}{l}\text { PRECIP. (CM.) } \\
\text { SR-90 (MCI/SQ.KM.) } \\
\text { SR-90 CONC. (PC/L) } \\
S R-89 / S R-90\end{array}$ & $\begin{array}{c}32.82 \\
0.00 \mathrm{C} \\
0.01 \\
-\end{array}$ & $\begin{array}{l}17.22 \\
0.00 C \\
0.01 \\
--\end{array}$ & $\begin{array}{l}6.20 \\
0.01 \\
0.17 \\
--\end{array}$ & $\begin{array}{l}3.91 \\
-- \\
--\end{array}$ & $\begin{array}{c}10.01 \\
* \\
--\end{array}$ & $\begin{array}{l}* \\
0.00 c \\
--\end{array}$ & $\begin{array}{l}* 0.000 \\
--\end{array}$ & $\begin{array}{l}0.10 \\
0.000 \\
0.01 \\
--\end{array}$ & $\begin{array}{l}* \\
0.00 C \\
--\end{array}$ & $\begin{array}{l}4.80 \\
0.02 C \\
C .42 \\
--\end{array}$ & $\begin{array}{l}13.41 \\
0.03 \\
0.23 \\
*\end{array}$ & $\begin{array}{l}34.93 \\
0.04 \\
0.12 \\
*\end{array}$ & $\begin{array}{r}123.40 \\
0.10\end{array}$ \\
\hline 1962 & $\begin{array}{l}\text { PRECIP. (CM.) } \\
\text { SR-9C I CCI/SQ.KM.) } \\
\text { SR-90 CONC. (PC/L) } \\
\text { SR-89/SR-90 }\end{array}$ & $\begin{array}{l}24.03 \\
0.01 \\
0.05 \\
\end{array}$ & $\begin{array}{r}16.81 \\
0.02 \\
0.12 \\
*\end{array}$ & $\begin{array}{r}29.92 \\
0.02 \\
0.07 \\
*\end{array}$ & $\begin{array}{l}9.40 \\
0.03 \mathrm{C} \\
0.32 \\
-.\end{array}$ & $\begin{array}{l}0.20 \\
0.00 C \\
0.01 \\
--\end{array}$ & $\begin{array}{l}* \\
0.00 c\end{array}$ & $\begin{array}{l}* \\
= \\
=\end{array}$ & $\begin{array}{r}0.10 \\
0.02 \\
20.01 \\
34.00\end{array}$ & $\begin{array}{r}8.51 \\
0.03 \\
0.36 \\
10.00\end{array}$ & $\begin{array}{r}21.21 \\
0.04 \\
0.19 \\
9.00\end{array}$ & $\begin{array}{r}30.02 \\
0.02 \\
0.07 \\
15.00\end{array}$ & $\begin{array}{r}22.81 \\
0.02 \\
0.09 \\
11.00\end{array}$ & $\begin{array}{r}163.01 \\
0.21\end{array}$ \\
\hline 1963 & $\begin{array}{l}\text { PRECIP. }(C M \cdot) \\
\text { SR-90 IMCI/SQ.KM }) \\
\text { SR-90 CONC. }(P C / L) \\
S R-89 / S R-90\end{array}$ & $\begin{array}{r}10.72 \\
0.03 \\
0.28 \\
11.00\end{array}$ & $\begin{array}{r}25.02 \\
0.04 \\
0.16 \\
17.00\end{array}$ & $\begin{array}{l}1.40 \\
0.04 \\
2.86 \\
--\end{array}$ & $\begin{array}{r}13.21 \\
0.26 \\
1.97 \\
22.00\end{array}$ & $\begin{array}{r}0.10 \\
0.02 \\
20.01 \\
*\end{array}$ & $\begin{array}{l}* \\
0.02 \\
*\end{array}$ & $\begin{array}{l}0.01 \\
-\end{array}$ & $=0.01$ & $=$ & $\begin{array}{l}c .02 \\
=\end{array}$ & $\begin{array}{l}-- \\
0.02 \\
--\end{array}$ & $\begin{array}{l}-\overline{0.02} \\
\overline{2 .} \\
\overline{2.20}\end{array}$ & $\begin{array}{r}50.45 \\
0.49\end{array}$ \\
\hline 1964 & $\begin{array}{l}\text { PRECIP. }\left(C M M_{-}\right) \\
\text {SR-90 (MCI/SQ-KM.) } \\
\text { SR-90 CONC. }(P C / L) \\
S R-89 / S R-90\end{array}$ & $\overrightarrow{0.02}$ & $\begin{array}{l}-- \\
--\end{array}$ & 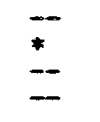 & $\begin{array}{l}-- \\
-- \\
--\end{array}$ & $\begin{array}{l}-- \\
-\infty\end{array}$ & $\begin{array}{l}-- \\
-- \\
--\end{array}$ & + & $\ddot{*}$ & 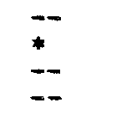 & $\vec{*}$ & $\begin{array}{l}-\infty \\
-\infty\end{array}$ & $\begin{array}{l}-- \\
0.02 \\
-\infty\end{array}$ & $\begin{array}{l}0.0 \\
0.05\end{array}$ \\
\hline 1965 & $\begin{array}{l}\text { PRECIP. (CM.) } \\
\text { SR-90 (MCI/SQ.KM.) } \\
\text { SR-90 CONC. (PC/L) } \\
\text { SR-89/SR-90 }\end{array}$ & $\begin{array}{l}m \\
--\end{array}$ & 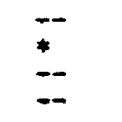 & $\begin{array}{l}- \\
0.01 \\
-\end{array}$ & $\begin{array}{l}-- \\
--\end{array}$ & $\begin{array}{l}-- \\
*-\end{array}$ & $=$ & $\begin{array}{l}-- \\
+- \\
--\end{array}$ & $\begin{array}{l}-- \\
--\end{array}$ & 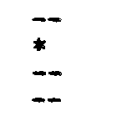 & $\begin{array}{l}39.42 \\
\div \\
0.00\end{array}$ & $\begin{array}{l}55.17 \\
* \\
--\end{array}$ & $\begin{array}{c}18.07 \\
*- \\
--\end{array}$ & $\begin{array}{r}112.66 \\
0.01\end{array}$ \\
\hline 1966 & $\begin{array}{l}\text { PRECIP, }(C M .) \\
\text { SR-90 IMCI/SQ.KM }) \\
S R-90 \text { CONC. }(P C / L) \\
S R-89 / S R=90\end{array}$ & $\begin{array}{l}34.42 \\
* \\
=\end{array}$ & $\begin{array}{l}32.92 \\
*- \\
=-\end{array}$ & $\begin{array}{l}-- \\
-\end{array}$ & $\overline{-}$ & $\begin{array}{l}-- \\
-- \\
-\end{array}$ & $\begin{array}{l}-- \\
-- \\
--\end{array}$ & $\ddot{--}$ & $=-$ & 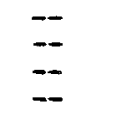 & $\begin{array}{l}-- \\
--\end{array}$ & $\begin{array}{l}3.20 \\
0.01 \\
0.32 \\
*\end{array}$ & $\begin{array}{l}3.00 \\
0.01 \\
0.34 \\
*\end{array}$ & $\begin{array}{r}73.54 \\
0.02\end{array}$ \\
\hline \multicolumn{15}{|c|}{$\begin{array}{l}\text { NOTES } \\
--: \text { DATA NOT AVAILABLE } \\
\text { *: ZERO OR TRACE } \\
\text { A: APPROXIMATE } \\
\text { B: LOWER LIMIT OF REPORTED DATA } \\
\text { C: PROPORTIONED FROM ORIGINALLY CONSOL IDATED DATA }\end{array}$} \\
\hline
\end{tabular}




$$
\begin{aligned}
& \text { JAN. FEB. MAR. APR. MAY JUNE JULY AUG. SEP. OCT. NOV. DEC. CUM. TOTAL }
\end{aligned}
$$

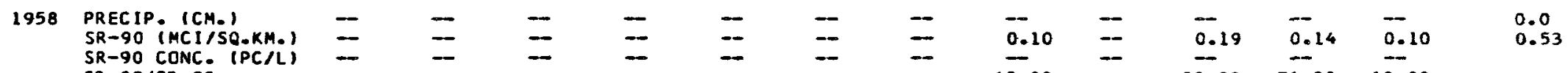

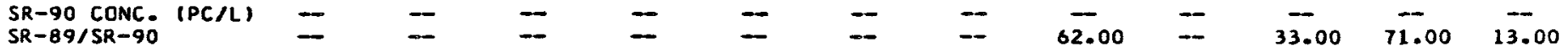

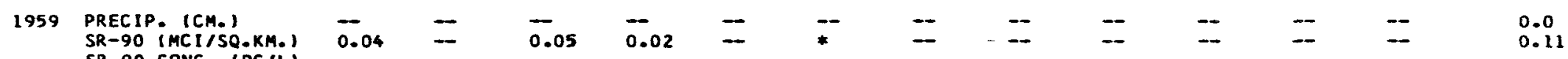

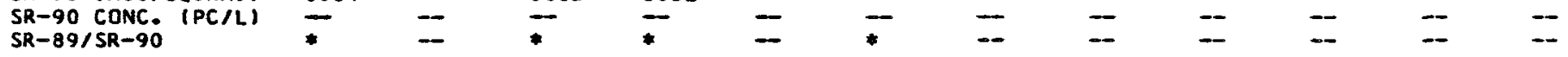

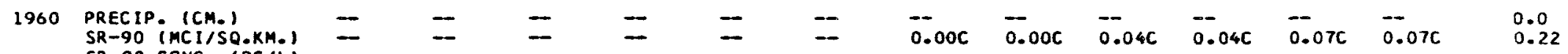

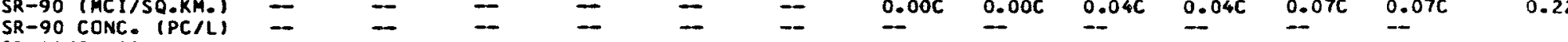

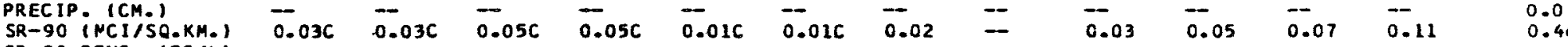




\section{MONTHLY FALLOUT DEPOSITION COLLECTIONS}

SITE: BRAZIL, MANAUS

LAT. 325 LONG. 60 IW ALT. 29M. (POT)

SOURCE: INSTITUTO DE FISICA, PONTIFICIA UNIVERS IDADE CATOLICA COLLECTIONS TERMINATED IN SEPTEMBER 1964

\begin{tabular}{|c|c|c|c|c|c|c|c|c|c|c|c|c|c|c|}
\hline & & JAN. & FEB. & MAR. & $\underline{A P R}$. & $\stackrel{\text { MAY }}{--}$ & JUNE & JUL.Y & AUG. & SEP. & OCT. & NOV. & DEC. & CUM. TOTAL \\
\hline 1959 & $\begin{array}{l}\text { PRECIP. (CM.) } \\
S R-9 C \text { (NCI/SQ.KM.) } \\
\text { SR-90 CQNC. }(P C / L) \\
S R-89 / S R-90\end{array}$ & $\begin{array}{r}216.26 \\
0.31 \\
0.15 \\
1.70\end{array}$ & $\begin{array}{r}22.10 \\
0.26 \\
1.18 \\
41.00\end{array}$ & $\begin{array}{l}25.81 \\
0.24 \\
0.93 \\
-\end{array}$ & $\begin{array}{l}18.80 \\
0.02 \\
0.11 \\
-.\end{array}$ & $\begin{array}{l}12.17 \\
0.05 \\
0.42 \\
--\end{array}$ & $\begin{array}{l}10.87 \\
0.01 \\
0.10 \\
--\end{array}$ & $\begin{array}{l}3.28 \\
0.03 \\
0.92 \\
-.\end{array}$ & $\begin{array}{l}5.74 \\
0.02 \\
0.35 \\
-.\end{array}$ & $\begin{array}{l}4.06 \\
* \\
--\end{array}$ & $\begin{array}{l}1.02 \\
0.03 \\
2.95 \\
\end{array}$ & $\begin{array}{l}21.34 \\
=- \\
-\end{array}$ & $\begin{array}{l}15.09 \\
=- \\
=-\end{array}$ & $\begin{array}{r}356.54 \\
0.97\end{array}$ \\
\hline 1960 & $\begin{array}{l}\text { PRECIP. (CM-) } \\
\text { SR-90 (MCI/SQ.KMH) } \\
\text { SR-90 CONC. (PC/L) } \\
\text { SR-89/SR-9Q }\end{array}$ & $\stackrel{*}{-}$ & $\begin{array}{l}19.10 \\
=- \\
=-\end{array}$ & $\begin{array}{l}22.91 \\
= \\
=\end{array}$ & $\begin{array}{l}41.02 \\
0.20 \\
0.49 \\
-\end{array}$ & $\begin{array}{l}11.86 \\
=- \\
=-\end{array}$ & $\begin{array}{l}17.45 \\
-- \\
--\end{array}$ & $\begin{array}{l}0.81 \\
0.01 \\
0.15 \\
-.\end{array}$ & $\begin{array}{l}5.92 \\
--\end{array}$ & $\begin{array}{l}8.05 \\
-- \\
--\end{array}$ & $\begin{array}{l}22.61 \\
-- \\
=-\end{array}$ & $\begin{array}{l}24.00 \\
0.02 \mathrm{C} \\
0.09 \\
-.\end{array}$ & $\begin{array}{l}29.97 \\
0.02 \mathrm{C} \\
0.07 \\
--\end{array}$ & $\begin{array}{r}209.70 \\
0.25\end{array}$ \\
\hline 1961 & $\begin{array}{l}\text { PRECIP. }\left(C M_{-}\right) \\
S R-90\left(N C I / S Q-K M_{-}\right) \\
S R-90 \text { CCNC. }(P C / L) \\
S R-89 / S R-90\end{array}$ & $\begin{array}{l}29.26 \\
0.036 \\
0.11 \\
-\end{array}$ & $\begin{array}{l}33.25 \\
0.03 \mathrm{C} \\
0.10 \\
-.\end{array}$ & $\begin{array}{l}23.98 \\
0.08 \\
0.34 \\
-\end{array}$ & $\begin{array}{l}35.31 \\
0.03 \mathrm{C} \\
0.09 \\
=-\end{array}$ & $\begin{array}{l}32.77 \\
C .03 \mathrm{C} \\
0.10 \\
--\end{array}$ & $\begin{array}{l}10.21 \\
=- \\
=-\end{array}$ & $\begin{array}{l}3.33 \\
+ \\
--\end{array}$ & $\begin{array}{l}0.69 \\
=- \\
=\end{array}$ & $\begin{array}{l}5.18 \\
C . C 16 \\
0.20 \\
0.00 C\end{array}$ & $\begin{array}{l}12.32 \\
C .03 \mathrm{C} \\
0.25 \\
0.00 \mathrm{C}\end{array}$ & $\begin{array}{r}14.68 \\
0.51 \\
3.48 \\
49.00\end{array}$ & $\begin{array}{r}35.03 \\
0.13 \\
0.38 \\
50.00\end{array}$ & $\begin{array}{r}236.01 \\
0.88\end{array}$ \\
\hline 1962 & $\begin{array}{l}\text { PRECIP. }(C M-) \\
\text { SR-90 IMCI/SQ-KM-) } \\
S R-90 \text { CONC. }(P C / L) \\
\text { SR-89/SR-90 }\end{array}$ & $\begin{array}{l}40.69 \\
=- \\
=-\end{array}$ & $\begin{array}{l}40.08 \\
-- \\
=-\end{array}$ & $\begin{array}{l}30.18 \\
=- \\
=\end{array}$ & $\begin{array}{l}29.92 \\
=- \\
=-\end{array}$ & $\begin{array}{l}26.06 \\
=- \\
=-\end{array}$ & $\begin{array}{c}10.36 A \\
0.03 A \\
0.29 \\
--\end{array}$ & $\begin{array}{l}8.64 A \\
0.07 A \\
0.82 \\
--\end{array}$ & $\begin{array}{l}4.11 A \\
0.05 A \\
1.22 \\
--\end{array}$ & $\begin{array}{l}1.304 \\
0.014 \\
0.77 \\
--\end{array}$ & $\begin{array}{l}12.50 \mathrm{~A} \\
0.01 \mathrm{~A} \\
0.09 \\
-.\end{array}$ & $\begin{array}{l}18.75 A \\
0.03 A \\
0.17 \\
-.\end{array}$ & $\begin{array}{l}9.80 \mathrm{~A} \\
0.06 \mathrm{~A} \\
0.62 \\
-.\end{array}$ & $\begin{array}{r}232.39 \\
0.26\end{array}$ \\
\hline 1963 & $\begin{array}{l}\text { PRECIP. }(C M-) \\
\text { SR-90 (MCI/SQ.KM.) } \\
\text { SR-90 CONC. }(P C / L) \\
\text { SR-89/SR-SC }\end{array}$ & $\begin{array}{l}22.86 \mathrm{~A} \\
0.25 \mathrm{~A} \\
1.10 \\
=-\end{array}$ & $\begin{array}{l}17.48 \mathrm{~A} \\
0.27 \mathrm{~A} \\
1.55 \\
--\end{array}$ & $\begin{array}{l}20.88 \mathrm{~A} \\
0.20 \mathrm{~A} \\
0.96 \\
-\end{array}$ & $\begin{array}{l}28.194 \\
0.124 \\
0.43 \\
--\end{array}$ & $\begin{array}{l}30.024 \\
0.204 \\
0.67 \\
=-\end{array}$ & $\begin{array}{l}20.52 A \\
0.07 A \\
0.35 \\
--\end{array}$ & $\begin{array}{l}11.61 \mathrm{~A} \\
0.06 \mathrm{~A} \\
0.52 \\
-\end{array}$ & $\begin{array}{l}-- \\
0.05 \mathrm{~A} \\
--\end{array}$ & $\begin{array}{l}-- \\
-- \\
--\end{array}$ & $\begin{array}{l}-- \\
-- \\
--\end{array}$ & $\begin{array}{l}-- \\
=- \\
--\end{array}$ & $=$ & $\begin{array}{r}151.56 \\
1.22\end{array}$ \\
\hline 1964 & $\begin{array}{l}\text { PRECIP. (CM.) } \\
\text { SR-9O ( } M C I / S Q . K M-) \\
S R-90 \text { CONC }(P C / L) \\
\text { SR-89/SR-90 }\end{array}$ & $\begin{array}{l}- \\
- \\
-\end{array}$ & $=$ & $\vec{m}=$ & $\overrightarrow{0.054}$ & $\begin{array}{l}-- \\
\overline{0} \\
-\end{array}$ & $\begin{array}{l}-\infty \\
0 . \\
--\end{array}$ & $\begin{array}{l}-- \\
-- \\
--\end{array}$ & $\frac{-0}{0}$ & $\begin{array}{l}- \\
-\infty \\
-\end{array}$ & $\begin{array}{l}-- \\
-- \\
--\end{array}$ & $\begin{array}{l}-- \\
-- \\
-\end{array}$ & $\begin{array}{l}-- \\
-- \\
--\end{array}$ & $\begin{array}{l}0.0 \\
0.46\end{array}$ \\
\hline $\begin{array}{l}\text { NOTES } \\
\text { A: } \\
*: 2 \\
A: \\
B: \\
C:\end{array}$ & $\begin{array}{l}\text { ATA NOT AVAILABLE } \\
\text { IERD OR TRACE } \\
\text { PPPROXIMATE } \\
\text { OWER LIMIT OF REPOF } \\
\text { PROPORTI ONED FROM OF }\end{array}$ & $\begin{array}{l}\text { DATA } \\
\text { NALLLY }\end{array}$ & & & & & & & & & & & & \\
\hline
\end{tabular}




\begin{tabular}{|c|c|c|c|c|c|c|c|c|c|c|c|c|c|c|}
\hline & & JAN. & FEB. & MAR. & APR. & MAY & JUNE & JULY & AUG. & SEP. & OCT. & NOV. & DEC. & CUM. TOTAL \\
\hline 1958 & $\begin{array}{l}\text { PRECIP. }\left(C M_{-}\right) \\
\text {SR-90 }\left(M C I / S Q_{-K M}\right) \\
S R-90 \text { CONC. }(P C / L) \\
\text { SR-89/SR-90 }\end{array}$ & $\begin{array}{l}10.97 \\
= \\
=\end{array}$ & $\begin{array}{l}12.65 \\
= \\
=\end{array}$ & $\begin{array}{c}12.34 \\
=- \\
=\end{array}$ & $\begin{array}{l}12.22 \\
= \\
=\end{array}$ & $\begin{array}{l}6.93 \\
-- \\
--\end{array}$ & $\begin{array}{l}2.11 \\
=- \\
=\end{array}$ & $\begin{array}{l}5.99 \\
-- \\
-\infty\end{array}$ & $\begin{array}{r}0.28 \\
0.05 \\
17.86 \\
21.00\end{array}$ & $\begin{array}{r}18.21 \\
0.19 \\
1.05 \\
65.00\end{array}$ & $\begin{array}{r}11.07 \\
0.32 \\
2.90 \\
16.00\end{array}$ & $\begin{array}{l}38.23 \\
=- \\
=\end{array}$ & $\begin{array}{r}20.12 \\
0.14 \\
0.70 \\
18.00\end{array}$ & $\begin{array}{r}151.12 \\
0.70\end{array}$ \\
\hline 1959 & $\begin{array}{l}\text { PRECIP. (CM-) } \\
S R-90 \text { (MCI/SQ.KM.) } \\
S R-9 O C O N C \text {. }(P C / L) \\
S R-89 / S R-S O\end{array}$ & $\begin{array}{r}21.69 \\
0.08 \\
0.37 \\
13.00\end{array}$ & $\begin{array}{l}9.93 \\
0.08 \\
0.81 \\
9.80\end{array}$ & $\begin{array}{l}7.54 \\
0.07 \\
0.93 \\
3.80\end{array}$ & $\begin{array}{l}0.99 \\
0.00 \\
0.01 \\
*\end{array}$ & $\begin{array}{l}1.93 \\
= \\
-\infty\end{array}$ & $\begin{array}{l}+ \\
0.02 \\
1.70\end{array}$ & $\begin{array}{l}1.32 \\
0.01 \\
C .76 \\
*\end{array}$ & $\begin{array}{r}11.48 \\
0.06 \\
0.53 \\
2.10\end{array}$ & $\begin{array}{l}0.30 \\
\neq \\
--\end{array}$ & $\begin{array}{l}6.43 \\
0.04 \\
C .63 \\
+\end{array}$ & $\begin{array}{l}36.91 \\
0.11 \\
0.30 \\
*\end{array}$ & $\bar{m}=$ & $\begin{array}{r}98.52 \\
0.47\end{array}$ \\
\hline 1960 & $\begin{array}{l}\text { PRECIP. }(C M-) \\
S R-90 \text { (KCI/SQ-KM.) } \\
S R-90 \text { CONC. }(P C / L) \\
S R-89 / S R-90\end{array}$ & $\begin{array}{l}29.18 \\
0.00 \mathrm{C} \\
0.01 \\
--\end{array}$ & $\begin{array}{l}39.90 \\
0.01 \mathrm{C} \\
0.03 \\
--\end{array}$ & $\begin{array}{l}66.17 \\
0.05 \\
0.08 \\
-\end{array}$ & $\begin{array}{l}19.43 \\
* \\
=\end{array}$ & $\begin{array}{l}1.19 \\
0.00 \mathrm{C} \\
0.01 \\
-\end{array}$ & $\begin{array}{c}15.01 \\
0.02 C \\
0.14 \\
-\end{array}$ & $\begin{array}{l}0.89 \\
0.036 \\
3.38 \\
--\end{array}$ & $\begin{array}{l}2.67 \\
0.076 \\
2.63 \\
-\end{array}$ & $\begin{array}{l}0.91 \\
0.050 \\
5.50 \\
--\end{array}$ & $\overline{0.05 C}$ & $\begin{array}{l}36.04 \\
0.14 \\
0.39 \\
--\end{array}$ & $\begin{array}{l}30.15 \\
=- \\
=-\end{array}$ & $\begin{array}{r}241.54 \\
0.42\end{array}$ \\
\hline 1961 & $\begin{array}{l}\text { PRECIP. }(C M-) \\
\text { SR-90 IMCI/SQ.KM-) } \\
S R-90 \text { CONC. }(P C / L) \\
S R-89 / S R-90\end{array}$ & $\begin{array}{l}39.27 \\
0.076 \\
0.18 \\
-.\end{array}$ & $\begin{array}{l}24.41 \\
0.03 \mathrm{C} \\
0.13 \\
=-\end{array}$ & $\begin{array}{l}21.82 \\
0.05 \mathrm{C} \\
0.23 \\
-\end{array}$ & $\begin{array}{l}7.92 \\
0.02 C \\
0.26 \\
-\end{array}$ & $\begin{array}{l}6.53 \\
0.02 C \\
0.31 \\
--\end{array}$ & $\begin{array}{l}4.06 \\
0.026 \\
0.50 \\
-\infty\end{array}$ & $\begin{array}{l}0.81 \\
0.05 C \\
6.18 \\
-.\end{array}$ & $\begin{array}{l}0.10 \\
0.006 \\
0.01 \\
-0\end{array}$ & $\begin{array}{l}0.69 \\
* \\
-\infty \\
*\end{array}$ & $\begin{array}{l}3.48 \\
* \\
--\end{array}$ & $\begin{array}{l}18.82 \\
0.17 \\
0.91 \\
*\end{array}$ & $\begin{array}{l}12.95 \\
0.11 \\
0.85 \\
*\end{array}$ & $\begin{array}{r}140.86 \\
0.54\end{array}$ \\
\hline 1962 & $\begin{array}{l}\text { PREC IP. (CM.) } \\
S R-9 C \text { (HCI/SQ.KM.) } \\
S R-90 \text { CONC. }(P C / L) \\
S R-89 / S R-90\end{array}$ & $\begin{array}{r}27.18 \\
0.10 \\
0.37 \\
\end{array}$ & $\begin{array}{r}22.61 \\
0.07 \\
0.31 \\
3.00\end{array}$ & $\begin{array}{r}7.04 \\
0.04 \\
0.57 \\
12.00\end{array}$ & $\begin{array}{r}10.06 \\
0.04 \\
0.40 \\
*\end{array}$ & $\begin{array}{r}4.83 \\
0.08 \\
1.66 \\
79.00\end{array}$ & $\begin{array}{r}0.25 \\
0.02 \\
8.01 \\
57.00\end{array}$ & $\begin{array}{r}2.79 \\
0.06 \\
2.16 \\
41.00\end{array}$ & $\begin{array}{r}1.30 \\
0.05 \\
3.85 \\
17.00\end{array}$ & $\begin{array}{r}5.16 \\
0.08 \\
1.56 \\
23.00\end{array}$ & $\begin{array}{r}27.78 \\
0.09 \\
0.51 \\
18.00\end{array}$ & $\begin{array}{r}24.05 \\
0.01 \\
0.05 \\
14.00\end{array}$ & $\begin{array}{l}20.98 \\
0.01 \\
0.05 \\
*\end{array}$ & $\begin{array}{r}144.03 \\
0.65\end{array}$ \\
\hline 1963 & $\begin{array}{l}\text { PRECIP. (CM.) } \\
\text { SR-90 IMCI/SQ.KM.) } \\
S R-90 \text { CONC. }(P C / L) \\
S R-89 / S R-90\end{array}$ & $=-$ & $\begin{array}{r}11.13 \\
0.10 \\
0.90 \\
8.00\end{array}$ & $\begin{array}{l}11.35 \\
= \\
=\end{array}$ & $\begin{array}{l}2.74 \\
0.02 \\
0.73 \\
3.00\end{array}$ & $\begin{array}{l}1.32 \\
0.02 \\
1.52 \\
+\end{array}$ & $\begin{array}{l}0.28 \\
0.02 \\
7.15 \\
*\end{array}$ & $\begin{array}{l}-\infty \\
0.02 \\
*\end{array}$ & $\begin{array}{l}0.61 \\
0.05 \\
0.20 \\
*\end{array}$ & $\begin{array}{l}- \\
0.02 \\
*\end{array}$ & $\begin{array}{l}6.12 \\
0.10 \\
1.64 \\
+\end{array}$ & $\begin{array}{l}15.19 \\
0.06 \\
0.40 \\
*\end{array}$ & $\begin{array}{l}- \\
\overline{-} \\
-\end{array}$ & $\begin{array}{r}48.74 \\
0.41\end{array}$ \\
\hline 1964 & $\begin{array}{l}P R E C I P \text {. }(C M-) \\
S R-9 C \text { IMCIISQ.KM.) } \\
S R-90 \text { CONC. }(P C / L) \\
S R-89 / S R-90\end{array}$ & $=-$ & $\begin{array}{l}-- \\
-- \\
--\end{array}$ & $\begin{array}{l}17.81 \\
0.07 \\
0.40 \\
*\end{array}$ & $\begin{array}{r}23.80 \\
0.06 \\
0.26 \\
*\end{array}$ & $\begin{array}{r}12.50 \\
6.05 \\
0.41 \\
*\end{array}$ & $\begin{array}{r}2.29 \\
0.95 \\
41.49 \\
-\end{array}$ & $\begin{array}{l}12.01 \\
0.13 \\
1.09 \\
-\end{array}$ & $\begin{array}{l}6.58 \\
0.06 \\
0.92 \\
--\end{array}$ & $\begin{array}{l}9.78 \\
0.14 \\
1.44 \\
-.\end{array}$ & $\begin{array}{l}54.00 \\
0.23 \\
0.43 \\
*\end{array}$ & $\begin{array}{l}47.09 \\
0.24 \\
0.51 \\
*\end{array}$ & $\begin{array}{r}109.68 \\
0.14 \\
0.13 \\
-\end{array}$ & $\begin{array}{r}295.54 \\
2.07\end{array}$ \\
\hline 1965 & $\begin{array}{l}\text { PRECIP. (CM.) } \\
5 R-90 \text { (MCI/SQ.KM.) } \\
S R-90 \text { CDNC. (PC/L) } \\
\text { SR-89/SR-SO }\end{array}$ & $\overline{-}$ & $\begin{array}{r}111.68 \\
0.15 \\
0.14 \\
0 .\end{array}$ & $\begin{array}{r}35.28 \\
0.10 \\
0.29 \\
-.\end{array}$ & $\begin{array}{l}29.82 \\
0.08 \\
0.27 \\
--\end{array}$ & $\begin{array}{r}20.02 \\
0.03 \\
0.15 \\
-\end{array}$ & $\begin{array}{l}5.31 \\
0.02 \\
0.38 \\
--\end{array}$ & $\begin{array}{l}19.61 \\
0.01 \\
0.06 \\
--\end{array}$ & $\begin{array}{r}18.52 \\
0.07 \\
0.38 \\
--\end{array}$ & $\begin{array}{l}6.20 \\
0.06 \\
0.97 \\
--\end{array}$ & $\begin{array}{l}41.02 \\
0.18 \\
0.44 \\
--\end{array}$ & $\begin{array}{l}90.70 \\
* \\
=-\end{array}$ & $\begin{array}{l}79.25 \\
0.28 \\
0.36 \\
--\end{array}$ & $\begin{array}{r}457.41 \\
0.98\end{array}$ \\
\hline 1966 & $\begin{array}{l}\text { PREC I P. (CM.) } \\
\text { SR-90 IMCI/SQ.KM-) } \\
\text { SR-90 CONC. (PC/L) } \\
\text { SR-89/SR-90 }\end{array}$ & $\begin{array}{l}62.05 \\
0.08 \\
0.13 \\
-\end{array}$ & $\begin{array}{l}22.52 C \\
0.06 C \\
0.27 \\
=-\end{array}$ & $\begin{array}{l}22.52 C \\
0.07 C \\
0.32 \\
=-\end{array}$ & $\begin{array}{r}42.24 \\
0.09 \\
0.22 \\
-.\end{array}$ & $\begin{array}{l}6.20 \\
* \\
*\end{array}$ & $*$ & $\begin{array}{r}16.30 \\
0.03 \\
0.19 \\
37.00\end{array}$ & $\begin{array}{l}5.20 \\
0.15 \\
2.89 \\
*\end{array}$ & $\begin{array}{r}0.46 \\
0.08 \\
17.40 \\
65.00\end{array}$ & $\begin{array}{r}1.94 \\
0.28 \\
14.44 \\
158.00\end{array}$ & $\begin{array}{r}4.34 \\
0.29 \\
6.69 \\
35.60\end{array}$ & $\begin{array}{r}0.53 \\
0.16 \\
2.46 \\
13.30\end{array}$ & $\begin{array}{r}190.20 \\
1.29\end{array}$ \\
\hline 1967 & $\begin{array}{l}\text { PRECIP. (CM.) } \\
\text { SR-90 IMCI/SQ.KM.) } \\
\text { SR-90 CONC. (PCIL) } \\
\text { SR-89/SR-90 }\end{array}$ & $\begin{array}{l}3.20 \\
0.07 \\
2.19 \\
6.80\end{array}$ & $\begin{array}{l}2.72 \\
0.03 \\
1.11 \\
*\end{array}$ & $\begin{array}{l}2.55 \\
0.03 \\
1.18 \\
*\end{array}$ & $\begin{array}{l}0.85 \\
0.01 \\
1.18 \\
*\end{array}$ & $\begin{array}{l}=- \\
\overline{--} \\
-\end{array}$ & $\begin{array}{l}= \\
= \\
=\end{array}$ & $\overline{--}$ & $\overline{--}$ & $\overline{--}$ & $=-$ & $=$ & $\overline{--}$ & $\begin{array}{l}9.32 \\
0.14\end{array}$ \\
\hline $\begin{array}{l}\text { NOTES } \\
-=: \\
*: 2 \\
A: \\
B= \\
C:\end{array}$ & $\begin{array}{l}\text { IA NOT AVAILABLE } \\
\text { IO OR TRACE } \\
\text { ROXIMATE } \\
\text { EER LIMIT OF REPO } \\
\text { JPORTIQNED FROM O }\end{array}$ & 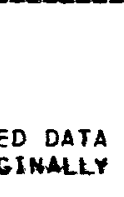 & & Ell & & & & & & & & & & \\
\hline
\end{tabular}


MONTHLY FALLOUT DEPOSITION COLLECTIONS

SITE: BRAZIL, RECIFE

LAT. 825 LONG. $3458 \mathrm{~W}$ ALT.

3M. (COLUMN)

SOURCE: INSTITUTO DE FISICA, PONTIFICIA UNIVERSIDADE CATOLICA COLLECTIONS TERMIMATED IN NOVEMBER 1964

\begin{tabular}{|c|c|c|c|c|c|c|c|c|c|c|c|c|c|c|}
\hline & & JAN。 & FEB. & MAR. & APR. & $\begin{array}{l}\text { MAY } \\
--\end{array}$ & $\underset{\rightarrow}{\longrightarrow}$ & $\begin{array}{l}\text { JuLY } \\
-\end{array}$ & AUG. & SEP. & SCr. & NOV. & DEC. & CUM. TOTAL \\
\hline 1959 & 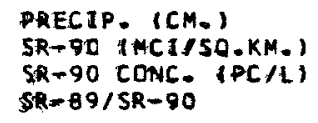 & $\begin{array}{l}2.59 \\
-- \\
-\end{array}$ & $\frac{13.61}{--}$ & $\begin{array}{l}4.29 \\
= \\
=\end{array}$ & $\begin{array}{l}20.83 \\
=- \\
=\end{array}$ & $\begin{array}{l}30.33 \\
= \\
=\end{array}$ & $\begin{array}{l}52.04 \\
= \\
=\end{array}$ & $\begin{array}{l}25.91 \\
=- \\
=\end{array}$ & $\begin{array}{l}8.31 \\
= \\
-\end{array}$ & $\begin{array}{l}9.62 \\
0.05 \\
0.56 \\
-\end{array}$ & $\begin{array}{l}0.71 \\
0.02 \\
2.82 \\
-\end{array}$ & $\begin{array}{l}3.20 \\
0.03 \\
0.94 \\
-\infty\end{array}$ & $\begin{array}{l}0.81 \\
0.01 \\
1.24 \\
=0\end{array}$ & $\begin{array}{r}171.65 \\
0.11\end{array}$ \\
\hline 1960 & $\begin{array}{l}\text { PREC IP. }\left(C M_{-}\right) \\
S R=90 \text { (MCI/SO-KM-) } \\
S R=90 \text { CONC. (PC/L) } \\
S R=89 / S R=90\end{array}$ & $\begin{array}{l}6.50 \\
0.02 \\
0.31 \\
=-\end{array}$ & $\begin{array}{l}1.30 \\
0.02 \\
1.54 \\
--\end{array}$ & $\begin{array}{l}49.05 \\
0.21 \\
0.43 \\
-\end{array}$ & $\begin{array}{r}23.42 \\
0.05 \\
0.22 \\
-7\end{array}$ & $\begin{array}{l}27.53 \\
0.22 \\
0.80 \\
=-\end{array}$ & $\begin{array}{l}29.21 \\
=- \\
=\end{array}$ & $\begin{array}{r}23.42 \\
0.08 \\
0.35 \\
\end{array}$ & $\stackrel{16.21}{-}$ & $\begin{array}{l}5.89 \\
=- \\
=\end{array}$ & $\begin{array}{l}4.01 \\
-- \\
-\infty\end{array}$ & $\begin{array}{l}0.99 \\
=- \\
=\end{array}$ & $\begin{array}{l}5.69 \\
-- \\
-- \\
--\end{array}$ & $\begin{array}{r}193.22 \\
0.60\end{array}$ \\
\hline 1861 & $\begin{array}{l}\text { PRECIP. (CM-) } \\
S R=90 \text { iMCI/SQ-KM-) } \\
3 R-90 \text { CONC. (PCIL) } \\
\text { SR=89/SR-90 }\end{array}$ & ${ }^{30.33}$ & $\begin{array}{l}6.30 \\
-\infty \\
-\end{array}$ & $\frac{35.43}{=}$ & $\stackrel{36.73}{=}$ & $\begin{array}{l}27.61 \\
=\end{array}$ & $\begin{array}{l}21.92 \\
\Rightarrow\end{array}$ & $\begin{array}{l}20.73 \\
=\end{array}$ & $\begin{array}{l}2.79 \\
-\end{array}$ & $\frac{10.11}{=}$ & $\begin{array}{l}4.60 \\
= \\
=\end{array}$ & $\begin{array}{l}0.20 \\
-- \\
-\infty\end{array}$ & $\begin{array}{l}1.19 \\
-- \\
-\infty\end{array}$ & $\begin{array}{c}197.94 \\
0.0\end{array}$ \\
\hline $29 \times 2$ & $\begin{array}{l}\text { PRECIP. }(C M-) \\
\$ R=90 \text { (MCIISQ,KM-) } \\
\$ R=90 \text { CONC. (PC/L) } \\
S R-99 / S R-90\end{array}$ & $\begin{array}{l}0-89 \\
=- \\
=\end{array}$ & $\begin{array}{l}8.66 \\
=- \\
=\end{array}$ & $\frac{13.11}{-}$ & $\begin{array}{l}14.20 \\
= \\
=\end{array}$ & $\begin{array}{l}13.11 \\
-\infty \\
=\end{array}$ & $\begin{array}{r}4.33 \\
0.10 \\
0.42 \\
40.00\end{array}$ & $\begin{array}{r}17.22 \\
0.13 \\
0.76 \\
31.00\end{array}$ & $\begin{array}{r}7.52 \\
0.10 \\
1.33 \\
38.00\end{array}$ & $\begin{array}{r}10.52 \\
0.08 \\
0.77 \\
18.00\end{array}$ & $\begin{array}{r}2.11 \\
0.04 \\
1.90 \\
23.00\end{array}$ & $\begin{array}{r}0.51 \\
0.02 \\
3.93 \\
46.00\end{array}$ & $\begin{array}{r}6.10 \\
0.07 \\
1.15 \\
22.00\end{array}$ & $\begin{array}{r}140.58 \\
0.63\end{array}$ \\
\hline 1963 & $\begin{array}{l}\text { PRECIP. (CM.) } \\
S R-90 \text { (MCIHSO.KM.) } \\
S R=90 \text { CONC. (PCAL) } \\
S R=691 S R-40\end{array}$ & $\begin{array}{r}1.91 \\
0.06 \\
3.15 \\
11.00\end{array}$ & $\begin{array}{r}17.32 \\
0.05 \\
0.29 \\
8.00\end{array}$ & $\begin{array}{r}34.72 \\
0.12 \\
0.35 \\
10.00\end{array}$ & $\begin{array}{r}24.13 \\
0.03 \\
0.13 \\
+\end{array}$ & $\begin{array}{l}12.01 \\
0.05 \\
0.42 \\
\end{array}$ & $\begin{array}{r}12.40 \\
0.07 \\
0.57 \\
1.00\end{array}$ & $=$ & $=$ & $\begin{array}{l}-- \\
- \\
-\end{array}$ & $=$ & $=$ & $\begin{array}{l}7.98 \\
0.07 \\
0.88 \\
*\end{array}$ & $\begin{array}{r}110.47 \\
0.45\end{array}$ \\
\hline 1964 & $\begin{array}{l}\text { PREC IP. (CME) } \\
S R=90 \text { IMCI/SQ.KM.) } \\
S R=90 \text { CONC. (PCAL) } \\
5 R=9,5 R-90\end{array}$ & $=$ & $\overline{-}$ & $=$ & $=$ & $=$ & $\overline{-}$ & $\begin{array}{r}21.97 \\
0.24 \\
1.10 \\
\end{array}$ & $\begin{array}{r}15.67 \\
0.24 \\
1.54 \\
\end{array}$ & $\begin{array}{l}11.61 \\
0.22 \\
1.90 \\
-.\end{array}$ & $\begin{array}{l}4.57 \\
0.20 \\
4.38 \\
-.\end{array}$ & $=$ & $\begin{array}{l}-\infty \\
-\infty\end{array}$ & $\begin{array}{r}53.82 \\
0.90\end{array}$ \\
\hline $\begin{array}{l}\text { NOTES } \\
-:= \\
*: \\
A: \\
B: \\
C:\end{array}$ & $\begin{array}{l}\text { ATA NOT AVAILABLE } \\
\text { ERO OR TRACE } \\
\text { PPROXIMATE } \\
\text { OWER LIMIT OF REPO } \\
\text { ROPORTIONED FROM O }\end{array}$ & $\begin{array}{l}\text { DATA } \\
\text { VALLY }\end{array}$ & & & & & & & & & & & & \\
\hline
\end{tabular}


MONTHLY FALLDUT DEPOSITION COLLECTIONS

SITE: BRAZIL, RIO DE JANEIRO

LAT. $2254 S$ LONG. $4313 \mathrm{H}$ ALT.

9M. (COLUMN)

SOURCE: INSTITUTO DE FISICA, PONTIFICIA UNIVERSIDADE CATOLICA

\begin{tabular}{|c|c|c|c|c|c|c|c|c|c|c|c|c|c|c|}
\hline & & JAN. & FEB. & MAR. & APR. & MAY & SUNE & $\underline{\text { JULY }}$ & AUG. & SEP. & OCT. & NOV. & DEC. & CUM. TOTAL \\
\hline 1960 & $\begin{array}{l}\text { PRECIP. (CM.) } \\
\text { SR-90 IMCI/SQ.KM-) } \\
S R-90 \text { CONC. }(P C / L) \\
\text { SR-89/SR-90 }\end{array}$ & $=$ & $=$ & $\begin{array}{l}-- \\
-- \\
--\end{array}$ & $\begin{array}{l}7.47 \\
0.00 \\
0.01 \\
-=\end{array}$ & $\begin{array}{l}22.76 \\
0.04 \mathrm{C} \\
0.18 \\
-.\end{array}$ & $\begin{array}{c}15.11 \\
0.03 C \\
0.20 \\
--\end{array}$ & $\begin{array}{l}6.88 \\
0.00 \mathrm{C} \\
0.01 \\
-.\end{array}$ & $\begin{array}{l}12.57 \\
0.01 \mathrm{C} \\
0.08 \\
--\end{array}$ & $\begin{array}{l}8.51 \\
0.01 \mathrm{C} \\
0.12 \\
-.\end{array}$ & $\begin{array}{l}2.67 \\
0.00 C \\
0.01 \\
--\end{array}$ & $\begin{array}{l}12.85 \\
=- \\
=-\end{array}$ & $\begin{array}{l}17.17 \\
=- \\
--\end{array}$ & $\begin{array}{r}105.99 \\
0.09\end{array}$ \\
\hline 1961 & $\begin{array}{l}\text { PREC IP. (CM.) } \\
\text { SR-9O INCI/SQ-KM-) } \\
S R-90 \text { CONC. (PC/L) } \\
\text { SR-99/SR-90 }\end{array}$ & $\begin{array}{l}17.48 \\
0.061 \\
0.35 \\
.\end{array}$ & $\begin{array}{l}9.40 \\
0.036 \\
0.32 \\
--\end{array}$ & $\begin{array}{c}12.73 \\
0.08 \mathrm{C} \\
0.63 \\
.\end{array}$ & $\begin{array}{c}17.27 \\
0.126 \\
0.70 \\
-\end{array}$ & $\begin{array}{l}14.55 \\
0.03 C \\
0.21 \\
--\end{array}$ & $\begin{array}{l}20.35 \\
0.04 \mathrm{C} \\
0.20 \\
-\end{array}$ & $\begin{array}{l}8.00 \\
0.100 \\
1.26 \\
-\end{array}$ & $\begin{array}{l}0.99 \\
0.016 \\
1.02 \\
--\end{array}$ & $\begin{array}{l}3.35 \\
0.08 \\
1.80 \\
3.30\end{array}$ & $\begin{array}{r}0.56 \\
0.02 \\
3.50 \\
21.00\end{array}$ & $\begin{array}{r}11.71 \\
0.06 \\
0.52 \\
*\end{array}$ & $\begin{array}{l}17.30 \\
0.14 \\
0.81 \\
*\end{array}$ & $\begin{array}{r}133.69 \\
0.75\end{array}$ \\
\hline 1962 & 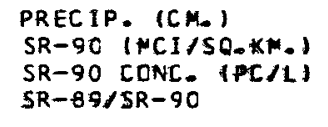 & $\begin{array}{r}46.99 \\
0.19 \\
0.41 \\
1.04\end{array}$ & $\begin{array}{r}32.46 \\
0.11 \\
0.34 \\
4.00\end{array}$ & $\begin{array}{l}1.83 \\
0.02 \\
1.10 \\
7.00\end{array}$ & $\begin{array}{l}3.86 \\
0.04 \\
1.04 \\
*\end{array}$ & $\begin{array}{r}14.43 \\
0.19 \\
1.32 \\
42.00\end{array}$ & $\begin{array}{r}8.69 \\
0.09 \\
1.04 \\
30.00\end{array}$ & $\begin{array}{r}9.40 \\
6.19 \\
2.03 \\
21.00\end{array}$ & $\begin{array}{r}6.10 \\
0.08 \\
1.32 \\
18.00\end{array}$ & $\begin{array}{r}15.50 \\
0.22 \\
1.39 \\
13.00\end{array}$ & $\begin{array}{r}24.71 \\
0.42 \\
1.70 \\
11.00\end{array}$ & $\begin{array}{r}18.42 \\
0.34 \\
1.85 \\
10.00\end{array}$ & $\begin{array}{r}12.50 \\
0.29 \\
2.33 \\
5.80\end{array}$ & $\begin{array}{r}195.29 \\
2.18\end{array}$ \\
\hline 1963 & $\begin{array}{l}\text { PRECIP. (CM.) } \\
\text { SR-90 (MCI/SQEKM-) } \\
\text { SR-90 CONC. (PC/L) } \\
\text { SR-QS/SR-QO }\end{array}$ & $\begin{array}{r}1.52 \\
0.09 \\
5.93 \\
10.00\end{array}$ & $\begin{array}{r}15.77 \\
0.15 \\
0.96 \\
6.00\end{array}$ & $\begin{array}{l}9.60 \\
0.05 \\
0.53 \\
6.00\end{array}$ & $\begin{array}{l}6.53 \\
0.05 \\
0.77 \\
3.00\end{array}$ & $\begin{array}{l}8.26 \\
0.13 \\
1.58 \\
-\end{array}$ & $\begin{array}{l}4.09 \\
0.13 \\
3.18 \\
+\end{array}$ & $\begin{array}{l}4.29 \\
0.05 \\
1.17 \\
*\end{array}$ & $\begin{array}{l}3.99 \\
0.09 \\
2.26 \\
*\end{array}$ & $\begin{array}{l}1.09 \\
0.03 \\
2.76 \\
*\end{array}$ & $\begin{array}{r}13.41 \\
0.28 \\
2.09 \\
*\end{array}$ & $\begin{array}{l}9.88 \\
0.14 \\
1.42 \\
*\end{array}$ & $\begin{array}{l}0.68 \\
0.06 \\
0.90 \\
*\end{array}$ & $\begin{array}{r}85.11 \\
1.25\end{array}$ \\
\hline 1964 & $\begin{array}{l}P R E C I P \text {. }(C M-) \\
S R-9 C \text { IFCI/SO.KM-) } \\
S R-9 C \text { CONC }(P C / L) \\
S R-89 / S R \rightarrow 90\end{array}$ & $\begin{array}{l}2.34 \\
0.08 \\
3.42 \\
0\end{array}$ & $\begin{array}{l}25.96 \\
0.11 \\
0.43 \\
*\end{array}$ & $\begin{array}{r}16.51 \\
0.20 \\
1.22 \\
*\end{array}$ & $\begin{array}{l}9.04 \\
0.10 \\
1.11 \\
2.40\end{array}$ & $\begin{array}{l}9.27 \\
0.15 \\
1.62 \\
0\end{array}$ & $\begin{array}{r}14.15 \\
0.36 \\
2.55 \\
=-\end{array}$ & $\begin{array}{r}11.20 \\
0.35 \\
3.13 \\
-\end{array}$ & $\begin{array}{l}8.33 \\
0.38 \\
4.57 \\
-\infty\end{array}$ & $\begin{array}{r}11.02 \\
0.43 \\
3.91 \\
-\end{array}$ & $\begin{array}{r}24.61 \\
C .14 \\
0.57 \\
*\end{array}$ & $\begin{array}{l}18.54 \\
0.29 \\
1.57 \\
*\end{array}$ & $\begin{array}{r}27.56 \\
0.56 \\
2.04 \\
\end{array}$ & $\begin{array}{r}178.53 \\
3.15\end{array}$ \\
\hline 1965 & $\begin{array}{l}\text { PRECIP. }\left(C M_{-}\right) \\
\text {SR-90 INCIISQ.KM.) } \\
\text { SR-90 CCNC. (PC/L) } \\
S R-89 / S R=90\end{array}$ & $\begin{array}{r}17.09 \\
0.30 \\
1.76 \\
-.\end{array}$ & $\begin{array}{l}31.55 \\
0.24 \\
0.77 \\
-\end{array}$ & $\begin{array}{r}12.07 \\
0.21 \\
1.74 \\
\end{array}$ & $\begin{array}{r}12.55 \\
0.15 \\
1.20 \\
-.\end{array}$ & $\begin{array}{l}26.39 \\
C .25 \\
0.95 \\
-.\end{array}$ & $\begin{array}{l}4.75 \\
C .10 \\
2.11 \\
--\end{array}$ & $\begin{array}{l}20.50 \\
0.27 \\
1.32 \\
--\end{array}$ & $\begin{array}{r}6.25 \\
0.86 \\
13.99 \\
--\end{array}$ & $\begin{array}{r}12.83 \\
0.31 \\
2.42 \\
--\end{array}$ & $\begin{array}{r}15.01 \\
0.21 \\
1.40 \\
\end{array}$ & $\begin{array}{l}6.88 \\
0.04 \\
0.59 \\
--\end{array}$ & $\begin{array}{r}24.61 \\
0.13 \\
0.53 \\
-.\end{array}$ & $\begin{array}{r}190.38 \\
3.07\end{array}$ \\
\hline 1966 & $\begin{array}{l}\text { PRECIP. (CM.) } \\
S R-90 \text { (MCI/SO.KM.) } \\
S R-9 C \text { CONC. (PC/L) } \\
S R-89 / S R-90\end{array}$ & $\begin{array}{r}68.05 \\
0.06 \\
0.09 \\
-.\end{array}$ & $\begin{array}{r}12.67 \\
0.19 \\
1.50 \\
--\end{array}$ & $\begin{array}{l}32.51 \\
0.26 \\
0.80 \\
=-\end{array}$ & $\begin{array}{l}14.05 \\
0.10 \\
0.72 \\
-.0\end{array}$ & $\begin{array}{l}10.39 \\
0.10 \\
0.97 \\
*\end{array}$ & $\begin{array}{l}3.48 \\
0.06 \\
1.73 \\
+\end{array}$ & $\begin{array}{r}11.35 \\
0.09 \\
0.00 \\
73.00\end{array}$ & $\begin{array}{l}21.41 \\
0.23 \\
1.08 \\
*\end{array}$ & $\begin{array}{r}16.84 \\
0.20 \\
1.19 \\
74.00\end{array}$ & $\begin{array}{r}29.97 \\
0.86 \\
2.87 \\
40.00\end{array}$ & $\begin{array}{r}20.52 \\
0.19 \\
0.93 \\
24.00\end{array}$ & $\begin{array}{r}18.14 \\
0.08 \\
0.45 \\
10.20\end{array}$ & $\begin{array}{r}259.38 \\
2.42\end{array}$ \\
\hline 1967 & $\begin{array}{l}\text { PRECIP. (CM.) } \\
S R-90 \text { (NCI/SQ.KM.) } \\
S R-90 \text { CONC. (PC/L) } \\
S R-89 / S R-90\end{array}$ & $\begin{array}{r}17.65 \\
0.04 \\
0.23 \\
*\end{array}$ & 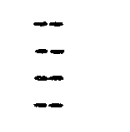 & $\bar{z}$ & $\overline{-}$ & $\overline{-}$ & $\overline{-}$ & $\begin{array}{l}-- \\
-- \\
--\end{array}$ & $\overrightarrow{-}$ & $\begin{array}{l}-- \\
-- \\
--\end{array}$ & $\begin{array}{l}-- \\
=- \\
--\end{array}$ & $\begin{array}{l}-- \\
--\end{array}$ & $\begin{array}{l}-- \\
-- \\
--\end{array}$ & $\begin{array}{r}17.65 \\
0.04\end{array}$ \\
\hline \begin{tabular}{cc} 
NOTES \\
\hdashline$-:$ & $D$ \\
$*:$ & 2 \\
$A:$ & $A$ \\
$B:$ & $L$ \\
$C:$ & $P$
\end{tabular} & $\begin{array}{l}\text { ATA NOT AVAILABLE } \\
\text { IERO OR TRACE } \\
\text { PPROXIMATE } \\
\text { OWER LIMIT OF REPOR } \\
\text { ROPORTI ONED FROM OR }\end{array}$ & DATA & & & & & & & & & & & & \\
\hline
\end{tabular}




\begin{tabular}{|c|c|c|c|c|c|c|c|c|c|c|c|c|c|c|}
\hline & & JAN. & FEB. & MAR. & $A P R$ & MAY & JUNE & JULY & AUG. & SEP. & OCT. & NOV. & DEC. & CUM. TOTAL \\
\hline 1959 & $\begin{array}{l}\text { PRECIP. (CM.) } \\
\text { SR-90 (MCI/SO.KM.) } \\
\text { SR-90 CONC. (PC/L) } \\
\text { SR-89/SR-90 }\end{array}$ & $\overline{=}$ & $=$ & $=$ & $=$ & $\bar{z}$ & $=$ & $\begin{array}{l}=- \\
=\end{array}$ & $\frac{-}{0.10}$ & $\frac{-1}{0.12}$ & 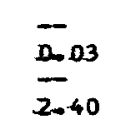 & $\overline{0.02}$ & $\overline{0.08}_{2.20}$ & $\begin{array}{l}0.0 \\
0.35\end{array}$ \\
\hline 1960 & $\begin{array}{l}\text { PRECIP. (CM.) } \\
\text { SR-90 IMCI/SQ-KM.) } \\
S R-90 \text { CONC. (PC/LL) } \\
\text { SR-89/SR-SO }\end{array}$ & $\begin{array}{l}-- \\
0.05 \\
-\end{array}$ & $\begin{array}{l}0.05 \\
-\infty\end{array}$ & 0.09 & $\begin{array}{l}2.21 \\
0.06 \\
2.72 \\
\end{array}$ & $\begin{array}{l}-\infty \\
0.15 C \\
--\end{array}$ & $\begin{array}{l}\overline{0.150} \\
=\end{array}$ & $\overline{0.15 C}$ & $\overline{0.15 c}$ & $\bar{D}=$ & $\overline{0.08 c}$ & $\overline{0.08 C}$ & $\begin{array}{l}\overline{0.08 \tau} \\
=\end{array}$ & $\begin{array}{l}2.21 \\
1.17\end{array}$ \\
\hline 1961 & $\begin{array}{l}\text { PRECIP. (CM-) } \\
\text { SR-9O (MCI/SQ.KM-) } \\
\text { SR-90 CONC. }(P C / L) \\
S R-89 / S R-90\end{array}$ & $\begin{array}{l}- \\
0.05 C \\
=-\end{array}$ & $\overrightarrow{0.05 C}$ & $\underline{0.04 C}$ & $\begin{array}{l}-0.04 C \\
-\end{array}$ & $\overline{0.06 c}$ & $\overline{0.06 c}$ & $\bar{m}$ & $\overline{0.05}$ & $\bar{*}$ & $\overline{0.21}$ & $\frac{-}{0.19}$ & $\overline{0.11}$ & $\begin{array}{l}0.0 \\
0.86\end{array}$ \\
\hline 1962 & $\begin{array}{l}\text { PRECIP, (CM.) } \\
\text { SR-9O IMCI/SO.KM.) } \\
\text { SR-9O CONC. }(P C / L) \\
S R-89 / S R-90\end{array}$ & $\begin{array}{l}-\overline{0.08} \\
\bar{*}\end{array}$ & $\overrightarrow{0.06}$ & $\begin{array}{l}-0.05 \\
+\end{array}$ & $\overrightarrow{0.02}$ & $\overline{0.06}$ & $\frac{\overline{0.08}}{38.00}$ & $\begin{array}{l}\overline{0.05} \\
\overline{50.00}\end{array}$ & $\begin{array}{l}\overline{0.16} \\
\overline{19.00}\end{array}$ & $\begin{array}{l}\overline{0.17} \\
\overline{16.00}\end{array}$ & $\begin{array}{l}\overline{0.09} \\
\overline{11.00}\end{array}$ & $\begin{array}{c}-.13 \\
0.0 \\
105.00\end{array}$ & $\begin{array}{l}-- \\
\overline{0.07} \\
14.00\end{array}$ & $\begin{array}{l}0.0 \\
1.02\end{array}$ \\
\hline 1963 & $\begin{array}{l}\text { PRECIP. (CM.) } \\
S R-9 C \text { I } M C I / S Q . K M-) \\
S R-90 \text { CONC. }(P C / L) \\
S R-89 / S R-90\end{array}$ & $\begin{array}{l}-\overline{0.07} \\
6.00\end{array}$ & $=$ & $\bar{E}$ & $\frac{0.13}{*}$ & $\overline{0.02}$ & $\overline{0.04}$ & $\frac{\overline{0.13}}{1.00}$ & $\overline{0.56}^{*}$ & $\overline{0}^{0.90}$ & $\frac{0.19}{*}$ & $\begin{array}{l}- \\
0.25 \\
*\end{array}$ & $\begin{array}{l}3.71 \\
0.08 \\
2.16 \\
*\end{array}$ & $\begin{array}{l}3.71 \\
1.77\end{array}$ \\
\hline 1964 & $\begin{array}{l}\text { PRECIP. (CM.) } \\
\text { SR-9C IMCI/SQ.KM-) } \\
\text { SR-90 CONC. }(P C / L) \\
S R-89 / S R \rightarrow 90\end{array}$ & $\begin{array}{l}3.10 \\
-- \\
--\end{array}$ & $\begin{array}{l}14.48 \\
0.10 \\
0.70 \\
*\end{array}$ & $\begin{array}{l}10.74 \\
0.07 \\
0.66 \\
*\end{array}$ & $\begin{array}{l}6.40 \\
0.09 \\
1.41 \\
*\end{array}$ & $\begin{array}{l}5.99 \\
0.05 \\
0.84 \\
--\end{array}$ & $\begin{array}{l}6.40 \\
0.12 \\
1.88 \\
\end{array}$ & $\begin{array}{r}12.80 \\
0.24 \\
1.88 \\
--\end{array}$ & $\begin{array}{r}15.09 \\
0.44 \\
2.92 \\
-\end{array}$ & $\begin{array}{l}10.32 \\
0.09 \\
0.88 \\
--\end{array}$ & $=$ & $\begin{array}{l}-- \\
--\end{array}$ & $\begin{array}{r}14.66 \\
0.14 \\
0.96 \\
\end{array}$ & $\begin{array}{r}99.97 \\
1.34\end{array}$ \\
\hline 1965 & $\begin{array}{l}\text { PRECIP. (CM.) } \\
\text { SR-9O INCI/SQ.KM-) } \\
\text { SR-9O CONC. }(P C / L) \\
\text { SR-g9/SR-90 }\end{array}$ & $\begin{array}{l}5.46 \\
0.25 \\
4.58 \\
--\end{array}$ & $\begin{array}{l}7.75 \\
0.09 \\
1.17 \\
-.\end{array}$ & $\begin{array}{l}13.36 \\
0.20 \\
1.50 \\
-\end{array}$ & $\begin{array}{l}6.32 \\
0.09 \\
1.43 \\
-.\end{array}$ & $\begin{array}{l}5.94 \\
0.06 \\
1.02 \\
\end{array}$ & $\begin{array}{r}10.74 \\
0.16 \\
1.49 \\
\end{array}$ & $\begin{array}{r}11.07 \\
0.12 \\
1.09 \\
\end{array}$ & $\begin{array}{r}42.60 \\
0.16 \\
0.38 \\
-\end{array}$ & $\begin{array}{r}30.43 \\
0.37 \\
1.22 \\
-\end{array}$ & $\begin{array}{l}14.00 \\
= \\
=\end{array}$ & $\begin{array}{r}11.10 \\
0.13 \\
1.18 \\
\end{array}$ & $\begin{array}{l}-13 \\
--\end{array}$ & $\begin{array}{r}158-77 \\
1-76\end{array}$ \\
\hline 1966 & $\begin{array}{l}\text { PRECIP. (CMA) } \\
\text { SR-9O IMCI/SQ.KM-) } \\
S R-90 \text { CCNC. (PC/L) } \\
S R-89 / S R-90\end{array}$ & $\begin{array}{l}17.17 \\
0.16 \\
0.94 \\
-\end{array}$ & $\begin{array}{l}21.13 \\
0.14 \\
0.67 \\
--\end{array}$ & $\begin{array}{l}9.63 \\
0.13 \\
1.35 \\
-.\end{array}$ & $\begin{array}{l}3.53 \\
0.05 \\
1.42 \\
--\end{array}$ & $\frac{-0}{0.02}$ & $\begin{array}{l}17.64 \\
0.04 \\
0.23 \\
*\end{array}$ & $\begin{array}{r}26.28 \\
6.12 \\
6.43 \\
120.00\end{array}$ & $\begin{array}{r}26.024 \\
0.14 \\
0.54 \\
31.00\end{array}$ & $\begin{array}{r}11.14 \\
0.07 \\
0.63 \\
15.00\end{array}$ & $\begin{array}{r}20.0 .3 \\
0.0 .03 \\
0.40 \\
230.00\end{array}$ & $\begin{array}{r}1.74 \\
0.13 \\
1.34 \\
37.80\end{array}$ & $\overline{-}$ & $\begin{array}{r}164.53 \\
1.08\end{array}$ \\
\hline 1967 & $\begin{array}{l}\text { PRECIP. (CM.) } \\
\text { SR-90 (MCI/SQ.KM.) } \\
S R-90 \text { CONC. (PC/L) } \\
\text { SR-89/SR-90 }\end{array}$ & $\begin{array}{r}14.30 \\
0.26 \\
1.82 \\
5.40\end{array}$ & $\begin{array}{l}7.65 \\
0.11 \\
1.44 \\
+\end{array}$ & $\begin{array}{l}7.09 \\
0.15 \\
2.12 \\
*\end{array}$ & $\begin{array}{l}3.05 \\
0.01 \\
0.33 \\
*\end{array}$ & $\overline{-}$ & $=$ & $\begin{array}{l}-- \\
-\infty\end{array}$ & $=$ & $=$ & $\overline{-}$ & $\bar{m}$ & $=$ & $\begin{array}{r}32.09 \\
0.53\end{array}$ \\
\hline $\begin{array}{c}\text { NOTES } \\
\text { NO: } \\
*: Z \\
A: A \\
B: \\
C:\end{array}$ & $\begin{array}{l}\text { ATA NOT AVAILABLE } \\
\text { ERO OR TRACE } \\
\text { PPROXIMATE } \\
\text { OWER LIMIT OF REPOR } \\
\text { ROPORTI ONEO FROM OR }\end{array}$ & $\begin{array}{l}\text { DATA } \\
\text { NALIT }\end{array}$ & NSOL & ED 0 & & & & & & & & & & \\
\hline
\end{tabular}


MONTHR FALLOUT OEPOSITION COLLECTIONS

SITE: BRAZIL, TRINDADE ISLAND

LAT. 20315 LONG. 29 20W ALT.

92\%. (COLUMN)

SOURCE: DIRETORIA DE HIDROGRAFIA E NAVEGACAO

JAN. FEB. MAR. APR. MAY JUNE JULY AUG. SEP. OCT. NOV. OEC. CUM. TOTAL

\begin{tabular}{|c|c|c|c|c|c|c|c|c|c|c|c|c|c|c|}
\hline 1962 & $\begin{array}{l}\text { PRECIP. (CM.) } \\
\text { SR-90 (MCI/SQ.KM.) } \\
\text { SR-90 CONC. }(P C / L) \\
\text { SR-89/SR-90 }\end{array}$ & $=$ & $\begin{array}{l}-- \\
-- \\
-\end{array}$ & $=$ & $=$ & $\begin{array}{l}-\infty \\
0.09 \\
3.00\end{array}$ & $\begin{array}{c}0.02 \\
112.0 \mathrm{C}\end{array}$ & $\begin{array}{l}\overline{0.05} \\
\overline{15.00}\end{array}$ & $\begin{array}{l}\overline{0.10} \\
\overline{11.00}\end{array}$ & $\begin{array}{c}\overline{0.11} \\
\overline{13.00}\end{array}$ & $\begin{array}{l}0.10 \\
6.00\end{array}$ & $\begin{array}{c}-- \\
0.07 \\
10.00\end{array}$ & $\begin{array}{l}-- \\
0.10 \\
\overline{7.00}\end{array}$ & $\begin{array}{l}0.0 \\
0.64\end{array}$ \\
\hline 1963 & $\begin{array}{l}\text { PRECIP. }\left(C M_{-}\right) \\
\text {SR-90 (MCI/SQ.KM.) } \\
S R-90 \text { CONC. }(P C / L) \\
S R-89 / S R-90\end{array}$ & $\begin{array}{l}-- \\
0.05 \\
5.00\end{array}$ & $\begin{array}{l}-. \\
0.10 \\
-. \\
6.30\end{array}$ & $\begin{array}{c}\overline{0.11} \\
10.00\end{array}$ & $=0.05$ & +10 & $\begin{array}{l}-\infty \\
0.16 \\
*\end{array}$ & $\begin{array}{l}0.08 \\
--\end{array}$ & $\begin{array}{l}-\infty \\
0.44\end{array}$ & $=0.05$ & $\begin{array}{l}-- \\
0 . \\
--\end{array}$ & $\begin{array}{l}-- \\
0.32 \mathrm{C} \\
--\end{array}$ & $\begin{array}{l}-- \\
0.03 \\
*\end{array}$ & $\begin{array}{l}0.0 \\
1.81\end{array}$ \\
\hline 1964 & $\begin{array}{l}\text { PRECIP. }\left(C M_{-}\right) \\
\text {SR-90 (MCI/SQ.KM.) } \\
\text { SR-90 CONC. }(P C / L) \\
\text { SR-89/SR-SC }\end{array}$ & $\begin{array}{l}-- \\
0.12 \\
--\end{array}$ & $\frac{\overline{0.07}}{\overline{0}}$ & $\frac{-08}{0}$ & $\begin{array}{r}3.18 \\
0.01 \\
0.32 \\
12.80\end{array}$ & $\begin{array}{l}0.99 \\
0.08 \\
8.09 \\
*\end{array}$ & $\begin{array}{l}-- \\
0.59 \\
--\end{array}$ & $\begin{array}{l}-- \\
0.16 \\
--\end{array}$ & $\begin{array}{l}-- \\
0.19 \\
--\end{array}$ & $\begin{array}{l}-- \\
0.29 \\
-- \\
--\end{array}$ & $\begin{array}{l}-- \\
0.23 \\
--\end{array}$ & $\begin{array}{l}-\overline{0.26} \\
-\bar{t}\end{array}$ & $\begin{array}{l}-- \\
-- \\
--\end{array}$ & $\begin{array}{l}4.17 \\
2.08\end{array}$ \\
\hline 1965 & $\begin{array}{l}\text { PREC IP. (CM.) } \\
\text { SR-9O (PCI/SQ.KM.) } \\
S R=90 \text { CONC, }(P C / L) \\
S R-89 / S R-90\end{array}$ & $=$ & $=$ & $\overrightarrow{0.22}$ & $=0.02$ & $=0.17$ & $=0.05$ & 0.05 & $\overrightarrow{0.12}$ & $\begin{array}{l}--10 \\
0 . \\
=-\end{array}$ & $\begin{array}{l}5.13 \\
* \\
-- \\
--\end{array}$ & $\begin{array}{l}-- \\
-- \\
--\end{array}$ & $\begin{array}{l}-- \\
0.11 \\
--\end{array}$ & $\begin{array}{l}5.13 \\
0.84\end{array}$ \\
\hline 1966 & $\begin{array}{l}\text { PRECIP, (CM.) } \\
\text { SR-90 (NCI/SO.KM.) } \\
\text { SR-90 CONC. (PC/L) } \\
S R-8 S / S R-90\end{array}$ & $\begin{array}{l}2.44 \\
0.03 \\
1.23 \\
-.\end{array}$ & $\begin{array}{l}1.47 \\
0.05 \\
3.41 \\
-\end{array}$ & $\begin{array}{l}39.10 \\
*- \\
=-\end{array}$ & $\begin{array}{r}49.50 \\
0.04 \\
0.09 \\
*\end{array}$ & $\begin{array}{r}33.80 \\
0.04 \\
0.12 \\
+\end{array}$ & $\begin{array}{l}26.00 \\
0.04 \\
0.16\end{array}$ & $\begin{array}{l}0.04 \\
33.00\end{array}$ & $\begin{array}{l}\overrightarrow{0.03} \\
19.00\end{array}$ & $\begin{array}{l}-\infty \\
0.06 \\
6.00\end{array}$ & $\begin{array}{r}14.50 \\
0.12 \\
0.83 \\
66.00\end{array}$ & $\begin{array}{l}-0.05 \\
\overrightarrow{0.00}\end{array}$ & $\begin{array}{l}1.90 \\
0.02 \\
1.06 \\
*\end{array}$ & $\begin{array}{r}168.71 \\
0.52\end{array}$ \\
\hline 1967 & $\begin{array}{l}\text { PRECIP. }\left(C M_{-}\right) \\
S R-90 \text { (NCI/SO.KM. }) \\
\text { SR-90 CONC. }(P C / L) \\
S R-89 / S R-90\end{array}$ & $\begin{array}{l}4.70 \\
0.05 \\
1.07\end{array}$ & $\begin{array}{l}3.20 \\
0.02 \\
0.63 \\
*\end{array}$ & $\frac{\overrightarrow{0.03}}{*}$ & $=0.01$ & $=$ & $\begin{array}{l}=- \\
=-\end{array}$ & $=$ & $=$ & $=$ & $=$ & $=$ & $=$ & $\begin{array}{l}7.90 \\
0.11\end{array}$ \\
\hline
\end{tabular}

NOTES

--: DATA NOT AVAILABLE

*: LERO OR TRACE

A: APPROXIMATE

B: LOHER LIMIT OF REPORTED DATA

C: PROPORTIONED FROM ORIGINALLY CONSOLIDATED DATA 
SOURCE: ERNST HARMON AIR BASE, U. S. AIR WEATHER SERVICE

COLLECTIONS TERMIMAITD IN APRIL 1966

\begin{tabular}{|c|c|c|c|c|c|c|c|c|c|c|c|c|c|c|}
\hline & & JAN. & PEO- & MAR. & APR. & MAY & JUNE & JULY & AUG. & SEP. & OCT. & NDN. & $D E C$ & CUM. TOTAL \\
\hline 1959 & $\begin{array}{l}\text { PREC IP. (CM) } \\
\text { SR-9C (MCI/SO-KM-) } \\
\text { SR-9O CONC (PC/L) } \\
\text { SR-89/SR-90 }\end{array}$ & $\begin{array}{l}23.62 \\
=\end{array}$ & $\begin{array}{l}14.17 \\
=\end{array}$ & $\begin{array}{l}16.31 \\
--\end{array}$ & $\begin{array}{l}1.57 \\
= \\
-\end{array}$ & $\begin{array}{l}4.55 \\
-- \\
--\end{array}$ & $\begin{array}{l}4.62 \\
-- \\
-\end{array}$ & $=$ & $\begin{array}{l}9.30 \\
0.15 \\
1.62 \\
0.16\end{array}$ & $\begin{array}{l}8.20 \\
0.07 \\
0.86 \\
--\end{array}$ & $\begin{array}{l}10.29 \\
= \\
=\end{array}$ & $\overline{0.13}$ & $\begin{array}{l}6.10 \\
-- \\
--\end{array}$ & $\begin{array}{r}105.92 \\
0.35\end{array}$ \\
\hline 1960 & $\begin{array}{l}\text { PRECIP- }\left(C M_{-}\right) \\
S R-90\left(M C I / S Q_{\left.-K M_{-}\right)}\right) \\
S R-B D \text { CONC. }(P C / L) \\
S R-89 / S R-90\end{array}$ & $\begin{array}{l}18.16 \\
0.03 \\
0.17 \\
-0\end{array}$ & $\begin{array}{l}2.59 \\
0.15 \\
5.80 \\
-.\end{array}$ & $\begin{array}{l}5.11 \\
0.07 \\
1.37 \\
-\end{array}$ & $\begin{array}{l}5.92 \\
0.21 \\
3.55 \\
\end{array}$ & $\begin{array}{l}3.86 \\
0.12 C \\
2.85 \\
-\end{array}$ & $\begin{array}{l}7.06 \\
0.200 \\
2.84 \\
-\end{array}$ & $\begin{array}{l}7-67 \\
0.10 \mathrm{C} \\
1.31 \\
--\end{array}$ & $\begin{array}{l}3.30 \\
0.04 C \\
1.22 \\
-\end{array}$ & $\begin{array}{l}7.87 \\
0.026 \\
0.26 \\
-\end{array}$ & $\begin{array}{c}12.09 \\
0.03 \mathrm{C} \\
0.25 \\
-\end{array}$ & $\begin{array}{l}10.69 \\
=-\end{array}$ & $\stackrel{11.53}{ \pm}$ & $\begin{array}{r}95.85 \\
0.96\end{array}$ \\
\hline 19.61 & $\begin{array}{l}P R E C I P \text {. (CM) } \\
S R-90 \text { (MCI/SD-KH-) } \\
S R-90 \text { CRNC. }(P C / L) \\
S R-89 / S R-90\end{array}$ & $\begin{array}{c}2.1 .69 \\
0.1 .12 \\
C .51 \\
\end{array}$ & $\begin{array}{l}7.59 \\
0.04 C \\
0.53 \\
--\end{array}$ & $\begin{array}{l}10.41 \\
0.34 C \\
3.27 \\
--\end{array}$ & $\begin{array}{l}1.17 \\
0.04 C \\
3.42 \\
-.\end{array}$ & $\begin{array}{l}2.97 \\
0.14 C \\
4.72 \\
--\end{array}$ & $\begin{array}{l}7.87 \\
0.376 \\
4.71 \\
-=\end{array}$ & ${ }^{13-13}$ & $\begin{array}{l}7.8 .2 \\
-- \\
--\end{array}$ & $\begin{array}{l}5.44 \\
=- \\
--\end{array}$ & $\begin{array}{r}16.36 \\
0.20 \\
1.23 \\
80.00\end{array}$ & $\begin{array}{r}8.56 \\
0.21 \\
2.46 \\
100.00\end{array}$ & $\begin{array}{r}11.51 \\
0.14 \\
1.22 \\
66.00\end{array}$ & $\begin{array}{r}114.52 \\
1.59\end{array}$ \\
\hline 1962 & $\begin{array}{l}\text { PRECIP }\left(T M_{-}\right) \\
S R-90 \text { (MCI/SOLKM-) } \\
\text { SR-90 CLNC. }(P C / L) \\
S R-89 / S R \rightarrow O 0\end{array}$ & $\begin{array}{r}24.56 \\
0.33 \\
1.35 \\
44.00\end{array}$ & $\begin{array}{r}3.3 .93 \\
0.23 \\
0.68 \\
35.00\end{array}$ & $\begin{array}{r}5.00 \\
0.17 \\
3.41 \\
18.00\end{array}$ & $\begin{array}{r}11.10 \\
0.83 \\
7.48 \\
6.00\end{array}$ & $\begin{array}{r}9.12 \\
0.94 \\
10.31 \\
9.00\end{array}$ & $\begin{array}{r}10.44 \\
1.49 \\
14.28 \\
9.00\end{array}$ & $\begin{array}{r}8.23 \\
0.85 \\
10-33 \\
6.00\end{array}$ & $\begin{array}{r}11.10 \\
1.34 \\
12.08 \\
4.00\end{array}$ & $\begin{array}{r}7.06 \\
0.41 \\
5.81 \\
31.0 .0\end{array}$ & $\begin{array}{r}8.23 \\
0.04 \\
0.49 \\
15.00\end{array}$ & $\begin{array}{r}9.55 \\
0.72 \\
7.54 \\
42.00\end{array}$ & $\begin{array}{r}12.04 \\
0.27 \\
2.25 \\
50.00\end{array}$ & $\begin{array}{r}150.36 \\
7.62\end{array}$ \\
\hline 1963 & $\begin{array}{l}\text { PRECIP. }\left(C M_{-}\right) \\
S R-90\left(M C I / S K_{-} K M_{-}\right) \\
S R-9 C \text { CCNC. }(P C / L) \\
S R-89 / S R-90\end{array}$ & $\begin{array}{r}9.02 \\
0.33 \\
3.66 \\
28.00\end{array}$ & $\begin{array}{r}7-67 \\
0.08 \\
1.05 \\
24.00\end{array}$ & $\begin{array}{r}2.97 \\
0.27 \\
9 .-43 \\
18-00\end{array}$ & $\begin{array}{r}2.72 \\
0.64 \\
23.53 \\
6.00\end{array}$ & $\begin{array}{r}7.92 \\
3.96 \\
5 C .0 D \\
6.10\end{array}$ & $\begin{array}{r}7.52 \\
2.80 \\
37.24 \\
*\end{array}$ & $\begin{array}{r}8.00 \\
2.48 \\
31.00 \\
2.00\end{array}$ & $\begin{array}{r}14.07 \\
2.31 \\
16.42 \\
1.00\end{array}$ & $\begin{array}{r}10.49 \\
1.35 \\
12.87 \\
0.80\end{array}$ & $\begin{array}{r}5.03 \\
0.79 \\
15.71 \\
0.50\end{array}$ & $\begin{array}{l}16.13 \\
0.08 \\
0.50 \\
*\end{array}$ & $\begin{array}{r}14.07 \\
0.09 \\
0.64 \\
*\end{array}$ & $\begin{array}{r}105.61 \\
15.19\end{array}$ \\
\hline 1964 & $\begin{array}{l}\text { PRECIP. }\left(C M_{-}\right) \\
S R-9 C\left(M C I / S Q_{. K M}\right) \\
S R-90 \text { CONC. }(P C / L) \\
S R \rightarrow-9 / S R-90\end{array}$ & $\begin{array}{l}9.73 \\
0.05 \\
0.52 \\
+\end{array}$ & $\begin{array}{r}10-11 \\
0.37 \\
3-66 \\
*\end{array}$ & $\begin{array}{l}7.42 \\
0.34 \\
4.59 \\
*\end{array}$ & $\begin{array}{r}13.00 \\
1.27 \\
9.77 \\
*\end{array}$ & $\begin{array}{r}3.18 \\
1.67 \\
52.52 \\
+\end{array}$ & $\begin{array}{r}11.86 \\
2.22 \\
18.72 \\
--\end{array}$ & $\begin{array}{l}12.12 \\
1.71 \\
14.11 \\
--\end{array}$ & $\begin{array}{l}7.44 \\
0.67 \\
9.01 \\
\end{array}$ & $\begin{array}{l}8.59 \\
0.37 \\
4.31 \\
--\end{array}$ & $\begin{array}{l}9.47 \\
0.33 \\
3.49 \\
8.10\end{array}$ & $\begin{array}{l}6.30 \\
0.17 \\
2.70 \\
+\end{array}$ & $\begin{array}{l}9.17 \\
0.14 \\
1.53 \\
-\end{array}$ & $\begin{array}{r}108.39 \\
9.31\end{array}$ \\
\hline 1965 & $\begin{array}{l}\text { PRECIP. }\left(C M_{-}\right) \\
S R-90 \text { (RCI/SO-KM-) } \\
S R-90 \text { CDNC. }(P C / L) \\
S R-99 / S R-90\end{array}$ & $\begin{array}{l}11 . .96 \\
0.12 \\
1.01 \\
-\end{array}$ & $\begin{array}{l}1.78 \\
0.04 \\
2.25 \\
--\end{array}$ & $\begin{array}{l}\pi-80 \\
0.04 \\
0.52 \\
--\end{array}$ & $\begin{array}{l}4.47 \\
0.08 \\
1.79 \\
-.\end{array}$ & $\begin{array}{l}2.04 \\
0.08 \\
2.82 \\
-.\end{array}$ & $\begin{array}{l}5.89 \\
0.55 \\
9.34 \\
-.\end{array}$ & $\begin{array}{l}9.19 \\
0.29 \\
3.16 \\
-\end{array}$ & $\begin{array}{r}15.19 \\
0.38 \\
2.51 \\
-\end{array}$ & $\begin{array}{l}6.68 \\
0.28 \\
4.20 \\
-\end{array}$ & $\begin{array}{r}12.80 \\
0.15 \\
1.18 \\
-\end{array}$ & $\begin{array}{c}10.92 \\
+- \\
=-\end{array}$ & $\begin{array}{l}9.65 \\
0.07 \\
0.73 \\
--\end{array}$ & $\begin{array}{r}99.17 \\
2.08\end{array}$ \\
\hline 1966 & $\begin{array}{l}\text { PRECIP (CM.) } \\
\text { SR }-90 \text { IMCI/SO.KM-) } \\
\text { SR-90 CONC. }(P C / L) \\
S R-89 / S R-90\end{array}$ & $\begin{array}{l}5.05 \\
0.01 \\
0.20 \\
-\end{array}$ & $\begin{array}{l}8.00 \\
+ \\
- \\
-\end{array}$ & $\begin{array}{l}2.26 \\
0.05 \\
2.22 \\
--\end{array}$ & $\overline{-}$ & $=-$ & $\begin{array}{l}-- \\
--\end{array}$ & $\overline{-}$ & $\begin{array}{l}- \\
-\end{array}$ & $=$ & $\overline{-}$ & $=$ & $\overline{-}$ & $\begin{array}{r}15.31 \\
0.06\end{array}$ \\
\hline 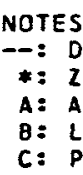 & $\begin{array}{l}\text { A NOT AVAILABLE } \\
\text { O OR TRACE } \\
\text { ROXIMATE } \\
\text { IER LIMIT OF REPO } \\
\text { PORTIONEO FROM O }\end{array}$ & & & & & & & & & & & & & \\
\hline
\end{tabular}




\section{MONTHLY FALLOUT DEPOSITION COLLECTIONS}

SOURCE: GOOSE AIR BASE, U. S. AIR HEATHER SERVICE COLLECTIONS TERMINATED IN MARCH 1964

$$
\begin{aligned}
& \text { JAN. FEB. MAR. APR. MAY JUNE JULY AUG. SEP. DLT. NOV. DEC. CUM. TOTAL }
\end{aligned}
$$

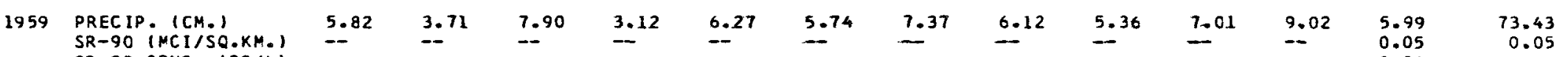

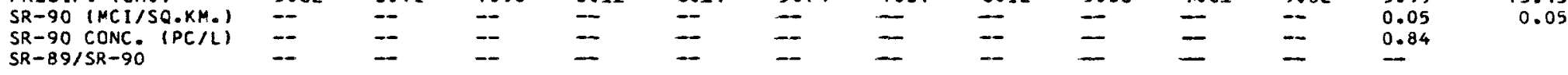

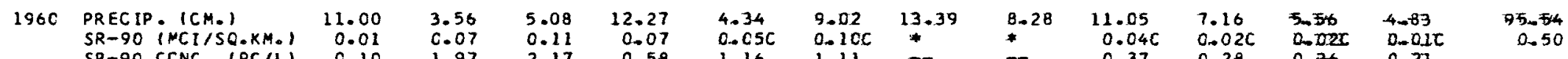

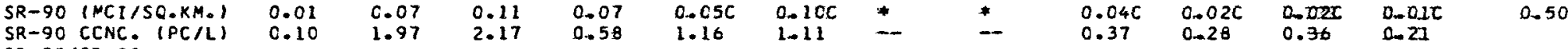

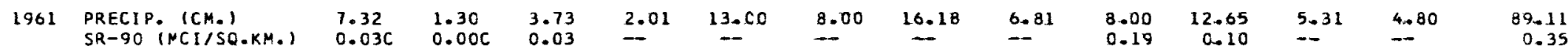

$$
\begin{aligned}
& 0.41 \quad 0.01 \quad 0.81
\end{aligned}
$$

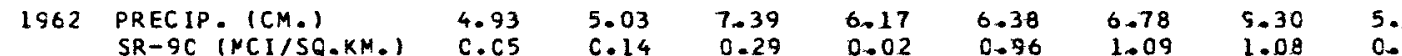$$
S R-90 \text { CONC. (PC/L) }
$$

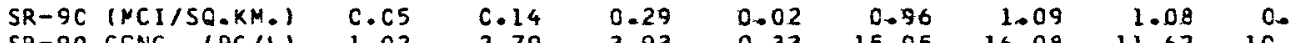

-: DATA NOT AVAILABLE

* 2ERO OR TRACE

B: LOWER LIMIT OF REPORTED DATA

C: PROPORTIONED FROM CRIGINALLY CONSOLICATEO DATA 
MONTHLY FALLOUT DEPOSITION GOLLECTIONS

SITE: CANADA, ONTARID, MODSONEE

LAT. 51 16N LONG. 80 89N ALT.

10M. (GOLUMN) SURFACE AIR SAMPLING STATION

SOURCE: METEOROLOGICAL BRANCH, DEPARTMENT OF TRANSPORT, TORONTO, ONTARIO

\begin{tabular}{|c|c|c|c|c|c|c|c|c|c|c|c|c|c|c|}
\hline & & JAN. & FEB. & MAR. & APR. & MAY & $\begin{array}{l}\text { JUNE } \\
---\end{array}$ & JULY & AUG, & SEP. & OCT. & NOV. & DEC. & CUM. TDTAL \\
\hline 1963 & $\begin{array}{l}\text { PRECIP. (CM.) } \\
\text { SR-9C INCI/SQ.KM) } \\
S R-90 \text { CCNC. (PC/L) } \\
S R-89 / S R-90\end{array}$ & $\begin{array}{l}-- \\
--\end{array}$ & $\begin{array}{l}-- \\
-- \\
--\end{array}$ & $\begin{array}{r}2.36 \\
0.29 \\
12.29 \\
19.00\end{array}$ & $\begin{array}{r}3.66 \\
1.46 \\
39.90 \\
12.00\end{array}$ & $\begin{array}{r}8.69 \\
3.10 \\
35.68 \\
6.50\end{array}$ & $\begin{array}{r}5.54 \\
3.55 \\
64.08 \\
3.10\end{array}$ & $\begin{array}{r}5.11 \\
2.19 \\
42.86 \\
3.00\end{array}$ & $\begin{array}{r}4.27 \\
1.80 \\
42.16 \\
4\end{array}$ & $\begin{array}{r}5.74 \\
C .70 \\
12.20 \\
*\end{array}$ & $\begin{array}{r}2.82 \\
0.52 \\
18.44 \\
C .80\end{array}$ & $\begin{array}{l}8.36 \\
0.14 \\
1.68 \\
*\end{array}$ & $\begin{array}{l}1.73 \\
0.07 \\
4.05 \\
*\end{array}$ & $\begin{array}{l}48.28 \\
13.82\end{array}$ \\
\hline 1964 & $\begin{array}{l}\text { PRECIP : (CM.) } \\
S R-9 C\left(N C I / S K_{-K M .)}\right. \\
S R-S C \text { CCNC. }(P C / L) \\
S R-89 / S R-90\end{array}$ & $\begin{array}{l}6.35 \\
0.43 \\
6.78 \\
*\end{array}$ & $\begin{array}{l}3.66 \\
0.14 \\
3.83 \\
0.30\end{array}$ & $\begin{array}{l}3.30 \\
0.10 \\
3.04 \\
*\end{array}$ & $\begin{array}{r}3.94 \\
1.06 \\
26.91 \\
*\end{array}$ & $\begin{array}{r}9.47 \\
2.54 \\
26.83 \\
*\end{array}$ & $\begin{array}{r}11.07 \\
3.13 \\
28.28 \\
--\end{array}$ & $\begin{array}{l}4.37 \\
1.16 \\
26.55 \\
-. .\end{array}$ & $\begin{array}{l}17.35 \\
1.37 \\
7.90 \\
-.\end{array}$ & $\begin{array}{l}8.66 \\
C .46 \\
5.32 \\
-.\end{array}$ & $\begin{array}{l}5.66 \\
C .26 \\
4.60 \\
2.40\end{array}$ & $\begin{array}{l}8.03 \\
0.15 \\
1.87 \\
7\end{array}$ & $\begin{array}{l}3.86 \\
0.06 \\
1.56 \\
-.\end{array}$ & $\begin{array}{l}85.72 \\
10.86\end{array}$ \\
\hline 1965 & $\begin{array}{l}\text { PRECIP }\{C M \cdot) \\
S R-9 C(M C I / S Q . K M=\} \\
S R-S C \text { CCNC. }(P C / L) \\
S R-89 / S R-90\end{array}$ & $\begin{array}{l}4.57 \\
C .02 \\
0.44 \\
--\end{array}$ & $\begin{array}{l}3.53 \\
0.03 \\
0.85 \\
-.\end{array}$ & $\begin{array}{l}0.66 \\
0.01 \\
1.52 \\
-.\end{array}$ & $\begin{array}{r}1.50 \\
0.16 \\
10.67 \\
*\end{array}$ & $\begin{array}{l}7.59 \\
0.66 \\
8.70 \\
-.\end{array}$ & $\begin{array}{r}3.20 \\
0.59 \\
18.44 \\
-.\end{array}$ & $\begin{array}{l}8.48 \\
C .71 \\
E .38 \\
--\end{array}$ & $\begin{array}{r}10.00 \\
0.40 \\
3.98 \\
-.\end{array}$ & $\begin{array}{l}8.38 \\
C .2 C \\
2.39 \\
--\end{array}$ & $\begin{array}{r}10.64 \\
6.29 \\
2.73 \\
-.\end{array}$ & $\begin{array}{l}3.99 \\
0.08 \\
2.01 \\
-.\end{array}$ & $\begin{array}{l}2.67 \\
0.03 \\
1.13 \\
-. .\end{array}$ & $\begin{array}{r}05.27 \\
3.16\end{array}$ \\
\hline 1966 & $\begin{array}{l}\text { PRECIP. }\left(C M_{-}\right) \\
S R-9 C \text { IMCI/SQ.KM.) } \\
S R-9 O C O N C .(P C / L) \\
S R-89 / S R-90\end{array}$ & $\begin{array}{l}5.56 \\
0.20 \\
3.60 \\
-.\end{array}$ & $\begin{array}{l}3.43 \\
0.05 \\
1.46 \\
--\end{array}$ & $\begin{array}{l}8.71 \\
0.10 \\
1.15 \\
--\end{array}$ & $\begin{array}{l}4.24 \\
0.06 \\
1.42 \\
-.\end{array}$ & $\begin{array}{l}5.46 \\
0.10 \\
1.84 \\
--\end{array}$ & $\begin{array}{l}7.75 \\
0.02 \\
0.26 \\
*\end{array}$ & $\begin{array}{r}15.26 \\
0.12 \\
0.79 \\
*\end{array}$ & $\begin{array}{l}7.16 \\
0.03 \\
0.42 \\
*\end{array}$ & $\begin{array}{r}13.03 \\
0.02 \\
0.16 \\
.\end{array}$ & $\begin{array}{r}10.13 \\
0.01 \\
0.10 \\
*\end{array}$ & $\begin{array}{l}7.700 \\
0.016 \\
0.13 \\
*\end{array}$ & $\begin{array}{l}7.70 E \\
0.016 \\
0.13 \\
*\end{array}$ & $\begin{array}{r}96.13 \\
0.73\end{array}$ \\
\hline 1967 & $\begin{array}{l}\text { PREE IP. }\left(C M_{-}\right) \\
S R-90 \text { INCI/SQ.KM.) } \\
S R-90 \text { CONC. }(P C / L) \\
S R-89 / S R-S O\end{array}$ & $\begin{array}{l}3.07 \\
0.01 \\
0.33 \\
*\end{array}$ & $\begin{array}{l}3.25 \\
0.01 \\
c .32 \\
*\end{array}$ & $\begin{array}{l}3.35 \\
0.05 \\
1.50 \\
9.20\end{array}$ & $\begin{array}{l}5.20 \\
0.08 \\
1.57 \\
7.80\end{array}$ & $\begin{array}{l}2.59 \\
0.05 \\
1.94 \\
4.80\end{array}$ & $\begin{array}{l}2.90 \\
0.07 \\
2.42 \\
*\end{array}$ & $\begin{array}{r}10.08 \\
0.16 \\
1.59 \\
*\end{array}$ & $\begin{array}{l}-- \\
-- \\
--\end{array}$ & $\begin{array}{l}=- \\
\overline{--}\end{array}$ & $\begin{array}{l}=- \\
=- \\
=-\end{array}$ & $\begin{array}{l}-- \\
-- \\
--\end{array}$ & $=$ & $\begin{array}{r}30.24 \\
0.43\end{array}$ \\
\hline \begin{tabular}{l} 
NOTES \\
\hdashline$-: D$ \\
$*: 2$ \\
$A: A$ \\
$B: L$ \\
$C: A$
\end{tabular} & $\begin{array}{l}\text { S } \\
\text { DATA NOT AVAILABLE } \\
\text { ZERO OR TRACE } \\
\text { APPROXIMATE } \\
\text { LOWER LIMIT OF REPOR } \\
\text { PROPORTIONED FROM OR }\end{array}$ & DATA & & & & & & & & & & & & \\
\hline
\end{tabular}




\begin{tabular}{|c|c|c|c|c|c|c|c|c|c|c|c|c|c|c|}
\hline & & JAN. & FEB. & MAR. & APR. & MAY & JUNE & JULY & AUG. & SEP. & OCT. & NOV. & DEC. & CUM. TOTAL \\
\hline 1959 & $\begin{array}{l}\text { PRECIP. }(C M .) \\
\text { SR-9O INCI/SQ.KM.) } \\
S R-9 C \text { CCNC. }(P C / L) \\
S R-89 / S R-90\end{array}$ & $\begin{array}{l}4.45 \\
-- \\
=-\end{array}$ & $\begin{array}{l}6.81 \\
-- \\
--\end{array}$ & $\begin{array}{l}1.30 \\
-- \\
--\end{array}$ & $\begin{array}{l}46.66 \\
=- \\
=-\end{array}$ & $\begin{array}{l}30.61 \\
-- \\
--\end{array}$ & $\begin{array}{l}31.27 \\
=- \\
=\end{array}$ & $\begin{array}{l}18.64 \\
=- \\
=-\end{array}$ & $\begin{array}{l}17.98 \\
=- \\
=\end{array}$ & $\begin{array}{l}23.16 \\
C .05 \\
0.22 \\
*\end{array}$ & $\begin{array}{l}25.10 \\
=- \\
=-\end{array}$ & $\begin{array}{l}26.87 \\
0.01 \\
0.04 \\
*\end{array}$ & $\begin{array}{l}16.79 \\
0.02 \\
0.12 \\
*\end{array}$ & $\begin{array}{r}249.64 \\
0.08\end{array}$ \\
\hline 1960 & $\begin{array}{l}\text { PRECIP. (CM.) } \\
\text { SR-90 IMCI/SQ-KM-) } \\
\text { SR-90 CONC. }(P C / L) \\
\text { SR-89/SR-90 }\end{array}$ & $\begin{array}{l}4.67 \\
0.15 \\
3.22 \\
--\end{array}$ & $\begin{array}{r}17.32 \\
0.00 \\
0.01 \\
--\end{array}$ & $\begin{array}{l}6.93 \\
0.06 \\
0.87 \\
--\end{array}$ & $\begin{array}{l}19.84 \\
0.05 \\
0.26 \\
-2\end{array}$ & $\begin{array}{c}35.69 \\
0.016 \\
0.03 \\
-.\end{array}$ & $\begin{array}{l}12.60 \\
0.00 \mathrm{C} \\
0.01 \\
--\end{array}$ & $\begin{array}{l}46.69 \\
- \\
--\end{array}$ & $\begin{array}{l}4.19 \\
+- \\
--\end{array}$ & $\begin{array}{l}11.56 \\
0.016 \\
0.09 \\
--\end{array}$ & $\begin{array}{l}35.28 \\
0.02 C \\
C .06 \\
--\end{array}$ & $\begin{array}{l}42.55 \\
* \\
--\end{array}$ & $\begin{array}{l}5.33 \\
* \\
--\end{array}$ & $\begin{array}{r}242.65 \\
0.30\end{array}$ \\
\hline 1961 & $\begin{array}{l}\text { PRECIP. (CM.) } \\
S R-90 \text { (MCI/SO.KM.) } \\
S R-9 C \text { CONC }(P C / L) \\
S R-89 / S R-90\end{array}$ & $\begin{array}{l}3.02 \\
-- \\
--\end{array}$ & $\begin{array}{l}4.88 \\
* \\
-- \\
--\end{array}$ & $\begin{array}{l}26.87 \\
0.03 \mathrm{C} \\
0.12 \\
\ldots\end{array}$ & $\begin{array}{l}46.79 \\
0.05 C \\
0.11 \\
--\end{array}$ & $\begin{array}{l}51.05 \\
0.06 \mathrm{C} \\
0.12 \\
--\end{array}$ & $\begin{array}{l}22.28 \\
0.02 C \\
0.09 \\
-\end{array}$ & $\begin{array}{l}22.45 \\
=- \\
-\end{array}$ & $\begin{array}{l}23.01 \\
0.12 \\
0.53 \\
-\end{array}$ & $\begin{array}{r}42.65 \\
6.02 \\
6.05 \\
12.00\end{array}$ & $\begin{array}{r}48.44 \\
0.03 \\
0.07 \\
39.00\end{array}$ & $\begin{array}{r}50.39 \\
0.03 \\
0.06 \\
67.00\end{array}$ & $\begin{array}{r}26.04 \\
0.02 \\
0.08 \\
68.00\end{array}$ & $\begin{array}{r}367.87 \\
0.38\end{array}$ \\
\hline 1962 & $\begin{array}{l}\text { PREC IP. }\left(C M_{-}\right) \\
S R-S C \text { (NCI/SO.KM-) } \\
S R-90 \text { CONC. }(P C / L) \\
S R-89 / S R-90\end{array}$ & $\begin{array}{l}7.90 \\
0.00 \\
0.01 \\
+\end{array}$ & $\begin{array}{l}3.86 \\
+ \\
--\end{array}$ & $\begin{array}{r}9.80 \\
0.24 \\
2.45 \\
18.00\end{array}$ & $\begin{array}{l}-- \\
--\end{array}$ & $\begin{array}{r}49.38 \\
0.13 \\
0.27 \\
11.00\end{array}$ & $\begin{array}{r}13.08 \\
0.24 \\
1.84 \\
17.0 C\end{array}$ & $\begin{array}{r}16.95 \\
0.27 \\
2.47 \\
8.00\end{array}$ & $\begin{array}{r}43.51 \\
0.17 \\
0.40 \\
9.00\end{array}$ & $\begin{array}{r}15.59 \\
0.08 \\
0.41 \\
9.00\end{array}$ & $\begin{array}{r}41.68 \\
0.30 \\
C .72 \\
23.00\end{array}$ & $\begin{array}{r}22.02 \\
0.07 \\
0.32 \\
33.00\end{array}$ & $\begin{array}{r}8.48 \\
0.03 \\
0.36 \\
32.00\end{array}$ & $\begin{array}{r}230.65 \\
1.53\end{array}$ \\
\hline 1963 & $\begin{array}{l}\text { PRECIP. }\left(C M_{-}\right) \\
S R-S C \text { INCI/SO.KM-) } \\
S R-90 C C N C . \quad(P C / L) \\
S R-89 / S R-90\end{array}$ & $\begin{array}{l}7.49 \\
-- \\
-- \\
--\end{array}$ & $\begin{array}{r}22.17 \\
0.26 \\
1.18 \\
22.00\end{array}$ & $\begin{array}{r}12.07 \\
0.11 \\
0.92 \\
14.00\end{array}$ & $\begin{array}{r}32.87 \\
0.44 \\
1.34 \\
11.00\end{array}$ & $\begin{array}{r}54.66 \\
0.25 \\
0.46 \\
12.90\end{array}$ & $\begin{array}{l}18.36 \\
1.03 \\
5.62 \\
-\end{array}$ & $\begin{array}{r}35.34 \\
0.58 \\
1.48 \\
*\end{array}$ & $\begin{array}{l}8.92 \\
0.08 \\
0.90 \\
--\end{array}$ & $\begin{array}{r}53.29 \\
C .44 \\
0.83 \\
*\end{array}$ & $\begin{array}{r}70.71 \\
0.28 \\
0.40 \\
0.90\end{array}$ & $\begin{array}{l}43.56 \\
0.12 \\
0.28 \\
*\end{array}$ & $\begin{array}{l}30.68 \\
1.63 \\
5.32 \\
*\end{array}$ & $\begin{array}{r}394.12 \\
5.22\end{array}$ \\
\hline 1964 & $\begin{array}{l}\text { PRECIP. }(C M-) \\
S R-90 \text { INCI/SQ.KM.) } \\
S R-9 O C O N C . \quad(P C / L) \\
S R-89 / S R-90\end{array}$ & $\begin{array}{l}4.27 \\
0.10 \\
2.35 \\
*\end{array}$ & $\begin{array}{l}17.32 \\
0.14 \\
0.81 \\
*\end{array}$ & $\begin{array}{r}12.19 \\
0.02 \\
0.17 \\
1.20\end{array}$ & $\begin{array}{r}15.65 \\
0.03 \\
0.20 \\
*\end{array}$ & $\begin{array}{l}50.70 \\
0.06 \\
0.12 \\
*\end{array}$ & $\begin{array}{r}13.67 \\
0.32 \\
2.35 \\
--\end{array}$ & $\begin{array}{r}17.17 \\
0.04 \\
0.24 \\
--\end{array}$ & $\begin{array}{l}6.53 \\
0.22 \\
3.37 \\
-\end{array}$ & $\begin{array}{l}12.93 \\
0.056 \\
0.39 \\
-.\end{array}$ & $\begin{array}{l}46.36 \\
C .18 C \\
0.39 \\
*\end{array}$ & $\begin{array}{l}19.89 \\
0.08 \mathrm{C} \\
0.41 \\
*\end{array}$ & $\begin{array}{l}7.26 \\
0.18 \\
2.48 \\
--\end{array}$ & $\begin{array}{r}223.94 \\
1.42\end{array}$ \\
\hline 1965 & $\begin{array}{l}\text { PREC IP }(C M-) \\
S R-9 C \text { ( } M C I / S Q . K M-) \\
S R-90 C C N C . \quad(P C / L) \\
S R-89 / S R-90\end{array}$ & $\begin{array}{l}4.24 \\
-- \\
-- \\
-\end{array}$ & $\begin{array}{l}10.54 \\
0.03 \\
0.29 \\
--\end{array}$ & $\begin{array}{l}4.88 \\
0.06 \\
1.23 \\
--\end{array}$ & $\begin{array}{l}35.48 \\
0.02 C \\
0.06 \\
=-\end{array}$ & $\begin{array}{l}6 C .96 \\
0.026 \\
0.04 \\
--\end{array}$ & $\begin{array}{l}7.42 \\
=- \\
--\end{array}$ & $\begin{array}{l}5.51 \\
0.02 \\
0.37 \\
--\end{array}$ & $\begin{array}{l}17.78 \\
0.07 \\
0.40 \\
--\end{array}$ & $\begin{array}{l}22.81 \\
0.08 \\
0.36 \\
-.\end{array}$ & $\begin{array}{l}5 C .75 \\
C .05 \\
C .10 \\
--\end{array}$ & $\begin{array}{l}-- \\
0.02 \\
--\end{array}$ & $\begin{array}{l}15.24 \\
0.10 \\
0.66 \\
--\end{array}$ & $\begin{array}{r}235.61 \\
0.47\end{array}$ \\
\hline 1966 & $\begin{array}{l}\text { PRECIP. (CM.) } \\
\text { SR-90 (MCI/SQ.KM.) } \\
\text { SR-90 CONC. (PC/L) } \\
\text { SR-89/SR-90 }\end{array}$ & $\begin{array}{c}16.60 \\
= \\
=\end{array}$ & $\begin{array}{l}2.60 \\
- \\
-\end{array}$ & $\begin{array}{r}22.30 \\
0.12 \\
0.54 \\
--\end{array}$ & $\begin{array}{r}33.50 \\
0.32 \\
0.96 \\
--\end{array}$ & $\begin{array}{r}14.70 \\
0.12 \\
0.82 \\
--\end{array}$ & $\begin{array}{r}13.60 \\
0.10 \\
0.74 \\
8.70\end{array}$ & $\begin{array}{l}10.20 \\
=- \\
=-\end{array}$ & $\begin{array}{l}-- \\
* \\
*\end{array}$ & $\begin{array}{l}20.32 \\
0.11 \\
0.55 \\
*\end{array}$ & $\begin{array}{r}17.78 \\
6.03 \\
0.17 \\
11.00\end{array}$ & $\begin{array}{l}-- \\
0.02 \\
--\end{array}$ & $\begin{array}{l}-- \\
0.02 \\
--\end{array}$ & $\begin{array}{r}151.60 \\
0.84\end{array}$ \\
\hline 1967 & $\begin{array}{l}\text { PRECIP. (CM.) } \\
\text { SR-90 IMCI/SQ.KM.) } \\
\text { SR-90 CONC. (PC/L) } \\
\text { SR-89/SR-90 }\end{array}$ & $\begin{array}{r}20.32 \\
0.01 \\
0.05 \\
*\end{array}$ & $\begin{array}{r}22.86 \\
0.02 \\
0.09 \\
*\end{array}$ & 11.50 & $\begin{array}{r}10.84 \\
0.04 \\
0.37 \\
*\end{array}$ & $\begin{array}{c}59.30 \\
= \\
=\end{array}$ & $\begin{array}{l}15.65 \\
0.02 \\
0.13 \\
\end{array}$ & $=$ & $\begin{array}{l}-- \\
--\end{array}$ & $\begin{array}{l}-- \\
-- \\
--\end{array}$ & $\begin{array}{l}-- \\
-- \\
--\end{array}$ & $\begin{array}{l}-- \\
=-\end{array}$ & $\begin{array}{l}-- \\
-- \\
--\end{array}$ & $\begin{array}{r}140.47 \\
0.09\end{array}$ \\
\hline
\end{tabular}


MONTHLY FALLOUT DEPOSITION COLLECTIONS

SITE: CHILE, ANTARCTICA, ISLA DEC

LAT. 62565 LONG. $6036 \mathrm{~W}$ ALT.

16M. (COLUMN) SURFACE AIR SAMPLING STATION

SOURCE: OFICINA MEIEOROLOGICA DE CHILE

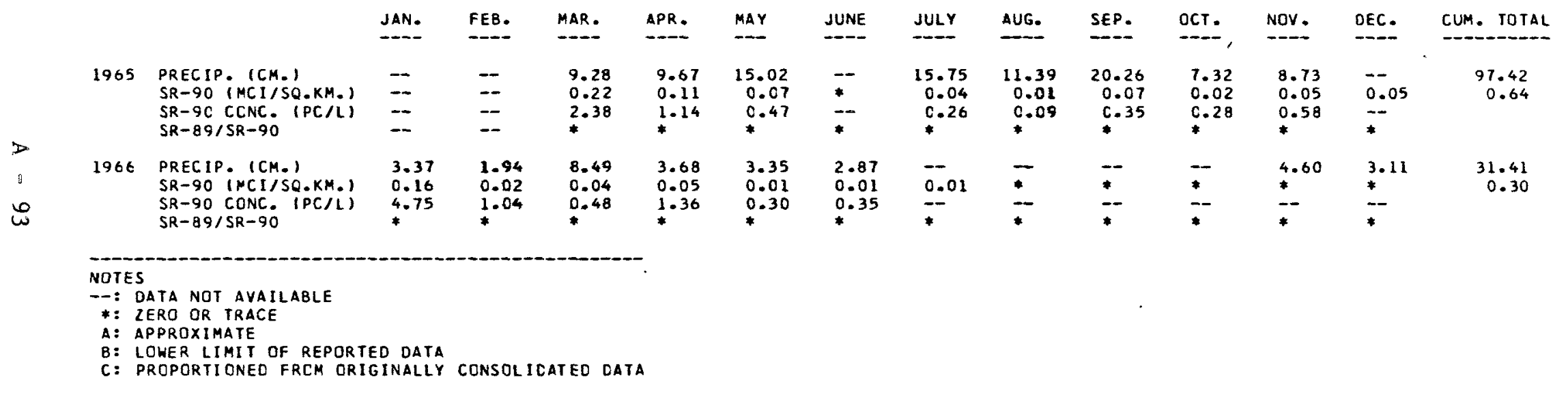


MONTHLY FALLOUT DEPOSITION COLLECTIONS

SITE: CHILE, ANTOFAGASTA

LAT. 23375 LONG. $7016 \mathrm{~W}$ ALT.

519M. (COLUMN) SURFACE AIR SAMPLING STATION

SOURCE : DEPARTAMENTO de InVESTIGACIONES DE LA UNIVERS IDAD DEL NORTE

\begin{tabular}{|c|c|c|c|c|c|c|c|c|c|c|c|c|c|c|}
\hline & & JAN. & FEB. & MAR. & APR. & MAY & JUNE & JULY & AUG. & SEP. & OCT. & NOV. & DEC. & CUM. TOTAL \\
\hline 1963 & $\begin{array}{l}\text { PRECIP. }\left(C M_{*}\right) \\
\text { SR-9O (MCI/SQ.KM.) } \\
S R-90 \text { CONC. }(P C / L) \\
S R-89 / S R-90\end{array}$ & $\begin{array}{l}* \\
-- \\
--\end{array}$ & $\stackrel{*}{--}$ & $\begin{array}{l}* \\
0.00 \\
10.00\end{array}$ & $\begin{array}{l}* \\
0.01 \\
--\end{array}$ & $\begin{array}{l}* \\
0.01 \\
--\end{array}$ & $\begin{array}{l}* \\
0.02 \\
--\end{array}$ & $\begin{array}{l}* \\
-- \\
--\end{array}$ & $\begin{array}{l}* \\
-- \\
--\end{array}$ & $\begin{array}{r}0.05 \\
0.01 \\
20.01 \\
*\end{array}$ & $\begin{array}{l}* \\
0.17 \\
-0\end{array}$ & $\begin{array}{l}* \\
0.02 \\
*\end{array}$ & $\begin{array}{l}0.02 \\
* \\
*\end{array}$ & $\begin{array}{l}0.05 \\
0.26\end{array}$ \\
\hline 1964 & $\begin{array}{l}\text { PRECIP. (CM.) } \\
\text { SR-90 INCI/SQ-KM.) } \\
S R-90 \text { CONC. }(P C / L) \\
S R-89 / S R-90\end{array}$ & $\begin{array}{l}* \\
0.03 \\
*\end{array}$ & $\begin{array}{l}* \\
0.02 \\
1.00\end{array}$ & $\begin{array}{l}* \\
0.01 \\
*\end{array}$ & $\begin{array}{l}* \\
0.02 \\
*\end{array}$ & $\begin{array}{l}* \\
0.02 \\
*\end{array}$ & $\begin{array}{l}* \\
0.02 \\
-- \\
--\end{array}$ & $\begin{array}{l}* \\
0.02 \\
-- \\
--\end{array}$ & $\begin{array}{l}* \\
0.02 \\
=-\end{array}$ & $\begin{array}{l}* \\
0.01 \\
-\infty\end{array}$ & $\begin{array}{l}* \\
0.01 \\
-\infty\end{array}$ & $\begin{array}{l}* \\
0.02 \\
--\end{array}$ & $\begin{array}{l}* \\
* \\
-- \\
--\end{array}$ & $\begin{array}{l}0.0 \\
0.20\end{array}$ \\
\hline 1965 & $\begin{array}{l}\text { PRECIP. (CM.) } \\
\text { SR-90 IMCI/SO.KM.) } \\
\text { SR-90 CONC. }(P C / L) \\
S R-89 / S R-90\end{array}$ & $\begin{array}{l}* \\
* \\
--\end{array}$ & $\begin{array}{l}* \\
* \\
--\end{array}$ & $\begin{array}{l}* \\
0.00 \\
--\end{array}$ & $\begin{array}{l}* \\
* \\
--\end{array}$ & $\begin{array}{l}* \\
0.00 \\
--\end{array}$ & $\begin{array}{l}* \\
0.03 \\
--\end{array}$ & $\begin{array}{l}* \\
* \\
=-\end{array}$ & $\begin{array}{r}0.05 \\
0.02 \\
40.01 \\
-\infty\end{array}$ & $\stackrel{*}{*}+$ & $\begin{array}{l}-- \\
-- \\
--\end{array}$ & $\overline{--}$ & $\begin{array}{l}-- \\
0.03 \\
--\end{array}$ & $\begin{array}{l}0.05 \\
0.08\end{array}$ \\
\hline 1966 & $\begin{array}{l}\text { PRECIP. }(C M-) \\
\text { SR-90 IMCI/SQ.KM-) } \\
\text { SR-90 CONC. }(P C / L) \\
S R-89 / S R-90\end{array}$ & $\begin{array}{l}* \\
0.02 \\
--\end{array}$ & $\begin{array}{l}* \\
* \\
--\end{array}$ & $\begin{array}{l}* \\
0.01 \\
-\end{array}$ & $\begin{array}{l}4 \\
0.01 \\
-- \\
--\end{array}$ & $\begin{array}{l}* \\
0.05 \\
*\end{array}$ & $\stackrel{*}{*}$ & $\frac{-}{0.01}$ & $\begin{array}{l}-- \\
--\end{array}$ & $\begin{array}{l}+ \\
0.01 \\
--\end{array}$ & $\begin{array}{l}-\infty \\
0.01 \\
*\end{array}$ & $\begin{array}{l}* \\
0.01 \\
--\end{array}$ & $\begin{array}{l}* \\
0.01 \\
-\end{array}$ & $\begin{array}{l}0.0 \\
0.14\end{array}$ \\
\hline 1967 & $\begin{array}{l}\text { PRECIP. (CM.) } \\
S R-90 \text { (MCI/SQ.KM.) } \\
S R-90 \text { CONC. }(P C / L) \\
S R-89 / S R-90\end{array}$ & $\begin{array}{l}\# \\
0.01 \\
--\end{array}$ & $\begin{array}{l}* \\
-- \\
--\end{array}$ & $\begin{array}{l}* \\
--\end{array}$ & $\begin{array}{l}-- \\
- \\
-\end{array}$ & $\begin{array}{l}* \\
--\end{array}$ & $\begin{array}{l}* \\
-- \\
--\end{array}$ & $=-$ & $=$ & $\begin{array}{l}-- \\
-- \\
--\end{array}$ & $\begin{array}{l}-- \\
-- \\
--\end{array}$ & $\begin{array}{l}-- \\
--\end{array}$ & $\begin{array}{l}-- \\
--\end{array}$ & $\begin{array}{l}0.0 \\
0.01\end{array}$ \\
\hline $\begin{array}{c}\text { NOTES } \\
--:=0 \\
*: \\
A: \\
B: A \\
C: L \\
C: P\end{array}$ & $\begin{array}{l}\text { ATA NOT AVAILABLE } \\
\text { ERO OR TRACE } \\
\text { PPROXIMATE } \\
\text { OWER LIMIT OF REPOR } \\
\text { ROPORTIONED FROM OR }\end{array}$ & $\begin{array}{l}\text { D DATA } \\
\text { INALLY }\end{array}$ & ONSOL & $T E D$ & & & & & & & & & & \\
\hline
\end{tabular}


MONTHLY FALLOUT DEPOSITION COLLECTIONS

SITE: CHILE, EASTER ISLAND

SOURCE: OFICINA METEOROLOGICA DE CHILE
LAT. 27 IOS LONG.109 26H ALT. T4H. (OOLLMN)

\begin{tabular}{|c|c|c|c|c|c|c|c|c|c|c|c|c|c|c|}
\hline & & JAN. & FEB. & MAR. & APR. & MAY & JUNE & JULY & AUG. & SER, & UCT. & NOK. & IIEC & CUM. TOTAL \\
\hline 1963 & $\begin{array}{l}\text { PRECIP. (CM.) } \\
\text { SR-9O INCI/SQ.KM.) } \\
\text { SR-90 CONC. (PC/L) } \\
\text { SR-89/SR-90 }\end{array}$ & $\begin{array}{l}7.77 \\
-- \\
--\end{array}$ & $\begin{array}{l}6.55 \\
-- \\
--\end{array}$ & $\begin{array}{l}2.59 \\
0.07 \\
2.71 \\
*\end{array}$ & $\begin{array}{r}10.39 \\
0.08 \\
0.77 \\
22.00\end{array}$ & $\begin{array}{l}32.59 \\
0.15 \\
0.47 \\
*\end{array}$ & $\begin{array}{r}14.07 \\
0.09 \\
0.64 \\
5.00\end{array}$ & $\begin{array}{l}5.66 \\
C .06 \\
1.07\end{array}$ & $\begin{array}{l}2.59 \\
0.06 \\
2.12 \\
+\end{array}$ & $\begin{array}{l}8+148 \\
0 \leqslant 100 \\
b-23\end{array}$ & $\begin{array}{l}4: 159 \\
0: 06 \\
1.44\end{array}$ & $\begin{array}{l}3.99 \\
0.07 \\
1.76 \\
*\end{array}$ & $\begin{array}{l}6.10 \\
0.17 \\
2.79 \\
--\end{array}$ & $\begin{array}{r}104.67 \\
0.91\end{array}$ \\
\hline 1964 & $\begin{array}{l}\text { PRECIP. }(C M-) \\
\text { SR-9O (MCI/SQ.KM.) } \\
S R-90 \text { CONC. }(P C / L) \\
S R-8 S / S R-S O\end{array}$ & $\begin{array}{l}0.40 \\
0.01 \\
0.16\end{array}$ & $\begin{array}{l}9.70 \\
0.11 \\
1.14 \\
-.\end{array}$ & $\begin{array}{l}11.40 \\
0.14 \\
1.23 \\
-.\end{array}$ & $\begin{array}{l}- \\
0.14 \\
=\end{array}$ & $\overline{-}$ & $\begin{array}{l}9.04 \\
0.15 \\
1.66 \\
-\end{array}$ & $\begin{array}{r}17.81 \\
0.17 \\
0.96 \\
\end{array}$ & $\begin{array}{l}3.43 \\
0.19 \\
5.54 \\
-\end{array}$ & $\begin{array}{l}4.55 \\
0.11 \\
2.42 \\
\end{array}$ & $\begin{array}{l}9.55 \\
0.18 \\
1.89 \\
-.\end{array}$ & $\begin{array}{r}21.39 \\
0.36 \\
1.69 \\
\end{array}$ & $\begin{array}{l}6.43 \\
0.21 \\
3.27 \\
-.\end{array}$ & $\begin{array}{r}99.70 \\
1.77\end{array}$ \\
\hline 1965 & $\begin{array}{l}\text { PRECIP. }(C M .) \\
\text { SR-90 (NCI/SO.KM.) } \\
\text { SR-90 CONC. }(P C / L) \\
\text { SR-89/SR-9O }\end{array}$ & $\begin{array}{l}11.84 \\
0.19 \\
1.61 \\
--\end{array}$ & $\begin{array}{l}7.92 \\
0.13 \\
1.65 \\
--\end{array}$ & $\begin{array}{l}10.92 \\
0.26 \\
2.39 \\
--\end{array}$ & $\begin{array}{l}6.07 \\
0.16 \\
2.64 \\
-.\end{array}$ & $\begin{array}{l}22.07 \\
0.22 \\
1.00 \\
--\end{array}$ & $\begin{array}{l}6.40 \\
0.06 \\
0.94 \\
\end{array}$ & $\begin{array}{l}5.49 \\
0.10 \\
1.83 \\
--\end{array}$ & $\begin{array}{r}9.12 \\
-0.100 \\
-1.10 \\
-\end{array}$ & $\begin{array}{l}7.57 \\
0.08 \\
b .66 \\
\end{array}$ & $\begin{array}{l}3.89 \\
0.04 \\
1.03 \\
\end{array}$ & $\begin{array}{l}9.32 \\
0.13 \\
1.40 \\
--\end{array}$ & $\begin{array}{l}9.52 \\
0.13 \\
1.37 \\
-\end{array}$ & $\begin{array}{r}110.13 \\
1.60\end{array}$ \\
\hline 1966 & $\begin{array}{l}\text { PRECIP. }\left(C M_{-}\right) \\
\text {SR-9C }(R C I / S Q-K M-) \\
S R-90 \text { CONC. }(P C / L) \\
S R-89 / S R-90\end{array}$ & $\begin{array}{l}9.68 \\
0.06 \\
0.62 \\
-.\end{array}$ & $\begin{array}{l}4.47 \\
0.06 \\
1.35 \\
-.\end{array}$ & $\begin{array}{l}8.87 \\
0.06 \\
0.68 \\
*\end{array}$ & $\begin{array}{l}10.25 \\
.0 .03 \\
0.30 \\
*\end{array}$ & $\begin{array}{l}20.08 \\
0.05 \\
c .25 \\
*\end{array}$ & $\begin{array}{r}3.39 \\
0.04 \\
1.18 \\
34.0 \mathrm{C}\end{array}$ & $\begin{array}{l}7.59 \\
0.06 \\
0.80 \\
3.60\end{array}$ & $\begin{array}{l}0.97 \\
0.05 \\
0.56 \\
*\end{array}$ & $\begin{array}{l}5.9 .0 \\
0.0 .6 \\
1.62 \\
*\end{array}$ & $\frac{.0 .07}{17.00}$ & $\begin{array}{r}28.16 \\
0.24 \\
0.66 \\
30.000\end{array}$ & $\begin{array}{l}3.55 \\
0.09 \\
.2 .54 \\
.0 .20\end{array}$ & $\begin{array}{r}110.91 \\
0.87\end{array}$ \\
\hline 1967 & $\begin{array}{l}\text { PRECIP. (CM-) } \\
\text { SR-90 INCI/SO-KM.) } \\
\text { SR-90 CONC. }(P C / L) \\
S R-89 / S R-90\end{array}$ & $\begin{array}{l}2.84 C \\
0.03 C \\
1.06 \\
5.60\end{array}$ & $\begin{array}{l}2.84 \mathrm{C} \\
0.03 \mathrm{C} \\
1.06 \\
5.60\end{array}$ & $\begin{array}{l}6.13 \\
0.02 \\
0.33 \\
*\end{array}$ & $\begin{array}{l}6.94 \\
0.04 \\
0.58 \\
+\end{array}$ & $\begin{array}{l}-- \\
0.02\end{array}$ & $\overline{-}$ & $\bar{E}$ & $\bar{z}$ & $=$ & $\underline{E}$ & $=$ & $\overline{-}$ & $\begin{array}{r}32.50 \\
0.21\end{array}$ \\
\hline $\begin{array}{c}\text { NOTES } \\
--: \text { D } \\
*: \\
A: \\
B: A \\
C: P\end{array}$ & $\begin{array}{l}\text { ATA NOT AVAILABLE } \\
\text { ERD OR TRACE } \\
\text { PPROXIMATE } \\
\text { OWER LIMIT OF REPOR } \\
\text { ROPORTIIONED FROM OR }\end{array}$ & $\begin{array}{l}\text { DATA } \\
\text { INALLY }\end{array}$ & 15 & & & & & & & & & & & \\
\hline
\end{tabular}


MONTHLY FALLOUT DEPOSITION COLLECTIONS

SITE: CHILE, ISLA ROB. CRUSOE

LAT. 33375 LONG. $7852 \mathrm{~W}$ ALT. 6M. (COLUMN)

SOURCE: OFICINA METEOROLOGICA DE CHILE

\begin{tabular}{|c|c|c|c|c|c|c|c|c|c|c|c|c|c|c|}
\hline & & JAN. & FEB. & MAR. & APR. & MAY & JUNE & JULY & AUG. & SEP. & OCT. & NOV. & OEC. & CUM. TOTAL \\
\hline 1964 & $\begin{array}{l}\text { PRECIP. (CM.) } \\
\text { SR-90 (MCI/SQ.KM.) } \\
\text { SR-90 CONC. }(P C / L) \\
S R-89 / S R-90\end{array}$ & $\begin{array}{l}4.39 \\
- \\
=\end{array}$ & $\begin{array}{l}1.35 \\
--\end{array}$ & $\begin{array}{l}8.15 \\
-- \\
--\end{array}$ & $\frac{8.36}{--}$ & $\begin{array}{l}11.96 \\
0.21 \\
1.76 \\
--\end{array}$ & $\begin{array}{l}9.60 \\
0.36 \\
3.76 \\
--\end{array}$ & $\begin{array}{r}12.60 \\
0.37 \\
2.94 \\
--\end{array}$ & $\begin{array}{r}10.52 \\
0.19 \\
1.81 \\
-.\end{array}$ & $\begin{array}{l}0.38 \\
0.03 \\
0.48 \\
--\end{array}$ & $\ddot{*}$ & $\begin{array}{l}3.20 \\
0.14 \\
4.38 \\
--\end{array}$ & $\begin{array}{l}5.61 \\
0.36 \\
6.42 \\
--\end{array}$ & $\begin{array}{r}82.12 \\
1.66\end{array}$ \\
\hline 1965 & $\begin{array}{l}\text { PRECIP. (CM.) } \\
\text { SR-90 (MCI/SQ.KM.) } \\
\text { SR-90 CONC. (PC/L) } \\
\text { SR-89/SR-90 }\end{array}$ & $\begin{array}{l}1.63 \\
0.13 \\
7.98 \\
-.\end{array}$ & $\begin{array}{l}1.40 \\
0.05 \\
3.56 \\
\end{array}$ & $\begin{array}{l}3.78 \\
0.17 \\
4.50 \\
--\end{array}$ & $\begin{array}{r}18.64 \\
0.15 \\
0.81 \\
--\end{array}$ & $\begin{array}{r}10.49 \\
0.45 \\
4.29 \\
-\end{array}$ & $\begin{array}{l}17.78 \\
0.03 \\
0.17 \\
--\end{array}$ & $\begin{array}{l}17.83 \\
\div- \\
--\end{array}$ & $\begin{array}{r}23.85 \\
0.69 \\
2.90 \\
-\end{array}$ & $\begin{array}{l}5.79 \\
0.10 \\
1.73\end{array}$ & $\begin{array}{l}3.76 \\
0.22 \\
5.36 \\
-=\end{array}$ & $\begin{array}{l}2.79 \\
0.08 \\
2.87 \\
--\end{array}$ & $\begin{array}{l}10.03 \\
=- \\
=\end{array}$ & $\begin{array}{r}117.77 \\
2.07\end{array}$ \\
\hline 1966 & $\begin{array}{l}\text { PRECIP. (CM.) } \\
\text { SR-90 (MCI/SQ-KM.) } \\
\text { SR-90 CONC. (PC/L) } \\
\text { SR-89/SR-90 }\end{array}$ & $\begin{array}{l}2.13 \\
0.06 \\
2.82 \\
-\end{array}$ & $\begin{array}{l}1.50 \\
= \\
=\end{array}$ & $\begin{array}{l}3.53 \\
0.10 \\
2.84 \\
\end{array}$ & $\begin{array}{l}-- \\
0.11 \\
-\end{array}$ & $=-$ & $=$ & $m+$ & $\begin{array}{r}11.07 \\
0.11 \\
1.00 \\
4.60\end{array}$ & $\begin{array}{l}8.81 \\
0.11 \\
1.25 \\
2.80\end{array}$ & $\begin{array}{r}2.34 \\
0.03 \\
1.29 \\
13.00\end{array}$ & $\begin{array}{r}5.62 \\
0.08 \\
1.43 \\
19.00\end{array}$ & $\begin{array}{r}8.39 \\
0.09 \\
1.08 \\
10.00\end{array}$ & $\begin{array}{r}43.39 \\
0.69\end{array}$ \\
\hline 1967 & $\begin{array}{l}\text { PRECIP. (CM.) } \\
\text { SR-90 IMCI/SQ-KM.) } \\
S R-90 \text { CONC. }(P C / L) \\
S R-89 / S R-90\end{array}$ & $\begin{array}{l}1.54 \\
0.04 \\
2.60 \\
*\end{array}$ & - & $\overline{-}$ & $m+$ & $=$ & $\overline{-}$ & $\begin{array}{l}-- \\
-- \\
--\end{array}$ & $=$ & $=$ & $=-$ & $=-$ & $=$ & $\begin{array}{l}1.54 \\
0.04\end{array}$ \\
\hline 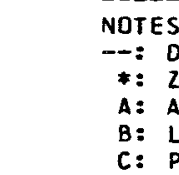 & $\begin{array}{l}\text { S } \\
\text { OATA NOT AVAILABLE } \\
\text { ZERO OR TRACE } \\
\text { APPROXIMATE } \\
\text { LOWER LIMIT OF REPOR } \\
\text { PROPORTI ONED FROM OR }\end{array}$ & $\begin{array}{l}\text { DATA } \\
\text { NALLY }\end{array}$ & & $=0$ & & & & & & & & & & \\
\hline
\end{tabular}


MONTHLY FALLOUT DEPOSITION COLLECTIONS

SITE: CHILE, PORTILLO

LAT. $3225 \$$ LONG.70 $08 \mathrm{~W}$ ALT. 2850M. (COLUMN) SURFACE AIR SAMPLING STATION SOURCE: OFICINA METEOROLOGICA DE CHILE

JAN. FEB. MAR. APR. MAY JUNE JULY AUG. SEP. OCT. NOV. DEC. CUM. TOTAL

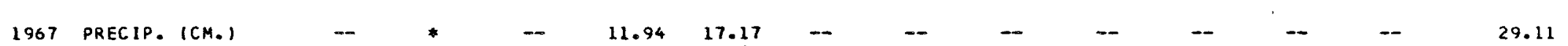

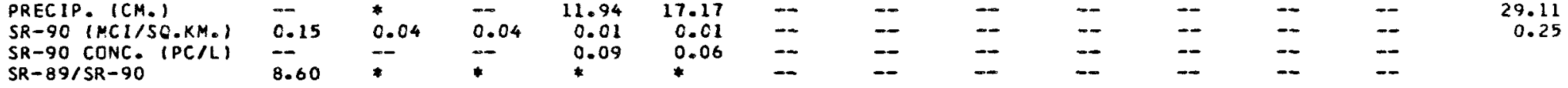
NOTES

-: DATA NOT AVAILABLE

*: ZERO OR TRACE

A: APPROXIMATE

B: LOHER LIMIT OF REPORTED DATA

$C$ : PROPORTI ONED FROM ORIGINALLY CONSOLIDATED DATA 
MONTHLY FALLOUT OEPOSITION COLLECTIONS

SITE: CHILE, PUERTO MONTT

SOURCE: OFICINA METEOROLOGICA DE CHILE
LAT. 41265 LONG. 73 OTW ALT. HOM. (COLUMN) SURFACE AIR SAMPLING STATION

\begin{tabular}{|c|c|c|c|c|c|c|c|c|c|c|c|c|c|c|}
\hline & & JAN. & FEB. & MAR: & APR. & MAY & JUNE & JULY & AUG. & SEP. & OCT. & NOV. & DEC. & CUM. TOTAL \\
\hline I $s \in 3$ & 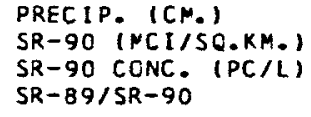 & $\begin{array}{l}9.63 \\
-- \\
--\end{array}$ & $\begin{array}{l}4.88 \\
-- \\
--\end{array}$ & $\begin{array}{l}16.89 \\
-- \\
--\end{array}$ & $\begin{array}{r}26.42 \\
0.37 \\
1.41 \\
3.00\end{array}$ & $\begin{array}{r}18.72 \\
0.30 \\
1.61 \\
*\end{array}$ & $\begin{array}{l}6.10 \\
0.37 \\
6.07 \\
*\end{array}$ & $\begin{array}{r}25.93 \\
0.22 \\
0.85 \\
*\end{array}$ & $\begin{array}{r}18.06 \\
0.23 \\
1.28 \\
*\end{array}$ & $\begin{array}{r}11.94 \\
0.01 \\
0.09 \\
*\end{array}$ & $\begin{array}{l}6.71 \\
0.05 \\
0.75 \\
*\end{array}$ & $\begin{array}{l}17.42 \\
-- \\
=-\end{array}$ & $\begin{array}{l}8.33 \\
0.18 \\
2.17 \\
*\end{array}$ & $\begin{array}{r}171.03 \\
1.73\end{array}$ \\
\hline 1964 & $\begin{array}{l}\text { PRECIP. (CM.) } \\
\text { SR-9O INCI/SQ.KM.) } \\
\text { SR-9C CONC. }(P C / L) \\
\text { SR-89/SR-SO }\end{array}$ & $\begin{array}{l}8.18 \\
0.02 \\
0.25 \\
6.10\end{array}$ & $\begin{array}{r}11.13 \\
0.14 \\
1.26 \\
\end{array}$ & $\begin{array}{l}19.05 \\
0.16 \\
0.84 \\
*\end{array}$ & $\begin{array}{r}12.47 \\
0.10 \\
0.81 \\
1.80\end{array}$ & $\begin{array}{r}37.06 \\
0.36 \\
0.98 \\
--\end{array}$ & $\begin{array}{l}25.17 \\
0.27 \\
1.08 \\
--\end{array}$ & $\begin{array}{l}22.30 \\
C .32 \\
1.44 \\
--\end{array}$ & $\begin{array}{l}26.77 \\
0.24 \\
0.90 \\
-.\end{array}$ & $\begin{array}{l}-- \\
=- \\
=-\end{array}$ & $\begin{array}{l}7.72 \\
0.16 \\
2.08 \\
-\end{array}$ & $\begin{array}{r}16.92 \\
0.33 \\
1.96 \\
*\end{array}$ & $\begin{array}{r}14.38 \\
0.52 \\
3.62 \\
--\end{array}$ & $\begin{array}{r}201.15 \\
2.62\end{array}$ \\
\hline 1965 & $\begin{array}{l}\text { PRECIP. (CM.) } \\
\text { SR-9O (MCI/SQ.KM.) } \\
\text { SR-90 CCNC. }(P C / L) \\
\text { SR-89/SR-90 }\end{array}$ & $\begin{array}{r}10.19 \\
0.29 \\
2.85 \\
--\end{array}$ & $\begin{array}{r}19.79 \\
0.32 \\
1.62 \\
--\end{array}$ & $\begin{array}{l}7.29 \\
0.09 \\
1.24 \\
--\end{array}$ & $\begin{array}{r}22.89 \\
0.25 \\
1.10 \\
-.\end{array}$ & $\begin{array}{l}16.92 \\
0.27 \\
1.60 \\
--\end{array}$ & $\begin{array}{r}34.26 \\
0.19 \\
0.56 \\
-.\end{array}$ & $\begin{array}{r}22.91 \\
0.26 \\
1.14 \\
-.\end{array}$ & $\begin{array}{r}27.76 \\
0.36 \\
1.30 \\
--\end{array}$ & $\begin{array}{l}9.47 \\
0.11 \\
1.17 \\
--\end{array}$ & $\begin{array}{l}15.46 \\
0.22 \\
1.43 \\
--\end{array}$ & $\begin{array}{l}10.59 \\
* \\
-- \\
--\end{array}$ & $\begin{array}{l}-- \\
--\end{array}$ & $\begin{array}{r}197.53 \\
2.36\end{array}$ \\
\hline 1966 & $\begin{array}{l}\text { PRECIP. }(C M-) \\
\text { SR-9O (NCI/SQ.KM-) } \\
\text { SR-9C CONC. (PC/L) } \\
\text { SR-89/SR-9O }\end{array}$ & $\begin{array}{l}0.07 \\
0.06 \\
0.99 \\
-.\end{array}$ & $\begin{array}{l}6.15 \\
0.11 \\
1.79 \\
--\end{array}$ & $\begin{array}{r}16.56 \\
0.14 \\
0.85 \\
--\end{array}$ & $\begin{array}{r}21.28 \\
0.09 \\
0.43 \\
--\end{array}$ & $\begin{array}{r}32.56 \\
0.20 \\
0.62 \\
--\end{array}$ & $\begin{array}{r}35.48 \\
0.10 \\
0.29 \\
*\end{array}$ & $\begin{array}{l}24.63 \\
0.24 \\
C .98 \\
*\end{array}$ & $\begin{array}{r}13.82 \\
0.11 \\
0.80 \\
4.00\end{array}$ & $\begin{array}{r}16.36 \\
0.22 \\
1.35 \\
20.00\end{array}$ & $\begin{array}{r}11.97 \\
0.14 \\
1.17 \\
78.00\end{array}$ & $\begin{array}{r}8.80 \\
0.11 \\
1.26 \\
24.00\end{array}$ & $\begin{array}{r}20.43 \\
0.24 \\
1.18 \\
11.00\end{array}$ & $\begin{array}{r}214.11 \\
1.76\end{array}$ \\
\hline 1967 & $\begin{array}{l}\text { PRECIP. (CM.) } \\
\text { SR-90 (NCI/SQ.KM.) } \\
\text { SR-90 CONC. (PC/L) } \\
\text { SR-89/SR-90 }\end{array}$ & $\begin{array}{l}-- \\
--\end{array}$ & $\begin{array}{r}14.32 \\
0.08 \\
0.56 \\
*\end{array}$ & $\begin{array}{l}12.51 \\
0.08 \\
0.64 \\
*\end{array}$ & $\begin{array}{l}10.22 \\
0.05 \\
0.49 \\
\$\end{array}$ & $\begin{array}{l}34.59 \\
6.13 \\
0.38 \\
*\end{array}$ & $=$ & $\begin{array}{l}=- \\
=- \\
=-\end{array}$ & $\begin{array}{l}= \\
= \\
=\end{array}$ & $\begin{array}{l}=- \\
=-\end{array}$ & $\begin{array}{l}=- \\
= \\
=\end{array}$ & $\begin{array}{l}-- \\
-- \\
--\end{array}$ & 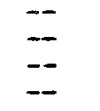 & $\begin{array}{r}71.64 \\
0.34\end{array}$ \\
\hline $\begin{array}{l}\text { NOTES } \\
-=: \\
*: \\
A= \\
B=1 \\
C:\end{array}$ & $\begin{array}{l}\text { ATA NDI AVAILABLE } \\
\text { ERO OR TRACE } \\
\text { OPROXIMATE } \\
\text { OWER LIMIT OF REPOR } \\
\text { ROPORTIONED FROM OR }\end{array}$ & $\begin{array}{l}\text { DATA } \\
\text { NALLY }\end{array}$ & & & & & & & & & & & & \\
\hline
\end{tabular}




\section{SITE: CHILE, PUNTA ARENAS}

SOURCE: OFICINA METEOROLOGICA DE CHILE
MONTHLY FALLOUT DEPOSITION COLLECTIONS
LAT. 53 OOS
LONG. 70 5OW
ALT.
74M. (COLUMN) SURFACE AIR SAMPLING STATION

\begin{tabular}{|c|c|c|c|c|c|c|c|c|c|c|c|c|c|c|}
\hline & & JAN. & FEB. & MAR. & APR. & MAY & JUNE & JULY & AUG. & SEP. & OCT. & NOV. & DEC. & CUM. TOTAL \\
\hline 1963 & $\begin{array}{l}\text { PRECIP. (CM.) } \\
S R-90 \text { (NCI/SQ.KM-) } \\
S R-90 \text { CONC. }(P C / L) \\
S R-89 / S R-90\end{array}$ & $\begin{array}{l}1.45 \\
-- \\
--\end{array}$ & $\begin{array}{l}2.16 \\
0.07 \mathrm{~B} \\
3.25 \\
-.\end{array}$ & $\begin{array}{r}0.46 \\
0.05 \\
10.87 \\
3.40\end{array}$ & $\begin{array}{l}8.10 \\
0.09 \\
1.12 \\
2.00\end{array}$ & $\begin{array}{l}3.40 \\
0.08 \\
2.36 \\
*\end{array}$ & $\begin{array}{l}5.92 \\
0.08 \\
1.36 \\
+\end{array}$ & $\begin{array}{l}4.78 \\
= \\
-\end{array}$ & $\begin{array}{l}6.994 \\
0.064 \\
0.86 \\
--\end{array}$ & $\begin{array}{l}1.12 \\
0.05 \\
4.47\end{array}$ & $\begin{array}{l}1.14 \\
=- \\
=-\end{array}$ & $\begin{array}{l}1.27 \\
0.04 \\
3.15 \\
-\infty\end{array}$ & $\begin{array}{l}3.66 \\
0.15 \\
4.10 \\
*\end{array}$ & $\begin{array}{r}40.45 \\
0.67\end{array}$ \\
\hline 1964 & $\begin{array}{l}\text { PRECIP. }(C M .) \\
\text { SR-90 INCI/SQ.KM.) } \\
S R-90 \text { CCNC. }(P C / L) \\
\text { SR-89/SR-90 }\end{array}$ & $\begin{array}{l}4.19 \\
0.05 \\
1.20\end{array}$ & $\begin{array}{l}1.98 \\
0.09 \\
4.55\end{array}$ & $\begin{array}{l}3.00 \\
0.13 \\
4.34 \\
*\end{array}$ & $\begin{array}{l}1.98 \\
0.03 \\
1.52 \\
--\end{array}$ & $\begin{array}{l}3.63 \\
0.10 \\
2.76 \\
--\end{array}$ & $\begin{array}{l}2.69 \\
0.02 \\
0.75 \\
--\end{array}$ & 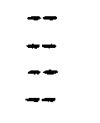 & $\begin{array}{l}0.66 \\
* \\
-\end{array}$ & $\begin{array}{l}2.44 \\
0.12 \\
4.92 \\
-\end{array}$ & $\begin{array}{l}0.58 \\
0.01 \\
1.73 \\
*\end{array}$ & $\begin{array}{l}- \\
-- \\
--\end{array}$ & $\begin{array}{l}4.37 \\
0.25 \\
5.73 \\
--\end{array}$ & $\begin{array}{r}25.52 \\
0.80\end{array}$ \\
\hline 1965 & $\begin{array}{l}\text { PRECIP. (CM.) } \\
\text { SR-9O (MCI/SQ.KM.) } \\
S R-9 C \text { CCNC. }(P C / L) \\
S R-9 S / S R-9 O\end{array}$ & $\begin{array}{l}2.13 \\
0.09 \\
4.23 \\
--\end{array}$ & $\begin{array}{l}4.11 \\
0.25 \\
6.09 \\
-.\end{array}$ & $\begin{array}{l}3.48 \\
0.12 \\
3.45 \\
--\end{array}$ & $\begin{array}{l}3.76 \\
0.08 \\
2.13 \\
--\end{array}$ & $\begin{array}{l}1.47 \\
0.07 \\
4.77 \\
--\end{array}$ & $\begin{array}{l}7.85 \\
-- \\
--\end{array}$ & $\begin{array}{l}1.04 \\
0.07 \\
6.74 \\
--\end{array}$ & $\begin{array}{l}3.71 \\
0.10 \\
2.70 \\
--\end{array}$ & $\begin{array}{l}2.03 \\
0.03 \\
1.48 \\
--\end{array}$ & $\begin{array}{l}1.55 \\
0.02 \\
1.30\end{array}$ & $\begin{array}{l}1.02 \\
0.08 \\
7.85 \\
--\end{array}$ & $\begin{array}{r}0.28 \\
0.05 \\
17.86 \\
--\end{array}$ & $\begin{array}{r}32.43 \\
0.96\end{array}$ \\
\hline lste & $\begin{array}{l}\text { PRECIP }\{(C M \cdot) \\
\text { SR-90 (NCI/SQ.KM.) } \\
S R-90 \text { CCNC. }(P C / L) \\
S R-89 / S R-90\end{array}$ & $\begin{array}{l}0.30 \\
= \\
=-\end{array}$ & $\begin{array}{l}0.86 \\
0.05 \\
5.82 \\
--\end{array}$ & $\begin{array}{l}4.29 \\
0.12 \\
2.80 \\
--\end{array}$ & $\begin{array}{l}1.09 \\
0.05 \\
4.59 \\
--\end{array}$ & $\begin{array}{l}2.06 \\
0.03 \\
1.46 \\
--\end{array}$ & $\begin{array}{l}1.82 \\
0.02 \\
1.10 \\
*\end{array}$ & $\begin{array}{l}16.60 \\
* \\
*\end{array}$ & $\begin{array}{l}1.44 \\
0.02 \\
1.39 \\
*\end{array}$ & $\begin{array}{l}2.48 \\
0.05 \\
2.02 \\
*\end{array}$ & $\begin{array}{r}1.82 \\
0.03 \\
1.65 \\
13.00\end{array}$ & $\begin{array}{r}1.16 \\
0.04 \\
3.45 \\
15.00\end{array}$ & $\begin{array}{l}2.99 \\
0.05 \\
1.68 \\
7.00\end{array}$ & $\begin{array}{r}36.91 \\
0.46\end{array}$ \\
\hline 1567 & $\begin{array}{l}\text { PRECIP. }(C M .) \\
S R-90(M C I / S Q . K M .) \\
S R-90 \text { CONC. }(P C / L) \\
S R-89 / S R-90\end{array}$ & $\begin{array}{l}3.73 \\
0.04 \\
1.08\end{array}$ & $\begin{array}{r}14.32 \\
0.03 \\
0.21 \\
*\end{array}$ & $\begin{array}{l}3.12 \\
0.04 \\
1.29 \\
*\end{array}$ & $\begin{array}{l}5.69 \\
0.02 \\
0.36 \\
*\end{array}$ & $\begin{array}{l}5.74 \\
0.06 \\
1.05\end{array}$ & $\begin{array}{l}-- \\
-- \\
--\end{array}$ & $\begin{array}{l}-- \\
--\end{array}$ & $\begin{array}{l}-- \\
--\end{array}$ & $\begin{array}{l}-- \\
-- \\
--\end{array}$ & $\begin{array}{l}=- \\
=- \\
=-\end{array}$ & $\begin{array}{l}-- \\
-- \\
--\end{array}$ & $m$ & $\begin{array}{r}32.60 \\
0.19\end{array}$ \\
\hline \begin{tabular}{c} 
NOTES \\
\hdashline$:$ \\
$\vdots:$ \\
$A:$ \\
$B:$ \\
$C:$
\end{tabular} & $\begin{array}{l}\text { ATA NOT AVAILABLE } \\
\text { ERO OR IRACE } \\
\text { PPROXIMATE } \\
\text { OWER LIMIT OF REPORT T T } \\
\text { ROPORTIONED FRCM ORI }\end{array}$ & $\begin{array}{l}\text { DATA } \\
\text { INALLLY }\end{array}$ & & & & & & & & & & & & \\
\hline
\end{tabular}


STE: CHILE, SANTIAGO LAT. 33295 LONG. 10 4OW ALT. 522M. IPOTI SOURCE: FACULTY OF CHEMISTRY, UNIVERSITY OF CHILE

\begin{tabular}{|c|c|c|c|c|c|c|c|c|c|c|c|c|c|c|}
\hline & & JAN. & FEB. & MAR. & APR. & MAY & JUNE & JULY & AUG. & SEP. & OCT. & NOV. & DEC. & CUM. TOTAL \\
\hline 1958 & $\begin{array}{l}\text { PRECIP. }\left(C M_{-}\right) \\
\text {SR-90 }\left(M C I / S Q . K M_{-}\right) \\
\text {SR-90 CONC. }(P C / L) \\
\text { SR-89/SR-90 }\end{array}$ & $\begin{array}{l}-- \\
-- \\
--\end{array}$ & $\begin{array}{l}-- \\
--\end{array}$ & $=$ & $\begin{array}{l}-- \\
-- \\
-\infty\end{array}$ & $m+$ & $\begin{array}{l}11.00 \\
=- \\
=-\end{array}$ & $\begin{array}{r}2.01 \\
0.05 \\
2.49 \\
53.00\end{array}$ & $\begin{array}{r}8.99 \\
0.22 \\
2.45 \\
62.00\end{array}$ & $\begin{array}{r}0.99 \\
0.03 \\
3.04 \\
34.00\end{array}$ & $\begin{array}{l}* \\
0.10 \\
33.00\end{array}$ & $\begin{array}{r}0.99 \\
0.07 \\
7.08 \\
20.00\end{array}$ & $\begin{array}{l}* \\
0.02 \\
-- \\
45.00\end{array}$ & $\begin{array}{r}23.98 \\
0.49\end{array}$ \\
\hline 1959 & $\begin{array}{l}\text { PRECIP. (CM.) } \\
\text { SR-9C (MCI/SQ.KM.) } \\
\text { SR-SC CCNC. (PC/L) } \\
\text { SR- \& IISR-SO }\end{array}$ & $\begin{array}{c}* \\
0.01 \\
-- \\
20.00\end{array}$ & $\begin{array}{l}* \\
0.01 \\
--\end{array}$ & $\begin{array}{l}2.01 \\
0.04 \\
2.00 \\
*\end{array}$ & $\begin{array}{l}5.99 \\
0.08 \\
1.34 \\
2.70\end{array}$ & $\begin{array}{l}5.99 \\
0.06 \\
1.01 \\
*\end{array}$ & $\begin{array}{l}8.00 \\
0.11 \\
1.38\end{array}$ & $\begin{array}{l}5.00 \\
0.07 \\
1.41 \\
+\end{array}$ & $\begin{array}{l}4.01 \\
0.09 \\
2.25 \\
1.60\end{array}$ & $\begin{array}{l}0.99 \\
C . C 2 \\
2 . c 3 \\
*\end{array}$ & $\begin{array}{l}0.99 \\
0.02 \\
2.03\end{array}$ & $\begin{array}{l}* \\
0.01 \\
*\end{array}$ & $\begin{array}{l}* \\
0.01 \\
*\end{array}$ & $\begin{array}{r}32.98 \\
0.53\end{array}$ \\
\hline 1960 & $\begin{array}{l}\text { PRECIP. }(C M .) \\
\text { SR-90 INCI/SO.KM.) } \\
S R-90 \text { CONC. }(P C / L) \\
S R-89 / S R-90\end{array}$ & $\begin{array}{l}* \\
0.00 \\
--\end{array}$ & $\begin{array}{l}* \\
0.00 \\
--\end{array}$ & $\begin{array}{l}5.99 \\
0.01 \\
0.17 \\
--\end{array}$ & $\begin{array}{l}* \\
0.01 \\
-- \\
--\end{array}$ & $\begin{array}{l}2.01 \\
0.02 \mathrm{C} \\
1.00 \\
-.\end{array}$ & $\begin{array}{l}0.89 \\
0.016 \\
1.13 \\
=-\end{array}$ & $\begin{array}{l}5.00 \\
0.03 C \\
0.61 \\
--\end{array}$ & $\begin{array}{l}2.01 \\
0.016 \\
0.50 \\
--\end{array}$ & $\begin{array}{l}0.99 \\
0.04 C \\
4.05 \\
--\end{array}$ & $\begin{array}{l}* \\
0.00 C \\
--\end{array}$ & $\begin{array}{l}* \\
0.00 C \\
=-\end{array}$ & $\begin{array}{l}* \\
0.00 C \\
--\end{array}$ & $\begin{array}{r}16.89 \\
0.13\end{array}$ \\
\hline 1961 & $\begin{array}{l}\text { PRECIP. (CM.) } \\
\text { SR-9O (NCI/SQ.KM.) } \\
S R-9 C \text { CCNC. }(P C / L) \\
S R-89 / S R-90\end{array}$ & $\begin{array}{l}* \\
0.01 C \\
=- \\
--\end{array}$ & $\begin{array}{l}* \\
0.010 \\
--\end{array}$ & $\begin{array}{l}0.99 \\
0.036 \\
3.04 \\
-.\end{array}$ & $\begin{array}{l}* 0.00 C \\
-- \\
=-\end{array}$ & $\begin{array}{l}2.01 \\
0.03 C \\
1.50 \\
-.\end{array}$ & $\begin{array}{l}8.00 \\
0.14 C \\
1.7 t \\
-.\end{array}$ & $\begin{array}{l}2.01 \\
0.02 \mathrm{C} \\
1.00 \\
-.\end{array}$ & $\begin{array}{l}7.01 \\
0.08 \mathrm{C} \\
1.15 \\
-.\end{array}$ & $\begin{array}{l}3.00 \\
0.02 \\
0.67 \\
+\end{array}$ & $\begin{array}{l}3.00 \\
0.09 \\
3.01 \\
+\end{array}$ & $\begin{array}{l}* \\
* \\
--\end{array}$ & $\begin{array}{l}0.71 \\
0.01 \\
1.41\end{array}$ & $\begin{array}{r}26.73 \\
0.44\end{array}$ \\
\hline 1962 & $\begin{array}{l}P R E C 1 P \text {. (CM.) } \\
S R-9 C \text { INCI/SQ.KM.) } \\
S R-90 C C N C . \quad(P C / L) \\
S R-89 / S R-90\end{array}$ & $\begin{array}{l}* \\
0.02 \\
*\end{array}$ & $\begin{array}{l}* \\
* \\
*\end{array}$ & $\begin{array}{l}* \\
0.01 \\
*\end{array}$ & $\begin{array}{l}0.05 \\
0.00 \\
0.01 \\
*\end{array}$ & $\begin{array}{l}* \\
* \\
--\end{array}$ & $\begin{array}{r}14.99 \\
0.08 \\
0.54 \\
29.06\end{array}$ & $\begin{array}{r}1.42 \\
C .04 \\
2.82 \\
4 t .0 C\end{array}$ & $\begin{array}{r}3.20 \\
0.13 \\
4.07 \\
28.00\end{array}$ & $\begin{array}{l}0.61 \\
0.02 \\
3.28 \\
8.00\end{array}$ & $\begin{array}{l}1.12 \\
0.00 \\
0.01 \\
*\end{array}$ & $=$ & $\begin{array}{c}* \\
0.05 \\
31.00\end{array}$ & $\begin{array}{r}21.39 \\
0.35\end{array}$ \\
\hline 1963 & $\begin{array}{l}\text { PRECIP ( ICM.) } \\
\text { SR-90 (MCI/SQ.KM.) } \\
\text { SR-9O CCNC. (PC/L) } \\
\text { SR-89/SR-90 }\end{array}$ & $\begin{array}{l}0.03 \\
0.00 \\
0.01 \\
7.00\end{array}$ & $\frac{*}{0.02}$ & $\begin{array}{r}0.02 \\
1 \overline{11.00}\end{array}$ & $\begin{array}{r}0.05 \\
0.05 \\
100.00 \\
10.00\end{array}$ & $\begin{array}{r}0.43 \\
0.06 \\
13.96 \\
3.70\end{array}$ & $\begin{array}{l}-.07 \\
0.07 \\
1.70\end{array}$ & $\begin{array}{l}-- \\
2.00\end{array}$ & $\begin{array}{l}-- \\
0.07 \\
--\end{array}$ & $\begin{array}{l}-0 \\
0.08 \\
1.60\end{array}$ & $\begin{array}{l}-- \\
0.04 \\
*\end{array}$ & $\begin{array}{l}-- \\
0.04 \\
--\end{array}$ & $\begin{array}{l}-- \\
0.04 \\
--\end{array}$ & $\begin{array}{l}0.51 \\
0.49\end{array}$ \\
\hline 1964 & $\begin{array}{l}\text { PRECIP. }(C M-) \\
\text { SR-90 (MCI/SQ.KM.) } \\
S R-90 \text { CONC. }(P C / L) \\
S R-89 / S R-90\end{array}$ & $\begin{array}{l}* \\
0.01 \\
--\end{array}$ & 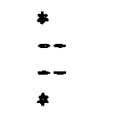 & $\begin{array}{l}* \\
0.03 \\
*\end{array}$ & $\begin{array}{l}0.02 \\
0 . \\
=-\end{array}$ & $\begin{array}{l}2.18 \\
0.03 \\
1.38 \\
-\end{array}$ & $\begin{array}{l}4.11 \\
0.05 \\
1.22 \\
-.\end{array}$ & $\begin{array}{l}4.01 \\
C .21 \\
5.24 \\
-.\end{array}$ & $\begin{array}{l}5.99 \\
0.15 \\
2.51 \\
*\end{array}$ & $\begin{array}{l}* \\
0.03 \\
*\end{array}$ & $\begin{array}{l}* \\
* \\
*-\end{array}$ & $\begin{array}{l}* \\
* \\
-- \\
--\end{array}$ & $\begin{array}{l}* \\
0.05 \\
-- \\
--\end{array}$ & $\begin{array}{r}16.29 \\
0.58\end{array}$ \\
\hline 1565 & $\begin{array}{l}\text { PRECIP. (CM.) } \\
5 R-90 \text { (MCI/SQ.KM.) } \\
\text { SR-90 CQNC. }(P C / L) \\
\text { SR-89/SR-90 }\end{array}$ & $\begin{array}{l}* \\
0.00 \\
--\end{array}$ & $\begin{array}{l}* \\
* \\
--\end{array}$ & $\begin{array}{l}* \\
1.00 \\
--\end{array}$ & $\begin{array}{l}4.01 \\
0.13 \\
3.25 \\
-.\end{array}$ & $\begin{array}{l}3.00 \\
0.11 \\
3.67 \\
--\end{array}$ & $\begin{array}{l}0.99 \\
0.00 \\
0.01 \\
--\end{array}$ & $\begin{array}{l}13.00 \\
0.39 \\
3.01 \\
--\end{array}$ & $\begin{array}{r}16.00 \\
0.05 \\
0.32 \\
--\end{array}$ & $\begin{array}{l}* \\
0.21 \\
--\end{array}$ & $\begin{array}{l}--18 \\
--\end{array}$ & $\begin{array}{l}-- \\
0.02 \\
--\end{array}$ & $\begin{array}{l}-- \\
0.05 \\
--\end{array}$ & $\begin{array}{r}37.00 \\
2.14\end{array}$ \\
\hline 1966 & $\begin{array}{l}\text { PRECIP. (CM-) } \\
\text { SR-90 (MCI/SQ.KM.) } \\
S R-90 \text { CCNC. }(P C / L) \\
\text { SR-89/SR-90 }\end{array}$ & $\begin{array}{l}-- \\
-- \\
--\end{array}$ & 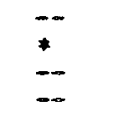 & $\begin{array}{l}-- \\
-\infty\end{array}$ & $\begin{array}{l}-\overline{0.02} \\
=-\end{array}$ & +- & $\begin{array}{l}-- \\
0.02 \\
--\end{array}$ & $\begin{array}{l}-- \\
0.04 \\
--\end{array}$ & $\begin{array}{l}-- \\
0.01 \\
--\end{array}$ & $\begin{array}{l}- \\
C .01 \\
--\end{array}$ & $\begin{array}{l}\overline{0.04} \\
52.00\end{array}$ & $\begin{array}{c}-- \\
0.02 \\
20.00\end{array}$ & $\begin{array}{l}-\overline{0} \\
\overline{0} 01\end{array}$ & $\begin{array}{l}0.0 \\
0.17\end{array}$ \\
\hline 1967 & $\begin{array}{l}\text { PRECIP. }\left(C M_{-}\right) \\
\text {SR-90 (MCI/SQ.KM, } \\
S R-90 \text { CCNC. }(P C / L) \\
\text { SR-89/SR-90 }\end{array}$ & $\begin{array}{l}* \\
0.01 \\
*\end{array}$ & $\begin{array}{l}* \\
0.01 \\
*\end{array}$ & $\begin{array}{l}* \\
0.01 \\
*\end{array}$ & $\overline{0.03}$ & $\begin{array}{l}1.30 \\
0.03 \\
2.31 \\
*\end{array}$ & $\begin{array}{l}3.60 \\
0.03 \\
0.84 \\
*\end{array}$ & $\begin{array}{l}-- \\
-- \\
--\end{array}$ & $m-$ & $=-$ & $m+$ & $\begin{array}{l}-- \\
-- \\
--\end{array}$ & $\begin{array}{l}-- \\
-- \\
--\end{array}$ & $\begin{array}{l}4.90 \\
0.12\end{array}$ \\
\hline \multicolumn{15}{|c|}{$\begin{array}{l}\text { NOTES } \\
\text { : : DATA NOT AVAILABLE } \\
\text { *: ZERO DR TRACE } \\
\text { A: APPROXIMATE } \\
\text { B: LOWER LIMIT OF REPORTED DATA } \\
\text { C: PROPORTIONED FRCM ORIGINALLY CONSOLICATED DATA }\end{array}$} \\
\hline
\end{tabular}




\section{MONTHLY FALLOUT DEPOSITION COLLECTIONS}

SITE: CHILE, SANTIAGO

LAT. 33275 LONG. $7042 \mathrm{~W}$ ALT.

52CM. (COLUMN) SURFACE ATR SAMPLING STATION

SOURCE : OFICINA METEOROLOGICA DE CHILE

\begin{tabular}{|c|c|c|c|c|c|c|c|c|c|c|c|c|c|c|}
\hline & & JAN. & FEB. & MAR - & APR. & MAY & JUNE & JULY & AUG. & SEP. & OCT. & NOV. & DEC. & CUM. TOTAL \\
\hline 1963 & $\begin{array}{l}\text { PRECIP. (CM.) } \\
S R-9 C \text { I } C I / S Q . K M .) \\
S R-9 O C O N C . \text { (PC/L) } \\
S R-89 / S R-90\end{array}$ & $\begin{array}{l}0.20 \\
-- \\
--\end{array}$ & $\stackrel{--}{--}$ & $\begin{array}{l}0.43 \\
0.03 \\
6.98 \\
4.00\end{array}$ & $\begin{array}{r}0.03 \\
0.01 \\
33.34 \\
6.00\end{array}$ & $\begin{array}{l}2.72 \\
C .65 \\
1.84 \\
3.20\end{array}$ & $\begin{array}{l}3.40 \\
0.10 \\
2.95 \\
*\end{array}$ & $\begin{array}{l}9.58 \\
0.17 \\
1.78 \\
+\end{array}$ & $\begin{array}{l}10.39 \\
0.24 C \\
2.31 \\
=-\end{array}$ & $\begin{array}{l}10.80 \\
0.24 C \\
2.23 \\
-\end{array}$ & $\begin{array}{l}2.87 \\
0.03 \\
1.05 \\
*\end{array}$ & $\begin{array}{l}0.58 \\
0.03 \\
5.18 \\
+\end{array}$ & $\begin{array}{l}0.12 \\
--\end{array}$ & $\begin{array}{r}41.00 \\
1.02\end{array}$ \\
\hline 1964 & $\begin{array}{l}\text { PRECIP. (CM.) } \\
\text { SR-90 IMCI/SQ.KM.) } \\
S R-90 \text { CONC. (PC/L) } \\
S R-89 / S R-90\end{array}$ & $\begin{array}{l}* \\
0.05 \\
-\end{array}$ & $\begin{array}{l}* .03 \\
-*\end{array}$ & $\begin{array}{l}* \\
0.04 \\
*\end{array}$ & $\begin{array}{r}0.05 \\
0.03 \\
60.01 \\
\end{array}$ & $\begin{array}{l}0.20 \\
-- \\
--\end{array}$ & $\begin{array}{l}8.48 \\
0.44 \\
5.19 \\
--\end{array}$ & $\begin{array}{r}12.07 \\
0.13 \\
1.08 \\
--\end{array}$ & $\begin{array}{l}6.12 \\
0.42 \\
6.87 \\
-\end{array}$ & $\begin{array}{l}.0 .03 \\
-- \\
--\end{array}$ & $\begin{array}{l}0.02 \\
-- \\
--\end{array}$ & $\begin{array}{l}-- \\
0.06 \\
--\end{array}$ & $\begin{array}{l}0.20 \\
0.02 \\
10.01 \\
-.\end{array}$ & $\begin{array}{r}27.12 \\
1.27\end{array}$ \\
\hline 1965 & $\begin{array}{l}\text { PRECIP. (CM.) } \\
\text { SR-9O I CCI/SQ.KM.) } \\
\text { SR-SC CCNC. (PC/L) } \\
\text { SR-89/SR-90 }\end{array}$ & $\begin{array}{l}0.20 \\
0.02 \\
10.01 \\
--\end{array}$ & $\begin{array}{l}* \\
0.03 \\
--\end{array}$ & $\begin{array}{l}* \\
0.02 \\
--\end{array}$ & $\begin{array}{l}3.63 \\
0.21 \\
5.79 \\
--\end{array}$ & $\begin{array}{l}3.33 \\
C .1 C \\
3.01 \\
=-\end{array}$ & $\begin{array}{r}1.47 \\
0.15 \\
10.21 \\
-.\end{array}$ & $\begin{array}{r}13.08 \\
C .48 \\
3.67 \\
-\end{array}$ & $\begin{array}{r}15.95 \\
0.17 \\
1.07 \\
-.\end{array}$ & $\begin{array}{l}0.36 \\
- \\
--\end{array}$ & $\begin{array}{l}1.80 \\
0.06 \\
3.34 \\
-.\end{array}$ & $\begin{array}{r}0.36 \\
0.28 \\
77.78 \\
--\end{array}$ & $\begin{array}{r}1.37 \\
0.45 \\
32.85 \\
--\end{array}$ & $\begin{array}{r}41.55 \\
1.97\end{array}$ \\
\hline 1966 & $\begin{array}{l}\text { PRECIP. ICM. } \\
\text { SR-90 } \text { IMCI/SO.KM.) } \\
S R-90 \text { CONC. (PC/L) } \\
S R-89 / S R-9 O\end{array}$ & $\begin{array}{l}0.16 \\
-- \\
--\end{array}$ & $\begin{array}{l}* \\
0.21 \\
--\end{array}$ & $\begin{array}{l}* \\
* \\
=-\end{array}$ & $\begin{array}{l}4.00 \\
-- \\
--\end{array}$ & $\begin{array}{l}1.00 \\
0.02 \\
2.01 \\
--\end{array}$ & $\begin{array}{r}10.00 \\
0.08 \\
0.81 \\
*\end{array}$ & $\begin{array}{r}10.36 \\
0.03 \\
0.29 \\
68.00\end{array}$ & $\begin{array}{l}4.51 \\
0.06 \\
1.34 \\
*\end{array}$ & $\begin{array}{l}-- \\
-- \\
--\end{array}$ & $\begin{array}{r}0.61 \\
0.09 \\
14.76 \\
12.00\end{array}$ & $\begin{array}{r}2.00 \\
0.01 \\
0.51 \\
11.00\end{array}$ & $\begin{array}{l}1.38 \\
0.01 \\
0.73 \\
+\end{array}$ & $\begin{array}{r}33.96 \\
0.67\end{array}$ \\
\hline 1967 & $\begin{array}{l}\text { PRECIP. (CM.) } \\
\text { SR-90 (NCI/SQ.KM.) } \\
S R-90 \text { CONC. (PC/L) } \\
\text { SR-89/SR-90 }\end{array}$ & $\begin{array}{l}* \\
0.01 \\
\neq\end{array}$ & $\begin{array}{l}0.01 \\
--\end{array}$ & $\begin{array}{l}0.02 \\
*\end{array}$ & $\begin{array}{l}0.13 \\
0.01 \\
7.70 \\
+\end{array}$ & $\begin{array}{l}1.24 \\
0.07 \\
5.65 \\
*\end{array}$ & $\begin{array}{l}3.59 \\
0.04 \\
1.12 \\
*\end{array}$ & $\begin{array}{l}-- \\
= \\
--\end{array}$ & $=$ & $=$ & $=$ & $\begin{array}{l}-- \\
-- \\
--\end{array}$ & $\begin{array}{l}-- \\
-- \\
-\infty\end{array}$ & $\begin{array}{l}4.96 \\
0.16\end{array}$ \\
\hline
\end{tabular}

NOTES

-: DATA NOT AVAILABLE

*: ZERO OR TRAC

A: APPROXIMATE

B: LOWER LIMIT OF REPORTED DATA

C: PROPORTIONED FROM ORIGINALLY CONSOL IDATED DATA 
MONTHLY FALLOUT DEPOSITION COLLECTIONS

LAT. 4 38N LONG. 74 5H ALT. $2632 \mathrm{M}$

(POT)

SDURCE: THE ROCKEFELLER FOUNOATION,BOGOTA

\begin{tabular}{|c|c|c|c|c|c|c|c|c|c|c|c|c|c|c|}
\hline & & JAN. & FEB. & MAR. & APR: & $\begin{array}{l}\text { MAY } \\
---\end{array}$ & JUNE & JULY & AUG. & SEP. & OCT. & NOV. & DEC. & CUM. TOTAL \\
\hline 1957 & $\begin{array}{l}\text { PRECIP. (CM.) } \\
S R-90 \text { (MCI/SQ.KM-) } \\
S R-90 \text { CONC. }(P C / L) \\
S R-89 / S R-90\end{array}$ & $\begin{array}{l}2.44 \\
=- \\
=-\end{array}$ & $\begin{array}{l}1.12 \\
=- \\
=-\end{array}$ & $\begin{array}{l}3.10 \\
-- \\
--\end{array}$ & $\begin{array}{c}10.77 \\
-- \\
--\end{array}$ & $\begin{array}{l}12.15 \\
-- \\
--\end{array}$ & $\begin{array}{l}3.53 \\
-- \\
-- \\
--\end{array}$ & $\begin{array}{l}2.82 \\
-- \\
=-\end{array}$ & $\begin{array}{l}2.59 \\
0.01 \\
0.39 \\
--\end{array}$ & $\begin{array}{l}4.01 \\
0.01 \\
0.25 \\
.-\end{array}$ & $\begin{array}{l}12.09 \\
* \\
=-\end{array}$ & $\begin{array}{l}4.45 \\
-- \\
-- \\
--\end{array}$ & $\begin{array}{l}1.09 \\
* \\
--\end{array}$ & $\begin{array}{r}59.16 \\
0.02\end{array}$ \\
\hline 1958 & $\begin{array}{l}\text { PRECIP }(C M .) \\
S R-90 \text { IMCI/SQ.KM.) } \\
S R-90 \text { CCNC. }(P C / L) \\
S R-89 / S R-90\end{array}$ & $\begin{array}{r}0.89 \\
0.02 \\
2.25 \\
12.00\end{array}$ & $\begin{array}{r}2.44 \\
C .02 \\
c .82 \\
15.00\end{array}$ & $\begin{array}{l}4.17 \\
0.05 \\
1.20 \\
4.00\end{array}$ & $\begin{array}{r}6.88 \\
0.01 \\
0.15 \\
33.00\end{array}$ & $\begin{array}{r}2.49 \\
0.05 \\
2.01 \\
13.00\end{array}$ & $\begin{array}{r}1.35 \\
0.01 \\
0.75 \\
133.0 \mathrm{C}\end{array}$ & $\begin{array}{r}0.56 \\
0.04 \\
7.15 \\
43.00\end{array}$ & $\begin{array}{r}5.66 \\
0.08 \\
1.42 \\
92.00\end{array}$ & $\begin{array}{r}1.88 \\
0.02 \\
1.07 \\
72.00\end{array}$ & $\begin{array}{r}7.04 \\
0.03 \\
0.43 \\
42.00\end{array}$ & $\begin{array}{r}10.24 \\
0.04 \\
0.40 \\
44.00\end{array}$ & $\begin{array}{r}4.67 \\
0.05 \\
1.08 \\
45.00\end{array}$ & $\begin{array}{r}48.27 \\
0.42\end{array}$ \\
\hline 1959 & $\begin{array}{l}\text { PRECIP. (CM.) } \\
S R-9 C \text { INCI/SQ.KN.) } \\
S R-90 \text { CONC. (PC/L) } \\
S R-89 / S R-90\end{array}$ & $\begin{array}{l}0.66 \\
=- \\
-- \\
--\end{array}$ & $\begin{array}{r}1.04 \\
0.02 \\
1.93 \\
25.00\end{array}$ & $\begin{array}{r}6.02 \\
0.03 \\
0.50 \\
19.00\end{array}$ & $\begin{array}{r}7.47 \\
0.10 \\
1.34 \\
12.00\end{array}$ & $\begin{array}{r}5.72 \\
C . C 1 \\
0.18 \\
10.00\end{array}$ & $\begin{array}{l}11.99 \\
-- \\
--\end{array}$ & $\begin{array}{l}6.83 \\
-- \\
--\end{array}$ & $\begin{array}{l}4.09 \\
0.02 \\
0.49 \\
*\end{array}$ & $\begin{array}{l}2.39 \\
0.01 \\
0.42 \\
*\end{array}$ & $\begin{array}{l}9.25 \\
-- \\
--\end{array}$ & $\begin{array}{l}4.52 \\
0.13 \\
2.88 \\
*\end{array}$ & $\begin{array}{l}1.85 \\
-- \\
--\end{array}$ & $\begin{array}{r}61.83 \\
0.32\end{array}$ \\
\hline 1960 & $\begin{array}{l}\text { PRECIP. (CM.) } \\
\text { SR-9O (NCI/SC.KM.) } \\
\text { SR-9O CONC. }(P C / L) \\
\text { SR-89/SR-9O }\end{array}$ & $\begin{array}{l}2.01 \\
-- \\
--\end{array}$ & $\begin{array}{l}5.08 \\
-- \\
-- \\
--\end{array}$ & $\begin{array}{l}2.84 \\
0.11 \\
3.88 \\
-.\end{array}$ & $\begin{array}{l}5.69 \\
0.02 \\
0.36 \\
--\end{array}$ & $\begin{array}{l}6.48 \\
0.016 \\
0.16 \\
--\end{array}$ & $\begin{array}{l}2.03 \\
0.00 \mathrm{C} \\
0.01 \\
-.\end{array}$ & $\begin{array}{l}7.49 \\
* \\
--\end{array}$ & $\begin{array}{l}4.01 \\
* \\
-- \\
--\end{array}$ & $\begin{array}{l}6.58 \\
-- \\
--\end{array}$ & $\begin{array}{l}11.38 \\
-- \\
--\end{array}$ & $\begin{array}{l}3.05 \\
=- \\
--\end{array}$ & $\begin{array}{l}8.08 \\
-- \\
-- \\
--\end{array}$ & $\begin{array}{r}64.72 \\
0.14\end{array}$ \\
\hline 1961 & $\begin{array}{l}\text { PRECIP. }\left(C M_{*}\right) \\
S R-90(K C I / S Q-K M)) \\
S R-90 \text { CONC. }(P C / L) \\
S R-89 / S R-90\end{array}$ & $\begin{array}{l}2.31 \\
-- \\
--\end{array}$ & $\begin{array}{l}0.64 \\
-- \\
-- \\
--\end{array}$ & $\begin{array}{l}5.64 \\
-- \\
=- \\
--\end{array}$ & $\begin{array}{l}10.08 \\
=- \\
=\end{array}$ & $\begin{array}{l}3.33 \\
-- \\
--\end{array}$ & $\begin{array}{l}6.55 \\
-- \\
-- \\
--\end{array}$ & $\begin{array}{l}4.37 \\
-- \\
-- \\
--\end{array}$ & $\begin{array}{l}2.49 \\
-- \\
--\end{array}$ & $\begin{array}{l}1.75 \\
0.04 \\
2.29 \\
--\end{array}$ & $\begin{array}{l}14.63 \\
* \\
--\end{array}$ & $\begin{array}{r}11.99 \\
0.10 \\
0.84 \\
65.00\end{array}$ & $\begin{array}{l}3.07 \\
-- \\
-- \\
--\end{array}$ & $\begin{array}{r}66.85 \\
0.14\end{array}$ \\
\hline 1962 & $\begin{array}{l}\text { PRECIP. (CM-) } \\
\text { SR-90 (MCI/SQ.KM.) } \\
S R-90 \text { CONC. }(P C / L) \\
S R-89 / S R-90\end{array}$ & $\begin{array}{l}-- \\
-- \\
--\end{array}$ & $\begin{array}{l}-- \\
-- \\
--\end{array}$ & $\begin{array}{l}-- \\
-- \\
--\end{array}$ & $\begin{array}{l}--0 \\
\overline{0.04} \\
19.00\end{array}$ & $\begin{array}{l}-- \\
*\end{array}$ & $\begin{array}{c}-- \\
0.04 \\
57.00\end{array}$ & $\begin{array}{l}-- \\
-- \\
--\end{array}$ & $\begin{array}{l}-- \\
-- \\
-- \\
--\end{array}$ & $\begin{array}{c}-- \\
0.06 \\
26.00\end{array}$ & $\frac{--}{0.02}$ & $\begin{array}{c}\overline{0.25} \\
\overline{22.00}\end{array}$ & $\begin{array}{l}--. \\
0.11 \\
-.00\end{array}$ & $\begin{array}{l}0.0 \\
0.52\end{array}$ \\
\hline 1963 & 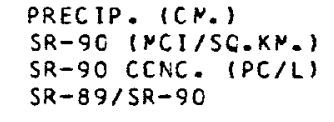 & $\begin{array}{c}\overline{0.08} \\
\overline{41.00}\end{array}$ & $\begin{array}{c}-- \\
0.16 \\
24.00\end{array}$ & $\begin{array}{r}52.58 \\
0.05 \\
0.10 \\
17.00\end{array}$ & $\begin{array}{r}7.32 \\
0.14 \\
1.92 \\
13.00\end{array}$ & $\begin{array}{l}11.38 \\
0.05 \\
0.44 \\
-.\end{array}$ & $\begin{array}{l}7.16 \\
0.02 \\
0.28 \\
*\end{array}$ & $\begin{array}{l}2.46 \\
0.04 \\
1.63 \\
*\end{array}$ & $\begin{array}{l}3.73 \\
0.03 \\
0.81 \\
*\end{array}$ & $\begin{array}{l}2.24 \\
0.02 \\
0.90 \\
*\end{array}$ & $\begin{array}{l}8.36 \\
C .03 \\
0.34 \\
*\end{array}$ & $\begin{array}{l}8.64 \\
0.05 \\
0.58 \\
*\end{array}$ & $\begin{array}{l}1.37 \\
0.02 \\
1.46 \\
1.00\end{array}$ & $\begin{array}{r}105.74 \\
0.69\end{array}$ \\
\hline 1964 & $\begin{array}{l}\text { PQECIP. (CM.) } \\
S R-S C \text { (NCI/SQ.KN.) } \\
S R-9 O C C N C \text { (PC/L) } \\
S R-89 / 5 R-90\end{array}$ & $\begin{array}{l}0.64 \\
0.03 \\
4.69 \\
*\end{array}$ & $\begin{array}{l}2.24 \\
0.07 \\
3.13 \\
*\end{array}$ & $\begin{array}{l}8.460 \\
0.10 C \\
1.19 \\
-.\end{array}$ & $\begin{array}{l}8.460 \\
0.100 \\
1.19 \\
-.\end{array}$ & $\begin{array}{l}7.26 \\
0.03 \\
0.42 \\
-.\end{array}$ & $\begin{array}{l}10.44 \\
0.07 \\
0.68 \\
--\end{array}$ & $\begin{array}{l}6.53 \\
C .03 \\
0.46 \\
-.\end{array}$ & $\begin{array}{l}4.09 \\
C .05 \\
1.23 \\
--\end{array}$ & $\begin{array}{l}6.73 \\
+ \\
-- \\
--\end{array}$ & $\begin{array}{l}7.44 \\
0.02 \\
0.27 \\
+\end{array}$ & $\begin{array}{l}5.46 \\
0.04 \\
0.74 \\
--\end{array}$ & $\begin{array}{l}1.19 \\
0.01 \\
0.85 \\
-.\end{array}$ & $\begin{array}{r}68.94 \\
0.55\end{array}$ \\
\hline 1965 & $\begin{array}{l}\text { PRECIP. }(C M-) \\
\text { SR-SO } \text { (NCI/SQ.KM.) } \\
\text { SR-90 CONC. }(P C / L) \\
\text { SR-89/SR-90 }\end{array}$ & $\begin{array}{l}2.31 \\
0.01 \\
0.44 \\
-.\end{array}$ & $\begin{array}{l}-- \\
-- \\
--\end{array}$ & $\begin{array}{l}2.34 \\
=- \\
=-\end{array}$ & $\begin{array}{l}21.21 \\
0.00 \\
0.01 \\
-.\end{array}$ & $\begin{array}{l}7.87 C \\
0.026 \\
0.26 \\
--\end{array}$ & $\begin{array}{l}7.876 \\
0.026 \\
0.26 \\
=-\end{array}$ & $\begin{array}{l}3.30 \\
0.00 \\
0.01 \\
--\end{array}$ & $\begin{array}{l}3.63 \\
0.01 \\
0.28 \\
--\end{array}$ & $\begin{array}{l}-- \\
-- \\
--\end{array}$ & $\begin{array}{l}-- \\
-- \\
--\end{array}$ & $\begin{array}{l}18.59 \\
0.01 \\
0.06 \\
-0\end{array}$ & $\begin{array}{l}-- \\
-- \\
--\end{array}$ & $\begin{array}{r}67.12 \\
0.07\end{array}$ \\
\hline $156 t$ & $\begin{array}{l}\text { PRECIP ( }(C M)) \\
S R-90 \text { (MCI/SQ.KM.) } \\
S R-90 \text { CONC. }(P C / L) \\
S R-89 / S R-90\end{array}$ & $\begin{array}{l}* \\
* \\
--\end{array}$ & $\begin{array}{l}1.65 \\
0.01 \\
0.61 \\
-\end{array}$ & $\begin{array}{l}-- \\
-- \\
--\end{array}$ & $\begin{array}{l}-- \\
-- \\
--\end{array}$ & $\begin{array}{l}-- \\
-- \\
--\end{array}$ & $\begin{array}{l}-- \\
-- \\
--\end{array}$ & $\begin{array}{l}4.88 \\
* \\
--\end{array}$ & $\begin{array}{l}6.02 \\
* \\
*\end{array}$ & $\begin{array}{l}3.86 \\
+ \\
--\end{array}$ & $\begin{array}{l}-- \\
-- \\
--\end{array}$ & $\begin{array}{l}-\infty \\
-- \\
--\end{array}$ & $\begin{array}{l}-- \\
-- \\
--\end{array}$ & $\begin{array}{r}16.41 \\
0.01\end{array}$ \\
\hline 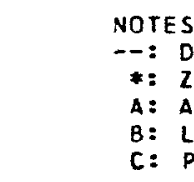 & $\begin{array}{l}\text { DATA NOT AVAILABLE } \\
\text { LERO OR TRACE } \\
\text { APPROXIMATE } \\
\text { LOWER LIMIT OF REPOR } \\
\text { PROPORTIONED FRCM CR }\end{array}$ & $\begin{array}{l}\text { DATA } \\
\text { NALLY }\end{array}$ & & & & & & & & & & & & \\
\hline
\end{tabular}


SITE: CONGO REP.KINSHASA SITE ? SOURCE: SERVICE METEOROLOGIQUE
MONTHEY FALLOUT DEPOSITION COLLECTIONS

LAT. $420 S$ LONG. $1518 E$ ALT. 305M. (COLUMN)
JAN. FEB. MAR. APR. MAY JUNE JULY AUG. SEP. OCT. NOV. DEC. CUM. TOIAL

1960 PRECIP. $1 C M$

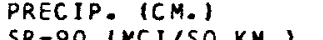
SR SR-89/5R-SO

1961 PRECIP. (CM.) SR-OO (NCISQ.KM.) 13.00 $S R-9 C$ CCNC.
$S R-89 / 5 R-90$

1962 PRECIP. (CM.) $\begin{array}{ll}(N C I / S Q . K H .) & 0.20\end{array}$ $S R-90$ CCNC. $(P C / L)$ $S R-89 / S R-S C$

1963 PRECIP. $\left\{C M_{-}\right\}$ SR-9O (NCI/SO.KN.) 14.15 $S R-90$ CCNC. $(P C / L)$ $S R-89 / S R-90$

1964 PRECIP. (CM.) SR-OC (NCIISG.KM.) 15.52 $\begin{array}{ll}S R-90 C C N C . & (P C / L) \\ S R-89 / S R-90 & 1.23 \\ & 0.50\end{array}$

1965 PRECIP. (CM.) SR-OC (KCI/SO,KM, 12.22 $S R-Q C$ CONC I $P C / L)$ $S R-8 S / S R-S O$ (PCJL) C.58 $\begin{array}{ll}-- & - \\ - & = \\ - & -\end{array}$ $=$ 0.61
0.002
0.02 0.01 $\begin{array}{lll}21.89 & 0.05 & * \\ 0.06 \mathrm{C} & 0.15 \mathrm{C} & 0.00 \mathrm{C}\end{array}$ 0.28300 .00 .000 $6.48 \quad 11.25$ 17.53 $\begin{array}{llll}1.24 & 1.25 & 0.80 & 0.84\end{array}$ 67.39
0.65 17.58 17.5

9.70

\subsection{8}

$-$ 0.11 35.48
$0.05 C$
0.15

17.98

$*$

0.23

$0.23 \quad *$

6.76

6.76 $-$ $-$ $-\infty$

\subsection{8}

0.19

24.64 5.92 $13.05=$ 0.45 0.00 $--$ 0.10 * $--$ 64.00 36.00

100.73 0.26

$\begin{array}{llllll}-- & - & 4.93 & 9.63 & 31.24 & 23.50\end{array}$ $\begin{array}{llllll}c .07 & 0.01 & 0.05 & 0.05 & 0.05 & 0.19\end{array}$ $\begin{array}{llllll}7.0 & * & 1.62 & 0.52 & 0.17 & 0.81\end{array}$ $\begin{array}{rrrr}1.02 & 0.52 & 0.05 & 0.19 \\ 23.00 & 18.00 & 14.00 & 29.00\end{array}$ $3 \mathrm{C} .00 \quad 18.00$

$0.07 \quad 0.16$
10.19 $\begin{array}{rr}1.19 & 0.96 \\ 12.00 & 135.00\end{array}$

\subsection{6}

22.63

0.44

22.02

2.63
0.56

0.56
2.48
18.00

20.62

20.62
0.05
0.25
18.00

11.89

0.04
0.34
3.50

(-

1.52

\section{$--$}

1.40

1.40
0.04

$6.68 \quad 30.20 \quad 28.58$ $\begin{array}{lll}0.06 & 0.14 & 0.58\end{array}$ $0.90 \quad 0.47 \quad 2.03$
$*$

9. $78 \quad 14.50$

55.88 $19.1 \mathrm{C}$

$\begin{array}{llll}0.07 & 0.01 & 0.03 & 0.04 \\ 0.01 & 0.82 & -- & 2.86 \\ * & * & * & \#\end{array}$ 1.54 2.21

5.88
0.14

0.14

$19.1 \mathrm{C}$
0.10

*

*

0.53

0.1

0.03

$\begin{array}{ll}1.17 & 0.58\end{array}$

7.42

$29.74 \quad 12.75$

$0.10 \quad 0.07$

$\begin{array}{lll}0.54 & 0.34 & 0.55\end{array}$

166.44

1.30

$6.88 \quad 33.91$

$9.83 \quad 13.72$

$*$

*

3.45

*

$0.02 \quad 0.12$

$0.30 \quad 0.36$

0.41

0.09
0.66

0.0

$0.03 \quad 0.13$

4.34

23.65

2.98

$\begin{array}{lll}4.06 & 0.03 \quad 0.03 \quad 12.98\end{array}$

$\begin{array}{llll}1.39 & 0.24 & 0.09 & 0.47\end{array}$

0.47

130.26

l9EE PRECIP. (CM.)

$\begin{array}{llll} & 1 & & \end{array}$

SR-90 INCI/SQ.KM.) $S R-90$ CONC.
$S R-89 / S R-90$

1967 PRECIP. (CM.) PRECIP. ICM.), 24.31 $\begin{array}{lll}S R-90 & (N C I / S Q . K M .) & 0.07 \\ S R-90 & C O N C .(P C / L) & 0.29\end{array}$ $S R-89 / S R-90$

31.52

3.29

2.54

*.0

$=01$

$*$
+
+
--
--

$$
1.97
$$$$
--
$$$$
\text { -- }
$$ 
SOURCE: INSTITUTO INTERAMERICANA DE CIENCAS AGRICOLAS

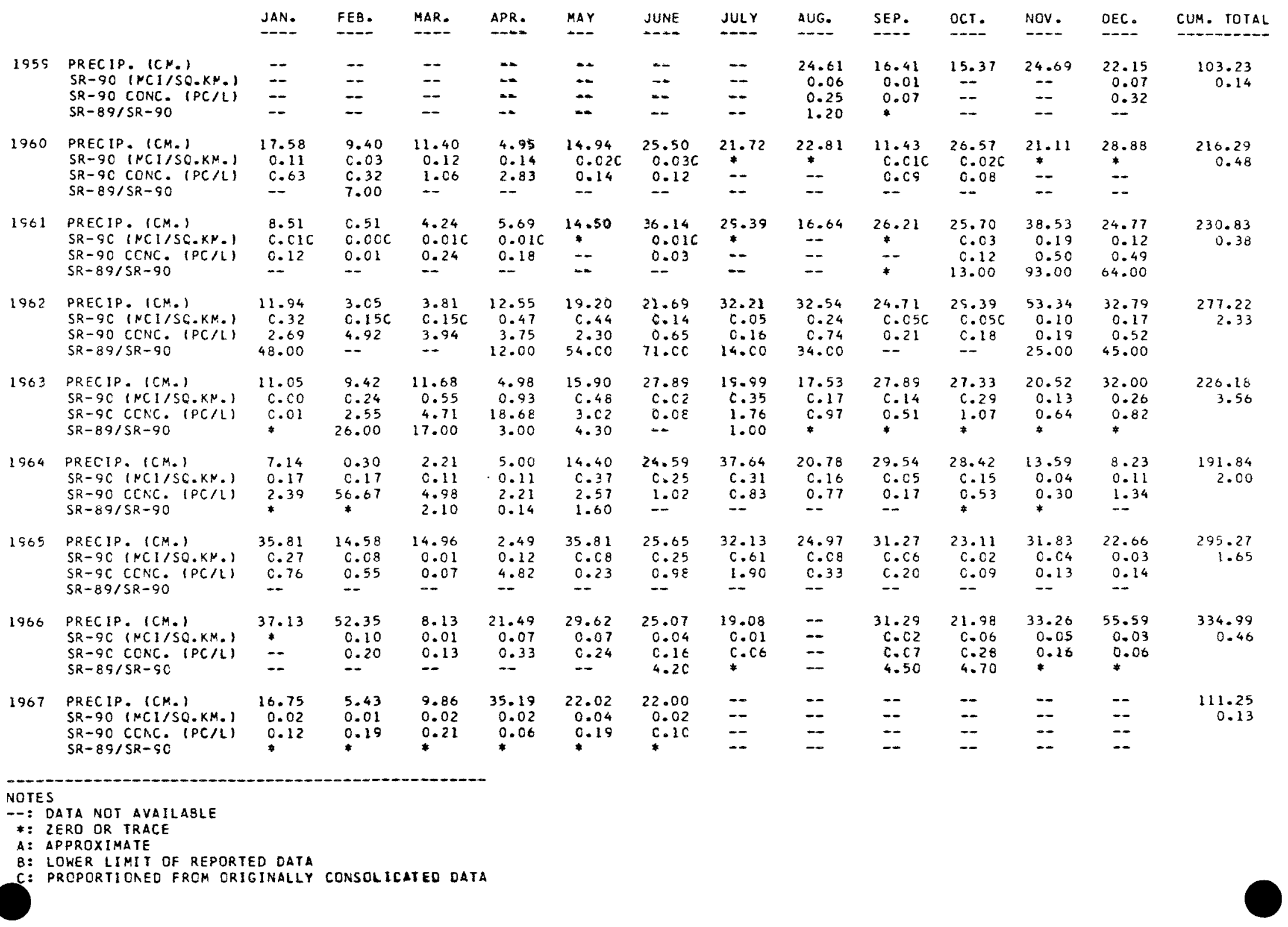


MONTHLY FALLCUT DEPOSITION COLLECTIONS

SITE: ECUADOR, GUAYAQUIL

LAT. 2 IOS LONG. $7952 \mathrm{~W}$ ALT.

7M. (COLUMN) SURFACE AIR SAMPLING STATION

SOURCE: ESTACION METEOROLOGICA, SUBOIRECION DE AVIACION CIVIL

\begin{tabular}{|c|c|c|c|c|c|c|c|c|c|c|c|c|c|c|}
\hline & & JAN. & FEB. & MAR & APR: & $\begin{array}{l}\text { MAY } \\
-\ldots\end{array}$ & JUNE & JULY & AUG. & SEP. & OCI. & NOV. & DEC. & CUM. TOIAL \\
\hline 1963 & $\begin{array}{l}\text { PRECIP. (CM.) } \\
S R-S C \text { (NCI/SQ.KM.) } \\
S R-90 \text { CCNC. (PC/L) } \\
S R-89 / S R-90\end{array}$ & $\begin{array}{l}5.11 \\
-- \\
-- \\
--\end{array}$ & $\begin{array}{c}35.36 \\
=- \\
=-\end{array}$ & $\begin{array}{l}18.59 \\
-- \\
--\end{array}$ & $\begin{array}{l}4.72 \\
0.04 \\
0.85 \\
6.00\end{array}$ & $\begin{array}{l}0.81 \\
0.01 \\
1.24 \\
*\end{array}$ & $\begin{array}{l}* \\
0.03 \\
*-\end{array}$ & $\begin{array}{l}* \\
0.02 \\
-\end{array}$ & $\begin{array}{l}* \\
0.01 \\
3.00\end{array}$ & $\begin{array}{l}* \\
0.01 \\
* \\
*\end{array}$ & $\begin{array}{l}* \\
0.05 \\
*-\end{array}$ & $\begin{array}{l}* \\
0.02 \\
--\end{array}$ & $\begin{array}{c}2.01 \\
0.22 \\
10.95 \\
*\end{array}$ & $\begin{array}{r}66.60 \\
0.41\end{array}$ \\
\hline 1964 & $\begin{array}{l}\text { PRECIP. }(C N .) \\
\text { SR-SC }(N C I / S Q . K N .) \\
S R-90 \text { CONC. }(P C / L) \\
S R-89 / S R-90\end{array}$ & $\begin{array}{l}21.67 \\
C .08 \\
0.37 \\
\end{array}$ & $\begin{array}{r}16.36 \\
0.08 \\
0.49 \\
0.90\end{array}$ & $\begin{array}{l}42.27 \\
0.79 \\
1.87 \\
*\end{array}$ & $\begin{array}{l}18.08 \\
0.06 \\
0.34 \\
*\end{array}$ & $\begin{array}{r}0.38 \\
0.08 \\
21.06 \\
*\end{array}$ & $\begin{array}{l}0.41 \\
0.01 \\
2.44 \\
--\end{array}$ & $\begin{array}{l}* \\
C .01 \\
-- \\
--\end{array}$ & $\begin{array}{l}* \\
0.01 \\
-- \\
--\end{array}$ & $\begin{array}{l}* \\
0.02 \\
-- \\
--\end{array}$ & $\begin{array}{l}* \\
C .01 \\
=-\end{array}$ & $\begin{array}{l}0.05 \\
* \\
*\end{array}$ & $\begin{array}{l}+ \\
0.03 \\
--\end{array}$ & $\begin{array}{r}99.22 \\
1.18\end{array}$ \\
\hline 1965 & $\begin{array}{l}\text { PRECIP (CM.) } \\
\text { SR-90 INCI/SQ.KM.) } \\
\text { SR-90 CONC. (PC/L) } \\
\text { SR-89/SR-90 }\end{array}$ & $\begin{array}{l}12.85 \\
0.07 \\
0.55 \\
--\end{array}$ & $\begin{array}{l}-- \\
-- \\
--\end{array}$ & $\begin{array}{l}20.29 C \\
0.10 C \\
0.50 \\
--\end{array}$ & $\begin{array}{c}20.29 C \\
0.10 C \\
0.50 \\
-.\end{array}$ & $\begin{array}{l}-- \\
-- \\
--\end{array}$ & $\begin{array}{l}-- \\
-- \\
--\end{array}$ & $\begin{array}{l}-- \\
-- \\
--\end{array}$ & $\begin{array}{l}-- \\
-- \\
--\end{array}$ & $\begin{array}{l}-- \\
-- \\
--\end{array}$ & $\begin{array}{l}-- \\
-- \\
--\end{array}$ & $\begin{array}{l}* \\
0.14 \\
=- \\
=-\end{array}$ & $\begin{array}{l}3.30 \\
0.11 \\
3.34 \\
=-\end{array}$ & $\begin{array}{r}56.73 \\
0.52\end{array}$ \\
\hline 8 & $\begin{array}{l}\text { PRECIP. (CN.) } \\
S R-9 C \text { (NCI/SQ.KN.) } \\
S R-90 \text { CONC. }(P C / L) \\
S R-89 / S R-90\end{array}$ & $\begin{array}{r}53.80 \\
0.06 \\
0.12 \\
-.\end{array}$ & $\begin{array}{l}13.87 \\
0.03 \\
0.22 \\
--\end{array}$ & $\begin{array}{l}27.81 \\
* \\
-- \\
--\end{array}$ & $\begin{array}{l}5.28 \\
+ \\
-- \\
--\end{array}$ & $\begin{array}{l}1.59 \\
0.03 \\
1.89 \\
--\end{array}$ & $\begin{array}{l}0.17 \\
* \\
--\end{array}$ & $\begin{array}{l}* \\
0.01 \\
--\end{array}$ & $\begin{array}{l}* \\
0.05 \\
--\end{array}$ & $\begin{array}{l}-- \\
-- \\
-- \\
--\end{array}$ & $\begin{array}{l}1.83 \\
0.01 \\
0.55 \\
*\end{array}$ & $\begin{array}{l}* 0.02 \\
0.00\end{array}$ & $\begin{array}{l}1.01 \\
0.01 \\
1.00 \\
4\end{array}$ & $\begin{array}{r}105.36 \\
0.22\end{array}$ \\
\hline 1967 & $\begin{array}{l}\text { PRECIP. (CM.) } \\
S R-90 \text { (NCI/SQ.KM.) } \\
S R-90 \text { CCNC. (PC/L) } \\
S R-89 / S R-90\end{array}$ & $\begin{array}{l}-- \\
-- \\
--\end{array}$ & $\begin{array}{l}40.30 \\
0.03 \\
0.08 \\
+\end{array}$ & $\begin{array}{l}11.26 \\
0.01 \\
0.09 \\
*\end{array}$ & $\begin{array}{l}* \\
0.01 \\
-- \\
*\end{array}$ & $\begin{array}{l}0.90 \\
0.01 \\
1.12 \\
*\end{array}$ & $\begin{array}{l}-- \\
-- \\
--\end{array}$ & $\begin{array}{l}-- \\
-- \\
-- \\
--\end{array}$ & $\begin{array}{l}-- \\
-- \\
--\end{array}$ & $\begin{array}{l}-- \\
-- \\
-- \\
--\end{array}$ & $\begin{array}{l}-- \\
-- \\
--\end{array}$ & $\begin{array}{l}-- \\
-- \\
--\end{array}$ & $\begin{array}{l}-- \\
=- \\
--\end{array}$ & $\begin{array}{r}52.46 \\
0.06\end{array}$ \\
\hline $\begin{array}{l}\text { NOTES } \\
--:: \\
*: \\
A: \\
B: 1 \\
C:\end{array}$ & $\begin{array}{l}\text { S } \\
\text { DATA NOI AVAILABLE } \\
\text { ZERO OR TRACE } \\
\text { APPROXIMATE } \\
\text { LOWER LIMIT OF REPORT } \\
\text { PROPORTIOAEO FROM CRI }\end{array}$ & $\begin{array}{l}\text { ED OATA } \\
\text { GINALLY }\end{array}$ & & $D$ & & & & & & & & & & \\
\hline
\end{tabular}


MONTHLY FALLOUT DEPOSITION COLLECTIONS

SITE: ENIWETOK ATOLL

LAT. $1121 \mathrm{~N}$ LONG.162 $21 \mathrm{E}$ ALT.

4M. (COLUMN:)

SOURCE: U. S. HEATHER BUREAU AIRPORT STATION

\begin{tabular}{|c|c|c|c|c|c|c|c|c|c|c|c|c|c|c|}
\hline & & JAN. & FEB. & MAR. & $A P R$. & MAY & JUNE & JULY & AUG. & SEP. & OCT. & NOV. & DEC. & CUM. TOTAL \\
\hline $1 s \in 2$ & $\begin{array}{l}\text { PRECIP. (CN.) } \\
\text { SR-SC INCI/SQ.KM.) } \\
S R-90 \text { CCNC. }(P C / L) \\
S R-89 / S R-90\end{array}$ & $\begin{array}{l}-- \\
-- \\
--\end{array}$ & $\begin{array}{l}-- \\
-- \\
--\end{array}$ & $\begin{array}{l}-- \\
--\end{array}$ & $\begin{array}{r}1.42 \\
0.11 \\
7.75 \\
18.00\end{array}$ & $\begin{array}{r}15.65 \\
0.34 \\
2.18 \\
12.00\end{array}$ & $\begin{array}{r}10.57 \\
0.17 \\
1.61 \\
33.00\end{array}$ & $\begin{array}{r}1 \varepsilon .21 \\
C .07 \\
0.39 \\
12.00\end{array}$ & $\begin{array}{r}16.33 \\
0.12 \\
0.74 \\
18.00\end{array}$ & $\begin{array}{r}31.09 \\
0.09 \\
0.29 \\
-\end{array}$ & $\begin{array}{r}28.75 \\
0.11 \\
0.39 \\
57.00\end{array}$ & $\begin{array}{l}12.95 \Delta \\
0.21 \Delta \\
1.63 \\
-\end{array}$ & $\begin{array}{l}19.794 \\
0.124 \\
0.61 \\
+-\end{array}$ & $\begin{array}{r}154.76 \\
1.34\end{array}$ \\
\hline 1963 & $\begin{array}{l}\text { PRECIP. }(C M .) \\
S R-9 O \text { INCI/SQ.KM.) } \\
S R-S C \text { CNC. }(P C / L) \\
S R-8 S / S R-S O\end{array}$ & $\begin{array}{l}1.96 C \\
0.27 C \\
13.78 \\
24.00\end{array}$ & $\begin{array}{l}1.96 C \\
C .27 C \\
13.78 \\
19.00\end{array}$ & $\begin{array}{l}1.96 C \\
0.27 C \\
13.78 \\
=.\end{array}$ & $\begin{array}{l}1.96 C \\
0.27 C \\
13.78 \\
-.\end{array}$ & $\begin{array}{r}34.32 \\
0.29 \\
0.85 \\
6.90\end{array}$ & $\begin{array}{r}23.11 \\
0.82 \\
3.55 \\
1.60\end{array}$ & $\begin{array}{r}21.51 \\
0.52 \\
2.42 \\
1.00\end{array}$ & $\begin{array}{r}22.58 \\
0.25 \\
1-11 \\
+\end{array}$ & $\begin{array}{r}19.51 \\
0.19 \\
0.98 \\
1.60\end{array}$ & $\begin{array}{l}30.00 \\
C .18 \\
c .61 \\
+\end{array}$ & $\begin{array}{l}10.64 \\
0.15 \\
1.41 \\
*\end{array}$ & $\begin{array}{l}1+6.38 \\
0.33 \\
2.02 \\
*\end{array}$ & $\begin{array}{r}185.89 \\
3.81\end{array}$ \\
\hline 1964 & $\begin{array}{l}\text { PRECIP. (CM.) } \\
S R-90 \text { (NCI/SQ.KM.) } \\
S R-90 \text { CONC. }(P C / L) \\
S R-89 / S R-S C\end{array}$ & $\begin{array}{l}5.13 \\
0.32 \\
6.24 \\
+\end{array}$ & $\begin{array}{l}2.77 \\
0.25 \\
9.03 \\
*\end{array}$ & $\begin{array}{r}0.33 \\
0.17 \\
51.52 \\
*\end{array}$ & $\begin{array}{l}25.83 \\
0.40 \\
1.55 \\
*\end{array}$ & $\begin{array}{l}6.93 \\
0.14 \\
2.03 \\
+\end{array}$ & $\begin{array}{r}12.14 \\
0.48 \\
3.96 \\
-.\end{array}$ & $\begin{array}{r}16.05 \\
0.13 \\
0.81 \\
-.\end{array}$ & $\begin{array}{r}12.83 \\
0.18 \\
1.41 \\
--\end{array}$ & $\begin{array}{r}30-12 \\
c=06 \\
t=20 \\
-\end{array}$ & $\begin{array}{l}26.42 \\
0.07 \\
0.27 \\
+\end{array}$ & $\begin{array}{l}18.04 \\
0.07 \\
0.38 \\
+\end{array}$ & $\begin{array}{l}3.89 \\
0.10 \\
2.58 \\
--\end{array}$ & $\begin{array}{r}161.08 \\
2.37\end{array}$ \\
\hline$\stackrel{\odot}{\infty}$ & $\begin{array}{l}\text { PRECIP. (CM.) } \\
\text { SR-9O (NCI/SQ.KM.) } \\
S R-90 \text { CCNC. }(P C / L) \\
S R-89 / S R-90\end{array}$ & $\begin{array}{l}3.89 \\
0.16 \\
4.12 \\
--\end{array}$ & $\begin{array}{l}1.50 \\
0.02 \\
1.34 \\
--.\end{array}$ & $\begin{array}{l}1.52 \\
0.00 \\
0.01 \\
-.\end{array}$ & $\begin{array}{l}0.91 \\
0.08 \\
8.90 \\
--\end{array}$ & $\begin{array}{l}2.95 \\
0.10 \\
3.39 \\
--\end{array}$ & $\begin{array}{l}4.62 \\
0.12 \\
2.60 \\
--\end{array}$ & $\begin{array}{l}22.05 \\
0.18 \\
0.82 \\
--\end{array}$ & $\begin{array}{r}11.73 \\
0.01 \\
0.09 \\
-\end{array}$ & $\begin{array}{r}27-08 \\
0.04 \\
0.15 \\
--\end{array}$ & $\begin{array}{r}62.33 \\
0.01 \\
0.02 \\
--\end{array}$ & $\begin{array}{l}9.60 \\
0.03 \\
0.32 \\
-\end{array}$ & $=-$ & $\begin{array}{r}148.18 \\
0.75\end{array}$ \\
\hline 1966 & $\begin{array}{l}\text { PRECIP. (CM.) } \\
S R-S O(N C I / S Q . K N .) \\
S R-9 C \text { CCNC. (PCI) } \\
S R-89 / S R-90\end{array}$ & $\begin{array}{l}-- \\
-- \\
--\end{array}$ & $\begin{array}{l}2.39 \\
0.03 \\
1.26 \\
--\end{array}$ & $\begin{array}{l}1.60 \\
0.04 \\
2.51 \\
--\end{array}$ & $\begin{array}{l}4.72 \\
0.09 \\
1.91 \\
--\end{array}$ & $\begin{array}{l}2.20 \\
0.02 \\
0.91 \\
--\end{array}$ & $\begin{array}{l}-- \\
\overline{--}\end{array}$ & $\begin{array}{l}9.70 \\
C .04 \\
C .42 \\
8.70\end{array}$ & $\begin{array}{l}29.62 \\
C .02 \\
0.07 \\
+\end{array}$ & $\begin{array}{l}23.85 \\
* \\
-\end{array}$ & $\begin{array}{l}23.54 \\
C .02 \\
0.09 \\
*\end{array}$ & $\begin{array}{l}9.78 \\
0.04 \\
0.41 \\
5.50\end{array}$ & $\begin{array}{l}9.75 \\
0.022 \\
0.21 \\
*\end{array}$ & $\begin{array}{r}117.15 \\
0.32\end{array}$ \\
\hline 1967 & $\begin{array}{l}\text { PRECIP. }\left(C M_{*}\right) \\
S R-90 \text { (NCI/SQ.KM.) } \\
S R-90 \text { CONC. }(P C / L) \\
S R-89 / S R-90\end{array}$ & $\begin{array}{l}5.79 \\
0.04 \\
0.70 \\
*\end{array}$ & $\begin{array}{l}3.76 \\
0.01 \\
0.27 \\
*\end{array}$ & $\begin{array}{r}11.40 \\
0.01 \\
0.09 \\
*\end{array}$ & $\begin{array}{l}15.14 \\
0.02 \\
0.14 \\
*\end{array}$ & $\begin{array}{l}17.96 \\
0.01 \\
0.06 \\
*\end{array}$ & $\begin{array}{r}19.40 \\
0.04 \\
0.21 \\
18.0 C\end{array}$ & $\begin{array}{l}29.97 \\
0.01 \\
0.04 \\
*\end{array}$ & $\begin{array}{l}-- \\
-- \\
--\end{array}$ & $\begin{array}{l}-- \\
-- \\
--\end{array}$ & $\begin{array}{l}-- \\
--\end{array}$ & $\begin{array}{l}-- \\
-- \\
--\end{array}$ & $\begin{array}{l}-- \\
-- \\
--\end{array}$ & $\begin{array}{r}103.42 \\
0.14\end{array}$ \\
\hline $\begin{array}{l}\text { NOTES } \\
--: \text { : } \\
\neq: \quad 2 \\
A: A \\
B: L \\
C: P\end{array}$ & $\begin{array}{l}\text { DATA NOT AVAILABLE } \\
\text { ZERO OR IRACE } \\
\text { APPROXIMATE } \\
\text { LOWER LIMIT OF REPORT } \\
\text { PROPORTIOAED FROM ORI }\end{array}$ & $\begin{array}{l}\text { DATA } \\
\text { INALLY }\end{array}$ & 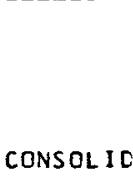 & & & & & & & & & & & \\
\hline
\end{tabular}




\begin{tabular}{|c|c|c|c|c|c|c|c|c|c|c|c|c|c|c|}
\hline & & JAN. & FEB. & MAR. & APR: & MAY & JUNE & JULY & AUG. & SEP. & OCT. & NOV. & DEC. & CUM. ROTAL \\
\hline $19 \in C$ & $\begin{array}{l}\text { PRECIP. (CN.) } \\
S R-90 \text { (NCI/SQ.KM.) } \\
S R-90 \text { CONC. }(P C / L) \\
S R-89 / S R-90\end{array}$ & $\begin{array}{l}0.43 \\
0.00 \\
0.01 \\
--\end{array}$ & $\begin{array}{l}2.31 \\
0.09 \\
3.90 \\
-.\end{array}$ & $\begin{array}{l}8.64 \\
0.07 \\
0.82 \\
-.\end{array}$ & $\begin{array}{l}3.99 \\
-- \\
--\end{array}$ & $\begin{array}{l}6.73 \\
-- \\
-- \\
--\end{array}$ & $\begin{array}{l}9.04 \\
-- \\
-- \\
--\end{array}$ & $\begin{array}{l}19.20 \\
* \\
-- \\
--\end{array}$ & $\begin{array}{c}28.22 \\
+ \\
--\end{array}$ & $\begin{array}{l}18.24 \\
0.016 \\
0.06 \\
--\end{array}$ & $\begin{array}{l}0.23 \\
0.016 \\
4.35 \\
--\end{array}$ & $\begin{array}{l}1.22 \\
0.02 \\
1.64 \\
--\end{array}$ & $\begin{array}{l}0.61 \\
0.04 \\
6.56 \\
--\end{array}$ & $\begin{array}{r}98.86 \\
0.24\end{array}$ \\
\hline 1961 & $\begin{array}{l}\text { PRECIP. (CN.) } \\
S R-90 \text { (NCI/SQ.KN=) } \\
S R-90 \text { CONC. (PC/L) } \\
\text { SR-89/SR-SO }\end{array}$ & $\begin{array}{l}* \\
0.04 C \\
-- \\
--\end{array}$ & $\begin{array}{l}0.36 \\
0.04 C \\
11.12 \\
=-\end{array}$ & $\begin{array}{l}7.98 \\
0.05 C \\
0.63 \\
--\end{array}$ & $\begin{array}{l}11.00 \\
0.05 \mathrm{C} \\
0.46 \\
--\end{array}$ & $\begin{array}{l}5.11 \\
0.130 \\
2.55 \\
--\end{array}$ & $\begin{array}{l}15.39 \\
0.13 C \\
0.85 \\
--\end{array}$ & $\begin{array}{l}38.10 \\
0.10 \mathrm{C} \\
\mathrm{C.27} \\
--\end{array}$ & $\begin{array}{l}26.21 \\
0.10 C \\
0.39 \\
--\end{array}$ & $\begin{array}{l}21.54 \\
0.03 C \\
C .14 \\
=-\end{array}$ & $\begin{array}{l}8.08 \\
C .03 C \\
C .38 \\
--\end{array}$ & $\begin{array}{l}5.89 \\
0.036 \\
0.51 \\
-.\end{array}$ & $\begin{array}{c}* \\
0.43 \\
-- \\
54.00\end{array}$ & $\begin{array}{r}139.66 \\
1.16\end{array}$ \\
\hline 1562 & $\begin{array}{l}\text { PRECIP. (CN.) } \\
\text { SR-9O (KCI/SG.KM.) } \\
S R-90 \text { CCAC. (PC/L) } \\
\text { SR-89/SR-SO }\end{array}$ & $\begin{array}{l}* \\
* \\
*-\end{array}$ & $\begin{array}{l}- \\
-- \\
--\end{array}$ & $\begin{array}{l}13.89 \\
-- \\
--\end{array}$ & $\begin{array}{r}3.23 \\
0.13 \\
4.03 \\
19.00\end{array}$ & $\begin{array}{r}1.19 \\
0.67 \\
56.31 \\
13.00\end{array}$ & $\begin{array}{l}5.0 \varepsilon \\
-- \\
-- \\
--\end{array}$ & $\begin{array}{r}22.68 \\
0.99 \\
4.37 \\
16.00\end{array}$ & $\begin{array}{r}22.48 \\
0.67 \\
2.99 \\
12.00\end{array}$ & $\begin{array}{r}21.64 \\
0.19 \\
0.88 \\
16.00\end{array}$ & $\begin{array}{l}1.24 \\
C .01 \\
0.81 \\
*\end{array}$ & $\begin{array}{r}1.42 \\
0.11 \\
7.85 \\
32.00\end{array}$ & $\begin{array}{r}0.94 \\
0.25 \\
26.60 \\
28.00\end{array}$ & $\begin{array}{r}93.79 \\
3.02\end{array}$ \\
\hline $15 \in 3$ & $\begin{array}{l}\text { PRECIP. (CM.) } \\
\text { SR-GC (NCI/SQ.KM.) } \\
\text { SR-9O CCRC. (PCIL) } \\
\text { SR-89/SR-90 }\end{array}$ & $\begin{array}{r}0.10 \\
6.34 \\
340.00 \\
36.00\end{array}$ & $\begin{array}{l}4.55 \\
-- \\
-- \\
--\end{array}$ & $\begin{array}{r}4.88 \\
0.92 \\
18.86 \\
15.00\end{array}$ & $\begin{array}{r}14.12 \\
0.82 \\
5.81 \\
8.00\end{array}$ & $\begin{array}{r}11.71 \\
1.73 \\
14.78 \\
8.60\end{array}$ & $\begin{array}{r}11.16 \\
2.77 \\
24.96 \\
3.00\end{array}$ & $\begin{array}{r}16.21 \\
3.59 \\
22.15 \\
*\end{array}$ & $\begin{array}{r}24.10 \\
3.14 \\
13.03 \\
0.80\end{array}$ & $\begin{array}{l}10.72 \\
0.05 \\
0.47 \\
*\end{array}$ & $\begin{array}{l}* \\
-- \\
--\end{array}$ & $\begin{array}{r}1.80 \\
0.31 \\
17.23 \\
*\end{array}$ & $\begin{array}{l}1.80 \\
0.06 \\
3.34 \\
+\end{array}$ & $\begin{array}{r}101.09 \\
13.73\end{array}$ \\
\hline 1964 & $\begin{array}{l}\text { PRECIP. (CM.) } \\
\text { SR-90 INCI/SG.KN.) } \\
\text { SR-9O CCNC. (PC/L) } \\
\text { SR-8S/SR-9O }\end{array}$ & $\begin{array}{l}* \\
\mathrm{C} . \mathrm{CH}_{4} \\
--\end{array}$ & $\begin{array}{l}* \\
0.10 \\
--\end{array}$ & $\begin{array}{r}12.17 \\
0.07 \\
0.58 \\
*\end{array}$ & $\begin{array}{l}6.81 \\
0.10 \\
1.47 \\
0.25\end{array}$ & $\begin{array}{l}11.86 \\
0.08 \\
0.68 \\
*\end{array}$ & $\begin{array}{l}19.84 \\
0.04 \\
0.21 \\
--\end{array}$ & $\begin{array}{l}27.03 \\
0.06 \\
0.23 \\
--\end{array}$ & $\begin{array}{r}12.37 \\
0.05 \\
0.41 \\
--\end{array}$ & $\begin{array}{l}19.59 \\
0.07 \\
0.36 \\
--\end{array}$ & $\begin{array}{l}5.38 \\
0.02 \\
C .38 \\
¥\end{array}$ & $\begin{array}{r}0.15 \\
0.06 \\
40.01 \\
--\end{array}$ & $\begin{array}{l}5.03 \\
0.03 \\
0.60 \\
--\end{array}$ & $\begin{array}{r}120.63 \\
0.72\end{array}$ \\
\hline 1965 & $\begin{array}{l}P R E C I P \text { : }(C M .) \\
S R-9 C \text { (NCI/SQ-KM.) } \\
S R-S C \text { CCNC. }(P C / L) \\
S R-89 / S R-90\end{array}$ & $\begin{array}{l}0.97 \\
0.07 \\
7.22 \\
-.\end{array}$ & $\begin{array}{l}0.64 \\
\pm \\
-- \\
--\end{array}$ & $\begin{array}{l}3.81 \\
0.13 \\
3.42 \\
-.\end{array}$ & $\begin{array}{l}5.33 \\
0.02 \\
0.38 \\
--\end{array}$ & $\begin{array}{r}0.91 \\
0.20 \\
21.98 \\
--\end{array}$ & $\begin{array}{l}6.17 \\
0.34 \\
5.52 \\
--\end{array}$ & $\begin{array}{l}27.46 \\
C .86 \\
2.41 \\
-.\end{array}$ & $\begin{array}{l}27.58 \\
C .54 \\
0.15 \\
-.\end{array}$ & $\begin{array}{r}11.61 \\
6.14 \\
1.21 \\
-.\end{array}$ & $\begin{array}{l}11.25 \\
6.05 \\
C .45 \\
--\end{array}$ & $\begin{array}{r}0.48 \\
0.15 \\
31.26 \\
-.\end{array}$ & $\begin{array}{l}4 \\
0.05 \\
--\end{array}$ & $\begin{array}{r}90.21 \\
1.85\end{array}$ \\
\hline 1966 & $\begin{array}{l}\text { PRECIP. (CM.) } \\
S R-9 C \text { (NCI/SQ.KN.) } \\
S R-90 \text { CCNC. }(P C / L) \\
S R-89 / S R-90\end{array}$ & $\begin{array}{r}0.05 \\
0.08 \\
160.00 \\
--\end{array}$ & $\begin{array}{l}-- \\
0.07 \\
-- \\
--\end{array}$ & $\begin{array}{l}8.13 \\
0.17 \\
2.10 \\
--\end{array}$ & $\begin{array}{l}8.38 \\
0.15 \\
1.79 \\
--\end{array}$ & $\begin{array}{l}0.38 \\
0.06 \\
15.75 \\
--.\end{array}$ & $\begin{array}{l}13.75 \\
0.31 \\
2.2 t \\
6.90\end{array}$ & $\begin{array}{r}29.22 \\
0.10 \\
0.35 \\
5.30\end{array}$ & $\begin{array}{r}32.97 \\
0.18 \\
0.55 \\
3.80\end{array}$ & $\begin{array}{l}15.00 \\
0.16 \\
1.07 \\
*\end{array}$ & $\begin{array}{l}5.70 \\
C .05 \\
c .88 \\
+\end{array}$ & $\begin{array}{r}0.32 \\
0.04 \\
12.51 \\
\$\end{array}$ & $\begin{array}{l}7 \\
0.01 \\
-- \\
+\end{array}$ & $\begin{array}{r}113.90 \\
1.36\end{array}$ \\
\hline 1967 & $\begin{array}{l}\text { PRECIP. (CM.) } \\
\text { SR-9C (NCI/SQ.KN.) } \\
\text { SR-90 CONC. (PC/L) } \\
\text { SR-89/SR-90 }\end{array}$ & $\begin{array}{l}* \\
\mathrm{C} . \mathrm{Cl} \\
--\end{array}$ & $\begin{array}{r}0.50 \\
0.07 \\
14.01 \\
*\end{array}$ & $\begin{array}{l}7.69 \\
0.05 \\
0.66 \\
+\end{array}$ & $\begin{array}{l}2.28 \\
0.05 \\
2.20 \\
4\end{array}$ & $\begin{array}{l}12.89 \\
0.03 \\
0.24 \\
*\end{array}$ & $\begin{array}{l}8.77 \\
0.11 \\
1.26\end{array}$ & $\begin{array}{l}-- \\
-- \\
--\end{array}$ & $\begin{array}{l}-- \\
-- \\
--\end{array}$ & $\begin{array}{l}-- \\
-- \\
--\end{array}$ & $\begin{array}{l}-\infty \\
-- \\
--\end{array}$ & $\begin{array}{l}-- \\
-- \\
--\end{array}$ & $\begin{array}{l}-- \\
-- \\
-- \\
--\end{array}$ & $\begin{array}{r}32.13 \\
0.32\end{array}$ \\
\hline \begin{aligned} & NOTES \\
& \hdashline$: D \\
& *: 2 \\
& A: A \\
& B: L \\
& C: P\end{aligned}$ & $\begin{array}{l}\text { SATA NOT AVAILABLE } \\
\text { IERO OR TRACE } \\
\text { APPROXIMATE } \\
\text { OWER LIMIT OF REPORT } \\
\text { PROPORTIOAED FROM ORI }\end{array}$ & $\begin{array}{l}\text { TED DATA } \\
\text { IGINALLY }\end{array}$ & & 0 & & & & & & & & & & \\
\hline
\end{tabular}


FEB. MAR. APR. MAY

1959 PRECIP. ICM

SR-90 (MCI/SQ.KH.)
PRECIP A ICM.)

33.86

21.08

SR-90 CONC. (PC/L

$-$

$S R-89 / S R-90$

ISGC PRECIP. ICM.)

(CM.) 39.27

SR-

39.27

$S R-90$ CONC. $(P C / L)$

0.09
0.23

$S R-89 / S R-90$

$-$.

1961 PRECIP. ICM.) $S R-S C(N C I / S O . K N$.

32.56 SR-GC CCNC. (PC/L) $C . C 2 C$
$C .07$ $S R-89 / S R-90$ $--$

1902 PRECIP. (CM.) SR-SC INCI/SC.KN.) $\quad 53.11$ $\begin{array}{ll}S R-9 C \text { CCAC. }(P C / L) & C .14 \\ S R-89 / S R-90 & 2.20\end{array}$

$-$

24.10 0.08 0.34

26.77 $0.02 \mathrm{C}$ C. 08

1963 PRECIP. (CN.) 39.40
$S R-90(N C I / S C . K M)$ SR-90 CCNC. (PC/L) 0.39

54.97

C. 12 0.22
3.00

44.27

44.40

mar

JUNE

JULY

AUG.

SEP

OCT

nov.

DEC.

CUM. TOTAL

16.87

0.14
0.83

7.00$$
=
$$$$
-
$$$$
\text { -- }
$$

3.35

48.7
0.1
0.2

3.89

22.94

$-$

$\because$

275.08 0.12

$26.92 \quad 12.37$

$\begin{array}{lll}0.35 & 12.37 & 16.38\end{array}$

16.38
$0.05 \mathrm{C}$

27.41

$-$

0.38

$* 01$

--

$-$

$-$

--

0.31

0.33

29.01
0.100

$11.10 \quad 11.99$

13.51

$45.57 \quad 23.7$

$0.11 \mathrm{C} \quad 0.05 \mathrm{C}$

$\begin{array}{lll}0.30 & 0.25 & 0.22\end{array}$

281.35
0.75

0.26

$\begin{array}{ccccccc}0.67 & 34.54 & 18.01 & 17.70 & 15.70 & 12.93 & 35.64 \\ 0.03 \mathrm{C} & 0.12 \mathrm{C} & 0.01 \mathrm{C} & 0.01 \mathrm{C} & 0.07 \mathrm{C} & 0.05 \mathrm{C} & 0.08\end{array}$

$0.03 \mathrm{C} \quad 0.12 \mathrm{C}$

5.64
0.08

0.67
$C .26$

$63.47 \quad 48.90$

0.320 .01

$\begin{array}{rr}0.51 & 0.03 \\ 0.50 & 32.00\end{array}$

-

-.

$-\infty$

$--4$

$-$

3.00

95.00

23.85

$\begin{array}{lr}0.25 & 0.22\end{array}$

$0.93 \quad 0.93$

9.00

1.47

$\begin{array}{ll}.05 & 0.14 \\ 4.00 & 4.00\end{array}$

$\begin{array}{lll}0.03 & 0.28 & 0.17 \\ 0.18 & 0.86 & 1.35\end{array}$

$\begin{array}{rrr}8.18 & 12.65 & 11.99 \\ -- & 0.12 & 0.16\end{array}$

0.95
12.00

$\stackrel{1.34}{*}$

51.59

$5.74 \quad 50.57 \quad 27.66$

10.31

0.49

0.18
0.35
$*$

50.63

$\begin{array}{rrrr}0.07 & 0.04 & -- & 0.1 \mathrm{C} \\ 0.24 & 0.08 & -- & 0.89\end{array}$

1.75

0.36
0.72
$*$

0.91

1.17

2.06

PRECIP. (CM.)

$\begin{array}{rrrrr}45.69 & 43.71 & 34.04 & 24.61 & 31.39 \\ 0.11 & 0.09 & 0.09 & 0.19 & 0.18\end{array}$

$3.48 \quad 15.9$

SR-9O (NCI/SQ.KM.)

$S R-9 C$ CCNC. $(P C / L) \quad C .54$

C. 2

0.21

0.27

0.78

0.58

5.25

PRECIP. (CM.)

21.53

53.49

50.75

$23.83 \quad 50.50$

SR-SC CCNC (PCIL)

$S R-8 S / S R-90$

C. 33

C. 0

0.2

$\begin{array}{rrr}23.83 & 50.50 & 3.58 \\ 0.37 & 0.12 & 0.25 \\ 1.56 & 0.24 & 8.11\end{array}$

-

0.24

8.11

$30.86 \quad 38.40$

21.16

0.14

0.67

319.67

*.4

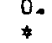

$--$

$\begin{array}{llllll}7.01 & 23.55 & 13.21 & 11.61 C & 11.61 C & 0.76\end{array}$

2.72

PRECIP. (CM.)

8.74

19.68

11.4

0.22

1.26
1.97

0.08

0.08

0.76
0.06

0.89

277.13

1.86 $S R-8 S / S R-S O$

0.61

0.35

C. 04

$0.04 \mathrm{C}$

$0.04 \mathrm{C}$

0.06
0.31
+

$0.07 \quad 9.55$

3.30

34

24.03

$\begin{array}{lllll}0.84 & 1.22 & 0.21 & 0.12 & 0.16 \\ 16.00 & 1.52 & 0.73 & 0.67\end{array}$ $\begin{array}{llllll}.5 C & 16.0 \mathrm{C} & 1 \mathrm{C} . \mathrm{CO} & 30.00 & 6.00 & 14.00\end{array}$

117.10

- $20.02 \quad-$

25.40

$\begin{array}{lll}S R-90 \text { (NCI/SO.KM.) } & C . C 7 \\ \text { SR-9O CCAC (PCIL) } & 0.28\end{array}$ SR- $-80 / 2 R-2$ C

$27.94 \quad--$

0.05
0.18

0.

$-$

$-$

--

-- -

$--$

NOTES

--: DATA NOT AVAILABLE

* : ZERO OR TRACE

A: APPROXIMATE

B: LOWER LIMIT OF REPCRTED DATA

C: PRCPORTICNED FROM CRIGINALLY CONSOLICATEO CATA 


\begin{tabular}{|c|c|c|c|c|c|c|c|c|c|c|c|c|c|c|}
\hline & & JAN. & FEB. & MAR. & APR. & MAY & JUNE & JULY & AUG. & SEP. & OCT. & NOY. & DEC. & CUM. TOTAL \\
\hline 1959 & $\begin{array}{l}\text { PRECIP. (CM.) } \\
\text { SR-90 IMCI/SQ.KM.) } \\
\text { SR-90 CONC. }(P C / L) \\
\text { SR-89/SR-90 }\end{array}$ & 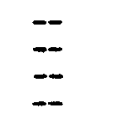 & $\ddot{m}=$ & $\begin{array}{l}-- \\
--\end{array}$ & $=$ & $\overline{-}$ & $\bar{m}$ & $\begin{array}{l}-- \\
--\end{array}$ & $\begin{array}{l}-- \\
-- \\
--\end{array}$ & $=$ & $\begin{array}{l}2.74 \\
0.02 \\
0.73 \\
+\end{array}$ & $\begin{array}{l}0.56 \\
0.02 \\
3.58 \\
*\end{array}$ & $\begin{array}{l}0.64 \\
0.01 \\
1.57 \\
+\end{array}$ & $\begin{array}{l}3.94 \\
0.05\end{array}$ \\
\hline 1960 & $\begin{array}{l}\text { PRECIP. (CM.) } \\
\text { SR-9C IMCI/SQ.KM.) } \\
\text { SR-9O CONC. (PC/L) } \\
\text { SR-99/SR-9O }\end{array}$ & $\begin{array}{l}0.58 \\
0.07 \\
12.07 \\
--\end{array}$ & $\begin{array}{l}0.61 \\
0.02 \\
3.28 \\
-.-\end{array}$ & $\begin{array}{l}1.45 \\
0.06 \\
4.14 \\
-.\end{array}$ & $\begin{array}{l}0.18 \\
0.08 \\
44.45 \\
--\end{array}$ & $\begin{array}{l}0.58 \\
0.06 \mathrm{C} \\
1 \mathrm{C} .35 \\
--\end{array}$ & $\begin{array}{l}0.38 \\
0.036 \\
7.96 \\
--\end{array}$ & $\begin{array}{l}1.70 \\
0.08 C \\
4.71 \\
--\end{array}$ & $\begin{array}{l}0.30 \\
0.026 \\
6.67 \\
--\end{array}$ & $\begin{array}{l}1.73 \\
C .016 \\
C .58 \\
--\end{array}$ & $\begin{array}{l}0.94 \\
0.01 \mathrm{C} \\
1.07 \\
-.\end{array}$ & $\begin{array}{l}2.34 \\
0.05 C \\
2.14 \\
-.\end{array}$ & $\begin{array}{l}0.38 \\
0.016 \\
2.64 \\
--\end{array}$ & $\begin{array}{r}11.17 \\
0.50\end{array}$ \\
\hline 1961 & $\begin{array}{l}\text { PRECIP. (CM.) } \\
\text { SR-90 IMCI/SQ.KM-) } \\
S R-90 \text { CONC. (PC/L) } \\
\text { SR-8S/SR-9O }\end{array}$ & $\begin{array}{l}0.41 \\
0.016 \\
2.44 \\
-.\end{array}$ & $\stackrel{*}{0.00 c}$ & $\begin{array}{l}0.20 \\
+ \\
--\end{array}$ & $\begin{array}{l}0.41 \\
-- \\
=-\end{array}$ & $\begin{array}{l}0.99 \\
0.036 \\
3.04 \\
--\end{array}$ & $\begin{array}{l}1.52 \\
0.05 C \\
3.29 \\
--\end{array}$ & $\begin{array}{l}1.57 \\
0.036 \\
1.92 \\
-.\end{array}$ & $\begin{array}{l}2.01 \\
0.036 \\
1.50 \\
-.\end{array}$ & $\begin{array}{r}0.84 \\
0.01 \\
1.2 \mathrm{C} \\
22.00\end{array}$ & $\begin{array}{r}0.56 \\
0.02 \\
3.58 \\
40.00\end{array}$ & $\begin{array}{r}0.41 \\
0.41 \\
100.00 \\
89.00\end{array}$ & $\begin{array}{r}0.58 \\
0.02 \\
3.45 \\
47.00\end{array}$ & $\begin{array}{l}9.50 \\
0.61\end{array}$ \\
\hline 1962 & $\begin{array}{l}\text { PRECIP ( }(C M .) \\
\text { SR-9O }\left(M C I / S Q_{-K M}\right) \\
S R-9 O \text { CONC. }(P C / L) \\
S R-89 / S R-S O\end{array}$ & $\begin{array}{l}0.51 \\
* \\
-- \\
--\end{array}$ & $\begin{array}{l}* \\
* \\
--\end{array}$ & $\begin{array}{l}1.04 \\
- \\
-\end{array}$ & $\begin{array}{l}1.68 \\
0.04 \\
2.39 \\
9.00\end{array}$ & $\begin{array}{l}1.32 \\
0.05 \\
3.79 \\
6.00\end{array}$ & $\stackrel{*}{*}$ & $\begin{array}{r}0.51 \\
0.12 \\
23.53 \\
11.00\end{array}$ & $\begin{array}{l}2.62 \\
0.25 \\
9.55 \\
7.00\end{array}$ & $\begin{array}{r}1.37 \\
0.03 \\
2.19 \\
21.00\end{array}$ & $\begin{array}{r}1.22 \\
0.09 \\
7.38 \\
18.00\end{array}$ & $\begin{array}{r}0.84 \\
0.03 \\
3.58 \\
26.00\end{array}$ & $\begin{array}{r}0.53 \\
0.02 \\
3.78 \\
49.00\end{array}$ & $\begin{array}{r}11.64 \\
0.63\end{array}$ \\
\hline 1963 & $\begin{array}{l}\text { PRECIP. (CM.) } \\
\text { SR-9O INCI/SQ.KM.) } \\
\text { SR-9OCONC. (PC/L) } \\
\text { SR-QS/SR-SO }\end{array}$ & $\begin{array}{r}2.72 \\
0.03 \\
1.11 \\
22.00\end{array}$ & $\begin{array}{r}1.91 \\
0.06 \\
3.15 \\
27.00\end{array}$ & $\begin{array}{r}0.28 \\
0.00 \\
0.01 \\
10.00\end{array}$ & $\begin{array}{l}0.38 \\
0.02 \\
5.27 \\
7.70\end{array}$ & $\begin{array}{l}0.81 \\
0.07 \\
8.65 \\
7.10\end{array}$ & $\begin{array}{r}0.25 \\
0.20 \\
80.01 \\
1.40\end{array}$ & $\begin{array}{r}3.02 \\
C .83 \\
27.49 \\
--\end{array}$ & $\begin{array}{r}3.53 \\
2.02 \\
57.23 \\
*\end{array}$ & $\begin{array}{l}1.65 \\
0.15 \\
9.10 \\
*\end{array}$ & $\begin{array}{r}C .97 \\
C .88 \\
9 C .73 \\
*\end{array}$ & $\begin{array}{l}1.22 \\
0.03 \\
2.46 \\
*\end{array}$ & $\begin{array}{l}0.66 \\
0.04 \\
6.07 \\
*\end{array}$ & $\begin{array}{r}17.40 \\
4.33\end{array}$ \\
\hline 1964 & $\begin{array}{l}\text { PRECIP. (CM.) } \\
\text { SR-90 (NCI/SQ.KM.) } \\
S R-90 \text { CCNC. }(P C / L) \\
\text { SR-89/SR-90 }\end{array}$ & $\begin{array}{l}3.00 \\
0.10 \\
3.34 \\
*\end{array}$ & $\begin{array}{l}3.00 \\
0.05 \\
1.67 \\
0.50\end{array}$ & $\begin{array}{l}0.48 \\
0.02 \\
4.17 \\
+\end{array}$ & $\begin{array}{l}0.51 \\
0.13 \\
25.50 \\
*\end{array}$ & $\begin{array}{l}0.69 \\
0.67 \\
10.15 \\
*\end{array}$ & $\begin{array}{r}0.05 \\
0.19 \\
380.00 \\
--\end{array}$ & $\begin{array}{l}1.14 \\
0.63 \\
55.27 \\
--\end{array}$ & $\begin{array}{l}1.17 \\
0.13 \\
11.12 \\
--\end{array}$ & $\begin{array}{l}1.65 \\
-\infty \\
-\infty\end{array}$ & $\begin{array}{l}2.29 \\
0.06 \\
2.63 \\
*\end{array}$ & $\begin{array}{l}2.29 \\
0.04 \\
1.75 \\
*\end{array}$ & $\begin{array}{l}1.14 \\
0.04 \\
3.51 \\
-.\end{array}$ & $\begin{array}{r}17.41 \\
1.46\end{array}$ \\
\hline 1965 & $\begin{array}{l}\text { PRECIP. (CM.) } \\
\text { SR-90 (MCI/SQ.KM.) } \\
\text { SR-9O CONC. (PC/L) } \\
\text { SR-89/SR-9O }\end{array}$ & $\begin{array}{l}1.14 \\
0.01 \\
0.88 \\
-.\end{array}$ & $\begin{array}{l}3.38 \\
0.03 \\
0.89 \\
--\end{array}$ & $\begin{array}{l}1.37 \\
0.02 \\
1.46 \\
-\end{array}$ & $\begin{array}{l}0.79 \\
0.02 \\
2.54 \\
--\end{array}$ & $\begin{array}{r}1.45 \\
0.42 \\
28.97 \\
--\end{array}$ & $\begin{array}{l}1.30 \\
0.07 \\
5.39 \\
-.\end{array}$ & $\begin{array}{l}1.85 \\
0.17 \\
9.19 \\
--\end{array}$ & $\begin{array}{c}0.36 \\
0.13 \\
36.12 \\
--\end{array}$ & $\begin{array}{l}1.70 \\
0.11 \\
6.48 \\
-.\end{array}$ & $\begin{array}{l}1.80 \\
C .01 \\
0.56 \\
--\end{array}$ & $\begin{array}{l}0.79 \\
0.02 \\
2.54 \\
-\infty\end{array}$ & $\begin{array}{l}0.67 C \\
0.016 \\
1.50 \\
-.\end{array}$ & $\begin{array}{r}16.60 \\
1.02\end{array}$ \\
\hline 1966 & $\begin{array}{l}\text { PRECIP (CM.) } \\
\text { SR-9C (MCI/SQ.KM.) } \\
\text { SR-9O CONC. (PC/L) } \\
\text { SR-89/SR-90 }\end{array}$ & $\begin{array}{l}0.67 C \\
0.016 \\
1.50 \\
--\end{array}$ & $\begin{array}{l}0.67 \mathrm{C} \\
0.01 \mathrm{C} \\
1.50 \\
--\end{array}$ & $\begin{array}{l}0.67 C \\
0.02 C \\
2.99 \\
-.\end{array}$ & $\begin{array}{l}0.58 \mathrm{C} \\
0.02 \mathrm{C} \\
3.45 \\
\end{array}$ & $\begin{array}{l}0.58 \mathrm{C} \\
0.02 \mathrm{C} \\
3.45 \\
-.\end{array}$ & $\begin{array}{l}0.86 \\
0.07 \\
8.14 \\
--\end{array}$ & $\begin{array}{r}2.18 \\
0.04 \\
1.84 \\
15.00\end{array}$ & $\begin{array}{l}4.82 \\
0.12 \\
2.49 \\
7.10\end{array}$ & $\begin{array}{l}2.63 \\
0.02 \\
0.77 \\
+\end{array}$ & $\begin{array}{l}1.55 \\
0.02 \\
1.30 \\
\end{array}$ & $\begin{array}{r}0.08 \\
0.01 \\
12.51 \\
*\end{array}$ & $\begin{array}{l}-- \\
0.01 \\
*\end{array}$ & $\begin{array}{r}15.29 \\
0.37\end{array}$ \\
\hline 1967 & $\begin{array}{l}\text { PRECIP. (CM.) } \\
\text { SR-90 (MCI/SQ.KM.) } \\
\text { SR-90 CONC. (PC/L) } \\
\text { SR-89/SR-90 }\end{array}$ & $\begin{array}{l}0.18 \\
0.01 \\
5.56 \\
*\end{array}$ & $\begin{array}{l}0.25 \\
0.01 \\
4.01 \\
+\end{array}$ & $\begin{array}{l}0.23 \\
0.01 \\
4.35 \\
*\end{array}$ & $\begin{array}{l}0.15 \\
0.01 \\
6.67 \\
\end{array}$ & $\begin{array}{l}1.88 \\
0.05 \\
2.66 \\
5.20\end{array}$ & $\begin{array}{l}=- \\
=- \\
--\end{array}$ & $\begin{array}{l}-- \\
-- \\
--\end{array}$ & $=$ & $\begin{array}{l}-- \\
-- \\
-\end{array}$ & $\begin{array}{l}= \\
=- \\
--\end{array}$ & $\begin{array}{l}=- \\
=- \\
=-\end{array}$ & $\begin{array}{l}-- \\
-- \\
--\end{array}$ & $\begin{array}{l}2.69 \\
0.09\end{array}$ \\
\hline $\begin{array}{l}\text { NOTES } \\
--:=0 \\
*: 2 \\
A: A \\
B: 1 \\
C: P\end{array}$ & $\begin{array}{l}\text { S } \\
\text { DATA NOT AVAILABLE } \\
\text { ZERD OR TRACE } \\
\text { APPROXIMATE } \\
\text { LOWER LIMIT OF REPDRT } \\
\text { PROPORTI ONEO FROM ORI }\end{array}$ & $\begin{array}{l}\text { DATA } \\
\text { NALIY }\end{array}$ & & & & & & & & & & & & \\
\hline
\end{tabular}


SOURCE: UNITED STATES CONSULATE

\begin{tabular}{|c|c|c|c|c|c|c|c|c|c|c|c|c|c|c|}
\hline & & JAN. & FEB. & MAR. & APR. & MAY & JUNE & JULY & AUG. & SEP. & OCT. & NOV. & DEC. & CUM. TOTAL \\
\hline 1960 & $\begin{array}{l}\text { PRECIP. (CH.) } \\
\text { SR-90 (MCI/SQ.KM-) } \\
\text { SR-90 CONC. (PC/L) } \\
\text { SR-89/SR-90. }\end{array}$ & $\begin{array}{l}0.99 \\
0.05 \\
5.06 \\
-\end{array}$ & $\stackrel{-}{-}$ & $\begin{array}{l}24.99 \\
0.07 \\
0.29 \\
-\end{array}$ & $\begin{array}{l}3.99 \\
- \\
-\end{array}$ & $\begin{array}{l}24.99 \\
0.09 \\
0.37 \\
--\end{array}$ & $\begin{array}{l}70.00 \\
=- \\
=\end{array}$ & $\begin{array}{l}16.00 \\
=- \\
=\end{array}$ & $\begin{array}{l}54.00 \\
= \\
=\end{array}$ & $\begin{array}{l}31.09 \\
=- \\
=\end{array}$ & $\begin{array}{l}25.98 \\
-- \\
=\end{array}$ & $\begin{array}{l}13.79 \\
=- \\
=\end{array}$ & $\begin{array}{l}0.13 \\
=- \\
-\end{array}$ & $\begin{array}{r}265.95 \\
0.21\end{array}$ \\
\hline 1961 & $\begin{array}{l}\text { PRECIP, (CM.) } \\
\text { SR-90 (NCI/SQ-KM-) } \\
S R-90 \text { CONC. (PC/L) } \\
S R-89 / S R-90\end{array}$ & $\begin{array}{l}0.38 \\
-- \\
--\end{array}$ & $\begin{array}{l}4.62 \\
-- \\
--\end{array}$ & $\begin{array}{l}5.13 \\
0.08 \mathrm{C} \\
1.56 \\
0.61\end{array}$ & $\begin{array}{c}17.22 \\
0.116 \\
0.64 \\
0.61\end{array}$ & $\begin{array}{l}21.39 \\
0.03 C \\
0.15 \\
0.15\end{array}$ & $\begin{array}{c}12.09 \\
0.05 C \\
0.42 \\
0.15\end{array}$ & $\begin{array}{l}49.20 \\
=- \\
=-\end{array}$ & $\begin{array}{l}46.20 \\
0.05 \\
0.11 \\
--\end{array}$ & $\begin{array}{l}53.49 \\
* \\
*\end{array}$ & $\begin{array}{l}3.12 \\
-- \\
--\end{array}$ & $\begin{array}{l}9.14 \\
-- \\
--\end{array}$ & $\begin{array}{l}1.22 \\
-- \\
--\end{array}$ & $\begin{array}{r}223.20 \\
0.32\end{array}$ \\
\hline 1962 & $\begin{array}{l}\text { PRECIP, (CM.) } \\
\text { SR-90 (MCI/SQ-KM.) } \\
\text { SR-90 CCNC. }(P C / L) \\
\text { SR-89/SR-9O }\end{array}$ & $\begin{array}{l}0.71 \\
-- \\
--\end{array}$ & $\begin{array}{l}8.26 \\
-- \\
--\end{array}$ & $\begin{array}{r}1.04 \\
0.54 \\
51.93 \\
18.00\end{array}$ & $\begin{array}{r}5.05 \\
0.27 \\
5.35 \\
13.00\end{array}$ & $\begin{array}{r}18.47 \\
0.38 \\
2.06 \\
-\end{array}$ & $\begin{array}{r}50.50 \\
0.40 \\
0.80 \\
21.00\end{array}$ & $\begin{array}{r}15.77 \\
6.15 \\
0.96 \\
10.00\end{array}$ & $\begin{array}{r}8.48 \\
0.03 \\
0.36 \\
12.00\end{array}$ & $\begin{array}{l}45.59 \\
=- \\
=-\end{array}$ & $\begin{array}{r}16.84 \\
C .38 \\
2.26 \\
--\end{array}$ & $\begin{array}{l}3.38 \\
0.05 C \\
1.48 \\
-.\end{array}$ & $\begin{array}{l}* \\
0.05 C \\
--\end{array}$ & $\begin{array}{r}174.09 \\
2.25\end{array}$ \\
\hline 1963 & $\begin{array}{l}\text { PRECIP. (CM.) } \\
\text { SR-9O IMCI/SQ.KM.) } \\
\text { SR-9O CONC. (PCIL) } \\
\text { SR-89/SR-90 }\end{array}$ & $\begin{array}{r}0.99 \\
0.11 \\
11.12 \\
40.00\end{array}$ & $\begin{array}{c}0.15 \\
0.35 \mathrm{C} \\
233.34 \\
--\end{array}$ & $\begin{array}{l}0.91 \\
0.35 C \\
38.47 \\
=-\end{array}$ & $\begin{array}{l}1.37 \\
0.35 C \\
25.55 \\
=-\end{array}$ & $\begin{array}{l}0.61 \\
0.35 C \\
57.38 \\
--\end{array}$ & $\begin{array}{l}20.47 \\
0.35 C \\
1.71 \\
--\end{array}$ & $\begin{array}{l}32.36 \\
0.10 \mathrm{C} \\
c .31 \\
--\end{array}$ & $\begin{array}{l}18.21 \\
0.10 \mathrm{C} \\
0.55 \\
--\end{array}$ & $\begin{array}{r}8.31 \\
0.89 \\
10.71 \\
*\end{array}$ & $\begin{array}{l}3.51 \\
0.53 \\
15.10 \\
*\end{array}$ & $\begin{array}{l}3.15 \\
-- \\
--\end{array}$ & $\begin{array}{r}0.10 \\
0.02 \\
20.01 \\
2.50\end{array}$ & $\begin{array}{r}90.14 \\
3.50\end{array}$ \\
\hline 1964 & $\begin{array}{l}\text { PRECIP. (CM-) } \\
\text { SR }-90 \text { IMCI/SQ.KM-) } \\
S R-90 \text { CONC. }(P C / L) \\
\text { SR-89/SR-90 }\end{array}$ & $\begin{array}{c}10.06 \\
1.04 \\
10.34 \\
*\end{array}$ & $\begin{array}{l}1.12 \\
-- \\
--\end{array}$ & $\begin{array}{l}3.71 \\
0.12 \\
3.24 \\
*\end{array}$ & $\begin{array}{r}1.73 \\
0.25 \\
14.46 \\
1.80\end{array}$ & $\begin{array}{l}41.30 \\
0.24 \\
C .59 \\
*\end{array}$ & $\begin{array}{l}26.39 \\
0.54 \\
2.05 \\
--\end{array}$ & $\begin{array}{r}10.36 \\
0.18 \\
1.74 \\
-.\end{array}$ & $\begin{array}{l}44.50 \\
0.02 \\
0.05 \\
--\end{array}$ & $\begin{array}{l}51.84 \\
-- \\
--\end{array}$ & $\begin{array}{l}51.49 \\
=- \\
--\end{array}$ & $\begin{array}{l}0.41 \\
-- \\
--\end{array}$ & $\begin{array}{l}0.30 \\
-- \\
--\end{array}$ & $\begin{array}{r}243.21 \\
2.39\end{array}$ \\
\hline 1965 & $\begin{array}{l}\text { PRECIP. (CM.) } \\
\text { SR-9O (MCI/SQ.KM-) } \\
\text { SR-SC CONC: }(P C / L) \\
S R-89 / S R-90\end{array}$ & $\begin{array}{l}1.30 \\
= \\
=\end{array}$ & $\begin{array}{l}1.60 \\
0.026 \\
1.26 \\
-\infty\end{array}$ & $\begin{array}{l}0.89 \\
0.036 \\
3.38 \\
-.\end{array}$ & $\begin{array}{l}25.32 \\
0.00 \\
0.01 \\
--\end{array}$ & $\begin{array}{l}16.41 \\
* \\
--\end{array}$ & $\begin{array}{l}33.12 \\
0.05 \\
0.16 \\
-\end{array}$ & $\begin{array}{l}34.32 \\
6.02 \\
0.06 \\
--\end{array}$ & $\begin{array}{l}17.02 \\
0.02 \\
0.06 \\
--\end{array}$ & $\begin{array}{l}79.86 \\
0.01 \\
0.02 \\
-.\end{array}$ & $\begin{array}{l}15.62 \\
* \\
=-\end{array}$ & $\begin{array}{l}-- \\
0.04 \\
--\end{array}$ & $\begin{array}{l}= \\
=- \\
=-\end{array}$ & $\begin{array}{r}225.46 \\
0.18\end{array}$ \\
\hline 1966 & $\begin{array}{l}\text { PRECIP. (CM.) } \\
\text { SR-90 (MCI/SQ.KM.) } \\
\text { SR-90 CONC. (PC/L) } \\
\text { SR-89/SR-90 }\end{array}$ & $\begin{array}{l}* \\
0.06 \\
--\end{array}$ & $\begin{array}{r}10.00 \\
0.16 \\
1.61 \\
-\end{array}$ & $\begin{array}{r}12.60 \\
0.03 \\
0.26 \\
--\end{array}$ & $\begin{array}{l}33.70 \\
\div-\end{array}$ & $\begin{array}{l}-0 \\
- \\
-\end{array}$ & $\begin{array}{l}96.30 \\
0.01 \\
0.02 \\
*\end{array}$ & $\begin{array}{r}47.40 \\
0.01 \\
C .03 \\
*\end{array}$ & $\ddot{*}$ & +- & $\overline{0.01}$ & $\begin{array}{l}-\overline{0.01} \\
-- \\
*\end{array}$ & $\begin{array}{l}-- \\
0.01 \\
+-\end{array}$ & $\begin{array}{r}199.00 \\
0.32\end{array}$ \\
\hline 1967 & $\begin{array}{l}\text { PRECIP. (CM.) } \\
\text { SR-90 IMCI/SQ.KM.) } \\
\text { SR-90 CONC. (PC/L) } \\
\text { SR-89/SR-90 }\end{array}$ & $\begin{array}{l}0.80 \\
0.02 \\
2.51\end{array}$ & $\begin{array}{l}3.80 \\
0.02 \\
0.53 \\
*\end{array}$ & $\begin{array}{r}0.30 \\
0.07 \\
23.34 \\
*\end{array}$ & $\begin{array}{l}-- \\
0.04\end{array}$ & $\begin{array}{l}2.90 \\
0.01 \\
0.35 \\
*\end{array}$ & $\begin{array}{l}35.70 \\
0.01 \\
0.03 \\
*\end{array}$ & $\ddot{--}$ & $m-$ & $=$ & $\overline{--}$ & $\begin{array}{l}-- \\
-- \\
-\end{array}$ & 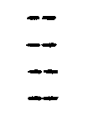 & $\begin{array}{r}43.50 \\
0.17\end{array}$ \\
\hline 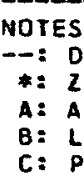 & $\begin{array}{l}\text { ATA NOT AVAILABLE } \\
\text { ERO OR TRACE } \\
\text { PPROXIMATE } \\
\text { OHER LIMIT OF REPOR } \\
\text { ROPORTI ONED FROM OR }\end{array}$ & $\begin{array}{l}\text { DATA } \\
\text { NALLY }\end{array}$ & & 8 & & & & & & & & & & \\
\hline
\end{tabular}


MONTHLY FALLCUT DEPOSITION COLLECTIONS

SITE: IRAN, TEHRAN

LAT. $3544 \mathrm{~N}$ LONG. $5123 \mathrm{E}$ ALT. 1362M. (POT)

SOURCE: TEHRAN UNIVERSITY NUCLEAR CENTER

\begin{tabular}{|c|c|c|c|c|c|c|c|c|c|c|c|c|c|c|}
\hline & & JAN. & FEB. & MAR. & APR. & MAY & JUNE & JULY & AUG. & SEP. & OCT. & NOV. & DEC. & CUM. TOTAL \\
\hline 1962 & $\begin{array}{l}\text { PRECIP. (CM.) } \\
S R-90 \text { (MCI/SQ.KM.) } \\
S R-90 \text { CONC. (PCAL) } \\
S R-89 / S R-90\end{array}$ & $\begin{array}{l}1.42 \\
-- \\
--\end{array}$ & $\begin{array}{l}11.00 \\
=- \\
=-\end{array}$ & $\begin{array}{l}0.46 \\
-- \\
--\end{array}$ & $\begin{array}{l}13.28 \\
=- \\
=-\end{array}$ & $\begin{array}{l}0.38 \\
-- \\
--\end{array}$ & $\begin{array}{r}0.38 \\
0.25 \\
65.79 \\
8.0 C\end{array}$ & $\begin{array}{l}* \\
0.03 \\
13.00\end{array}$ & $\frac{0.07}{13.00}$ & $\begin{array}{r}0.23 \\
0.08 \\
34.79 \\
16.00\end{array}$ & $\begin{array}{r}1.55 \\
0.42 \\
27.10 \\
11.00\end{array}$ & $\begin{array}{r}0.53 \\
0.16 \\
30.19 \\
29.00\end{array}$ & $\begin{array}{r}1.30 \\
0.29 \\
22.31 \\
47.00\end{array}$ & $\begin{array}{r}30.53 \\
1.30\end{array}$ \\
\hline 1963 & $\begin{array}{l}\text { PRECIP. (CM.) } \\
\text { SR-90 (MCI/SQ.KM.) } \\
\text { SR-90 CONC. (PC/L) } \\
\text { SR-89/SR-9C }\end{array}$ & $\begin{array}{r}1.09 \\
0.63 \\
57.80 \\
47.00\end{array}$ & $\begin{array}{r}12.19 \\
0.68 \\
5.58 \\
24.00\end{array}$ & $\begin{array}{r}0.91 \\
1.26 \\
138.47 \\
15.00\end{array}$ & $\begin{array}{r}3.96 \\
3.18 \\
80.31 \\
5.20\end{array}$ & $\begin{array}{r}4.04 \\
2.87 \\
71.04 \\
9.50\end{array}$ & $\begin{array}{r}0.08 \\
0.41 \\
512.5 C \\
*\end{array}$ & $\begin{array}{l}* \\
0.10 \\
+\end{array}$ & $\begin{array}{l}1.52 \\
0.09 \\
5.93 \\
*\end{array}$ & $\begin{array}{r}0.10 \\
0.05 \\
50.01 \\
*\end{array}$ & $\begin{array}{r}0.33 \\
0.26 \\
78.79 \\
+\end{array}$ & $\begin{array}{r}2.06 \\
0.30 \\
14.57 \\
*\end{array}$ & $\begin{array}{r}6.25 \\
0.90 \\
14.41 \\
*\end{array}$ & $\begin{array}{l}32.53 \\
10.73\end{array}$ \\
\hline 1964 & $\begin{array}{l}\text { PRECIP. (CM.) } \\
\text { SR-90 IMCI/SQ.KM-) } \\
\text { SR-9O CCNC. }(P C / L) \\
S R-89 / S R-90\end{array}$ & $\begin{array}{r}0.33 \\
0.14 \\
42.43 \\
*\end{array}$ & $\begin{array}{l}3.33 \\
0.29 \\
8.71 \\
*\end{array}$ & $\begin{array}{r}0.94 \\
0.66 \\
70.22 \\
6.50\end{array}$ & $\begin{array}{r}1.68 \\
2.25 \\
133.93 \\
*\end{array}$ & $\begin{array}{l}* \\
0.22 \\
--\end{array}$ & $\begin{array}{l}* \\
0.24 \\
=-\end{array}$ & $\begin{array}{l}* \\
C .05 \\
-\infty\end{array}$ & $\begin{array}{l}* \\
0.03 \\
--\end{array}$ & $\begin{array}{l}* \\
* \\
--\end{array}$ & $\begin{array}{l}* \\
0.01 \\
*\end{array}$ & $\begin{array}{l}1.12 \\
0.12 \\
10.72 \\
--\end{array}$ & $\begin{array}{l}2.54 \\
0.03 \\
1.19 \\
--\end{array}$ & $\begin{array}{l}9.94 \\
4.04\end{array}$ \\
\hline 1965 & $\begin{array}{l}\text { PRECIP. (CM.) } \\
\text { SR-9O IMCI/SQ.KM-) } \\
S R-90 \text { CONC. }(P C / L) \\
S R-89 / S R-90\end{array}$ & $\begin{array}{l}11.51 \\
0.07 \\
C .61 \\
--\end{array}$ & $\begin{array}{c}0.91 \\
0.33 \\
36.27 \\
--\end{array}$ & $\begin{array}{l}3.07 \\
0.77 \\
25.09 \\
--\end{array}$ & $\begin{array}{l}1.60 \\
0.23 \\
14.38 \\
--\end{array}$ & $\begin{array}{r}0.66 \\
0.27 \\
40.91 \\
-\end{array}$ & $\begin{array}{l}-15 \\
--\end{array}$ & $\begin{array}{l}-- \\
--\end{array}$ & $\begin{array}{l}* 05 \\
-\infty\end{array}$ & $\begin{array}{l}1.02 \\
C .29 \\
28.44 \\
--\end{array}$ & $\begin{array}{l}-- \\
-- \\
-\end{array}$ & $\begin{array}{l}2.03 \\
0.06 \\
2.96 \\
--\end{array}$ & $\begin{array}{l}2.03 \\
0.04 \\
1.98 \\
--\end{array}$ & $\begin{array}{r}22.83 \\
2.26\end{array}$ \\
\hline 1966 & $\begin{array}{l}\text { PRECIP. }\left(C M_{-}\right) \\
\text {SR-9O }\left(M C I / S Q_{-K M}\right) \\
S R-90 \text { CONC. }(P C / L) \\
S R-89 / S R-90\end{array}$ & $\begin{array}{l}1.42 \\
0.03 \\
2.12 \\
-.\end{array}$ & $\begin{array}{l}3.38 \\
0.12 \\
3.56 \\
--.\end{array}$ & $\begin{array}{l}4.06 \\
0.04 \\
0.99 \\
--\end{array}$ & $\begin{array}{l}1.33 C \\
=- \\
--\end{array}$ & $\begin{array}{l}1.33 C \\
-- \\
--\end{array}$ & $\begin{array}{l}0.15 \\
-- \\
-\infty\end{array}$ & $\begin{array}{r}0.10 \\
0.04 \\
4 C .01 \\
*\end{array}$ & * & $\begin{array}{l}0.06 \\
* \\
--\end{array}$ & $\begin{array}{l}2.21 \\
0.04 \\
1.81 \\
*\end{array}$ & $\begin{array}{l}0.95 \\
0.01 \\
1.06 \\
1\end{array}$ & $\begin{array}{r}0.10 \\
0.08 \\
80.01 \\
*\end{array}$ & $\begin{array}{r}15.09 \\
0.36\end{array}$ \\
\hline 1967 & $\begin{array}{l}\text { PRECIP. }\left(C M M_{-}\right) \\
\text {SR-90 (MCI/SQ.KM.) } \\
\text { SR-90 CONC. (PC/L) } \\
\text { SR-89/SR-90 }\end{array}$ & $\begin{array}{l}0.89 \\
0.07 \\
7.87 \\
*\end{array}$ & $\begin{array}{r}2.03 \\
0.07 \\
3.45 \\
11.50\end{array}$ & $\begin{array}{r}0.08 \\
0.05 \\
62.51 \\
10.30\end{array}$ & $\begin{array}{l}-- \\
-- \\
--\end{array}$ & $=$ & $\begin{array}{l}-- \\
-- \\
--\end{array}$ & $\begin{array}{l}-- \\
--\end{array}$ & $m$ & $\begin{array}{l}-- \\
--\end{array}$ & $=$ & $\begin{array}{l}-- \\
= \\
=\end{array}$ & -- & $\begin{array}{l}3.00 \\
0.19\end{array}$ \\
\hline $\begin{aligned} \text { NOTES } & \\
-: & D \\
t: & Z \\
A: & A \\
B= & 1 \\
C: & P\end{aligned}$ & $\begin{array}{l}\text { ATA NOT AVAILABLE } \\
\text { ERO OR TRACE } \\
\text { PPROXIMATE } \\
\text { OWER LIMIT OF REPOR } \\
\text { ROPORTIONED FROM OR }\end{array}$ & $\begin{array}{l}\text { DATA } \\
\text { NALLY }\end{array}$ & & & & & & & & & & & & \\
\hline
\end{tabular}




\begin{tabular}{|c|c|c|c|c|c|c|c|c|c|c|c|c|}
\hline JAN. & FEB. & MAR. & APR. & $\begin{array}{l}\text { MAY } \\
---\end{array}$ & JUNE & JULY & AUG. & SEP. & OCT. & NOV. & DEC. & CUM. TOIAL \\
\hline $\begin{array}{l}-- \\
-- \\
-\end{array}$ & $=$ & $\begin{array}{l}- \\
=- \\
-\end{array}$ & $\begin{array}{l}-- \\
--\end{array}$ & $\begin{array}{l}= \\
=- \\
=\end{array}$ & $\begin{array}{l}-- \\
-- \\
-\infty\end{array}$ & $\begin{array}{l}= \\
=- \\
=\end{array}$ & $=$ & $\begin{array}{l}-- \\
-- \\
--\end{array}$ & $\begin{array}{l}2.51 \\
0.05 \\
2.00 \\
1.50\end{array}$ & $\begin{array}{l}-- \\
0.13 \\
--\end{array}$ & $\begin{array}{r}12.47 \\
0.17 \\
1.37 \\
0.80\end{array}$ & $\begin{array}{r}14.98 \\
0.35\end{array}$ \\
\hline $\begin{array}{l}16.79 \\
0.10 \\
0.60 \\
--\end{array}$ & $\begin{array}{l}6.71 \\
0.25 \\
3.73 \\
0.30\end{array}$ & $\begin{array}{l}12.37 \\
0.24 \\
1.95 \\
--\end{array}$ & $\begin{array}{l}4.90 \\
0.14 \\
2.86 \\
--\end{array}$ & $\begin{array}{l}0.18 \\
0.01 C \\
5.56 \\
-.\end{array}$ & $\begin{array}{l}4.47 \\
0.316 \\
6.94 \\
-.\end{array}$ & $\begin{array}{c}10.95 \\
0.410 \\
3.75 \\
=-\end{array}$ & $\begin{array}{l}0.86 \\
0.03 \mathrm{C} \\
3.49 \\
--\end{array}$ & $\begin{array}{l}11.20 \\
0.06 \\
0.54 \\
--\end{array}$ & $\begin{array}{c}22.48 \\
C .12 C \\
0.54 \\
-\end{array}$ & $\begin{array}{c}11.61 \\
0.07 \mathrm{C} \\
0.61 \\
--\end{array}$ & $\begin{array}{l}17.30 \\
0.15 \mathrm{C} \\
0.87 \\
-\end{array}$ & $\begin{array}{r}119.82 \\
1.89\end{array}$ \\
\hline $\begin{array}{l}11.46 \\
0.126 \\
1.05 \\
=\end{array}$ & $\begin{array}{l}2.77 \\
0.036 \\
1.09 \\
--\end{array}$ & $\begin{array}{l}* \\
0.000 \\
--\end{array}$ & $\begin{array}{c}10.57 \\
0.376 \\
3.51 \\
--\end{array}$ & $\begin{array}{l}3.56 \\
0.07 C \\
1.97 \\
-.\end{array}$ & $\begin{array}{l}8.46 \\
0.166 \\
1.90 \\
=-\end{array}$ & $\begin{array}{l}0.76 \\
0.036 \\
3.95 \\
--\end{array}$ & $\begin{array}{l}1.02 \\
0.04 C \\
3.93 \\
-\end{array}$ & $\begin{array}{l}6.07 \\
0.10 \\
1.65 \\
1.80\end{array}$ & $\begin{array}{r}10.67 \\
0.26 \\
2.44 \\
95.00\end{array}$ & $\begin{array}{r}11.02 \\
0.25 \\
2.27 \\
101.00\end{array}$ & $\begin{array}{r}8.41 \\
0.74 \\
8.80 \\
70.00\end{array}$ & $\begin{array}{r}74.77 \\
2.17\end{array}$ \\
\hline $\begin{array}{r}14.10 \\
0.52 \\
3.69 \\
48.00\end{array}$ & $\begin{array}{r}7.80 \\
0.52 \\
6.67 \\
29.00\end{array}$ & $\begin{array}{r}8.64 \\
1.17 \\
13.55 \\
22.00\end{array}$ & $\begin{array}{r}5.05 \\
1.68 \\
33.27 \\
12.00\end{array}$ & $\begin{array}{r}5.31 \\
1.17 \\
22.04 \\
7.00\end{array}$ & $\begin{array}{r}5.16 \\
1.30 \\
25.2 C \\
8.00\end{array}$ & $\begin{array}{r}0.08 \\
0.05 \\
62.51 \\
8.00\end{array}$ & $\begin{array}{c}0.27 \\
-\overline{0.00} \\
28.00\end{array}$ & $\begin{array}{r}3.07 \\
0.19 \\
6.19 \\
28.00\end{array}$ & $\begin{array}{r}8.46 \\
0.42 \\
4.97 \\
52.00\end{array}$ & $\begin{array}{r}16.00 \\
0.98 \\
6.13 \\
43.00\end{array}$ & $\begin{array}{r}5.84 \\
0.70 \\
11.99 \\
44.00\end{array}$ & $\begin{array}{r}79.51 \\
8.97\end{array}$ \\
\hline $\begin{array}{r}4.57 \\
1.20 \\
26.26 \\
36.00\end{array}$ & $\begin{array}{r}1.22 \\
0.71 \\
58.20 \\
25.00\end{array}$ & $\begin{array}{r}7.12 \\
0.28 \\
3.94 \\
12.00\end{array}$ & $\begin{array}{r}7.98 \\
3.05 \\
38.23 \\
12.00\end{array}$ & $\begin{array}{r}8.38 \\
5.20 \\
62.06 \\
7.10\end{array}$ & $\begin{array}{r}5.66 \\
3.58 \\
63.26 \\
1.10\end{array}$ & $\begin{array}{r}7.47 \\
2.35 \\
31.46 \\
3.00\end{array}$ & $\begin{array}{l}7.37 \\
3.13 \\
42.47 \\
*\end{array}$ & $\begin{array}{l}6.91 \\
0.07 \\
1.03 \\
*\end{array}$ & $\begin{array}{r}2.64 \\
0.56 \\
21.22 \\
*\end{array}$ & $\begin{array}{c}8.15 \\
1.11 \\
13.62 \\
*\end{array}$ & $\begin{array}{l}7.57 \\
0.32 \\
4.23 \\
\end{array}$ & $\begin{array}{l}74.93 \\
21.56\end{array}$ \\
\hline $\begin{array}{l}2.64 \\
0.15 \\
5.69 \\
\end{array}$ & $\begin{array}{r}0.33 \\
0.84 \\
10.09 \\
0.20\end{array}$ & $\begin{array}{r}15.54 \\
2.08 \\
13.39 \\
*\end{array}$ & $\begin{array}{c}4.52 \\
1.18 \\
26.11 \\
*\end{array}$ & $\begin{array}{r}11.94 \\
2.57 \\
21.53 \\
*\end{array}$ & $\begin{array}{r}5.38 \\
2.01 \\
37.37 \\
-\end{array}$ & $\begin{array}{r}9.19 \\
4.40 \\
47.98 \\
--\end{array}$ & $\begin{array}{r}3.48 \\
1.05 \\
30.18 \\
--\end{array}$ & $\begin{array}{l}3.45 \\
- \\
=\end{array}$ & $\begin{array}{r}21.46 \\
1.37 \\
6.39 \\
3.10\end{array}$ & $\begin{array}{l}6.93 \\
0.11 \\
1.59\end{array}$ & $\begin{array}{l}8.92 \\
0.34 \\
3.82 \\
--\end{array}$ & $\begin{array}{r}101.78 \\
16.10\end{array}$ \\
\hline $\begin{array}{l}7.77 \\
0.13 \\
1.68 \\
--\end{array}$ & $\begin{array}{l}0.05 \\
=- \\
=-\end{array}$ & $\begin{array}{l}0.25 \\
0.12 \\
1.93 \\
--\end{array}$ & $\begin{array}{r}7.37 \\
1.16 \\
15.74 \\
--\end{array}$ & $\begin{array}{l}8.43 \\
1.33 \\
15.78 \\
--\end{array}$ & $\begin{array}{r}14.71 \\
0.76 \\
4.76 \\
--\end{array}$ & $\begin{array}{r}3.30 \\
C .96 \\
29.10 \\
--\end{array}$ & $\begin{array}{l}7.54 \\
0.63 \\
8.36 \\
--\end{array}$ & $\begin{array}{r}13.26 \\
0.64 \\
4.83 \\
-.\end{array}$ & $\begin{array}{l}0.76 \\
0.05 \\
6.58 \\
-\end{array}$ & $\begin{array}{r}17.86 \\
0.30 \\
1.68 \\
--\end{array}$ & $\begin{array}{l}9.78 \\
0.27 \\
2.77 \\
--\end{array}$ & $\begin{array}{r}97.08 \\
6.29\end{array}$ \\
\hline $\begin{array}{l}6.43 \\
0.11 \\
1.72 \\
--\end{array}$ & $\begin{array}{l}6.20 \\
0.30 \\
4.84 \\
-.\end{array}$ & $\begin{array}{l}1.93 \\
0.17 \\
8.81 \\
-\end{array}$ & $\begin{array}{l}5.44 \\
0.30 \\
5.52 \\
--\end{array}$ & $\begin{array}{l}3.20 \\
0.12 \\
3.76 \\
=-\end{array}$ & $\begin{array}{l}6.21 C \\
0.23 \mathrm{C} \\
3.71 \\
1.2 \mathrm{CC}\end{array}$ & $\begin{array}{l}6.216 \\
0.230 \\
3.71 \\
1.200\end{array}$ & $\begin{array}{r}12.10 \\
0.23 \\
1.91 \\
2.60\end{array}$ & $\begin{array}{l}9.16 \\
0.14 \\
1.53 \\
*\end{array}$ & $\begin{array}{c}14.24 \\
0.09 \\
0.64 \\
*\end{array}$ & $\begin{array}{r}27.10 \\
0.17 \\
0.63 \\
9.70\end{array}$ & $\begin{array}{l}7.45 C \\
0.05 C \\
0.68 \\
\end{array}$ & $\begin{array}{r}105.67 \\
2.14\end{array}$ \\
\hline $\begin{array}{l}7.45 C \\
0.04 C \\
0.54 \\
2.00\end{array}$ & $\begin{array}{l}2.08 \\
0.08 \\
2.89 \\
7.10\end{array}$ & $\begin{array}{l}4.92 \\
0.11 \\
2.24 \\
7.40\end{array}$ & $\begin{array}{r}1.26 \\
0.14 \\
11.12 \\
5.10\end{array}$ & $\begin{array}{r}12.34 \\
0.14 \\
1.14 \\
3.90\end{array}$ & $\begin{array}{l}7.06 \\
0.12 \\
1.70 \\
2.40\end{array}$ & $\begin{array}{l}- \\
=- \\
=\end{array}$ & $=$ & $\begin{array}{l}-- \\
-- \\
--\end{array}$ & $\begin{array}{l}\overline{-} \\
\overline{-}\end{array}$ & $\begin{array}{l}-- \\
-- \\
--\end{array}$ & $\begin{array}{l}-- \\
-- \\
--\end{array}$ & $\begin{array}{r}35.11 \\
0.61\end{array}$ \\
\hline
\end{tabular}

\section{NOTES}

\section{--: DATA nOt avaILABLE}

*: ZERO OR TRACE

A: APPROXIMATE

B: LOWER LIMIT OF REPORTED DATA

C: PROPORTIONED FROM ORIGINALLY CONSOLICATEO DATA

PREC 1P. (CM.) SR-90 THCITSQ.KMEI $S R-89 / S R-90$ $S R-89 / S R-90$

PRECIP. (CM.) $S R-89 / S R-S O$
$S R-90$ SR $S$ S 9 (MCI/SQ.KM. $S R-89 / S R-90$

PRECIP. ICM.)

$S R-90\left(N C I / 5 Q-K^{\mu} \cdot\right)$ $S R-89 / S R-90$

PRECIP. (CM.) $S R-89 / S R-90$

PRECIP. (CM.) $S R-89 / S R \rightarrow 90$

PRECIP. (CM-)

SR-90 (MCI/SQ.KM.) SR-9O CONC. (PC/L)

2.00

$$
\text { COLUMNI }
$$




\begin{tabular}{|c|c|c|c|c|c|c|c|c|c|c|c|c|c|c|}
\hline & & JAN, & EEB, & MAR. & ABR & $\begin{array}{l}\operatorname{may} \\
=-\infty\end{array}$ & JUNE & JULY & AUG. & SEP. & OCT. & Nov. & DEC. & CUM. TOTAL \\
\hline 1959 & $\begin{array}{l}\text { PRECIP, }\left(C H_{+}\right) \\
\text {SR-90 (MCI/SO.KH,) } \\
\text { SR-90 CONC. }(P C / L) \\
S R-89 / S R-90\end{array}$ & $\begin{array}{l}=- \\
-- \\
-\end{array}$ & $=$ & $\begin{array}{l}- \\
= \\
= \\
=\end{array}$ & $\begin{array}{l}\pi \\
= \\
=\end{array}$ & $=$ & $=$ & $=$ & $=$ & $\begin{array}{l}9.09 \\
0.01 \\
0.12 \\
.\end{array}$ & $\begin{array}{l}2.34 \\
0.01 \\
0.43 \\
\end{array}$ & $\begin{array}{r}17.30 \\
0.04 \\
0.24 \\
+\end{array}$ & $\begin{array}{l}23.62 \\
0.01 \\
0.05 \\
.\end{array}$ & $\begin{array}{r}52.35 \\
0.07\end{array}$ \\
\hline 1960 & $\begin{array}{l}\text { PRECIP. (CM-) } \\
\text { SR-9O (KCI/SQ.KM-) } \\
S R-9 C \text { CONC. }(P C / L) \\
S R-89 / S R-S O\end{array}$ & $\begin{array}{l}8.71 \\
0.08 \\
0.92 \\
-.\end{array}$ & $\begin{array}{l}9.02 \\
0.15 \\
1.67 \\
=\end{array}$ & $\begin{array}{l}10.01 \\
0.07 \\
0.70 \\
=-\end{array}$ & $\begin{array}{l}4.29 \\
0.13 \\
3.04 \\
+\end{array}$ & $\begin{array}{l}2.44 \\
0.056 \\
2.05 \\
=+\end{array}$ & $\begin{array}{l}17.27 \\
0.39 C \\
2.26 \\
==\end{array}$ & $\begin{array}{l}2.01 \\
0.02 \mathrm{C} \\
1.00 \\
=\end{array}$ & $\begin{array}{l}11.00 \\
0.10 \mathrm{C} \\
0.91 \\
=\end{array}$ & $\begin{array}{l}17.83 \\
0.13 C \\
6.73 \\
-=\end{array}$ & $\begin{array}{c}24.84 \\
0.186 \\
C .73 \\
=-1\end{array}$ & $\begin{array}{l}12.70 \\
0.176 \\
1.34 \\
=-\infty\end{array}$ & $\begin{array}{l}6.35 \\
0.08 C \\
1.26 \\
-.\end{array}$ & $\begin{array}{r}126.47 \\
1.55\end{array}$ \\
\hline 1961 & $\begin{array}{l}\text { PRECIP. (CM.) } \\
\text { SR-90 (YCI/SO.KM, } \\
\text { SR-90 CCNC. (PC/L) } \\
\text { SR-89/SR-90 }\end{array}$ & $\begin{array}{l}3.10 \\
0.026 \\
0.65 \\
=\end{array}$ & $\begin{array}{l}3.68 \\
0.03 \mathrm{C} \\
0.82 \\
=.\end{array}$ & $\begin{array}{l}+ \\
0.006 \\
-\end{array}$ & $\begin{array}{l}13.56 \\
0.326 \\
2.36 \\
==\end{array}$ & $\begin{array}{l}5.84 \\
0.166 \\
2.14 \\
=1\end{array}$ & $\begin{array}{l}19.03 \\
0.276 \\
2.70 \\
==\end{array}$ & $\begin{array}{l}9.14 \\
0.160 \\
1.76 \\
=0\end{array}$ & $\begin{array}{l}1.70 \\
0.036 \\
1.77 \\
+1\end{array}$ & $\begin{array}{l}1.27 \\
-= \\
=-\end{array}$ & $\begin{array}{r}7.57 \\
0.10 \\
1.33 \\
84.00\end{array}$ & $\begin{array}{r}17.68 \\
0.52 \\
2.95 \\
82.00\end{array}$ & $\begin{array}{r}5.69 \\
0.16 \\
2.82 \\
68.00\end{array}$ & $\begin{array}{r}79.26 \\
1.77\end{array}$ \\
\hline 1962 & $\begin{array}{l}\text { PRECIP. (CM.) } \\
\text { SR-9O IMCI/SQ,KM, } \\
\text { SR-9C CCNC. IPCAL) } \\
\text { SR-89/SR-SO }\end{array}$ & $\begin{array}{r}1.91 \\
0.48 \\
25.16 \\
46.00\end{array}$ & $\begin{array}{r}5.74 \\
0.68 \\
11.85 \\
25.00\end{array}$ & $\begin{array}{r}6.88 \\
0.13 \\
1.89 \\
18,00\end{array}$ & $\begin{array}{r}10,13 \\
1.13 \\
11.16 \\
11.00\end{array}$ & $\begin{array}{l}9.12 \\
0.88 \\
9.65 \\
9.00\end{array}$ & $\begin{array}{r}7.29 \\
0.90 \\
12.35 \\
6.00\end{array}$ & $\begin{array}{r}1.83 \\
0.34 \\
18.58 \\
18.00\end{array}$ & $\begin{array}{l}4.47 \\
-\rightarrow \\
=\end{array}$ & $\begin{array}{r}7.39 \\
0.34 \\
4.61 \\
22.00\end{array}$ & $\begin{array}{r}8.71 \\
0.41 \\
4.71 \\
34.00\end{array}$ & $\begin{array}{r}15.19 \\
0.73 \\
4.81 \\
18.00\end{array}$ & $\begin{array}{r}42.93 \\
0.13 \\
0.31 \\
38.00\end{array}$ & $\begin{array}{r}121.59 \\
6.15\end{array}$ \\
\hline 1963 & $\begin{array}{l}\text { PRECIP. ICM.) } \\
\text { SR-SO IMCI/SQ.KME) } \\
S R-9 C \text { CCNC. (PCILI) } \\
S R-89 / S R-90\end{array}$ & $\begin{array}{r}10.24 \\
0.52 \\
5,08 \\
28,00\end{array}$ & $\begin{array}{r}7.32 \\
0.29 \\
3,97 \\
34,00\end{array}$ & $\begin{array}{r}8.64 \\
1.09 \\
12.62 \\
12.00\end{array}$ & $\begin{array}{r}8.64 \\
1.52 \\
17.60 \\
13.00\end{array}$ & $\begin{array}{r}8.99 \\
1.32 \\
14.69 \\
6.70\end{array}$ & $\begin{array}{l}6.27 \\
0.23 \\
3.67 \\
2.00\end{array}$ & $\begin{array}{r}5.23 \\
2.54 \\
48.57 \\
2.00\end{array}$ & $\begin{array}{r}13.97 \\
3.73 \\
26.71 \\
*\end{array}$ & $\begin{array}{r}12.09 \\
6.72 \\
5.96 \\
0.90\end{array}$ & $\begin{array}{l}3.91 \\
0.36 \\
9.21 \\
.\end{array}$ & $\begin{array}{l}22.28 \\
1.59 \\
7.14 \\
*\end{array}$ & $\begin{array}{l}5.61 \\
0.27 \\
4.82 \\
\end{array}$ & $\begin{array}{r}113.19 \\
14.18\end{array}$ \\
\hline 1964 & $\begin{array}{l}\text { PRECIP. (CM, } \\
\text { SR-9C IMCI/SQ,KM, } \\
S R-9 C \text { CCNC (PCAL) } \\
\text { SR-89/SR-90 }\end{array}$ & $\begin{array}{r}1.40 \\
1.55 \\
140.72 \\
+\end{array}$ & $\begin{array}{l}8,51 \\
0,66 \\
7,76 \\
7\end{array}$ & $\begin{array}{r}18.39 \\
2.05 \\
11.15 \\
*\end{array}$ & $\begin{array}{l}7.80 \\
0.60 \\
7.70 \\
1.80\end{array}$ & $\begin{array}{r}8.48 \\
2.06 \\
24.30 \\
+\end{array}$ & $\begin{array}{r}2.21 \\
2.76 \\
124.89 \\
--\end{array}$ & $\begin{array}{r}6.20 \\
2.21 \\
35.65 \\
-.\end{array}$ & $\begin{array}{r}3.20 \\
1.13 \\
35.32 \\
-\end{array}$ & $\begin{array}{l}1.30 \\
.0 .19 \\
14.62 \\
-.-\end{array}$ & $\begin{array}{r}11.51 \\
0.76 \\
6.61 \\
4.50\end{array}$ & $\begin{array}{l}4.39 \\
0.31 \\
7.07 \\
.\end{array}$ & $\begin{array}{l}8.41 \\
0.42 \\
5.00 \\
-.\end{array}$ & $\begin{array}{l}81.80 \\
14.70\end{array}$ \\
\hline 1965 & $\begin{array}{l}\text { PRECIP. (CM.) } \\
S R-90 \text { (NCI/SQ.KM.) } \\
S R-90 \text { CONC. (PC/L) } \\
S R-89 / S R-90\end{array}$ & $\begin{array}{l}6.20 \\
0.60 \\
9.60 \\
-=\end{array}$ & $\begin{array}{l}0.03 \\
=- \\
=\end{array}$ & $\begin{array}{l}4.29 \\
0.40 \\
9.33 \\
=-\end{array}$ & $\begin{array}{l}1.19 \\
0.12 \\
10.09 \\
3=\end{array}$ & $\begin{array}{l}9.68 \\
0.26 \\
2.69 \\
-\end{array}$ & $\begin{array}{l}15.04 \\
1.45 \\
9.65 \\
--\end{array}$ & $\begin{array}{l}7.62 \\
6.22 \\
2.89 \\
--\end{array}$ & $\begin{array}{l}16.28 \\
0.69 \\
4.24 \\
-\end{array}$ & $\begin{array}{l}9.73 \\
c .01 \\
0.11 \\
--\end{array}$ & $\begin{array}{r}0.03 \\
0.01 \\
33.34 \\
-.\end{array}$ & $\begin{array}{r}11.73 \\
0.42 \\
3.59 \\
--\end{array}$ & $\begin{array}{l}3.40 \\
0.15 \\
4.42 \\
-. .\end{array}$ & $\begin{array}{r}85.19 \\
4.36\end{array}$ \\
\hline 1966 & $\begin{array}{l}\text { PRECIP. (CM.) } \\
\text { SR-9O IMCI/SQ.KM.) } \\
\text { SR-GO CCNC. (PC/L) } \\
\text { SR- } 99 / S R-9.0\end{array}$ & $\begin{array}{l}2.29 \\
0.09 \\
3.94 \\
-\end{array}$ & $\begin{array}{r}4.88 \\
0.50 \\
10.25 \\
--\end{array}$ & $\begin{array}{c}20.61 C \\
0.31 C \\
1.51 \\
-.\end{array}$ & $\begin{array}{l}20.61 \mathrm{C} \\
0.32 \mathrm{C} \\
1.56 \\
-\end{array}$ & $\begin{array}{l}4.01 \\
0.19 \\
4.74 \\
-.\end{array}$ & $\begin{array}{r}10.94 \\
0.26 \\
2.38 \\
4.30\end{array}$ & $\begin{array}{l}6.82 \\
C .36 \\
5.28 \\
1.50\end{array}$ & $\begin{array}{l}7.45 \\
0.36 \\
4.84 \\
*\end{array}$ & $\begin{array}{l}9.44 \\
* \\
-- \\
*\end{array}$ & $\begin{array}{l}24.20 \\
C .14 \\
0.58 \\
*\end{array}$ & $\begin{array}{r}14.08 \\
0.07 \\
0.50 \\
6.30\end{array}$ & $\begin{array}{l}1.07 \\
0.02 \\
1.87 \\
+\end{array}$ & $\begin{array}{r}147.01 \\
2.62\end{array}$ \\
\hline 1967 & $\begin{array}{l}\text { PRECIP. (CM.) } \\
\text { SR-90 IMCI/SQ.KM.) } \\
\text { SR-90 CONC. (PC/L) } \\
\text { SR-89/SR-90 }\end{array}$ & $\begin{array}{l}1.10 \\
9.83 \\
2.73 \\
1.73\end{array}$ & $\begin{array}{l}4.80 \\
0.06 \\
1.26 \\
8.40\end{array}$ & $\begin{array}{l}4.02 \\
0.13 \\
3.24 \\
8.20\end{array}$ & $\begin{array}{l}5.32 \\
0.14 \\
2.64 \\
9.30\end{array}$ & $\begin{array}{l}3.80 \\
0.12 \\
3.16 \\
3.40\end{array}$ & $\begin{array}{l}7.68 \\
0.13 \\
1.70 \\
*\end{array}$ & $\begin{array}{l}6.76 \\
0.13 \\
1.93 \\
\end{array}$ & $\begin{array}{l}- \\
-- \\
-\end{array}$ & $\begin{array}{l}m \\
=- \\
=-\end{array}$ & $=$ & $\begin{array}{l}= \\
= \\
-\end{array}$ & $=$ & $\begin{array}{r}33.48 \\
0.74\end{array}$ \\
\hline \multicolumn{15}{|c|}{$\begin{array}{l}\text { NOTES } \\
\text { *: DAFA NOT AVAILABLE } \\
\text { *: 2ERO OR TRACE } \\
\text { A: APPROXEMATE } \\
\text { B: LOWER LIMIT OF REPORTEO DAFA } \\
\text { C: PROPORTIONED FROM ORIGLNALLY }\end{array}$} \\
\hline
\end{tabular}




\begin{tabular}{|c|c|c|c|c|c|c|c|c|c|c|c|c|c|c|}
\hline & & JAN. & FEB. & MAR. & APR. & HAY & JUNE & JULY & AUG. & SEP. & OCT. & NOV. & DEC. & CUM. TOTAL \\
\hline 1959 & $\begin{array}{l}\text { PRECIP. (CM.) } \\
\text { SR-90 INCI/SQ.KM.) } \\
S R-90 \text { CONC. (PC/L) } \\
S R-89 / S R-90\end{array}$ & $\begin{array}{l}7.19 \\
-- \\
--\end{array}$ & $\begin{array}{l}2.36 \\
=- \\
=-\end{array}$ & $\begin{array}{l}2.90 \\
=- \\
=-\end{array}$ & $\begin{array}{l}14.45 \\
=- \\
--\end{array}$ & $\begin{array}{l}13.54 \\
-- \\
--\end{array}$ & $\begin{array}{l}4.06 \\
=- \\
=\end{array}$ & $\begin{array}{l}5.33 \\
=- \\
=-\end{array}$ & $\begin{array}{l}13.41 \\
0.04 \\
0.30 \\
2.10\end{array}$ & $\begin{array}{l}13.92 \\
0.05 \\
0.36 \\
*\end{array}$ & $\begin{array}{r}17.15 \\
0.03 \\
0.18 \\
*\end{array}$ & $\begin{array}{l}7.32 \\
0.02 \\
0.28 \\
*\end{array}$ & $\begin{array}{l}8.81 \\
-- \\
-- \\
--\end{array}$ & $\begin{array}{r}110.44 \\
0.14\end{array}$ \\
\hline 1960 & $\begin{array}{l}\text { PRECIP. (CM.) } \\
\text { SR-9C INCI/SO.KM-) } \\
\text { SR-90 CCNC. (PC/L) } \\
\text { SR-89/SR-90 }\end{array}$ & $\begin{array}{l}17.81 \\
C .19 \\
1.07 \\
-\end{array}$ & $\begin{array}{l}18.01 \\
0.09 \\
0.50 \\
--\end{array}$ & $\begin{array}{l}3.81 \\
0.13 \\
3.42 \\
--\end{array}$ & $\begin{array}{l}29.72 \\
0.14 \\
0.48 \\
--\end{array}$ & $\begin{array}{l}3.33 \\
0.050 \\
1.51 \\
--\end{array}$ & $\begin{array}{l}6.68 \\
0.096 \\
1.35 \\
--\end{array}$ & $\begin{array}{l}13.11 \\
0.03 C \\
0.23 \\
--\end{array}$ & $\begin{array}{l}29.90 \\
0.07 C \\
0.24 \\
=-\end{array}$ & $\begin{array}{l}* \\
0.00 C \\
=-\end{array}$ & $\begin{array}{l}3 C .00 \\
C .02 C \\
0.07 \\
--\end{array}$ & $\begin{array}{l}* \\
0.00 C \\
--\end{array}$ & $\begin{array}{l}7.59 \\
0.046 \\
0.53 \\
-.\end{array}$ & $\begin{array}{r}159.96 \\
0.85\end{array}$ \\
\hline 1961 & $\begin{array}{l}\text { PRECIP, (CM.) } \\
\text { SR-GC IMCI/SQ.KM-) } \\
S R-9 O C O N C \text { (PC/L) } \\
S R-89 / S R-S C\end{array}$ & $\begin{array}{l}6.88 \\
0.07 C \\
1.02 \\
--\end{array}$ & $\begin{array}{l}9.27 \\
0.106 \\
1.08 \\
--\end{array}$ & $\begin{array}{l}17.48 \\
0.25 C \\
1.44 \\
--\end{array}$ & $\begin{array}{l}7.24 \\
0.10 \mathrm{C} \\
1.39 \\
-\end{array}$ & $\begin{array}{l}7.29 \\
0.086 \\
1.10 \\
--\end{array}$ & $\begin{array}{l}7.92 \\
0.08 \mathrm{C} \\
1.02 \\
--\end{array}$ & $\begin{array}{l}34.37 \\
C .02 C \\
C .06 \\
--\end{array}$ & $\begin{array}{l}16.43 \\
0.01 C \\
0.07 \\
--\end{array}$ & $\begin{array}{r}20.47 \\
0.06 \\
C .30 \\
14.00\end{array}$ & $\begin{array}{r}15.46 \\
C .04 \\
0.21 \\
90.00\end{array}$ & $\begin{array}{r}14.22 \\
0.12 \\
0.85 \\
72.00\end{array}$ & $\begin{array}{r}6.91 \\
0.76 \\
11.00 \\
69.00\end{array}$ & $\begin{array}{r}167.94 \\
1.69\end{array}$ \\
\hline 1962 & $\begin{array}{l}\text { PRECIP, (CM.) } \\
\text { SR-9C INCI/SQ.KM.) } \\
\text { SR-9O CONC. (PC/L) } \\
\text { SR-89/SR-90 }\end{array}$ & $\begin{array}{r}18.75 \\
0.64 \\
3.42 \\
47.00\end{array}$ & $\begin{array}{r}0.84 \\
0.25 \\
29.77 \\
29.00\end{array}$ & $\begin{array}{r}5.16 \\
0.32 \\
6.21 \\
22.00\end{array}$ & $\begin{array}{r}6.86 \\
0.42 \\
6.13 \\
14.00\end{array}$ & $\begin{array}{r}19.43 \\
0.17 \\
0.88 \\
8.00\end{array}$ & $\begin{array}{r}22.25 \\
0.54 \\
2.43 \\
7.0 \mathrm{C}\end{array}$ & $\begin{array}{l}4.57 \\
0.15 \\
3.29 \\
5.00\end{array}$ & $\begin{array}{r}28.45 \\
0.30 \\
1.06 \\
12.00\end{array}$ & $\begin{array}{l}5.51 \\
0.11 \\
2.00 \\
16.00\end{array}$ & $\begin{array}{r}33.71 \\
0.31 \\
0.92 \\
30.00\end{array}$ & $\begin{array}{r}8.43 \\
0.13 \\
1.55 \\
49.00\end{array}$ & $\begin{array}{r}14.40 \\
0.87 \\
6.05 \\
50.00\end{array}$ & $\begin{array}{r}168.36 \\
4.21\end{array}$ \\
\hline 1963 & $\begin{array}{l}\text { PRECIP. (CM.) } \\
\text { SR-9O (NCI/SQ.KM.) } \\
S R-90 \text { CCNC. (PC/L) } \\
\text { SR-89/SR-9O }\end{array}$ & $\begin{array}{r}3.66 \\
0.56 \\
15.31 \\
45.00\end{array}$ & $\begin{array}{r}0.79 \\
0.28 \\
35.45 \\
35.00\end{array}$ & $\begin{array}{l}1.47 \\
0.02 \\
1.37 \\
20.00\end{array}$ & $\begin{array}{r}3.07 \\
0.25 \\
8.15 \\
12.00\end{array}$ & $\begin{array}{r}11.20 \\
C .58 \\
5.18 \\
12.20\end{array}$ & $\begin{array}{l}5.16 \\
1.06 \\
20.55 \\
*\end{array}$ & $\begin{array}{r}10.31 \\
C .58 \\
5.63 \\
c .20\end{array}$ & $\begin{array}{r}13.64 \\
0.54 \\
3.96 \\
*\end{array}$ & $\begin{array}{r}17.60 \\
0.32 \\
1.82 \\
1.00\end{array}$ & $\begin{array}{r}19.63 \\
0.24 \\
1.23\end{array}$ & $\begin{array}{r}15.39 \\
0.46 \\
2.99 \\
*\end{array}$ & $\begin{array}{l}23.29 \\
0.48 \\
2.07 \\
*\end{array}$ & $\begin{array}{r}125.21 \\
5.37\end{array}$ \\
\hline 1964 & $\begin{array}{l}\text { PRECIP. (CM.) } \\
\text { SR-9O INCI/SQ.KM.) } \\
S R-9 C \text { CONC. (PC/L) } \\
S R-89 / S R-90\end{array}$ & $\begin{array}{r}3.15 \\
0.49 \\
15.56 \\
*\end{array}$ & $\begin{array}{r}1.83 \\
0.22 \\
12.03 \\
*\end{array}$ & $\begin{array}{l}1.35 \\
0.10 \\
7.41 \\
*\end{array}$ & $\begin{array}{r}0.58 \\
0.24 \\
41.38 \\
\end{array}$ & $\begin{array}{r}11.89 \\
0.41 \\
3.45 \\
*\end{array}$ & $\begin{array}{l}2.97 \\
0.22 \\
7.41 \\
-.\end{array}$ & $\begin{array}{l}22.43 \\
0.72 \\
3.21 \\
--\end{array}$ & $\begin{array}{l}24.92 \\
0.17 \\
0.69 \\
--\end{array}$ & $\begin{array}{l}-\infty \\
--\end{array}$ & $\begin{array}{l}2 c .27 \\
0.25 \\
1.24 \\
6.80\end{array}$ & $\begin{array}{l}10.01 \\
0.60 \\
6.00 \\
*\end{array}$ & $\begin{array}{l}19.99 \\
0.52 \\
2.61 \\
--\end{array}$ & $\begin{array}{r}119.39 \\
3.96\end{array}$ \\
\hline 1965 & $\begin{array}{l}\text { PRECIP. (CM.) } \\
\text { SR-9O IMCI/SQ.KM.) } \\
S R-90 \text { CONC. (PC/L) } \\
\text { SR-89/SR-9O }\end{array}$ & $\begin{array}{l}8.89 \\
0.17 \\
1.92 \\
-.\end{array}$ & $\begin{array}{l}-- \\
-- \\
--\end{array}$ & $\begin{array}{r}10.52 \\
1.15 \\
10.94 \\
-.\end{array}$ & $\begin{array}{l}-- \\
-- \\
--\end{array}$ & $\begin{array}{l}8.81 \\
0.33 \\
3.75 \\
-0\end{array}$ & $\begin{array}{l}4.93 \\
0.39 \\
7.92 \\
-.\end{array}$ & $\begin{array}{l}7.77 \\
0.14 \\
1.81 \\
-.\end{array}$ & $\begin{array}{l}9.55 \\
C .08 \\
0.84 \\
--\end{array}$ & $\begin{array}{l}17.91 \\
C .27 \\
1.51 \\
-.-\end{array}$ & $\begin{array}{l}-- \\
c .08 \\
--\end{array}$ & $\begin{array}{l}4.14 \\
0.12 \\
2.90 \\
--\end{array}$ & $\begin{array}{r}0.76 \\
0.46 \\
60.53 \\
-\end{array}$ & $\begin{array}{r}73.28 \\
3.19\end{array}$ \\
\hline 1966 & $\begin{array}{l}\text { PRECIP }(\text { ICM.) } \\
\text { SR-9O }(M C I / S Q-K M-) \\
S R-90 \text { CCNC. }(P C / L) \\
S R-89 / S R-90\end{array}$ & $\begin{array}{l}3.56 \\
0.08 \\
2.25 \\
-\end{array}$ & $\begin{array}{l}2.11 \\
0.16 \\
7.59 \\
-.\end{array}$ & $\begin{array}{l}3.81 \\
0.14 \\
3.68 \\
--\end{array}$ & $\begin{array}{l}3.81 \\
0.12 \\
3.15 \\
--\end{array}$ & $\begin{array}{l}-- \\
0.18 \\
--\end{array}$ & 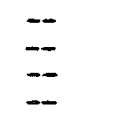 & $\begin{array}{r}3.81 \\
0.53 \\
13.92 \\
2.30\end{array}$ & 0.13 & $\begin{array}{l}10.97 \\
0.13 \\
1.19 \\
*\end{array}$ & $\begin{array}{r}11.00 \\
0.14 \\
1.28 \\
*\end{array}$ & $\begin{array}{l}2.21 \\
0.04 \\
1.81 \\
*\end{array}$ & $\begin{array}{l}-. \\
0.08 \\
25.00\end{array}$ & $\begin{array}{r}41.28 \\
1.73\end{array}$ \\
\hline 1967 & $\begin{array}{l}\text { PRECIP. ICM+) } \\
\text { SR-9O INCI/SO.KM.) } \\
\text { SR-90 CCNC. (PC/L) } \\
\text { SR-89/SR-90 }\end{array}$ & $\begin{array}{r}5.94 \\
0.06 \\
1.02 \\
22.70\end{array}$ & $\begin{array}{r}7.95 \\
0.06 \\
6.76 \\
13.50\end{array}$ & $\frac{-}{0}$ & $\begin{array}{l}5.41 \\
0.07 \\
1.30 \\
3.60\end{array}$ & $\begin{array}{l}31.24 \\
0.12 \\
0.39 \\
*\end{array}$ & $\begin{array}{l}--11 \\
0.1\end{array}$ & $\frac{-}{0.01}$ & $\begin{array}{l}= \\
=- \\
--\end{array}$ & $\begin{array}{l}-- \\
-- \\
--\end{array}$ & $\begin{array}{l}-- \\
-- \\
--\end{array}$ & $\begin{array}{l}-- \\
-- \\
--\end{array}$ & $\begin{array}{l}-- \\
-- \\
--\end{array}$ & $\begin{array}{r}50.54 \\
0.44\end{array}$ \\
\hline $\begin{array}{c}\text { NOTES } \\
\text { O: } \\
*: \\
A: \\
B: \\
C:\end{array}$ & $\begin{array}{l}\text { ATA NOT AVAILABLE } \\
\text { ERO OR IRACE } \\
\text { PPROXIMATE } \\
\text { OWER LIMIT OF REPOR } \\
\text { ROPORTIONED FROM OR }\end{array}$ & $\begin{array}{l}\text { DATA } \\
\text { NALLY }\end{array}$ & ys & 0 & & & & & & & & & & \\
\hline
\end{tabular}




\begin{tabular}{|c|c|c|c|c|c|c|c|c|c|c|c|c|c|c|}
\hline & & JAN. & FEB. & MAR. & APR. & MaY & JUNE & JULY & AUG. & SEP. & OCT. & nov. & DEC. & CUM. TOTAL \\
\hline 1956 & $\begin{array}{l}\text { PRECIP. (CM.) } \\
\text { SR-90 (MCI/SO.KM.) } \\
\text { SR-90 CONC. (PCAL) } \\
\text { SR-89/SR-90 }\end{array}$ & $\begin{array}{l}\bar{z} \\
\overline{-}\end{array}$ & $\bar{z}$ & $\bar{z}$ & $\bar{z}$ & $\begin{array}{l}\overline{-} \\
\overline{-}\end{array}$ & $\begin{array}{l}\bar{z} \\
\bar{z}\end{array}$ & $\bar{z}$ & $\begin{array}{l}30.30 \\
0.19 \\
0.63 \\
-\end{array}$ & $\begin{array}{l}24.97 \\
=- \\
=-\end{array}$ & $\begin{array}{l}8.92 \\
0.10 \\
1.13 \\
--\end{array}$ & $\begin{array}{l}4.17 \\
0.04 \\
0.96 \\
--\end{array}$ & $\begin{array}{l}0.58 \\
0.02 \\
3.45 \\
--\end{array}$ & $\begin{array}{r}68.94 \\
0.35\end{array}$ \\
\hline 1957 & $\begin{array}{l}\text { PREC1P. (CM-) } \\
\text { SR-SC irC1/SQ.KM.) } \\
S R-90 \text { CONC. (PCAL) } \\
S R-89 / S R \rightarrow 90\end{array}$ & $\begin{array}{l}5.46 \\
0.11 \\
2.02 \\
--\end{array}$ & $\begin{array}{l}5.74 \\
0.20 \\
3.49 \\
--\end{array}$ & $\begin{array}{l}3.28 \\
0.09 \\
2.75 \\
--\end{array}$ & $\begin{array}{l}27.94 \\
0.43 \\
1.54 \\
--\end{array}$ & $\begin{array}{r}16.36 \\
0.22 \\
1.35 \\
-.\end{array}$ & $\begin{array}{l}25.96 \\
0.19 \\
0.74 \\
--\end{array}$ & $\begin{array}{l}53.59 \\
0.32 \\
0.60 \\
--\end{array}$ & $\begin{array}{r}11.38 \\
0.02 \\
0.18 \\
-.-\end{array}$ & $\begin{array}{l}27.74 \\
0.11 \\
0.40 \\
--\end{array}$ & $\begin{array}{l}5.26 \\
-- \\
=-\end{array}$ & $\begin{array}{l}6.25 \\
0.05 \\
0.81 \\
--\end{array}$ & $\begin{array}{l}4.85 \\
0.14 \\
2.89 \\
--\end{array}$ & $\begin{array}{r}193.81 \\
1.88\end{array}$ \\
\hline 1958 & $\begin{array}{l}\text { PRECIP_ (CM.) } \\
\text { SR-9C IKCI/SO.KM.) } \\
\text { SR-90 CCNC. (PCIL) } \\
\text { SR-89/SR-9O }\end{array}$ & $\begin{array}{r}6.73 \\
0.09 \\
1.34 \\
13.00\end{array}$ & $\begin{array}{r}7.44 \\
0.10 \\
1.35 \\
12.00\end{array}$ & $\begin{array}{r}12.40 \\
0.36 \\
2.91 \\
11.00\end{array}$ & $\begin{array}{r}26.64 \\
1.30 \\
4.88 \\
10.00\end{array}$ & $\begin{array}{r}9.50 \\
0.41 \\
4.32 \\
14.00\end{array}$ & $\begin{array}{r}10.11 \\
0.25 \\
2.48 \\
11.00\end{array}$ & $\begin{array}{r}17.53 \\
6.17 \\
6.97 \\
3.20\end{array}$ & $\begin{array}{r}25.07 \\
1.03 \\
4.11 \\
17.00\end{array}$ & $\begin{array}{r}9.12 \\
0.16 \\
1.76 \\
52.00\end{array}$ & $\begin{array}{r}12.40 \\
0.30 \\
2.42 \\
48.00\end{array}$ & $\begin{array}{l}6.05 \\
0.27 \\
4.47 \\
8.00\end{array}$ & $\begin{array}{r}4.95 \\
0.22 \\
4.45 \\
62.00\end{array}$ & $\begin{array}{r}147.94 \\
4.66\end{array}$ \\
\hline 1959 & $\begin{array}{l}\text { PREC IP. (CM.) } \\
\text { SR-9C (MCI/SC.KM-) } \\
5 R-9 C \text { CONC. (PC/L) } \\
S R-89 / S R-90\end{array}$ & $\begin{array}{r}6.71 \\
c .70 \\
16.44 \\
31.00\end{array}$ & $\begin{array}{r}13.31 \\
0.95 \\
7.14 \\
28.00\end{array}$ & $\begin{array}{r}6.02 \\
2.14 \\
35.55 \\
10.00\end{array}$ & $\begin{array}{r}20.62 \\
2.52 \\
12.23 \\
7.00\end{array}$ & $\begin{array}{r}15.32 \\
0.15 \\
c .98 \\
-\end{array}$ & $\begin{array}{l}6.60 \\
0.42 \\
6.37 \\
5.60\end{array}$ & $\begin{array}{r}33.22 \\
0.53 \\
1.60 \\
3.20\end{array}$ & $\begin{array}{l}12.12 \\
-- \\
--\end{array}$ & $\begin{array}{l}12.52 \\
c . c 2 \\
c .16 \\
*\end{array}$ & $\begin{array}{r}11.00 \\
6.10 \\
c .91 \\
c .50\end{array}$ & $\begin{array}{l}5.69 \\
0.05 \\
0.88 \\
*\end{array}$ & $\begin{array}{l}9.40 \\
0.06 \\
0.64 \\
*\end{array}$ & $\begin{array}{r}152.53 \\
7.64\end{array}$ \\
\hline 1960 & 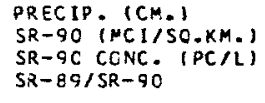 & $\begin{array}{l}4.01 \\
c .09 \\
2.25 \\
--\end{array}$ & $\begin{array}{l}0.99 \\
0.03 \\
3.04 \\
--\end{array}$ & $\begin{array}{l}5.33 \\
0.32 \\
6.01 \\
--\end{array}$ & $\begin{array}{l}13.00 \\
0.22 \\
1.70 \\
--\end{array}$ & $\begin{array}{l}21.82 \\
c .24 C \\
1.10 \\
-.\end{array}$ & $\begin{array}{l}24.10 \\
0.276 \\
1.13 \\
-=\end{array}$ & $\begin{array}{l}18.08 \\
C .12 \mathrm{C} \\
0.67 \\
--\end{array}$ & $\begin{array}{l}10.39 \\
c . c 7 c \\
0.68 \\
--\end{array}$ & $\begin{array}{l}27.51 \\
C .086 \\
c .30 \\
--\end{array}$ & $\begin{array}{l}7.11 \\
c .03 \mathrm{C} \\
0.43 \\
--\end{array}$ & $\begin{array}{l}7.49 \\
0.022 \\
0.27 \\
--\end{array}$ & $\begin{array}{l}2.01 \\
0.016 \\
0.50 \\
--\end{array}$ & $\begin{array}{r}141.84 \\
1.50\end{array}$ \\
\hline 1961 & $\begin{array}{l}\text { PRECIP. (CM.) } \\
\text { SR-9C (KCI/SC.KN.) } \\
\text { SR-90 CONC. }(P C / L) \\
S R-89 / S R-90\end{array}$ & $\begin{array}{l}5.49 \\
0.056 \\
0.92 \\
-.-\end{array}$ & $\begin{array}{l}3.99 \\
0.04 C \\
1.01 \\
--\end{array}$ & $\begin{array}{l}8.41 \\
0.14 C \\
1.67 \\
-\end{array}$ & $\begin{array}{c}16.99 \\
0.276 \\
1.59 \\
-\end{array}$ & $\begin{array}{l}13.59 \\
0.15 \mathrm{C} \\
1.11 \\
--\end{array}$ & $\begin{array}{l}12.70 \\
0.14 C \\
1.11\end{array}$ & $\begin{array}{l}17.30 \\
0.02 c \\
c .12 \\
-.\end{array}$ & $\begin{array}{l}11.71 \\
0.02 C \\
c .18 \\
--\end{array}$ & $\begin{array}{l}7.90 \\
=- \\
=-\end{array}$ & $\begin{array}{r}18.08 \\
0.11 \\
0.61 \\
6.70\end{array}$ & $\begin{array}{r}11.71 \\
0.22 \\
1.88 \\
74.00\end{array}$ & $\begin{array}{l}4.70 \\
=- \\
=-\end{array}$ & $\begin{array}{r}132.57 \\
1.10\end{array}$ \\
\hline 1962 & $\begin{array}{l}\text { PRECIP } \text { (CM.) } \\
\text { SR-9C } \text { (KCI/SO.KM.) } \\
S R-S C \text { CCNC. (PC/L) } \\
S R-89 / S R-9 O\end{array}$ & $\begin{array}{r}4.01 \\
c .43 \\
1 C .73 \\
38.00\end{array}$ & $\begin{array}{r}3.00 \\
c .64 \\
21.34 \\
23.00\end{array}$ & $\begin{array}{r}2.39 \\
1.35 \\
56.49 \\
17.00\end{array}$ & $\begin{array}{r}15.19 \\
1.33 \\
8.76 \\
14.00\end{array}$ & $\begin{array}{r}18.59 \\
1.37 \\
7.37 \\
8.00\end{array}$ & $\begin{array}{r}24.41 \\
0.66 \\
2.71 \\
7.00\end{array}$ & $\begin{array}{r}47.19 \\
6.05 \\
6.11 \\
10.00\end{array}$ & $\begin{array}{r}11.51 \\
0.11 \\
0.56 \\
22.00\end{array}$ & $\begin{array}{r}12.19 \\
0.45 \\
3.70 \\
16.00\end{array}$ & $\begin{array}{r}22.10 \\
0.37 \\
1.68 \\
25.60\end{array}$ & $\begin{array}{r}9.40 \\
0.36 \\
3.83 \\
37.00\end{array}$ & $\begin{array}{r}5.69 \\
0.65 \\
11.43 \\
36.00\end{array}$ & $\begin{array}{r}175.67 \\
7.77\end{array}$ \\
\hline 1963 & $\begin{array}{l}\text { PRECIP. (CM.) } \\
\text { SR-90 (NC1/SQ.KM.) } \\
\text { SR-90 CCNC. (PC/L) } \\
\text { SR-89/SR-9O }\end{array}$ & $\begin{array}{r}3.51 \\
0.44 \\
12.54 \\
32.00\end{array}$ & $\begin{array}{r}2.19 \\
0.51 \\
18.28 \\
36.00\end{array}$ & $\begin{array}{r}6.20 \\
1.37 \\
22.10 \\
16.00\end{array}$ & $\begin{array}{l}14.91 \\
3.38 \\
22.67 \\
11.00\end{array}$ & $\begin{array}{r}39.98 \\
3.54 \\
8.86 \\
11.00\end{array}$ & $\begin{array}{l}25.40 \\
4.67 \\
18.39 \\
*\end{array}$ & $\begin{array}{r}16.10 \\
0.02 \\
0.13 \\
1.00\end{array}$ & $\begin{array}{r}30.00 \\
0.90 \\
3.01 \\
*\end{array}$ & $\begin{array}{l}26.39 \\
=- \\
=-\end{array}$ & $\begin{array}{r}9.68 \\
1.20 \\
12.40 \\
c .20\end{array}$ & $\begin{array}{l}5.08 \\
0.28 \\
5.52 \\
*\end{array}$ & $\begin{array}{r}1.30 \\
0.22 \\
16.93 \\
*\end{array}$ & $\begin{array}{r}181.34 \\
16.53\end{array}$ \\
\hline 1964 & $\begin{array}{l}\text { PRECIP } \text { (CM.) } \\
\text { SR-9C } \text { INCI/SQ.KY) } \\
\text { SR-90 CCNC. }(P C / L) \\
\text { SR-89/SR-90 }\end{array}$ & $\begin{array}{l}9.69 \\
0.46 \\
5.07 \\
*\end{array}$ & $\begin{array}{r}5.31 \\
0.76 \\
14.32 \\
*\end{array}$ & $\begin{array}{r}9.60 \\
0.99 \\
10.32 \\
1.10\end{array}$ & $\begin{array}{l}17.53 \\
1.13 \\
6.45 \\
0.06\end{array}$ & $\begin{array}{l}5.31 \\
0.78 \\
14.69 \\
*\end{array}$ & $\begin{array}{l}37.13 \\
2.03 \\
5.47 \\
-.\end{array}$ & $\begin{array}{l}8.71 \\
0.52 \\
5.98 \\
--\end{array}$ & $\begin{array}{l}10.11 \\
0.09 \\
0.90 \\
--\end{array}$ & $\begin{array}{l}9.30 \\
0.19 \\
2.05 \\
--\end{array}$ & $\begin{array}{l}9.02 \\
0.18 \\
2.00 \\
2.00\end{array}$ & $\begin{array}{l}5.61 \\
0.11 \\
1.97 \\
*\end{array}$ & $\begin{array}{l}4.01 \\
0.03 \\
0.75 \\
-.\end{array}$ & $\begin{array}{r}130.73 \\
7.27\end{array}$ \\
\hline 1965 & $\begin{array}{l}\text { PRECIP. (CM.) } \\
\text { SR-9C } \text { irCI/SQ.KM-) } \\
\text { SR-90 CCNC. (PC/L) } \\
\text { SR-99/SR-90 }\end{array}$ & $\begin{array}{l}6.25 \\
0.39 \\
6.25 \\
-.2\end{array}$ & $\begin{array}{l}3.05 \\
c .23 \\
7.55 \\
--\end{array}$ & $\begin{array}{l}3.45 \\
-- \\
--\end{array}$ & $\begin{array}{r}14.38 \\
0.40 \\
2.79 \\
--\end{array}$ & $\begin{array}{r}25.96 \\
0.94 \\
3.63 \\
--\end{array}$ & $\begin{array}{l}38.43 \\
0.47 \\
1.23 \\
-.\end{array}$ & $\begin{array}{l}43.43 \\
0.33 \\
0.76 \\
--\end{array}$ & $\begin{array}{l}4.50 \\
=- \\
=-\end{array}$ & $\begin{array}{l}28.22 \\
0.54 \\
1.92 \\
--\end{array}$ & $\begin{array}{l}10.52 \\
0.11 \\
1.05 \\
--\end{array}$ & $\begin{array}{l}\overline{0.18} \\
--\end{array}$ & $\begin{array}{l}=- \\
=-\end{array}$ & $\begin{array}{r}178.19 \\
3.59\end{array}$ \\
\hline 1966 & $\begin{array}{l}\text { PRECIP. (CM-1 } \\
\text { SR-SO INCI/SC.KR.) } \\
\text { SR-90 CONC. (PC/L) } \\
\text { SR-89/SR-90 }\end{array}$ & $\begin{array}{l}4.90 \\
0.24 \\
4.90 \\
--\end{array}$ & $\begin{array}{l}7.40 \\
c: 32 \\
4.19 \\
-:\end{array}$ & $\begin{array}{l}23.80 \\
= \\
=\end{array}$ & $\begin{array}{r}14.60 \\
0.67 \\
4.59 \\
--\end{array}$ & $\begin{array}{l}17.50 \\
0.14 \\
0.81 \\
--\end{array}$ & $\begin{array}{r}32.7 c \\
0.42 \\
1.29 \\
6.10\end{array}$ & $\begin{array}{r}17.40 \\
6.10 \\
0.58 \\
2.20\end{array}$ & $\frac{11.30}{*}$ & $\begin{array}{l}-- \\
z-\end{array}$ & $\begin{array}{l}-- \\
0.03 \\
-\end{array}$ & $\begin{array}{l}\overrightarrow{0.07} \\
\overrightarrow{10.00}\end{array}$ & $\begin{array}{c}-- \\
0.17 \\
-- \\
39.00\end{array}$ & $\begin{array}{r}129.60 \\
2.15\end{array}$ \\
\hline 1967 & $\begin{array}{l}\text { PRECIP. (CM-) } \\
\text { SR-90 iMC1/SQ.KM-) } \\
\text { SR-90 CONC. (PC/L) } \\
\text { SR-8S/SR-90 }\end{array}$ & $\begin{array}{r}6.00 \\
0.09 \\
1.51 \\
12.00\end{array}$ & $\begin{array}{l}3.20 \\
0.16 \\
5.01 \\
2.30\end{array}$ & $\begin{array}{r}20.60 \\
0.23 \\
1.12 \\
9.40\end{array}$ & $\begin{array}{l}-\overline{0.21} \\
-- \\
6.00\end{array}$ & $\begin{array}{l}8.50 \\
0.03 \\
0.36 \\
*\end{array}$ & $\begin{array}{r}15.80 \\
0.07 \\
0.45 \\
4.90\end{array}$ & $\begin{array}{l}\bar{z} \\
\bar{z}\end{array}$ & $\begin{array}{l}=- \\
=-\end{array}$ & $\begin{array}{l}=- \\
=-\end{array}$ & $\begin{array}{l}= \\
= \\
=\end{array}$ & $\begin{array}{l}= \\
= \\
=-\end{array}$ & $\begin{array}{l}= \\
= \\
-\end{array}$ & $\begin{array}{r}54.10 \\
0.79\end{array}$ \\
\hline \multicolumn{15}{|c|}{$\begin{array}{l}\text { NOTES } \\
\text { :- DATA NOT AVATLABLE } \\
\text { \&: ZERO OR TRACE } \\
\text { A: APPROXIMATE } \\
\text { B: LOHER LIMIT OF REPORTED DATA } \\
\text { C: PROPORTIONED FRCM ORIGINALLY CONSOLICATED DATA }\end{array}$} \\
\hline
\end{tabular}




\begin{tabular}{|c|c|c|c|c|c|c|c|c|c|c|c|c|c|c|}
\hline & & JAN. & FEB. & MAR. & APR. & MAY & JUNE & JULY & AUG. & SEP. & OCT. & Nov. & DEC. & CUM. TOTAL \\
\hline 1959 & $\begin{array}{l}\text { PRECIP. (CM.) } \\
\text { SR-9O (MCI/SQ.KM.) } \\
\text { SR-9C CONC. (PC/L) } \\
\text { SR-89/SR-90 }\end{array}$ & $\begin{array}{l}9.60 \\
-- \\
--\end{array}$ & $\begin{array}{l}4-88 \\
=- \\
=-\end{array}$ & $\begin{array}{l}11.25 \\
-- \\
--\end{array}$ & $\begin{array}{l}4.88 \\
-2 \\
--\end{array}$ & $\begin{array}{l}7.19 \\
-- \\
--\end{array}$ & $\begin{array}{l}13.56 \\
=- \\
--\end{array}$ & $\begin{array}{l}11.38 \\
=- \\
=-\end{array}$ & $\begin{array}{c}10.49 \\
=- \\
=-\end{array}$ & $\begin{array}{r}18.14 \\
0.16 \\
0.89 \\
0.46\end{array}$ & $\begin{array}{l}8.74 \\
0.09 \\
1.03 \\
1.00\end{array}$ & $\begin{array}{l}7.42 \\
0.06 \\
0.81 \\
*\end{array}$ & $\begin{array}{l}5.36 \\
0.10 \\
1.87 \\
*\end{array}$ & $\begin{array}{r}112.89 \\
0.41\end{array}$ \\
\hline 1960 & $\begin{array}{l}\text { PRECIP. (CM-) } \\
S R-9 C \text { INCI/SQ-KM-) } \\
S R-90 \text { CONC. (PC/L) } \\
S R-89 / S R-90\end{array}$ & $\begin{array}{r}23.50 \\
c .05 \\
0.22 \\
-\end{array}$ & $\begin{array}{l}9.93 \\
C .20 \\
2.02 \\
--\end{array}$ & $\begin{array}{l}6.76 \\
-- \\
=-\end{array}$ & $\begin{array}{l}10.03 \\
0.11 \\
1.10 \\
-.\end{array}$ & $\begin{array}{c}10.44 \\
\div- \\
--\end{array}$ & $\begin{array}{l}4.67 \\
* \\
--\end{array}$ & $\begin{array}{l}5.36 \\
- \\
--\end{array}$ & $\begin{array}{c}12 . c 1 \\
* \\
=\end{array}$ & $\begin{array}{l}8.36 \\
C .03 \\
0.36 \\
--\end{array}$ & $\begin{array}{l}16.66 \\
0.05 C \\
0.31 \\
-.\end{array}$ & $\begin{array}{l}9.65 \\
0.00 \mathrm{C} \\
0.01 \\
--\end{array}$ & $\begin{array}{l}11.63 \\
0.01 \mathrm{C} \\
0.09 \\
-.\end{array}$ & $\begin{array}{r}129.00 \\
0.45\end{array}$ \\
\hline 1961 & $\begin{array}{l}\text { PRECIP. (CM.) } \\
\text { SR-SO IMCI/SQ.KM.) } \\
\text { SR-9OCCNC. (PC/L) } \\
\text { SR-QS/SR-SO }\end{array}$ & $\begin{array}{l}6.05 \\
0.070 \\
1.16 \\
-.\end{array}$ & $\begin{array}{l}8.48 \\
0.09 \mathrm{C} \\
1.07 \\
-.\end{array}$ & $\begin{array}{l}2.01 \\
0.08 C \\
3.99 \\
=-\end{array}$ & $\begin{array}{l}6.99 \\
0.26 C \\
3.72 \\
--\end{array}$ & $\begin{array}{l}2.31 \\
0.04 C \\
1.74 \\
--\end{array}$ & $\begin{array}{l}9.45 \\
0.176 \\
1.8 C \\
--\end{array}$ & $\begin{array}{l}11.56 \\
0.05 C \\
C .44 \\
--\end{array}$ & $\begin{array}{l}8.18 \\
0.03 C \\
0.37 \\
-.\end{array}$ & $\begin{array}{c}14.68 \\
* \\
--\end{array}$ & $\begin{array}{r}12.42 \\
0.20 \\
1.62 \\
52.00\end{array}$ & $\begin{array}{r}4.85 \\
0.19 \\
3.92 \\
94.00\end{array}$ & $\begin{array}{r}7.77 \\
0.42 \\
5.41 \\
62.00\end{array}$ & $\begin{array}{r}94.75 \\
1.60\end{array}$ \\
\hline 1962 & $\begin{array}{l}\text { PRECIP. }(C M-) \\
S R-90 \text { (PCI/SQ.KM.) } \\
S R-90 \text { CONC. }(P C / L) \\
S R-89 / S R-90\end{array}$ & $\begin{array}{r}8.48 \\
0.33 \\
3.90 \\
19.00\end{array}$ & $\begin{array}{l}7.44 \\
--\end{array}$ & $\begin{array}{l}5.61 C \\
0.69 C \\
12.30 \\
--\end{array}$ & $\begin{array}{l}5.61 C \\
0.69 C \\
12.30 \\
-.\end{array}$ & $\begin{array}{r}5.66 \\
1.20 \\
21.21 \\
12.00\end{array}$ & $\begin{array}{r}5.84 \\
0.72 \\
12.33 \\
1 C . C C\end{array}$ & $\begin{array}{r}3.68 \\
0.02 \\
C .55 \\
12.0 C\end{array}$ & $\begin{array}{r}12.73 \\
0.34 \\
2.68 \\
33.60\end{array}$ & $\begin{array}{r}19.66 \\
1.10 \\
5.60 \\
17.00\end{array}$ & $\begin{array}{r}10.26 \\
1.22 \\
11.90 \\
43.00\end{array}$ & $\begin{array}{r}5.99 \\
0.39 \\
6.52 \\
51.00\end{array}$ & $\begin{array}{r}3.05 \\
0.29 \\
9.51 \\
39.00\end{array}$ & $\begin{array}{r}94.01 \\
6.99\end{array}$ \\
\hline 1963 & $\begin{array}{l}\text { PRECIP. }(C M .) \\
5 R-9 O \text { (MCI/SC.KM.) } \\
S R-9 C \text { CONC. }(P C / L) \\
S R-Q 9 / 5 R-90\end{array}$ & $\begin{array}{r}15.49 \\
0.39 \\
2.52 \\
55.00\end{array}$ & $\begin{array}{r}5.36 \\
1.01 \\
18.85 \\
26.00\end{array}$ & $\begin{array}{r}7.01 \\
0.81 \\
11.56 \\
30.00\end{array}$ & $\begin{array}{r}5.26 \\
1.64 \\
31.18 \\
10.00\end{array}$ & $\begin{array}{l}8.15 \\
0.59 \\
7.24 \\
8.60\end{array}$ & $\begin{array}{r}9.83 \\
1.71 \\
17.40 \\
1.10\end{array}$ & $\begin{array}{r}18.01 \\
2.79 \\
15.50 \\
2.00\end{array}$ & $\begin{array}{r}15.72 \\
1.72 \\
10.95 \\
3.00\end{array}$ & $\begin{array}{r}5.00 \\
6.79 \\
15.81 \\
0.60\end{array}$ & $\begin{array}{l}9.30 \\
C .84 \\
9.04 \\
+\end{array}$ & $\begin{array}{l}4.45 \\
0.35 \\
7.87 \\
*\end{array}$ & $\begin{array}{l}5.05 \\
0.30 \\
5.95 \\
+\end{array}$ & $\begin{array}{r}108.63 \\
12.94\end{array}$ \\
\hline 1964 & $\begin{array}{l}\text { PRECIP. (CM.) } \\
\text { SR-9C INCI/SQ.KM.) } \\
\text { SR-90 CONC. (PC/L) } \\
\text { SR-89/SR-90 }\end{array}$ & $\begin{array}{l}9.14 \\
0.55 \\
6.02 \\
*\end{array}$ & $\begin{array}{r}14.10 \\
6.76 \\
5.40 \\
*\end{array}$ & $\begin{array}{r}4.37 \\
0.64 \\
14.65 \\
1.60\end{array}$ & $\begin{array}{l}18.16 \\
1.93 \\
10.63 \\
*\end{array}$ & $\begin{array}{c}8.28 \\
1.71 \\
20.66 \\
*\end{array}$ & $\begin{array}{l}6.02 \\
1.91 \\
31.73 \\
--\end{array}$ & $\begin{array}{r}15.06 \\
0.24 \\
1.60 \\
--\end{array}$ & $\begin{array}{l}19.53 \\
0.18 \\
0.93 \\
--\end{array}$ & $\begin{array}{r}18.19 \\
0.64 \\
3.52 \\
-.\end{array}$ & $\begin{array}{l}4.60 \\
0.20 \\
4.35 \\
3.50\end{array}$ & $\begin{array}{r}15.32 \\
0.85 \\
5.55 \\
1.80\end{array}$ & $\begin{array}{l}2.64 \\
0.19 \\
7.20 \\
--\end{array}$ & $\begin{array}{r}135.41 \\
9.80\end{array}$ \\
\hline 1965 & $\begin{array}{l}\text { PRECIP }(C M-) \\
S R-90 \text { (NCI/SQ.KM.) } \\
S R-90 \text { CONC. }(P C / L) \\
S R-89 / S R-S O\end{array}$ & $\begin{array}{l}11.71 \\
0.24 \\
2.05 \\
-.\end{array}$ & $\begin{array}{l}6.40 \\
0.32 \\
5.01 \\
-.\end{array}$ & $\begin{array}{l}7.59 \\
0.30 \\
3.96 \\
--\end{array}$ & $\begin{array}{l}4.88 \\
0.32 \\
6.56 \\
--\end{array}$ & $\begin{array}{l}7.92 \\
0.36 \\
4.55 \\
--\end{array}$ & $\begin{array}{l}-- \\
--\end{array}$ & $\begin{array}{l}19.66 \\
0.60 \\
3.06 \\
-.\end{array}$ & $\begin{array}{l}5.26 \\
0.13 \\
2.48 \\
-.\end{array}$ & $\begin{array}{r}15.90 \\
0.35 \\
2.21 \\
--\end{array}$ & $\begin{array}{l}1.17 \\
0.06 \\
5.13 \\
-.\end{array}$ & $\begin{array}{l}12.34 \\
0.15 \\
1.22 \\
--\end{array}$ & $\begin{array}{l}7.29 \\
0.08 \\
1.10 \\
-.\end{array}$ & $\begin{array}{r}100.12 \\
2.91\end{array}$ \\
\hline 1966 & $\begin{array}{l}\text { PRECIP. (CM.) } \\
\text { SR-9O IMCI/SO.KM.) } \\
S R-90 \text { CCNC. (PC/L) } \\
S R-89 / 5 R-90\end{array}$ & $\begin{array}{l}7.92 \\
0.11 \\
1.39 \\
-0\end{array}$ & $\begin{array}{l}7.39 \\
0.13 \\
1.76 \\
-.\end{array}$ & $\begin{array}{l}7.57 \\
0.20 \\
2.65 \\
-.\end{array}$ & $\begin{array}{l}8.23 \\
0.07 \\
0.86 \\
--\end{array}$ & $\begin{array}{l}8.48 \\
0.29 \\
3.42 \\
--\end{array}$ & $\begin{array}{r}19.20 \\
0.12 \\
0.63 \\
*\end{array}$ & $\begin{array}{r}21.11 \\
0.13 \\
0.62 \\
2.80\end{array}$ & $\begin{array}{l}9.75 \\
0.10 \\
1.03 \\
+\end{array}$ & $\begin{array}{l}20.98 \\
0.09 \\
0.43 \\
*\end{array}$ & $\begin{array}{r}16.26 \\
0.12 \\
0.74 \\
+\end{array}$ & $\begin{array}{l}2.29 \\
0.03 \\
1.32 \\
8.70\end{array}$ & $\begin{array}{l}4.75 \\
0.02 \\
0.43 \\
*\end{array}$ & $\begin{array}{r}133.93 \\
1.41\end{array}$ \\
\hline 1967 & $\begin{array}{l}\text { PRECIP. (CM.) } \\
\text { SR-9C }\left(N C 1 / S Q . K M_{-}\right) \\
S R-90 \text { CONC }(P C / L) \\
S R-89 / S R-90\end{array}$ & $\begin{array}{r}12.52 \\
0.06 \\
0.48 \\
7.30\end{array}$ & $\begin{array}{l}2.77 \\
0.02 \\
0.73 \\
.\end{array}$ & $\begin{array}{r}3.89 \\
0.02 \\
0.52 \\
25.30\end{array}$ & $\begin{array}{l}4.11 \\
0.07 \\
1.71 \\
5.50\end{array}$ & $\begin{array}{l}6.56 \\
C .20 \\
3.05 \\
5.00\end{array}$ & $\begin{array}{l}5.26 \\
0.11 \\
2.10 \\
*\end{array}$ & $\begin{array}{l}9.02 \\
0.04 \\
0.45 \\
*\end{array}$ & $\begin{array}{l}-\infty \\
--\end{array}$ & $\begin{array}{l}-- \\
-- \\
--\end{array}$ & $\begin{array}{l}-- \\
-- \\
--\end{array}$ & $\begin{array}{l}-- \\
-- \\
--\end{array}$ & $\begin{array}{l}=- \\
=- \\
--\end{array}$ & $\begin{array}{r}44.13 \\
0.52\end{array}$ \\
\hline
\end{tabular}


JAN. FEB. MAR. APR. MAY JUNE JULY AUG. SEP. OCT. NOV. DEC. CUAM. TOTAL

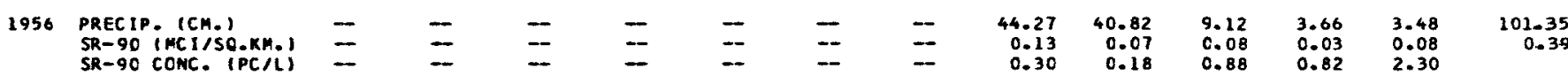

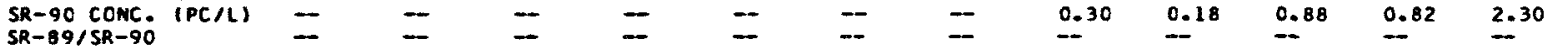
10.01 $\begin{array}{ll}S R-90 \text { (KCIISO.KM.) } & 0.39 \\ S R R-90 \text { CCNC. } & \text { (PCAL) } \\ S R-99 / S R-90 & 3.90\end{array}$

1958 PRECIP. (CM.) SR-90 (MCIISO.KM.) 6.35 $\begin{array}{lr}S R-90 \text { CONC. (PC/L) } & 3.63 \\ S R-B S / S R-90 & 16.00\end{array}$

1959 PRECIP ICH.I

8.51 $\begin{array}{ll}S R-9 C \text { (FCLISSC.KM-) } & 1.16 \\ S R-90 \text { CONC. (PG/L) } & 13.64 \\ S R-89 / S R-90 & 31.00\end{array}$

$8.33 \quad 3.56$

28.63

$\begin{array}{ll}6.36 & 25 . \\ 0.28 & 0.18\end{array}$

\begin{tabular}{rr}
.86 & 72.82 \\
.10 & 0.41 \\
.39 & 0.57 \\
\hline
\end{tabular}

$13.67 \quad 37.95$
$0.04 \quad 18$

$\begin{array}{llll}7.04 & 0.64 & 0.97 & 18.52\end{array}$

$\begin{array}{rrr}2.99 & 3.22 & 2.56 \\ 11.00 & 14.00 & 15.00\end{array}$

PRECIP. (CM.)

$14.81 \quad 9.02$

$\begin{array}{rr}0.83 & 1.44 \\ 5.61 & 15.97\end{array}$

1960 $\begin{array}{ll}\text { SR-9O CONC. (PC/L) } & 0.84\end{array}$

1961 PRECIP. (CM.) 8.79 SR-90 CONC. (PC $/ 1) 1.26$
SR-89/5R-90

1962 PRECIP. ICM.) $\begin{array}{ll}S R-90 \text { (MCI/SQ.KH.) } & 1.21 \\ S R-9 C \text { CCNC. (PCAL) } & 8.65\end{array}$

$\begin{array}{rrr}2.01 & 11.00 & 14.30 \\ 0.08 & 0.33 & 0.22\end{array}$ \begin{tabular}{rr}
0.08 & 0.33 \\
3.99 & 3.01 \\
\hline
\end{tabular} $\begin{array}{lcc}4.70 & 10.59 & 10.69 \\ 0.06 \mathrm{C} & 0.01 \mathrm{C} & 0.01 \mathrm{C}\end{array}$ $\begin{array}{lcc}4.70 & 10.59 & 10.69 \\ 0.06 \mathrm{C} & 0.01 \mathrm{C} & 0.01 \mathrm{C}\end{array}$ $1.28 \quad 0.10 \quad 0$. $S R-89 / S R+90$

\subsection{9}

6.20

$\begin{array}{rr}1.28 & 1.63 \\ 22.50 & 26.30 \\ 29.00 & 23.00\end{array}$

ISE 3 PRECIP. (CM.), 11.00 $S R-90$ (NC1/SC.KM.) 1.33 $\begin{array}{ll}S R-90 \text { CONC. }(P C / L) & 12.10 \\ S R-89 / S R-90 & 29.00\end{array}$

$3.10 \quad 10.90 \quad 20.19$

$\begin{array}{rrr}0.78 & 2.43 & 0.35 \\ 25.17 & 22.30 & 1.74\end{array}$

SR-89/SR-90

$35.00 \quad 15.00$

11.84
$S R-90$ irC1/SO.KM.)
0.76 SR-SO CCNC. (PC/L) 6.42 $S R-89 / S R-90$

1965 PRECIP. (CM.) (MRM.) 7.09 SR-GC CLNC. (PC/i)

7.09
0.32 $89 / S R-90$

1966 PRECIP. (CM.) 5.70 $S R-90$ (MC1/SQ.KMH)
$S R-90$ CONC. (PC/L) $S R-89 / S R-90$

1567 PRECIP. (CM.) SR-90 (MCI/SC.KK.) $\begin{aligned} & 9.70 \\ & 0.15\end{aligned}$ $\begin{array}{lr}\text { SR-90 CONC. (PC/L) } \\ \text { SR-89/SR-90 } & 1.55 \\ & 14.00\end{array}$

$\begin{array}{rr}7.44 & 27.13 \\ 0.89 & 0.29 \\ 1.97 & 1.07\end{array}$

0.34

$\begin{array}{rr}3.68 & 2.26 \\ 4.00 & 35.0 C\end{array}$

19.91

10.62

$\begin{array}{lll}2 & 28.83 & 37 \\ 1 & 0.18 & 04 \\ 7 & 0.63 & 0 \\ & - & \\ & 36.25\end{array}$

$\begin{array}{rr}33.97 & 0.53 \\ 6.00 & 60.00\end{array}$

.44

$\begin{array}{lll}5.36 & 6.15 \quad 13.67\end{array}$

257.02
2.66 5.88
7.30

0.026

$30.89 \quad 3.81$ 0.09

0.026
0.07

22.30

0.86

0.8

$\begin{array}{llllll}0.02 C & 0.036 & 0.01 & 0.05 & 0.41 & 0.78\end{array}$

$\begin{array}{llllll}0.15 & 0.17 & 6.10 & 0.33 & 3.29 & 12.01\end{array}$

$\begin{array}{llllllll}19.20 & 31.39 & 48.82 & 32.69 & 19.00 & 11.99 & 12.50 & 7.90\end{array}$

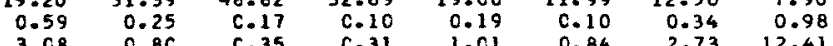
1.00 $\begin{array}{rrrr}9.8 \mathrm{C} & 0.35 & 0.31 & 1.01 \\ 9.0 \mathrm{C} & 14.00 & 15.00 & 15.00\end{array}$

34.49
0.26

0.26
0.76

$2.40 \quad 4 c .41$

0.48
2.15

0.19

1.40
0.21
1.85

33
0
0
-

$\begin{array}{llll}63 & 6.40 & 14.50 & 10.72\end{array}$

$\begin{array}{llll}84 & 5.99 & 5.64 & 8.05 \\ 20 & 0.34 & 0.45 & 1.01\end{array}$

$\begin{array}{lll}5.34 & 0.45 & 1.01 \\ 5.68 & 7.98 & 12.55\end{array}$

$\begin{array}{rrr}9.91 & 10.01 & 3.61 \\ 0.05 & 0.25 & 0.03\end{array}$

$\begin{array}{lll}0.51 & 2.50 \quad 0.84\end{array}$

$\begin{array}{llllllll}0.27 & 5.26 & 17.20 & 21.16 & 44.04 & 50.34 & 13.41 & 9.80\end{array}$

$6.40 * 0.0$

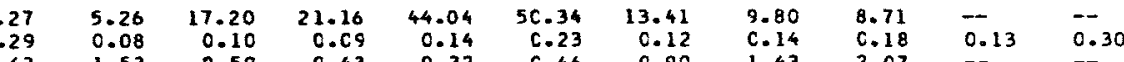

$\begin{array}{lll}.63 & 1.53 & 0.59\end{array}$ 0.43

c. 32

2.0

$\begin{array}{rrrr}5.50 & 12.90 & 13.50 & 14.20 \\ 0.28 & 0.34 & 0.27 & 0.25 \\ 5.10 & 2.64 & 2.01 & 1.06\end{array}$

$0.16 \quad-$

-

2.12

2.642 .01

$-$

$=-$

$\begin{array}{lll}-0.03 & \overline{0.07} & \overline{0.32} \\ -- & \overline{-} & \overline{-}\end{array}$

51.80
1.73

43.80
1.06

NOTES

-: OATA not available

* ZERO OR TRACE

B: LOWER LIMIT OF REPORTED DATA
C: PROPORTIOKEO FROM ORIGINALIY CONSOLIOATEO OATA 
JAN. FEB. MAR. APR. MAY JUNE JULY AUG. SEP. OCT. NOV. DEC. CUM. TOTAL

\begin{tabular}{|c|c|c|c|c|c|c|c|c|c|c|c|c|c|c|}
\hline & & & & & & & & & & & & & & \\
\hline 1959 & $\begin{array}{l}\text { PRECIP. (CM.) } \\
\text { SR-90 (NCIISQ.KM.) } \\
\text { SR-90 CONC. (PC/L) } \\
\text { SR-89/SR-90 }\end{array}$ & $\bar{m}=$ & $m$ & $=$ & $\begin{array}{l}-- \\
\overline{-} \\
--\end{array}$ & $\begin{array}{l}-- \\
--\end{array}$ & $=$ & $=$ & $\begin{array}{l}-- \\
--\end{array}$ & $\begin{array}{l}-- \\
-- \\
--\end{array}$ & $\begin{array}{l}--13 \\
--\end{array}$ & $\begin{array}{l}7.04 \\
-- \\
--\end{array}$ & $\begin{array}{l}9.53 \\
0.05 \\
0.53 \\
*\end{array}$ & $\begin{array}{r}16.57 \\
0.18\end{array}$ \\
\hline 1960 & $\begin{array}{l}\text { PRECIP. (CM.) } \\
\text { SR-9O IMCI/SQ.KM-) } \\
\text { SR-9O CONC. (PC/L) } \\
\text { SR-QS/SR-SO }\end{array}$ & $\begin{array}{l}4.57 \\
0.08 \\
1.76 \\
-2\end{array}$ & $\begin{array}{r}0.28 \\
0.05 \\
17.86 \\
-.\end{array}$ & $\begin{array}{l}5.33 \\
0.15 \\
2.82 \\
--\end{array}$ & $\begin{array}{l}16.31 \\
0.37 \\
2.27 \\
--\end{array}$ & $\begin{array}{l}13.28 \\
0.29 \mathrm{C} \\
2.19 \\
--\end{array}$ & $\begin{array}{l}8.51 \\
0.18 \mathrm{C} \\
2.12 \\
--\end{array}$ & $\begin{array}{l}3.63 \\
* \\
--\end{array}$ & $\begin{array}{l}40.69 \\
* \\
=-\end{array}$ & $\begin{array}{l}16.41 \\
0.05 \mathrm{C} \\
0.31 \\
--\end{array}$ & $\begin{array}{l}12.88 \\
C .040 \\
c .32 \\
--\end{array}$ & $\begin{array}{l}6.96 \\
0.116 \\
1.59 \\
=-\end{array}$ & $\begin{array}{l}7.09 \\
0.116 \\
1.56 \\
-.\end{array}$ & $\begin{array}{r}135.94 \\
1.43\end{array}$ \\
\hline 1961 & $\begin{array}{l}\text { PRECIP. ICM.) } \\
\text { SR-9O IPCI/SC.KM.) } \\
\text { SR-9O CCNC. (PC/L) } \\
\text { SR-89/SR-SO }\end{array}$ & $\begin{array}{l}1.73 \\
0.176 \\
9.83 \\
-.\end{array}$ & $\begin{array}{l}3.40 \\
0.17 \mathrm{C} \\
5.01 \\
-.\end{array}$ & $\begin{array}{l}8.79 \\
0.13 \mathrm{C} \\
1.48 \\
-.\end{array}$ & $\begin{array}{l}15.01 \\
0.23 \mathrm{C} \\
1.54 \\
-.\end{array}$ & $\begin{array}{l}4.85 \\
0.04 \mathrm{C} \\
0.83 \\
--\end{array}$ & $\begin{array}{l}33.60 \\
0.28 C \\
0.84 \\
=-\end{array}$ & $\begin{array}{l}3.18 \\
C .02 C \\
C .03 \\
--\end{array}$ & $\begin{array}{l}9.88 \\
0.06 C \\
0.61 \\
--\end{array}$ & $\begin{array}{l}7.29 \\
+ \\
--\end{array}$ & $\begin{array}{r}29.62 \\
0.03 \\
0.11 \\
80.00\end{array}$ & $\begin{array}{r}4.83 \\
0.07 \\
1.45 \\
78.00\end{array}$ & $\begin{array}{r}3.68 \\
0.18 \\
4.90 \\
66.00\end{array}$ & $\begin{array}{r}125.86 \\
1.38\end{array}$ \\
\hline 1962 & $\begin{array}{l}\text { PRECIP. }\left(C M_{-}\right) \\
\text {SR-SO (KCI/SQ.KM.) } \\
S R-90 \text { CCNC. (PC/L) } \\
S R-89 / S R-S C\end{array}$ & $\begin{array}{r}2.16 \\
0.14 \\
6.49 \\
44.00\end{array}$ & $\begin{array}{r}1.96 \\
0.19 \\
9.70 \\
27.00\end{array}$ & $\begin{array}{l}5.18 \\
* \\
-- \\
--\end{array}$ & $\begin{array}{r}9.83 \\
0.68 \\
6.92 \\
12.00\end{array}$ & $\begin{array}{r}14.22 \\
0.77 \\
5.42 \\
10.00\end{array}$ & $\begin{array}{r}19.23 \\
1.63 \\
8.48 \\
7.00\end{array}$ & $\begin{array}{r}27.25 \\
0.87 \\
3.20 \\
7.00\end{array}$ & $\begin{array}{r}22.28 \\
0.15 \\
0.68 \\
13.00\end{array}$ & $\begin{array}{r}1.75 \\
0.06 \\
3.43 \\
15.00\end{array}$ & $\begin{array}{r}10.39 \\
0.46 \\
4.43 \\
37.00\end{array}$ & $\begin{array}{r}13.87 \\
0.58 \\
4.19 \\
46.00\end{array}$ & $\begin{array}{r}6.32 \\
0.19 \\
3.01 \\
51.00\end{array}$ & $\begin{array}{r}134.44 \\
5.72\end{array}$ \\
\hline 1963 & $\begin{array}{l}\text { PRECIP. (CM.) } \\
\text { SR-90 (NCI/SQ.KM.) } \\
\text { SR-90 CONC. IPC/L) } \\
\text { SR-89/SR-9O }\end{array}$ & $\begin{array}{r}0.13 \\
0.10 \\
76.93 \\
17.00\end{array}$ & $\begin{array}{r}0.84 \\
0.14 \\
16.67 \\
28.00\end{array}$ & $\begin{array}{r}6.12 \\
1.19 \\
19.45 \\
12.00\end{array}$ & $\begin{array}{r}6.58 \\
1.39 \\
21.13 \\
9.00\end{array}$ & $\begin{array}{r}9.47 \\
2.38 \\
25.14 \\
4.70\end{array}$ & $\begin{array}{r}21.06 \\
2.84 \\
13.49 \\
2.40\end{array}$ & $\begin{array}{r}8.00 \\
1.33 \\
16.63 \\
2.00\end{array}$ & $\begin{array}{l}27.97 \\
0.74 \\
2.65 \\
*\end{array}$ & $\begin{array}{l}7.16 \\
1.08 \\
15.09 \\
*\end{array}$ & $\begin{array}{r}25.02 \\
0.77 \\
3.08 \\
1.00\end{array}$ & $\begin{array}{l}7.09 \\
0.56 \\
7.90 \\
*\end{array}$ & $\begin{array}{l}2.59 \\
0.15 \\
5.80 \\
*\end{array}$ & $\begin{array}{r}122.03 \\
12.67\end{array}$ \\
\hline 1964 & $\begin{array}{l}\text { PRECIP. (CM.) } \\
\text { SR-9C (PCI/SQ.KM) } \\
5 R-90 \text { CCNC. (PC/L) } \\
S R-89 / S R-90\end{array}$ & $\begin{array}{l}10.92 \\
c .03 \\
0.28 \\
*\end{array}$ & $\begin{array}{l}6.43 \\
0.24 \\
3.74 \\
0.50\end{array}$ & $\begin{array}{l}8.26 \\
0.28 \\
3.39 \\
4.20\end{array}$ & $\begin{array}{l}8.74 \\
0.62 \\
7.10 \\
*\end{array}$ & $\begin{array}{l}7.85 \\
C .65 \\
8.29 \\
*\end{array}$ & $\begin{array}{r}9.04 \\
1.08 \\
11.95 \\
--\end{array}$ & $\begin{array}{l}4.04 \\
1.04 \\
25.75 \\
--\end{array}$ & $\begin{array}{l}35.74 \\
0.17 \\
0.48 \\
-.\end{array}$ & $\begin{array}{l}12.37 \\
0.10 \\
0.81 \\
--\end{array}$ & $\begin{array}{l}9.04 \\
* \\
*-\end{array}$ & $\begin{array}{l}4.17 \\
* \\
*\end{array}$ & $\begin{array}{l}4.32 \\
0.08 \\
1.86\end{array}$ & $\begin{array}{r}120.92 \\
4.29\end{array}$ \\
\hline 1985 & $\begin{array}{l}\text { PRECIP. (CM.) } \\
\text { SR-9C (NCI/SQ-KM) } \\
S R-90 \text { CONC. (PC/L) } \\
\text { SR-89/SR-90 }\end{array}$ & $\begin{array}{l}1.60 \\
0.04 \\
2.51 \\
--\end{array}$ & $\begin{array}{l}0.94 \\
C .04 \\
4.26 \\
-.\end{array}$ & $\begin{array}{l}3.56 \\
0.11 \\
3.09 \\
-.\end{array}$ & $\begin{array}{r}14.33 \\
0.45 \\
3.15 \\
-.\end{array}$ & $\begin{array}{l}29.03 \\
C .82 \\
2.83 \\
--\end{array}$ & $\begin{array}{r}22.10 \\
0.35 \\
1.77 \\
-.\end{array}$ & $\begin{array}{r}41.43 \\
C .45 \\
1.09 \\
-\end{array}$ & $\begin{array}{l}33.25 \\
1.01 \\
3.04 \\
-.\end{array}$ & $\begin{array}{l}20.22 \\
C .13 \\
0.65 \\
-.\end{array}$ & $\begin{array}{l}4.93 \\
0.17 \\
3.45 \\
-. .\end{array}$ & $\begin{array}{l}8.64 \\
=- \\
--\end{array}$ & $\begin{array}{l}7.16 \\
0.05 \\
0.70 \\
-.\end{array}$ & $\begin{array}{r}187.19 \\
3.66\end{array}$ \\
\hline 1966 & $\begin{array}{l}\text { PRECIP }(C M-) \\
S R-90 \text { (NCI/SQ.KM.) } \\
S R-90 \text { CONC. }(P C / L) \\
S R-89 / S R-90\end{array}$ & $\begin{array}{l}1.90 \\
* \\
-- \\
--\end{array}$ & $\begin{array}{l}9.65 \\
0.12 \\
1.25 \\
-. .\end{array}$ & $\begin{array}{l}9.22 \\
0.36 \\
3.91 \\
--\end{array}$ & $\begin{array}{l}11.94 \\
0.31 \\
2.60 \\
--\end{array}$ & $\begin{array}{l}15.62 \\
0.14 \\
0.90 \\
--\end{array}$ & $\begin{array}{r}48.54 \\
0.35 \\
0.73 \\
2.10\end{array}$ & $\begin{array}{l}16.54 \\
* \\
-\end{array}$ & $\begin{array}{l}5.10 \\
* \\
*\end{array}$ & $\begin{array}{l}-- \\
--\end{array}$ & $\begin{array}{r}12.12 \\
6.07 \\
0.58 \\
.\end{array}$ & $\begin{array}{l}1.85 \\
0.01 \\
0.55 \\
*\end{array}$ & $\begin{array}{r}0.79 \\
0.01 \\
1.27 \\
15.00\end{array}$ & $\begin{array}{r}133.27 \\
1.37\end{array}$ \\
\hline 1967 & $\begin{array}{l}\text { PRECIP. (CM.) } \\
\text { SR-9O (NCI/SO.KM) } \\
\text { SR-9O CCNC. (PC/L) }\end{array}$ & $\begin{array}{l}2.06 \\
0.04 \\
1.95\end{array}$ & $\begin{array}{l}3.68 \\
0.01 \\
0.28\end{array}$ & $\begin{array}{l}7.26 \\
0.01 \\
0.14\end{array}$ & $\begin{array}{l}8.58 \\
0.01 \\
0.12\end{array}$ & $\begin{array}{l}7.67 \\
0.11 \\
1.44\end{array}$ & $\begin{array}{l}8.71 \\
0.07 \\
0.81\end{array}$ & $\overline{--}$ & 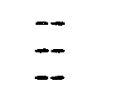 & $\begin{array}{l}-- \\
--\end{array}$ & $\begin{array}{l}-- \\
--\end{array}$ & $\overline{-}$ & $=$ & $\begin{array}{r}37.96 \\
0.25\end{array}$ \\
\hline
\end{tabular}

NOTES

-- DATA NOT AVAILABLE

*: ZERO DR TRACE

: LOWER LIMIT OF REPORTED DATA

C: PROPORTIONED FROM CRIGINALLY CONSOLICATEC DATA 
MONTHLY FALLOUT DEPOSITION COLLECTIONS

SOURCE: U. S. WEATHER BUREAU AIRPORT STATION

\begin{tabular}{|c|c|c|c|c|c|c|c|c|c|c|c|c|c|c|c|}
\hline & & & JAN. & FEB. & MAR. & APR. & MAY & JUNE & JULY & AUG. & SEP. & OCT & NOV. & DEC. & CUM. TOTAL \\
\hline & 1960 & $\begin{array}{l}\text { PRECIP. (CM.) } \\
S R-90(M C I / S Q-K M-) \\
S R-90 \text { CONC }(P C / L) \\
S R-89 / S R-90\end{array}$ & $\bar{m}=$ & $\begin{array}{r}0.99 \\
0.12 \\
12.13 \\
-.\end{array}$ & $\begin{array}{l}13.41 \\
0.05 \\
0.38 \\
-.\end{array}$ & $\begin{array}{l}2.21 \\
0.07 \\
3.27 \\
-.\end{array}$ & $\begin{array}{l}2.62 \\
0.10 \mathrm{C} \\
3.82 \\
-\end{array}$ & $\begin{array}{l}2.01 \\
0.080 \\
3.95 \\
-.\end{array}$ & $\begin{array}{l}2.11 \\
0.000 \\
c .01 \\
-.\end{array}$ & $\begin{array}{l}4.19 \\
0.006 \\
0.01 \\
-\end{array}$ & $\begin{array}{l}9.70 \\
0.016 \\
0.11 \\
--\end{array}$ & $\begin{array}{l}17.70 \\
0.02 \mathrm{C} \\
0.12 \\
=-\end{array}$ & $\begin{array}{l}7.92 \\
0.026 \\
0.26 \\
-.\end{array}$ & $\begin{array}{l}7.92 \\
0.02 \mathrm{C} \\
0.26 \\
-.\end{array}$ & $\begin{array}{r}70.78 \\
0.49\end{array}$ \\
\hline & 1961 & $\begin{array}{l}\text { PRECIP. (CM.) } \\
\text { SR-9O (MCI/SO.KM.) } \\
S R-90 \text { CONC. }(P C / L) \\
S R-89 / S R-90\end{array}$ & $\begin{array}{l}1.63 \\
0.026 \\
1.23 \\
-.\end{array}$ & $\begin{array}{l}2.79 \\
0.036 \\
1.08 \\
--\end{array}$ & $\begin{array}{l}1.98 \\
=- \\
=\end{array}$ & $\begin{array}{l}8.05 \\
0.08 \\
1.00 \\
-\end{array}$ & $\begin{array}{l}0.99 \\
0.00 \mathrm{C} \\
0.01 \\
--\end{array}$ & $\begin{array}{l}2.11 \\
0.01 \mathrm{C} \\
0.4 \mathrm{e} \\
-.\end{array}$ & $\begin{array}{l}4.32 \\
0.01 \\
0.24 \\
-.\end{array}$ & $\begin{array}{l}9.60 \\
0.026 \\
0.21 \\
--\end{array}$ & $\begin{array}{r}2.87 \\
0.02 \\
0.70 \\
19.00\end{array}$ & $\begin{array}{r}32.31 \\
C .03 \\
0.10 \\
111.00\end{array}$ & $\begin{array}{r}7.70 \\
0.27 \\
3.51 \\
86.00\end{array}$ & $\begin{array}{r}4.42 \\
0.11 \\
2.49 \\
58.00\end{array}$ & $\begin{array}{r}78.77 \\
0.60\end{array}$ \\
\hline & 1962 & $\begin{array}{l}\text { PREC IP. (CM.) } \\
\text { SR-9C (NCI/SC.KM-) } \\
S R-90 \text { CCAC. (PC/L) } \\
S R-89 / 5 R-90\end{array}$ & $\begin{array}{r}4.95 \\
6.18 \\
3.64 \\
38.00\end{array}$ & $\begin{array}{r}9.93 \\
0.46 \\
4.64 \\
28.00\end{array}$ & $\begin{array}{c}\overline{1.08} \\
19.00\end{array}$ & $\begin{array}{r}9.73 \\
0.03 \\
0.31 \\
17.00\end{array}$ & $\begin{array}{r}4.60 \\
0.05 \\
1.09 \\
12.00\end{array}$ & $\begin{array}{l}2.92 \\
0.27 \\
9.25 \\
8.00\end{array}$ & $\begin{array}{r}1.30 \\
C .51 \\
35.24 \\
3.00\end{array}$ & $\begin{array}{r}37.59 \\
0.02 \\
0.06 \\
8.00\end{array}$ & $\begin{array}{r}5.28 \\
0.14 \\
2.86 \\
11.00\end{array}$ & $\begin{array}{r}2.18 \\
0.02 \\
0.92 \\
16.00\end{array}$ & $\begin{array}{r}4.47 \\
0.02 \\
0.45 \\
31.00\end{array}$ & $\begin{array}{r}1.57 \\
0.01 \\
0.64 \\
50.00\end{array}$ & $\begin{array}{r}94.52 \\
2.79\end{array}$ \\
\hline p & 1963 & $\begin{array}{l}\text { PRECIP. }\left(C M_{-}\right) \\
S R-90 \text { (INCI/SQ.KM.) } \\
S R-9 C \text { CCNC. (PC/L) } \\
S R-8 S / S R-S C\end{array}$ & $\begin{array}{r}0.74 \\
0.02 \\
2.71 \\
26.00\end{array}$ & $\begin{array}{r}1.04 \\
0.03 \\
2.89 \\
25.00\end{array}$ & $\begin{array}{r}5.89 \\
0.09 \\
1.53 \\
13.00\end{array}$ & $\begin{array}{r}19.35 \\
0.14 \\
0.73 \\
11.00\end{array}$ & $\begin{array}{r}30.58 \\
0.22 \\
0.72 \\
7.60\end{array}$ & $\begin{array}{l}5.33 \\
0.13 \\
2.44 \\
4.00\end{array}$ & $\begin{array}{l}3.05 \\
0.12 \\
3.94 \\
3.00\end{array}$ & $\begin{array}{l}8.38 \\
0.02 \\
0.24 \\
*\end{array}$ & $\begin{array}{l}5.28 \\
C .02 \\
C .38 \\
+\end{array}$ & $\begin{array}{l}2.79 \\
0.05 \\
1.80 \\
+\end{array}$ & $\begin{array}{l}4.27 \\
0.01 \\
0.24 \\
*\end{array}$ & $\begin{array}{l}2.49 \\
0.03 \\
1.21 \\
*\end{array}$ & $\begin{array}{r}89.19 \\
0.88\end{array}$ \\
\hline 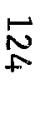 & $19 \in 4$ & $\begin{array}{l}\text { PRECIP. (CM.) } \\
S R-9 C \text { INCI/SQ.KM.) } \\
S R-90 \text { CCNC. }(P C / L) \\
S R-89 / S R-90\end{array}$ & $\begin{array}{l}2.62 \\
C .07 \\
2.68 \\
*\end{array}$ & $\begin{array}{l}2.95 \\
0.19 \\
6.45 \\
0.90\end{array}$ & $\begin{array}{l}1.93 \\
0.08 \\
4.15 \\
*\end{array}$ & $\begin{array}{r}21.06 \\
0.08 \\
0.38 \\
0.38\end{array}$ & $\begin{array}{l}9.07 \\
0.02 \\
0.23 \\
2.60\end{array}$ & $\begin{array}{l}3.81 \\
0.03 \\
0.84 \\
-.\end{array}$ & $\begin{array}{l}2.08 \\
C .11 \\
5.29 \\
--\end{array}$ & $\begin{array}{l}2.44 \\
0.08 \\
3.28 \\
--\end{array}$ & $\begin{array}{l}2.69 \\
0.05 \\
1.86 \\
--\end{array}$ & $\begin{array}{r}5.92 \\
6.05 \\
C .85 \\
18.00\end{array}$ & $\begin{array}{l}4.14 \\
0.07 \\
1.70 \\
+\end{array}$ & $\begin{array}{l}33.12 \\
0.64 \\
1.94 \\
-\end{array}$ & $\begin{array}{r}91.63 \\
1.47 .\end{array}$ \\
\hline & 1965 & $\begin{array}{l}\text { PRECIP. (CM.) } \\
S R-9 C \text { (MCI/SO.KM.) } \\
S R-9 C \text { CCNC. (PC/L) } \\
S R-S G / S R-S O\end{array}$ & $\begin{array}{l}3.96 \\
0.12 \\
3.04 \\
=-\end{array}$ & $\begin{array}{r}2.46 \\
0.53 \\
21.55 \\
-.\end{array}$ & $\begin{array}{r}2.67 \\
0.30 \\
11.24 \\
--\end{array}$ & $\begin{array}{l}1.37 \\
0.10 \\
7.30 \\
-.\end{array}$ & $\begin{array}{r}11.35 \\
0.22 \\
1.94 \\
-.\end{array}$ & $\begin{array}{l}2.74 \\
0.15 \\
6.94 \\
--\end{array}$ & $\begin{array}{l}1.57 \\
C .10 \\
6.37 \\
--\end{array}$ & $\begin{array}{l}3.71 \\
0.08 \\
2.16 \\
-.\end{array}$ & $\begin{array}{l}4.57 \\
0.3 C \\
6.57 \\
--\end{array}$ & $\begin{array}{l}8.28 \\
C .07 \\
0.85 \\
--\end{array}$ & $\begin{array}{l}4.29 \\
0.16 \\
3.73 \\
-.\end{array}$ & $\begin{array}{l}5.69 \\
0.05 \\
0.88 \\
--\end{array}$ & $\begin{array}{r}52.66 \\
2.22\end{array}$ \\
\hline & 1966 & $\begin{array}{l}\text { PRECIP. (CM.) } \\
S R-90 \text { (NCI/SO-KM.) } \\
S R-90 \text { CCNC. }(P C / L) \\
S R-89 / S R-90\end{array}$ & $\begin{array}{l}1.80 \\
C .08 \\
4.45 \\
-.-\end{array}$ & $\begin{array}{l}13.56 \\
C .03 \\
0.23 \\
--\end{array}$ & $\begin{array}{l}0.89 \\
0.06 \\
6.75 \\
-.\end{array}$ & $\begin{array}{l}0.79 \\
0.10 \\
12.66 \\
-\end{array}$ & $\begin{array}{c}0.28 \\
0.03 \\
10.72 \\
-.\end{array}$ & $\begin{array}{r}1.07 \\
0.02 \\
1.87 \\
27.06\end{array}$ & $\begin{array}{l}3.18 \\
C .04 \\
1.26 \\
*\end{array}$ & $\begin{array}{l}5.41 \\
0.02 \\
0.37 \\
*\end{array}$ & $\begin{array}{l}1.24 \\
* \\
*\end{array}$ & $\begin{array}{l}21.03 \\
0.01 \\
c .05 \\
*\end{array}$ & $\begin{array}{r}10.90 \\
0.01 \\
0.10 \\
51.00\end{array}$ & $\begin{array}{l}3.07 \\
0.02 \\
0.66 \\
*\end{array}$ & $\begin{array}{r}63.22 \\
0.42\end{array}$ \\
\hline & 1967 & $\begin{array}{l}\text { PRECIP. (CM.) } \\
\text { SR-9O (NCI/SC.KM-) } \\
S R-90 \text { CONC. }(P C / L) \\
S R-89 / S R-90\end{array}$ & $\begin{array}{l}2.21 \\
0.03 \\
1.36 \\
+\end{array}$ & $\begin{array}{l}6.45 \\
0.02 \\
0.32 \\
+\end{array}$ & $\begin{array}{r}16.99 \\
0.05 \\
0.30 \\
7.10\end{array}$ & $\begin{array}{l}1.30 \\
0.02 \\
1.54 \\
+\end{array}$ & $\begin{array}{l}5.31 \\
0.01 \\
0.19 \\
+\end{array}$ & $\begin{array}{l}4.70 \\
0.01 \\
0.22 \\
.\end{array}$ & $\begin{array}{l}-- \\
-- \\
--\end{array}$ & $\begin{array}{l}-- \\
--\end{array}$ & $\begin{array}{l}-- \\
-- \\
--\end{array}$ & $\begin{array}{l}-- \\
--\end{array}$ & $\begin{array}{l}-- \\
-- \\
--\end{array}$ & $\begin{array}{l}-- \\
--\end{array}$ & $\begin{array}{r}36.96 \\
0.14\end{array}$ \\
\hline & 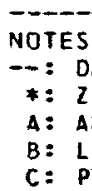 & $\begin{array}{l}\text { ATA NOT AVAILABLE } \\
\text { ERD OR TRACE } \\
\text { PPROXIMATE } \\
\text { OWER LIMIT OF REPOR } \\
\text { ROPORTIONED FRCM CR }\end{array}$ & $\begin{array}{l}\text { DATA } \\
\text { INALLYY }\end{array}$ & & & & & & & & & & & & \\
\hline
\end{tabular}


MONTHLY FALLOUT DEPOSITION COLLECTIONS

SITE: KENYA, KIKUYU

LAT. 1135 LONG. $3638 E$

ALT.

2074M. (POT)

SOURCE: EAST AFRICAN AGRICULTURAL ANO FORESTRY RESEARCH ORGANIZATION

\begin{tabular}{|c|c|c|c|c|c|c|c|c|c|c|c|c|c|c|}
\hline & & JAN. & FE8. & MAR . & APR. & MAY & JUNE & JULY & AUG. & SEP. & OCT. & Nov. & OEC. & CUM. TOTAL \\
\hline 1957 & $\begin{array}{l}\text { PRECIP. (CM.) } \\
\text { SR-SC INCI/SQ.KM.) } \\
\text { SR-SC CCNC. (PC/L) } \\
S R-89 / S R-90\end{array}$ & $\begin{array}{l}12.98 \\
0.05 \\
c .39 \\
--\end{array}$ & $\begin{array}{l}5.08 \\
0.10 \\
1.97 \\
-.\end{array}$ & $\begin{array}{l}1.75 \\
0.01 \\
0.58 \\
-.\end{array}$ & $\begin{array}{l}26.11 \\
0.01 \\
0.04 \\
-.\end{array}$ & $\begin{array}{l}28.93 \\
0.05 \\
0.18 \\
--\end{array}$ & $\begin{array}{r}14.45 \\
0.07 \\
0.45 \\
-\end{array}$ & $\begin{array}{l}1.96 \\
c .06 \\
3.07 \\
--\end{array}$ & $\begin{array}{l}0.23 \\
0.01 \\
4.35 \\
-\end{array}$ & $\begin{array}{l}3.20 \\
c .02 \\
c .63\end{array}$ & $\begin{array}{l}5.92 \\
0.03 \\
0.51 \\
-\end{array}$ & $\begin{array}{l}12.40 \\
0.02 \\
0.17 \\
\ldots\end{array}$ & $\begin{array}{l}8.33 \\
0.06 \\
0.73 \\
--\end{array}$ & $\begin{array}{r}121.34 \\
0.49\end{array}$ \\
\hline 1958 & 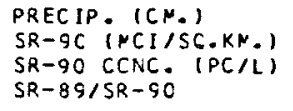 & $\begin{array}{r}2.29 \\
C .01 \\
6.44 \\
24.00\end{array}$ & $\begin{array}{r}13.87 \\
0.05 \\
0.37 \\
12.00\end{array}$ & $\begin{array}{l}8.66 \\
0.08 \\
0.93 \\
5.00\end{array}$ & $\begin{array}{r}12.17 \\
0.05 \\
0.42 \\
6.00\end{array}$ & $\begin{array}{r}3 C . C 2 \\
0.35 \\
1.17 \\
5.00\end{array}$ & $\begin{array}{l}7.34 \\
0.08 \\
1.09 \\
30.0 \mathrm{C}\end{array}$ & $\begin{array}{r}6.12 \\
0.10 \\
1.64 \\
48.00\end{array}$ & $\begin{array}{r}0.71 \\
0.05 \\
7.05 \\
24.00\end{array}$ & $\begin{array}{r}0.20 \\
C .03 \\
15.01 \\
5 C .0 C\end{array}$ & $\begin{array}{r}5.92 \\
0.10 \\
1.69 \\
26.00\end{array}$ & $\begin{array}{r}6.07 \\
0.06 \\
0.99 \\
37.00\end{array}$ & $\begin{array}{r}0.45 \\
0.05 \\
0.78 \\
02.00\end{array}$ & $\begin{array}{r}99.82 \\
1.01\end{array}$ \\
\hline 1959 & $\begin{array}{l}\text { PRECIP. (CM.) } \\
S R-9 O \text { (MCI/SQ.KM.) } \\
S R-9 C \text { CCAC. }(P C / L) \\
S R-89 / S K-90\end{array}$ & $\begin{array}{r}1.65 \\
0.09 \\
5.46 \\
32.00\end{array}$ & $\begin{array}{r}4.34 \\
0.08 \\
1.85 \\
28.00\end{array}$ & $\begin{array}{r}6.25 \\
0.10 \\
1.81 \\
19.00\end{array}$ & $\begin{array}{r}10.29 \\
0.14 \\
1.37 \\
12.00\end{array}$ & $\begin{array}{r}15.82 \\
C . C 8 \\
C .51 \\
6.60\end{array}$ & $\begin{array}{l}0.15 \\
0.01 \\
6.67 \\
7.0 \mathrm{C}\end{array}$ & $\begin{array}{l}1.32 \\
c .00 \\
c .01 \\
*\end{array}$ & $\begin{array}{l}3.18 \\
0.02 \\
0.63 \\
*\end{array}$ & $\begin{array}{l}2.08 \\
0.03 \\
1.45 \\
1.70\end{array}$ & $\begin{array}{l}2.03 \\
c .01 \\
c .50 \\
\end{array}$ & $\begin{array}{l}24.97 \\
0.04 \\
0.17 \\
\end{array}$ & $\begin{array}{l}2.59 \\
0.02 \\
0.36 \\
+\end{array}$ & $\begin{array}{r}77.67 \\
0.62\end{array}$ \\
\hline 1960 & $\begin{array}{l}\text { PRECIP. (CM.) } \\
S R-90 \text { (NCI/SQ.KH.) } \\
S R-90 \text { CENC. }(P C / L) \\
S R-89 / S R-90\end{array}$ & $\begin{array}{l}4.60 \\
0.04 \\
0.87 \\
-.\end{array}$ & $\begin{array}{l}0.69 \\
C .00 \\
0.01 \\
-.\end{array}$ & $\begin{array}{l}14.94 \\
0.05 \\
0.34 \\
--\end{array}$ & $\begin{array}{l}13.64 \\
0.03 \\
0.22 \\
--\end{array}$ & $\begin{array}{l}8.23 \\
C .08 \\
0.98 \\
--.\end{array}$ & $\begin{array}{l}3.28 \\
-- \\
--\end{array}$ & $\begin{array}{l}0.43 \\
C .6 C C \\
0.01 \\
--\end{array}$ & $\begin{array}{l}1.52 \\
0.016 \\
0.66 \\
-.\end{array}$ & $\begin{array}{l}2.03 \\
0.020 \\
0.59 \\
-.\end{array}$ & $\begin{array}{l}2.84 \\
C .05 C \\
0.86 \\
-.\end{array}$ & $\begin{array}{l}0.68 \\
0.036 \\
0.45 \\
-.\end{array}$ & $\begin{array}{l}2.26 \\
0.010 \\
0.45 \\
=-\end{array}$ & $\begin{array}{r}64.14 \\
0.32\end{array}$ \\
\hline 1961 & $\begin{array}{l}\text { PRECIP, (CM.) } \\
S R-S C \text { (NCI/SG.KN.) } \\
S R-9 C \text { CCNC. }(P C / L) \\
S R-8 S / S R-S C\end{array}$ & $\begin{array}{l}0.56 \\
0.016 \\
1.52 \\
-.\end{array}$ & $\begin{array}{l}0.71 \\
0.010 \\
1.41 \\
-.\end{array}$ & $\begin{array}{l}6.96 \\
0.016 \\
0.15 \\
--\end{array}$ & $\begin{array}{l}16.81 \\
0.03 C \\
0.18 \\
-.\end{array}$ & $\begin{array}{l}9.78 \\
C .28 \mathrm{C} \\
2.87 \\
-.\end{array}$ & $\begin{array}{l}1.57 \\
0.056 \\
3.15 \\
-.\end{array}$ & $\begin{array}{l}2.67 \\
C .02 C \\
c .75 \\
-.\end{array}$ & $\begin{array}{l}2.54 \\
0.02 C \\
C .79 \\
\cdots\end{array}$ & $\begin{array}{r}3.28 \\
C .15 \\
4.58 \\
28 . C C\end{array}$ & $\begin{array}{l}16.41 \\
0.01 \\
c .07 \\
\end{array}$ & $\begin{array}{l}55.70 \\
0.06 \\
0.11 \\
\end{array}$ & $\begin{array}{r}34.47 \\
0.18 \\
0.53 \\
65.00\end{array}$ & $\begin{array}{r}151.50 \\
0.83\end{array}$ \\
\hline 1962 & $\begin{array}{l}\text { PRECIP. ICN.) } \\
S R-9 O \text { (NCI/SC.KM.) } \\
S Q-9 O C O N C \text { (PCIL) } \\
S R-8 S / S R-S C\end{array}$ & $\begin{array}{r}16.13 \\
0.21 \\
1.31 \\
4 C . C 0\end{array}$ & $\begin{array}{r}1.73 \\
0.05 \\
2.90 \\
33.00\end{array}$ & $\begin{array}{r}3.28 \\
0.05 \\
1.53 \\
20.00\end{array}$ & $\begin{array}{r}14.40 \\
0.64 \\
4.45 \\
9.00\end{array}$ & $\begin{array}{r}37.59 \\
0.30 \\
0.80 \\
4.00\end{array}$ & $\begin{array}{l}3.30 \\
-- \\
7.00\end{array}$ & $\begin{array}{r}0.13 \\
0.02 \\
15.39 \\
34.0 C\end{array}$ & $\begin{array}{r}3.28 \\
0.04 \\
1.22 \\
31.00\end{array}$ & $\begin{array}{l}0.31 \\
0.01 \\
1.24 \\
7.0 C\end{array}$ & $\begin{array}{r}7.01 \\
C .07 \\
1.00 \\
16.00\end{array}$ & $\begin{array}{r}11.28 \\
0.06 \\
0.54 \\
26.00\end{array}$ & $\begin{array}{r}8.97 \\
0.11 \\
1.23 \\
54.00\end{array}$ & $\begin{array}{r}107.91 \\
1.56\end{array}$ \\
\hline 1963 & $\begin{array}{l}\text { PRECIP. ICM.) } \\
\text { SR-GC INCI/SC.KN.) } \\
S R-9 C \text { CONC. (PC/L) } \\
S R-8 S / S R-S C\end{array}$ & $\begin{array}{r}8.81 \\
0.15 \\
1.71 \\
37.00\end{array}$ & $\begin{array}{r}5.38 \\
C .17 \\
3.16 \\
34.00\end{array}$ & $\begin{array}{r}4.65 \\
0.13 \\
2.80 \\
24.00\end{array}$ & $\begin{array}{r}39.09 \\
0.04 \\
0.11 \\
3.00\end{array}$ & $\begin{array}{r}23.24 \\
0.50 \\
2.16 \\
7.10\end{array}$ & $\begin{array}{l}3.61 \\
C .09 \\
2.5 C \\
*\end{array}$ & $\begin{array}{l}0.46 \\
0.04 \\
8.70 \\
2.00\end{array}$ & $\begin{array}{l}5.08 \\
0.06 \\
1.19\end{array}$ & $\begin{array}{r}0.23 \\
0.03 \\
13.05 \\
*\end{array}$ & $\begin{array}{l}1.40 \\
C .02 \\
1.43\end{array}$ & $\begin{array}{r}19.43 \\
0.08 \\
0.42 \\
\end{array}$ & $\begin{array}{r}24.43 \\
0.17 \\
C .70 \\
*\end{array}$ & $\begin{array}{r}135.81 \\
1.48\end{array}$ \\
\hline 1564 & $\begin{array}{l}\text { PRECIP. (CM.) } \\
S R-90 \text { (KCI/SC.KM.) } \\
S R-90 \text { CONC. (PC/L) } \\
S R-89 / S R-90\end{array}$ & $\begin{array}{l}3.66 \\
0.02 \\
0.55 \\
1.00\end{array}$ & $\begin{array}{l}8.69 \\
0.41 \\
4.72 \\
*\end{array}$ & $\begin{array}{l}10.36 \\
\cdots \\
--\end{array}$ & $\begin{array}{l}35.38 \\
-- \\
-- \\
0.36\end{array}$ & $\begin{array}{l}7.65 \\
0.12 \\
1.57 \\
\end{array}$ & $\begin{array}{l}1.35 \\
0.14 \\
10.38 \\
--\end{array}$ & $\begin{array}{l}6.12 \\
0.22 \\
3.60 \\
--\end{array}$ & $\begin{array}{l}6.12 \\
0.22 \\
3.60 \\
--\end{array}$ & $\begin{array}{l}1.75 \\
C .05 \\
2.86 \\
--\end{array}$ & $\begin{array}{l}2.72 \\
0.03 \\
1.11 \\
*\end{array}$ & $\begin{array}{l}8.46 \\
\therefore- \\
\because-\end{array}$ & $\begin{array}{l}4.50 \\
4 \\
--\end{array}$ & $\begin{array}{r}96.76 \\
1.21\end{array}$ \\
\hline 1965 & $\begin{array}{l}\text { PRECIP. ICM.) } \\
\text { SR-SC INCI/SQ.KN.) } \\
S R-9 C \text { CCNC. }(P C A L) \\
S R-8 S / S R-9 O\end{array}$ & $\begin{array}{l}11.91 \\
0.12 \\
1.01 \\
--\end{array}$ & $\begin{array}{l}1.32 \\
0.05 \\
3.79 \\
-.-\end{array}$ & $\begin{array}{l}5.72 \\
\pm \\
=- \\
=-\end{array}$ & $\begin{array}{l}22.25 \\
0.08 \\
0.36 \\
-.\end{array}$ & $\begin{array}{l}7.39 \\
0.07 \\
0.95 \\
=-\end{array}$ & $\begin{array}{l}1.63 \\
0.08 \\
4.91 \\
-.\end{array}$ & $\begin{array}{l}1.09 \\
C .08 \\
7.34 \\
-.\end{array}$ & $\begin{array}{l}0.64 \\
0.02 \\
3.13 \\
--\end{array}$ & $\begin{array}{l}0.91 \\
0.03 \\
3.3 C \\
--\end{array}$ & $\begin{array}{l}4.17 \\
c .02 \\
0.48 \\
-.\end{array}$ & $\begin{array}{l}14.50 \\
0.01 \\
0.07 \\
-.\end{array}$ & $\begin{array}{l}10.44 \\
0.08 \\
0.77 \\
--\end{array}$ & $\begin{array}{r}81.97 \\
0.64\end{array}$ \\
\hline 1966 & $\begin{array}{l}\text { PRECIP. }(C M-) \\
\text { SR-9O INCI/SO.KM }) \\
S R-90 \text { CCNC. }(P C / L) \\
S R-89 / S R-90\end{array}$ & $\begin{array}{l}10.49 \\
\vdots \\
--\end{array}$ & $\begin{array}{l}3.94 \\
0.04 \\
1.02 \\
--\end{array}$ & $\begin{array}{l}14.50 \\
0.06 \\
0.42 \\
-.\end{array}$ & $\begin{array}{l}20.32 \\
0.05 \\
0.25 \\
-.\end{array}$ & $\begin{array}{l}8.66 \\
\pm \\
--\end{array}$ & $\begin{array}{l}2.06 \\
0.01 \\
0.49 \\
-.\end{array}$ & $\begin{array}{l}0.94 \\
0.01 \\
1.07\end{array}$ & $\begin{array}{l}3.15 \\
0.04 \\
1.27 \\
14.00\end{array}$ & $\begin{array}{l}3.00 \\
0.03 \\
1.01 \\
*\end{array}$ & $\begin{array}{r}4.75 \\
C .02 \\
c .43 \\
38.00\end{array}$ & $\begin{array}{r}8.89 \\
0.02 \\
0.23 \\
29.00\end{array}$ & $\begin{array}{l}2.13 \\
0.01 \\
0.47 \\
*\end{array}$ & $\begin{array}{r}82.83 \\
0.29\end{array}$ \\
\hline 1967 & $\begin{array}{l}\text { PRECIP. (CM.) } \\
S R-90 \text { (MCI/SO.KM-) } \\
S R-90 \text { CONC. (PC/L) } \\
\text { SR-89/SR-90 }\end{array}$ & $\begin{array}{l}* \\
0.01 \\
--\end{array}$ & $\begin{array}{r}0.15 \\
0.02 \\
13.34 \\
*\end{array}$ & $\begin{array}{l}2.16 \\
0.02 \\
0.93 \\
*\end{array}$ & $\begin{array}{l}41.91 \\
0.01 \\
0.03 \\
*\end{array}$ & $\begin{array}{l}37.57 \\
0.04 \\
0.11 \\
*\end{array}$ & $\begin{array}{l}-- \\
-- \\
--\end{array}$ & $\begin{array}{l}=- \\
=- \\
--\end{array}$ & $\begin{array}{l}-- \\
\overline{-} \\
-\end{array}$ & $\begin{array}{l}-- \\
= \\
--\end{array}$ & $\begin{array}{l}-- \\
\because- \\
-\end{array}$ & $=$ & $\begin{array}{l}-- \\
-- \\
--\end{array}$ & $\begin{array}{r}31.79 \\
0.10\end{array}$ \\
\hline \multicolumn{15}{|c|}{$\begin{array}{l}\text { NOTES } \\
\text { *: DATA NOT AVAILABLE } \\
\text { *: 2ERO OR TRACE } \\
\text { A: APPROXIMATE } \\
\text { B: LOHER LIMIT OF REPORTED DATA } \\
\text { C: PROPQRTIONEO FRCM CRIGINALLY CONSOLICATEO DATA }\end{array}$} \\
\hline
\end{tabular}


MONTHLY FALLOUT DEPOSITION COLLECTIONS

SITE: KENYA, NAIROBI

SOURCE: UNITED STATES EMBASSY
LAT. 1175 LONG. 36 A9E ALT. 1663M. (COLUMN)

\begin{tabular}{|c|c|c|c|c|c|c|c|c|c|c|c|c|c|c|}
\hline & & JAK. & FEB. & MAR. & APR. & $\operatorname{may}$ & JUNE & JuLY & AUG. & SEP. & OCT. & Nov. & DEC. & CUN. TOTAL \\
\hline 1959 & $\begin{array}{l}\text { PRECIP. ICH.) } \\
\text { SR-90 IMCI/SQ.KM.) } \\
S R-90 \text { CONC. }(P C / L) \\
S R-89 / S R-90\end{array}$ & $\begin{array}{l}1.09 \\
= \\
=\end{array}$ & $\begin{array}{l}8.99 \\
=\end{array}$ & $\begin{array}{c}18.92 \\
=- \\
=\end{array}$ & 10.24 & $\begin{array}{l}8.48 \\
= \\
=\end{array}$ & $\begin{array}{l}0.20 \\
=- \\
=\end{array}$ & $\begin{array}{l}0.13 \\
= \\
=\end{array}$ & $\begin{array}{l}3.12 \\
-- \\
-\end{array}$ & $\begin{array}{l}6.20 \\
0.06 \\
0.97 \\
*\end{array}$ & $\begin{array}{l}3.05 \\
0.03 \\
0.99 \\
0\end{array}$ & $\begin{array}{l}23.14 \\
0.05 \\
0.22 \\
.\end{array}$ & $\begin{array}{l}3.48 \\
-- \\
--\end{array}$ & $\begin{array}{r}87.04 \\
0.14\end{array}$ \\
\hline 1960 & $\begin{array}{l}\text { PREC IP }\left(C M_{-}\right) \\
S R-9 C \text { (NCI/SO.KM,) } \\
S R-9 C \text { CCNC. }(P C / L) \\
S R-89 / S R-90\end{array}$ & $\begin{array}{l}4.37 \\
0.05 \\
1.15 \\
-.\end{array}$ & $\begin{array}{l}1.19 \\
0.07 \\
5.89 \\
-\end{array}$ & $\begin{array}{l}29.21 \\
0.10 \\
0.35 \\
\end{array}$ & $\begin{array}{l}15.62 \\
0.08 \\
0.52 \\
\cdots\end{array}$ & $\begin{array}{c}13.16 \\
0.026 \\
0.16 \\
-0\end{array}$ & $\begin{array}{l}3.73 \\
0 . c c c \\
0.01 \\
--\end{array}$ & $\begin{array}{l}0.64 \\
= \\
=-\end{array}$ & $\begin{array}{l}0.53 \\
* \\
--\end{array}$ & $\begin{array}{l}2.41 \\
C .016 \\
0.42 \\
--\end{array}$ & $\begin{array}{l}7.06 \\
6.026 \\
0.29 \\
--.\end{array}$ & $\begin{array}{l}7.37 \\
0.006 \\
0.01 \\
-.\end{array}$ & $\begin{array}{l}5.31 \\
0.00 c \\
0.01 \\
--\end{array}$ & $\begin{array}{r}90.60 \\
0.35\end{array}$ \\
\hline 1961 & $\begin{array}{l}\text { PRECIP. }(C M .) \\
\text { SR-9C IMCI/SO.KM.) } \\
S R-90 \text { CONC. }(P C / L) \\
S R-89 / S R-90\end{array}$ & $\begin{array}{l}0.84 \\
* \\
--\end{array}$ & $\begin{array}{l}1.57 \\
-- \\
--\end{array}$ & $\begin{array}{l}7.57 \\
0.056 \\
0.67 \\
-.\end{array}$ & $\begin{array}{l}15.70 \\
0.10 \mathrm{C} \\
0.64 \\
=-\end{array}$ & $\begin{array}{l}11.51 \\
C .07 C \\
0.61 \\
-\infty\end{array}$ & $\begin{array}{l}2.49 \\
0.02 \mathrm{C} \\
0.81 \\
-.\end{array}$ & $\begin{array}{l}0.69 \\
c .00 C \\
0.01 \\
--\end{array}$ & $\begin{array}{l}2.69 \\
0.026 \\
0.75 \\
--\end{array}$ & $\begin{array}{l}3.56 \\
+ \\
--\end{array}$ & $\begin{array}{r}16.41 \\
0.02 \\
0.13 \\
6.70\end{array}$ & $\begin{array}{r}74.96 \\
0.08 \\
0.11 \\
49.00\end{array}$ & $\begin{array}{r}37.95 \\
0.25 \\
0.66 \\
69.00\end{array}$ & $\begin{array}{r}175.94 \\
0.61\end{array}$ \\
\hline 1962 & $\begin{array}{l}\text { PRECIP. }(C M-) \\
\text { SR-90 (MCI/SQ.KM.) } \\
\text { SR-90 CCNC. (PC/L) } \\
\text { SR-89/SR-90 }\end{array}$ & $\begin{array}{r}19.91 \\
0.49 \\
2.47 \\
38.00\end{array}$ & $\begin{array}{l}4.45 \\
0.11 \\
2.48 \\
28.00\end{array}$ & $\begin{array}{r}2.34 \\
0.08 \\
3.42 \\
17.00\end{array}$ & $\begin{array}{r}14.61 \\
0.09 \\
0.62 \\
17.00\end{array}$ & $\begin{array}{r}25.58 \\
0.31 \\
1.22 \\
2.00\end{array}$ & $\begin{array}{c}-\overline{0.26} \\
\overline{25.00}\end{array}$ & $\begin{array}{r}0.18 \\
C .07 \\
38.89 \\
20.00\end{array}$ & $\begin{array}{r}4.24 \\
0.05 \\
1.18 \\
18.00\end{array}$ & $\begin{array}{r}1.57 \\
0.12 \\
7.65 \\
11.00\end{array}$ & $\begin{array}{l}8.33 \\
0.15 \\
1.81 \\
8.00\end{array}$ & $\begin{array}{r}10.44 \\
0.10 \\
0.96 \\
28.00\end{array}$ & $\begin{array}{l}9.53 \\
0.12 \\
1.26 \\
47.00\end{array}$ & $\begin{array}{r}101.18 \\
1.95\end{array}$ \\
\hline 1963 & $\begin{array}{l}\text { PRECIP. }(C M .) \\
\text { SR-90 (MCI/SQ-KM.) } \\
\text { SR-90 CONC. (PC/L) } \\
S R-89 / S R-90\end{array}$ & $\begin{array}{r}19.30 \\
0.59 \\
3.06 \\
39.00\end{array}$ & $\begin{array}{r}5.84 \\
0.25 \\
4.29 \\
21.00\end{array}$ & $\begin{array}{r}11.40 \\
0.24 \\
2.11 \\
15.00\end{array}$ & $\begin{array}{r}12.34 \\
0.08 \\
0.65 \\
8.00\end{array}$ & $\begin{array}{r}25.37 \\
0.02 \\
0.08 \\
13.30\end{array}$ & $\begin{array}{l}3.58 \\
-2 \\
6.4 \mathrm{C}\end{array}$ & $\begin{array}{l}0.334 \\
0.024 \\
6.07 \\
--\end{array}$ & $\begin{array}{l}5.13 \\
0.02 \\
0.39 \\
2.00\end{array}$ & $\begin{array}{r}0.18 \\
0.03 \\
16.67 \\
*\end{array}$ & $\begin{array}{l}1.37 \\
0.02 \\
1.46 \\
\neq\end{array}$ & $\begin{array}{r}20.22 \\
0.04 \\
0.20 \\
\end{array}$ & $\begin{array}{l}30.91 \\
0.18 \\
0.59 \\
*\end{array}$ & $\begin{array}{r}135.97 \\
1.49\end{array}$ \\
\hline 1564 & $\begin{array}{l}\text { PRECIP. (CM.) } \\
\text { SR-90 (NCI/SQ.KM.) } \\
S R-90 \text { CONC. (PC/L) } \\
\text { SR-89/SR-90 }\end{array}$ & $\begin{array}{l}4.728 \\
0.078 \\
1.49 \\
-.\end{array}$ & $\begin{array}{l}8.53 \\
0.11 \\
1.29 \\
0.20\end{array}$ & $\begin{array}{r}10.36 \\
0.36 \\
3.48 \\
--\end{array}$ & $\begin{array}{r}42.67 \\
0.19 \\
0.45 \\
0.48\end{array}$ & $\begin{array}{r}11.20 \\
0.13 \\
1.17 \\
*\end{array}$ & $\begin{array}{l}1.35 \\
0.20 \\
14.82 \\
-.\end{array}$ & $\begin{array}{l}3.30 \\
0.15 \\
4.55 \\
--\end{array}$ & $\begin{array}{l}6.73 \\
0.15 \\
2.23 \\
--\end{array}$ & $\begin{array}{l}2.59 \\
0.27 \\
10.43 \\
-.\end{array}$ & $\begin{array}{l}3.96 \\
0.10 \\
2.53\end{array}$ & $\begin{array}{l}8.84 \\
0.14 \\
1.59 \\
1\end{array}$ & $\begin{array}{l}6.07 \\
0.15 \\
2.48 \\
-.\end{array}$ & $\begin{array}{r}110.32 \\
2.02\end{array}$ \\
\hline 1965 & $\begin{array}{l}\text { PRECIP }\left(C M_{-}\right) \\
S R-90\left(M C I / S Q_{. K M}\right) \\
S R-90 \text { CONC. }(P C / L) \\
S R-89 / 5 R-90\end{array}$ & $\begin{array}{l}5.04 \\
0.22 \\
2.44 \\
--\end{array}$ & $\begin{array}{l}0.15 \\
0.01 \\
6.67 \\
-.\end{array}$ & $\begin{array}{l}5.61 \\
0.07 \\
1.25 \\
-.\end{array}$ & $\begin{array}{l}30.61 \\
0.13 \\
0.43 \\
-\end{array}$ & $\begin{array}{l}8.92 \\
0.12 \\
1.35 \\
--.\end{array}$ & $\begin{array}{l}9.42 \\
0.47 \\
4.99 \\
-.\end{array}$ & $\begin{array}{l}4.62 \\
0.06 \\
1.30 \\
--\end{array}$ & $\begin{array}{l}2.16 \\
0.04 \\
1.86 \\
--\end{array}$ & $\begin{array}{r}0.48 \\
0.36 \\
75.01 \\
--\end{array}$ & $\begin{array}{l}7.37 \\
0.03 \\
0.41 \\
-0\end{array}$ & $\begin{array}{r}10.41 \\
0.07 \\
0.68 \\
--\end{array}$ & $\begin{array}{r}15.52 \\
0.04 \\
0.26 \\
--\end{array}$ & $\begin{array}{r}104.31 \\
1.62\end{array}$ \\
\hline 1966 & $\begin{array}{l}\text { PRECIP. (CM.) } \\
S R-9 C \text { (NCI/SQ.KM.) } \\
S R-9 C \text { CCNC. }(P C / L) \\
S R-89 / S R-90\end{array}$ & $\begin{array}{l}9.40 \\
+ \\
--\end{array}$ & $\begin{array}{l}3.84 \\
0.12 \\
3.13 \\
-.\end{array}$ & $\begin{array}{r}12.52 \\
0.05 \\
0.40 \\
-.\end{array}$ & $\begin{array}{r}16.46 \\
0.10 \\
0.61 \\
--\end{array}$ & $\begin{array}{l}10.34 \\
0.02 \\
0.20 \\
-\end{array}$ & $\begin{array}{l}2.36 \\
0.02 \\
0.85 \\
+\end{array}$ & $\begin{array}{l}0.25 \\
C .01 \\
4.01 \\
*\end{array}$ & $\begin{array}{l}5.200 \\
0.060 \\
1.16 \\
*\end{array}$ & $\begin{array}{l}5.206 \\
C .06 C \\
1.16 \\
.\end{array}$ & $\begin{array}{l}5.20 C \\
C .06 C \\
1.16 \\
*\end{array}$ & $\begin{array}{r}12.04 \\
0.04 \\
0.34 \\
15.00\end{array}$ & $\begin{array}{l}2.26 \\
0.01 \\
0.45 \\
+\end{array}$ & $\begin{array}{r}85.07 \\
0.55\end{array}$ \\
\hline 1967 & $\begin{array}{l}\text { PRECIP. (CM.) } \\
S R \rightarrow 90 \text { (MCI/SO.KM.) } \\
S R-90 \text { CCNC. }(P C M L) \\
S R-89 / S R-90\end{array}$ & $\begin{array}{l}* \\
0.01 \\
*\end{array}$ & $\begin{array}{l}* 01 \\
0\end{array}$ & $\begin{array}{l}3.96 \\
0.04 \\
1.02 \\
*\end{array}$ & $\begin{array}{l}27.84 \\
0.03 \\
0.11 \\
*\end{array}$ & $\begin{array}{r}38.81 \\
0.03 \\
0.08 \\
*\end{array}$ & $\begin{array}{l}2.56 \\
0.01 \\
0.40 \\
-\end{array}$ & $\begin{array}{l}-- \\
--\end{array}$ & $\begin{array}{l}-- \\
-- \\
--\end{array}$ & $=$ & $\begin{array}{l}-- \\
-- \\
--\end{array}$ & $\begin{array}{l}- \\
-- \\
--\end{array}$ & $\begin{array}{l}= \\
=- \\
=-\end{array}$ & $\begin{array}{r}73.17 \\
0.13\end{array}$ \\
\hline $\begin{aligned} \text { NOTES } & \\
2-: & 0 \\
*: & 2 \\
A: & A \\
B: & 1 \\
C: & P\end{aligned}$ & $\begin{array}{l}\text { S } \\
\text { DATA NOT AVAILABLE } \\
\text { ZERO OR TRACE } \\
\text { APPROXIMATE } \\
\text { LOHER LIMIT OF REPOR } \\
\text { PROPORTIONEO FROM OR }\end{array}$ & $\begin{array}{l}\text { DATA } \\
\text { NALLY }\end{array}$ & & & & & & & & & & & & \\
\hline
\end{tabular}


SOURCE: OB SERVATORY AND METECROLOGICAL STATION. AMER ICAN UNIVERSITY TD JAN. 1966

U. S. EMBASSY FROM AUGUST 1966

\begin{tabular}{|c|c|c|c|c|c|c|c|c|c|c|c|c|c|c|}
\hline & & JAN. & FEB. & MAR. & APR. & MAY & JUNE & JULY & AUG. & SEP. & CCT. & NOV. & DEC. & CUM. TOTAL \\
\hline 1959 & $\begin{array}{l}\text { PREC IP. (CM.) } \\
\text { SR-90 (MCI/SQ.KM.) } \\
\text { SR-90 CONC }(P C / L) \\
\text { SR-89/SR-90 }\end{array}$ & $\begin{array}{l}-- \\
-- \\
--\end{array}$ & $=$ & $\begin{array}{l}-- \\
--\end{array}$ & $\bar{m}=$ & $\begin{array}{l}-- \\
-- \\
--\end{array}$ & 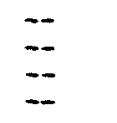 & $\begin{array}{l}-- \\
-- \\
--\end{array}$ & $\begin{array}{l}-- \\
-- \\
--\end{array}$ & $\begin{array}{l}-- \\
-- \\
--\end{array}$ & $\begin{array}{l}14.02 \\
0.11 \\
0.79 \\
*\end{array}$ & $\begin{array}{l}11.56 \\
=- \\
=-\end{array}$ & $\begin{array}{l}5.99 \\
0.24 \\
4.01 \\
.\end{array}$ & $\begin{array}{r}31.57 \\
0.35\end{array}$ \\
\hline 1960 & $\begin{array}{l}\text { PRECIP. (CM.) } \\
\text { SR-9C INCI/SQ-KM.) } \\
\text { SR-90 CONC. (PC/L) } \\
\text { SR-89/SR-90 }\end{array}$ & $\begin{array}{l}14.27 \\
C .03 \\
C .22 \\
--\end{array}$ & $\begin{array}{l}-- \\
c .12 \\
--\end{array}$ & $\begin{array}{l}11.99 \\
-- \\
--\end{array}$ & $\begin{array}{l}2.97 \\
-- \\
-- \\
--\end{array}$ & $\begin{array}{l}0.05 \\
-- \\
-- \\
--\end{array}$ & $\begin{array}{l}* \\
-- \\
--\end{array}$ & $\begin{array}{l}-- \\
+- \\
--\end{array}$ & $\begin{array}{l}-- \\
-- \\
--\end{array}$ & $\begin{array}{l}1.80 \\
0.02 C \\
1.12 \\
--\end{array}$ & $\begin{array}{l}1.19 \\
0.02 C \\
1.69 \\
-.\end{array}$ & $\begin{array}{l}0.99 \\
0.056 \\
5.06 \\
--\end{array}$ & $\begin{array}{l}3.00 \\
0.176 \\
5.67 \\
-.\end{array}$ & $\begin{array}{r}36.26 \\
0.41\end{array}$ \\
\hline 1961 & $\begin{array}{l}\text { PRECIP. ICM.) } \\
\text { SR-9C IMCI/SQ.KN.) } \\
S R-90 \text { CCNC. }(P C / L) \\
S R-89 / S R-90\end{array}$ & $\begin{array}{l}16.33 \\
c .20 C \\
1.23 \\
--\end{array}$ & $\begin{array}{l}26.82 \\
C .33 C \\
1.24 \\
=-\end{array}$ & $\begin{array}{l}9.30 \\
0.20 \mathrm{C} \\
2.16 \\
--\end{array}$ & $\begin{array}{l}0.28 \\
0.016 \\
3.58 \\
--\end{array}$ & $\begin{array}{l}15.65 \\
0.08 \\
0.52 \\
-.\end{array}$ & $\begin{array}{l}* \\
c . c c c \\
--\end{array}$ & $\begin{array}{l}* \\
\mathrm{c} .02 \mathrm{C} \\
-- \\
--\end{array}$ & $\begin{array}{l}* \\
0.02 C \\
=-\end{array}$ & $\begin{array}{l}33.53 \\
0.07 \\
0.21 \\
--\end{array}$ & $\begin{array}{r}14.81 \\
0.05 \\
0.34 \\
62.00\end{array}$ & $\begin{array}{r}7.67 \\
0.29 \\
3.79 \\
51.00\end{array}$ & $\begin{array}{r}14.00 \\
1.98 \\
13.43 \\
53.00\end{array}$ & $\begin{array}{r}138.39 \\
3.15\end{array}$ \\
\hline 1962 & $\begin{array}{l}P R E C I P \text { (CM.) } \\
\text { SR-9O INCI/SQ.KM.) } \\
S R-9 C \text { CCNC. (PC/L) } \\
S R-89 / S R-90\end{array}$ & $\begin{array}{r}17.78 \\
1.94 \\
16.92 \\
43.00\end{array}$ & $\begin{array}{r}19.20 \\
3.28 \\
17.05 \\
31.00\end{array}$ & $\begin{array}{r}1.35 \\
0.52 \\
38.52 \\
30.00\end{array}$ & $\begin{array}{r}5.79 \\
1.03 \\
17.79 \\
17.00\end{array}$ & $\begin{array}{r}0.74 \\
0.42 \\
56.76 \\
8.00\end{array}$ & $\begin{array}{l}* \\
0.01 \\
*\end{array}$ & $\begin{array}{l}--0 . \\
8.02 \\
8.00\end{array}$ & $\begin{array}{l}-\overline{c .03} \\
\overline{7.00}\end{array}$ & $\begin{array}{r}31.09 \\
0.01 \\
0.04 \\
17.00\end{array}$ & $\begin{array}{r}13.79 \\
6.06 \\
6.44 \\
15.00\end{array}$ & $\begin{array}{r}0.46 \\
0.13 \\
28.27 \\
14.00\end{array}$ & $\begin{array}{r}28.58 \\
1.43 \\
5.01 \\
43.00\end{array}$ & $\begin{array}{r}118.78 \\
8.88\end{array}$ \\
\hline$\underset{\infty}{\sim}$ & $\begin{array}{l}\text { PRECIP. }\left(C M M_{-}\right) \\
\text {SR-9O INCISSO.KM.) } \\
\text { SR-9O CONC. }(P C I L) \\
\text { SR-89/SR-9O }\end{array}$ & $\begin{array}{l}25.65 \\
4.05 \\
15.79 \\
50.00\end{array}$ & $\begin{array}{r}15.75 \\
0.96 \\
6.10 \\
37.00\end{array}$ & $\begin{array}{r}7.11 \\
0.70 \\
9.85 \\
16.60\end{array}$ & $\begin{array}{r}9.40 \\
0.67 \\
7.13 \\
11.40\end{array}$ & $\begin{array}{r}7.67 \\
1.75 \\
22.82 \\
6.8 C\end{array}$ & $\begin{array}{l}* \\
0.31 \\
*\end{array}$ & $\begin{array}{l}-- \\
0.04 \\
1.00\end{array}$ & $\begin{array}{l}-\infty \\
0.05 \\
+\end{array}$ & $\begin{array}{r}2.21 \\
0.44 \\
19.91 \\
+\end{array}$ & $\begin{array}{r}9.45 \\
1.09 \\
11.54 \\
1.70\end{array}$ & $\begin{array}{l}13.67 \\
0.98 \\
7.17 \\
*\end{array}$ & $\begin{array}{r}13.69 \\
2.27 \\
16.59 \\
*\end{array}$ & $\begin{array}{r}104.60 \\
13.31\end{array}$ \\
\hline 1964 & $\begin{array}{l}\text { PRECIP. }(C M-) \\
S R-9 C(N C I / S Q . K M .) \\
S R-90 \text { CONC. (PCIL) } \\
S R-89 / S R-90\end{array}$ & $\begin{array}{l}48.67 \\
1.45 \\
2.98 \\
*\end{array}$ & $\begin{array}{r}31.95 \\
4.06 \\
12.71 \\
*\end{array}$ & $\begin{array}{l}9.12 \\
2.79 \\
30.60 \\
*\end{array}$ & $\begin{array}{l}2.11 \\
0.38 \\
18.01 \\
*\end{array}$ & $\begin{array}{l}-- \\
-- \\
--\end{array}$ & $\begin{array}{l}0.63 \\
0 \\
+\end{array}$ & $\begin{array}{l}+ \\
C .02 \\
*-\end{array}$ & $\begin{array}{l}* \\
0.02 \\
--\end{array}$ & $\begin{array}{c}0.20 \\
0.21 \\
105.60 \\
--\end{array}$ & $\begin{array}{l}* \\
0.06 \\
--\end{array}$ & $\begin{array}{r}23.22 \\
0.55 \\
2.37 \\
1.10\end{array}$ & $\begin{array}{l}9.55 \\
0.05 \\
0.53 \\
--\end{array}$ & $\begin{array}{r}124.82 \\
10.22\end{array}$ \\
\hline 1965 & $\begin{array}{l}\text { PRECIP. (CM.) } \\
\text { SR-90 INCI/SQ.KN.) } \\
\text { SR-90 CONC. }(P C / L) \\
S R-89 / S R-90\end{array}$ & $\begin{array}{l}15.72 \\
1.04 \\
6.62 \\
-.\end{array}$ & $\begin{array}{l}-- \\
1.18 \\
--\end{array}$ & $\begin{array}{l}10.62 \\
0.84 \\
7.91 \\
--\end{array}$ & $\begin{array}{r}9.09 \\
1.06 \\
11.67 \\
--\end{array}$ & $\begin{array}{r}1.91 \\
0.37 \\
19.38 \\
--\end{array}$ & $\begin{array}{r}0.38 \\
0.20 \\
52.64 \\
--\end{array}$ & $\begin{array}{l}+ \\
C .03 \\
-- \\
--\end{array}$ & $\begin{array}{l}0.15 \\
0.06 \\
40.01 \\
--\end{array}$ & $\begin{array}{l}+ \\
0.03 \\
-- \\
--\end{array}$ & $\begin{array}{l}15.19 \\
0.31 \\
2.05 \\
--\end{array}$ & $\begin{array}{l}3.68 \\
0.20 \\
5.44 \\
-.\end{array}$ & $\begin{array}{l}24.59 \\
0.44 \\
1.79 \\
--\end{array}$ & $\begin{array}{r}81.33 \\
5.76\end{array}$ \\
\hline 1966 & $\begin{array}{l}\text { PRECIP. (CM.) } \\
\text { SR-90 (NCI/SQ-KM.) } \\
\text { SR-90 CONC. (PC/L) } \\
\text { SR-89/SR-90 }\end{array}$ & $\begin{array}{r}18.59 \\
0.51 \\
2.75 \\
--\end{array}$ & $\begin{array}{l}-- \\
-- \\
--\end{array}$ & $\begin{array}{l}-- \\
--\end{array}$ & $\ddot{--}$ & $\begin{array}{l}-- \\
-- \\
--\end{array}$ & $\begin{array}{l}-- \\
=- \\
--\end{array}$ & $\begin{array}{l}-- \\
-- \\
--\end{array}$ & $\begin{array}{l}0.52 \\
* \\
* \\
*\end{array}$ & $+\infty$ & $\begin{array}{l}4.26 \\
0.04 \\
C .94\end{array}$ & $\begin{array}{l}43.60 \\
0.04 \\
0.10 \\
*\end{array}$ & $\begin{array}{l}24.92 \\
0.05 \\
0.21 \\
\$\end{array}$ & $\begin{array}{r}91.89 \\
0.65\end{array}$ \\
\hline 1967 & $\begin{array}{l}\text { PRECIP. }\left(C M_{-}\right) \\
\text {SR-9O (MCI/SQ-KM.) } \\
\text { SR-90 CONC. (PC/L) } \\
\text { SR-B9/SR-90 }\end{array}$ & $\begin{array}{r}25.20 \\
0.34 \\
1.35 \\
6.20\end{array}$ & $\begin{array}{r}17.27 \\
C .45 \\
2.61 \\
13.50\end{array}$ & $\begin{array}{r}26.52 \\
0.28 \\
1.06 \\
8.20\end{array}$ & $=$ & $\begin{array}{l}-- \\
-- \\
--\end{array}$ & $\begin{array}{l}-- \\
=- \\
=-\end{array}$ & $\begin{array}{l}-- \\
-- \\
-\end{array}$ & $m$ & $\begin{array}{l}-- \\
-- \\
--\end{array}$ & $\begin{array}{l}-- \\
-- \\
--\end{array}$ & $\begin{array}{l}-- \\
-- \\
--\end{array}$ & $=$ & $\begin{array}{r}68.99 \\
1.07\end{array}$ \\
\hline $\begin{array}{l}\text { NOTES } \\
--: D \\
*:=2 \\
A: A \\
B: L \\
C: P\end{array}$ & $\begin{array}{l}\text { DATA NOT AVAILABLE } \\
\text { ERO OR TRACE } \\
\text { IPPROXIMATE } \\
\text { OWER LIMIT OF REPOR } \\
\text { ROPORTICAEO FROM OR }\end{array}$ & $\begin{array}{l}\text { D DATA } \\
\text { INALLYY }\end{array}$ & & ED D & & & & & & & & & & \\
\hline
\end{tabular}




\begin{tabular}{|c|c|c|c|c|c|c|c|c|c|c|c|c|}
\hline JAN. & FEB. & MAR. & APR. & MAY & JUNE & JULY & AUG. & SEP. & OCT. & NOV. & DEC. & CUM. TOTAL \\
\hline $\begin{array}{l}= \\
- \\
-\end{array}$ & $\begin{array}{l}-- \\
--\end{array}$ & $\begin{array}{l}-- \\
-- \\
--\end{array}$ & $\begin{array}{l}= \\
\overline{-}\end{array}$ & $\begin{array}{l}-- \\
-- \\
--\end{array}$ & $\begin{array}{l}-- \\
=- \\
--\end{array}$ & $\begin{array}{l}7.628 \\
0.12 \\
1.58 \\
*\end{array}$ & $\begin{array}{l}= \\
= \\
=\end{array}$ & $\begin{array}{l}12.73 \\
0.06 \\
0.48 \\
*\end{array}$ & $\begin{array}{l}2.57 \\
0.02 \\
0.78 \\
--\end{array}$ & $\begin{array}{l}-- \\
0.04 \\
- \\
*\end{array}$ & $\begin{array}{l}-- \\
0.00 \\
--\end{array}$ & $\begin{array}{r}22.92 \\
0.24\end{array}$ \\
\hline $\begin{array}{l}3.00 \\
0.00 \\
0.01 \\
-.\end{array}$ & $\begin{array}{l}2.57 \\
=- \\
=-\end{array}$ & $\begin{array}{c}12.73 \\
-- \\
--\end{array}$ & $\begin{array}{l}3.68 \\
0.07 \\
1.91 \\
-.\end{array}$ & $\begin{array}{l}28.12 \\
0.05 \\
0.18 \\
--\end{array}$ & $\begin{array}{c}145.24 \\
0.02 \\
0.06 \\
-\end{array}$ & $\begin{array}{c}49.02 \\
0.01 C \\
0.03 \\
-\end{array}$ & $\begin{array}{c}59.46 \\
0.02 C \\
0.04 \\
-\end{array}$ & $\begin{array}{c}65.41 \\
0.03 \mathrm{C} \\
0.05 \\
-\end{array}$ & $\begin{array}{l}-- \\
0.036 \\
--\end{array}$ & $\begin{array}{l}-- \\
0.036 \\
-- \\
--\end{array}$ & $\begin{array}{l}-- \\
0.03 c \\
-- \\
--\end{array}$ & $\begin{array}{r}369.23 \\
0.35\end{array}$ \\
\hline $\begin{array}{l}-- \\
0.01 \\
--\end{array}$ & $\begin{array}{l}2.29 \\
0.14 \\
6.12 \\
-\end{array}$ & $\begin{array}{c}12.45 \\
0.17 \mathrm{C} \\
1.37 \\
=-\end{array}$ & $\begin{array}{l}9.47 \\
0.136 \\
1.38 \\
-\end{array}$ & $\begin{array}{l}-- \\
-- \\
--\end{array}$ & $\begin{array}{l}= \\
-- \\
-\end{array}$ & $\begin{array}{l}-- \\
-- \\
--\end{array}$ & $\begin{array}{l}-- \\
-- \\
--\end{array}$ & $\begin{array}{l}-- \\
-- \\
--\end{array}$ & $\begin{array}{l}-- \\
-- \\
--\end{array}$ & $\begin{array}{l}-- \\
--\end{array}$ & $\begin{array}{l}-- \\
-- \\
=-\end{array}$ & $\begin{array}{r}24.21 \\
0.45\end{array}$ \\
\hline-- & $\begin{array}{l}-- \\
--\end{array}$ & $\begin{array}{l}-- \\
-- \\
-- \\
--\end{array}$ & $\begin{array}{l}-- \\
-- \\
--\end{array}$ & $\begin{array}{l}-- \\
-- \\
-\end{array}$ & $\begin{array}{l}-- \\
-- \\
--\end{array}$ & $\begin{array}{l}-- \\
--\end{array}$ & $\begin{array}{l}-- \\
-- \\
--\end{array}$ & $\begin{array}{l}-- \\
-- \\
--\end{array}$ & $\begin{array}{l}-- \\
-- \\
--\end{array}$ & $\begin{array}{l}-- \\
--\end{array}$ & $\begin{array}{l}-- \\
-- \\
--\end{array}$ & $\begin{array}{l}0.0 \\
0.0\end{array}$ \\
\hline $\begin{array}{l}-- \\
-- \\
--\end{array}$ & $\begin{array}{l}-- \\
0.174 \\
-- \\
--\end{array}$ & $\begin{array}{l}-- \\
0.174 \\
--\end{array}$ & $\begin{array}{l}0.174 \\
--\end{array}$ & $\begin{array}{l}-- \\
0.174 \\
--\end{array}$ & $\begin{array}{l}-\overline{1.18 \mathrm{~A}} \\
\overline{2.9 \mathrm{CA}}\end{array}$ & $\begin{array}{l}-- \\
1.12 \mathrm{~A} \\
2.00 \mathrm{~A}\end{array}$ & $\begin{array}{l}-\overline{0.564} \\
\bar{*}\end{array}$ & $\begin{array}{l}=- \\
=-\end{array}$ & $\begin{array}{l}0.93 \\
--\end{array}$ & $\begin{array}{l}-- \\
0.08 \\
-- \\
-\end{array}$ & $\begin{array}{l}-- \\
-- \\
--\end{array}$ & $\begin{array}{l}0.0 \\
4.55\end{array}$ \\
\hline $\begin{array}{l}-\overline{0.02} \\
\overline{0}\end{array}$ & $\begin{array}{l}- \\
0.03 \\
--\end{array}$ & $\begin{array}{l}-- \\
0.07 \\
--\end{array}$ & $\begin{array}{l}-\infty \\
0.46 \\
-\end{array}$ & $\begin{array}{l}-- \\
0.63 \\
* \\
*\end{array}$ & $\begin{array}{l}-- \\
0.21 \\
--\end{array}$ & $\begin{array}{l}-- \\
0.13 \\
--\end{array}$ & $\begin{array}{l}--39 \\
--\end{array}$ & $\begin{array}{l}-- \\
0.19 \\
=-\end{array}$ & $\begin{array}{l}-- \\
0.57 \\
--\end{array}$ & $\begin{array}{l}- \\
0.37 \\
--\end{array}$ & $\begin{array}{l}-- \\
0.13 \\
--\end{array}$ & $\begin{array}{l}0.0 \\
3.20\end{array}$ \\
\hline $\begin{array}{l}-- \\
0.03 \\
--\end{array}$ & $\begin{array}{l}-- \\
--\end{array}$ & $\begin{array}{l}-- \\
0.08 \\
=-\end{array}$ & $\begin{array}{l}- \\
0.19 \\
-\end{array}$ & $\begin{array}{l}-- \\
0.14 \\
=- \\
--\end{array}$ & $\begin{array}{l}-- \\
0.53 \\
=\end{array}$ & $\begin{array}{l}-- \\
0.74 \\
--\end{array}$ & $\begin{array}{l}-- \\
0.14 \\
--\end{array}$ & $\begin{array}{l}-- \\
0.15 \\
=-\end{array}$ & $\begin{array}{l}-- \\
0.02 \\
=-\end{array}$ & $\begin{array}{l}-- \\
0.39 \\
-- \\
--\end{array}$ & $\begin{array}{l}-- \\
0.18 \\
--\end{array}$ & $\begin{array}{l}0.0 \\
2.59\end{array}$ \\
\hline $\begin{array}{l}-- \\
0.05 \\
--\end{array}$ & $\begin{array}{l}-\overline{0} \\
0.02 C \\
=- \\
=-\end{array}$ & $\begin{array}{l}-- \\
0.02 \mathrm{C} \\
=-\end{array}$ & $\begin{array}{l}0.09 \\
-\end{array}$ & $\begin{array}{l}-.16 \\
0 .-\end{array}$ & $\frac{-0}{0.076}$ & $\begin{array}{l}-0 \\
0.08 C \\
*\end{array}$ & $\begin{array}{l}-\overline{0.106} \\
\overline{11.00}\end{array}$ & $\begin{array}{l}--100 \\
0\end{array}$ & $\begin{array}{c}-- \\
0.07 \\
19.00\end{array}$ & $\begin{array}{c}-- \\
0.04 \\
14.00\end{array}$ & $\begin{array}{l}-- \\
0.01 \\
+-\end{array}$ & $\begin{array}{l}0.0 \\
0.81\end{array}$ \\
\hline$\overline{0.01}$ & $\begin{array}{l}-- \\
0.01 \\
-\end{array}$ & $\frac{-0}{0.01}$ & $\begin{array}{c}15.95 \\
0.14 \\
0.88 \\
*\end{array}$ & $\begin{array}{l}24.79 \\
0.06 \\
0.25 \\
*\end{array}$ & $\begin{array}{l}-- \\
0.06 \\
-.40\end{array}$ & $\begin{array}{l}-- \\
--\end{array}$ & $\begin{array}{l}-- \\
-- \\
--\end{array}$ & $\begin{array}{l}=- \\
= \\
--\end{array}$ & $\begin{array}{l}-- \\
=- \\
--\end{array}$ & $\begin{array}{l}-- \\
-- \\
--\end{array}$ & $\begin{array}{l}-- \\
-- \\
--\end{array}$ & $\begin{array}{r}40.74 \\
0.29\end{array}$ \\
\hline
\end{tabular}


MONTHLY FALLOUT DEPOSITION COLLECTIONS

SITE: LIBYA, BEN GASHIR TRIPOLI LAT. 32 54N LONG. 13 IIE ALT. SM. ICOLUMNI

SOURCE: LIBYAN MINISTRY OF COMMUNICATIONS, METEOROLOGICAL DEPARTMENT

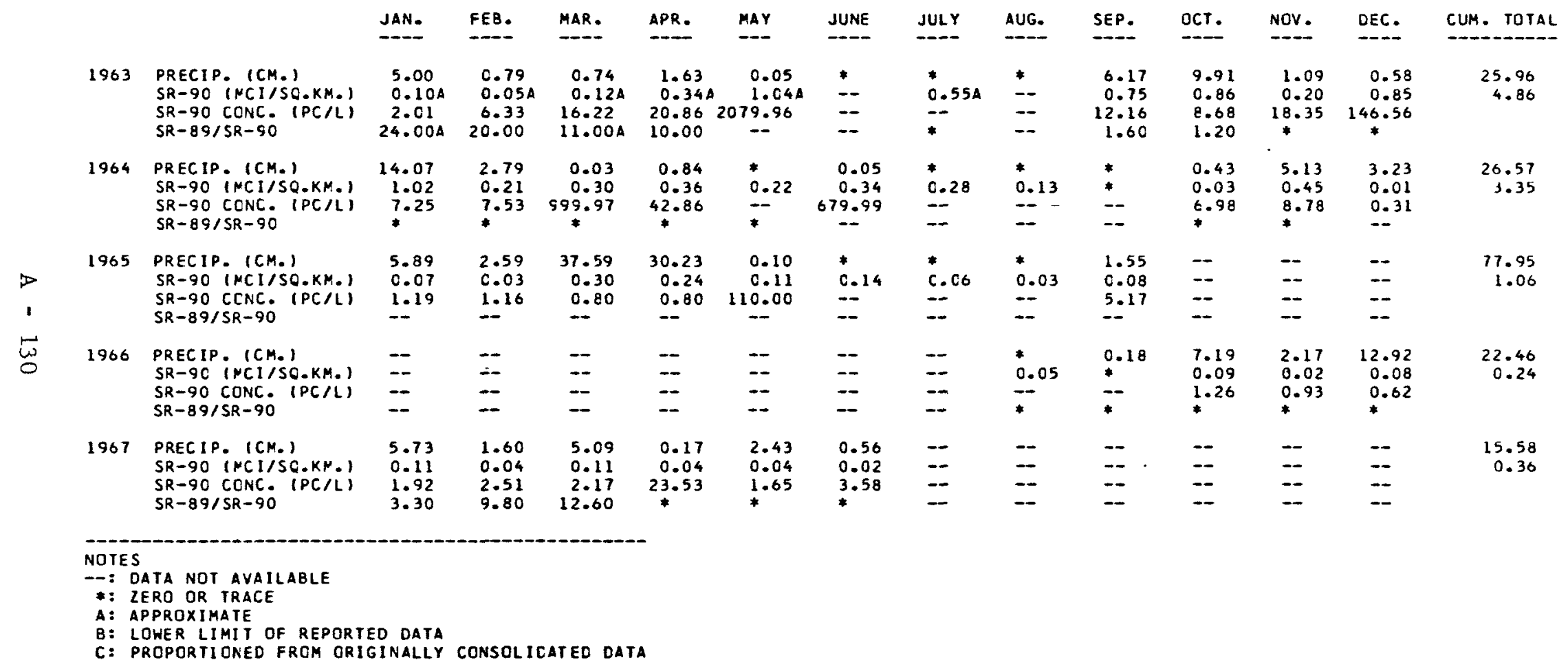


MONTHLY FALLOUT DEPOSITION COLLECTIONS

SITE: LIBYA, CYRENE

LAT. $3249 \mathrm{~N}$ LONG. $2151 E$ ALT. 621M. (COLUMN)

SOURCE: LIBYAN MINISTRY OF COMMUNICATIONS, METEOROLOGICAL OEPARTMENT COLLECTIONS TERMINATED IN MARCH 1964

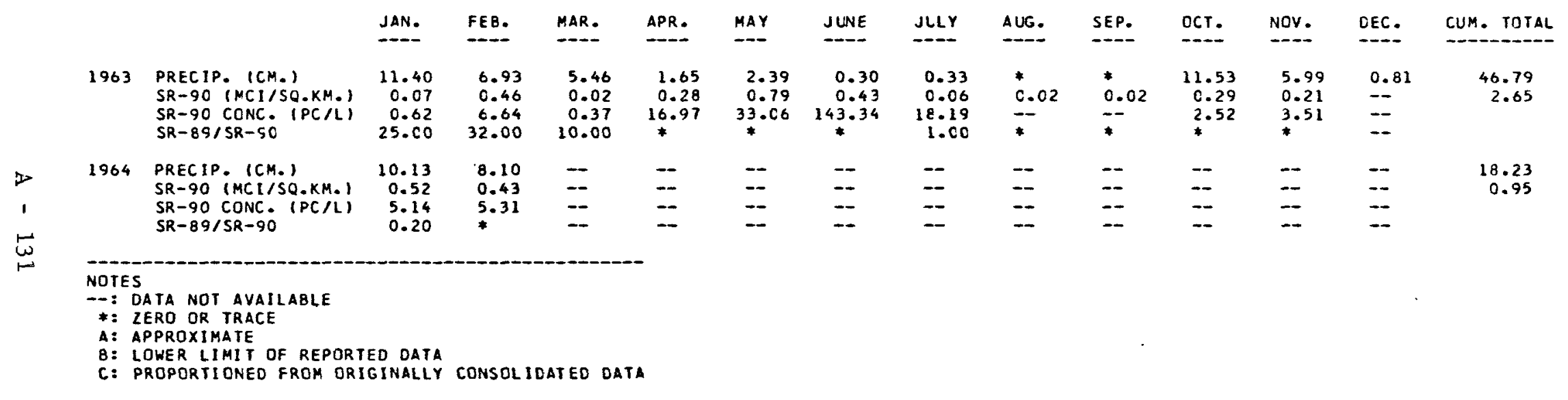


MONTHLY FALLCUT DEPOSITION COLLECTIONS

SIIE: MAJURO I SLAND

SOURCE: U. S. WEATHER BUREAU AIRPORT STATION
LAT. $75 \mathrm{~N}$ LONG.17I $23 E$ ALT. 3M. (COLUMN)

\begin{tabular}{|c|c|c|c|c|c|c|c|c|c|c|c|c|c|c|}
\hline & & JAN. & FE8. & MAR : & APR. & MAY & JUNE & JULY & AUG. & SEP. & DCT. & NOV. & OEC. & CUM. TOTAL \\
\hline 1960 & $\begin{array}{l}\text { PRECIP ICM.) } \\
S R-9 C \text { (MCI/SC.KM.) } \\
\text { SR-SC CCNC. }(P C / L) \\
S R-89 / S R-90\end{array}$ & $\begin{array}{c}23.29 \\
-- \\
=\end{array}$ & $\begin{array}{l}9.14 \\
C .07 \\
C .77 \\
--\end{array}$ & $\begin{array}{l}28.37 \\
0.08 \\
0.29 \\
--\end{array}$ & $\begin{array}{r}59.46 \\
0.17 \\
0.29 \\
--\end{array}$ & $\begin{array}{l}36.25 \\
C .07 C \\
0.20 \\
--\end{array}$ & $\begin{array}{l}33.58 \\
0.07 \mathrm{C} \\
0.21 \\
--\end{array}$ & $\begin{array}{l}35.81 \\
C .05 C \\
C .14 \\
=-\end{array}$ & $\begin{array}{l}37.06 \\
0.056 \\
0.14 \\
-.\end{array}$ & $\begin{array}{l}43.00 \\
C .02 \mathrm{C} \\
0.05 \\
--\end{array}$ & $\begin{array}{l}24.66 \\
C .01 \mathrm{C} \\
C .05 \\
--\end{array}$ & $\begin{array}{l}41.45 \\
0.06 \mathrm{C} \\
0.15 \\
=-\end{array}$ & $\begin{array}{l}16.61 \\
0.03 \mathrm{C} \\
0.19 \\
--\end{array}$ & $\begin{array}{r}388.68 \\
0.68\end{array}$ \\
\hline 1961 & $\begin{array}{l}\text { PRECIP. (CM.) } \\
S R-9 C \text { (NCI/SQ.KM.) } \\
S R-90 \text { CONC. (PC/L) } \\
S R-89 / S R-90\end{array}$ & $\begin{array}{l}55.80 \\
C .07 C \\
0.13 \\
--\end{array}$ & $\begin{array}{l}16.51 \\
0.02 C \\
0.13 \\
=-\end{array}$ & $\begin{array}{l}10.77 \\
0.04 C \\
0.38 \\
=-\end{array}$ & $\begin{array}{l}21.59 \\
0.08 \mathrm{C} \\
0.38 \\
--\end{array}$ & $\begin{array}{l}21.18 \\
* \\
--\end{array}$ & $\begin{array}{l}35.31 \\
*- \\
--\end{array}$ & $\begin{array}{l}13.56 \\
\div- \\
--\end{array}$ & $\begin{array}{c}28.73 \\
*- \\
--\end{array}$ & $\begin{array}{c}28 \cdot 3 C \\
\div \\
--\end{array}$ & $\begin{array}{r}29.21 \\
0.02 \\
0.07 \\
12.00\end{array}$ & $\begin{array}{r}30.58 \\
0.03 \\
0.10 \\
72.00\end{array}$ & $\begin{array}{r}42.98 \\
0.20 \\
0.47 \\
70.00\end{array}$ & $\begin{array}{r}334.52 \\
0.46\end{array}$ \\
\hline 1962 & $\begin{array}{l}\text { PRECIP }(C M .) \\
S R-9 C \text { INCI/SQ.KM.) } \\
S R-9 C \text { CONC. }(P C / L) \\
S R-89 / S R-S O\end{array}$ & $\begin{array}{r}44.58 \\
C .21 \\
0.48 \\
40.00\end{array}$ & $\begin{array}{r}13.08 \\
0.14 \\
1.08 \\
34.00\end{array}$ & $\begin{array}{r}29.16 \\
0.84 \\
2.89 \\
20.00\end{array}$ & $\begin{array}{r}15.11 \\
0.33 \\
2.19 \\
13.00\end{array}$ & $\begin{array}{r}30.51 \\
0.14 \\
0.46 \\
36.00\end{array}$ & $\begin{array}{r}19.15 \\
0.25 \\
1.31 \\
54.0 \mathrm{C}\end{array}$ & $\begin{array}{r}27.59 \\
0.14 \\
0.51 \\
30.00\end{array}$ & $\begin{array}{r}22.63 \\
0.12 \\
0.54 \\
22.00\end{array}$ & $\begin{array}{r}53.42 \\
0.05 \\
0.10 \\
19.00\end{array}$ & $\begin{array}{r}41.55 \\
0.08 \\
0.20 \\
30.00\end{array}$ & $\begin{array}{r}57.63 \\
0.12 \\
0.21 \\
99.00\end{array}$ & $\begin{array}{r}29.74 \\
0.12 \\
0.41 \\
55.00\end{array}$ & $\begin{array}{r}384.55 \\
2.54\end{array}$ \\
\hline 1963 & $\begin{array}{l}\text { PRECIP. (CM.) } \\
\text { SR-SC INCI/SQ.KM.) } \\
\text { SR-SO CONC. (PC/L) } \\
\text { SR-8Q/SR-SO }\end{array}$ & $\begin{array}{r}44.35 \\
0.14 \\
0.32 \\
25.00\end{array}$ & $\begin{array}{r}24.31 \\
0.38 \\
1.57 \\
24.00\end{array}$ & $\begin{array}{r}31.57 \\
0.53 \\
1.68 \\
18.00\end{array}$ & $\begin{array}{r}15.72 \\
0.50 \\
3.19 \\
3.00\end{array}$ & $\begin{array}{r}28.73 \\
0.08 \\
0.28 \\
9.20\end{array}$ & $\begin{array}{r}30.38 \\
0.30 \\
0.99 \\
3.00\end{array}$ & $\begin{array}{r}29.69 \\
0.19 \\
0.64 \\
5.00\end{array}$ & $\begin{array}{l}27.36 \\
0.15 \\
0.55 \\
*\end{array}$ & $\begin{array}{l}17.35 \\
0.06 \\
0.35 \\
*\end{array}$ & $\begin{array}{r}33.35 \\
C .09 \\
c .27 \\
*\end{array}$ & $\begin{array}{l}29.46 \\
0.12 \\
0.41 \\
+\end{array}$ & $\begin{array}{l}21.77 \\
=- \\
+\end{array}$ & $\begin{array}{r}334.04 \\
2.54\end{array}$ \\
\hline 1964 & $\begin{array}{l}\text { PREC IP. (CM.) } \\
\text { SR-9C INCI/SQ.KN.) } \\
S R-90 \text { CCNC. }(P C / L) \\
\text { SR-89/SR-90 }\end{array}$ & $\begin{array}{l}3.56 \\
0.24 \\
6.75 \\
*\end{array}$ & $\begin{array}{r}17.75 \\
0.39 \\
2.20 \\
+\end{array}$ & $\begin{array}{r}18.36 \\
0.24 \\
1.31 \\
+\end{array}$ & $\begin{array}{r}28.98 \\
0.20 \\
0.70 \\
*\end{array}$ & $\begin{array}{l}55.93 \\
C .1 C \\
0.18 \\
*\end{array}$ & $\begin{array}{l}28.35 \\
0.15 \\
0.53 \\
--\end{array}$ & $\begin{array}{l}47.47 \\
C .22 \\
0.47 \\
-.\end{array}$ & $\begin{array}{l}39.57 \\
0.13 \\
0.33 \\
--\end{array}$ & $\begin{array}{l}53.62 \\
0.10 \\
0.19 \\
-.\end{array}$ & $\begin{array}{r}57.89 \\
6.10 \\
0.18 \\
+\end{array}$ & $\begin{array}{r}42.80 \\
0.10 \\
0.24 \\
*\end{array}$ & $\begin{array}{r}18.85 \\
0.10 \\
0.54 \\
--\end{array}$ & $\begin{array}{r}413.13 \\
2.07\end{array}$ \\
\hline 1965 & $\begin{array}{l}\text { PRECIP. }(C M-) \\
S R-9 C \text { INCI/SQ.KM.) } \\
S R-9 C \text { CCNC. }(P C / L) \\
S R-89 / S R-90\end{array}$ & $\begin{array}{r}25.02 \\
0.03 \\
0.12 \\
--\end{array}$ & $\begin{array}{l}13.51 \\
0.09 \\
0.67 \\
--\end{array}$ & $\begin{array}{l}5.03 \\
0.07 \\
1.40 \\
--\end{array}$ & $\begin{array}{r}11.91 \\
0.05 \\
0.42 \\
-.\end{array}$ & $\begin{array}{l}20.14 \\
0.01 \\
0.05 \\
--\end{array}$ & $\begin{array}{r}29.08 \\
0.14 \\
0.49 \\
--\end{array}$ & $\begin{array}{l}37.72 \\
C . c 8 \\
C .22 \\
--\end{array}$ & $\begin{array}{l}17.58 \\
0.03 \\
0.18 \\
--\end{array}$ & $\begin{array}{l}39.01 \\
C .31 \\
0.80 \\
--\end{array}$ & $\begin{array}{l}37.13 \\
c .13 \\
0.36 \\
--\end{array}$ & $\begin{array}{l}30.78 \\
* \\
--\end{array}$ & $\begin{array}{l}24.36 \\
0.15 \\
0.62 \\
--\end{array}$ & $\begin{array}{r}291.27 \\
1.09\end{array}$ \\
\hline 1966 & $\begin{array}{l}\text { PRECIP. (CM.) } \\
\text { SR-90 INCI/SQ.KN.) } \\
\text { SR-90 CONC. }(P C / L) \\
\text { SR-89/SR-90 }\end{array}$ & $\begin{array}{l}9.63 \\
0.08 \\
0.84 \\
--\end{array}$ & $\begin{array}{r}11.23 \\
0.05 \\
0.45 \\
--\end{array}$ & $\begin{array}{l}14.73 \\
0.02 \\
0.14 \\
--\end{array}$ & $\begin{array}{l}40.69 \\
0.16 \\
0.40 \\
-.\end{array}$ & $\begin{array}{r}22.22 \\
C . c 7 \\
0.32 \\
--\end{array}$ & $\begin{array}{r}23.88 \\
0.09 \\
0.38 \\
17.00\end{array}$ & $\begin{array}{r}37.95 \\
C .02 \\
0.06 \\
13.00\end{array}$ & $\begin{array}{l}16.56 \\
0.02 \\
0.13 \\
*\end{array}$ & $\begin{array}{l}-- \\
-- \\
--\end{array}$ & $\begin{array}{r}34.37 \\
0.03 \\
0.09 \\
40.00\end{array}$ & $\begin{array}{r}30.99 \\
0.04 \\
0.13 \\
28.00\end{array}$ & $\begin{array}{r}49.38 \\
0.03 \\
0.07 \\
6.50\end{array}$ & $\begin{array}{r}291.63 \\
0.61\end{array}$ \\
\hline 1967 & $\begin{array}{l}\text { PRECIP. (CM.) } \\
\text { SR-SC INCI/SQ.KN.) } \\
\text { SR-9O CONC. (PC/L) } \\
\text { SR-89/SR-90 }\end{array}$ & $\begin{array}{l}30.18 \\
0.02 \\
0.07 \\
*\end{array}$ & $\begin{array}{r}24.69 \\
0.09 \\
0.37 \\
*\end{array}$ & $\begin{array}{r}31.65 \\
0.04 \\
0.13 \\
4.10\end{array}$ & $\begin{array}{l}19.40 \\
0.03 \\
0.16 \\
*\end{array}$ & $\begin{array}{l}12.52 \\
0.01 \\
0.08 \\
*\end{array}$ & $\begin{array}{r}27.91 \\
0.04 \\
0.15 \\
13.00\end{array}$ & $=$ & $\overline{--}$ & $\begin{array}{l}-- \\
-- \\
--\end{array}$ & $\begin{array}{l}-- \\
-- \\
--\end{array}$ & $\begin{array}{l}-- \\
-- \\
--\end{array}$ & $\begin{array}{l}-- \\
-- \\
--\end{array}$ & $\begin{array}{r}146.35 \\
0.23\end{array}$ \\
\hline
\end{tabular}

NOTES

--: DATA NOT aVAILABLE

*: ZERO OR TRACE

A: APPROXIMATE

B: LOWER LIMIT OF REPORTED DATA

C: PROPORTIUNEO FREM ORIGINALLY CONSOLICATED DATA 


\begin{tabular}{|c|c|c|c|c|c|c|c|c|c|c|c|c|c|c|}
\hline & & JAN. & FEB. & MAR. & APR. & $\begin{array}{l}\text { MAY } \\
---\end{array}$ & JUNE & JULY & AUG. & SEP. & OCT. & NOV. & OEC. & CUM. TOTAL \\
\hline 1959 & $\begin{array}{l}\text { PRECIP. (CM.) } \\
\text { SR-90 iNCH/SQ.KM-) } \\
\text { SR-SC CCNC. (PC/L) } \\
\text { SR-89/SR-90 }\end{array}$ & $\begin{array}{l}0.20 \\
=- \\
=-\end{array}$ & $\begin{array}{l}0.05 \\
=- \\
=-\end{array}$ & $\begin{array}{l}1.14 \\
=- \\
=-\end{array}$ & $\begin{array}{l}4.06 \\
=- \\
=-\end{array}$ & $\begin{array}{l}3.23 \\
=- \\
=-\end{array}$ & $\begin{array}{l}12.62 \\
=-- \\
--\end{array}$ & $\begin{array}{l}22.86 \\
=- \\
=-\end{array}$ & $\begin{array}{r}16.51 \\
0.08 \\
0.49 \\
2.10\end{array}$ & $\begin{array}{r}12.70 \\
0.02 \\
0.16 \\
*\end{array}$ & $\begin{array}{r}15.01 \\
0.03 \\
0.20 \\
1.50\end{array}$ & $\begin{array}{l}0.20 \\
-- \\
--\end{array}$ & $\begin{array}{l}0.10 \\
0.06 \\
60.01\end{array}$ & $\begin{array}{r}88.68 \\
0.19\end{array}$ \\
\hline 1960 & 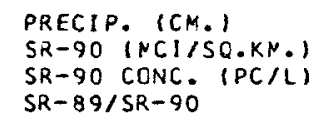 & $\begin{array}{l}0.51 \\
0.05 \\
9.81 \\
--\end{array}$ & $\begin{array}{l}* \\
0.05 \\
--\end{array}$ & $\begin{array}{l}1.27 \\
0.01 \\
0.79 \\
--\end{array}$ & $\begin{array}{r}0.20 \\
0.10 \\
50.01\end{array}$ & $\begin{array}{l}4.45 \\
-- \\
--\end{array}$ & $\begin{array}{l}7.01 \\
=- \\
=-\end{array}$ & $\begin{array}{l}18.01 \\
-\therefore \\
--\end{array}$ & $\begin{array}{l}15.01 \\
-- \\
--\end{array}$ & $\begin{array}{l}12.60 \\
0.026 \\
0.16 \\
-\end{array}$ & $\begin{array}{l}4.52 \\
C .01 C \\
c .23 \\
-.\end{array}$ & $\begin{array}{l}2.11 \\
0.016 \\
0.48 \\
--\end{array}$ & $\begin{array}{l}1.75 \\
0.000 \\
0.01 \\
--\end{array}$ & $\begin{array}{r}67.44 \\
0.25\end{array}$ \\
\hline 1961 & $\begin{array}{l}\text { PRECIP. (CM.) } \\
\text { SR-9O (NCI/SO.KM.) } \\
\text { SR-9O CONC. (PC/L) } \\
S R-89 / S R-S O\end{array}$ & $\begin{array}{l}0.74 \\
0.06 \mathrm{C} \\
8.11 \\
--\end{array}$ & $\begin{array}{l}* \\
0.000 \\
--\end{array}$ & $\begin{array}{l}7.62 \\
0.056 \\
0.66 \\
--\end{array}$ & $\begin{array}{l}15.75 \\
0.11 \mathrm{C} \\
0.70 \\
--\end{array}$ & $\begin{array}{l}1.42 \\
0.01 \mathrm{C} \\
0.71 \\
--\end{array}$ & $\begin{array}{c}22.25 \\
0.18 C \\
0.81 \\
--\end{array}$ & $\begin{array}{l}11.66 \\
0.04 C \\
C .35 \\
--\end{array}$ & $\begin{array}{c}14.91 \\
c .056 \\
0.34 \\
--\end{array}$ & $\begin{array}{l}12.78 \\
0.02 \\
0.16 \\
*\end{array}$ & $\begin{array}{l}2.69 \\
*- \\
--\end{array}$ & $\begin{array}{l}0.36 \\
0.01 \\
2.78 \\
8.60\end{array}$ & $\begin{array}{r}0.33 \\
0.02 \\
6.07 \\
56.00\end{array}$ & $\begin{array}{r}90.51 \\
0.55\end{array}$ \\
\hline 1962 & $\begin{array}{l}\text { PRECIP. }\left(C M_{-}\right) \\
\text {SR-90 (NCIISQ-KM) } \\
S R-S C \text { CCAC. }(P C / L) \\
S R-39 / S R-90\end{array}$ & $\begin{array}{l}* \\
\vdots \\
--\end{array}$ & $\begin{array}{l}* \\
0.01 \\
17.00\end{array}$ & $\begin{array}{l}0.41 \\
* \\
--\end{array}$ & $\begin{array}{r}9.96 \\
1.05 \\
10.55 \\
13.00\end{array}$ & $\begin{array}{r}3.71 \\
C .23 \\
6.20 \\
19.00\end{array}$ & $\begin{array}{r}14.07 \\
0.31 \\
2.21 \\
13.00\end{array}$ & $\begin{array}{l}14.58 \\
=- \\
=-\end{array}$ & $\begin{array}{r}12.90 \\
0.34 \\
2.64 \\
17.00\end{array}$ & $\begin{array}{r}12.55 \\
0.06 \\
0.48 \\
15.00\end{array}$ & $\begin{array}{r}9.04 \\
0.08 \\
0.89 \\
13.00\end{array}$ & $\begin{array}{r}13.21 \\
0.03 \\
0.23 \\
31.00\end{array}$ & $\begin{array}{r}0.30 \\
0.03 \\
10.01 \\
19.00\end{array}$ & $\begin{array}{r}90.73 \\
2.14\end{array}$ \\
\hline $15 \in 3$ & $\begin{array}{l}\text { PRECIP. }\left(C M_{-}\right) \\
\text {SR-9O (NCI/SO.KN-) } \\
\text { SR-g0 CCNC. (PC/L) } \\
S R-89 / S R-90\end{array}$ & $\begin{array}{l}* \\
0.01 \\
--\end{array}$ & $\begin{array}{l}* \\
0.02 \\
0.00\end{array}$ & $\begin{array}{r}1.50 \\
0.03 \\
2.01 \\
13.00\end{array}$ & $\begin{array}{r}0.18 \\
0.91 \\
505.56 \\
12.00\end{array}$ & $\begin{array}{l}4.24 \\
0.16 \\
3.78 \\
7.30\end{array}$ & $\begin{array}{r}10.29 \\
1.56 \\
15.17 \\
2.1 \mathrm{C}\end{array}$ & $\begin{array}{r}24.17 \\
1.00 \\
4.04 \\
1.00\end{array}$ & $\begin{array}{l}19.41 \\
1.03 \\
5.31 \\
*\end{array}$ & $\begin{array}{r}16.05 \\
0.29 \\
1.81 \\
1.20\end{array}$ & $\begin{array}{l}8.59 \\
0.23 \\
2.68 \\
*\end{array}$ & $\begin{array}{l}0.18 \\
0.04 \\
22.23 \\
*\end{array}$ & $\begin{array}{l}0.56 \\
-- \\
\#\end{array}$ & $\begin{array}{r}85.77 \\
5.28\end{array}$ \\
\hline 1984 & $\begin{array}{l}\text { PRECIP. }(C N-1) \\
\text { SR-9O } \text { (NCI/SO.KM.) } \\
\text { SR-90 CONC. }(P C / L) \\
\text { SR-89/SR-90 }\end{array}$ & $\begin{array}{l}1.55 C \\
0.03 C \\
1.94 \\
--\end{array}$ & $\begin{array}{l}1.55 C \\
0.036 \\
1.94 \\
--\end{array}$ & $\begin{array}{l}2.03 \\
\because- \\
--\end{array}$ & $\begin{array}{l}5.84 \\
=- \\
--\end{array}$ & $\begin{array}{l}6.45 \\
\because- \\
=\end{array}$ & $\begin{array}{l}27.53 \\
= \\
=\end{array}$ & $\begin{array}{l}12.70 \\
=- \\
=-\end{array}$ & $\begin{array}{l}11.00 \\
=- \\
=-\end{array}$ & $\begin{array}{l}=- \\
z \\
z\end{array}$ & $\begin{array}{l}* \\
\text { c.or } \\
-- \\
*\end{array}$ & $\begin{array}{l}-- \\
0.02 \\
--\end{array}$ & $\begin{array}{l}-- \\
0.01 \\
--\end{array}$ & $\begin{array}{r}68.65 \\
0.16\end{array}$ \\
\hline 1965 & $\begin{array}{l}\text { PREC IP. }(C M-) \\
\text { SR-90 (NCI/SQ.KN.) } \\
\text { SR-90 CCNC. (PC/L) } \\
\text { SR-89/SR-90 }\end{array}$ & $\begin{array}{l}* \\
0.03 c \\
-\therefore\end{array}$ & $\begin{array}{l}\text { c.04c } \\
-\therefore\end{array}$ & $\begin{array}{l}2.54 \\
0.02 \\
0.79 \\
--\end{array}$ & $\begin{array}{l}1.04 \\
0.07 \\
6.74 \\
--\end{array}$ & $\begin{array}{l}2.95 \\
0.04 \\
1.36 \\
--\end{array}$ & $\begin{array}{l}12.24 \\
0.19 \\
1.56 \\
--\end{array}$ & $\begin{array}{l}14.20 \\
0.17 \\
1.20\end{array}$ & $\begin{array}{l}25.96 \\
0.06 \\
0.24 \\
--\end{array}$ & $\begin{array}{r}17.25 \\
0.01 \\
0.06 \\
--\end{array}$ & $\begin{array}{l}6.65 \\
0.09 \\
1.36 \\
--\end{array}$ & $\begin{array}{l}0.08 \\
* \\
-- \\
--\end{array}$ & $\begin{array}{l}0.61 \\
0.03 \\
4.92 \\
--\end{array}$ & $\begin{array}{r}83.52 \\
0.75\end{array}$ \\
\hline 1966 & $\begin{array}{l}\text { PRECIP. }\left(C \mu_{-}\right) \\
\text {SR-SC INCI/SG.KM.) } \\
\text { SR-90 CCNC. (PC/L) } \\
\text { SR-89/SR-90 }\end{array}$ & $\begin{array}{l}0.81 \\
0.07 \\
8.65 \\
--\end{array}$ & $\begin{array}{r}0.13 \\
0.16 \\
123.08\end{array}$ & $\begin{array}{l}5.05 \\
0.18 \\
3.57 \\
\cdots-\end{array}$ & $\begin{array}{l}5.28 \\
* \\
-- \\
--\end{array}$ & $\begin{array}{l}4.11 \\
0.07 \\
1.71 \\
--\end{array}$ & $\begin{array}{l}7.58 \\
\pm \\
--\end{array}$ & $\begin{array}{l}13.25 \\
6.09 \\
0.68 \\
*\end{array}$ & $\begin{array}{r}22.27 \\
0.09 \\
0.41 \\
*\end{array}$ & $\begin{array}{l}11.15 \\
0.02 \\
0.18 \\
+\end{array}$ & $\begin{array}{l}2.41 \\
0.02 \\
0.83 \\
\end{array}$ & $\begin{array}{l}* \\
0.01 \\
--\end{array}$ & $\begin{array}{l}0.42 \\
0.01 \\
2.39 \\
*\end{array}$ & $\begin{array}{r}72.46 \\
0.72\end{array}$ \\
\hline 1967 & $\begin{array}{l}\text { PRECIP. (CM.) } \\
\text { SR-90 (NC1/SQ.KM.) } \\
\text { SR-90 CONC. (PC/L) } \\
\text { SR-89/SR-90 }\end{array}$ & $\begin{array}{l}5.39 \\
0.02 \\
0.38 \\
*\end{array}$ & $\frac{*}{0.01}$ & $\begin{array}{l}* \\
0.01 \\
*\end{array}$ & $\begin{array}{l}0.97 \\
0.03 \\
3.10 \\
*\end{array}$ & $\begin{array}{l}-- \\
0.05 \\
--\end{array}$ & $\begin{array}{l}5.10 \\
0.03 \\
0.59 \\
8.10\end{array}$ & $\begin{array}{l}=- \\
=- \\
=-\end{array}$ & $\begin{array}{l}= \\
=\end{array}$ & $\begin{array}{l}=- \\
\bar{z}\end{array}$ & $\begin{array}{l}=- \\
=- \\
=-\end{array}$ & $\begin{array}{l}= \\
= \\
=\end{array}$ & $\begin{array}{l}= \\
\overline{-} \\
=\end{array}$ & $\begin{array}{r}11.46 \\
0.15\end{array}$ \\
\hline $\begin{array}{c}\text { NOTE } \\
--: \\
*: \\
A: \\
B: \\
C:\end{array}$ & $\begin{array}{l}\text { OXIMATE } \\
\text { R LIMIT OF REP } \\
\text { ORTIONED FRCM }\end{array}$ & & & & & & & & & & & & & \\
\hline
\end{tabular}


SITE: MOROCCO, RABAT

SOURCE: UNITED STATES EMBASSY

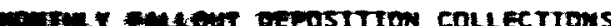
LONG.
51W ALT.
OH. (COLUMm)

\begin{tabular}{|c|c|c|c|c|c|c|c|c|c|c|c|c|c|c|}
\hline & & JAN. & FEB. & MAR. & APR. & MAY & JUNE & JULY & AUG. & SEP. & OCT. & NOV. & DEC. & CUM. TOJAL \\
\hline 1963 & $\begin{array}{l}\text { PRECIP. ICM-1 } \\
\text { SR-90 IMCI/SO.KM_) } \\
\text { SR-90 CONC. (PC/L) } \\
\text { SR-B9/SR-90 }\end{array}$ & $\begin{array}{l}19.56 \\
= \\
=\end{array}$ & $\begin{array}{l}14.48 \\
=- \\
=\end{array}$ & $\begin{array}{l}7.62 \\
=- \\
=-\end{array}$ & $\begin{array}{l}2.03 \\
=\end{array}$ & $\begin{array}{l}4.06 \\
= \\
=\end{array}$ & $\begin{array}{l}1.02 \\
= \\
=\end{array}$ & $\frac{0.02}{1.00}$ & $\frac{0.12}{*}$ & $\begin{array}{l}0.25 \\
0.07 \\
28.01 \\
*\end{array}$ & $\begin{array}{r}0.25 \\
0.13 \\
52.01 \\
*\end{array}$ & $\begin{array}{l}5.08 \\
0.42 \\
8.27 \\
*\end{array}$ & $\begin{array}{l}17.78 \\
2.66 \\
14.97 \\
*\end{array}$ & $\begin{array}{r}72.13 \\
3.42\end{array}$ \\
\hline 1964 & $\begin{array}{l}\text { PRECIP. (CM.) } \\
\text { SR-90 (MCI/SQ.KM.) } \\
\text { SR-90 CONC. (PC/L) } \\
\text { SR-B9/SR-90 }\end{array}$ & $\begin{array}{r}0.51 \\
11.08 \\
211.77\end{array}$ & $\begin{array}{r}5.08 \\
1.08 \\
21.26\end{array}$ & $\begin{array}{r}10.16 \\
1.13 \\
11.13 \\
1.00\end{array}$ & $\begin{array}{r}0.99 \\
2.12 \\
214.15 \\
*\end{array}$ & $\begin{array}{l}5.08 \\
0.26 \\
5.12 \\
--\end{array}$ & $\begin{array}{l}* \\
0.25 \\
-\end{array}$ & $\begin{array}{l}0.30 \\
=\end{array}$ & $\frac{0.06}{=}$ & $\begin{array}{l}0.25 \\
0.19 \\
76.01\end{array}$ & $\frac{0.15}{2.10}$ & $\begin{array}{r}1.27 \\
0.20 \\
15.75 \\
1.60\end{array}$ & $\begin{array}{l}7.62 \\
0.64 \\
8.40 \\
--\end{array}$ & $\begin{array}{r}30.96 \\
7.46\end{array}$ \\
\hline 1965 & $\begin{array}{l}\text { PREC IP. }\left(C M_{-}\right) \\
\text {SR-90 (MCI/SO.KM-) } \\
\text { SR-90 CCNC. }(P C / L) \\
\text { SR-89/SR-90 }\end{array}$ & $\begin{array}{l}10.16 \\
0.43 \\
4.24 \\
--\end{array}$ & $\begin{array}{r}10.16 \\
0.35 \\
3.45 \\
--\end{array}$ & $\begin{array}{r}2.54 \\
0.37 \\
14.57 \\
-\end{array}$ & $\begin{array}{r}2.54 \\
0.47 \\
18.51 \\
-\end{array}$ & $\begin{array}{r}2.54 \\
0.47 \\
18.51\end{array}$ & $\begin{array}{l}0.25 \\
0.21 \\
84.01 \\
\end{array}$ & $\begin{array}{l}* 0.060 \\
=-\end{array}$ & $\begin{array}{l}* \\
0.066 \\
--\end{array}$ & $\begin{array}{l}2.54 \\
0.05 \\
1.97\end{array}$ & $\begin{array}{l}2.54 \\
0.25 \\
9.85 \\
--\end{array}$ & $\begin{array}{l}1.50 \\
0.12 \\
8.01 \\
--\end{array}$ & $\begin{array}{l}2.54 \\
-- \\
--\end{array}$ & $\begin{array}{r}37.31 \\
2.84\end{array}$ \\
\hline 1966 & $\begin{array}{l}\text { PRECIP. (CM.) } \\
\text { SR-90 iMCI/SO.KM-) } \\
\text { SR-90 CONC. (PC/L) } \\
\text { SR-89/SR-90 }\end{array}$ & $\begin{array}{l}1.80 \\
\pm \\
-\end{array}$ & $\begin{array}{r}3.50 \\
0.44 \\
12.58\end{array}$ & $\begin{array}{l}\overline{0.026} \\
=-\end{array}$ & $\begin{array}{l}\overline{0.03 c} \\
=-\end{array}$ & $\begin{array}{l}1.27 \mathrm{C} \\
0.09 \mathrm{C} \\
7.09 \\
-.\end{array}$ & $\begin{array}{l}1.27 c \\
0.08 c \\
6.30 \\
--\end{array}$ & $\frac{*}{0.026}$ & $\frac{0.02 c}{*}$ & $\begin{array}{r}0.20 \\
0.02 \\
10.01 \\
* \quad .\end{array}$ & $\begin{array}{l}0.80 \\
0.07 \\
8.76 \\
*\end{array}$ & $\overline{0.02 c}$ & $\begin{array}{l}-\overline{0.03 c} \\
-\end{array}$ & $\begin{array}{r}8.84 \\
.0 .84\end{array}$ \\
\hline 1967 & $\begin{array}{l}\text { PREC1P. (CM-) } \\
\text { SR-90 (MCI/SQ.KM_) } \\
\text { SR-90 CONC. (PC/L) } \\
\text { SR-89/SR-90 }\end{array}$ & $\begin{array}{l}1.60 \\
0.02 \\
1.26 \\
4\end{array}$ & $\begin{array}{r}3.00 \\
0.08 \\
2.67 \\
11.80\end{array}$ & $\begin{array}{l}1.00 \\
0.06 \\
6.01 \\
5.90\end{array}$ & $\begin{array}{l}3.20 \\
0.08 \\
2.51 \\
7.20\end{array}$ & $\begin{array}{l}1.00 \\
0.02 \\
2.01 \\
*\end{array}$ & $\begin{array}{l}\overline{0.02} \\
\bar{*}\end{array}$ & $\frac{0.01}{*}$ & $\bar{z}$ & $\begin{array}{l}=- \\
=\end{array}$ & $\bar{z}$ & $\bar{z}$ & $\begin{array}{l}\overline{-} \\
\overline{--}\end{array}$ & $\begin{array}{l}9.80 \\
0.29\end{array}$ \\
\hline 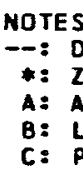 & $\begin{array}{l}\text { ATA NOT AVAILABLE } \\
\text { ERD OR TRACE } \\
\text { PPROXIMATE } \\
\text { OWER LIMIT OF REPO } \\
\text { ROPORTIONED FROM }\end{array}$ & INal & & & & & & & & & & & & \\
\hline
\end{tabular}


MONTHLY FALLOUT DEPOSITION COLLECTIONS

SITE: MOROCCO, SIDI SLI AFB

LAT. 32 5IN LONG. $32 \mathrm{~N}$ ALT.

OM. (COLUMN)

SOURCE: U. S. AIR WEATHER SERVICE

COLLECTIONS TERMINATED JULY 1963

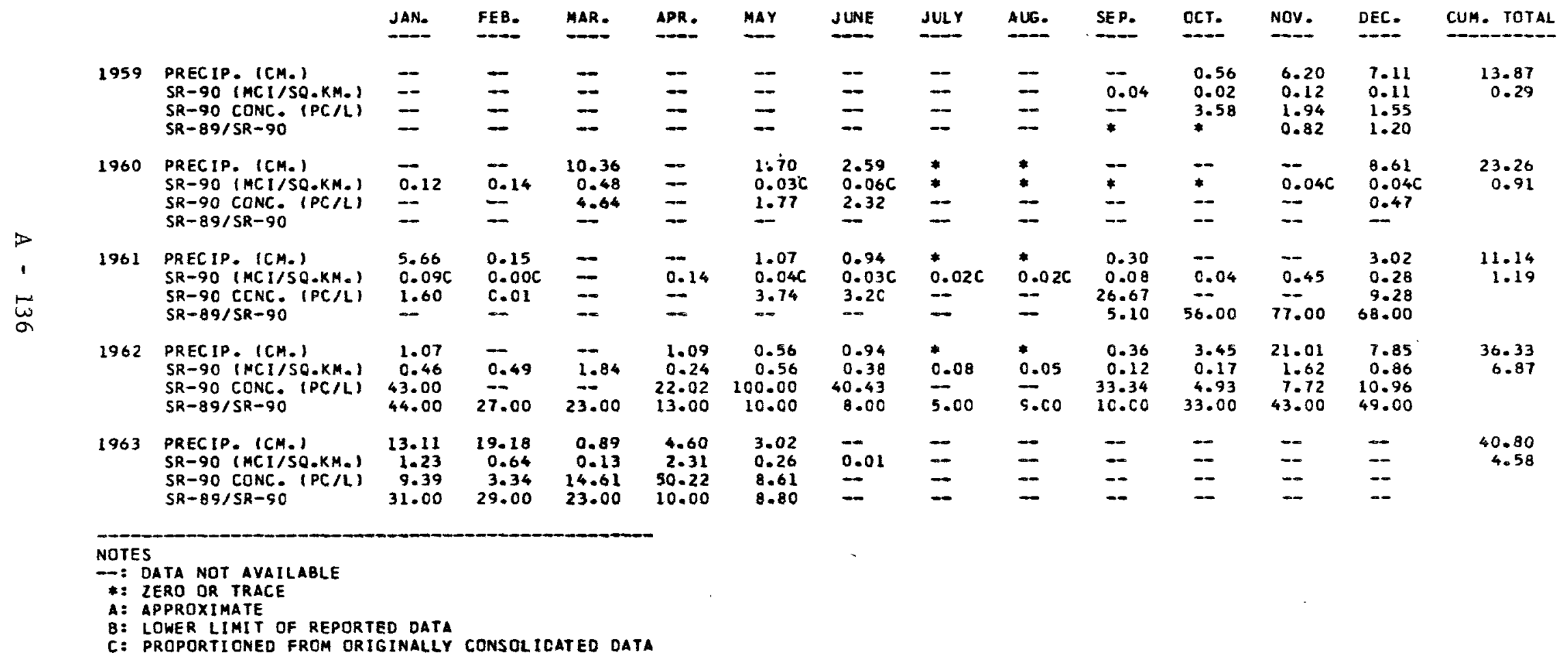




\begin{tabular}{|c|c|c|c|c|c|c|c|c|c|c|c|c|c|c|}
\hline & & JAN. & FEB. & MAR . & APR : & Mar & JUNE & JULY & AUG. & SEP. & OCT. & NOV. & DEC. & CUM. TOTAL \\
\hline 1959 & $\begin{array}{l}\text { PRECIP. (CM.) } \\
\text { SR-90 (MCI/SQ.KM.) } \\
\text { SR-90 CONC. (PC/L) } \\
\text { SR-89/SR-90 }\end{array}$ & $=$ & $\begin{array}{l}- \\
= \\
-\end{array}$ & $=$ & $\begin{array}{l}\ddot{\infty} \\
\approx\end{array}$ & $=$ & $\overline{-}$ & $=$ & $\bar{z}$ & $\begin{array}{l}2.92 \\
0.07 \\
2.40 \\
0.67\end{array}$ & $\begin{array}{l}11.00 \\
0.06 \\
0.55 \\
*\end{array}$ & $\begin{array}{l}5.00 \\
0.03 \\
0.61 \\
+\end{array}$ & $\begin{array}{l}5.00 \\
0.04 \\
0.81 \\
*\end{array}$ & $\begin{array}{r}23.92 \\
0.20\end{array}$ \\
\hline 1960 & $\begin{array}{l}\text { PRECIP. (CM.) } \\
\text { SR-9O (MEI/SO,KM, } \\
S R-90 \text { CONE. }(P C / L) \\
S R-89 / S R-90\end{array}$ & $\begin{array}{l}4.01 \\
0.10 \\
2.50 \\
-.\end{array}$ & $\begin{array}{l}11.00 \\
0.14 \\
1.28 \\
-\end{array}$ & $\begin{array}{l}9.02 \\
0.12 \\
1.34\end{array}$ & $\begin{array}{l}4.14 \\
0.11 \\
2.66 \\
=-\infty\end{array}$ & $\begin{array}{l}8.99 \\
-- \\
--\end{array}$ & $\begin{array}{l}18.19 \\
0.07 \\
0.39 \\
\end{array}$ & $\begin{array}{l}19.30 \\
0.12 \mathrm{C} \\
0.63 \\
--\end{array}$ & $\begin{array}{c}12.52 \\
0.08 \mathrm{C} \\
0.64 \\
-\end{array}$ & $\begin{array}{l}15.65 \\
0.14 \mathrm{C} \\
0.90 \\
-\end{array}$ & $\begin{array}{l}9.32 \\
0.086 \\
0.86 \\
-.\end{array}$ & $\begin{array}{l}3.99 \\
0.116 \\
2.76 \\
-.\end{array}$ & $\begin{array}{l}3.91 \\
0.116 \\
2.82 \\
-\end{array}$ & $\begin{array}{r}120.04 \\
1.18\end{array}$ \\
\hline 1961 & 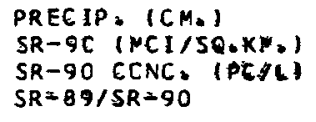 & $\begin{array}{l}9.91 \\
6.06 \mathrm{C} \\
0.61 \\
-\end{array}$ & $\begin{array}{l}6.78 \\
0.04 C \\
0.59 \\
--\end{array}$ & $\begin{array}{c}13.00 \\
0.126 \\
0.93 \\
-\end{array}$ & $\begin{array}{l}5.11 \\
0.05 C \\
0.98 \\
\end{array}$ & $\begin{array}{l}19.05 \\
0.12 \mathrm{C} \\
0.63 \\
--\end{array}$ & $\begin{array}{l}19.05 \\
0.12 \mathrm{C} \\
0.63 \\
--\end{array}$ & $\begin{array}{l}5.92 \\
0.00 \mathrm{C} \\
0.01 \\
--\end{array}$ & $\begin{array}{l}7.75 \\
0.00 \mathrm{C} \\
0.01 \\
--\end{array}$ & $\begin{array}{l}16.92 \\
0.02 \\
0.12 \\
*\end{array}$ & $\begin{array}{l}1.96 \\
0.03 \\
1.54 \\
5.00\end{array}$ & $\begin{array}{l}5.33 \\
0.04 \\
0.76 \\
*\end{array}$ & $\begin{array}{l}3.15 \\
0.06 \\
1.91 \\
1.00\end{array}$ & $\begin{array}{r}113.93 \\
0.66\end{array}$ \\
\hline 1562 & $\begin{array}{l}\text { PRECIP. }\left(C M_{V}\right) \\
S R-90 \text { (NCI/SQRKHW } \\
S R-90 \text { CONC. }(P C / L) \\
S R-89 / S R-9 O\end{array}$ & $\begin{array}{r}25.04 \\
0.61 \\
2.44 \\
1.40\end{array}$ & $\begin{array}{l}5.18 \\
0.03 \\
0.58 \\
+\end{array}$ & $\begin{array}{l}30.52 \\
0.11 \\
1.05 \\
*\end{array}$ & $\begin{array}{r}21.21 \\
0.06 \\
0.29 \\
\quad\end{array}$ & $\begin{array}{r}12.78 \\
0.08 \\
0.63 \\
20.00\end{array}$ & $\begin{array}{r}16.13 \\
0.20 \\
0.62 \\
\times 1.00\end{array}$ & $\begin{array}{r}13.64 \\
0.08 \\
0.59 \\
15.60\end{array}$ & $\begin{array}{r}15.39 \\
0.08 \\
0.52 \\
12.00\end{array}$ & $\begin{array}{r}5.79 \\
0.05 \\
0.87 \\
10.00\end{array}$ & $\begin{array}{r}17.55 \\
0.22 \\
1.26 \\
8.00\end{array}$ & $\begin{array}{r}7.26 \\
0.10 \\
1.38 \\
20.00\end{array}$ & $\begin{array}{r}8.41 \\
0.08 \\
0.96 \\
15.00\end{array}$ & $\begin{array}{r}150.90 \\
1.60\end{array}$ \\
\hline 1963 & $\begin{array}{l}P R E C I P \text {. (CM-) } \\
S R=90 \text { IPCI/SO-KM-) } \\
S R-90 \text { CONC= (PC/L) } \\
S R-89 / S R=90\end{array}$ & $\begin{array}{l}6.17 \\
0.10 \\
1.63 \\
8.00\end{array}$ & $\begin{array}{l}8.79 \\
0.10 \\
1.14 \\
4.00\end{array}$ & $\begin{array}{l}6.73 \\
0.13 \\
1.94 \\
2.80\end{array}$ & $\begin{array}{l}5.97 \\
0.13 \\
2.18 \\
2.00\end{array}$ & $\begin{array}{l}8.92 \\
0.08 \\
0.90 \\
2.00\end{array}$ & $\begin{array}{l}13.84 \\
0.14 \\
1.02 \\
=-\end{array}$ & $\begin{array}{l}8.10 \\
0.09 \\
1.12\end{array}$ & $\begin{array}{l}23.95 \\
0.22 \\
0.92 \\
*\end{array}$ & $\begin{array}{l}7.39 \\
0.01 \\
0.16 \\
*\end{array}$ & $\begin{array}{l}0.76 \\
0.07 \\
5.22 \\
*\end{array}$ & $\begin{array}{l}8.51 \\
0.12 \\
1.42 \\
*\end{array}$ & $\begin{array}{r}3.40 \\
0.43 \\
12.65 \\
*\end{array}$ & $\begin{array}{r}102.53 \\
1.62\end{array}$ \\
\hline 1964 & $\begin{array}{l}\text { PRECIP. }(C M=) \\
S R=90 \text { (MCI/SOSKM ) } \\
S R=90 \text { CONC. }(P C / L) \\
S R=89 / S R-S O\end{array}$ & $\begin{array}{l}7.52 \\
0.19 \\
2.53 \\
\end{array}$ & $\begin{array}{l}2.49 \\
0.08 \\
3.22 \\
0.80\end{array}$ & $\begin{array}{l}9.17 \\
0.08 \\
0.88 \\
+\end{array}$ & $\begin{array}{l}4.11 \\
0.06 \\
1.46 \\
+\end{array}$ & $\begin{array}{r}10.16 \\
0.13 \\
1.28 \\
+\end{array}$ & $\begin{array}{r}15.72 \\
0.26 \\
1.66 \\
-0\end{array}$ & $\begin{array}{r}22.78 \\
0.63 \\
2.77 \\
-0\end{array}$ & $\begin{array}{l}5.46 \\
0.16 \\
2.54 \\
\end{array}$ & $\begin{array}{l}9.65 \\
0.40 \\
4.15 \\
-.\end{array}$ & $\begin{array}{l}3.40 \\
0.11 \\
3.24\end{array}$ & $\begin{array}{l}6.35 \\
0.21 \\
3.31 \\
*\end{array}$ & $\begin{array}{l}9.04 \\
0.34 \\
3.77 \\
-.\end{array}$ & $\begin{array}{r}105.85 \\
2.65\end{array}$ \\
\hline 1965 & $\begin{array}{l}\text { PRECIP, }\left(C M_{2}\right) \\
S R-90 \text { (MCI/SQ } X M=) \\
S R-9 C \text { CONC. }(P C / L) \\
S R-89 / S R-90^{\circ}\end{array}$ & $\begin{array}{l}5.84 \\
0.29 \\
4.97 \\
2 .\end{array}$ & $\begin{array}{l}6.60 \\
0.24 \\
3.64 \\
=-\end{array}$ & $\begin{array}{l}22.83 \\
0.67 \\
2.94 \\
-2\end{array}$ & $\begin{array}{l}6.10 \\
0.24 \\
2.30 \\
=-10\end{array}$ & $\begin{array}{l}6.91 \\
0.07 \\
1.22 \\
=0\end{array}$ & $\begin{array}{l}20.80 \\
0.26 \\
1.26 \\
=-\end{array}$ & $\begin{array}{l}15.42 \\
C .0 .2 \\
c=13 \\
=\end{array}$ & $\begin{array}{l}17.45 \\
0.18 \\
1.04 \\
-\end{array}$ & $\begin{array}{l}7.34 \\
0.22 \\
3.0 \mathrm{C} \\
--\end{array}$ & $\begin{array}{l}6.73 \\
0.14 \\
2.09 \\
-.\end{array}$ & $\begin{array}{l}22.61 \\
0.15 \\
0.67 \\
-.\end{array}$ & $\begin{array}{l}9.52 \\
0.23 \\
2.42 \\
-.\end{array}$ & $\begin{array}{r}148.15 \\
2.61\end{array}$ \\
\hline 1566 & $\begin{array}{l}\text { PRECIP. }(C M+) \\
S R-90 \text { (NCI/SO.KM-) } \\
S R=90 \text { CCNC }(P C / L) \\
S R=89 / S R^{2}=90\end{array}$ & $\begin{array}{r}13.18 \\
0.16 \\
1.22 \\
-6\end{array}$ & $\begin{array}{l}0.06 \\
=-\end{array}$ & $\begin{array}{l}4.67 \\
0.07 \\
1.50 \\
--\end{array}$ & $\begin{array}{l}18.49 \\
0.12 \\
0.65 \\
-\end{array}$ & $\begin{array}{l}22.20 \\
0.06 \\
0.28 \\
-.\end{array}$ & $\begin{array}{l}9.47 \\
0.05 \\
0.53 \\
+\end{array}$ & $\begin{array}{l}16.41 \\
0.05 \\
C .31 \\
*\end{array}$ & $\begin{array}{l}12.09 \\
0.06 \\
0.50 \\
*\end{array}$ & $\begin{array}{l}10.49 \\
0.07 \\
0.67 \\
*\end{array}$ & $\begin{array}{r}4.06 \\
0.07 \\
1.73 \\
11.00\end{array}$ & $\begin{array}{l}- \\
0.03 \\
-\end{array}$ & $\begin{array}{l}-. \\
0.15 \\
8.40\end{array}$ & $\begin{array}{r}111.06 \\
0.95\end{array}$ \\
\hline 1967 & $\begin{array}{l}\text { PRECIP. }\left(C M_{3}\right) \\
S R=90 \text { (MCI/SOLKM) } \\
S R=90 \text { CONC. }(P C / L) \\
S R=89 / S R=90\end{array}$ & $\begin{array}{l}-. \\
0.05 \\
7.20\end{array}$ & $\frac{0.06}{2.70}$ & $\begin{array}{l}0.06 \\
+\infty\end{array}$ & $\begin{array}{r}12.98 \\
0.05 \\
0.39 \\
*\end{array}$ & $\underset{*}{0.05}$ & $\begin{array}{l}0.01 \\
-\infty\end{array}$ & $\frac{\overline{0.06}}{\overline{13.00}}$ & $=$ & $=$ & $=$ & $\begin{array}{l}= \\
=- \\
=-\end{array}$ & $\begin{array}{l}-- \\
=- \\
--\end{array}$ & $\begin{array}{r}12.98 \\
0.34\end{array}$ \\
\hline
\end{tabular}

MOTES

NO: DATA NOT AVAILABLE

*: ZERO OR TRACE

A: APPROXIMATE

B: LOHER LIMIT OF REPORTED DATA

C: PROPLRTIONED FROM ORIGINALIY CONSOL TOATED DATA 
MONTHLY FALLOUT DEPOSITION COLLECTIONS

SITE: NIGERIA, LAGOS

SOURCE: UNITEO STATES CONSULATE
LAT. $626 \mathrm{~N}$ LONG. $324 \mathrm{E}$ ALT. 1M. (COLUMN)

\begin{tabular}{|c|c|c|c|c|c|c|c|c|c|c|c|c|c|c|}
\hline & & JAN, & FEB. & MAR: & $A P R_{0}$ & MAY & JUNE & JULY & AUG. & SEP. & OCT. & NOV. & DEC. & CUM. TOTAL \\
\hline 1959 & $\begin{array}{l}\text { PRECIP, (CM,) } \\
S R-90 \text { IMCI/SO.KM,) } \\
S R-90 \text { CONC. (PC/L) } \\
S R-89 / S R-90\end{array}$ & $=$ & $=$ & $=$ & $=$ & $=$ & $=$ & $=$ & $\begin{array}{l}8.00 \\
-- \\
-\end{array}$ & $\begin{array}{r}11.00 \\
0.03 \\
0.28 \\
*\end{array}$ & $\begin{array}{l}2.01 \\
0.03 \\
1.50 \\
1.20\end{array}$ & $\begin{array}{l}14.02 \\
*\end{array}$ & $\begin{array}{l}3.00 \\
* \\
*\end{array}$ & $\begin{array}{r}38.03 \\
0.06\end{array}$ \\
\hline 1960 & $\begin{array}{l}P R E C I P,(C M,) \\
\$ R=9 C \text { IMCI/SQ,KM.) } \\
\$ R=90 \text { CONC. (PC/L) } \\
\$ R=89 / S R \rightarrow 90\end{array}$ & $\begin{array}{l}2.01 \\
0.05 \\
2.49 \\
-.\end{array}$ & $\begin{array}{l}5.99 \\
0.06 \\
1.01 \\
-.0\end{array}$ & $\begin{array}{l}4.01 \\
0.08 \\
2.00 \\
-\end{array}$ & $\begin{array}{l}22.00 \\
= \\
=\end{array}$ & $\begin{array}{l}23.01 \\
0.020 \\
0.09 \\
-\end{array}$ & $\begin{array}{l}33.99 \\
0.04 \mathrm{C} \\
0.12 \\
-\end{array}$ & $\begin{array}{c}10.01 \\
0.04 C \\
0.40 \\
-\end{array}$ & $\begin{array}{l}11.00 \\
0.056 \\
0.46 \\
-.\end{array}$ & $\begin{array}{l}23.01 \\
C .01 \\
0.05 \\
--\end{array}$ & $\begin{array}{l}16.00 \\
0.01 \\
0.07 \\
--\end{array}$ & $\begin{array}{l}7.01 \\
0.026 \\
0.29 \\
-.\end{array}$ & $\begin{array}{l}10.01 \\
0.036 \\
0.30 \\
--\end{array}$ & $\begin{array}{r}168.05 \\
0.41\end{array}$ \\
\hline 1961 & $\begin{array}{l}\text { PRECIP (CM.) } \\
S R=9 C \text { (PCI/SQ-KM.) } \\
S R=90 \text { CONC. }(P C / L) \\
S R=89 / S R=90\end{array}$ & $\begin{array}{l}3.00 \\
0.04 C \\
1.34 \\
=-\end{array}$ & $\begin{array}{l}0.30 \\
0.000 \\
0.01 \\
=-\end{array}$ & $\begin{array}{l}14.99 \\
0.08 \mathrm{C} \\
0.54 \\
=-\end{array}$ & $\begin{array}{l}29.01 \\
0.16 \mathrm{C} \\
0.56 \\
=-\end{array}$ & $\begin{array}{l}+\overrightarrow{0.090} \\
=-\end{array}$ & $\begin{array}{l}49.00 \\
0.09 C \\
0.19 \\
-\end{array}$ & $\begin{array}{l}32.00 \\
0.066 \\
0.19 \\
=\end{array}$ & $\begin{array}{l}0.30 \\
0.00 C \\
0.01 \\
-\infty\end{array}$ & $\begin{array}{l}10.01 \\
--\end{array}$ & $\begin{array}{r}26.01 \\
0.03 \\
0.12 \\
67.00\end{array}$ & $\begin{array}{r}10.01 \\
0.01 \\
0.10 \\
60.00\end{array}$ & $\begin{array}{r}0.99 \\
0.05 \\
5.06 \\
58.00\end{array}$ & $\begin{array}{r}175.62 \\
0.61\end{array}$ \\
\hline 1962 & $\begin{array}{l}\text { PRECIP. }\left(C M_{*}\right) \\
S R=90 \text { i } M C I / S Q . K M=) \\
S R=90 \text { CONC. }(P C / L) \\
S R-89 / 5 R=90\end{array}$ & $\begin{array}{r}0.30 \\
0.05 \\
16.67 \\
46.00\end{array}$ & $\begin{array}{l}7.62 \\
3.10 C \\
40.69 \\
--\end{array}$ & $\begin{array}{l}0.000 \\
=-\end{array}$ & $\begin{array}{l}0.18 \\
12.00\end{array}$ & $\begin{array}{l}\overline{0.25} \\
22.00\end{array}$ & $\begin{array}{r}3.81 \\
0.69 \\
18.12 \\
30.0 \mathrm{C}\end{array}$ & $\begin{array}{l}-- \\
0.26 \\
3 c . c 0\end{array}$ & $\begin{array}{l}-\overline{0.10} \\
-- \\
16.00\end{array}$ & $\begin{array}{c}0.08 \\
13.00\end{array}$ & $\begin{array}{l}0.12 \mathrm{~A} \\
27.00 \mathrm{~A}\end{array}$ & $\begin{array}{l}-\overline{0.074} \\
\overline{25.004}\end{array}$ & $\begin{array}{l}-0.05 \mathrm{~A} \\
20.00 \mathrm{~A}\end{array}$ & $\begin{array}{r}11.73 \\
4.95\end{array}$ \\
\hline 1963 & $\begin{array}{l}\text { PRECIP. }\left(C M_{0}\right) \\
S R-90 \text { (NCI/SQ.KM.) } \\
S R-9 C \text { CONC. (PC/L) } \\
S R-8 S / S R=S C\end{array}$ & $\begin{array}{l}0.184 \\
0.074 \\
38.89 \\
18.004\end{array}$ & $\begin{array}{l}1.80 A \\
0.12 \mathrm{~A} \\
6.67 \\
26.00 \mathrm{~A}\end{array}$ & $\begin{array}{l}4.654 \\
0.394 \\
8.39 \\
14.004\end{array}$ & $\begin{array}{c}13.764 \\
0.40 A \\
3.41 \\
28.004\end{array}$ & $\begin{array}{l}30.12 \\
1.57 \\
5.22 \\
9.104\end{array}$ & $\begin{array}{l}30.384 \\
0.02 A \\
0.07 \\
--\end{array}$ & $\begin{array}{l}30.38 \\
0.300 \\
C .99 \\
-\end{array}$ & $\begin{array}{l}49.30 \\
0.48 C \\
0.98 \\
-.\end{array}$ & $\begin{array}{l}37.03 \\
0.01 \\
0.03 \\
\end{array}$ & $\begin{array}{l}-\infty \\
0.04 \\
*\end{array}$ & $\begin{array}{l}--13 \\
0.13 \\
*\end{array}$ & $\begin{array}{l}-- \\
0.03 \\
--0 \\
1.00\end{array}$ & $\begin{array}{r}195.60 \\
3.56\end{array}$ \\
\hline 1964 & $\begin{array}{l}\text { PRECIP, (CM.) } \\
\text { SR-90 (MCI/SQ.KH,) } \\
\text { SR-90 CONC. (PC/L) } \\
\text { SR-89/SR-90 }\end{array}$ & $\begin{array}{r}0.10 \\
0.07 \\
70.01 \\
*\end{array}$ & $\begin{array}{r}10.01 \\
0.29 \\
2.90\end{array}$ & $\begin{array}{l}-- \\
0.22 \\
-\end{array}$ & $\begin{array}{r}15.01 \\
0.50 \\
3.34 \\
*\end{array}$ & $\begin{array}{l}45.03 \\
0.74 \\
1.65 \\
-.\end{array}$ & $\begin{array}{l}-1.99 \\
-\end{array}$ & $=$ & $=$ & $\ddot{m}=$ & $\begin{array}{l}11.40 \\
0.11 \mathrm{C} \\
0.97 \\
--\end{array}$ & $\begin{array}{l}4.50 \\
0.04 C \\
0.89 \\
--\end{array}$ & $\begin{array}{l}0.81 \\
0.016 \\
1.24 \\
--\end{array}$ & $\begin{array}{r}86.86 \\
3.97\end{array}$ \\
\hline 1965 & $\begin{array}{l}\text { PREC IP. (CM.) } \\
\text { SR-90 IMCI/SQ.KM.) } \\
S R-90 \text { CONC. }(P C / L) \\
S R-89 / S R-90\end{array}$ & $\begin{array}{r}1.80 \\
0.30 \\
16.67 \\
--\end{array}$ & $\begin{array}{l}2.79 \\
0.19 \\
6.82 \\
-.\end{array}$ & $\begin{array}{l}25.02 \\
-- \\
=\end{array}$ & $\begin{array}{l}18.01 \\
0.296 \\
1.62 \\
=\end{array}$ & $\begin{array}{l}18.31 \\
0.29 C \\
1.59 \\
--\end{array}$ & $\begin{array}{l}32.82 \\
1.19 \\
3.63 \\
--\end{array}$ & $\begin{array}{l}46.74 \\
=- \\
=-\end{array}$ & $\begin{array}{l}15.70 \\
= \\
=\end{array}$ & $\begin{array}{l}21.11 \\
=- \\
=\end{array}$ & $\begin{array}{l}10.90 \\
=- \\
=\end{array}$ & $=$ & $\begin{array}{l}-- \\
-- \\
--\end{array}$ & $\begin{array}{r}193.20 \\
2.26\end{array}$ \\
\hline $\begin{array}{c}\text { NOTE } \\
-: \\
A: \\
B: \\
B: \\
C:\end{array}$ & $\begin{array}{l}\text { S } \\
\text { DATA NOT AVAILABLE } \\
\text { ZERO OR TRACE } \\
\text { APPROXIMATE } \\
\text { LOHER LIMIT OF REPOR R } \\
\text { PROPORTI ONED FROM OR }\end{array}$ & $\begin{array}{l}\text { DATA } \\
\text { NALLY }\end{array}$ & & & & & & & & & & & & \\
\hline
\end{tabular}




\section{MDNTHLY FALLDUT DEPOSITION COLLECTIONS}

\section{SITE: PAKISTAN, KARACHI}

LAT. $2451 N$ LONG. 67 2E ALT.

15M. (COLUMN)

SOURCE: PAKISTAN ATOMIC ENERGY COMMISSION

COLLECTIONS AT THIS SITE TERMINATED

JAN. FEB. MAR. APR. MAY

\begin{tabular}{|c|c|c|c|c|c|c|c|c|c|c|c|c|c|c|}
\hline 1958 & $\begin{array}{l}\text { PRECIP. }\left(C M_{*}\right) \\
S R-90 \text { (MCI/SQ.KM.) } \\
S R-90 \text { CONC. }(P C / L) \\
S R-89 / S R-90\end{array}$ & $\overline{--}$ & $\begin{array}{r}0.99 \\
0.01 \\
1.02 \\
45.00\end{array}$ & $\begin{array}{l}* \\
0.03 \\
-- \\
40.00\end{array}$ & $\begin{array}{l}* \\
0.05 \\
4.00\end{array}$ & $\begin{array}{l}* \\
0.15 \\
3.00\end{array}$ & $\begin{array}{c}* \\
0.03 \\
13.00\end{array}$ & $\begin{array}{r}8.00 \\
0.11 \\
1.38 \\
53.00\end{array}$ & $\begin{array}{l}\overline{0.06} \\
\overline{31.00}\end{array}$ & $\begin{array}{c}\overline{0.22} \\
\overline{61.00}\end{array}$ & $\begin{array}{c}\overline{0.02} \\
\overline{16.00}\end{array}$ & $\begin{array}{l}* \\
0.02 \\
9.00\end{array}$ & $\begin{array}{r}0.10 \\
0.25 \\
250.00 \\
49.00\end{array}$ & $\begin{array}{l}9.09 \\
0.95\end{array}$ \\
\hline 1959 & $\begin{array}{l}\text { PRECIP (CM.) } \\
\text { SR-90 IMCI/SQ.KM-) } \\
\text { SR-90 CONC. (PC/L) } \\
S R-89 / S R-90\end{array}$ & $\begin{array}{c}* \\
0.11 \\
37.00\end{array}$ & $\begin{array}{l} \pm \\
-- \\
-\end{array}$ & $\begin{array}{l}* \\
0.06 \\
18.00\end{array}$ & $\begin{array}{l}* \\
0.18 \\
5.30\end{array}$ & $\begin{array}{l}-- \\
--\end{array}$ & $\begin{array}{l}* \\
-- \\
--\end{array}$ & $\begin{array}{r}16.00 \\
0.18 \\
1.13 \\
2.40\end{array}$ & $\begin{array}{l}0.20 \\
0.02 \\
10.01 \\
--\end{array}$ & $\begin{array}{r}25.02 \\
0.04 \\
0.16 \\
4.50\end{array}$ & $\begin{array}{l}* \\
-- \\
--\end{array}$ & $\begin{array}{l}4.01 \\
0.05 \\
1.25 \\
+\end{array}$ & $\begin{array}{l}* \\
-- \\
--\end{array}$ & $\begin{array}{r}45.23 \\
0.64\end{array}$ \\
\hline $196 \mathrm{C}$ & $\begin{array}{l}\text { PRECIP }\left(C M_{-}\right) \\
S R-90 \text { IMCI/SQ.KM.) } \\
S R-90 \text { CONC. }(P C / L) \\
S R-89 / S R-90\end{array}$ & $\begin{array}{r}0.41 \\
0.15 \\
36.59 \\
-\end{array}$ & $\begin{array}{l}* \\
0.01 \\
--\end{array}$ & $\begin{array}{l}3.00 \\
0.05 \\
1.67 \\
--\end{array}$ & $\begin{array}{l}* \\
0.01 \\
--\end{array}$ & $\begin{array}{l}* \\
-- \\
--\end{array}$ & $\begin{array}{l}* \\
-- \\
--\end{array}$ & $\begin{array}{l}3.00 \\
0.016 \\
0.34 \\
--\end{array}$ & $\begin{array}{l}0.51 \\
0.00 \mathrm{C} \\
0.01 \\
-.\end{array}$ & $\begin{array}{l}* \\
0.02 C \\
=-\end{array}$ & $\begin{array}{l}* \\
0.02 C \\
--\end{array}$ & $\begin{array}{l}* \\
0.02 C \\
--\end{array}$ & $\begin{array}{l}* \\
0.02 C \\
--\end{array}$ & $\begin{array}{l}6.92 \\
0.31\end{array}$ \\
\hline 1961 & $\begin{array}{l}\text { PRECIP. (CM.) } \\
\text { SR-SO INCI/SO.KM-) } \\
S R-90 \text { CCNC. (PC/L) } \\
S R-89 / S R-90\end{array}$ & $\begin{array}{l}0.10 \\
0.04 \mathrm{C} \\
40.01 \\
--\end{array}$ & $\begin{array}{l}* \\
0.00 C \\
=-\end{array}$ & $\begin{array}{l}* \\
0.04 \\
--\end{array}$ & $\begin{array}{l}0.61 \\
0.02 \\
3.28 \\
-.\end{array}$ & $\begin{array}{l}* \\
* \\
\cdots\end{array}$ & $\begin{array}{l}0.51 \\
-- \\
-- \\
--\end{array}$ & $\begin{array}{l}9.02 \\
-- \\
--\end{array}$ & $\begin{array}{l}5.00 \\
=- \\
=\end{array}$ & $\begin{array}{l}10.01 \\
=- \\
-\end{array}$ & $\begin{array}{l}2.44 \\
-- \\
--\end{array}$ & $\begin{array}{l}0.05 \\
-- \\
--\end{array}$ & $\begin{array}{l}0.66 \\
-- \\
--\end{array}$ & $\begin{array}{r}28.40 \\
0.10\end{array}$ \\
\hline 1962 & $\begin{array}{l}\text { PRECIP. ICM.) } \\
\text { SR-90 IMCI/SQ.KM.) } \\
\text { SR-90 CONC. (PC/L) } \\
\text { SR-89/SR-90 }\end{array}$ & $\overline{--}$ & $=-$ & $=$ & $\begin{array}{l}-- \\
--\end{array}$ & $\ddot{-}$ & $=$ & $\begin{array}{l}-- \\
-- \\
--\end{array}$ & $\begin{array}{l}-- \\
--\end{array}$ & $\ddot{-}$ & $\begin{array}{l}-- \\
-- \\
--\end{array}$ & $\begin{array}{l}-- \\
--\end{array}$ & $\begin{array}{l}-- \\
-- \\
--\end{array}$ & $\begin{array}{l}0.0 \\
0.0\end{array}$ \\
\hline 1963 & $\begin{array}{l}\text { PRECIP. (CM.) } \\
\text { SR-90 (PCI/SQ.KM.) } \\
S R-90 \text { CONC. }(P C / L) \\
S R-89 / S R-90\end{array}$ & $\begin{array}{l}* \\
0.04 \\
18.00\end{array}$ & $\ddot{--}$ & $\frac{0.06}{11.00}$ & $\begin{array}{r}2.54 \\
0.15 \\
5.91 \\
11.00\end{array}$ & $\overrightarrow{0.07}$ & $\begin{array}{l}-- \\
--\end{array}$ & $\begin{array}{l}-- \\
--\end{array}$ & 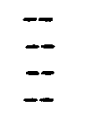 & $=-$ & $\begin{array}{l}-- \\
=- \\
--\end{array}$ & $\begin{array}{l}-- \\
--\end{array}$ & $\begin{array}{l}m \\
m \\
m\end{array}$ & $\begin{array}{l}2.54 \\
0.32\end{array}$ \\
\hline 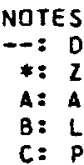 & $\begin{array}{l}\text { ATA NOT AVAILABLE } \\
\text { ERO OR TRACE } \\
\text { PPROXIMATE } \\
\text { OWER LIMIT OF REPOR } \\
\text { ROPORTIONED FROM OR }\end{array}$ & $\begin{array}{l}\text { OATA } \\
\text { NALLLY }\end{array}$ & & & & & & & & & & & & \\
\hline
\end{tabular}




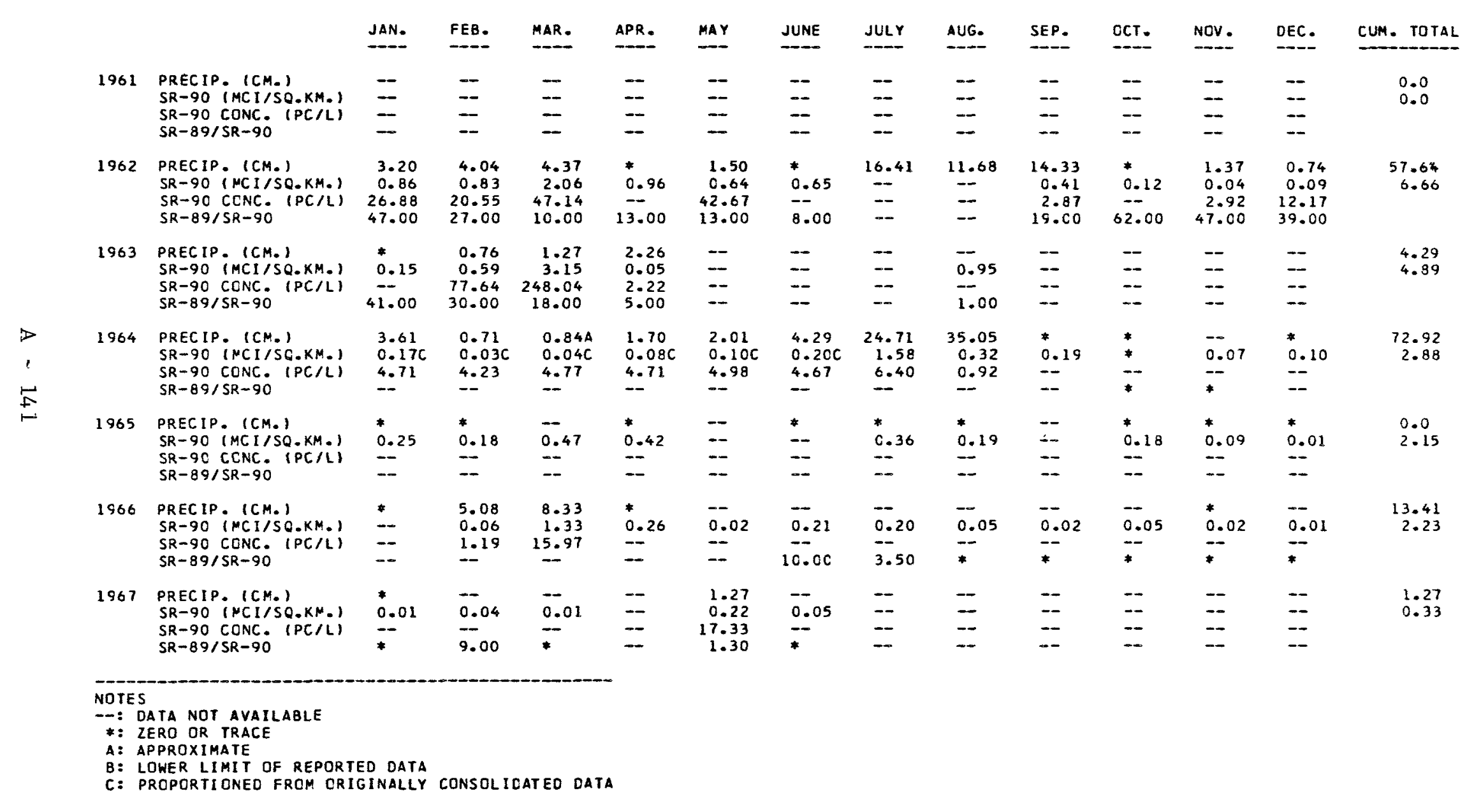


MONTHLY FALLOUT DEPOSITION COLLECTIONS

SITE: PAKISTAN, RAWALPINDI

LAT. 33 4ON LONG. 73 OE ALT. OM. (COLUMN)

SOURCE: ATOMIC ENERGY CENTRE (PINSTECH SITE)

\begin{tabular}{|c|c|c|c|c|c|c|c|c|c|c|c|c|c|c|}
\hline & & JAN. & FEB. & MAR. & APR : & MAY & JUNE & JULY & AUG. & SEP. & OCT. & NOV. & DEC. & CUM. TOTAL \\
\hline 1964 & $\begin{array}{l}\text { PRECIP. (CM.) } \\
\text { SR-90 IMCI/SQ.KM.) } \\
\text { SR-90 CONC. }(P C / L) \\
\text { SR-89/SR-90 }\end{array}$ & $\bar{m}$ & $=$ & $=$ & $=$ & $=$ & 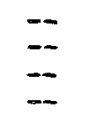 & $\begin{array}{l}= \\
=-\end{array}$ & $\begin{array}{l}-- \\
--\end{array}$ & $=-$ & $\begin{array}{l}=- \\
=- \\
=-\end{array}$ & $\begin{array}{l}-- \\
-- \\
--\end{array}$ & $\begin{array}{c}0.91 \\
0.10 \\
10.99 \\
--\end{array}$ & $\begin{array}{l}0.91 \\
0.10\end{array}$ \\
\hline 1965 & $\begin{array}{l}\text { PRECIP. }\left(C M_{-}\right) \\
\text {SR-9O (NCI/SQ.KM.) } \\
\text { SR-9O CONC. }(P C / L) \\
S R-89 / S R-90\end{array}$ & $\begin{array}{l}4.32 \\
0.09 \\
2.09 \\
-0\end{array}$ & $\begin{array}{l}11.25 \\
1.35 \\
12.00 \\
-=\end{array}$ & $\begin{array}{r}12.45 \\
1.61 \\
12.94 \\
--\end{array}$ & $\begin{array}{l}19.23 \\
1.20 \\
6.25 \\
=-\end{array}$ & $\begin{array}{r}12.55 \\
0.76 \\
6.06 \\
--\end{array}$ & $\begin{array}{r}2.03 \\
0.69 \\
34.00 \\
-.-\end{array}$ & $\begin{array}{r}17.65 \\
0.52 \\
2.95 \\
--\end{array}$ & $\begin{array}{r}15.88 \\
0.59 \\
3.72 \\
--\end{array}$ & 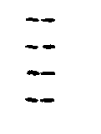 & $\begin{array}{l}-- \\
C .08 \\
--\end{array}$ & $\begin{array}{l}1.96 \\
0.04 \\
2.05 \\
-.\end{array}$ & $\begin{array}{l}1.12 \\
0.17 \\
15.18 \\
--\end{array}$ & $\begin{array}{r}98.44 \\
7.10\end{array}$ \\
\hline 点 & $\begin{array}{l}\text { PRECIP. (CM.) } \\
\text { SR-90 IMCI/SO.KM.) } \\
S R-90 \text { CONC. }(P C / L) \\
S R-89 / S R-90\end{array}$ & $\begin{array}{l}* \\
0.04 \\
--\end{array}$ & $\begin{array}{l}15.75 \\
0.61 \\
3.88 \\
--\end{array}$ & $\begin{array}{l}-- \\
0.29 \\
-- \\
-\infty\end{array}$ & $\begin{array}{r}4.27 \\
0.45 \\
10.54 \\
--\end{array}$ & $\begin{array}{r}5.08 \\
0.28 \\
5.52 \\
21.00\end{array}$ & $\begin{array}{l}-. \\
0.29 \\
0.10\end{array}$ & $\begin{array}{l}-\overline{0} \\
\bar{c}-14 \mathrm{C} \\
\end{array}$ & $\begin{array}{l}-\overline{0.150} \\
--\end{array}$ & $\begin{array}{l}-\infty \\
0.13 \\
*\end{array}$ & $\begin{array}{l}-- \\
\mathrm{C} .08 \\
*\end{array}$ & $\begin{array}{l}-- \\
0.02 \\
*\end{array}$ & $\begin{array}{l}-- \\
0.06 \\
+\end{array}$ & $\begin{array}{r}25.10 \\
2.54\end{array}$ \\
\hline 1967 & $\begin{array}{l}\text { PRECIP. (CM.) } \\
\text { SR-90 IMCI/SQ.KM.) } \\
\text { SR-90 CONC. (PC/L) } \\
\text { SR-89/SR-90 }\end{array}$ & $\begin{array}{l}-0.02 \\
*\end{array}$ & $\begin{array}{r}3.81 \\
0.19 \\
4.99 \\
11.40\end{array}$ & $\begin{array}{l}-. \\
0.17 \\
-8.80\end{array}$ & $\begin{array}{l}0.06 \\
5.90\end{array}$ & $\begin{array}{l}-.10 \\
0.10 \\
3.20\end{array}$ & +0.02 & $=-$ & $\begin{array}{l}=- \\
=-\end{array}$ & $\begin{array}{l}-- \\
--\end{array}$ & $=$ & $m$ & $\begin{array}{l}-- \\
-- \\
--\end{array}$ & $\begin{array}{l}3.81 \\
0.56\end{array}$ \\
\hline $\begin{array}{l}\text { NOTES } \\
-=: \\
*: 2 \\
A: \\
8: 1 \\
C:\end{array}$ & $\begin{array}{l}\text { S } \\
\text { DATA NOT AVAILABLE } \\
\text { ZERO OR TRACE } \\
\text { APPROXIMATE } \\
\text { LOWER LIMIT OF REPOR } \\
\text { PROPORTIONEO FROM CR }\end{array}$ & $\begin{array}{l}\text { D DATA } \\
\text { INALLY }\end{array}$ & NSOL & FO & & & & & & & & & & \\
\hline
\end{tabular}




\begin{tabular}{|c|c|c|c|c|c|c|c|c|c|c|c|c|c|c|}
\hline & & JAN. & FEB: & MAR: & APR . & $\operatorname{miY}$ & JUNE & JuL & AUG. & SEP. & OCT. & Nov. & DEC. & CUM. TOTAL \\
\hline 1959 & $\begin{array}{l}\text { PRECIP (CM.) } \\
S R-90 \text { IMCI/SQ.KMG) } \\
S R-90 \text { CONC. }(P C / L) \\
S R-89 / S R-90\end{array}$ & $\begin{array}{l}=- \\
= \\
=\infty\end{array}$ & $\stackrel{-}{=}$ & $\underset{-\infty}{m}$ & $\begin{array}{l}-- \\
+ \\
+\end{array}$ & 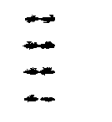 & $=$ & $\ddot{m}$ & $=-$ & $\begin{array}{r}11.05 \\
0.03 \\
0.28 \\
*\end{array}$ & $\begin{array}{l}48.01 \\
0.02 \\
0.05 \\
*\end{array}$ & $\begin{array}{r}23.50 \\
0.03 \\
0.13 \\
0.90\end{array}$ & $\begin{array}{r}20.75 \\
0.07 \\
0.34 \\
1.20\end{array}$ & $\begin{array}{r}103.31 \\
0.15\end{array}$ \\
\hline 1960 & $\begin{array}{l}\text { PRECIP. (CM.) } \\
S R-90 \text { INCI/SGAKM) } \\
S R=90 \text { CONC. (PE/K) } \\
S R-89 / 5 R-90\end{array}$ & $\begin{array}{l}8.46 \\
0.05 \\
0.60 \\
--\end{array}$ & $\begin{array}{l}2.90 \\
0.08 \\
2.76 \\
--\end{array}$ & $\begin{array}{l}2.01 \\
0.07 \\
3.49 \\
\cdots\end{array}$ & $\begin{array}{l}21.01 \\
0.19 \\
0.91 \\
-1\end{array}$ & $\begin{array}{l}22.63 \\
0.64 C \\
0.18 \\
=\end{array}$ & $\begin{array}{l}17.01 \\
0.036 \\
0.17 \\
--\end{array}$ & $\begin{array}{l}16.33 \\
6.01 \\
0.07 \\
-.0\end{array}$ & $\begin{array}{l}17.40 \\
0.01 \\
0.06 \\
=.\end{array}$ & $\begin{array}{l}18.47 \\
0.036 \\
0.17 \\
=\end{array}$ & $\begin{array}{l}19.08 \\
0.03 C \\
0.16 \\
=-\end{array}$ & $\begin{array}{l}38.58 \\
0.02 C \\
0.06 \\
\ldots\end{array}$ & $\begin{array}{l}25.91 \\
0.01 C \\
0.04 \\
--\end{array}$ & $\begin{array}{r}210.59 \\
0.57\end{array}$ \\
\hline 1961 & 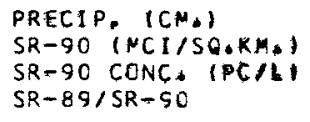 & $\begin{array}{l}0.48 \\
0.016 \\
2.09 \\
=.\end{array}$ & $\begin{array}{l}2.18 \\
0.03 C \\
1.38 \\
=-\end{array}$ & $\begin{array}{l}* \\
=- \\
--\end{array}$ & $\begin{array}{l}4.55 \\
0.06 \\
1.32 \\
+-\end{array}$ & $\begin{array}{l}10.64 \\
0.03 C \\
0.29 \\
--\end{array}$ & $\begin{array}{l}26.19 \\
0.07 C \\
0.27 \\
-.\end{array}$ & $\begin{array}{c}25.22 \\
0.016 \\
0.04 \\
--\end{array}$ & $\begin{array}{c}15.01 \\
0.00 \mathrm{C} \\
0.01 \\
-\end{array}$ & $\begin{array}{r}18.12 \\
0.00 \\
0.01 \\
14.00\end{array}$ & $\begin{array}{r}27.76 \\
C .00 \\
C .01 \\
46.00\end{array}$ & $\begin{array}{r}30.28 \\
0.10 \\
0.34 \\
102.00\end{array}$ & $\begin{array}{r}10.57 \\
0.11 \\
1.05 \\
56.00\end{array}$ & $\begin{array}{r}170.99 \\
0.42\end{array}$ \\
\hline 1962 & $\begin{array}{l}P R E C 1 P \text { (CHA) } \\
S R-9 C \text { (MCI/SO+KM.) } \\
S R-9 C \text { CONC, }(P C / L) \\
S R-89 / S R=S O\end{array}$ & $\begin{array}{r}2.62 \\
0.16 \\
6.11 \\
41.00\end{array}$ & $\begin{array}{l}* \\
* \\
-- \\
--\end{array}$ & $\begin{array}{r}0.08 \\
0.04 \\
50.01 \\
22.00\end{array}$ & $\begin{array}{l}8.92 \\
= \\
= \\
=\end{array}$ & $\begin{array}{l}20.04 \\
+- \\
+-\end{array}$ & $\begin{array}{r}22.53 \\
0.73 \\
3.25 \\
34.0 \mathrm{C}\end{array}$ & $\begin{array}{l}20.09 \\
\therefore \\
=\end{array}$ & $\begin{array}{r}20.55 \\
0.59 \\
2.88 \\
29.00\end{array}$ & $\begin{array}{r}17.91 \\
0.12 \\
0.68 \\
22.00\end{array}$ & $\begin{array}{r}38.68 \\
0.11 \\
0.29 \\
18.00\end{array}$ & $\begin{array}{r}23.32 \\
0.18 \\
0.78 \\
46.00\end{array}$ & $\begin{array}{r}17.27 \\
0.22 \\
1.28 \\
39.00\end{array}$ & $\begin{array}{r}192.01 \\
2.15\end{array}$ \\
\hline 1963 & 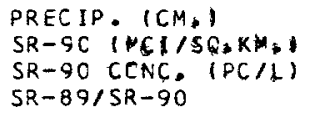 & $\begin{array}{r}5.87 \\
C .14 \\
2.39 \\
50.00\end{array}$ & $\begin{array}{r}0.27 \\
0.14 \\
2.24 \\
20.00\end{array}$ & $\begin{array}{c}* \\
0.03 \\
15.00\end{array}$ & $\begin{array}{r}12.29 \\
0.80 \\
7.00 \\
9.00\end{array}$ & $\begin{array}{r}41.33 \\
1.59 \\
3.85 \\
10.00\end{array}$ & $\begin{array}{r}19.74 \\
0.58 \\
2.94 \\
*\end{array}$ & $\begin{array}{r}26.09 \\
c .37 \\
1.85 \\
1.00\end{array}$ & $\begin{array}{r}2.77 \\
0.32 \\
11.56 \\
1.00\end{array}$ & $\begin{array}{l}14.02 \\
0.11 \\
0.79 \\
*\end{array}$ & $\begin{array}{r}21.64 \\
C .11 \\
0.51 \\
2.30\end{array}$ & $\begin{array}{l}26.82 \\
0.57 \\
2.13 \\
*\end{array}$ & $\begin{array}{l}2.79 \\
0.10 \\
3.59 \\
+\end{array}$ & $\begin{array}{r}173.63 \\
4.92\end{array}$ \\
\hline 1964 & $\begin{array}{l}\text { PRECIP (CME) } \\
\text { SR-90 (NCI/SG.KM.) } \\
S R-90 \text { CONC, (PCIL) } \\
S R-89 / S R=90\end{array}$ & $\begin{array}{l}0.84 \\
C .08 \\
9.53 \\
4\end{array}$ & $\begin{array}{l}3.40 \\
0.17 \\
5.01 \\
*\end{array}$ & $\begin{array}{l}0.51 \\
0.03 \\
5.89 \\
+\end{array}$ & $\begin{array}{r}14.63 \\
0.24 \\
1.65 \\
2.10\end{array}$ & $\begin{array}{r}17.86 \\
0.34 \\
1.91 \\
4\end{array}$ & $\begin{array}{l}17.40 \\
0.04 \\
0.23 \\
-=\end{array}$ & $\begin{array}{l}15.06 \\
0.17 \\
1.13 \\
=\end{array}$ & $\begin{array}{l}22.33 \\
0.15 \\
0.68 \\
=-\end{array}$ & $\begin{array}{l}21.26 \\
0.13 \\
0.62 \\
-.\end{array}$ & $\begin{array}{l}* \\
0.05 \\
- \\
*\end{array}$ & $\begin{array}{l}29.46 \\
0.09 \\
0.31 \\
4\end{array}$ & $\begin{array}{l}7.70 \\
0.09 \\
1.17 \\
-.\end{array}$ & $\begin{array}{r}150.45 \\
1.58\end{array}$ \\
\hline 1965 & $\begin{array}{l}\text { PRECIP }(C M .) \\
\text { SR-GC }(M C I / S Q, K M) \\
S R-9 O C C N C .(P C / L) \\
\text { SR-89/SR-90 }\end{array}$ & $\begin{array}{l}1.57 \\
+- \\
=-\end{array}$ & $\begin{array}{l}0.97 \\
0.04 \\
4.13 \\
--\end{array}$ & $\begin{array}{l}* \\
0.00 \\
--\end{array}$ & 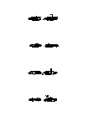 & $\begin{array}{l}29.16 \\
C .12 \\
0.42 \\
--\end{array}$ & $\begin{array}{l}16.61 \\
0.70 \\
4.22 \\
-\end{array}$ & $\begin{array}{l}7.06 \\
0.05 \\
0.71 \\
-\end{array}$ & $\begin{array}{l}18.08 \\
0.07 \\
0.39 \\
--\end{array}$ & $\begin{array}{l}21.13 \\
0.07 \\
0.34 \\
-.\end{array}$ & $\begin{array}{l}21.41 \\
0.05 \\
0.24 \\
=-\end{array}$ & $\begin{array}{l}34.04 \\
0.04 \\
0.12 \\
-.\end{array}$ & $\begin{array}{l}8.71 \\
0.21 \\
2.42 \\
-.\end{array}$ & $\begin{array}{r}158.74 \\
1.35\end{array}$ \\
\hline 1966 & $\begin{array}{l}\text { PRECIP. }(C M \cdot) \\
S R-90 \text { (NCI/SQ,KH, } \\
S R-90 \text { CQNC. }(P C / L) \\
S R-89 / S R-90\end{array}$ & $\begin{array}{l}6.25 \\
0.06 \\
0.97 \\
=\end{array}$ & $\begin{array}{l}* \\
* \\
-- \\
--\end{array}$ & $\begin{array}{r}0.15 \\
0.04 \\
26.67 \\
-.\end{array}$ & $\begin{array}{l}8.53 \\
0.27 \\
3.17 \\
--\end{array}$ & $\begin{array}{r}33.22 \\
0.09 \\
0.28 \\
-.\end{array}$ & $\begin{array}{c}33.25 \\
+ \\
*\end{array}$ & $\begin{array}{r}24.33 \\
C .02 \\
0.09 \\
14.00\end{array}$ & $\begin{array}{l}18.54 \\
* \\
*\end{array}$ & $\begin{array}{l}21.82 \\
+ \\
-\end{array}$ & $\begin{array}{r}43.33 \\
0.02 \\
0.05 \\
20.00\end{array}$ & $\begin{array}{r}27.20 \\
0.04 \\
0.15 \\
8.60\end{array}$ & $\begin{array}{l}14.45 \\
0.03 \\
0.21 \\
*\end{array}$ & $\begin{array}{r}231.07 \\
0.57\end{array}$ \\
\hline 1967 & $\begin{array}{l}\text { PRECIP. }(C N .) \\
S R-90\left(N C I / S Q_{-K M}\right) \\
S R-90 \text { CONC. }(P C / L) \\
S R-89 / S R-90\end{array}$ & $\begin{array}{l}1.40 \\
0.02 \\
1.43 \\
4\end{array}$ & $\begin{array}{l}0.58 \\
0.02 \\
3.45 \\
*\end{array}$ & $\begin{array}{l}0.71 \\
0.02 \\
2.82 \\
+\end{array}$ & $\begin{array}{r}19.08 \\
0.07 \\
0.37 \\
2.60\end{array}$ & $\begin{array}{r}14.22 \\
0.04 \\
0.29 \\
*\end{array}$ & $\begin{array}{r}15.54 \\
0.01 \\
0.07 \\
*\end{array}$ & $\begin{array}{r}21.92 \\
0.06 \\
0.28 \\
8.00\end{array}$ & $\begin{array}{l}-- \\
-- \\
--\end{array}$ & $\begin{array}{l}-- \\
-\infty \\
-\end{array}$ & $\begin{array}{l}-- \\
=-\end{array}$ & $\begin{array}{l}-- \\
-- \\
--\end{array}$ & $\begin{array}{l}-- \\
-- \\
--\end{array}$ & $\begin{array}{r}73.45 \\
0.24\end{array}$ \\
\hline
\end{tabular}




\begin{tabular}{|c|c|c|c|c|c|c|c|c|c|c|c|c|c|c|}
\hline & & JAN. & FEB. & MAR & APR. & MAY & JUNE & JuL & AUG. & SEP. & OCI . & NOX. & DEC. & CUM. TOIAL \\
\hline 1959 & $\begin{array}{l}\text { PRECIP. }\left(C M_{-}\right) \\
\text {SR-9O INCI/SQ.KM.) } \\
\text { SR-90 CONC. }(P C / L) \\
\text { SR-89/SR-90 }\end{array}$ & $\begin{array}{l}-- \\
= \\
--\end{array}$ & $=$ & $\overline{-}$ & $=$ & $=$ & $\begin{array}{l}-- \\
--\end{array}$ & $\begin{array}{l}-- \\
--\end{array}$ & $\begin{array}{l}-- \\
-\infty\end{array}$ & $\begin{array}{l}0.41 \\
-- \\
=-\end{array}$ & $\begin{array}{l}2.01 \\
0.01 \\
0.50 \\
*\end{array}$ & $\begin{array}{l}0.15 \\
-- \\
-\end{array}$ & $\begin{array}{r}0.20 \\
0.05 \\
25.01 \\
*\end{array}$ & $\begin{array}{l}2.77 \\
0.06\end{array}$ \\
\hline 1960 & $\begin{array}{l}\text { PRECIP. (CM.) } \\
\text { SR-9O (MCI/SQ.KM.) } \\
S R-9 C \text { CONC. }(P C / L) \\
S R-99 / S R-9 O\end{array}$ & $\begin{array}{l}* \\
0.01 \\
-- \\
--\end{array}$ & $\stackrel{-}{--}$ & $\stackrel{*}{--}$ & $\begin{array}{l}* \\
0.03 \\
--\end{array}$ & $\begin{array}{l}0.25 \\
0.03 \mathrm{C} \\
12.01 \\
--\end{array}$ & $\begin{array}{l}0.51 \\
0.06 \mathrm{C} \\
11.77 \\
-\end{array}$ & $\begin{array}{l}0.51 \\
-- \\
--\end{array}$ & $\begin{array}{l}0.99 \\
= \\
=\end{array}$ & $\begin{array}{l}5.99 \\
0.05 \\
0.84 \\
--\end{array}$ & $\begin{array}{l}0.61 \\
-\end{array}$ & $\begin{array}{l}0.20 \\
0.036 \\
15.01 \\
--\end{array}$ & $\begin{array}{l}* \\
0.000 \\
=-\end{array}$ & $\begin{array}{l}9.06 \\
0.21\end{array}$ \\
\hline 1961 & $\begin{array}{l}\text { PRECIP. (CM.) } \\
S R-90 \text { (NCI/SQ.KM.) } \\
S R-9 C \text { CCNC. (PC/L) } \\
S R-89 / S R-90\end{array}$ & $\begin{array}{l}* \\
0.01 \mathrm{C} \\
--\end{array}$ & $\begin{array}{l}* \\
0.01 C \\
=\end{array}$ & $\begin{array}{l}* \\
0.04 C \\
--\end{array}$ & $\begin{array}{l}* \\
0.04 C \\
--\end{array}$ & $\begin{array}{l}0.03 C \\
--\end{array}$ & $\begin{array}{l}* \\
0.03 C \\
=\end{array}$ & $\begin{array}{l}0.99 \\
* \\
-- \\
--\end{array}$ & $\begin{array}{l}0.99 \\
* \\
--\end{array}$ & $\begin{array}{l}0.99 \\
* \\
-- \\
*\end{array}$ & $\begin{array}{l}0.30 \\
0.02 \\
6.67 \\
+\end{array}$ & $\begin{array}{l}* \\
0.02 \\
-- \\
*\end{array}$ & $\begin{array}{l}* \\
* \\
--\end{array}$ & $\begin{array}{l}3.27 \\
0.20\end{array}$ \\
\hline 1962 & $\begin{array}{l}\text { PRECIP. (CM-) } \\
S R-90 \text { (NCI/SQ.KM-) } \\
S R-90 \text { CCNC. }(P C / L) \\
S R-8 S / S R-S O\end{array}$ & $\stackrel{*}{*}$ & $\begin{array}{l}* \\
* \\
*\end{array}$ & $\begin{array}{l}* \\
* \\
-1.70\end{array}$ & * & $\begin{array}{l}* \\
0.01 \\
-\overline{40.00}\end{array}$ & $\begin{array}{l}\frac{1}{0.03} \\
-- \\
45.00\end{array}$ & $\begin{array}{l}0.51 \\
0.03 \\
5.89 \\
5.00\end{array}$ & $\begin{array}{r}0.05 \\
0.10 \\
200.00 \\
10.00\end{array}$ & $\begin{array}{r}0.25 \\
0.14 \\
56.01 \\
27.00\end{array}$ & $\begin{array}{l}-. \\
0.04 \\
9.00\end{array}$ & $\begin{array}{l}-. \\
0.03 \\
14.00\end{array}$ & $\begin{array}{l}0.25 \\
0.02 \\
8.01 \\
2.00\end{array}$ & $\begin{array}{l}1.06 \\
0.40\end{array}$ \\
\hline 1963 & $\begin{array}{l}\text { PRECIP. (CM.) } \\
\text { SR-9O (NCI/SG.KM.) } \\
S R-90 \text { CONC. }(P C / L) \\
\text { SR-89/SR-90 }\end{array}$ & $\overline{-}$ & $\frac{0.01}{0}$ & $\begin{array}{l}* \\
0.00 \\
-- \\
--\end{array}$ & $\begin{array}{l}. \\
0.01 \\
3.00\end{array}$ & $\begin{array}{l}* \\
0.00 \\
--\end{array}$ & $\begin{array}{r}0.03 \\
0.02 \\
66.07 \\
4.60\end{array}$ & $\begin{array}{l}* \\
0.01 \\
-\infty\end{array}$ & $\begin{array}{l}0.10 \\
0.06 \\
60.01 \\
*\end{array}$ & $\begin{array}{r}0.08 \\
0.04 \\
50.01 \\
*\end{array}$ & $\begin{array}{r}0.05 \\
0.07 A \\
140.00 \\
\end{array}$ & $\begin{array}{l}* \\
0.06 \mathrm{~A} \\
=\end{array}$ & $\begin{array}{l}\neq \\
0.044 \\
=-\end{array}$ & $\begin{array}{l}0.26 \\
0.32\end{array}$ \\
\hline 1964 & $\begin{array}{l}\text { PRECIP. }\left(C M_{-}\right) \\
\text {SR-9O }\left(M C I / S Q . K M_{-}\right) \\
S R-9 O C O N C . \quad(P C / L) \\
S R-89 / S R-S O\end{array}$ & $\begin{array}{c}0.03 \\
0.044 \\
133.34 \\
-\end{array}$ & $\begin{array}{l}0.03 \\
0.02 \mathrm{~A} \\
66.67 \\
-\end{array}$ & $\stackrel{+}{*}$ & $\begin{array}{l}* \\
0.02 \\
--\end{array}$ & $\begin{array}{r}0.05 \\
0.02 \\
40.01 \\
--\end{array}$ & $\begin{array}{l}0.10 \\
0.03 \\
30.01 \\
-\end{array}$ & $\begin{array}{l}0.81 \\
0.05 \\
6.18 \\
-\end{array}$ & $\begin{array}{l}0.97 \\
=\end{array}$ & $\stackrel{*}{--}$ & $\begin{array}{l}* \\
C .08 \\
--\end{array}$ & $\frac{+}{0.05}$ & $\begin{array}{l}* \\
0.04 \\
--\end{array}$ & $\begin{array}{l}1.99 \\
0.35\end{array}$ \\
\hline 1965 & $\begin{array}{l}\text { PRECIP. (CM.) } \\
S R-90 \text { IMCI/SQ.KM) } \\
S R-90 \text { CONC. }(P C / L) \\
S R-89 / S R-90\end{array}$ & $=$ & $=$ & $\begin{array}{l}- \\
0.02 \\
-\end{array}$ & $\begin{array}{l}-- \\
0.026 \\
--\end{array}$ & $\begin{array}{l}-0.02 C \\
=- \\
=\end{array}$ & $\begin{array}{l}-- \\
0.0 .10 \\
=-\end{array}$ & $\begin{array}{l}* \\
= \\
=\end{array}$ & $\begin{array}{l}* \\
* \\
-\end{array}$ & $\begin{array}{r}0.36 \\
0.13 \\
36.12 \\
\end{array}$ & $\frac{-05}{-}$ & $\overline{0.04}$ & $\begin{array}{l}-- \\
0.15 \\
=-\end{array}$ & $\begin{array}{l}0.36 \\
0.51\end{array}$ \\
\hline 1966 & $\begin{array}{l}\text { PRECIP. }\left(C M_{-}\right) \\
\text {SR-90 (NCI/SQ.KM.) } \\
S R-90 \text { CONC. }(P C / L) \\
S R-89 / 5 R-90\end{array}$ & $\overline{0.03}$ & $\begin{array}{l}* \\
* \\
--\end{array}$ & $\begin{array}{l}-0.01 \\
*\end{array}$ & $\frac{-}{*}$ & $\ddot{-}$ & $\begin{array}{l}- \\
0=02 \\
+\end{array}$ & $\begin{array}{l}- \\
0.01 \\
-\infty\end{array}$ & $\begin{array}{l}- \\
0.016\end{array}$ & $\frac{\overline{0.011}}{12.00}$ & $\frac{0.04}{1}$ & $\frac{\overline{0.05}}{22.00}$ & $\begin{array}{l}\overline{0.02} \\
\overline{16.90}\end{array}$ & $\begin{array}{l}0.0 \\
0.20\end{array}$ \\
\hline 1967 & $\begin{array}{l}\text { PRECIP. }\left(C M_{-}\right) \\
S R-90 \text { (MCI/SQ.KM.) } \\
\text { SR-90 CONC. (PC/L) } \\
S R-89 / S R-90\end{array}$ & $\begin{array}{l}-- \\
0.02 \\
-- \\
8.60\end{array}$ & $=$ & $\bar{m}$ & $\begin{array}{l}-- \\
0.01 \\
-\end{array}$ & $\begin{array}{l}-- \\
-- \\
--\end{array}$ & $\begin{array}{l}-- \\
-- \\
--\end{array}$ & $\begin{array}{l}-- \\
-- \\
-\end{array}$ & $\begin{array}{l}- \\
-- \\
-\end{array}$ & $\begin{array}{l}=- \\
=- \\
=\end{array}$ & $\begin{array}{l}- \\
-- \\
--\end{array}$ & $\begin{array}{l}- \\
-- \\
-\end{array}$ & $\begin{array}{l}-- \\
-- \\
--\end{array}$ & $\begin{array}{l}0.0 \\
0.03\end{array}$ \\
\hline \multicolumn{15}{|c|}{$\begin{array}{l}\text { NOTES } \\
\text { :- DATA NOT AVAILABLE } \\
\text { *: ZERO OR TRACE } \\
\text { A: APPROXIMATE } \\
\text { B: LOWER LIMIT OF REPORTED DATA } \\
\text { C: PROPCRTIONED FRCM CRIGINALLY }\end{array}$} \\
\hline
\end{tabular}


mathar fallout derosition coLlections

SITE= MEaun, LIm

Lat. 12 is Lowc. 77 Ty MT.

30R. (COLUMM) SLRFACE AIR SAMPLIMG STATION

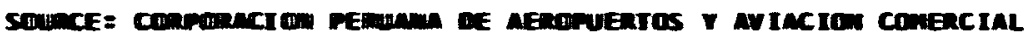

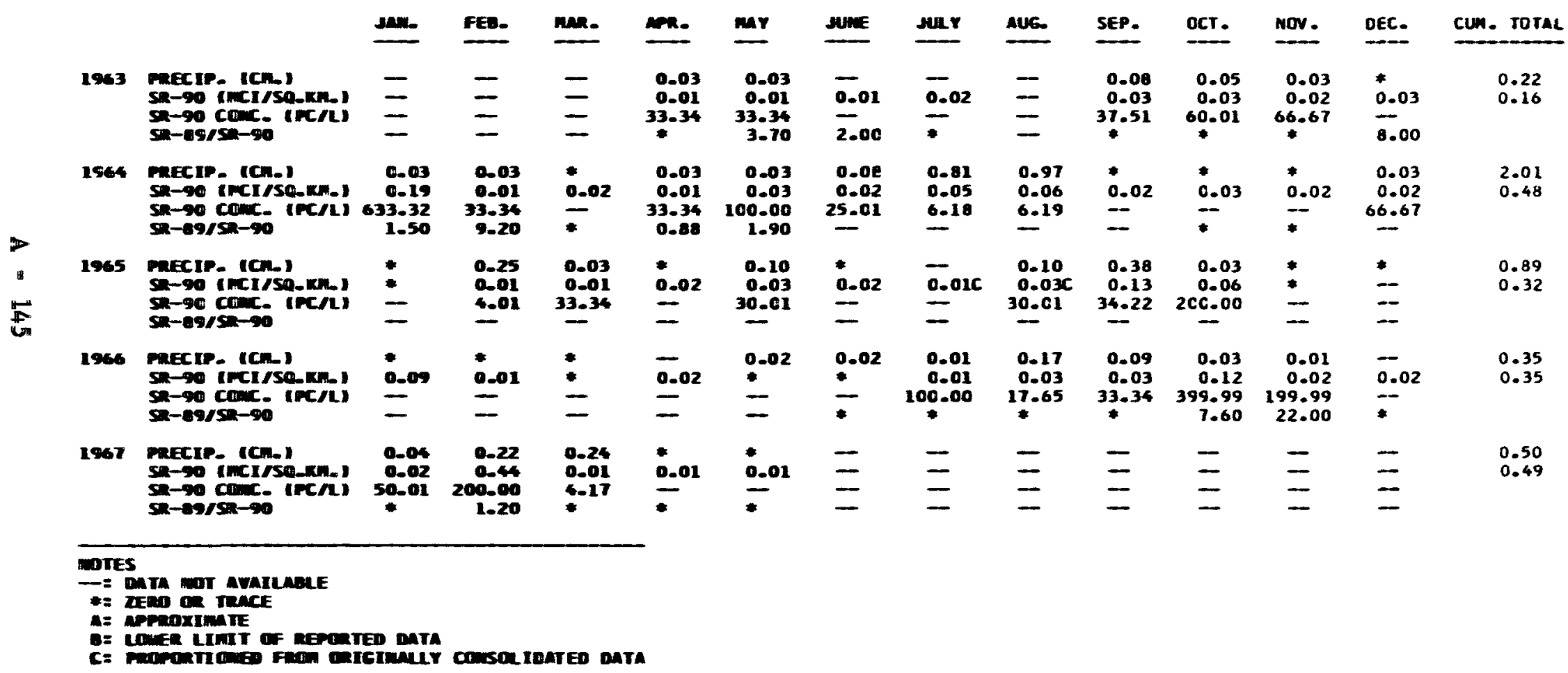


MONTHLY FALLOUT DEPOSITION COLLECTIONS

SITE: PHILIPPINE I., CEBU CITY

LAT. $1020 \mathrm{~N}$ LONG.123 S4E ALT.

34M. (COLUMM)

SOURCE: PHILIPPINES ATOMIC ENERGY COMMISSION, NATIONAL SCIENCE DEVELOPMENT BOARD

\begin{tabular}{|c|c|c|c|c|c|c|c|c|c|c|c|c|c|c|}
\hline & & JAN. & FEB. & MAR. & APR: & MAY & JUNE & JULY & AUG. & SEP. & OCT. & Nov. & DEC . & CUM. TOTAL \\
\hline 1961 & $\begin{array}{l}\text { PRECIP. }\left(C M_{-}\right) \\
\text {SR-90 (NCI/SQ.KM. }) \\
S R-90 \text { CCNC. }(P C / L) \\
\text { SR-89/SR-90 }\end{array}$ & $\begin{array}{l}-- \\
m \\
--\end{array}$ & $\begin{array}{l}- \\
- \\
-\end{array}$ & $=$ & $\begin{array}{l}7.01 \\
0.14 \\
2.00 \\
-0\end{array}$ & $\begin{array}{l}12.78 \\
* \\
--\end{array}$ & $\begin{array}{c}16.43 \\
- \\
-\end{array}$ & $\begin{array}{l}25.35 \\
* \\
=-\end{array}$ & $\begin{array}{l}7.52 \\
* \\
--\end{array}$ & $\begin{array}{l}=- \\
= \\
-\end{array}$ & $\begin{array}{r}26.11 \\
0.01 \\
0.04 \\
38.00\end{array}$ & $\begin{array}{l}11.33 \\
+\infty \\
--\end{array}$ & $\begin{array}{l}10.24 \\
0.06 \\
0.59 \\
-.\end{array}$ & $\begin{array}{r}116.77 \\
0.21\end{array}$ \\
\hline 1962 & $\begin{array}{l}\text { PRECIP, (CM.) } \\
\text { SR-9O IMCI/SQ.KM-) } \\
S R-9 O \text { CONC. }(P C / L) \\
S R-89 / S R-90\end{array}$ & $\begin{array}{l}5.28 \\
+ \\
=- \\
=-\end{array}$ & $\begin{array}{r}18.08 \\
0.58 \\
3.21 \\
30.00\end{array}$ & $\begin{array}{r}8.28 \\
0.03 \\
0.37 \\
25.00\end{array}$ & $\begin{array}{r}3.20 \\
0.06 \\
1.88 \\
15.00\end{array}$ & $\begin{array}{l}8.31 \\
0.11 \\
1.33 \\
4.00\end{array}$ & $\begin{array}{r}17.68 \\
0.11 \\
0.63 \\
15.00\end{array}$ & $\begin{array}{r}21.08 \\
0.18 \\
0.86 \\
37.00\end{array}$ & $\begin{array}{l}33.10 \\
=- \\
=-\end{array}$ & $\begin{array}{l}13.97 \\
0.00 \\
0.01 \\
+\end{array}$ & $\begin{array}{r}11.20 \\
0.03 \\
0.27 \\
22.00\end{array}$ & $\begin{array}{r}13.87 \\
0.05 \\
0.37 \\
46.00\end{array}$ & $\begin{array}{l}6.71 \\
0.07 \\
1.05 \\
40.00\end{array}$ & $\begin{array}{r}160.76 \\
1.22\end{array}$ \\
\hline 1963 & $\begin{array}{l}\text { PREC IP }(C M-) \\
S R-90 \text { IMCI/SQ.KM.) } \\
S R-90 \text { CONC. }(P C / L) \\
S R-89 / S R=90\end{array}$ & $\begin{array}{r}6.91 \\
0.04 \\
0.58 \\
22.00\end{array}$ & $\begin{array}{r}2.59 \\
0.10 \\
3.87 \\
15.00\end{array}$ & $\begin{array}{r}7.85 \\
0.11 \\
1.41 \\
15.00\end{array}$ & $\begin{array}{r}8.86 \\
0.24 \\
2.71 \\
15.00\end{array}$ & $\begin{array}{l}8.13 \\
0.37 \\
4.56 \\
1.50\end{array}$ & $\begin{array}{r}12.88 \\
0.29 \\
2.26 \\
*\end{array}$ & $\begin{array}{r}25.32 \\
0.30 \\
1.19 \\
1.00\end{array}$ & $\begin{array}{r}22.00 \\
0.12 \\
0.55 \\
\$\end{array}$ & $\begin{array}{l}23.85 \\
0.02 \\
0.09 \\
*\end{array}$ & $\begin{array}{r}21.06 \\
0.19 \\
0.91 \\
14.00\end{array}$ & $\begin{array}{r}10.13 \\
0.11 \\
1.09 \\
+\end{array}$ & $=-$ & $\begin{array}{r}149.58 \\
1.89\end{array}$ \\
\hline 1964 & $\begin{array}{l}\text { PRECIP. (CM.) } \\
S R-90 \text { (MCI/SQ.KM.) } \\
S R-90 \text { CONC }(P C / L) \\
S R-89 / S R-90\end{array}$ & $=$ & $=$ & 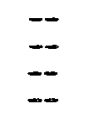 & $-\infty$ & $\begin{array}{l}-\infty \\
-\infty\end{array}$ & $=$ & $=$ & $\begin{array}{l}- \\
-- \\
-\end{array}$ & $\begin{array}{l}-- \\
-- \\
--\end{array}$ & $\ddot{-}$ & $m$ & $\begin{array}{l}-- \\
=- \\
--\end{array}$ & $\begin{array}{l}0.0 \\
0.0\end{array}$ \\
\hline 1965 & $\begin{array}{l}\text { PRECIP. }\left(C M_{0}\right) \\
\text { SR-90 }\left(M C I / S Q_{\left.-K M_{0}\right)}\right) \\
S R-90 \text { CONC. }(P C / L) \\
S R-89 / S R-90\end{array}$ & $=-$ & $=$ & $=$ & $\begin{array}{l}-- \\
-\infty\end{array}$ & $=$ & $m$ & $=$ & $=$ & $\begin{array}{l}-- \\
-- \\
--\end{array}$ & $\begin{array}{l}-- \\
- \\
-\end{array}$ & $=$ & $\begin{array}{l}- \\
= \\
-\end{array}$ & $\begin{array}{l}0.0 \\
0.0\end{array}$ \\
\hline 1966 & $\begin{array}{l}\text { PRECIP. (CM.) } \\
S R-90 \text { (NCI/SQ.KM.) } \\
S R-90 \text { CONC. }(P C / L) \\
S R=89 / S R-90\end{array}$ & $\begin{array}{l}- \\
-\infty\end{array}$ & $\begin{array}{l}-- \\
-- \\
--\end{array}$ & $\overline{-\infty}$ & $=$ & $=$ & $=$ & $\begin{array}{r}36.32 \\
0.04 \\
0.12 \\
+\end{array}$ & $\begin{array}{l}21.48 \\
0.02 \\
0.10 \\
*\end{array}$ & $\begin{array}{r}20.22 \\
0.02 \\
0.10 \\
*\end{array}$ & $\begin{array}{r}14.25 \\
0.03 \\
0.22 \\
*\end{array}$ & $\begin{array}{r}20.90 \\
0.02 \\
0.10 \\
*\end{array}$ & $\begin{array}{l}16.18 \\
0.01 \\
0.07 \\
*\end{array}$ & $\begin{array}{r}129.35 \\
0.14\end{array}$ \\
\hline 1967 & $\begin{array}{l}\text { PRECIP. ICM. } \\
\text { SR-9O IMCI/SQ.KM-) } \\
\text { SR-90 CONC. }(P C / L) \\
\text { SR-89/SR-90 }\end{array}$ & $\begin{array}{l}46.38 \\
0.01 \\
0.03 \\
*\end{array}$ & $\begin{array}{l}7.35 \\
0.01 \\
0.24 \\
+\end{array}$ & $\begin{array}{l}7.78 \\
0.02 \\
0.26 \\
*\end{array}$ & $\begin{array}{l}1.24 \\
0.01 \\
0.81 \\
+\end{array}$ & $\begin{array}{l}5.76 \\
0.01 \\
0.18 \\
*\end{array}$ & $-\infty$ & $\begin{array}{l}=- \\
=- \\
=-\end{array}$ & $=$ & $=$ & $=$ & $=$ & $\begin{array}{l}- \\
-\infty \\
-\infty\end{array}$ & $\begin{array}{r}68.51 \\
0.06\end{array}$ \\
\hline \begin{aligned} NOTES & \\
\hdashline$-: & D \\
A: & Z \\
A: & A \\
B: & L \\
C: & P\end{aligned}$ & $\begin{array}{l}\text { SATA NOT AVAILABLE } \\
\text { ZERO OR TRACE } \\
\text { APPROXIMATE } \\
\text { LOWER LIMIT OF REPOR } \\
\text { PROPORTI ONED FROM OR }\end{array}$ & $\begin{array}{l}\text { DATA } \\
\text { NALLY }\end{array}$ & & $=0$ & & & & & & & & & & \\
\hline
\end{tabular}


MONTHLY FALLOUT DEPOSITION COLLECTIONS

SITE: PHILIPPINE I. .QUEZON CITY LAT. 14 4ON LONG.121 5E ALT. TOM. ICOLUMN)

SOURCE: PHILIPPINES ATOMIC ENERGY COMMISSICN, NATIONAL SCIENCE CEVELOPMENT BOARD

\begin{tabular}{|c|c|c|c|c|c|c|c|c|c|c|c|c|c|c|}
\hline & & JAN. & FEB. & MAR. & APR : & MAY & JUNE & JULY & AUG. & SEP. & OCT. & NOV. & DEC. & CUM. IOTAL \\
\hline 1961 & $\begin{array}{l}\text { PRECIP. (CM.) } \\
\text { SR-9O (KCI/SQ.KM-) } \\
\text { SR-90 CONC. }(P C / L) \\
S R-89 / 5 R-90\end{array}$ & $=-$ & $=$ & $\begin{array}{l}6.35 \\
0.17 C \\
2.68 \\
--\end{array}$ & $\begin{array}{l}3.89 \\
0.116 \\
2.83 \\
--\end{array}$ & $\begin{array}{l}12.50 \\
0.02 \mathrm{C} \\
0.17 \\
--\end{array}$ & $\begin{array}{l}58.06 \\
0.06 \mathrm{C} \\
0.11 \\
.-\end{array}$ & $\begin{array}{l}41.83 \\
-- \\
--\end{array}$ & $\begin{array}{l}59.36 \\
0.00 \\
0.01 \\
--\end{array}$ & $\begin{array}{l}\ddot{*} \\
--\end{array}$ & $\begin{array}{r}27.51 \\
0.03 \\
0.11 \\
79.00\end{array}$ & $\begin{array}{r}10.95 \\
0.02 \\
0.19 \\
43.00\end{array}$ & $\begin{array}{r}1.17 \\
0.01 \\
0.86 \\
27.00\end{array}$ & $\begin{array}{r}221.62 \\
0.42\end{array}$ \\
\hline 1962 & $\begin{array}{l}\text { PRECIP. }\left(C M_{-}\right) \\
\text {SR-9C (MCI/SO.KM.) } \\
S R-90 \text { CGNC. (PC/L) } \\
S R-89 / S R-90\end{array}$ & $\begin{array}{l}3.18 \\
+ \\
-- \\
--\end{array}$ & $\begin{array}{l}0.05 \\
* \\
-- \\
--\end{array}$ & $\begin{array}{l}0.69 \\
* \\
-- \\
=-\end{array}$ & $\begin{array}{r}8.33 \\
0.10 \\
1.21 \\
16.00\end{array}$ & $\begin{array}{l}-- \\
-- \\
--\end{array}$ & $\begin{array}{r}18.69 \\
0.24 \\
1.29 \\
--\end{array}$ & $\begin{array}{r}96.11 \\
0.38 \\
6.40 \\
16.00\end{array}$ & $\begin{array}{l}33.12 \\
0.11 \\
0.34 \\
*\end{array}$ & $\begin{array}{r}35.66 \\
0.08 \\
0.23 \\
14.00\end{array}$ & $\begin{array}{r}5.03 \\
0.05 \\
1.00 \\
13.00\end{array}$ & $\begin{array}{r}9.22 \\
0.03 \\
0.33 \\
35.00\end{array}$ & $\begin{array}{l}1.04 \\
0.02 \\
1.93 \\
19.00\end{array}$ & $\begin{array}{r}211.12 \\
1.01\end{array}$ \\
\hline 1963 & $\begin{array}{l}\text { PRECIP. }(C M .) \\
\text { SR-9C INCI/SQ.KM.) } \\
S R-90 \text { CQNC. }(P C / L) \\
S R-89 / S R-90\end{array}$ & $\begin{array}{r}0.30 \\
0.02 \\
6.67 \\
29.00\end{array}$ & $\begin{array}{c}* \\
0.02 \\
15.00\end{array}$ & $\begin{array}{r}0.66 \\
0.05 \\
7.58 \\
11.00\end{array}$ & $\begin{array}{l}22.91 \\
0.02 \\
0.09 \\
*\end{array}$ & $\begin{array}{r}8.59 \\
6.31 \\
3.61 \\
11.20\end{array}$ & $\begin{array}{r}57.66 \\
1.78 \\
3.09 \\
0.70\end{array}$ & $\begin{array}{r}33.20 \\
0.29 \\
0.88 \\
1.00\end{array}$ & $\begin{array}{l}-\infty \\
0.31\end{array}$ & $\begin{array}{l}49.07 \\
0.16 \\
0.33 \\
+\end{array}$ & $\begin{array}{r}15.32 \\
0.15 \\
0.98 \\
1.20\end{array}$ & $\begin{array}{l}4.85 \\
0.04 \\
0.83 \\
+\end{array}$ & $\begin{array}{l}9.53 \\
0.02 \\
0.21 \\
*\end{array}$ & $\begin{array}{r}202.09 \\
3.17\end{array}$ \\
\hline 1964 & $\begin{array}{l}\text { PRECIP }\left(C M_{-}\right) \\
\text {SR-9C IMCI/SQ.KM.) } \\
\text { SR-GC CCNC. }(P C / L) \\
S R-89 / S R-90\end{array}$ & $\begin{array}{l}3.23 \\
0.02 \\
0.62 \\
*\end{array}$ & $\begin{array}{r}0.20 \\
0.02 \\
10.01 \\
0.80\end{array}$ & $\begin{array}{l}1.60 \\
0.02 \\
1.26 \\
*\end{array}$ & $\begin{array}{l}2.01 \\
0.05 \\
2.49 \\
0.19\end{array}$ & $\begin{array}{l}17.96 \\
0.12 \\
0.67 \\
*\end{array}$ & $\begin{array}{l}51.05 \\
0.08 \\
0.16 \\
--\end{array}$ & $\begin{array}{l}30.48 \\
C .14 \\
0.46 \\
--\end{array}$ & $\begin{array}{l}44.91 \\
0.08 \\
0.18 \\
-.0\end{array}$ & $\begin{array}{l}25.25 \\
C .05 \\
0.20 \\
-.\end{array}$ & $\begin{array}{l}20.12 \\
0.02 \\
0.10 \\
*\end{array}$ & $\begin{array}{l}-- \\
0.02 \\
+\end{array}$ & $\begin{array}{l}11.71 \\
0.02 \\
0.18 \\
-.\end{array}$ & $\begin{array}{r}208.52 \\
0.64\end{array}$ \\
\hline 1965 & $\begin{array}{l}\text { PRECIP. (CM.) } \\
\text { SR-90 (KCI/SQ.KM.) } \\
\text { SR-90 CONC. (PC/L) } \\
\text { SR-89/SR-9O }\end{array}$ & $\begin{array}{l}0.46 \\
0.02 \\
4.35 \\
-.\end{array}$ & $\begin{array}{l}1.19 \\
0.01 \\
0.85 \\
-.\end{array}$ & $\begin{array}{l}* \\
0.01 \\
-- \\
--\end{array}$ & $\overline{-}$ & $\begin{array}{r}15.44 \\
0.02 \\
0.13 \\
--\end{array}$ & 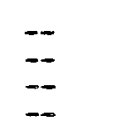 & $\begin{array}{l}1.42 \\
0.20 \\
14.09 \\
--\end{array}$ & $\begin{array}{r}15.62 \\
0.17 \\
1.09 \\
-\end{array}$ & $\begin{array}{l}-- \\
--\end{array}$ & $\begin{array}{l}5.84 \\
0.06 \\
1.03 \\
--\end{array}$ & $=$ & $\begin{array}{l}3.71 \\
* \\
--\end{array}$ & $\begin{array}{r}43.68 \\
0.49\end{array}$ \\
\hline 1966 & $\begin{array}{l}\text { PRECIP. (CM.) } \\
\text { SR-90 (NCI/SQ.KM.) } \\
S R=90 \text { CONC. (PC/L) } \\
\text { SR-89/SR-90 }\end{array}$ & $\begin{array}{l}-- \\
-- \\
--\end{array}$ & $\begin{array}{l}3.40 \\
* \\
-- \\
--\end{array}$ & $=$ & $\begin{array}{l}0.84 \\
0.05 \\
5.96 \\
-\end{array}$ & $\begin{array}{l}67.01 \\
0.03 \\
0.05 \\
--\end{array}$ & $\begin{array}{l}1.59 \\
0.05 \\
3.15 \\
+\end{array}$ & $\begin{array}{l}28.29 \\
0.01 \\
C .04 \\
*\end{array}$ & $\begin{array}{l}27.91 \\
0.03 \\
0.11 \\
*\end{array}$ & $\begin{array}{l}66.00 \\
0.07 \\
0.11 \\
+\end{array}$ & $\begin{array}{l}10.72 \\
0.02 \\
0.19 \\
*\end{array}$ & $\begin{array}{l}35.90 \\
0.02 \\
0.06 \\
*\end{array}$ & $\begin{array}{l}9.46 \\
0.01 \\
0.11 \\
*\end{array}$ & $\begin{array}{r}251.12 \\
0.29\end{array}$ \\
\hline 1967 & $\begin{array}{l}\text { PRECIP. }\left(C M M_{-}\right) \\
S R-90 \text { (MCI/SQ.KM.) } \\
\text { SR-90 CONC. }(P C / L) \\
\text { SR-89/SR-90 }\end{array}$ & $\begin{array}{l}3.34 \\
0.01 \\
0.30 \\
+\end{array}$ & $\begin{array}{l}0.79 \\
0.01 \\
1.27 \\
+\end{array}$ & $\begin{array}{l}0.64 \\
0.01 \\
1.57 \\
+\end{array}$ & $\begin{array}{l}0.46 \\
0.02 \\
4.35 \\
*\end{array}$ & $\begin{array}{l}=- \\
-- \\
=-\end{array}$ & $\begin{array}{l}=- \\
=- \\
=-\end{array}$ & $\begin{array}{l}= \\
-- \\
-\end{array}$ & $\begin{array}{l}=- \\
=- \\
--\end{array}$ & $\begin{array}{l}=- \\
-- \\
--\end{array}$ & $\begin{array}{l}-- \\
-- \\
--\end{array}$ & $\begin{array}{l}-- \\
-- \\
--\end{array}$ & $=$ & $\begin{array}{l}5.23 \\
0.05\end{array}$ \\
\hline $\begin{array}{l}\text { NOTES } \\
*:= \\
*: 2 \\
A: A \\
B: 1 \\
C: F\end{array}$ & $\begin{array}{l}\text { DSTA NOT AVAILABLE } \\
\text { ZERO OR TRACE } \\
\text { APPROXIMATE } \\
\text { LOWER LIMIT OF REPORT } \\
\text { PROPORTIONED FROM ORI }\end{array}$ & $\begin{array}{l}\text { DATA } \\
\text { NALLY }\end{array}$ & & $-\infty-\infty$ & & & & & & & & & & \\
\hline
\end{tabular}




\begin{tabular}{|c|c|c|c|c|c|c|c|c|c|c|c|c|c|c|}
\hline & & JAN. & FEB. & MAR - & APR . & MAY & JUNE & JULY & AUG. & SEP. & OCT. & NOV. & DEC. & CUM. TOTAL \\
\hline 1960 & $\begin{array}{l}\text { PRECIP }\left(C M_{-}\right) \\
\text {SR-9O }\left(M C I / S K_{-K M}\right) \\
\text { SR-90 CONC. }(P C / L) \\
S R-89 / S R-90\end{array}$ & $\bar{m}=$ & $\begin{array}{l}7.95 \\
0.07 \\
0.89 \\
-\infty\end{array}$ & $\begin{array}{l}6.10 \\
0.12 \\
1.97 \\
--\end{array}$ & $\begin{array}{r}14.63 \\
0.21 \\
1.44 \\
-.\end{array}$ & $\begin{array}{l}56.11 \\
= \\
=\end{array}$ & $\begin{array}{l}46.61 \\
=- \\
--\end{array}$ & $\begin{array}{l}12.90 \\
0.08 \mathrm{C} \\
0.63 \\
--\end{array}$ & $\begin{array}{c}21.56 \\
0.15 C \\
0.70 \\
--\end{array}$ & $\begin{array}{l}32.94 \\
0.10 \\
0.31 \\
-.\end{array}$ & $\begin{array}{l}41.10 \\
-- \\
--\end{array}$ & $\begin{array}{l}48.01 \\
-- \\
--\end{array}$ & $\begin{array}{l}53.01 \\
0.09 \mathrm{C} \\
0.17 \\
--\end{array}$ & $\begin{array}{r}340.92 \\
0.82\end{array}$ \\
\hline 1961 & $\begin{array}{l}\text { PRECIP. }\left(C M_{-}\right) \\
\text {SR- } 9 C \text { IMCI/SQ.KM-) } \\
\text { SR-9OCONC. (PC/L) } \\
\text { SR-89/SR-90 }\end{array}$ & $\begin{array}{l}8.92 \\
0.02 \mathrm{C} \\
0.23 \\
--\end{array}$ & $\begin{array}{l}3.76 C \\
0.06 C \\
1.60 \\
--\end{array}$ & $\begin{array}{l}3.76 \mathrm{C} \\
0.06 \mathrm{C} \\
1.60 \\
-.\end{array}$ & $\begin{array}{l}7.16 \\
0.12 \mathrm{C} \\
1.68 \\
=-\end{array}$ & $\begin{array}{l}4.50 \\
0.11 \\
2.45 \\
--\end{array}$ & $\begin{array}{l}13.41 \\
0.07 \mathrm{C} \\
0.53 \\
--\end{array}$ & $\begin{array}{l}23.70 \\
0.13 C \\
0.55 \\
--\end{array}$ & $\begin{array}{l}13.21 \\
-- \\
=-\end{array}$ & $\begin{array}{l}6.65 \\
0.12 \\
1.81 \\
0.80\end{array}$ & $\begin{array}{r}21.51 \\
0.08 \mathrm{C} \\
0.38 \\
03.00\end{array}$ & $\begin{array}{c}23.52 \\
0.10 \mathrm{C} \\
0.43 \\
63.00\end{array}$ & $\begin{array}{r}25.40 \\
0.33 \\
1.30 \\
64.00\end{array}$ & $\begin{array}{r}155.50 \\
1.20\end{array}$ \\
\hline 1962 & $\begin{array}{l}\text { PRECIP, }\left(C M_{-}\right) \\
\text {SR- } 9 C \text { IMCI/SQ.KM- }) \\
S R-9 C \text { CONC. }(P C / L) \\
S R-8 S / S R-90\end{array}$ & $\begin{array}{r}10.77 \\
0.59 \\
5.48 \\
42.00\end{array}$ & $\begin{array}{r}6.78 \\
0.39 \\
5.76 \\
34.00\end{array}$ & $=-$ & $\begin{array}{r}9.40 \\
1.34 \\
14.26 \\
12.00\end{array}$ & $\begin{array}{r}19.13 \\
1.01 \\
5.28 \\
20.00\end{array}$ & $\begin{array}{r}18.57 \\
0.61 \\
3.29 \\
11.00\end{array}$ & $\begin{array}{c}15.54 C \\
C .39 C \\
2.51 \\
-\end{array}$ & $\begin{array}{c}15.54 C \\
0.39 C \\
2.51 \\
-.\end{array}$ & $\begin{array}{r}12.32 \\
-0.21 \\
1.71 \\
9.00\end{array}$ & $\begin{array}{r}13.94 \\
6.12 \\
0.87 \\
63.00\end{array}$ & $\begin{array}{r}9.75 \\
0.14 \\
1.44 \\
62.00\end{array}$ & $\begin{array}{r}10.44 \\
0.36 \\
3.45 \\
46.00\end{array}$ & $\begin{array}{r}142.18 \\
5.55\end{array}$ \\
\hline 1963 & $\begin{array}{l}\text { PRECIP. (CM.) } \\
S R-90 \text { (MCI/SQ.KM-) } \\
5 R-90 \text { CONC. }(P C / L) \\
S R-89 / S R-90\end{array}$ & $\begin{array}{r}7.95 \\
0.34 \\
4.28 \\
37.00\end{array}$ & $\begin{array}{r}3.53 \\
0.24 \\
6.80 \\
33.00\end{array}$ & $\begin{array}{r}11.89 \\
1.79 \\
15.06 \\
13.00\end{array}$ & $\begin{array}{r}13.23 \\
0.93 \\
7.03 \\
8.10\end{array}$ & $\begin{array}{l}11.76 C \\
0.84 C \\
7.15 \\
=-\end{array}$ & $\begin{array}{l}11.76 \mathrm{C} \\
0.84 \mathrm{C} \\
7.15 \\
--\end{array}$ & $\begin{array}{l}11.76 C \\
0.84 C \\
7.15 \\
--\end{array}$ & $\begin{array}{l}11.76 C \\
0.84 C \\
7.15 \\
--\end{array}$ & $\begin{array}{r}27.89 \\
0.72 \\
2.59 \\
0.50\end{array}$ & $\begin{array}{l}4.14 \\
0.13 \\
3.15 \\
0.90\end{array}$ & $\begin{array}{l}7.62 \\
0.39 \\
5.12 \\
*\end{array}$ & $\begin{array}{r}1.73 \\
0.20 \\
11.57 \\
*\end{array}$ & $\begin{array}{r}125.02 \\
8.10\end{array}$ \\
\hline 1964 & $\begin{array}{l}\text { PRECIP. (CM-) } \\
\text { SR-SC (NCI/SQ.KM.) } \\
\text { SR-9OCONC. (PC/L) } \\
S R-89 / S R-90\end{array}$ & $\begin{array}{l}5.13 \\
0.26 \\
5.07 \\
+\end{array}$ & $\begin{array}{l}4.45 \\
0.29 \\
6.52 \\
*\end{array}$ & $\begin{array}{r}3.23 \\
0.41 \\
12.70 \\
*\end{array}$ & $\begin{array}{l}16.26 \\
1.23 \\
7.57 \\
*\end{array}$ & $\begin{array}{l}10.06 \\
0.76 \\
7.56 \\
*\end{array}$ & $\begin{array}{r}11.46 \\
0.87 \\
7.60 \\
-.\end{array}$ & $\begin{array}{l}17.91 \\
0.666 \\
3.69 \\
--\end{array}$ & $\begin{array}{l}17.02 \\
0.63 C \\
3.71 \\
=-\end{array}$ & $\begin{array}{r}12.93 \\
0.21 \\
1.63 \\
\end{array}$ & $\begin{array}{l}7.95 \\
0.15 \\
1.89 \\
*\end{array}$ & $\begin{array}{l}8.61 \\
0.15 \\
1.75 \\
*\end{array}$ & $\begin{array}{l}5.97 \\
0.32 \\
5.37 \\
--\end{array}$ & $\begin{array}{r}120.98 \\
5.94\end{array}$ \\
\hline 1965 & $\begin{array}{l}\text { PRECIP ( }\left(C M_{-}\right) \\
\text {SR-9O (MCI/SQ.KM) } \\
\text { SR-90 CONC. }(P C / L) \\
S R-89 / S R-90\end{array}$ & $\begin{array}{l}6.65 \\
* \\
--\end{array}$ & $\begin{array}{l}2.01 \\
0.02 \\
1.00 \\
-\end{array}$ & $\begin{array}{l}2.18 \\
0.11 \\
5.05 \\
-.\end{array}$ & $\begin{array}{l}5.56 \\
0.26 \\
4.68 \\
-\cdots\end{array}$ & $\begin{array}{l}38.07 \\
1.22 \\
3.21 \\
--\end{array}$ & $\begin{array}{l}27.84 \\
0.99 \\
3.56 \\
-.\end{array}$ & $\begin{array}{l}14.94 \\
0.32 \\
2.15 \\
--\end{array}$ & $\begin{array}{r}22.00 \\
0.32 \\
1.46 \\
-.\end{array}$ & $\begin{array}{l}12.19 \\
0.20 \\
1.65 \\
-\end{array}$ & $\begin{array}{l}12.90 \\
0.10 \\
0.78 \\
--\end{array}$ & $\begin{array}{l}9.98 \\
0.05 \\
0.51 \\
--\end{array}$ & $\begin{array}{r}14.22 \\
0.14 \\
0.99 \\
-\end{array}$ & $\begin{array}{r}168.54 \\
3.73\end{array}$ \\
\hline 1966 & $\begin{array}{l}\text { PRECIP. (CM.) } \\
S R-90 \text { (MCI/SO.KM.) } \\
S R-90 \text { CCNC. }(P C / L) \\
S R-89 / S R-90\end{array}$ & $\begin{array}{l}3.40 \\
0.06 \\
1.77 \\
--\end{array}$ & $\begin{array}{l}4.16 \\
0.28 \\
6.74 \\
--\end{array}$ & $\begin{array}{r}11.76 \\
0.15 \\
1.28 \\
-0\end{array}$ & $\begin{array}{r}14.27 \\
0.18 \\
1.27 \\
-.\end{array}$ & $\begin{array}{l}14.45 \\
0.17 \\
1.18 \\
--\end{array}$ & $\begin{array}{r}8.28 \\
0.07 \\
0.85 \\
21.00\end{array}$ & $\begin{array}{r}10.69 \\
0.10 \\
0.94 \\
4.70\end{array}$ & $\begin{array}{l}8.66 \\
0.06 \\
0.70 \\
*\end{array}$ & $\begin{array}{r}17.91 \\
-0.07 \\
0.40 \\
*\end{array}$ & $\begin{array}{r}22.83 \\
0.03 \\
0.14 \\
8.40\end{array}$ & $\begin{array}{r}20.29 \\
0.04 \\
0.20 \\
11.00\end{array}$ & $\begin{array}{l}15.77 \\
0.06 \\
0.39 \\
*\end{array}$ & $\begin{array}{r}152.47 \\
1.27\end{array}$ \\
\hline 1967 & $\begin{array}{l}\text { PRECIP. (CM.) } \\
S R-90 \text { (MCI/SO.KM.) } \\
S R-90 \text { CONC. (PC/L) } \\
\text { SR-89/SR-90 }\end{array}$ & $\begin{array}{l}7.80 \\
0.04 \\
0.52 \\
6.70\end{array}$ & $\begin{array}{l}8.20 \\
0.06 \\
0.74 \\
7.60\end{array}$ & $\begin{array}{l}3.68 \\
0.06 \\
1.64 \\
9.60\end{array}$ & $\begin{array}{l}2.16 \\
0.04 \\
1.86 \\
4.20\end{array}$ & $\begin{array}{r}10.54 \\
0.05 \\
0.48 \\
*\end{array}$ & $\begin{array}{l}8.58 \\
0.06 \\
0.70 \\
+\end{array}$ & $\begin{array}{r}12.50 \\
0.06 \\
0.49 \\
*\end{array}$ & $=$ & $\begin{array}{l}-- \\
--\end{array}$ & $\begin{array}{l}-- \\
-- \\
-\end{array}$ & $=-$ & $\begin{array}{l}-- \\
-- \\
--\end{array}$ & $\begin{array}{r}53.46 \\
0.37\end{array}$ \\
\hline \multicolumn{15}{|c|}{$\begin{array}{l}\text { NOTES } \\
\text { :- DATA NOT AVAILABLE } \\
\text { *: ZERO OR TRACE } \\
\text { A: APPROXIMATE } \\
\text { B: LOWER LIMIT OF REPORTEO DATA } \\
\text { C: PROPORTI ONED FROM ORIGINALLY }\end{array}$} \\
\hline
\end{tabular}




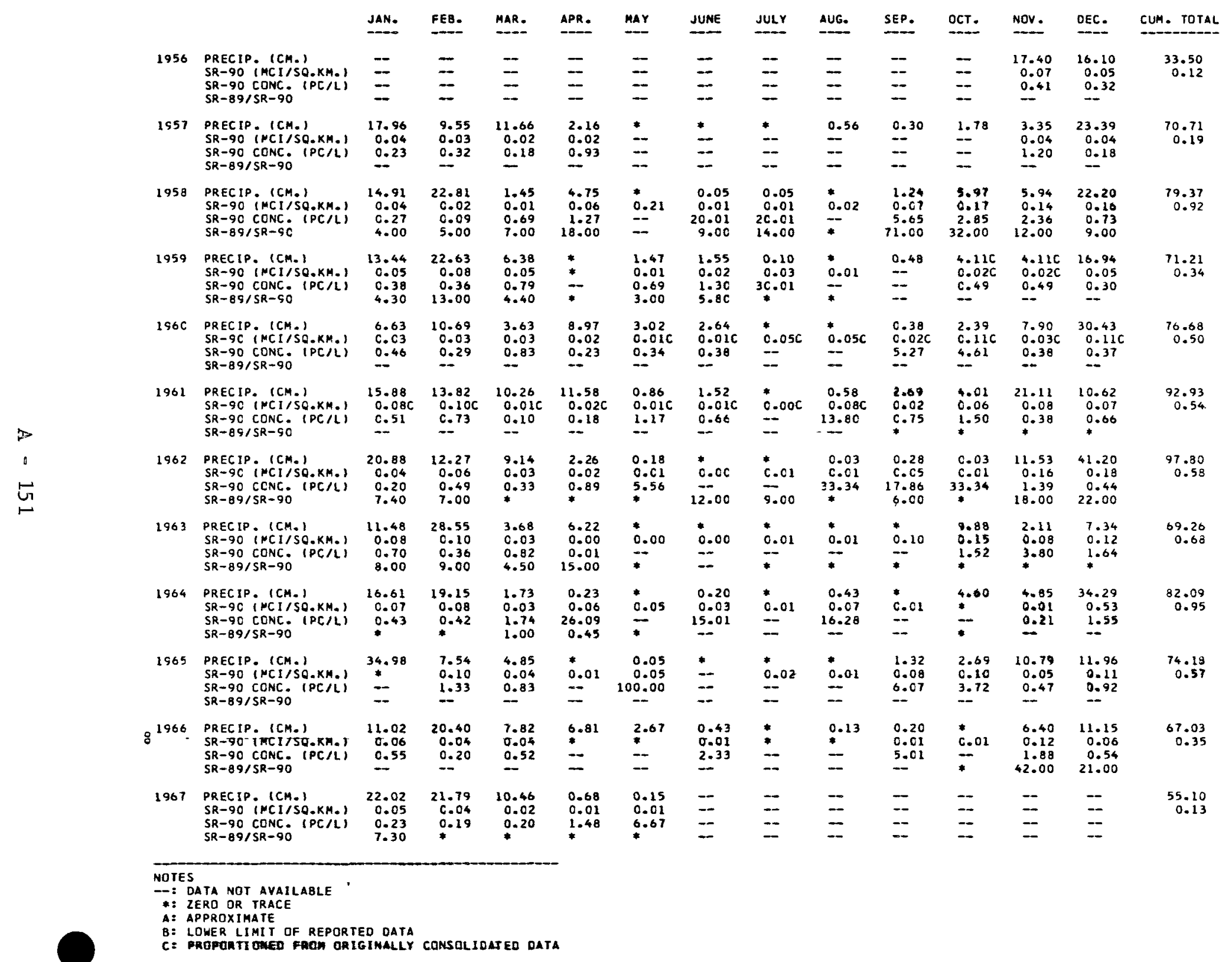




\begin{tabular}{|c|c|c|c|c|c|c|c|c|c|c|c|c|}
\hline JAN. & FEB. & MAR. & APR : & MAY & JUNE & JULY & AUG. & SEP. & OCT. & NOV. & DEC . & CUM. TOTAL \\
\hline-- & - & -- & - & - & - & -- & $\cdots$ & -- & - & -- & - & 0.0 \\
\hline-- & - & -- & - & - & -- & - & - & - & - & -- & 0.01 & 0.01 \\
\hline-- & - & - & -- & - & - & -- & - & -- & - & - & -- & \\
\hline- & -- & -- & -- & - & -- & - & -- & - & -- & -- & * & \\
\hline-- & -- & - & 0.13 & * & -- & $*$ & $*$ & $*$ & $*$ & $=-$ & 0.10 & 0.23 \\
\hline 0.05 & 0.02 & 0.18 & 0.09 & 0.10 & - & C.04C & $0.04 \mathrm{C}$ & $0.11 C$ & $0.11 C$ & $0.02 C$ & $0.02 C$ & 0.78 \\
\hline- & - & -- & 69.24 & -- & - & - & -- & -- & $-\infty$ & -- & 20.01 & \\
\hline-- & - & -- & -- & -- & - & - & - & - & -- & -- & -- & \\
\hline $\begin{array}{l}1.27 \\
0.05 \mathrm{C} \\
3.94\end{array}$ & $\begin{array}{l}1.65 \\
0.07 C \\
4.25\end{array}$ & $\begin{array}{l}1.27 \\
0.076 \\
5.52\end{array}$ & $\begin{array}{l}1.98 \\
0.10 \mathrm{C} \\
5.06\end{array}$ & * & * & * & * & $\begin{array}{l}* \\
* \\
--\end{array}$ & $\stackrel{*}{0.01}$ & $\begin{array}{l}2.59 \\
0.05 \\
1.94\end{array}$ & $\begin{array}{r}0.48 \\
0.18 \\
37.51\end{array}$ & $\begin{array}{l}9.24 \\
0.53\end{array}$ \\
\hline- & -- & - & - & -- & -- & - & - & - & 22.00 & 11.00 & 60.00 & \\
\hline$*$ & * & * & - & -- & -- & - & -- & -- & - & - & -- & 0.0 \\
\hline 0.04 & 0.05 & 0.15 & - & - & - & - & - & -- & - & -- & -- & 0.24 \\
\hline- & -- & -- & - & -- & - & - & - & -- & - & - & -- & \\
\hline 50.00 & 24.00 & 19.00 & - & - & -- & -- & - & - & - & - & -- & \\
\hline$*$ & 0.03 & $*$ & 1.45 & 2.44 & $*$ & $*$ & $*$ & $*$ & $*$ & 1.22 & 0.20 & 5.34 \\
\hline-- & -- & $-\infty$ & -- & -- & - & - & - & - & -- & 0.22 & 0.36 & 0.58 \\
\hline- & -- & - & - & - & -- & - & - & - & - & 18.04 & 180.00 & \\
\hline-- & $-\infty$ & -- & - & - & - & - & -- & -- & - & * & * & \\
\hline 3.30 & 5.08 & * & * 050 & * & * & * & $* 07$ & $*$ & 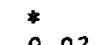 & $1.27 \mathrm{C}$ & $1.27 C$ & 10.92 \\
\hline 0.65 & 1.35 & 0.06 & $0.05 \mathrm{C}$ & $0.05 \mathrm{C}$ & $0.05 \mathrm{C}$ & 0.10 & 0.0 .7 & 0.03 & 0.02 & $0.11 \mathrm{C}$ & $0.11 \mathrm{C}$ & 2.65 \\
\hline 19.70 & 26.58 & -- & -- & - & - & - & - & -- & - & 8.67 & 8.67 & \\
\hline$*$ & 0.70 & 3.50 & -- & -- & - & - & -- & -- & $*$ & -- & - & \\
\hline $1.27 C$ & * & $0.18 \mathrm{C}$ & $0.18 \mathrm{C}$ & $*$ & - & * & * & -- & - & - & - & 1.63 \\
\hline $0.11 \mathrm{C}$ & 0.01 & 0.19 & 0.01 & 0.02 & -- & $0.04 E$ & $0.04 \mathrm{C}$ & -- & -- & -- & -- & 0.42 \\
\hline 8.67 & -- & 105.56 & 5.56 & -- & - & - & -- & - & -- & -- & - & \\
\hline-- & - & -- & - & - & - & - & -- & - & - & - & -- & \\
\hline-- & - & - & 0.23 & $*$ & * & * & * & $*$ & * & * & $*$ & 0.23 \\
\hline 0.05 & -- & - & 0.09 & 0.05 & $*$ & $*$ & 0.04 & * & * & 0.01 & 0.01 & 0.25 \\
\hline-- & -- & -- & 39.14 & - & - & - & -- & -- & -- & -- & -- & \\
\hline-- & -- & - & -- & - & * & * & * & $*$ & * & * & * & \\
\hline $\begin{array}{l}0.10 \\
0.02\end{array}$ & $\begin{array}{l}0.37 \\
0.05\end{array}$ & 0.58 & 1.80 & 0.30 & * & $\overline{-}$ & $=-$ & $\overline{-}$ & $=$ & $=$ & $=$ & 3.15 \\
\hline 20.01 & 13.52 & $\begin{array}{r}0.06 \\
10.35\end{array}$ & $\begin{array}{l}0.10 \\
5.56\end{array}$ & $\begin{array}{r}0.03 \\
10.01\end{array}$ & 0.01 & - & - & $=$ & $=$ & $=$ & -. & \\
\hline * & 4.40 & * & 3.00 & $\star$ & * & - & - & - & -- & -- & -- & \\
\hline
\end{tabular}


SOURCE: PRESTWICK AFB, U. S. AIR WEATHER SERVICE

COLLECTIONS TERMINATED IN JUNE 1966

\begin{tabular}{|c|c|c|c|c|c|c|c|c|c|c|c|c|c|c|}
\hline & & JAN. & FEB. & MAR. & APR . & MAY & JUNE & JULY & AUG. & SEP. & OCT & NOV. & DEC. & CUM. TOTAL \\
\hline 1959 & $\begin{array}{l}P R E C I P \text {. (CM.) } \\
\text { SR-90 (MCI/SQ.KM.) } \\
S R-90 \text { CONC. }(P C / L) \\
S R-89 / S R-90\end{array}$ & $=$ & $\overline{--}$ & $\overline{-}$ & $\overline{-}$ & $\overline{--}$ & $=$ & $\overline{--}$ & $\begin{array}{l}-- \\
--\end{array}$ & $\begin{array}{l}3.63 \\
.0 .05 \\
1.38 \\
4.30\end{array}$ & $\begin{array}{l}14.25 \\
=- \\
-\end{array}$ & $\begin{array}{l}9.98 \\
0.10 \\
1.01 \\
0.70\end{array}$ & $\begin{array}{l}10.80 \\
0.10 \\
0.93 \\
*\end{array}$ & $\begin{array}{r}38.66 \\
0.25\end{array}$ \\
\hline 1960 & $\begin{array}{l}\text { PREC IP. (CM-) } \\
S R-90 \text { (MCI/SQ.KM ) } \\
S R-90 \text { CONC. (PC/L) } \\
S R-89 / 5 R-90\end{array}$ & $\begin{array}{l}10.57 \\
0.01 \\
0.10 \\
--\end{array}$ & $\begin{array}{l}7.21 \\
0.10 \\
1.39 \\
-\end{array}$ & $\begin{array}{l}7.21 \\
0.17 \\
2.36 \\
\end{array}$ & $\begin{array}{l}5.59 \\
0.23 \\
4.12 \\
--\end{array}$ & $\begin{array}{l}3.40 \\
0.20 \\
5.89 \\
--\end{array}$ & $\begin{array}{l}6.55 \\
0.26 \\
3.97 \\
-\end{array}$ & $\begin{array}{l}5.46 \\
-- \\
--\end{array}$ & $\begin{array}{l}8.81 \\
= \\
=\end{array}$ & $\begin{array}{l}7.16 \\
0.12 \mathrm{C} \\
1.68 \\
--\end{array}$ & $\begin{array}{l}4.57 \\
0.08 \mathrm{C} \\
1.76 \\
-.\end{array}$ & $\begin{array}{c}10.31 \\
0.08 \mathrm{C} \\
0.78 \\
--\end{array}$ & $\begin{array}{l}9.14 \\
0.07 \mathrm{C} \\
0.77 \\
--\end{array}$ & $\begin{array}{r}85.98 \\
1.32\end{array}$ \\
\hline 1961 & $\begin{array}{l}\text { PRECIP. }(C M .) \\
\text { SR-9C (MCI/SQ.KM.) } \\
S R-90 \text { CONC. (PC/L) } \\
S R-89 / S R-90\end{array}$ & $\begin{array}{l}4.39 \\
0.076 \\
1.60 \\
--\end{array}$ & $\begin{array}{l}7.37 \\
0.136 \\
1.77 \\
--\end{array}$ & $\begin{array}{l}5.13 \\
0.03 C \\
0.59 \\
-\infty\end{array}$ & $\begin{array}{l}7.57 \\
0.04 C \\
0.53 \\
-\end{array}$ & $\begin{array}{l}2.29 \\
0.09 C \\
3.94 \\
-.\end{array}$ & $\begin{array}{l}3.99 \\
0.150 \\
3.76 \\
--\end{array}$ & $\begin{array}{l}7.14 \\
0.176 \\
2.39 \\
-.\end{array}$ & $\begin{array}{l}0.51 \\
0.016 \\
1.97 \\
-.\end{array}$ & $\begin{array}{r}14.43 \\
0.19 \\
1.32 \\
53.00\end{array}$ & $\begin{array}{r}13.41 \\
0.15 \\
1.12 \\
84.00\end{array}$ & $\begin{array}{l}5.66 \\
- \\
-\end{array}$ & $\begin{array}{r}7.90 \\
0.70 \\
8.87 \\
62.00\end{array}$ & $\begin{array}{r}79.79 \\
1.73\end{array}$ \\
\hline 1962 & $\begin{array}{l}\text { PRECIP. }\left(C M_{-}\right) \\
S R-90 \text { (MCI/SQ.KM.) } \\
S R-90 \text { CONC. }(P C / L) \\
S R-89 / S R-90\end{array}$ & $\begin{array}{r}12.78 \\
0.68 \\
5.33 \\
46.00\end{array}$ & $\begin{array}{r}5.56 \\
c .83 \\
14.93 \\
30.00\end{array}$ & $\begin{array}{r}2.72 \\
0.53 \\
19.49 \\
20.00\end{array}$ & $\begin{array}{l}4.80 \\
-- \\
--\end{array}$ & $\begin{array}{r}4.78 \\
1.10 \\
23.02 \\
8.00\end{array}$ & $\begin{array}{r}4.37 \\
0.7 \mathrm{C} \\
16.02 \\
10.00\end{array}$ & $\begin{array}{r}5.74 \\
6.62 \\
10.81 \\
7.00\end{array}$ & $\begin{array}{r}13.94 \\
1.11 \\
7.97 \\
8.00\end{array}$ & $\begin{array}{r}16.51 \\
0.62 \\
3.76 \\
17.00\end{array}$ & $\begin{array}{r}4.72 \\
0.30 \\
6.36 \\
25.00\end{array}$ & $\begin{array}{r}4.70 \\
0.49 \\
10.43 \\
9.00\end{array}$ & $\begin{array}{r}9.78 \\
0.60 \\
6.14 \\
45.00\end{array}$ & $\begin{array}{r}90.40 \\
7.58\end{array}$ \\
\hline 1963 & $\begin{array}{l}\text { PRECIP. }\left(C M_{-}\right) \\
\text {SR-9O (MCI/SQ.KM.) } \\
S R-90 \text { CONC. (PC/L) } \\
S R-89 / S R-90\end{array}$ & $\begin{array}{r}1.14 \\
0.14 \\
12.29 \\
28.00\end{array}$ & $\begin{array}{r}0.69 \\
1.00 \\
144.93 \\
21.00\end{array}$ & $\begin{array}{r}8.69 \\
0.97 \\
11.17 \\
23.00\end{array}$ & $\begin{array}{r}4.22 \\
7.30 \\
172.99 \\
14.00\end{array}$ & $\begin{array}{r}8.13 \\
2.00 \\
24.61 \\
8.70\end{array}$ & $\begin{array}{r}5.23 \\
2.05 \\
39.2 C \\
0.20\end{array}$ & $\begin{array}{r}5.33 \\
1.82 \\
34.15 \\
1.00\end{array}$ & $\begin{array}{r}0.94 \\
1.61 \\
18.01 \\
*\end{array}$ & $\begin{array}{r}6.96 \\
0.88 \\
12.65 \\
0.80\end{array}$ & $\begin{array}{r}11.84 \\
0.87 \\
7.35 \\
*\end{array}$ & $m$ & $\begin{array}{l}-- \\
-- \\
--\end{array}$ & $\begin{array}{l}61.17 \\
18.64\end{array}$ \\
\hline 1964 & $\begin{array}{l}\text { PRECIP. (CM.) } \\
\text { SR-9O (MCI/SO.KM.) } \\
\text { SR-90 CONC. (PC/L) } \\
\text { SR-89/SR-90 }\end{array}$ & $\begin{array}{r}6.30 \\
1.52 \\
24.13 \\
*\end{array}$ & $\begin{array}{r}1.37 \\
0.58 \\
42.34 \\
*\end{array}$ & $\begin{array}{r}2.34 \\
0.77 \\
32.91 \\
*\end{array}$ & $\begin{array}{r}4.32 \\
0.94 \\
21.76 \\
*\end{array}$ & $\begin{array}{r}6.68 \\
2.19 \\
32.79 \\
*\end{array}$ & $\begin{array}{l}6.25 \\
2.16 \\
34.56 \\
-=\end{array}$ & $\begin{array}{r}5.46 \\
1.29 \\
23.63 \\
--\end{array}$ & $\begin{array}{r}12.65 \\
0.96 \\
7.59 \\
-\end{array}$ & $\begin{array}{l}15.21 \\
0.68 \\
4.48 \\
-.\end{array}$ & $\begin{array}{l}4.67 \\
0.29 \\
6.21 \\
5.00\end{array}$ & $\begin{array}{l}8.99 \\
0.38 \\
4.23 \\
*\end{array}$ & $\begin{array}{l}10.24 \\
0.38 \\
3.72 \\
--\end{array}$ & $\begin{array}{l}84.48 \\
12.14\end{array}$ \\
\hline 1965 & $\begin{array}{l}\text { PRECIP. }(C M=) \\
\text { SR-90 (MCI/SO.KM.) } \\
\text { SR-90 CONC. }(P C / L) \\
\text { SR-89/SR-90 }\end{array}$ & $\begin{array}{l}11.76 \\
0.76 \\
6.47 \\
--\end{array}$ & $\begin{array}{l}1.78 \\
0.13 \\
7.31 \\
-.\end{array}$ & $\begin{array}{l}3.91 \\
0.23 \\
5.89 \\
-\end{array}$ & $\begin{array}{l}6.27 \\
0.53 \\
8.46 \\
-.\end{array}$ & $\begin{array}{l}7.34 \\
0.24 \\
3.27 \\
-\end{array}$ & $\begin{array}{l}9.27 \\
0.82 \\
8.85 \\
--\end{array}$ & $\begin{array}{l}8.84 \\
0.56 \\
6.34 \\
--\end{array}$ & $\begin{array}{l}10.46 \\
0.37 \\
3.54 \\
-\end{array}$ & $\begin{array}{l}11.81 \\
0.33 \\
2.80 \\
--\end{array}$ & $\begin{array}{l}7.67 \\
0.24 \\
3.13 \\
-.\end{array}$ & $\begin{array}{l}4.55 \\
0.09 \\
1.98 \\
\end{array}$ & $\begin{array}{l}11.51 \\
0.20 \\
1.74 \\
--\end{array}$ & $\begin{array}{r}95.17 \\
4.50\end{array}$ \\
\hline 1966 & $\begin{array}{l}\text { PRECIP. (CM.) } \\
\text { SR-90 (MCI/SQ.KM.) } \\
\text { SR-90 CONC. }(P C / L) \\
S R-89 / S R-90\end{array}$ & $\begin{array}{l}4.75 \\
* \\
--\end{array}$ & $\begin{array}{l}6.93 \\
0.06 \\
0.87 \\
-\end{array}$ & $\begin{array}{l}8.66 \\
0.29 \\
3.35 \\
-\end{array}$ & $\begin{array}{l}3.66 \\
0.11 \\
3.01 \\
--\end{array}$ & $\begin{array}{l}6.20 \\
0.28 \\
4.52 \\
-.-\end{array}$ & $\begin{array}{r}11.91 \\
0.23 \\
1.94 \\
1.80\end{array}$ & $\begin{array}{l}-- \\
--\end{array}$ & $\begin{array}{l}-- \\
--\end{array}$ & $\begin{array}{l}-- \\
--\end{array}$ & $=-$ & $=$ & $\begin{array}{l}-- \\
--\end{array}$ & $\begin{array}{r}42.11 \\
0.97\end{array}$ \\
\hline $\begin{array}{c}\text { NOTES } \\
-=: \\
*: \\
A: \\
B: A \\
C: L \\
C: D\end{array}$ & $\begin{array}{l}\text { TTA NOT AVAILABLE } \\
\text { ERO OR TRACE } \\
\text { OPROXIMATE } \\
\text { OHER LIIMIT OF REPOF } \\
\text { ROPORTI ONED FROM OI }\end{array}$ & $\begin{array}{l}\text { DATA } \\
\text { NALLLY }\end{array}$ & & & & & & & & & & & & \\
\hline
\end{tabular}


JAN. FEB. MAR.

\begin{tabular}{|c|c|c|c|c|c|c|c|c|c|c|c|c|c|c|}
\hline & & JAN. & FEB. & MAR. & APR : & MAY & JUNE & JULY & AUG. & SEP. & OCT. & NOV. & DEC. & CUM. TOTAL \\
\hline 1959 & $\begin{array}{l}\text { PRECIP. (CM.) } \\
\text { SR-90 (MCI/SQ-KM.) } \\
\text { SR-90 CONC. }(P C / L) \\
\text { SR-89/SR-90 }\end{array}$ & $=$ & $\begin{array}{l}=- \\
-- \\
--\end{array}$ & $\begin{array}{l}-- \\
-- \\
--\end{array}$ & $\begin{array}{l}-- \\
--\end{array}$ & $=$ & $\begin{array}{l}= \\
=- \\
--\end{array}$ & $\overline{-}$ & $\begin{array}{l}-- \\
--\end{array}$ & $\begin{array}{l}-- \\
0.02 \\
--\end{array}$ & $\begin{array}{l}16.18 \\
* \\
-\end{array}$ & $\begin{array}{l}22.20 \\
0.01 \\
0.05 \\
*\end{array}$ & $\begin{array}{l}51.69 \\
* \\
\vdots\end{array}$ & $\begin{array}{r}90.07 \\
0.03\end{array}$ \\
\hline 1960 & $\begin{array}{l}\text { PRECIP. (CM.) } \\
\text { SR-9C INCI/SQ.KM.) } \\
\text { SR-9C CONC. }(P C / L) \\
S R-89 / S R-90\end{array}$ & $\begin{array}{l}-- \\
-- \\
--\end{array}$ & $\begin{array}{l}21.21 \\
-- \\
--\end{array}$ & $\begin{array}{l}12.01 \\
0.04 \\
0.34 \\
--\end{array}$ & $\begin{array}{l}11.00 \\
0.01 \\
0.10 \\
-.\end{array}$ & $\begin{array}{l}14.02 \\
0.37 \\
2.64 \\
-.\end{array}$ & $\begin{array}{l}18.01 \\
=- \\
=\end{array}$ & $\begin{array}{l}10.95 \\
0.26 C \\
2.38 \\
--\end{array}$ & $\begin{array}{l}5.00 \\
0.11 C \\
2.21 \\
-2\end{array}$ & $\begin{array}{l}14.73 \\
0.02 C \\
0.14 \\
-.\end{array}$ & $\begin{array}{l}6.30 \\
0.016 \\
0.16 \\
-.\end{array}$ & $\begin{array}{l}10.49 \\
0.02 C \\
0.20 \\
--\end{array}$ & $\begin{array}{l}17.88 \\
0.02 \mathrm{C} \\
0.12 \\
--\end{array}$ & $\begin{array}{r}141.60 \\
0.86\end{array}$ \\
\hline 1961 & $\begin{array}{l}\text { PRECIP. }\left(C M_{-}\right) \\
S R-90 \text { (KCI/SQ.KM.) } \\
5 R-90 \text { CONC. }(P C / L) \\
S R-89 / S R-90\end{array}$ & $\begin{array}{c}15.37 \\
\star \\
--\end{array}$ & $\begin{array}{l}13.34 \\
* \\
--\end{array}$ & $\begin{array}{l}30.48 \\
0.07 \mathrm{C} \\
0.23 \\
--\end{array}$ & $\begin{array}{l}13.82 \\
0.03 \mathrm{C} \\
0.22 \\
-\end{array}$ & $\begin{array}{l}13.69 \\
0.01 \\
0.08 \\
-\end{array}$ & $\begin{array}{r}11.96 \\
0.50 \\
4.19 \\
-.-\end{array}$ & $\begin{array}{l}11.99 \\
0.03 C \\
0.26 \\
--\end{array}$ & $\begin{array}{l}6.60 \\
0.026 \\
0.31 \\
--\end{array}$ & $\begin{array}{r}14.50 \\
0.03 \\
0.21 \\
3.10\end{array}$ & $\begin{array}{r}5.26 \\
0.02 \\
0.39 \\
16.00\end{array}$ & $\begin{array}{r}21.97 \\
0.03 \\
0.14 \\
55.00\end{array}$ & $\begin{array}{r}28.52 \\
0.04 \\
0.15 \\
53.00\end{array}$ & $\begin{array}{r}187.50 \\
0.78\end{array}$ \\
\hline 1962 & $\begin{array}{l}\text { PRECIP. (CM-) } \\
\text { SR-9O (MCI/SQ.KM.) } \\
\text { SR-9O CONC. }(P C / L) \\
S R-89 / S R-90\end{array}$ & $\begin{array}{r}39.04 \\
0.05 \\
0.13 \\
43.00\end{array}$ & $\begin{array}{r}10.01 \\
0.07 \\
0.70 \\
22.00\end{array}$ & $\begin{array}{r}24.00 \\
0.05 \\
0.21 \\
18.00\end{array}$ & $\begin{array}{r}13.00 \\
0.10 \\
0.77 \\
14.00\end{array}$ & $\begin{array}{r}11.00 \\
0.02 \\
0.19 \\
12.00\end{array}$ & $\begin{array}{r}8.99 \\
0.08 \\
0.85 \\
56.0 C\end{array}$ & $\begin{array}{r}10.87 \\
0.05 \\
C .46 \\
32.00\end{array}$ & $\begin{array}{r}17.50 \\
0.19 \\
1.09 \\
12.00\end{array}$ & $\begin{array}{r}18.01 \\
0.09 \\
1.50 \\
10.00\end{array}$ & $\begin{array}{r}15.49 \\
0.02 \\
0.13 \\
28.00\end{array}$ & $\begin{array}{r}20.42 \\
0.05 \\
0.25 \\
25.00\end{array}$ & $\begin{array}{r}39.88 \\
0.08 \\
0.21 \\
41.00\end{array}$ & $\begin{array}{r}228.21 \\
0.85\end{array}$ \\
\hline 1963 & $\begin{array}{l}\text { PRECIP, (CM.) } \\
\text { SR-9O (NCI/SQ.KM.) } \\
\text { SR-9O CONC. }(P C / L) \\
S R-89 / S R-90\end{array}$ & $\begin{array}{r}22.63 \\
0.10 \\
0.45 \\
46.00\end{array}$ & $\begin{array}{r}15.14 \\
0.12 \\
0.80 \\
28.00\end{array}$ & $\begin{array}{r}4.55 \\
0.12 \\
2.64 \\
18.00\end{array}$ & $\begin{array}{l}4.78 \\
0.18 \\
3.77 \\
8.00\end{array}$ & $\begin{array}{r}15.88 \\
0.08 \\
0.51 \\
4.00\end{array}$ & $\begin{array}{r}17.02 \\
0.08 \\
0.48 \\
3.00\end{array}$ & $\begin{array}{l}16.64 \\
C .1 \mathrm{C} \\
C .61 \\
*\end{array}$ & $\begin{array}{l}7.70 \\
0.06 \\
0.78 \\
*\end{array}$ & $\begin{array}{l}17.42 \\
C .03 \\
C .18 \\
*\end{array}$ & $\begin{array}{l}7.34 \\
C .07 \\
C .96 \\
*\end{array}$ & $\begin{array}{l}15.19 \\
6.04 \\
0.27 \\
\$\end{array}$ & $\begin{array}{l}46.84 \\
0.06 \\
0.13 \\
\$\end{array}$ & $\begin{array}{r}191.13 \\
1.04\end{array}$ \\
\hline 1964 & $\begin{array}{l}\text { PRECIP, (CM-) } \\
S R-S C \text { (NCI/SQ.KM.) } \\
S R-90 \text { CONC. }(P C / L) \\
S R-89 / S R-90\end{array}$ & $\begin{array}{l}15.39 \\
0.10 \\
0.65 \\
+\end{array}$ & $\begin{array}{l}43.43 \\
0.02 \\
0.05 \\
+\end{array}$ & $\begin{array}{r}23.67 \\
0.19 \\
0.81 \\
4.70\end{array}$ & $\begin{array}{l}34.59 \\
0.22 \\
0.64 \\
*\end{array}$ & $\begin{array}{l}12.50 \\
0.09 \\
0.73 \\
*\end{array}$ & $\begin{array}{l}10.95 \\
0.08 \\
0.74 \\
--\end{array}$ & $\begin{array}{r}25.43 \\
C .04 \\
0.16 \\
--\end{array}$ & $\begin{array}{l}13.51 \\
0.01 \\
0.08 \\
-\end{array}$ & $\begin{array}{r}14.71 \\
6.07 \\
0.48 \\
0\end{array}$ & $\begin{array}{l}32.92 \\
0.02 \\
0.07 \\
*\end{array}$ & $\begin{array}{l}10.21 \\
0.02 \\
0.20 \\
*\end{array}$ & $\begin{array}{l}44.15 \\
0.06 \\
0.14 \\
-\end{array}$ & $\begin{array}{r}281.46 \\
0.92\end{array}$ \\
\hline 1965 & $\begin{array}{l}\text { PRECIP, (CM.) } \\
\text { SR-9O (NCI/SQ.KM.) } \\
\text { SR-9O CONC. (PC/L) } \\
\text { SR-8G/SR-9O }\end{array}$ & $\begin{array}{l}2.21 \\
0.01 \\
0.46 \\
=-\end{array}$ & $\begin{array}{l}29.51 \\
0.02 \\
0.07 \\
--\end{array}$ & $\begin{array}{l}19.20 \\
0.03 \\
0.16 \\
-.\end{array}$ & $\begin{array}{l}7.42 \\
0.03 \\
0.41 \\
-.\end{array}$ & $\begin{array}{l}26.52 \\
0.03 \\
0.12 \\
-.\end{array}$ & $\begin{array}{l}18.62 \\
0.03 \\
0.17 \\
--\end{array}$ & $\begin{array}{l}2.90 \\
0.01 \\
0.35 \\
--\end{array}$ & $\begin{array}{l}12.01 \\
0.03 \\
0.25 \\
-\end{array}$ & $\begin{array}{r}12.01 \\
0.40 \\
3.34 \\
--\end{array}$ & $\begin{array}{r}22.91 \\
0.05 \\
0.22 \\
--\end{array}$ & $\begin{array}{l}-\infty \\
-- \\
--\end{array}$ & $\begin{array}{l}-\infty \\
0.02 \\
--\end{array}$ & $\begin{array}{r}153.31 \\
0.66\end{array}$ \\
\hline 1966 & $\begin{array}{l}\text { PRECIP. (CM.) } \\
\text { SR-9O (MCI/SQ.KM.) } \\
\text { SR-9O CONC. (PC/L) } \\
\text { SR-89/SR-90 }\end{array}$ & $\begin{array}{l}26.20 \\
0.06 \\
0.23 \\
-.\end{array}$ & $\begin{array}{l}12.00 \\
0.12 \\
1.01 \\
--\end{array}$ & $\begin{array}{l}28.30 \\
* \\
=-\end{array}$ & $\begin{array}{l}16.40 \\
= \\
=-\end{array}$ & $\begin{array}{l}23.40 \\
0.05 \\
0.22 \\
*\end{array}$ & $\begin{array}{l}9.90 \\
0.02 \\
0.21 \\
+\end{array}$ & $\begin{array}{l}14.90 \\
0.01 \\
C .07 \\
*\end{array}$ & $\frac{0.02}{16.00}$ & $\begin{array}{l}-\infty \\
0.02 \\
*\end{array}$ & $\begin{array}{l}-\overline{0.03} \\
\overline{39.00}\end{array}$ & $\begin{array}{c}\overline{0.02} \\
\overline{33.00}\end{array}$ & $\begin{array}{l}27.94 \\
0.02 \\
0.08 \\
*\end{array}$ & $\begin{array}{r}159.04 \\
0.37\end{array}$ \\
\hline 1967 & $\begin{array}{l}\text { PRECIP, (CM.) } \\
\text { SR-90 (YCI/SQ.KM.) } \\
\text { SR-9O CCNC. (PC/L) } \\
\text { SR-89/SR-90 }\end{array}$ & $\begin{array}{l}49.02 \\
0.01 \\
0.03 \\
*\end{array}$ & $\begin{array}{l}48.26 \\
0.03 \\
0.07 \\
*\end{array}$ & $\begin{array}{l}35.56 \\
0.01 \\
0.03 \\
*\end{array}$ & $\begin{array}{l}25.40 \\
0.02 \\
0.08 \\
*\end{array}$ & $\begin{array}{l}25.40 \\
C .01 \\
0.04 \\
*\end{array}$ & $\begin{array}{l}14.20 \\
0.04 \\
0.29 \\
*\end{array}$ & $=$ & $\overline{-}$ & $\begin{array}{l}-- \\
-- \\
--\end{array}$ & $=$ & $\begin{array}{l}-- \\
-- \\
-\infty\end{array}$ & $\begin{array}{l}-- \\
--\end{array}$ & $\begin{array}{r}197.84 \\
0.12\end{array}$ \\
\hline $\begin{aligned} \text { NOTES } & \\
-D: & D \\
*: & Z \\
A: & A \\
B: & L \\
C: & P\end{aligned}$ & 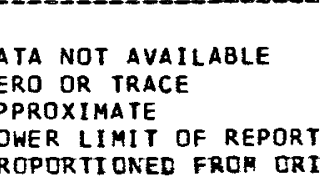 & DATA & & & & & & & & & & & & \\
\hline
\end{tabular}




\begin{tabular}{|c|c|c|c|c|c|c|c|c|c|c|c|c|c|c|}
\hline & & JAN. & FEB. & MAR. & APR. & MAY & JUNE & JULY & AUG. & SEP. & OCT. & NOV. & DEC. & CUM. TOTAL \\
\hline 1957 & $\begin{array}{l}\text { PRECIP. }\left(C M_{-}\right) \\
S R-90 \text { (MC I/SO.KM- }) \\
S R-90 \text { CONC. }(P C / L) \\
S R-89 / S R-90\end{array}$ & $\begin{array}{l}= \\
=- \\
=\end{array}$ & $=$ & $\begin{array}{l}= \\
=-\end{array}$ & $\bar{z}$ & $\bar{z}$ & $\begin{array}{l}0.99 \\
0.03 \\
3.04 \\
-.\end{array}$ & $\begin{array}{l}0.99 \\
-- \\
--\end{array}$ & $\begin{array}{l}1.98 \\
0.04 \\
2.03 \\
-\end{array}$ & $\begin{array}{l}11.79 \\
0.09 \\
0.77 \\
--\end{array}$ & $\begin{array}{l}8.92 \\
0.09 \\
1.01 \\
. .\end{array}$ & $\begin{array}{l}7.92 \\
0.12 \\
1.52 \\
--.\end{array}$ & $\begin{array}{l}14.86 \\
0.08 \\
0.54 \\
--\end{array}$ & $\begin{array}{r}47.45 \\
0.45\end{array}$ \\
\hline 1958 & $\begin{array}{l}\text { PRECIP. (CM.) } \\
\text { SR-SC IMCI/SO.KM.) } \\
S R-90 \text { CONC. }(P C / L) \\
S R-89 / S R-90\end{array}$ & $\begin{array}{l}24.77 \\
0.01 \\
0.05 \\
*\end{array}$ & $\begin{array}{r}26.75 \\
0.07 \\
0.27 \\
16.00\end{array}$ & $\begin{array}{r}11.89 \\
0.03 \\
0.26 \\
2.00\end{array}$ & $\begin{array}{l}29.72 \\
0.18 \\
0.61 \\
*\end{array}$ & $\begin{array}{r}0.99 \\
0.35 \\
35.36 \\
-\end{array}$ & $\begin{array}{r}1.98 \\
0.02 \\
1.02 \\
34.00\end{array}$ & $\begin{array}{l}1.98 \\
0.17 \\
8.59 \\
4.00\end{array}$ & $\begin{array}{r}0.64 \\
0.01 \\
1.57 \\
15.00\end{array}$ & $\begin{array}{l}7.92 \\
0.15 \\
1.90 \\
32.00\end{array}$ & $\begin{array}{r}2.97 \\
0.15 \\
5.00 \\
16.00\end{array}$ & $\begin{array}{r}10.90 \\
0.23 \\
2.12 \\
14.00\end{array}$ & $\begin{array}{r}9.02 \\
0.23 \\
2.55 \\
12.00\end{array}$ & $\begin{array}{r}129.53 \\
1.60\end{array}$ \\
\hline 1959 & $\begin{array}{l}\text { PRECIP. }\left(C M_{-}\right) \\
S R-9 C \text { (PCI/SQ-KM) } \\
S R-90 \text { CONC. }(P C / L) \\
S R-89 / S R-90\end{array}$ & $\begin{array}{r}13.00 \\
0.22 \\
1.70 \\
3.00\end{array}$ & $\begin{array}{l}7.01 \\
0.05 \\
0.72 \\
5.00\end{array}$ & $\begin{array}{l}2.01 \\
-- \\
--\end{array}$ & $\begin{array}{l}3.00 \\
0.07 \\
2.34 \\
3.00\end{array}$ & $\begin{array}{l}15.61 \\
0.09 \\
0.60 \\
2.30\end{array}$ & $\frac{*}{0.02}$ & $\begin{array}{r}11.00 \\
0.03 \\
0.28 \\
4.70\end{array}$ & $\begin{array}{l}0.00 \\
0.07 \\
0.88 \\
*\end{array}$ & $\begin{array}{l}4.01 \\
\therefore- \\
=\end{array}$ & $\begin{array}{l}8.00 \\
0.09 \\
2.13\end{array}$ & $\begin{array}{l}7.01 \\
0.17 \\
2.43 \\
*\end{array}$ & $\begin{array}{l}7.01 \\
0.10 \\
1.43 \\
2.90\end{array}$ & $\begin{array}{r}85.06 \\
0.91\end{array}$ \\
\hline 1960 & $\begin{array}{l}\text { PRECIP (CM.) } \\
\text { SR-9C (NCI/SQ-KM.) } \\
S R-9 C \text { CCNC. }(P C / L) \\
S R-89 / S R-90\end{array}$ & $\begin{array}{l}7.01 \\
0.11 \\
1.57 \\
-.\end{array}$ & $\begin{array}{l}9.02 \\
0.08 \\
0.89 \\
-.\end{array}$ & $\begin{array}{l}5.99 \\
0.03 \\
0.51 \\
-.-\end{array}$ & $\begin{array}{l}8.00 \\
-- \\
-\end{array}$ & $\begin{array}{l}0.99 \\
0.01 \mathrm{C} \\
1.02 \\
-.\end{array}$ & $\begin{array}{l}0.20 \\
0.0 c c \\
0.01 \\
-.\end{array}$ & $\begin{array}{l}0.99 \\
0.24 C \\
24.25 \\
-.\end{array}$ & $\begin{array}{l}0.10 \\
c . c 3 c \\
30.01 \\
-.\end{array}$ & $\begin{array}{l}5.00 \\
c .05 C \\
1.01 \\
-.\end{array}$ & $\begin{array}{l}8.99 \\
c .10 \mathrm{C} \\
1.12 \\
=-\end{array}$ & $\begin{array}{l}16.00 \\
-- \\
-\end{array}$ & $\begin{array}{l}16.99 \\
-- \\
=-\end{array}$ & $\begin{array}{r}79.28 \\
0.65\end{array}$ \\
\hline 1961 & $\begin{array}{l}\text { PRECIP. }(C M-1) \\
\text { SR-9C }(M C I / S Q . K M=) \\
S R-90 \text { CONC. }(P C / L) \\
S R-89 / S R-90\end{array}$ & $\begin{array}{l}11.99 \\
0.15 C \\
1.26 \\
-.\end{array}$ & $\begin{array}{l}7.01 \\
0.086 \\
1.15 \\
-.\end{array}$ & $\begin{array}{l}13.00 \\
C .08 \mathrm{C} \\
0.62 \\
=-\end{array}$ & $\begin{array}{l}27.00 \\
0.16 \mathrm{C} \\
0.60 \\
--\end{array}$ & $\begin{array}{l}7.01 \\
c .03 c \\
0.43 \\
-.\end{array}$ & $\begin{array}{l}14.00 \\
0.06 C \\
0.43 \\
=-\end{array}$ & $\begin{array}{l}5.00 \\
0.08 C \\
1.61 \\
-.\end{array}$ & $\begin{array}{l}4.01 \\
0.076 \\
1.75 \\
-=\end{array}$ & $\begin{array}{l}7.01 \\
0.11 \\
1.57 \\
+\end{array}$ & $\begin{array}{l}8.99 \\
0.21 \\
2.34 \\
\end{array}$ & $\begin{array}{l}7.98 \\
0.25 \\
3.14 \\
*\end{array}$ & $\begin{array}{l}7.98 \\
0.21 \\
2.64 \\
*\end{array}$ & $\begin{array}{r}120.98 \\
1.49\end{array}$ \\
\hline 1962 & $\begin{array}{l}\text { PRECIP. (CM.) } \\
\text { SR-9C (MCI/SQ-KM.) } \\
S R-90 \text { CCNC. }(P C / L) \\
\text { SR-89/SR-9O }\end{array}$ & $\begin{array}{l}9.02 \\
0.19 \\
2.11 \\
\end{array}$ & $\begin{array}{l}8.99 \\
0.21 \\
2.34 \\
\end{array}$ & $\begin{array}{r}11.99 \\
0.15 \\
1.26 \\
1.26\end{array}$ & $\begin{array}{l}3.99 \\
0.05 \\
1.26 \\
*\end{array}$ & $\begin{array}{l}5.00 \\
* \\
*\end{array}$ & $\begin{array}{r}0.10 \\
0.07 \\
70.01 \\
7.00\end{array}$ & $\begin{array}{l}-- \\
:- \\
*\end{array}$ & $\frac{\overline{0.09}}{25.00}$ & $\begin{array}{c}0.10 \\
17.00\end{array}$ & $\begin{array}{c}\overline{0.18} \\
\overline{12.00}\end{array}$ & $\begin{array}{l}\overline{0.29} \\
\overline{11.00}\end{array}$ & $\begin{array}{l}-\overline{0.17} \\
8.00\end{array}$ & $\begin{array}{r}39.09 \\
1.50\end{array}$ \\
\hline 1963 & $\begin{array}{l}\text { PRECIP. (CM.) } \\
\text { SR-9O INCI/SQ.KM.) } \\
\text { SR-9O CCNC. }(P C / L) \\
\text { SR-89/SR-9O }\end{array}$ & $\begin{array}{l}= \\
=\end{array}$ & $\begin{array}{l}0.20 \\
\overline{0.00}\end{array}$ & $\begin{array}{l}\overline{0.26} \\
\overline{24.00}\end{array}$ & $\begin{array}{l}\overline{0.04} \\
\overline{15.00}\end{array}$ & $\begin{array}{l}1.91 \\
0.03 \\
1.58 \\
2.20\end{array}$ & $\begin{array}{l}3.10 \\
0.05 \\
1.62 \\
+\end{array}$ & $\begin{array}{r}14.48 \\
0.20 \\
1.39 \\
*\end{array}$ & $\begin{array}{l}1.52 \\
0.04 \\
2.64 \\
*\end{array}$ & $\begin{array}{l}4.50 \\
0.02 \\
0.45 \\
*\end{array}$ & $\begin{array}{l}7.98 \\
0.25 \\
3.14 \\
*\end{array}$ & $\begin{array}{l}4.32 \\
0.15 \\
3.48 \\
*\end{array}$ & $\begin{array}{l}18.29 \\
0.14 \\
0.77 \\
*\end{array}$ & $\begin{array}{r}56.10 \\
1.38\end{array}$ \\
\hline 1964 & $\begin{array}{l}\text { PRECIP. (CM.) } \\
\text { SR-9O (MCI/SQ-KM-) } \\
\text { SR-90 CQNC. (PC/L) } \\
\text { SR-89/SR-9O }\end{array}$ & $\begin{array}{l}19.81 \\
0.31 \\
1.57 \\
*\end{array}$ & $\begin{array}{l}7.42 \\
0.41 \\
5.53 \\
+\end{array}$ & $\begin{array}{l}9.40 \\
0.06 \\
0.64 \\
+\end{array}$ & $\begin{array}{l}5.59 \\
0.12 \\
2.15 \\
*\end{array}$ & $\begin{array}{l}3.43 \\
0.05 \\
1.46 \\
-.\end{array}$ & $\begin{array}{l}5.51 \\
0.17 \\
3.09 \\
--\end{array}$ & $\begin{array}{l}3.45 \\
0.10 \\
2.90 \\
-.\end{array}$ & $\begin{array}{l}0.51 \\
0.07 \\
13.73 \\
--\end{array}$ & $\begin{array}{l}4.45 \\
0.22 \\
4.95 \\
-.\end{array}$ & $\begin{array}{l}24.69 \\
0.23 \\
0.94 \\
+\end{array}$ & $\begin{array}{l}8.64 \\
0.25 \\
2.90 \\
*\end{array}$ & $\begin{array}{l}8.13 \\
0.12 \\
1.48 \\
-\end{array}$ & $\begin{array}{r}101.03 \\
2.11\end{array}$ \\
\hline 1965 & $\begin{array}{l}\text { PRECIP. (CM.) } \\
\text { SR-90 (NCI/SQ-KM-) } \\
S R-90 \text { CONC. }(P C / L) \\
S R-89 / S R-90\end{array}$ & $\begin{array}{l}9.65 \\
0.44 \\
4.56 \\
--\end{array}$ & $\begin{array}{r}13.34 \\
0.12 \\
0.90 \\
\end{array}$ & $\begin{array}{l}1.27 \\
0.12 \\
9.45 \\
-\end{array}$ & $\begin{array}{l}1.91 \\
0.02 \\
1.05 \\
--\end{array}$ & $\begin{array}{l}6.99 \\
0.11 \\
1.58 \\
-.\end{array}$ & $\begin{array}{r}14.53 \\
0.34 \\
2.34 \\
\end{array}$ & $\begin{array}{l}4.17 \\
0.16 \\
3.84 \\
-\end{array}$ & $\begin{array}{l}10.31 \\
0.04 \\
0.39 \\
\end{array}$ & $\begin{array}{l}9.70 \\
0.39 \\
4.03 \\
--\end{array}$ & $\begin{array}{l}8.64 \\
0.27 \\
3.13 \\
-.\end{array}$ & $\begin{array}{l}13.00 \\
0.23 \\
1.77 \\
-.\end{array}$ & $\begin{array}{l}7.37 \\
0.21 \\
2.85 \\
--.\end{array}$ & $\begin{array}{r}100.68 \\
2.45\end{array}$ \\
\hline 1966 & $\begin{array}{l}\text { PRECIP. (CM.) } \\
S R-9 C \text { (NCI/SO.KM-) } \\
S R-90 \text { CONC. }(P C / L) \\
S R-89 / S R-90\end{array}$ & $\begin{array}{l}13.51 \\
0.13 \\
0.97 \\
\end{array}$ & $\begin{array}{r}10.03 \\
0.13 \\
1.30 \\
-\end{array}$ & $\begin{array}{l}2.13 \\
0.03 \\
1.41 \\
-.\end{array}$ & $\begin{array}{l}2.41 \\
0.07 \\
2.91 \\
-.\end{array}$ & $\begin{array}{l}10.41 \\
0.05 \\
0.49 \\
--\end{array}$ & $\begin{array}{l}2.92 \\
0.03 \\
1.03 \\
--\end{array}$ & $\begin{array}{l}1.65 \\
0.02 \\
1.22 \\
-\end{array}$ & $\begin{array}{l}1.65 \\
0.05 \\
3.04 \\
-\end{array}$ & $\begin{array}{l}4.11 \\
0.08 \\
1.95 \\
-.\end{array}$ & $\begin{array}{l}4.14 \\
0.18 \\
4.35 \\
-.\end{array}$ & $\begin{array}{r}13.00 \\
0.15 \\
1.16 \\
9.80\end{array}$ & $\begin{array}{r}6.22 \\
0.14 \\
2.26 \\
12.00\end{array}$ & $\begin{array}{r}72.18 \\
1.06\end{array}$ \\
\hline 1967 & $\begin{array}{l}\text { PRECIP. (CM.) } \\
\text { SR-90 (MCI/SQ.KM.) } \\
S R-90 \text { CCNC. (PCILL) } \\
\text { SR-89/SR-90 }\end{array}$ & $\begin{array}{l}6.81 \\
0.22 \\
3.24 \\
2.30\end{array}$ & $\begin{array}{r}21.21 \\
0.10 \\
0.48 \\
3.80\end{array}$ & $\begin{array}{r}24.71 \\
0.10 \\
0.41 \\
.\end{array}$ & $\begin{array}{l}10.21 \\
0.04 \\
0.40 \\
*\end{array}$ & $\begin{array}{l}1.90 \\
0.02 \\
1.06 \\
*\end{array}$ & $\begin{array}{l}-- \\
-- \\
--\end{array}$ & $\bar{m}$ & $\begin{array}{l}\bar{z} \\
\bar{z}\end{array}$ & $\begin{array}{l}-- \\
\overline{--} \\
--\end{array}$ & $=-$ & $\begin{array}{l}= \\
\overline{z-} \\
=\end{array}$ & $=$ & $\begin{array}{r}64.84 \\
0.48\end{array}$ \\
\hline \multicolumn{15}{|c|}{$\begin{array}{l}\text { NOTES } \\
\text { :: DATA NOT AVAILABLE } \\
\text { *: LERO OR TRACE } \\
\text { A: APPROXIMATE } \\
\text { B: LOHER LIMII OF REPORTED DATA } \\
\text { C: PROPORTIONED FROM ORIGINALLY }\end{array}$} \\
\hline
\end{tabular}


SITE: REP. S. AFRICA, PRETORIA

LAT. 25455 LONG. 28 14E ALT. 1369M. (POT) SOURCE: NATIONAL PHYSICAL RESEARCH LABORATORY TO MAY 1967
ATOMIC ENERGY BOARD AT PELINDABA FROM JUNE 1967

\begin{tabular}{|c|c|c|c|c|c|c|c|c|c|c|c|c|c|c|}
\hline & & JAN. & FEB. & MAR: & APR. & MAY & JUNE & JULY & AUG. & SEP. & OCT. & Nov. & OEC. & CUM. TOTAL \\
\hline 1957 & $\begin{array}{l}\text { PRECIP. (CM.) } \\
\text { SR-90 IMCI/SQ.KM.) } \\
\text { SR-90 CDNC. (PC/L) } \\
\text { SR-89/SR-90 }\end{array}$ & $=$ & $\bar{I}$ & $\begin{array}{l}=- \\
\bar{z}\end{array}$ & $\begin{array}{l}\overline{-} \\
\overline{-}\end{array}$ & $\begin{array}{l}= \\
=\end{array}$ & $\begin{array}{l}= \\
=-\end{array}$ & $\begin{array}{r}10.90 \\
0.02 \\
0.19 \\
\end{array}$ & $\begin{array}{l}3.96 \\
0.03 \\
0.76 \\
-\end{array}$ & $\begin{array}{r}11.89 \\
0.17 \\
1.43 \\
-\end{array}$ & $\begin{array}{l}7.92 \\
0.07 \\
0.89 \\
--\end{array}$ & $\overline{0}=$ & $\begin{array}{l}1.60 \\
0.05 \\
3.13 \\
-2\end{array}$ & $\begin{array}{r}36.27 \\
0.38\end{array}$ \\
\hline 1958 & $\begin{array}{l}\text { PRECIP. (CM-) } \\
\text { SR-9C (MCI/SO-KM-) } \\
\text { SR-9C CCNC. }(P C / L) \\
\text { SR-89/SR-90 }\end{array}$ & $\begin{array}{r}12.47 \\
0.04 \\
0.33 \\
29.00\end{array}$ & $\begin{array}{l}2.87 \\
0.02 \\
0.70 \\
3.00\end{array}$ & $\begin{array}{l}8.43 \\
0.04 \\
0.48 \\
3.00\end{array}$ & $\begin{array}{l}8.61 \\
0.07 \\
0.82 \\
3.00\end{array}$ & $\begin{array}{l}2.34 \\
0.19 \\
8.12 \\
--\end{array}$ & $\begin{array}{r}0.10 \\
0.01 \\
10.01 \\
38.00\end{array}$ & $\frac{0.02}{39.00}$ & $\begin{array}{l}* \\
0.00 \\
-\end{array}$ & $\begin{array}{r}3.96 \\
6.23 \\
5.81 \\
60.00\end{array}$ & $\begin{array}{r}5.94 \\
6.33 \\
5.56 \\
33.00\end{array}$ & $\begin{array}{r}10.90 \\
0.14 \\
1.29 \\
17.00\end{array}$ & $\begin{array}{r}19.02 \\
0.29 \\
1.53 \\
19.00\end{array}$ & $\begin{array}{r}74.64 \\
1.38\end{array}$ \\
\hline 1959 & $\begin{array}{l}\text { PRECIP. }\left(C M_{-}\right) \\
\text {SR-9O } \text { iNCI/SO.KM-) } \\
\text { SR-90 CONC. (PC/L) } \\
\text { SR-8S/SR-SO }\end{array}$ & $\begin{array}{r}19.02 \\
0.19 \\
1.00 \\
7.00\end{array}$ & $\begin{array}{r}10.21 \\
0.20 \\
1.96 \\
3.00\end{array}$ & $\begin{array}{r}0.46 \\
0.05 \\
10.87 \\
4.80\end{array}$ & $\begin{array}{l}4.01 \\
0.06 \\
1.50 \\
0.58\end{array}$ & $\begin{array}{l}2.01 \\
0.03 \\
1.50 \\
\end{array}$ & $\frac{*}{0.01}$ & $\begin{array}{l}0.99 \\
0.02 \\
2.03 \\
*\end{array}$ & $\begin{array}{l}* \\
*- \\
--\end{array}$ & $\begin{array}{l}0.99 \\
0.05 \\
5.06 \\
\end{array}$ & $\begin{array}{r}2.01 \\
0.03 \\
1.50 \\
97.00\end{array}$ & $\begin{array}{l}10.01 \\
0.07 \\
c .70 \\
*\end{array}$ & $\begin{array}{l}15.01 \\
0.09 \\
0.00 \\
*\end{array}$ & $\begin{array}{r}64.72 \\
0.80\end{array}$ \\
\hline 1960 & $\begin{array}{l}\text { PREC IP }\left(C M_{-}\right) \\
\text {SR-SC (MCI/SQ-KM.) } \\
\text { SR-9O CCNC (PC/L) } \\
\text { SR-89/SR-90 }\end{array}$ & $\begin{array}{l}7.62 \\
0.02 \\
0.27 \\
--\end{array}$ & $\begin{array}{l}5.99 \\
0.03 \\
0.51 \\
-\end{array}$ & $\begin{array}{l}7.01 \\
0.03 \\
0.43 \\
--\end{array}$ & $\begin{array}{l}7.01 \\
0.03 \\
0.43\end{array}$ & $\begin{array}{l}7.01 \\
0.08 \mathrm{C} \\
1.15\end{array}$ & $\begin{array}{l}c .2 c \\
0 . c c c \\
0.01 \\
--\end{array}$ & $\begin{array}{l}\begin{array}{l}0.10 \\
0.000 \\
0.01\end{array} \\
--\end{array}$ & $\begin{array}{l}0.99 \\
0.006 \\
0.01 \\
-0\end{array}$ & $\begin{array}{l}1.98 \\
c .02 C \\
1.02 \\
-.\end{array}$ & $\begin{array}{l}5.99 \\
c .076 \\
1.27 \\
--\end{array}$ & $\begin{array}{l}16.00 \\
0.10 \mathrm{C} \\
0.63\end{array}$ & $\begin{array}{l}11.00 \\
0.07 C \\
0.64 \\
--\end{array}$ & $\begin{array}{r}70.90 \\
0.45\end{array}$ \\
\hline 1961 & $\begin{array}{l}\text { PRECIP. }(C M-) \\
\text { SR-9O (NCI/SQ.KM-) } \\
\text { SR-SO CCNC. (PC/L) } \\
\text { SR-89/SR-SC }\end{array}$ & $\begin{array}{c}11.00 \\
0.10 \mathrm{C} \\
0.91 \\
-\end{array}$ & $\begin{array}{l}5.00 \\
0.05 C \\
1.01 \\
--\end{array}$ & $\begin{array}{l}8.00 \\
0.036 \\
0.38 \\
--\end{array}$ & $\begin{array}{l}11.00 \\
0.05 \mathrm{C} \\
0.46 \\
--\end{array}$ & $\begin{array}{l}3.48 \\
0.04 C \\
1.15 \\
--\end{array}$ & $\begin{array}{l}0.99 \\
0.016 \\
1.02 \\
--\end{array}$ & * & $\begin{array}{l}* \\
* \\
-\end{array}$ & $\begin{array}{l}3.99 \\
0.18 \\
4.52 \\
*\end{array}$ & $\begin{array}{l}16.01 \\
c .10 \\
1.00 \\
*\end{array}$ & $\begin{array}{l}11.00 \\
22.73\end{array}$ & $\begin{array}{l}7.44 \\
0.20 \\
2.69 \\
*\end{array}$ & $\begin{array}{r}71.91 \\
0.76\end{array}$ \\
\hline 1962 & $\begin{array}{l}\text { PRECIP. }(C M-) \\
\text { SR-SO (NCI/SO.KM.) } \\
\text { SR-SC CONC. }(P C / L) \\
\text { SR-89/SR-90 }\end{array}$ & $\begin{aligned} 10.01 \\
0.09 \\
0.90 \\
*\end{aligned}$ & $\begin{array}{r}12.62 \\
0.09 \\
0.72 \\
*\end{array}$ & $\begin{array}{l}6.60 \\
0.13 \\
1.97 \\
+\end{array}$ & $\begin{array}{l}3.40 \\
0.04 \\
1.18 \\
+\end{array}$ & $\frac{0.01}{*}$ & $\begin{array}{l}* \\
0.01 \\
85 . c c\end{array}$ & $\begin{array}{l}* \\
=- \\
=\end{array}$ & $\begin{array}{r}0.13 \\
0.05 \\
38.47 \\
19.00\end{array}$ & $\begin{array}{r}0.03 \\
0.02 \\
66.67 \\
14.00\end{array}$ & $\begin{array}{r}2.97 \\
6.26 \\
8.76 \\
25.00\end{array}$ & $\begin{array}{r}17.60 \\
0.19 \\
1.08 \\
12.00\end{array}$ & $\begin{array}{l}10.01 \\
-\therefore \\
--\end{array}$ & $\begin{array}{r}63.37 \\
0.89\end{array}$ \\
\hline 1963 & $\begin{array}{l}\text { PRECIP. (CM.) } \\
\text { SR-9C (NCI/SQ.KM-) } \\
\text { SR-SC CCNC. }(P C / L) \\
S R-89 / S R-S C\end{array}$ & $\begin{array}{r}13.03 \\
0.22 \\
1.69 \\
8.00\end{array}$ & $\begin{array}{l}1.30 \\
0.03 \\
2.31 \\
6.50\end{array}$ & $\begin{array}{r}1.09 \\
0.02 \\
1.84 \\
23.00\end{array}$ & $\begin{array}{r}12.93 \\
0.05 \\
0.39 \\
4.50\end{array}$ & $\begin{array}{l}0.74 \\
0.03 \\
4.06 \\
2.40\end{array}$ & $\begin{array}{l}5.51 \\
0.11 \\
2.00 \\
*\end{array}$ & $\begin{array}{l}1.07 \\
0.02 \\
1.87 \\
*\end{array}$ & $\frac{c}{c}$ & $\begin{array}{r}0.03 \\
c .03 \\
10 c .00 \\
*\end{array}$ & $\begin{array}{l}2.51 \\
c .10 \\
3.99 \\
*\end{array}$ & $\begin{array}{l}9.04 \\
0.12 \\
1.33 \\
*\end{array}$ & $\begin{array}{l}9.17 \\
0.34 \\
3.71 \\
*\end{array}$ & $\begin{array}{r}56.42 \\
1.12\end{array}$ \\
\hline 1964 & $\begin{array}{l}\text { PRECIP. }(C M-1 \\
\text { SR-9D (MCI/SQ-KM-) } \\
\text { SR-SC CCNC. }(P C / L) \\
\text { SR-S9/SR-9O }\end{array}$ & $\begin{array}{l}17.65 \\
0.26 \\
1.48 \\
*\end{array}$ & $\begin{array}{r}0.61 \\
C .07 \\
11.48 \\
0.60\end{array}$ & $\begin{array}{l}5.05 \\
0.03 \\
0.60 \\
*\end{array}$ & $\begin{array}{l}2.72 \\
0.19 \\
6.99 \\
*\end{array}$ & $\begin{array}{l}0.41 \\
0.03 \\
7.32 \\
*\end{array}$ & $\begin{array}{r}0.46 \\
0.16 \\
34.79 \\
--\end{array}$ & $\frac{0.02}{-}$ & $\frac{0.01}{--}$ & $\begin{array}{r}1.14 \\
0.22 \\
19.30 \\
-. .\end{array}$ & $\begin{array}{r}14.48 \\
C .44 \\
3.04 \\
1.90\end{array}$ & $\begin{array}{l}1.27 \\
0.02 \\
1.58 \\
*\end{array}$ & $\begin{array}{l}10.41 \\
0.08 \\
0.77 \\
-.\end{array}$ & $\begin{array}{r}54.20 \\
1.53\end{array}$ \\
\hline 1965 & $\begin{array}{l}\text { PRECIP }\left(C M_{-}\right) \\
S R-90 \text { iMCI/SQ-KM.) } \\
S R \rightarrow 90 \text { CONC. (PCAL) } \\
S R-89 / S R-90\end{array}$ & $\begin{array}{l}6.60 \\
0.29 \\
4.40 \\
-2\end{array}$ & $\begin{array}{l}3.56 \\
0.08 \\
2.25 \\
--\end{array}$ & $\begin{array}{l}2.34 \\
0.06 \\
2.57 \\
--\end{array}$ & $\begin{array}{l}7.92 \\
0.06 \\
0.76 \\
--\end{array}$ & $\begin{array}{l}* \\
0.03 \\
=-\end{array}$ & $\begin{array}{l}* \\
0.00 \\
--\end{array}$ & $\begin{array}{l}0.61 \\
0.02 \\
3.28 \\
--\end{array}$ & $\begin{array}{l}* \\
0.37 \\
--\end{array}$ & $\begin{array}{r}0.48 \\
0.11 \\
22.92 \\
-\end{array}$ & $\begin{array}{l}1.30 \\
0.12 \\
5.24 \\
--\end{array}$ & $\begin{array}{l}3.48 \\
0.08 \\
2.30 \\
--\end{array}$ & $\begin{array}{l}5.89 \\
0.17 \\
2.89 \\
--\end{array}$ & $\begin{array}{r}32.18 \\
1.39\end{array}$ \\
\hline 1966 & $\begin{array}{l}\text { PRECIP. (CM-) } \\
\text { SR-SC (MCI/SQ.KM.) } \\
\text { SR-SO CONC. (PC/L) } \\
\text { SR-89/SR-9O }\end{array}$ & $\begin{array}{r}1 C .03 \\
c .14 \\
1.40 \\
-=\end{array}$ & $\begin{array}{l}9.14 \\
0.07 \\
0.77 \\
--\end{array}$ & $\begin{array}{l}2.54 \\
0.62 \\
0.79 \\
-\end{array}$ & $\begin{array}{l}0.90 c \\
0.01 \\
1.12 \\
-\end{array}$ & $\begin{array}{l}0.90 \mathrm{C} \\
0.02 \\
2.23 \\
--\end{array}$ & $\begin{array}{l}* \\
0.01 \\
=-\end{array}$ & $\begin{array}{l}* \\
0.02 \\
--\end{array}$ & $\begin{array}{l}* \\
0.01 \\
=-\end{array}$ & $\begin{array}{l}0.66 \\
0.06 \\
9.10 \\
--\end{array}$ & $\begin{array}{l}13.46 \\
0.33 \\
2.46 \\
--\end{array}$ & $\begin{array}{r}6.60 \\
0.25 \\
3.79 \\
28.00\end{array}$ & $\begin{array}{r}26.67 \\
0.20 \\
0.75 \\
16.00\end{array}$ & $\begin{array}{r}70.90 \\
1.13\end{array}$ \\
\hline 1967 & $\begin{array}{l}\text { PRECIP. (CM.) } \\
\text { SR-9C (HCI/SO-KM.) } \\
\text { SR-90 CONC. (PC/L) } \\
\text { SR-89/SR-90 }\end{array}$ & $\begin{array}{r}16.89 \\
0.06 \\
0.36 \\
5.50\end{array}$ & $\begin{array}{l}3.38 \\
0.02 \\
0.60 \\
*\end{array}$ & $\begin{array}{l}12.19 \\
0.12 \\
0.99 \\
*\end{array}$ & $\begin{array}{l}9.60 \\
0.04 \\
0.42 \\
*\end{array}$ & $\begin{array}{l}1.70 \\
0.02 \\
1.18 \\
*\end{array}$ & $\frac{0.01}{26.00}$ & $\bar{z}=$ & $\begin{array}{l}= \\
=- \\
=\end{array}$ & $\begin{array}{l}= \\
= \\
=\end{array}$ & $\begin{array}{l}= \\
= \\
=\end{array}$ & $\begin{array}{l}=- \\
=- \\
=-\end{array}$ & $\begin{array}{l}= \\
=- \\
=\end{array}$ & $\begin{array}{r}43.76 \\
0.27\end{array}$ \\
\hline \multicolumn{15}{|c|}{ 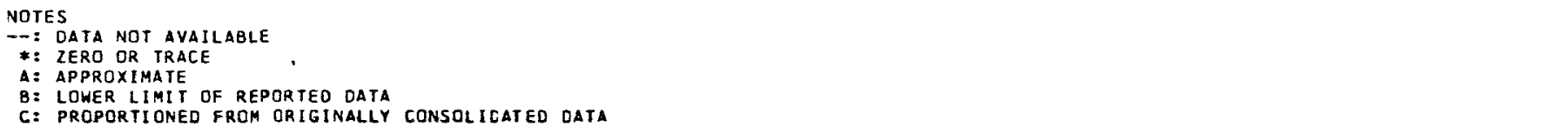 } \\
\hline
\end{tabular}




\section{MONTHLY FALLOUT DEPOSITION COLLECTIONS}
SITE: SYRIA, DAMASCUS
LAT. $3329 \mathrm{~N}$ LONG. 36 I4E ALT.
$734 m$.
(COLUAN)

SOURCE: SYRIAN MINISTRY OF DEFENSE, METEOROLOGICAL DEPARTMENT

\begin{tabular}{|c|c|c|c|c|c|c|c|c|c|c|c|c|c|c|}
\hline & & JAN. & FEB. & MAR. & APR . & MAY & JUNE & JULY & MUG. & SEP. & OCT. & NOV. & DEC. & cum. \\
\hline 1960 & $\begin{array}{l}\text { PRECIP. (CM.) } \\
\text { SR-90 IMCI/SQ.KM.) } \\
\text { SR-90 CONC. (PC/L) } \\
\text { SR-89/SR-90 }\end{array}$ & $=$ & $\begin{array}{l}* \\
0.006 \\
=-\end{array}$ & $\begin{array}{l}13.18 \\
0.06 \mathrm{C} \\
0.46 \\
-\end{array}$ & $\begin{array}{r}0.99 \\
0.20 \\
20.21 \\
-\end{array}$ & 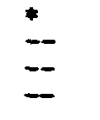 & $\begin{array}{l}\# \\
=- \\
--\end{array}$ & $\stackrel{-}{--}$ & $\overline{-}$ & 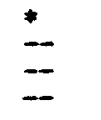 & $=$ & $\begin{array}{l}5.99 \\
0.02 C \\
0.34 \\
-\end{array}$ & $\begin{array}{l}3.00 \\
0.016 \\
0.34 \\
--\end{array}$ & $\begin{array}{r}23.16 \\
0.29\end{array}$ \\
\hline 1961 & $\begin{array}{l}\text { PRECIP. }\left(C M_{-}\right) \\
\text {SR-90 INCI/SQ.KM.) } \\
\text { SR-90 CONC. (PC/L) } \\
\text { SR-89/SR-90 }\end{array}$ & $\begin{array}{l}-\overline{0.19} \\
--\end{array}$ & $\begin{array}{l}-\overline{0.01} \\
--\end{array}$ & $=$ & $\overrightarrow{0.02}$ & $=$ & $=$ & $\bar{m}$ & $\overline{-}$ & $\overline{-}$ & $\begin{array}{l}\bar{z} \\
\bar{z}\end{array}$ & $=$ & $=$ & $\begin{array}{l}0.0 \\
0.22\end{array}$ \\
\hline 1962 & $\begin{array}{l}\text { PRECIP. }\left(C H_{-}\right) \\
\text {SR-90 (MCI/SQ.KM.) } \\
\text { SR-90 CONC. (PC/L) } \\
\text { SR-89/SR-90 }\end{array}$ & $\begin{array}{l}-- \\
--\end{array}$ & $\overline{--}$ & $\overline{-}$ & $\underline{z}$ & $=$ & $\overline{-}$ & $\overline{--}$ & $\overline{-}$ & $\overline{-}$ & $\bar{z}$ & $\overline{-}$ & $\overrightarrow{-}$ & $\begin{array}{l}0.0 \\
0.0\end{array}$ \\
\hline 1963 & $\begin{array}{l}\text { PRECIP, (CM.) } \\
\text { SR-90 (MCI/SO.KM.) } \\
\text { SR-90 CONC. (PC/L) } \\
\text { SR-89/SR-90 }\end{array}$ & $\bar{z}$ & $\overline{-}$ & $=$ & $=$ & $=$ & $\bar{z}$ & $\bar{z}$ & $\bar{z}$ & $\ddot{-}$ & $=$ & 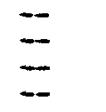 & $\begin{array}{l}\overline{-} \\
\overline{-}\end{array}$ & $\begin{array}{l}0.0 \\
0.0\end{array}$ \\
\hline 1964 & $\begin{array}{l}\text { PRECIP. (CM-) } \\
\text { SR-90 (MCI/SQ.KM.) } \\
\text { SR-90 CONC. }(P C / L) \\
\text { SR-89/SR-90 }\end{array}$ & $=$ & $\begin{array}{r}10.26 \\
1.20 \\
11.70 \\
*\end{array}$ & $\begin{array}{r}1.09 \\
0.54 \\
49.55 \\
*\end{array}$ & $\begin{array}{r}0.94 \\
0.76 \\
80.86 \\
0.06\end{array}$ & $\begin{array}{l}0.31 \\
--\end{array}$ & $\begin{array}{l}-\infty \\
-- \\
--\end{array}$ & $\begin{array}{l}0.03 \\
- \\
-\end{array}$ & $\begin{array}{l}0.02 \\
--\end{array}$ & $\stackrel{*}{*}+$ & $=$ & $\begin{array}{l}0.12 \\
0.23 \\
3.76 \\
*\end{array}$ & $\begin{array}{l}3.63 \\
0.29 \\
7.99 \\
-\end{array}$ & $\begin{array}{r}22.04 \\
3.40\end{array}$ \\
\hline 1965 & $\begin{array}{l}\text { PRECIP. (CM.) } \\
S R-9 C \text { IMCI/SQ-KM.) } \\
S R-90 \text { CCNC. }(P C / L) \\
S R-89 / S R-90\end{array}$ & $\begin{array}{r}13.64 \\
0.94 \\
6.90 \\
-\end{array}$ & $\begin{array}{l}1.19 \\
-- \\
-\end{array}$ & $\begin{array}{r}0.81 \\
0.21 \\
25.93 \\
-\end{array}$ & $\begin{array}{r}1.24 \\
0.27 \\
21.78 \\
-\end{array}$ & $\begin{array}{l}- \\
0.01 \\
-\end{array}$ & $\begin{array}{l}0.02 \\
-- \\
--\end{array}$ & $\begin{array}{l}* \\
0.03 \\
-- \\
-\end{array}$ & $\begin{array}{l}* \\
0.01 \\
-\end{array}$ & $\begin{array}{l}0.27 \\
-- \\
=-\end{array}$ & $\begin{array}{l}2.01 \\
0.17 \\
8.46 \\
-.\end{array}$ & $\begin{array}{l}1.83 \\
0.06 \\
3.28 \\
-.\end{array}$ & $\begin{array}{l}1.76 \\
0.06 \\
3.41 \\
-.\end{array}$ & $\begin{array}{r}22.48 \\
2.05\end{array}$ \\
\hline 1966 & $\begin{array}{l}\text { PRECIP }\left(C M_{-}\right) \\
S R-90 \text { (MCI/SQ.KM-) } \\
\text { SR-90 CONC. (PC/L) } \\
\text { SR-89/SR-90 }\end{array}$ & $\begin{array}{l}2.97 \\
0.11 \\
3.71 \\
--\end{array}$ & $\begin{array}{l}3.71 \\
0.16 \\
4.32 \\
-\end{array}$ & $\begin{array}{r}3.76 \\
0.40 \\
10.64 \\
\end{array}$ & $\begin{array}{l}0.03 \\
--\end{array}$ & $\begin{array}{l}* \\
0.03 \\
--\end{array}$ & $*$ & $\begin{array}{l}\bar{*} \\
\bar{*}\end{array}$ & 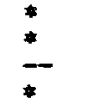 & 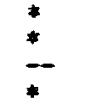 & $\begin{array}{l}0.73 \\
0.05 \\
6.85 \\
+\end{array}$ & $\begin{array}{l}1.73 \\
0.04 \\
2.32 \\
*\end{array}$ & $\begin{array}{l}6.94 \\
0.09 \\
1.30 \\
*\end{array}$ & $\begin{array}{r}19.84 \\
0.91\end{array}$ \\
\hline 1967 & $\begin{array}{l}\text { PRECIP, (CM.) } \\
\text { SR-9O (MCI/SQ-KM-) } \\
S R-90 \text { CONC. (PC/L) } \\
\text { SR-89/SR-90 }\end{array}$ & $\begin{array}{l}3.16 \\
0.06 \\
1.90\end{array}$ & $\begin{array}{l}2.08 \\
0.04 \\
1.93 \\
6.40\end{array}$ & $\begin{array}{r}2.50 \\
0.05 \\
2.01 \\
11.90\end{array}$ & $\begin{array}{r}0.20 \\
0.07 \\
35.01 \\
5.50\end{array}$ & $\overline{-}$ & $\overline{--}$ & $\begin{array}{l}-- \\
--\end{array}$ & $=$ & $=$ & $=$ & $=$ & $\begin{array}{l}-- \\
-- \\
--\end{array}$ & $\begin{array}{l}7.94 \\
0.22\end{array}$ \\
\hline
\end{tabular}

NOTES

-: DATA NOT availlable

*: ZERO OR TRACE

A: APPROXIMATE

B: LOHER LIMIT OF REPORTED DATA

C: PROPORTIONED FROM ORIGINALLY CONSOLICATED DATA 
MONTHLY FALLOUT DEPOSITION COLLECTIONS

SITE: SYRIA, KAMISHLY $\quad$ LAT. 37 3N LONG. 41 13E ALT. 452M. (COLUMN)

SOURCE: SYRIAN MINISTRY OF DEFENSE, METEOROLOGICAL DEPARTMENT

\begin{tabular}{|c|c|c|c|c|c|c|c|c|c|c|c|c|c|c|}
\hline & & JAN. & FEB. & MAR. & APR. & MAY & JUNE & JULY & AUG. & SEP. & OCT. & NOV. & DEC. & CUM. TOTAL \\
\hline 1960 & $\begin{array}{l}\text { PRECIP. (CM.) } \\
S R-90 \text { (MCI/SQ.KM-) } \\
\text { SR-90 CONC. (PC/L) } \\
S R-89 / S R-90\end{array}$ & $=$ & $\begin{array}{l}* \\
0.27 \\
--\end{array}$ & $\begin{array}{l}7.01 \\
- \\
-\end{array}$ & $\begin{array}{l}5.99 \\
-- \\
-\end{array}$ & $\begin{array}{l}-- \\
-- \\
-\end{array}$ & $\begin{array}{l}-- \\
-- \\
--\end{array}$ & $\begin{array}{l}* \\
=- \\
--\end{array}$ & $=$ & $\begin{array}{l}0.56 \\
0.006 \\
0.01 \\
+\end{array}$ & $\begin{array}{l}6.22 \\
0.00 \mathrm{C} \\
0.01 \\
-\end{array}$ & $\begin{array}{l}= \\
= \\
=\end{array}$ & 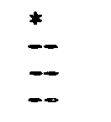 & $\begin{array}{r}19.78 \\
0.27\end{array}$ \\
\hline 1961 & $\begin{array}{l}\text { PRECIP. (CM.) } \\
\text { SR-90 (MCI/SQ.KM.) } \\
\text { SR-90 CONC. (PC/L) } \\
\text { SR-89/SR-90 }\end{array}$ & $\begin{array}{l}3.00 \\
-- \\
=\end{array}$ & $\begin{array}{l}2.39 \\
0.10 \\
4.19 \\
--\end{array}$ & $\begin{array}{l}8.00 \\
=- \\
-\end{array}$ & $\begin{array}{l}5.99 \\
0.24 \\
4.01 \\
-0\end{array}$ & $\begin{array}{l}-- \\
= \\
-\end{array}$ & $\begin{array}{l}-- \\
-- \\
--\end{array}$ & $\begin{array}{l}=- \\
=- \\
=-\end{array}$ & $\begin{array}{l}-- \\
-- \\
-\end{array}$ & $\begin{array}{l}-- \\
=- \\
--\end{array}$ & $=$ & $\begin{array}{l}-- \\
=- \\
=-\end{array}$ & $\begin{array}{l}-- \\
-- \\
--\end{array}$ & $\begin{array}{r}19.38 \\
0.34\end{array}$ \\
\hline 1962 & $\begin{array}{l}\text { PRECIP. (CM.) } \\
\text { SR-90 IMCI/SO-KM.) } \\
\text { SR-90 CONC. }(P C / L) \\
\text { SR-89/SR-90 }\end{array}$ & $\begin{array}{l}-- \\
-- \\
--\end{array}$ & $\begin{array}{l}-- \\
-- \\
--\end{array}$ & $\begin{array}{l}-- \\
-- \\
--\end{array}$ & $\begin{array}{l}-- \\
=- \\
--\end{array}$ & $=$ & $\begin{array}{l}-- \\
-- \\
--\end{array}$ & $=$ & 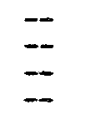 & $\begin{array}{l}-- \\
-- \\
-\end{array}$ & $\begin{array}{l}m \\
\ddot{-} \\
-\end{array}$ & $=$ & $\begin{array}{l}-- \\
--\end{array}$ & $\begin{array}{l}0.0 \\
0.0\end{array}$ \\
\hline 1963 & $\begin{array}{l}\text { PRECIP, (CM.) } \\
\text { SR-90 (MCI/SQ.KM.) } \\
S R-90 \text { CONC. }(P C / L) \\
S R-89 / S R-90\end{array}$ & $\begin{array}{l}-- \\
-- \\
-\end{array}$ & $\begin{array}{l}-- \\
=- \\
=-\end{array}$ & $=-$ & $\begin{array}{l}-- \\
-\end{array}$ & $=$ & $\begin{array}{l}-- \\
-- \\
-\end{array}$ & $=$ & $\begin{array}{l}-- \\
-- \\
--\end{array}$ & $\begin{array}{l}-- \\
-- \\
--\end{array}$ & $\begin{array}{l}m \\
m \\
--\end{array}$ & $\begin{array}{l}- \\
-- \\
--\end{array}$ & $\begin{array}{l}-- \\
-- \\
--\end{array}$ & $\begin{array}{l}0.0 \\
0.0\end{array}$ \\
\hline 1964 & $\begin{array}{l}\text { PRECIP. (CM.) } \\
\text { SR-90 (MCI/SQ.KM.) } \\
\text { SR-9O CONC. (PC/L) } \\
\text { SR-89/SR-90 }\end{array}$ & $=-$ & $\begin{array}{l}14.61 \\
1.23 \\
8.42 \\
*\end{array}$ & $\begin{array}{l}9.45 \\
2.88 \\
30.48 \\
*\end{array}$ & $\begin{array}{r}4.75 \\
2.06 \\
43.37 \\
*\end{array}$ & $\begin{array}{l}* \\
0.25 \\
*\end{array}$ & $\begin{array}{l}* \\
0.27 \\
--\end{array}$ & $\begin{array}{l}* \\
0.09 \\
--\end{array}$ & $\begin{array}{l}* \\
0.05 \\
-- \\
--\end{array}$ & $\begin{array}{l}* \\
0.02 \\
--\end{array}$ & $\begin{array}{l}0.02 \\
=-\end{array}$ & $\begin{array}{r}3.23 \\
0.36 \\
11.15 \\
+\end{array}$ & $\begin{array}{l}4.11 \\
0.32 \\
7.79 \\
-.\end{array}$ & $\begin{array}{r}36.15 \\
7.55\end{array}$ \\
\hline 1965 & $\begin{array}{l}\text { PRECIP. (CM.) } \\
\text { SR-9O (MCI/SQ.KM. }) \\
S R-90 \text { CONC. (PC/L) } \\
\text { SR-8S/SR-SO }\end{array}$ & $\begin{array}{l}8.84 \\
0.55 \\
6.23 \\
--\end{array}$ & $\begin{array}{l}10.67 \\
0.90 \\
8.44 \\
--\end{array}$ & $\begin{array}{l}6.35 \\
0.81 \\
12.76 \\
--\end{array}$ & $\begin{array}{r}11.00 \\
1.35 \\
12.28 \\
-\end{array}$ & $\begin{array}{l}0.38 \\
0.13 \\
34.22 \\
--\end{array}$ & $\begin{array}{r}0.13 \\
0.18 \\
138.47 \\
-0\end{array}$ & $\begin{array}{l}+ \\
0.07 \\
-- \\
--\end{array}$ & $\begin{array}{l}* \\
0.05 \\
--\end{array}$ & $\begin{array}{r}0.08 \\
0.01 \\
12.51 \\
--\end{array}$ & $\begin{array}{l}6.15 \\
0.20 \\
3.26 \\
--\end{array}$ & $\begin{array}{l}2.46 \\
0.09 \\
3.66 \\
-.\end{array}$ & $\begin{array}{l}5.18 \\
4 \\
-- \\
--\end{array}$ & $\begin{array}{r}51.24 \\
4.34\end{array}$ \\
\hline 1566 & $\begin{array}{l}\text { PRECIP. }(C N .) \\
5 R-90 \text { (NCI/SO.KM.) } \\
S R-90 \text { CONC. }(P C / L) \\
S R-89 / S R-90\end{array}$ & $\begin{array}{l}11.89 \\
0.27 \\
2.28 \\
-.\end{array}$ & $\begin{array}{l}2.18 \\
0.13 \\
5.97 \\
--\end{array}$ & $\begin{array}{l}4.85 \\
0.35 \\
7.22 \\
-.\end{array}$ & $\begin{array}{l}5.84 \\
0.47 \\
8.05 \\
--\end{array}$ & $\begin{array}{l}0.12 \\
0.01 \\
8.34 \\
--\end{array}$ & ${ }_{*}^{*}$ & $\begin{array}{l}-- \\
0.01 \\
\bar{*}\end{array}$ & $\begin{array}{l}* \\
0.04 \\
*\end{array}$ & $\begin{array}{r}0.16 \\
0.02 \\
12.51 \\
*\end{array}$ & $\begin{array}{l}2.80 \\
C .04 \\
1.43 \\
*\end{array}$ & $\begin{array}{l}0.79 \\
0.02 \\
2.54 \\
*\end{array}$ & $\begin{array}{l}7.51 \\
0.15 \\
2.00 \\
*\end{array}$ & $\begin{array}{r}36.14 \\
1.54\end{array}$ \\
\hline 1967 & $\begin{array}{l}\text { PRECIP. (CM.) } \\
\text { SR-90 (MCI/SQ.KM.) } \\
\text { SR-9O CONC. (PC/L) } \\
\text { SR-89/SR-90 }\end{array}$ & $\begin{array}{l}9.42 \\
0.17 \\
1.81 \\
6.30\end{array}$ & $\begin{array}{r}13.47 \\
0.30 \\
2.23 \\
6.50\end{array}$ & $\begin{array}{r}11.38 \\
0.40 \\
3.52 \\
11.70\end{array}$ & $\begin{array}{l}5.16 \\
0.22 \\
4.27 \\
7.10\end{array}$ & $=$ & $\begin{array}{l}=- \\
-- \\
-\infty\end{array}$ & $\begin{array}{l}-- \\
-- \\
--\end{array}$ & $\begin{array}{l}-\infty \\
--\end{array}$ & $\begin{array}{l}=- \\
-- \\
--\end{array}$ & $\begin{array}{l}-- \\
-- \\
--\end{array}$ & $=-$ & $\begin{array}{l}-- \\
=- \\
=-\end{array}$ & $\begin{array}{r}39.43 \\
1.09\end{array}$ \\
\hline \begin{tabular}{c} 
NOTES \\
\hdashline$:$ \\
$\$: Z$ \\
$A: A$ \\
$B: L$ \\
$C: P$
\end{tabular} & $\begin{array}{l}\text { ATA NOT AVAILABLE } \\
\text { ERO OR TRACE } \\
\text { PPROXIMATE } \\
\text { OWER IIIMI OF REPOR } \\
\text { ROPORTIONED FROM OR }\end{array}$ & $\begin{array}{l}\text { DATA } \\
\text { INALLYY }\end{array}$ & NSO & & & & & & & & & & & \\
\hline
\end{tabular}




\begin{tabular}{|c|c|c|c|c|c|c|c|c|c|c|c|c|c|c|}
\hline & & JAN. & FEB. & MAR. & APR. & MAY & JUNE & $\begin{array}{l}\text { JULY } \\
-\end{array}$ & AUG. & SEP. & OCT. & NOV. & DEC. & CUM. TOTAL \\
\hline 1958 & $\begin{array}{l}\text { PRECIP. (CM.) } \\
\text { SR-9O (MCI/SQ.KM.) } \\
\text { SR-9O CONC. (PCAL) } \\
\text { SR-QS/SR-SC }\end{array}$ & $\begin{array}{r}2.18 \\
0.01 \\
0.46 \\
25.00\end{array}$ & $\begin{array}{r}1.96 \\
0.03 \\
1.54 \\
12.00\end{array}$ & $\begin{array}{r}8.86 \\
0.07 \\
0.80 \\
10.00\end{array}$ & $\begin{array}{r}0.38 \\
0.02 \\
5.27 \\
12.00\end{array}$ & $\begin{array}{r}12.50 \\
0.16 \\
1.29 \\
58.00\end{array}$ & $\begin{array}{r}9.50 \\
0.08 \\
0.85 \\
47.00\end{array}$ & $\begin{array}{r}71.65 \\
0.39 \\
0.55 \\
65.00\end{array}$ & $\frac{-.12}{10.00}$ & $\begin{array}{c}\overline{0.14} \\
50.00\end{array}$ & $\begin{array}{c}0.00 \\
158.00\end{array}$ & $\begin{array}{c}-\overline{0.00} \\
110.00\end{array}$ & $\begin{array}{c}-- \\
0.21 \\
47.00\end{array}$ & $\begin{array}{r}107.03 \\
1.13\end{array}$ \\
\hline 1959 & $\begin{array}{l}\text { PRECIP. }\left(C M H_{-}\right) \\
\text {SR-90 IMCI/SQ.KM.) } \\
S R-90 \text { CONC. }(P C / L) \\
\text { SR-89/SR-90 }\end{array}$ & $\begin{array}{c}\overline{0.08} \\
\overline{35.00}\end{array}$ & $\begin{array}{c}-- \\
0.30 \\
29.00\end{array}$ & $\begin{array}{l}-- \\
0.05 \\
9.60\end{array}$ & $\begin{array}{c}-- \\
0.47 \\
15.00\end{array}$ & $\begin{array}{l}\overline{0.08} \\
\overline{8.30}\end{array}$ & $\begin{array}{l}-- \\
0.26 \\
--20\end{array}$ & $\begin{array}{l}-\overline{0.10} \\
-\overline{3.70}\end{array}$ & $\begin{array}{l}-\overline{0.07} \\
\overline{0.90}\end{array}$ & $\begin{array}{l}-- \\
-- \\
--\end{array}$ & $\begin{array}{l}-\overline{0.01} \\
-- \\
3.60\end{array}$ & $\begin{array}{l}-- \\
0.02 \\
*\end{array}$ & $\begin{array}{l}-\overline{0.02} \\
+\end{array}$ & $\begin{array}{l}0.0 \\
1.46\end{array}$ \\
\hline 1960 & $\begin{array}{l}\text { PRECIP. (CM.) } \\
\text { SR-9O (MCI/SQ.KM.) } \\
S R-90 \text { CCNC. }(P C / L) \\
\text { SR-89/SR-90 }\end{array}$ & $\begin{array}{l}-- \\
0.01 \\
-- \\
--\end{array}$ & $\begin{array}{l}2.01 \\
0.02 \\
1.00 \\
--\end{array}$ & $\begin{array}{r}14.02 \\
0.07 \\
0.50 \\
--\end{array}$ & $\begin{array}{l}23.01 \\
0.05 \\
0.22 \\
--\end{array}$ & $\begin{array}{l}-\overline{0 . C 6 C} \\
--\end{array}$ & $\begin{array}{l}-0 \\
0.060 \\
--\end{array}$ & $\begin{array}{l}\overline{c .06 C} \\
--\end{array}$ & $\begin{array}{l}0.06 C \\
=\end{array}$ & $\begin{array}{l}-- \\
0.010 \\
--\end{array}$ & $\begin{array}{l}-- \\
0.016 \\
=-\end{array}$ & $\begin{array}{l}-- \\
0.01 C \\
--\end{array}$ & $\begin{array}{l}-- \\
0.016 \\
--\end{array}$ & $\begin{array}{r}39.04 \\
0.43\end{array}$ \\
\hline 1961 & $\begin{array}{l}\text { PRECIP. (CM.) } \\
\text { SR }-9 C \text { (MCI/SO.KM.) } \\
S R-90 \text { CONC. (PC/L) } \\
S R-89 / S R-90\end{array}$ & $\begin{array}{l}-- \\
c .02 C \\
--\end{array}$ & $\begin{array}{l}-- \\
0.02 \mathrm{C} \\
--\end{array}$ & $\begin{array}{l}-- \\
0.016 \\
--\end{array}$ & $\begin{array}{l}-\infty \\
0.016 \\
=-\end{array}$ & $\begin{array}{l}-- \\
0.29 C \\
--\end{array}$ & $\begin{array}{l}-- \\
0.29 C \\
--\end{array}$ & $\begin{array}{l}-- \\
c .03 C \\
--\end{array}$ & $\begin{array}{l}-- \\
0.030 \\
--\end{array}$ & $\begin{array}{l}-- \\
* \\
--\end{array}$ & $\begin{array}{l}-\infty \\
\text { C. } 01\end{array}$ & $\begin{array}{c}-- \\
0.02 \\
\overline{-0.00}\end{array}$ & $\begin{array}{l}-- \\
0.05 \\
-- \\
69.00\end{array}$ & $\begin{array}{l}0.0 \\
0.78\end{array}$ \\
\hline 1962 & $\begin{array}{l}\text { PRECIP. (CM-) } \\
S R-90 \text { (MCI/SQ.KM.) } \\
S R-90 \text { CCNC. (PC/L) } \\
S R-89 / S R-90\end{array}$ & $\begin{array}{l}\overline{0.10} \\
\overline{38.00}\end{array}$ & $\begin{array}{c}-- \\
0.11 \\
\overline{34.00}\end{array}$ & $\frac{\overline{0.22}}{2 \overline{22.00}}$ & $\begin{array}{c}\overline{0.20} \\
\overline{11.00}\end{array}$ & $\begin{array}{l}-\overline{0.03} \\
\overline{8.00}\end{array}$ & $\begin{array}{l}-- \\
0.02 \\
40.00\end{array}$ & $\begin{array}{l}-- \\
0.18 \\
\overline{35.00}\end{array}$ & $\frac{0.07}{21.00}$ & $\begin{array}{c}-- \\
0.12 \\
25.00\end{array}$ & $\begin{array}{c}-- \\
0.03 \\
12.00\end{array}$ & $\begin{array}{c}-- \\
0.02 \\
20.00\end{array}$ & $\begin{array}{c}-- \\
0.02 \\
-- \\
51.00\end{array}$ & $\begin{array}{l}0.0 \\
1.12\end{array}$ \\
\hline 1963 & $\begin{array}{l}\text { PRECIP, (CM.) } \\
\text { SR-90 (NCI/SQ.KM,) } \\
S R-90 \text { COAC }(P C / L) \\
S R-89 / S R-90\end{array}$ & $\begin{array}{c}-- \\
0.12 \\
\overline{10.00}\end{array}$ & $\begin{array}{l}7.62 \\
0.07 \\
0.92 \\
8.20\end{array}$ & $\begin{array}{r}26.67 \\
0.29 \\
1.09 \\
46.00\end{array}$ & $\begin{array}{r}0.03 \\
0.07 \\
233.33 \\
14.00\end{array}$ & $\begin{array}{r}0.03 \\
0.04 \\
133.34 \\
7.60\end{array}$ & $\begin{array}{r}20.07 \\
0.25 \\
1.25 \\
4.60\end{array}$ & $\begin{array}{r}53.85 \\
0.66 \\
1.23 \\
0.40\end{array}$ & $\begin{array}{l}2.74 \\
0.11 \\
4.02 \\
4\end{array}$ & $\begin{array}{l}23.11 \\
0.18 \\
0.78 \\
*\end{array}$ & $\begin{array}{l}0.05 \\
0.03 \\
60.01 \\
*\end{array}$ & $\begin{array}{l}0.05 \\
0.04 \\
80.01 \\
*\end{array}$ & $\begin{array}{r}0.51 \\
0.06 \\
11.77 \\
*\end{array}$ & $\begin{array}{r}134.73 \\
1.92\end{array}$ \\
\hline 1564 & $\begin{array}{l}\text { PRECIP. }\left(C M_{-}\right) \\
\text {SR-9O }\left(M C I / S K_{-K M}\right) \\
S R-90 \text { CONC. }(P C / L) \\
S R-89 / S R-90\end{array}$ & $\begin{array}{l}4.90 \\
0.24 \\
4.90 \\
*\end{array}$ & $\begin{array}{l}0.08 \\
0.02 \\
25.01 \\
*\end{array}$ & $\begin{array}{l}7.87 \\
0.08 \\
1.02 \\
*\end{array}$ & $\begin{array}{l}-\infty \\
0.05 \\
*\end{array}$ & $\begin{array}{l}9.91 \\
0.18 \\
1.82\end{array}$ & $\begin{array}{l}30.38 \\
0.48 \\
1.58 \\
--\end{array}$ & $\begin{array}{l}15.65 \\
0.24 \\
1.54 \\
--\end{array}$ & $\begin{array}{l}10.54 \\
0.12 \\
1.14 \\
--\end{array}$ & $\begin{array}{l}16.76 \\
0.05 \\
0.30 \\
--\end{array}$ & $\begin{array}{l}0.46 \\
= \\
=\end{array}$ & $\begin{array}{l}0.05 \\
0.01 \\
20.01 \\
*\end{array}$ & $\begin{array}{l}* \\
0.01 \\
-- \\
--\end{array}$ & $\begin{array}{r}96.60 \\
1.48\end{array}$ \\
\hline 1965 & $\begin{array}{l}\text { PRECIP. (CM-) } \\
S R-90 \text { (MCI/SQ.KM-) } \\
S R-90 \text { CONC. }(P C / L) \\
S R-Q S / S R=9 O\end{array}$ & $\begin{array}{l}0.38 \\
0.02 \\
5.27 \\
--\end{array}$ & $\begin{array}{r}0.08 \\
0.02 \\
25.01 \\
--\end{array}$ & $\begin{array}{r}0.20 \\
0.29 \\
145.00 \\
--\end{array}$ & $\begin{array}{l}1.27 \\
0.00 \\
0.01 \\
--\end{array}$ & $\begin{array}{l}5.84 \\
0.08 \\
1.37 \\
--\end{array}$ & $\begin{array}{l}45.72 \\
-- \\
--\end{array}$ & $\begin{array}{r}19.30 \\
0.09 \\
0.47 \\
-.\end{array}$ & $\begin{array}{l}45.21 \\
0.13 \\
0.29 \\
--\end{array}$ & $\begin{array}{r}1.37 \\
0.15 \\
10.95 \\
--\end{array}$ & $\begin{array}{l}0.64 \\
0.03 \\
4.69 \\
--\end{array}$ & $\begin{array}{l}6.60 \\
0.05 \\
0.76 \\
--\end{array}$ & $\begin{array}{r}0.08 \\
0.19 \\
237.50 \\
--\end{array}$ & $\begin{array}{r}126.69 \\
1.05\end{array}$ \\
\hline 1966 & $\begin{array}{l}\text { PRECIP. }\left(C M_{-}\right) \\
\text {SR-9O (NCI/SQ.KM.) } \\
\text { SR-90 CONC. (PC/L) } \\
\text { SR-89/SR-90 }\end{array}$ & $\begin{array}{l}0.28 \\
=- \\
=-\end{array}$ & $\begin{array}{l}4.57 \\
0.03 \\
0.66 \\
--\end{array}$ & $\begin{array}{l}8.89 \\
0.13 \\
1.47 \\
-.\end{array}$ & $\begin{array}{l}4.52 \\
0.07 \\
1.55 \\
-\end{array}$ & $\begin{array}{r}32.54 \\
0.04 \\
0.13 \\
--\end{array}$ & $\begin{array}{l}52.58 \\
=- \\
=-\end{array}$ & $\begin{array}{r}30.48 \\
0.04 \\
0.14 \\
5.50\end{array}$ & $\begin{array}{l}16.84 \\
0.03 \\
0.18 \\
*\end{array}$ & $\begin{array}{l}5.74 \\
* \\
-- \\
*\end{array}$ & $\underset{*}{-\infty}$ & $\begin{array}{r}1.57 \\
0.01 \\
0.64 \\
23.00\end{array}$ & $\begin{array}{l}0.02 \\
0.01 \\
50.01 \\
*\end{array}$ & $\begin{array}{r}158.03 \\
0.37\end{array}$ \\
\hline 1967 & $\begin{array}{l}\text { PRECIP. (CM.) } \\
5 R-90 \text { (MCI/SQ.KM.) } \\
S R-90 \text { CONC. (PC/L) } \\
\text { SR-89/SR-90 }\end{array}$ & $\begin{array}{l}0.08 \\
0.01 \\
12.51 \\
*\end{array}$ & $\begin{array}{l}0.89 \\
0.02 \\
2.25 \\
*\end{array}$ & $\begin{array}{l}0.41 \\
0.01 \\
2.44 \\
*\end{array}$ & $\begin{array}{r}12.42 \\
0.01 \\
0.09 \\
\end{array}$ & $\begin{array}{r}24.28 \\
0.02 \\
0.09 \\
*\end{array}$ & $\begin{array}{l}39.95 \\
0.01 \\
0.03 \\
*\end{array}$ & $\begin{array}{l}-- \\
-- \\
--\end{array}$ & $=$ & $\begin{array}{l}-- \\
-- \\
--\end{array}$ & $\begin{array}{l}-- \\
-- \\
--\end{array}$ & $\begin{array}{l}-- \\
-- \\
--\end{array}$ & $\begin{array}{l}-- \\
-- \\
--\end{array}$ & $\begin{array}{r}78.03 \\
0.08\end{array}$ \\
\hline$:$ & $\begin{array}{l}\text { A NOT AVAILABLE } \\
\text { O OR TRACE } \\
\text { ROXIMATE } \\
\text { ER LIMIT OF REPO } \\
\text { PORTIONED FROM O }\end{array}$ & & & & & & & & & & & & & \\
\hline
\end{tabular}


MONTHLY FALLOUT DEPOSITION COLLECTIONS

LAT. 25 5N LONG.121 32E ALT.

OM. (POT)

SOURCE: THRU THE NATIONALIST CHINESE MISSION TO THE UNITEO NATIONS

\begin{tabular}{|c|c|c|c|c|c|c|c|c|c|c|c|c|c|c|}
\hline & & JAN. & FEB. & MAR. & APR. & MAY & JUNE & JULY & AUE. & SEP. & оCT. & NOV. & DEC. & CUM. TOTAL \\
\hline 1958 & $\begin{array}{l}\text { PREC IP }\left(C M_{.}\right) \\
\text {SR-90 (MCI/SQ-KM.) } \\
S R-90 \text { CCNC. }(P C / L) \\
S R-89 / S R-90\end{array}$ & $=$ & $\begin{array}{r}24.31 \\
0.06 \\
0.25 \\
9.00\end{array}$ & $\begin{array}{r}13.72 \\
0.04 \\
0.30 \\
14.00\end{array}$ & $\begin{array}{r}5.69 \\
0.22 \\
3.87 \\
11.00\end{array}$ & $\begin{array}{r}10.49 \\
0.12 \\
1.15 \\
37.00\end{array}$ & $\begin{array}{r}21.56 \\
0.39 \\
1.81 \\
34.00\end{array}$ & $\begin{array}{r}51.89 \\
0.03 \\
0.06 \\
327.00\end{array}$ & $\begin{array}{r}11.89 \\
0.22 \\
1.86 \\
51.00\end{array}$ & $\begin{array}{r}17.83 \\
0.09 \\
0.51 \\
47.00\end{array}$ & $\begin{array}{r}8.92 \\
0.31 \\
3.48 \\
54.00\end{array}$ & $\begin{array}{r}4.95 \\
0.44 \\
8.89 \\
44.00\end{array}$ & $\begin{array}{r}14.86 \\
0.78 \\
5.25 \\
32.00\end{array}$ & $\begin{array}{r}186.11 \\
2.70\end{array}$ \\
\hline 1959 & $\begin{array}{l}\text { PRECIP. (CM.) } \\
\text { SR-90 (MCI/SQ.KM.) } \\
\text { SR-90 CONC. }(P C / L) \\
\text { SR-89/SR-90 }\end{array}$ & $\begin{array}{r}8.00 \\
0.72 \\
9.01 \\
38.00\end{array}$ & $\begin{array}{l}6.20 \\
=- \\
--\end{array}$ & $\begin{array}{l}10.01 \\
1.10 \\
10.99 \\
17.00\end{array}$ & $\begin{array}{r}32.03 \\
1.53 \\
4.78 \\
12.00\end{array}$ & $\begin{array}{r}19.02 \\
0.45 \\
2.37 \\
7.60\end{array}$ & $\begin{array}{r}33.02 \\
0.05 \\
0.16 \\
3.9 C\end{array}$ & $\begin{array}{r}42.04 \\
C .10 \\
C .24 \\
1.90\end{array}$ & $\begin{array}{r}23.01 \\
0.08 \\
0.35 \\
1.70\end{array}$ & $\begin{array}{l}22.02 \\
0.03 \\
0.14 \\
\end{array}$ & $\begin{array}{l}12.01 \\
0.16 \\
1.34 \\
*\end{array}$ & $\begin{array}{r}13.00 \\
0.08 \\
0.62 \\
1.20\end{array}$ & $\begin{array}{l}38.02 \\
0.04 \\
0.11 \\
*\end{array}$ & $\begin{array}{r}258.38 \\
4.34\end{array}$ \\
\hline 1960 & $\begin{array}{l}\text { PRECIP. }\left(C M_{-}\right) \\
\text {SR-9C (MCI/SQ.KM.) } \\
S R-90 \text { CCNC. (PC/L) } \\
\text { SR-89/SR-90 }\end{array}$ & $\begin{array}{l}11.00 \\
=- \\
=-\end{array}$ & $\begin{array}{l}2.01 \\
0.12 \\
5.98 \\
-.\end{array}$ & $\begin{array}{l}14.02 \\
0.19 \\
1.36 \\
--\end{array}$ & $\begin{array}{l}23.01 \\
0.27 \\
1.18 \\
--\end{array}$ & $\begin{array}{l}20.02 \\
0.15 \\
C .75 \\
--\end{array}$ & $\begin{array}{l}24.03 \\
0.27 \\
1.13 \\
--\end{array}$ & $\begin{array}{l}24.03 \\
C .02 C \\
C .09 \\
--\end{array}$ & $\begin{array}{c}140.11 \\
0.09 C \\
0.07 \\
--\end{array}$ & $\begin{array}{l}19.02 \\
0.09 \mathrm{C} \\
0.48 \\
-\end{array}$ & $\begin{array}{l}7.01 \\
C .036 \\
0.43 \\
--\end{array}$ & $\begin{array}{l}7.01 \\
0.056 \\
0.72 \\
--\end{array}$ & $\begin{array}{l}7.01 \\
0.05 \mathrm{C} \\
0.72 \\
--\end{array}$ & $\begin{array}{r}298.28 \\
1.33\end{array}$ \\
\hline 1961 & $\begin{array}{l}\text { PRECIP. }(C M .) \\
\text { SR-90 (NCI/SQ.KM-) } \\
S R-90 \text { CONC. }(P C / L) \\
S R-89 / S R-90\end{array}$ & $\begin{array}{l}5.00 \\
0.056 \\
1.01 \\
--\end{array}$ & $\begin{array}{l}16.00 \\
0.14 C \\
0.88 \\
-.\end{array}$ & $\begin{array}{l}25.02 \\
0.02 C \\
0.08 \\
--\end{array}$ & $\begin{array}{l}11.00 \\
0.010 \\
0.10 \\
-\end{array}$ & $\begin{array}{l}19.02 \\
0.04 \mathrm{C} \\
0.22 \\
-.\end{array}$ & $\begin{array}{l}7.01 \\
0.016 \\
0.15 \\
-.\end{array}$ & $\begin{array}{l}40.03 \\
0.03 C \\
C .08 \\
--\end{array}$ & $\begin{array}{l}10.01 \\
0.016 \\
0.10 \\
--\end{array}$ & $\begin{array}{r}36.02 \\
0.05 \\
0.14 \\
121 . C C\end{array}$ & $\begin{array}{r}8.00 \\
C .20 \\
2.51 \\
79.00\end{array}$ & $\begin{array}{r}5.00 \\
0.12 \\
2.41 \\
85.00\end{array}$ & $\begin{array}{r}12.01 \\
0.39 \\
3.25 \\
59.00\end{array}$ & $\begin{array}{r}194.12 \\
1.07\end{array}$ \\
\hline 1962 & $\begin{array}{l}\text { PRECIP. (CM.) } \\
\text { SR-9O IMCI/SQ.KM-) } \\
S R-9 O \text { CCNC. }(P C / L) \\
S R-89 / S R-90\end{array}$ & $\begin{array}{r}11.00 \\
0.88 \\
8.01 \\
44.00\end{array}$ & $\begin{array}{r}8.00 \\
0.73 \\
9.13 \\
24.00\end{array}$ & $\begin{array}{r}29.01 \\
2.03 \\
7.00 \\
17.00\end{array}$ & $\begin{array}{r}11.99 \\
0.95 \\
7.93 \\
12.00\end{array}$ & $\begin{array}{r}18.01 \\
0.74 \\
4.11 \\
10.00\end{array}$ & $\begin{array}{l}-\overline{0.15} \\
\overline{9.00}\end{array}$ & $\frac{0 .}{0.14} \overline{16.00}$ & $\frac{--}{0.09}$ & $\overline{0.25}$ & $\begin{array}{l}-- \\
0.04 \\
\overline{17.000}\end{array}$ & $\begin{array}{c}-- \\
0.23 \\
42.00\end{array}$ & $\begin{array}{l}\overline{0.31} \\
\overline{47.00}\end{array}$ & $\begin{array}{r}78.01 \\
6.54\end{array}$ \\
\hline 1963 & $\begin{array}{l}\text { PRECIP. (CM.) } \\
\text { SR-90 IMCI/SQ.KM.) } \\
\text { SR-90 CONC. }(P C / L) \\
\text { SR-89/SR-90 }\end{array}$ & $\begin{array}{l}-\overline{0.17} \\
\overline{8.00}\end{array}$ & $\frac{\overline{1.05}}{2 \overline{22.00}}$ & $\frac{\overline{1.27}}{\overline{21.00}}$ & $\begin{array}{l}-. \\
0.68 \\
12.00\end{array}$ & $\begin{array}{c}\overline{2.67} \\
\overline{10.00}\end{array}$ & $\begin{array}{l}-\overline{0.90} \\
\overline{1.30}\end{array}$ & $\begin{array}{l}-8.87 \\
1.00\end{array}$ & $\begin{array}{l}-\overline{0.68} \\
\bar{*}\end{array}$ & $\frac{--}{0.52}$ & $\begin{array}{l}-- \\
0.52 \\
*\end{array}$ & $\frac{-}{0.48}$ & $\overline{0.76}$ & $\begin{array}{c}0.0 \\
10.57\end{array}$ \\
\hline 1964 & $\begin{array}{l}\text { PRECIP. }\left(C M_{.}\right) \\
\text {SR-90 (KCIISQ.KM.) } \\
\text { SR-90 CONC. (PC/L) } \\
\text { SR-89/SR-90 }\end{array}$ & $\frac{--99}{1.9}$ & $\begin{array}{l}-- \\
0.84 \\
*\end{array}$ & $\begin{array}{l}-- \\
3.26 \\
*\end{array}$ & $\begin{array}{l}-- \\
0.05 \\
-\bar{*}\end{array}$ & $\begin{array}{l}-- \\
0.23 \\
--\end{array}$ & $\overline{0.51}$ & $\begin{array}{l}-- \\
0.58 \\
--\end{array}$ & $\begin{array}{l}30.91 \\
0.20 \\
0.65 \\
--\end{array}$ & $\begin{array}{l}-- \\
0.08 \\
--\end{array}$ & $\begin{array}{l}-\overline{c .08} \\
\overline{8.30}\end{array}$ & $\begin{array}{l}-- \\
0.04 \\
=-\end{array}$ & $\begin{array}{l}-- \\
0.05 \\
--\end{array}$ & $\begin{array}{r}30.91 \\
5.91\end{array}$ \\
\hline 1965 & $\begin{array}{l}\text { PRECIP. (CM.) } \\
\text { SR-9C (NCI/SQ.KN.) } \\
S R-9 C \text { CCNC. }(P C / L) \\
S R-89 / S R-90\end{array}$ & $\begin{array}{l}14.40 \\
0.07 \\
0.49 \\
-\end{array}$ & $\begin{array}{l}2.90 \\
0.18 \\
6.21 \\
-.\end{array}$ & $\begin{array}{l}8.71 \\
0.12 \\
1.38 \\
-\end{array}$ & $\begin{array}{l}15.80 \\
0.04 \\
0.26 \\
--\end{array}$ & $\begin{array}{l}15.70 \\
C .37 \\
2.36 \\
--\end{array}$ & $\begin{array}{l}31.83 \\
0.04 \\
0.13 \\
--\end{array}$ & $\begin{array}{l}16.92 \\
6.07 \\
0.42 \\
-.\end{array}$ & $\begin{array}{l}18.01 \\
C .07 \\
0.39 \\
--\end{array}$ & $\begin{array}{l}5.41 \\
0.27 \\
5.00 \\
--\end{array}$ & $\begin{array}{l}9.70 \\
0.08 \\
0.83 \\
--\end{array}$ & $\begin{array}{l}-- \\
0.07 \\
--\end{array}$ & $\begin{array}{l}-- \\
0.14 \\
--\end{array}$ & $\begin{array}{r}139.38 \\
1.52\end{array}$ \\
\hline 1966 & $\begin{array}{l}\text { PRECIP. (CM.) } \\
\text { SR-90 IMCI/SQ.KM.) } \\
\text { SR-90 CONC. }(P C / L) \\
\text { SR-89/SR-90 }\end{array}$ & $\begin{array}{l}3.00 \\
0.22 \\
7.34 \\
-\end{array}$ & $\begin{array}{l}11.80 \\
0.06 \\
0.51 \\
-.\end{array}$ & $\begin{array}{l}13.90 \\
0.02 \\
0.15 \\
--\end{array}$ & $\begin{array}{l}19.80 \\
0.11 \\
0.56 \\
--\end{array}$ & $\begin{array}{l}6.50 \\
0.12 \\
1.85 \\
-. .\end{array}$ & $\begin{array}{l}59.60 \\
=- \\
=-\end{array}$ & $\begin{array}{r}11.40 \\
0.06 \\
0.53 \\
4.40\end{array}$ & $\begin{array}{l}-- \\
*- \\
-\end{array}$ & $\begin{array}{l}-- \\
0.09 \\
-\infty\end{array}$ & $\overline{0.01}$ & $\begin{array}{c}\overline{0.02} \\
\overline{21.00}\end{array}$ & $\begin{array}{l}-\overline{0} \\
*\end{array}$ & $\begin{array}{r}126.00 \\
0.76\end{array}$ \\
\hline 1967 & $\begin{array}{l}\text { PRECIP, (CM.) } \\
\text { SR-9O (MCI/SQ.KM.) } \\
\text { SR-9O CCNC. }(P C / L) \\
\text { SR-89/SR-90 }\end{array}$ & $\begin{array}{l}7.20 \\
0.10 \\
1.39 \\
*\end{array}$ & $\begin{array}{r}10.70 \\
0.09 \\
0.85 \\
6.90\end{array}$ & $\begin{array}{r}6.80 \\
0.08 \\
1.18 \\
10.40\end{array}$ & $\begin{array}{l}\overline{0.07} \\
\overline{8.10}\end{array}$ & $\begin{array}{r}23.20 \\
0.03 \\
0.13 \\
*\end{array}$ & $\begin{array}{l}15.70 \\
0.11 \\
0.71 \\
*\end{array}$ & $=$ & $\begin{array}{l}-- \\
=- \\
--\end{array}$ & $\begin{array}{l}-- \\
-- \\
--\end{array}$ & $=$ & $\begin{array}{l}-- \\
-- \\
--\end{array}$ & $\begin{array}{l}-- \\
-- \\
--\end{array}$ & $\begin{array}{r}63.60 \\
0.48\end{array}$ \\
\hline \multicolumn{15}{|c|}{$\begin{array}{l}\text { NOTES } \\
-=: \text { DATA NOT AVAILABLE } \\
\text { *: 2ERO OR TRACE } \\
\text { A: APPROXIMATE } \\
\text { B: LOWER LIMIT OF REPORTED DATA } \\
\text { C: PROPORTIONED FROM QRIGINALIY }\end{array}$} \\
\hline
\end{tabular}




\begin{tabular}{|c|c|c|c|c|c|c|c|c|c|c|c|c|c|c|c|}
\hline & & & JAN. & FEB. & MAR. & APR. & MAY & JUNE & JULY & AUG. & SEP. & OCT. & NOV. & DEC. & CUM. TOTAL \\
\hline & 1958 & $\begin{array}{l}\text { PRECIP. }\left(C M_{-}\right) \\
\text {SR-90 (NCI/SQ.KM.) } \\
\text { SR-9O CONC. }(P C / L) \\
\text { SR-89/SR-90 }\end{array}$ & $\begin{array}{l}= \\
= \\
=\end{array}$ & $\begin{array}{l}-- \\
-- \\
--\end{array}$ & $\bar{m}$ & $\begin{array}{r}2.97 \\
0.08 \\
2.70 \\
10.00\end{array}$ & $\begin{array}{r}17.42 \\
0.07 \\
0.41 \\
25.00\end{array}$ & $\begin{array}{r}10.92 \\
0.22 \\
2.02 \\
56.00\end{array}$ & $\begin{array}{r}39.95 \\
0.17 \\
0.43 \\
51.00\end{array}$ & $\begin{array}{l}-\overline{0.03} \\
\overline{1.00}\end{array}$ & $\begin{array}{c}\overline{0.01} \\
40.00\end{array}$ & $\begin{array}{l}-- \\
-- \\
--\end{array}$ & $\begin{array}{c}-- \\
0.03 \\
\overline{50.00}\end{array}$ & $\begin{array}{l}\overline{0.19} \\
\overline{41.00}\end{array}$ & $\begin{array}{r}71.26 \\
0.80\end{array}$ \\
\hline & 1959 & $\begin{array}{l}\text { PRECIP. (CM.) } \\
\text { SR-90 (NCI/SQ.KM-) } \\
S R-90 \text { CONC. (PC/L) } \\
\text { SR-89/SR-SO }\end{array}$ & $\frac{\overline{0.14}}{41.00}$ & $\overline{0.20}$ & $\overline{-}$ & $\begin{array}{l}-\overline{0.36} \\
\overline{9.40}\end{array}$ & $\begin{array}{l}-- \\
0.14 \\
8.90\end{array}$ & $\begin{array}{l}-- \\
0.08 \\
--\end{array}$ & $\begin{array}{l}-- \\
0.03 \\
--\end{array}$ & $\begin{array}{l}-- \\
0.02 \\
-\end{array}$ & $\begin{array}{l}-- \\
-- \\
--\end{array}$ & $\begin{array}{l}-- \\
0.01 \\
--\end{array}$ & $\begin{array}{l}-- \\
0.02 \\
*-\end{array}$ & $\begin{array}{l}-- \\
0.03 \\
-- \\
4.70\end{array}$ & $\begin{array}{l}0.0 \\
1.03\end{array}$ \\
\hline & 1960 & $\begin{array}{l}\text { PRECIP. (CM.) } \\
\text { SR-9O (MCI/SQ.KM-) } \\
S R-9 C \text { CONC. }(P C / L) \\
\text { SR-89/SR-90 }\end{array}$ & $\begin{array}{l}-- \\
0.02 \\
--\end{array}$ & $\begin{array}{l}-- \\
0.04 \\
-- \\
--\end{array}$ & $=0.02$ & $\begin{array}{l}-- \\
0.10 \\
=\end{array}$ & $\begin{array}{l}-- \\
0.05 C \\
=-\end{array}$ & $\begin{array}{l}=- \\
0.05 C \\
=-\end{array}$ & $\begin{array}{l}-- \\
c . c 2 c \\
--\end{array}$ & $\begin{array}{l}-- \\
0.02 C \\
-\end{array}$ & $\begin{array}{l}-- \\
0.010 \\
=-\end{array}$ & $\begin{array}{l}-- \\
0.01 c \\
--\end{array}$ & $\begin{array}{l}- \\
0.01 C \\
=-\end{array}$ & $\begin{array}{l}-\infty \\
0.01 C \\
--\end{array}$ & $\begin{array}{l}0.0 \\
0.36\end{array}$ \\
\hline & 1961 & $\begin{array}{l}\text { PRECIP: (CM.) } \\
S R-90 \text { (NCI/SQ-KM.) } \\
S R-90 \text { CONC. }(P C / L) \\
S R-89 / S R-90\end{array}$ & $\begin{array}{l}-- \\
0.04 C \\
=-\end{array}$ & $\begin{array}{l}\overline{0.04 C} \\
\overline{--}\end{array}$ & $\begin{array}{l}-\overline{0.10 C} \\
=-\end{array}$ & $\begin{array}{l}\ddot{0.100} \\
--\end{array}$ & $\begin{array}{l}-\overline{0.626} \\
--\end{array}$ & $\begin{array}{l}-\ddot{0.02 C} \\
--\end{array}$ & $\begin{array}{l}-- \\
c .016 \\
--\end{array}$ & $\begin{array}{l}-- \\
0.016 \\
=-\end{array}$ & $\begin{array}{l}-- \\
0.01 \\
-\end{array}$ & $\begin{array}{l}-- \\
* \\
*\end{array}$ & $\begin{array}{c}\overline{0.07} \\
\overline{31.00}\end{array}$ & $\begin{array}{c}\overline{0.14} \\
49.00\end{array}$ & $\begin{array}{l}0.0 \\
0.56\end{array}$ \\
\hline D & 1962 & $\begin{array}{l}\text { PRECIP. (CM.) } \\
\text { SR-90 (NCI/SQ.KM.) } \\
\text { SR-90 CONC. }(P C / L) \\
S R-89 / S R-S O\end{array}$ & $\begin{array}{l}\overline{0.17} \\
\overline{35.00}\end{array}$ & $\begin{array}{l}\overline{0.89} \\
\overline{33.00}\end{array}$ & $\begin{array}{l}-- \\
0.64 \\
17.00\end{array}$ & $\begin{array}{l}-- \\
1.20 \\
14.00\end{array}$ & $\begin{array}{l}-- \\
0.32 \\
--\end{array}$ & $\overline{0.01}+-$ & $\frac{--}{0.05}$ & $=$ & $\begin{array}{l}-- \\
\overline{0.06} \\
24.60\end{array}$ & $\begin{array}{l}0.80 \\
22.00\end{array}$ & $\begin{array}{l}\overline{0.08} \\
\overline{33.00}\end{array}$ & $\begin{array}{l}-- \\
\overline{0.12} \\
\overline{48.00}\end{array}$ & $\begin{array}{l}0.0 \\
4.34\end{array}$ \\
\hline$\sigma$ & 1963 & $\begin{array}{l}\text { PRECIP. (CM.) } \\
\text { SR-9C IMCI/SO.KM.) } \\
\text { SR-90 CONC. (PC/L) } \\
\text { SR-89/SR-90 }\end{array}$ & $\frac{\overline{c .54}}{26.00}$ & $\begin{array}{r}1.07 \\
0.25 \\
23.37 \\
18.00\end{array}$ & $\begin{array}{r}2.97 \\
0.89 \\
29.97 \\
17.00\end{array}$ & $\begin{array}{r}2.97 \\
0.81 \\
27.28 \\
19.00\end{array}$ & $\begin{array}{r}0.91 \\
0.16 \\
17.59 \\
7.70\end{array}$ & $\begin{array}{l}43.13 \\
0.17 \\
0.40 \\
*\end{array}$ & $\begin{array}{l}25.68 \\
C .19 \\
0.74 \\
*\end{array}$ & $\begin{array}{l}0.33 \\
0.02 \\
6.07 \\
*\end{array}$ & $\begin{array}{l}15.98 \\
1.61 \\
10.08 \\
*\end{array}$ & $\begin{array}{l}1.47 \\
0.11 \\
7.49 \\
*\end{array}$ & $\begin{array}{l}4.17 \\
0.10 \\
2.40 \\
1.10\end{array}$ & $\begin{array}{l}3.56 \\
0.07 \\
1.97\end{array}$ & $\begin{array}{r}102.24 \\
4.92\end{array}$ \\
\hline & 1564 & $\begin{array}{l}\text { PRECIP. }\left(C M_{-}\right) \\
S R-90 \text { (MCI/SO.KM.) } \\
S R-90 \text { CONC. }(P C / L) \\
S R-89 / S R-90\end{array}$ & $\begin{array}{l}3.81 \\
0.20 \\
5.25 \\
*\end{array}$ & $\begin{array}{l}4.27 \\
0.24 \\
5.63 \\
*\end{array}$ & $\begin{array}{l}5.89 \\
0.61 \\
10.36 \\
*\end{array}$ & $\begin{array}{l}4.67 \\
0.46 \\
9.86 \\
+\end{array}$ & $\begin{array}{l}12.24 \\
0.44 \\
3.60 \\
*\end{array}$ & $\begin{array}{l}15.62 \\
0.18 \\
1.16 \\
--\end{array}$ & $\begin{array}{l}7.57 \\
0.10 \\
1.33 \\
--\end{array}$ & $\begin{array}{l}38.61 \\
0.14 \\
0.37 \\
-.\end{array}$ & $\begin{array}{l}49.53 \\
0.06 \\
0.23 \\
--\end{array}$ & $\begin{array}{l}50.88 \\
0.23 \\
0.46 \\
*\end{array}$ & $\begin{array}{l}1.37 \\
0.01 \\
0.73 \\
*\end{array}$ & $\begin{array}{l}2.26 \\
* \\
=- \\
--\end{array}$ & $\begin{array}{r}196.72 \\
2.67\end{array}$ \\
\hline & 1965 & $\begin{array}{l}\text { PRECIP. (CM.) } \\
S R-90 \text { (MCI/SC.KM.) } \\
S R-90 \text { CCNC. }(P C / L) \\
S R-89 / S R-90\end{array}$ & $\begin{array}{l}1.85 \\
0.02 \\
1.09 \\
-.\end{array}$ & $\begin{array}{l}1.02 \\
0.05 \\
4.91 \\
-.\end{array}$ & $\begin{array}{l}1.30 \\
0.02 \\
1.54 \\
--\end{array}$ & $\begin{array}{l}6.35 \\
0.11 \\
1.74 \\
-.\end{array}$ & $\begin{array}{l}6.35 \\
0.09 \\
1.42 \\
-.\end{array}$ & $\begin{array}{l}31.27 \\
=- \\
=\end{array}$ & $\begin{array}{l}34.52 \\
0.03 \\
C .09 \\
-\end{array}$ & $\begin{array}{l}1.98 \\
0.02 \\
1.02 \\
\end{array}$ & $\begin{array}{l}5.46 \\
0.23 \\
4.22 \\
-.\end{array}$ & $\begin{array}{l}8.13 \\
0.05 \\
0.62 \\
-\end{array}$ & $\begin{array}{l}20.40 \\
0.20 \\
0.99 \\
--\end{array}$ & $\begin{array}{c}0.33 \\
0.26 \\
78.79 \\
-.\end{array}$ & $\begin{array}{r}118.96 \\
1.08\end{array}$ \\
\hline & 1966 & $\begin{array}{l}\text { PRECIP. (CM.) } \\
\text { SR-9O IMCI/SQ.KM-) } \\
\text { SR-9O CONC. (PC/L) } \\
\text { SR-89/SR-90 }\end{array}$ & $\begin{array}{l}4.06 \\
0.08 \\
1.98 \\
--\end{array}$ & $\begin{array}{l}4.11 \\
0.03 \\
0.73 \\
-\end{array}$ & $\begin{array}{l}5.97 \\
0.04 \\
0.68 \\
-\end{array}$ & $\begin{array}{l}8.41 \\
0.06 \\
0.72 \\
-\end{array}$ & $\begin{array}{c}39.12 \\
* \\
-\end{array}$ & $\begin{array}{l}17.53 \\
-- \\
--\end{array}$ & $\begin{array}{l}42.01 \\
* \\
*\end{array}$ & $\begin{array}{l}5.84 \\
* \\
*\end{array}$ & $\begin{array}{c}34.26 \\
* \\
*\end{array}$ & $\begin{array}{l}4.75 \\
0.01 \\
0.22 \\
+\end{array}$ & $\begin{array}{r}6.55 \\
0.03 \\
0.46 \\
19.00\end{array}$ & $\begin{array}{l}2.82 \\
0.02 \\
0.71 \\
*\end{array}$ & $\begin{array}{r}175.43 \\
0.27\end{array}$ \\
\hline & 1967 & $\begin{array}{l}\text { PRECIP. (CM.) } \\
\text { SR-9O IMCI/SQ.KM.) } \\
S R-90 \text { CONC. }(P C / L) \\
S R-89 / S R-90\end{array}$ & $\begin{array}{l}5.66 \\
0.03 \\
0.54 \\
*\end{array}$ & $\begin{array}{r}4.80 \\
0.04 \\
0.84 \\
12.80\end{array}$ & $\begin{array}{l}8.43 \\
0.01 \\
0.12 \\
.\end{array}$ & $\begin{array}{l}24.61 \\
0.01 \\
0.05 \\
*\end{array}$ & $\begin{array}{r}11.71 \\
0.02 \\
0.18 \\
*\end{array}$ & $\begin{array}{l}26.14 \\
0.01 \\
0.04 \\
*\end{array}$ & $\begin{array}{l}= \\
=- \\
=\end{array}$ & $\begin{array}{l}-- \\
-- \\
--\end{array}$ & $\begin{array}{l}-- \\
-- \\
-\end{array}$ & $\begin{array}{l}-- \\
-- \\
--\end{array}$ & $\begin{array}{l}-- \\
-- \\
--\end{array}$ & $\begin{array}{l}-- \\
-- \\
--\end{array}$ & $\begin{array}{r}81.35 \\
0.12\end{array}$ \\
\hline
\end{tabular}


MONTHLY FALLOUT DEPOSITION COLLECTIONS

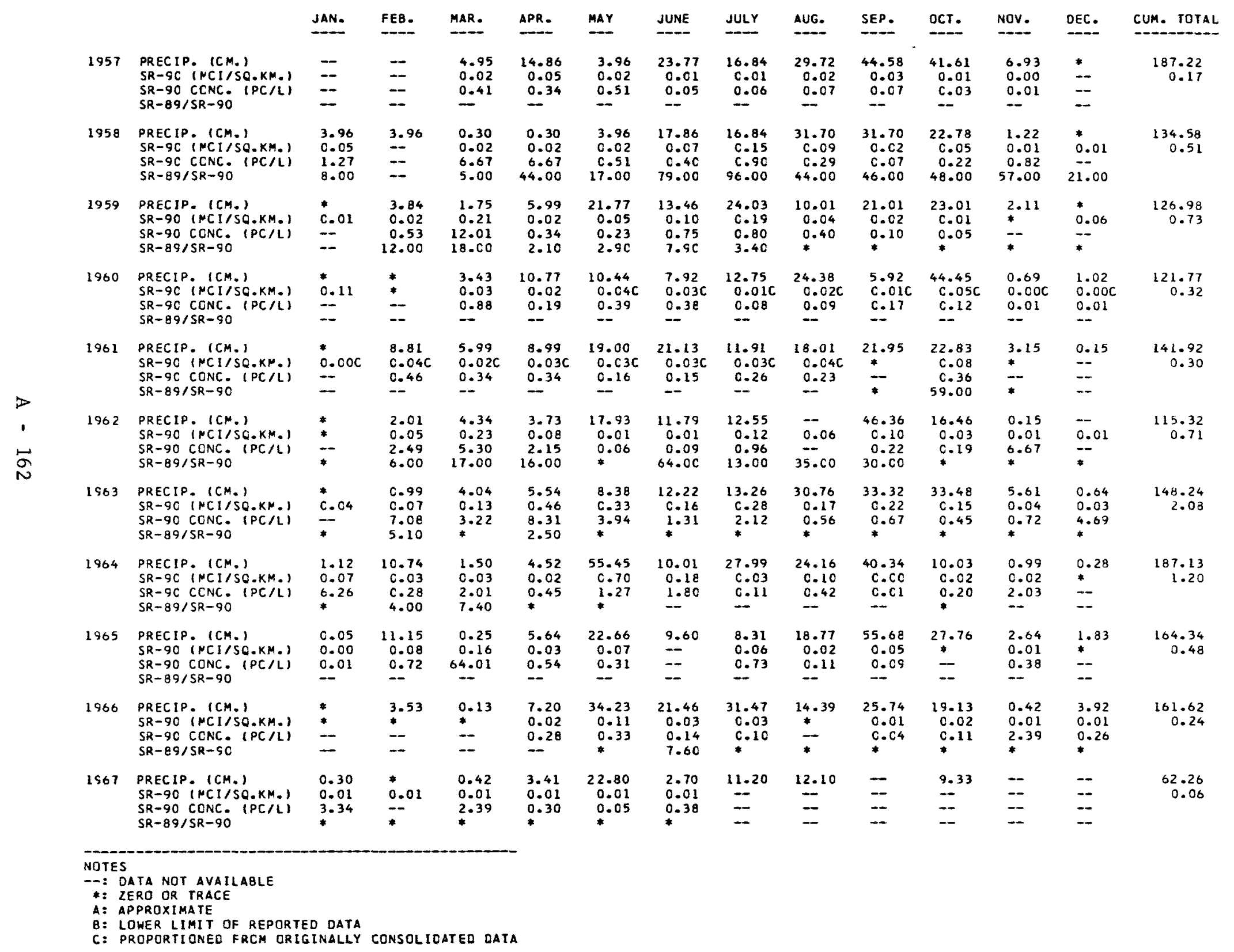


MONTHLY FALLOUT DEPOSITION COLLECTIONS

SITE: THAILAND, BANGKOK

LAT. $1344 \mathrm{~N}$ LONG.100 30E

ALT.

23M. (COLUMN)

SOURCE: THAI ATOMIC ENERGY COMMITTEE FOR PEACE

\begin{tabular}{|c|c|c|c|c|c|c|c|c|c|c|c|c|c|c|}
\hline & & JAN. & FEB. & MAR. & APR. & MAY & JUNE & JULY & AUG. & SEP. & OCT. & NOV. & DEC. & CUM. TOTAL \\
\hline 1963 & $\begin{array}{l}\text { PRECIP }\left(C M_{-}\right) \\
\text {SR-90 (MCI/SQ.KM-) } \\
S R-90 \text { CCNC. }(P C / L) \\
S R-89 / S R-90\end{array}$ & $\begin{array}{l}-- \\
--\end{array}$ & $\begin{array}{l}-- \\
--\end{array}$ & $\begin{array}{l}-- \\
--\end{array}$ & $\begin{array}{l}5.54 \\
0.24 \\
4.34 \\
--\end{array}$ & $\begin{array}{l}8.38 \\
0.72 \\
8.60 \\
*\end{array}$ & $\begin{array}{r}12.22 \\
0.40 \\
3.28 \\
*\end{array}$ & $\begin{array}{r}13.26 \\
0.00 \\
c .01 \\
1.00\end{array}$ & $\begin{array}{l}30.76 \\
0.10 \\
0.33 \\
*\end{array}$ & $\begin{array}{l}33.32 \\
0.22 \\
0.67 \\
\end{array}$ & $\begin{array}{r}33.48 \\
0.09 \\
0.27 \\
1.00\end{array}$ & $\begin{array}{l}5.61 \\
0.04 \\
0.72 \\
*\end{array}$ & $\begin{array}{r}0.64 \\
0.11 \\
17.19 \\
*\end{array}$ & $\begin{array}{r}143.21 \\
1.92\end{array}$ \\
\hline 1964 & $\begin{array}{l}\text { PRECIP, (CK.) } \\
\text { SR-90 IMCI/SQ.KM.) } \\
S R-90 \text { CONC. }(P C / L) \\
S R-89 / S R-90\end{array}$ & $\begin{array}{l}1.12 \\
0.02 \\
1.79 \\
1.50\end{array}$ & $\begin{array}{l}10.74 \\
0.04 \\
C .38 \\
--\end{array}$ & $\begin{array}{l}1.50 \\
0.20 \\
13.34 \\
*\end{array}$ & $\begin{array}{l}6.76 \\
0.09 \\
1.34 \\
3.50\end{array}$ & $\begin{array}{l}23.37 \\
0.27 \\
1.16 \\
*\end{array}$ & $\begin{array}{l}10.01 \\
0.14 \\
1.4 \mathrm{C} \\
--\end{array}$ & $\begin{array}{l}27.99 \\
0.17 \\
c .61 \\
-.\end{array}$ & $\begin{array}{l}15.65 \\
0.11 \\
0.71 \\
-.\end{array}$ & $\begin{array}{l}20.88 \\
0.05 \\
C .24 \\
-.\end{array}$ & $\begin{array}{l}10.03 \\
0.05 \\
c .50 \\
\end{array}$ & $\begin{array}{l}0.99 \\
0.02 \\
2.03 \\
+\end{array}$ & $\begin{array}{l}2.64 \\
0.02 \\
0.76 \\
--\end{array}$ & $\begin{array}{r}131.68 \\
1.18\end{array}$ \\
\hline 1965 & $\begin{array}{l}\text { PRECIP. (CM.) } \\
\text { SR-9O INCI/SO.KM.) } \\
\text { SR-90 CONC. }(P C / L) \\
S R-89 / S R-90\end{array}$ & $\begin{array}{l}-- \\
-\infty \\
--\end{array}$ & $\begin{array}{r}13.11 \\
2.96 \\
22.58 \\
-\end{array}$ & $\begin{array}{l}3.12 \\
0.07 \\
2.25 \\
--\end{array}$ & $\begin{array}{l}-- \\
0.03 \\
--\end{array}$ & $\begin{array}{l}12.83 \\
0.05 \\
0.39 \\
--\end{array}$ & $\begin{array}{l}5.21 \\
0.15 \\
2.88 \\
--\end{array}$ & $\begin{array}{l}8.31 \\
0.06 \\
0.73 \\
-.\end{array}$ & $\begin{array}{l}-- \\
= \\
--\end{array}$ & $\begin{array}{l}-- \\
--\end{array}$ & $\begin{array}{l}-- \\
-- \\
-\end{array}$ & $\begin{array}{l}0.20 \\
0.01 \\
5.01 \\
--\end{array}$ & $\begin{array}{l}-- \\
0.01 \\
--\end{array}$ & $\begin{array}{r}42.78 \\
3.36\end{array}$ \\
\hline 1966 & $\begin{array}{l}\text { PRECIP. (CM.) } \\
S R-90 \text { (NCI/SQ.KM.) } \\
S R-90 \text { CCNC. }(P C / L) \\
S R-89 / S R-90\end{array}$ & $\begin{array}{l}3.18 \\
0.05 \\
1.58 \\
-\end{array}$ & $\begin{array}{l}1.04 \\
-- \\
--\end{array}$ & $\begin{array}{l}-- \\
-- \\
--\end{array}$ & $\begin{array}{l}-- \\
-- \\
--\end{array}$ & $\begin{array}{l}29.53 \\
0.12 \\
0.41 \\
*\end{array}$ & $\begin{array}{l}9.70 \\
0.02 \\
0.21 \\
*\end{array}$ & $\begin{array}{l}26.95 \\
0.07 \\
C .26 \\
*\end{array}$ & $\begin{array}{l}14.53 \\
0.03 \\
0.21 \\
*\end{array}$ & $\begin{array}{l}15.21 \\
0.03 \\
0.20 \\
*\end{array}$ & $\begin{array}{r}15.03 \\
0.06 \\
0.40 \\
14.00\end{array}$ & $\begin{array}{l}0.33 \\
0.01 \\
3.04 \\
*\end{array}$ & $\begin{array}{l}-- \\
0.01 \\
--\end{array}$ & $\begin{array}{r}115.50 \\
0.40\end{array}$ \\
\hline 1967 & $\begin{array}{l}\text { PRECIP. (CM.) } \\
\text { SR-90 IMCI/SO.KM.) } \\
\text { SR-90 CONC. }(P C / L) \\
\text { SR-89/SR-9O }\end{array}$ & $\begin{array}{l}1.23 \\
0.01 \\
0.82 \\
*\end{array}$ & $\frac{--}{0.01}$ & $\begin{array}{l}- \\
* \\
*\end{array}$ & $\frac{-}{0.02}$ & $\begin{array}{l}28.65 \\
0.02 \\
0.07 \\
*\end{array}$ & $\begin{array}{l}10.71 \\
0.03 \\
0.29 \\
*\end{array}$ & $\begin{array}{l}-- \\
--\end{array}$ & 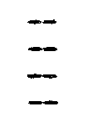 & $\begin{array}{l}-- \\
m \\
--\end{array}$ & $\begin{array}{l}-- \\
-- \\
--\end{array}$ & $\begin{array}{l}=- \\
=- \\
=-\end{array}$ & $\begin{array}{l}- \\
-- \\
--\end{array}$ & $\begin{array}{r}40.59 \\
0.09\end{array}$ \\
\hline \begin{tabular}{ll} 
NDTES \\
\hdashline$-:$ & $D$ \\
$*:$ & 2 \\
$A:$ & $A$ \\
$B:$ & $L$ \\
$C:$ & $P$
\end{tabular} & $\begin{array}{l}\text { S } \\
\text { DATA NOT AVAILABLE } \\
\text { ZERO OR TRACE } \\
\text { APPROXIMATE } \\
\text { LOWER LIMIT OF REPOR } \\
\text { PROPORTIONED FROM OR }\end{array}$ & $\begin{array}{l}\text { DATA } \\
\text { NALLYY }\end{array}$ & & $=0$ & & & & & & & & & & \\
\hline
\end{tabular}




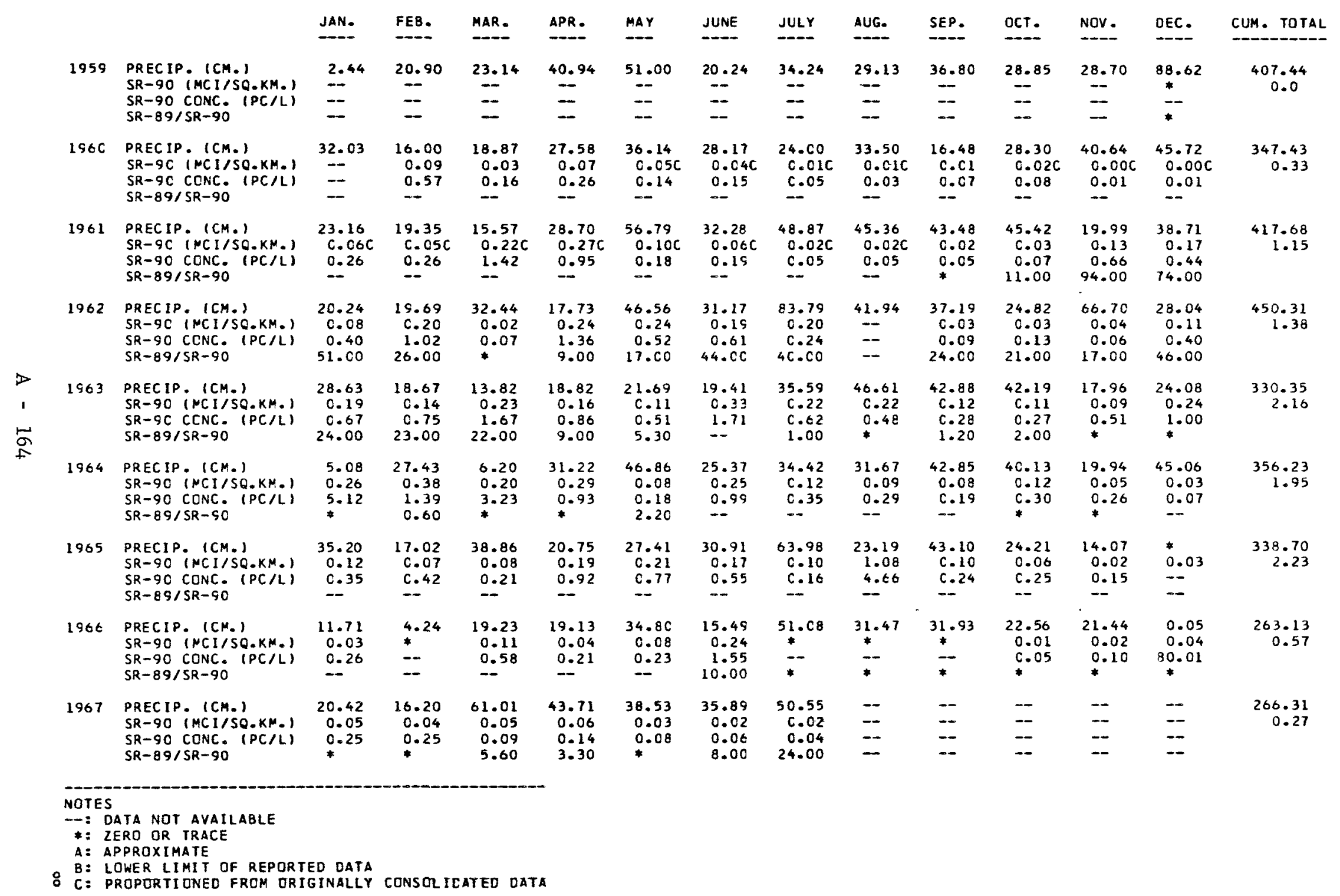




\section{MONTHLY FALLOUT DEPOSITION COLLECTIONS}
SITE: UAR, ALEXANDRIA
LAT. 31 13N LONG. 29 55E ALT.
8m. (COLUMN)

SOURCE: UAR ATOMIC ENERGY ESTABLISHMENT, HEALTH ANO SAFETY DIVISION

$D$

$$
\begin{aligned}
& \text { JAN. FEB. MAR. APR. MAY JUNE JULY AUG. SEP. OCT. NOV. DEC. CUM. TOTAL }
\end{aligned}
$$

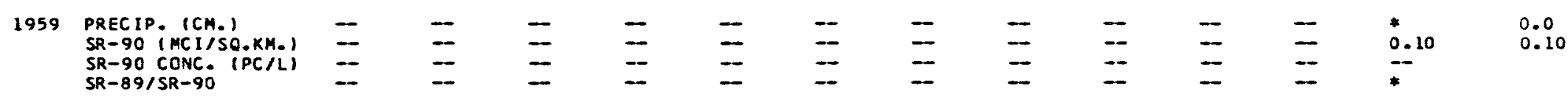

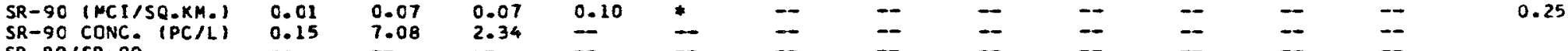

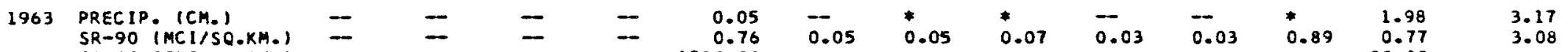

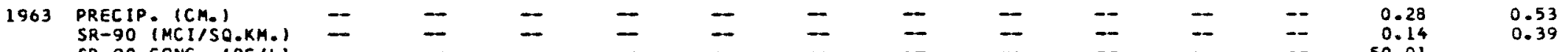

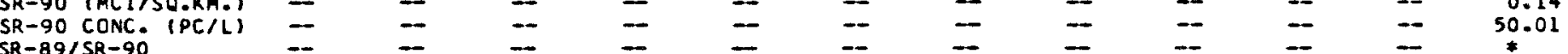

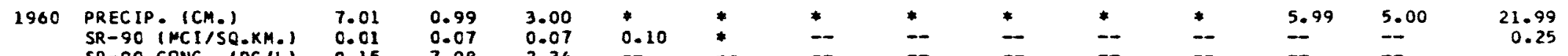

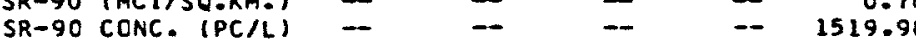

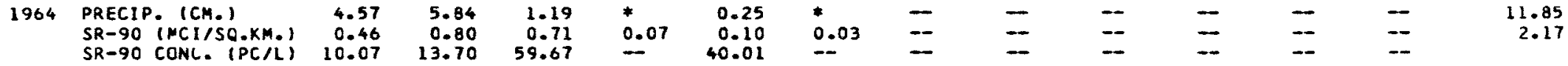

C: PROPORTIONED FROM ORIGINALLY COMSOL IOATED DATA 
MONTHLY FALLOUT DEPOSITION COLLECTIONS

SITE: UAR, CAIRO IINSHASI

LAT. $3023 \mathrm{~N}$ LONG. $3123 E$ ALT.

OM. (COLUMN)

SOURCE: UAR ATOMIC ENERGY ESTABLISHMENT, HEALTH ANO SAFETY DIVISION

JAN. FEB. MAR. APR. MAY JUNE JULY AUG. SEP. OCT. NOV. OEC. CUM. TOTAL

1963 PRECIP. (CM.)
SR-90 (MCI/SQ.KM.)

SR-90 CONC. (PC/L)

$\begin{array}{llll}- & -- & - & = \\ - & = & - & =\end{array}$

$0.05 \quad-0.05$

0.76
1519.98

0.05

$0.07 \quad 0$.

0.030 .03

03

0.28

0.33

1964 PRECIP. (CM.)
SR-9O (MCI/SQ.

$5 R-90$ CONC (PC/L)

$1.32 \quad 0 \quad 0.10 \quad 0.20$

6.00

*

2.02

$S R-89 / S R-90$

12.88

$0.10 \quad 0.20$

$*$

*

1965 PRECIP. (CM.)

SR-90 (MCI/SO.KM.) SR-90 CONC. (PC/L)

$-$

0.04

$*$

2.64

6.44

$\begin{array}{lll} & 0.16 & 0.41 \\ 0.04 & 0.06 & 0.10\end{array}$

$\begin{array}{rr}0.06 & 0.10 \\ 37.51 \quad 24.40\end{array}$

$-$

$\begin{array}{lll}* & * & * \\ 0.03 & 0.01 & - \\ -- & - & -\end{array}$

NOTES

-: DATA NOT AVAILABLE

*: ZERO OR IRACE

A: APPROXIMATE

$B$ : LOWER LIMIT OF REPORTED DATA

$C$ : PROPORTIONEO FROM ORIGINALLY CONSOLIDATED DATA 
MONTHLY FALLOUT DEPOSITION COLLECTIONS

SITE: VENEZUELA, CARACAS SITE 1

LAT. $1024 \mathrm{~N}$ LONG. 66 59W ALT. 175IH. (COLUMN)

SOURCE: INSTITUTO VENEZOLANO DE INYESTIGACIONES CIENTIFICAS

\begin{tabular}{|c|c|c|c|c|c|c|c|c|c|c|c|c|}
\hline JAN. & FE8. & MAR. & APR. & MAY & JUNE & JULY & AUG. & SEP. & OCT. & NOV. & DEC. & CUM. TOTAL \\
\hline $\begin{array}{l}2.01 \\
0.04 \\
2.00 \\
-\end{array}$ & $\begin{array}{l}3.71 \\
0.03 \\
0.81 \\
--\end{array}$ & $\begin{array}{l}0.13 \\
0.01 \\
7.70 \\
--\end{array}$ & $\begin{array}{l}0.08 \\
0.09 \\
1.12 \\
--\end{array}$ & $\begin{array}{l}7.98 \\
0.100 \\
1.26 \\
-\end{array}$ & $\begin{array}{c}16.38 \\
-0.2 C C \\
1.23 \\
-\end{array}$ & $\begin{array}{c}15.29 \\
C .06 C \\
C .40 \\
--\end{array}$ & $\begin{array}{c}12.37 \\
0.05 \mathrm{C} \\
0.41 \\
=-\end{array}$ & $\begin{array}{l}0.69 \\
0.036 \\
0.35 \\
--\end{array}$ & $\begin{array}{l}4.83 \\
0.026 \\
0.42 \\
-\end{array}$ & $\begin{array}{l}4.14 \\
0.036 \\
0.73 \\
-\end{array}$ & $\begin{array}{l}8.86 \\
0.06 \mathrm{C} \\
0.68 \\
--\end{array}$ & $\begin{array}{r}92.47 \\
0.72\end{array}$ \\
\hline $\begin{array}{l}0.94 \\
0.05 \mathrm{C} \\
5.32 \\
-.\end{array}$ & $\begin{array}{l}0.43 \\
0.026 \\
4.66 \\
-.\end{array}$ & $\begin{array}{l}0.33 \\
* \\
-- \\
--\end{array}$ & $\begin{array}{c}10.59 \\
* \\
=-\end{array}$ & $\begin{array}{l}0.30 \\
0.00 \mathrm{C} \\
0.01 \\
-\end{array}$ & $\begin{array}{l}8.46 \\
0.126 \\
1.42 \\
-.\end{array}$ & $\begin{array}{c}20.83 \\
0.02 C \\
c .10 \\
=-\end{array}$ & $\begin{array}{c}10.67 \\
0.01 C \\
0.10 \\
--\end{array}$ & $\begin{array}{c}12.80 \\
* \\
*\end{array}$ & $\begin{array}{c}9.45 \\
0.03 \mathrm{C} \\
0.32 \\
71.00 \mathrm{C}\end{array}$ & $\begin{array}{c}9.25 \\
0.03 \mathrm{C} \\
0.33 \\
71.00 \mathrm{C}\end{array}$ & $\begin{array}{r}3.76 \\
0.04 \\
1.07 \\
63.00\end{array}$ & $\begin{array}{r}87.81 \\
0.32\end{array}$ \\
\hline $\begin{array}{r}1.78 \\
C .21 \\
11.80 \\
46.00\end{array}$ & $\begin{array}{r}2.54 \\
0.11 \\
4.34 \\
27.00\end{array}$ & $\begin{array}{l}2.67 \mathrm{C} \\
0.09 \mathrm{C} \\
3.38 \\
-.\end{array}$ & $\begin{array}{l}2.67 C \\
0.09 C \\
3.38 \\
-.\end{array}$ & $\begin{array}{r}11.63 \\
0.22 \\
1.90 \\
28.00\end{array}$ & $\begin{array}{c}21.39 A \\
0.39 A \\
1.83 \\
14.00 A\end{array}$ & $\begin{array}{c}15.39 A \\
C .36 A \\
2.34 \\
--\end{array}$ & $\begin{array}{r}9.70 \\
0.13 \\
1.35 \\
23.00\end{array}$ & $\begin{array}{r}8.66 \\
C .24 \\
2.78 \\
15.60\end{array}$ & $\begin{array}{r}11.18 \\
0.07 \\
0.63 \\
26.00\end{array}$ & $\begin{array}{r}5.05 \\
0.10 \\
1.99 \\
58.00\end{array}$ & $\begin{array}{r}4.98 \\
0.10 \\
2.01 \\
37.00\end{array}$ & $\begin{array}{r}97.64 \\
2.11\end{array}$ \\
\hline $\begin{array}{r}2.21 \\
0.12 \\
5.43 \\
22.00\end{array}$ & $\begin{array}{r}1.65 \\
0.09 \\
5.46 \\
23.00\end{array}$ & $\begin{array}{r}3.84 \\
0.42 \\
10.94 \\
16.00\end{array}$ & $\begin{array}{l}3.25 \\
0.22 \\
6.77 \\
9.00\end{array}$ & $\begin{array}{r}35.20 \\
0.77 \\
2.19 \\
8.00\end{array}$ & $\begin{array}{l}22.73 \\
0.08 \\
0.36 \\
--\end{array}$ & $\begin{array}{l}7.98 \\
0.65 \\
8.15 \\
1.00\end{array}$ & $\begin{array}{l}6.22 \\
0.34 \\
5.47 \\
*\end{array}$ & $\begin{array}{l}14.73 \\
0.22 \\
1.50 \\
*\end{array}$ & $\begin{array}{r}13.67 \\
0.25 \\
1.83 \\
*\end{array}$ & $\begin{array}{r}14.96 \\
0.22 \\
1.48 \\
*\end{array}$ & $\begin{array}{l}3.48 \\
0.09 \\
2.59 \\
\end{array}$ & $\begin{array}{r}129.92 \\
3.47\end{array}$ \\
\hline $\begin{array}{l}1.19 \\
0.06 \\
5.05 \\
+\end{array}$ & $\begin{array}{r}0.15 \\
0.02 \\
13.34 \\
1.60\end{array}$ & $\begin{array}{r}0.38 \\
0.09 \\
23.69 \\
*\end{array}$ & $\begin{array}{r}1.22 \\
0.17 \\
13.94 \\
0.04\end{array}$ & $\begin{array}{l}5.44 \\
0.17 \\
3.13 \\
*\end{array}$ & $\begin{array}{c}18.24 \\
0.29 \\
1.59 \\
-\end{array}$ & $\begin{array}{c}26.54 \\
0.51 \\
1.93 \\
--\end{array}$ & $\begin{array}{c}14.61 \\
0.15 \\
1.03 \\
-\end{array}$ & $\begin{array}{l}9.25 \\
0.10 \\
1.09 \\
--\end{array}$ & $\begin{array}{r}12.88 \\
0.10 \\
0.78 \\
.\end{array}$ & $\begin{array}{l}3.89 \\
0.03 \\
0.78 \\
--\end{array}$ & $\begin{array}{l}3.76 \\
0.07 \\
1.87 \\
--\end{array}$ & $\begin{array}{r}97.55 \\
1.76\end{array}$ \\
\hline $\begin{array}{l}7.01 \\
0.05 \\
0.72 \\
-\end{array}$ & $\begin{array}{l}5.28 \\
0.02 \\
0.38 \\
--\end{array}$ & $\begin{array}{c}0.23 \\
0.03 \\
13.05 \\
--\end{array}$ & $\begin{array}{l}2.92 \\
0.07 \\
2.40 \\
-\end{array}$ & $\begin{array}{l}8.31 \\
0.03 \\
0.37 \\
-\end{array}$ & $\begin{array}{l}23.27 \\
0.19 \\
0.82 \\
-\end{array}$ & $\begin{array}{l}15.93 \\
0.12 \\
0.76 \\
0\end{array}$ & $\begin{array}{l}20.85 \\
0.16 \\
0.77 \\
\end{array}$ & $\begin{array}{r}3.20 \\
0.37 \\
11.57 \\
-\end{array}$ & $\begin{array}{l}6.93 \\
* \\
-- \\
--\end{array}$ & $\begin{array}{c}11.35 \\
+ \\
=\end{array}$ & 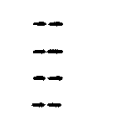 & $\begin{array}{r}105.23 \\
1.04\end{array}$ \\
\hline $\begin{array}{l}4.22 \\
0.15 \\
3.56 \\
--\end{array}$ & $\begin{array}{l}2.31 \\
* \\
-- \\
--\end{array}$ & $\begin{array}{c}0.13 \\
0.02 \\
15.39 \\
-\end{array}$ & $\begin{array}{l}5.26 \\
0.08 \\
1.53 \\
-\end{array}$ & $\begin{array}{l}8.92 \\
* \\
=- \\
-\end{array}$ & $\begin{array}{r}28.48 \\
0.05 \\
0.18 \\
21.00\end{array}$ & $\begin{array}{l}21.04 \\
0.06 \\
0.29 \\
*\end{array}$ & $\begin{array}{l}7.35 \\
0.02 \\
0.28 \\
+\end{array}$ & $\begin{array}{l}8.59 \\
0.02 \\
0.24 \\
*\end{array}$ & $\begin{array}{r}11.03 \\
0.03 \\
0.28 \\
+\end{array}$ & $\begin{array}{r}10.33 \\
0.02 \\
0.20 \\
18.00\end{array}$ & $\begin{array}{r}13.30 \\
0.03 \\
0.23 \\
*\end{array}$ & $\begin{array}{r}120.96 \\
0.48\end{array}$ \\
\hline $\begin{array}{l}5.70 \\
0.02 \\
0.36 \\
*\end{array}$ & $\begin{array}{l}1.27 \\
0.02 \\
1.58 \\
*\end{array}$ & $\begin{array}{l}2.83 \\
0.02 \\
0.71 \\
*\end{array}$ & $\begin{array}{l}9.16 \\
0.04 \\
0.44 \\
*\end{array}$ & $\begin{array}{l}5.84 \\
0.02 \\
0.35 \\
+\end{array}$ & $\begin{array}{r}13.04 \\
0.01 \\
0.08 \\
*\end{array}$ & $\begin{array}{l}-- \\
--\end{array}$ & $=$ & $=$ & $=$ & $\begin{array}{l}-- \\
-- \\
--\end{array}$ & $=$ & $\begin{array}{r}37.84 \\
0.13\end{array}$ \\
\hline
\end{tabular}

NOTES

-: DATA NOT AVAILABLE

*: ZERO OR TRACE

A: APPROXIMATE

C: PROPORTIONEO FROM ORIGINALLY CONSOLIDATEO DATA 
MONTHLY FALLOUT DEPOSITION COLLECTIONS

Site: VenezUela, caracas Site 2

LAT. 10 3N LONG. $6648 \mathrm{H}$ ALT.

926M. (COLUMN)

SOURCE: INSTITUTO VENEZOLANO DE INVESTIGACIONES CIENTIFICAS

\begin{tabular}{|c|c|c|c|c|c|c|c|c|c|c|c|c|c|c|}
\hline & & JAN. & FEB. & MAR. & APR. & MAY & JUNE & JULY & AUG. & SEP. & OCT. & Nov. & OEC . & CUM. TOTAL \\
\hline 1962 & $\begin{array}{l}\text { PRECIP. (CM-) } \\
\text { SR-90 (MCI/SQ.KM-) } \\
S R-90 \text { CONC, (PC/L) } \\
S R-89 / S R-90\end{array}$ & $\begin{array}{l}- \\
=-\end{array}$ & $\overline{-}$ & $\bar{m}$ & $\overline{-}$ & $\overline{-}$ & $\overline{-}$ & $\overline{-}$ & $\overline{-}$ & $=$ & $\frac{--}{0.07}$ & $\begin{array}{l}\overline{0.17} \\
30.00\end{array}$ & $\begin{array}{l}-- \\
-- \\
=\end{array}$ & $\begin{array}{l}0.0 \\
0.24\end{array}$ \\
\hline 1963 & $\begin{array}{l}\text { PREC IP. (CM.) } \\
\text { SR-90 (MCI/SO.KM.) } \\
\text { SR-9O CONC. (PC/L) } \\
S R-89 / S R-90\end{array}$ & $\begin{array}{l}-\overline{0.100} \\
\overline{-}\end{array}$ & $\begin{array}{l}- \\
0.100 \\
-\end{array}$ & $=$ & $\overline{0.100}$ & $\begin{array}{l}\overline{0.50} \\
\overline{9.40}\end{array}$ & $\begin{array}{l}-\infty \\
0.21 \\
+\end{array}$ & $\begin{array}{l}-- \\
0.50 \\
=\end{array}$ & $\overline{0.12}$ & $\begin{array}{l}-- \\
0.07 c \\
--\end{array}$ & $\begin{array}{l}-- \\
0.07 c \\
=-\end{array}$ & $\begin{array}{l}\overrightarrow{0.110} \\
--\end{array}$ & $\begin{array}{l}-- \\
0.11 C \\
=-\end{array}$ & $\begin{array}{l}0.0 \\
2.09\end{array}$ \\
\hline 1964 & $\begin{array}{l}\text { PRECIP. (CM.) } \\
\text { SR-90 IMCI/SQ.KM-) } \\
S R-90 \text { CONC. (PC/L) } \\
S R-89 / S R-90\end{array}$ & $\begin{array}{l}-- \\
0.46 \\
*\end{array}$ & $\begin{array}{l}-. \\
0.02 \\
1.00\end{array}$ & $\begin{array}{l}0.02 \\
*\end{array}$ & $\frac{-}{0.25}$ & $\overline{0.160}$ & $\begin{array}{l}-- \\
0.16 C\end{array}$ & $\overrightarrow{0.16 C}$ & $=0$ & $\overline{0.18}$ & $=0.04 C$ & $\begin{array}{l}\overline{0.04 C} \\
\bar{*}\end{array}$ & $\overrightarrow{0.06}$ & $\begin{array}{l}0.0 \\
1.65\end{array}$ \\
\hline 1965 & $\begin{array}{l}\text { PRECIP. (CM.) } \\
\text { SR-90 I MCI/SO.KH) } \\
\text { SR-90 CONC. }(P C / L) \\
S R-89 / S R-90\end{array}$ & $\overline{0.05}$ & $\begin{array}{l}\ddot{-} \\
--\end{array}$ & $\begin{array}{l}- \\
- \\
-\end{array}$ & $\bar{z}$ & $=$ & $=$ & $=$ & $=$ & $=0.07$ & $\begin{array}{l}-0.06 \\
--\end{array}$ & $\ddot{-}$ & $\begin{array}{l}-\infty \\
-\infty\end{array}$ & $\begin{array}{l}0.0 \\
0.23\end{array}$ \\
\hline 1966 & $\begin{array}{l}\text { PRECIP (CM.) } \\
\text { SR-9O IMCI/SC.KM-) } \\
S R-90 \text { CDNC. }(P C / L) \\
S R-89 / S R-90\end{array}$ & $=$ & $=$ & $=$ & $\ddot{-}$ & $\begin{array}{l}- \\
0.03 \\
-\end{array}$ & $\begin{array}{l}-- \\
0.03 \\
-\end{array}$ & $\begin{array}{l}-0.03 C \\
-\end{array}$ & $\begin{array}{l}\overline{0.02 C} \\
=-\end{array}$ & $\begin{array}{l}\overline{0.02} \\
\bar{*}\end{array}$ & $\overrightarrow{0.01}$ & $\begin{array}{l}-\overline{0.03} \\
--\end{array}$ & $\overline{0.01}$ & $\begin{array}{l}0.0 \\
0.18\end{array}$ \\
\hline 1967 & $\begin{array}{l}\text { PRECIP }(C M-) \\
S R-90 \text { IMCI/SO.KM.) } \\
S R-90 \text { CONC. }(P C / L) \\
S R-89 / S R-90\end{array}$ & $\frac{-}{0.01}$ & $\overline{0.01}$ & $\begin{array}{l}- \\
0.02\end{array}$ & $\frac{-}{0.02}$ & $\begin{array}{l}-0.02 \\
*\end{array}$ & $\begin{array}{r}16.90 \\
0.03 \\
0.18 \\
6.10\end{array}$ & $\overline{-}$ & $=$ & $\bar{z}$ & $\overline{-}$ & $=$ & $\overline{-}$ & $\begin{array}{r}16.90 \\
0.11\end{array}$ \\
\hline \begin{tabular}{c} 
NOTES \\
\hdashline$:$ \\
$*:$ \\
$A:$ \\
$B:$ \\
$C:$
\end{tabular} & $\begin{array}{l}\text { ATA NOT AVAILABLE } \\
\text { ERO OR TRACE } \\
\text { PPROXIMATE } \\
\text { OWER LIMI OF REPOR } \\
\text { ROPORTIONED FROM OR }\end{array}$ & DATA & & & & & & & & & & & & \\
\hline
\end{tabular}




\begin{tabular}{|c|c|c|c|c|c|c|c|c|c|c|c|c|c|c|}
\hline & & JAN. & FEB. & MAR. & APR. & MaY & JUNE & JULY & AUG. & SEP. & OCT. & Nov. & DEC. & CUM. TOTAL \\
\hline 1959 & $\begin{array}{l}\text { PRECIP. ICM-) } \\
\text { SR-90 (MCI/SO.KM.) } \\
\text { SR-90 CONC. (PC/L) } \\
\text { SR-89/SR-90 }\end{array}$ & $\begin{array}{l}2.59 \\
=- \\
=-\end{array}$ & $\begin{array}{l}1.45 \\
\because- \\
=-\end{array}$ & $\begin{array}{l}1.40 \\
= \\
=\end{array}$ & $\begin{array}{r}5.64 \\
0.75 \\
13.30 \\
12.00\end{array}$ & $\begin{array}{l}5.18 \\
0.40 \\
7.73 \\
8.40\end{array}$ & $\begin{array}{l}5.41 \\
0.03 \\
0.56 \\
5.70\end{array}$ & $\begin{array}{l}5.99 \\
0.09 \\
1.51 \\
2.60\end{array}$ & $\begin{array}{l}8.00 \\
0.07 \\
0.88 \\
1.20\end{array}$ & $\begin{array}{l}8.28 \\
0.04 \\
0.49 \\
*\end{array}$ & $\begin{array}{l}0.17 \\
0.02 \\
0.33 \\
*\end{array}$ & $\begin{array}{l}5.44 \\
0.10 \\
1.84 \\
*\end{array}$ & $\begin{array}{l}7.87 \\
0.08 \\
1.02 \\
2.90\end{array}$ & $\begin{array}{r}63.42 \\
1.58\end{array}$ \\
\hline $156 \mathrm{C}$ & $\begin{array}{l}\text { PRECIP. }(C M-) \\
\text { SR-9O iNCI/SO.KM.) } \\
\text { SR-90 CCNC. }(P C / L) \\
\text { SR-89/SR-9O }\end{array}$ & $\begin{array}{l}0.99 \\
0.06 \\
6.07 \\
-\end{array}$ & $\begin{array}{l}1.85 \\
* \\
--\end{array}$ & $\begin{array}{l}1.32 \\
0.09 \\
6.82 \\
--\end{array}$ & $\begin{array}{l}5.08 \\
0.09 \\
1.78 \\
--\end{array}$ & $\begin{array}{l}2.41 \\
0.08 \\
3.32 \\
-.\end{array}$ & $\begin{array}{l}13.89 \\
0.06 \\
0.44 \\
--\end{array}$ & $\begin{array}{l}8.00 \\
0.036 \\
0.38 \\
-.\end{array}$ & $\begin{array}{l}8.10 \\
0.03 c \\
0.38 \\
--\end{array}$ & $\begin{array}{l}12.37 \\
0.04 C \\
0.33 \\
--\end{array}$ & $\begin{array}{l}16.28 \\
0.056 \\
0.31 \\
--\end{array}$ & $\begin{array}{l}11.33 \\
0.04 \mathrm{C} \\
0.36 \\
--\end{array}$ & $\begin{array}{l}1.65 \\
0.006 \\
0.01 \\
--\end{array}$ & $\begin{array}{r}83.27 \\
0.57\end{array}$ \\
\hline 1961 & $\begin{array}{l}\text { PRECIP. (CM.) } \\
\text { SR-90 IMCI/SQ.KM-) } \\
\text { SR-9C CONC. (PC/L) } \\
\text { SR-8G/SR-9C }\end{array}$ & $\begin{array}{l}2.11 \\
0.11 \mathrm{C} \\
5.22\end{array}$ & $\begin{array}{l}1.42 \\
0.070 \\
4.93 \\
-9\end{array}$ & $\begin{array}{l}8.81 \\
0.06 C \\
0.69 \\
--\end{array}$ & $\begin{array}{l}2.77 \\
0.18 \mathrm{C} \\
6.50 \\
--\end{array}$ & $\begin{array}{l}1.27 \\
0.036 \\
2.37 \\
--\end{array}$ & $\begin{array}{l}2.77 \\
0.066 \\
2.17 \\
-=\end{array}$ & $\begin{array}{l}10.90 \\
0.06 C \\
0.56 \\
--\end{array}$ & $\begin{array}{l}20.40 \\
0.12 C \\
0.59 \\
--\end{array}$ & $\begin{array}{l}27.46 \\
.0 .11 \\
0.41 \\
3.80\end{array}$ & $\begin{array}{r}13.49 \\
0.05 \\
0.38 \\
53.00\end{array}$ & $\begin{array}{l}8.43 \\
+ \\
--\end{array}$ & $\begin{array}{r}1.93 \\
0.07 \\
3.63 \\
54.00\end{array}$ & $\begin{array}{r}101.76 \\
0.92\end{array}$ \\
\hline 1962 & $\begin{array}{l}\text { PRECIP. (CM.) } \\
\text { SR-9C INCI/SO-KM.) } \\
\text { SR-90 CONC. (PC/L) } \\
\text { SR-89/SR-90 }\end{array}$ & $\begin{array}{r}1.24 \\
1.06 \\
4.84 \\
51.00\end{array}$ & $\begin{array}{r}1.60 \\
0.16 \\
10.01 \\
32.00\end{array}$ & $\begin{array}{r}2.54 \\
0.04 \\
1.58 \\
34.00\end{array}$ & $\begin{array}{r}1.47 \\
0.24 \\
16.33 \\
12.00\end{array}$ & $\begin{array}{r}2.54 \\
0.19 \\
7.49 \\
13.00\end{array}$ & $\begin{array}{r}13.11 \\
0.68 \\
5.19 \\
11.00\end{array}$ & $\begin{array}{r}20.98 \\
0.54 \\
2.58 \\
13.00\end{array}$ & $\begin{array}{r}21.54 \\
0.44 \\
2.05 \\
10.00\end{array}$ & $\begin{array}{r}15.93 \\
0.08 \\
0.51 \\
23.60\end{array}$ & $\begin{array}{r}29.72 \\
0.06 \\
0.21 \\
22.00\end{array}$ & $\begin{array}{r}2.62 \\
0.07 \\
2.68 \\
48.00\end{array}$ & $\begin{array}{r}6.20 \\
0.22 \\
3.55 \\
47.00\end{array}$ & $\begin{array}{r}119.49 \\
2.78\end{array}$ \\
\hline 1963 & $\begin{array}{l}\text { PRECIP. (CM.) } \\
\text { SR-90 IMCI/SO-KM.) } \\
\text { SR-9C CONC. (PC/L) } \\
\text { SR-99/SR-9O }\end{array}$ & $\begin{array}{r}1.40 \\
0.62 \\
44.29 \\
28.00\end{array}$ & $\begin{array}{r}0.81 \\
0.22 \\
27.17 \\
22.00\end{array}$ & $\begin{array}{r}2.77 \\
0.15 \\
5.42 \\
23.00\end{array}$ & $\begin{array}{r}7.26 \\
0.47 \\
6.48 \\
16.00\end{array}$ & $\begin{array}{r}9.30 \\
1.89 \\
20.33 \\
9.70\end{array}$ & $\begin{array}{r}8.56 \\
1.25 \\
14.61 \\
*\end{array}$ & $\begin{array}{r}3.51 \\
6.49 \\
13.97 \\
3.00\end{array}$ & $\begin{array}{l}11.10 \\
0.33 \\
2.98 \\
*\end{array}$ & & $\begin{array}{l}14.07 \\
0.44 \\
3.13 \\
*\end{array}$ & $\begin{array}{l}7.92 \\
0.15 \\
1.90 \\
*\end{array}$ & $\begin{array}{l}4.37 \\
0.11 \\
2.52 \\
*\end{array}$ & $\begin{array}{r}76.38 \\
6.32\end{array}$ \\
\hline 1964 & $\begin{array}{l}\text { PRECIP. (CM.) } \\
\text { SR-90 iMCI/SQ.KM.) } \\
\text { SR-90 CCNC. (PC/L) } \\
\text { SR-89/SRR-90 }\end{array}$ & $\begin{array}{r}2.34 \\
0.44 \\
18.81 \\
*\end{array}$ & $\begin{array}{r}1.55 \\
0.24 \\
15.49 \\
1.00\end{array}$ & $\begin{array}{r}2.74 \\
0.58 \\
21.17\end{array}$ & $\begin{array}{r}9.35 \\
1.30 \\
13.91 \\
+\end{array}$ & $\begin{array}{r}1.07 \\
0.27 \\
25.24 \\
*\end{array}$ & $\begin{array}{r}12.83 \\
1.06 \\
8.27 \\
--\end{array}$ & $\begin{array}{l}11.33 \\
0.09 \\
0.80 \\
--\end{array}$ & $\begin{array}{l}14.86 \\
0.64 \\
4.31 \\
--\end{array}$ & $\begin{array}{l}17.22 \\
0.03 \\
0.18 \\
--\end{array}$ & $\begin{array}{l}7.06 \\
* \\
*\end{array}$ & $\begin{array}{l}5.99 \\
0.08 \\
1.34 \\
8.30\end{array}$ & $\begin{array}{l}4.24 \\
0.24 \\
5.67 \\
-\end{array}$ & $\begin{array}{r}90.58 \\
4.97\end{array}$ \\
\hline 1965 & $\begin{array}{l}\text { PRECIP. (CM.) } \\
\text { SR-90 iNCI/SC.KM.) } \\
\text { SR-90 CONC. (PC/L) } \\
\text { SR-89/SR-90 }\end{array}$ & $\begin{array}{r}0.15 \\
0.08 \\
53.34 \\
--\end{array}$ & $\begin{array}{l}2.74 \\
0.03 \\
1.10 \\
--\end{array}$ & $\begin{array}{l}3.51 \\
0.12 \\
3.42 \\
-\end{array}$ & $\begin{array}{l}8.36 \\
0.19 \\
2.28 \\
--\end{array}$ & $\begin{array}{l}5.61 \\
0.07 \\
1.25 \\
--\end{array}$ & $\begin{array}{l}3.58 \\
0.23 \\
8.43 \\
--\end{array}$ & $\begin{array}{l}1.98 \\
0.09 \\
4.55 \\
--\end{array}$ & $\begin{array}{l}10.21 \\
0.16 \\
1.57 \\
--\end{array}$ & $\begin{array}{l}3.66 \\
0.06 \\
1.64 \\
--\end{array}$ & $\begin{array}{l}19.15 \\
0.14 \\
0.74 \\
--\end{array}$ & $\begin{array}{l}4.83 \\
0.05 \\
1.04 \\
-\end{array}$ & $\begin{array}{r}1.30 \\
0.18 \\
13.85 \\
--\end{array}$ & $\begin{array}{r}65.08 \\
1.40\end{array}$ \\
\hline 1966 & $\begin{array}{l}\text { PRECIP. ICM-) } \\
\text { SR-90 iNCI/SO.KM-) } \\
\text { SR-90 CCNC. (PCIL) } \\
\text { SR-89/SR-90 }\end{array}$ & $\begin{array}{l}1.70 \\
\pm \\
-\end{array}$ & $\begin{array}{l}3.40 \\
\pm \\
=-\end{array}$ & $\begin{array}{l}1.62 \\
0.01 \\
0.62 \\
--\end{array}$ & $\begin{array}{l}3.30 \\
0.15 \\
4.55 \\
-\end{array}$ & $\begin{array}{l}8.18 \\
0.15 \\
1.84 \\
-1\end{array}$ & $\begin{array}{l}4.04 \\
0.08 \\
1.99 \\
13.00\end{array}$ & $\begin{array}{l}4.32 \\
0.06 \\
1.39 \\
*\end{array}$ & $\begin{array}{l}12.32 \\
0.03 \\
0.25 \\
*\end{array}$ & $\begin{array}{l}13.08 \\
0.03 \\
0.23 \\
*\end{array}$ & $\begin{array}{l}6.43 \\
0.02 \\
0.32 \\
*\end{array}$ & $\begin{array}{l}0.82 \\
0.16 \\
19.52 \\
*\end{array}$ & $\begin{array}{l}1.57 \\
0.02 \\
1.28 \\
*\end{array}$ & $\begin{array}{r}60.78 \\
0.71\end{array}$ \\
\hline 1967 & $\begin{array}{l}\text { PRECIP. (CM-) } \\
\text { SR-90 (MCI/SO-KM.) } \\
\text { SR-90 CCNC. (PC/L) } \\
\text { SR-89/SRR-90 }\end{array}$ & $\begin{array}{l}2.97 \\
0.04 \\
1.35 \\
8.20\end{array}$ & $\begin{array}{l}4.83 \\
0.05 \\
1.04 \\
8.30\end{array}$ & $=$ & $\begin{array}{l}9.65 \\
0.13 \\
1.35 \\
4.40\end{array}$ & $\begin{array}{l}5.72 \\
0.05 \\
0.88 \\
*\end{array}$ & $\begin{array}{l}10.62 \\
0.05 \\
0.48 \\
*\end{array}$ & $\begin{array}{l}26.82 \\
0.03 \\
0.12 \\
*\end{array}$ & $\begin{array}{l}=- \\
= \\
=-\end{array}$ & $\begin{array}{l}-- \\
= \\
--\end{array}$ & $\begin{array}{l}\overline{-} \\
= \\
=\end{array}$ & $\begin{array}{l}= \\
= \\
=\end{array}$ & $\bar{z}$ & $\begin{array}{r}60.61 \\
0.35\end{array}$ \\
\hline
\end{tabular}

\section{NOTES}

--: data not aVAILABLE

*: ZERO OR TRACE

A: APPROXIMATE

LDWER LIMIT OF REPORTED DATA

PROPORTIONED FROM ORIGINALLY CONSOL IDATED DATA 
MONTHR Y FALLOUT DEPOSITION COLLECTIONS

\begin{tabular}{|c|c|c|c|c|c|c|c|c|c|c|c|c|c|c|}
\hline & & JAN. & FEB. & MAR. & APR. & MAY & JUNE & JULY & AUG. & SEP. & $\mathrm{OCT}$ & NOV. & DEC. & CUM. TOTAL \\
\hline 1960 & $\begin{array}{l}\text { PRECIP (CM-) } \\
\text { SR-90 (MCI/SQ-KM-) } \\
\text { SR-90 CCNC. (PC/L) } \\
\text { SR-89/SR } 90\end{array}$ & $\begin{array}{l}4.37 \\
0.17 \\
3.90 \\
-.\end{array}$ & $\begin{array}{r}15.82 \\
C .05 \\
0.32 \\
-\end{array}$ & $\begin{array}{l}10.72 \\
0.09 \\
0.84 \\
-\end{array}$ & $\begin{array}{l}16.00 \\
0.09 \\
0.57 \\
-\end{array}$ & $\begin{array}{c}34.34 \\
0.10 \mathrm{C} \\
0.30 \\
-\end{array}$ & $\begin{array}{l}24.28 \\
0.076 \\
0.29 \\
--\end{array}$ & $\begin{array}{l}29.11 \\
0.02 \\
0.07 \\
--\end{array}$ & $\begin{array}{l}30.40 \\
0.02 \\
0.07 \\
-\end{array}$ & $\begin{array}{c}27.10 \\
0.03 C \\
0.12 \\
-\end{array}$ & $\begin{array}{c}45.90 \\
0.05 C \\
0.11 \\
=-\end{array}$ & $\begin{array}{l}52.71 \\
=- \\
=\end{array}$ & $\begin{array}{l}20.70 \\
0.01 \\
0.05 \\
--\end{array}$ & $\begin{array}{r}311.45 \\
0.70\end{array}$ \\
\hline 1961 & $\begin{array}{l}\text { PRECIP. (CM.) } \\
\text { SR } 90 \text { (MCI/SQ-KM.) } \\
\text { SR-90 CONC. }(P C / L) \\
\text { SR-89/SR-90 }\end{array}$ & $\begin{array}{l}29.59 \\
0.08 \mathrm{C} \\
0.28 \\
--\end{array}$ & $\begin{array}{c}14.40 \\
0.04 C \\
C .28 \\
-\end{array}$ & $\begin{array}{r}28.30 \\
0.11 \\
0.39 \\
-\end{array}$ & $\begin{array}{l}12.09 \\
-- \\
=\end{array}$ & $\begin{array}{l}45.90 \\
-- \\
-\end{array}$ & $\frac{31.29}{--}$ & $\begin{array}{l}32.31 \\
-- \\
--\end{array}$ & $\begin{array}{l}43.79 \\
- \\
-\end{array}$ & $\begin{array}{l}27.46 \\
* \\
=\end{array}$ & $\begin{array}{r}53.34 \\
0.05 \\
0.10 \\
31.00\end{array}$ & $\begin{array}{l}12.52 \\
= \\
-\end{array}$ & $\begin{array}{r}28.91 \\
0.14 \\
0.49 \\
62.00\end{array}$ & $\begin{array}{r}359.90 \\
0.42\end{array}$ \\
\hline 1962 & $\begin{array}{l}\text { PRECIP. (CH.) } \\
S R-90 \text { (FCI/SQ-KM.) } \\
S R-90 \text { CONC. }(P C / L) \\
S R-89 / S R \rightarrow 90\end{array}$ & $\begin{array}{r}21.67 \\
0.10 \\
0.47 \\
54.00\end{array}$ & $\begin{array}{r}33.93 \\
0.32 \\
0.95 \\
29.00\end{array}$ & $\begin{array}{r}19.05 \\
0.32 \\
1.68 \\
22.00\end{array}$ & $\begin{array}{r}40.51 \\
0.33 \\
0.82 \\
13.00\end{array}$ & $\begin{array}{r}36.65 \\
0.32 \\
0.88 \\
9.00\end{array}$ & $\begin{array}{r}19.74 \\
0.15 \\
0.76 \\
18.00\end{array}$ & $\begin{array}{r}49.38 \\
0.30 \\
0.61 \\
20.00\end{array}$ & $\begin{array}{l}44.50 \\
-- \\
--\end{array}$ & $\begin{array}{r}31.06 \\
0.09 \\
0.29 \\
15.00\end{array}$ & $\begin{array}{r}23.83 \\
0.06 \\
0.26 \\
17.00\end{array}$ & $\begin{array}{r}18.82 \\
0.10 \\
0.54 \\
32.00\end{array}$ & $\begin{array}{r}38.13 \\
0.25 \\
0.66 \\
39.00\end{array}$ & $\begin{array}{r}377.27 \\
2.34\end{array}$ \\
\hline 1963 & $\begin{array}{l}\text { PRECIP. (CM.) } \\
\text { SR-90 (KCI/SQ.KM.) } \\
S R-90 \text { CCNC. }(P C / L) \\
S R-89 / S R-90\end{array}$ & $\begin{array}{r}28.60 \\
0.14 \\
0.49 \\
44.00\end{array}$ & $\begin{array}{r}30.99 \\
0.24 \\
c .78 \\
29.00\end{array}$ & $\begin{array}{r}28.27 \\
0.36 \\
1.28 \\
17.60\end{array}$ & $\begin{array}{r}10.67 \\
0.96 \\
9.00 \\
10.00\end{array}$ & $\begin{array}{r}18.14 \\
0.36 \\
1.99 \\
5.90\end{array}$ & $\begin{array}{r}22.28 \\
0.64 \\
2.88 \\
4.50\end{array}$ & $\begin{array}{r}34.26 \\
0.18 \\
0.53 \\
2.00\end{array}$ & $\begin{array}{l}71.37 \\
0.33 \\
0.47 \\
*\end{array}$ & $\begin{array}{l}26.04 \\
0.19 \\
0.73 \\
*\end{array}$ & $\begin{array}{r}42.16 \\
0.33 \\
0.79 \\
1.80\end{array}$ & $\begin{array}{r}18.95 \\
0.19 \\
1.01 \\
*\end{array}$ & $\begin{array}{r}25.55 \\
0.20 \\
0.79 \\
1.00\end{array}$ & $\begin{array}{r}357.28 \\
4.12\end{array}$ \\
\hline 1964 & $\begin{array}{l}\text { PRECIP. }(C M .) \\
S R-90 \text { (KCI/SQ.KM.) } \\
S R-90 \text { CONC. }(P C / L) \\
S R \rightarrow 89 / S R-90\end{array}$ & $\begin{array}{l}6.02 \\
0.29 \\
4.82 \\
*\end{array}$ & $\begin{array}{r}17.55 \\
0.52 \\
2.97 \\
\end{array}$ & $\begin{array}{r}10.19 \\
0.61 \\
5.99 \\
*\end{array}$ & $\begin{array}{r}19.41 \\
0.32 \\
1.65 \\
*\end{array}$ & $\begin{array}{l}45.14 \\
0.30 \\
0.67 \\
*\end{array}$ & $\begin{array}{l}16.61 \\
0.23 \\
1.39 \\
--\end{array}$ & $\begin{array}{r}23.98 \\
0.22 \\
0.92 \\
--\end{array}$ & $\begin{array}{l}42.47 \\
0.15 \\
0.36 \\
--\end{array}$ & $\begin{array}{l}31.62 \\
0.12 \\
0.38 \\
-.\end{array}$ & $\begin{array}{r}29.69 \\
0.08 \\
0.27 \\
*\end{array}$ & $\begin{array}{r}15.72 \\
0.05 \\
0.32 \\
*\end{array}$ & $\begin{array}{r}29.49 \\
0.08 \\
0.28 \\
-.\end{array}$ & $\begin{array}{r}287.89 \\
2.97\end{array}$ \\
\hline 1965 & $\begin{array}{l}\text { PRECIP }\left(C M_{-}\right) \\
\text {SR-90 (KCI/SQ-KM.) } \\
S R-90 \text { CLNC. }(P C / L) \\
S R-89 / S R-90\end{array}$ & $\begin{array}{l}8.38 \\
0.06 \\
0.72 \\
--.\end{array}$ & $\begin{array}{l}15.27 \\
0.05 \\
0.33 \\
--\end{array}$ & $\begin{array}{l}19.38 \\
0.07 \\
0.37 \\
--\end{array}$ & $\begin{array}{l}10.80 \\
0.10 \\
0.93 \\
--\end{array}$ & $\begin{array}{l}20.52 \\
0.15 \\
0.74 \\
--\end{array}$ & $\begin{array}{l}27.64 \\
0.10 \\
0.37 \\
--\end{array}$ & $\begin{array}{l}67.21 \\
0.16 \\
0.24 \\
-.\end{array}$ & $\begin{array}{l}31.47 \\
0.01 \\
0.04 \\
--\end{array}$ & $\begin{array}{l}45.01 \\
0.25 \\
0.34 \\
--\end{array}$ & $\begin{array}{r}21.39 \\
0.08 \\
0.38 \\
-\end{array}$ & $\begin{array}{l}30.78 \\
0.11 \\
0.36 \\
--\end{array}$ & $\begin{array}{l}9.37 \\
0.10 \\
1.07 \\
-.\end{array}$ & $\begin{array}{r}307.22 \\
1.14\end{array}$ \\
\hline 1966 & $\begin{array}{l}P R E C I P \text {. (CM.) } \\
\text { SR-90 IKCI/SQ.KM.) } \\
S R-90 \text { CCNC. }(P C / L) \\
S R-89 / S R-90\end{array}$ & $\begin{array}{r}12.65 \\
0.03 \\
0.24 \\
--\end{array}$ & $\begin{array}{l}3.28 \\
0.13 \\
3.97 \\
--\end{array}$ & $\begin{array}{l}5.87 \\
0.06 \\
1.03 \\
--\end{array}$ & $\begin{array}{l}4.72 \\
0.06 \\
1.28 \\
-\end{array}$ & $\begin{array}{r}17.04 \\
0.08 \\
0.47 \\
-\end{array}$ & $\begin{array}{r}31.80 \\
0.03 \\
0.10 \\
19.00\end{array}$ & $\begin{array}{r}45.67 \\
0.04 \\
0.09 \\
15.00\end{array}$ & $\begin{array}{l}22.91 \\
* \\
-\end{array}$ & $\begin{array}{l}24.36 \\
0.02 \\
0.09 \\
+\end{array}$ & $\begin{array}{r}18.06 \\
0.04 \\
0.23 \\
*\end{array}$ & $\begin{array}{r}22.45 \\
0.02 \\
0.09 \\
*\end{array}$ & $\begin{array}{r}25.32 \\
0.02 \\
0.08 \\
*\end{array}$ & $\begin{array}{r}234.13 \\
0.53\end{array}$ \\
\hline 1967 & $\begin{array}{l}\text { PRECIP. (CM-) } \\
\text { SR-9O (YCI/SO.KM-) } \\
\text { SR-9O CCNC. (PC/L) } \\
S R-89 / 5 R-90\end{array}$ & $\begin{array}{l}30.53 \\
0.03 \\
0.10 \\
*\end{array}$ & $\begin{array}{l}15.88 \\
0.03 \\
0.19 \\
+\end{array}$ & $\begin{array}{r}13.64 \\
6.04 \\
0.30 \\
7.10\end{array}$ & $\begin{array}{l}29.87 \\
0.05 \\
0.17 \\
*\end{array}$ & $\begin{array}{l}40.18 \\
C .03 \\
0.08 \\
*\end{array}$ & $\begin{array}{l}42.49 \\
0.01 \\
0.03 \\
\end{array}$ & $\begin{array}{l}-- \\
- \\
-\end{array}$ & $\overline{-}$ & $=$ & $\begin{array}{l}-- \\
--\end{array}$ & $\bar{m}=$ & $\overline{-}$ & $\begin{array}{r}172.59 \\
0.19\end{array}$ \\
\hline $\begin{aligned} \text { NOTES } & \\
--: & 0 \\
*: & Z \\
A: & A \\
B: & L \\
C: & P\end{aligned}$ & $\begin{array}{l}\text { ATA NOT AVAILABLE } \\
\text { ERO OR TRACE } \\
\text { PPROXIMATE } \\
\text { OWER LIMIT OF REPO } \\
\text { ROPORTI ONEO FROM O }\end{array}$ & DATA & & & & & & & & & & & & \\
\hline
\end{tabular}


Fisston Product and Activation Product Radionuclides in Monthly

Deposition at selected Sites

Other radianuclides in addition to strontium-90 and strontium-89 have been analyzed in monthly preclpitation at selected sites. These sites and the associated analytical laboratory are given below:

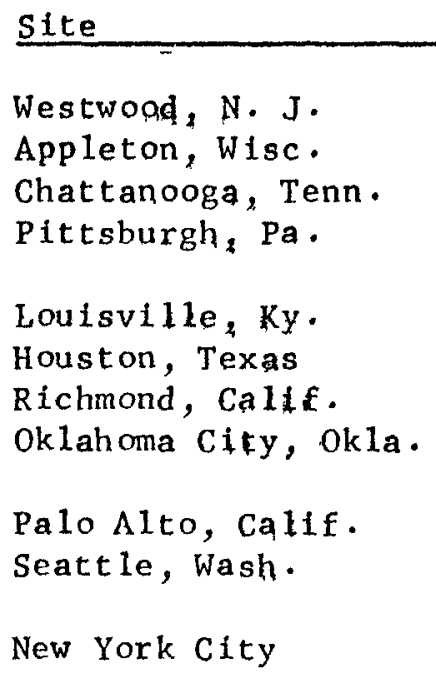

Laboratory

$\begin{array}{cccc}\text { Is otopes, Inc. (Westwaod, N. J.) } \\ \text { " } & \text { " } & \text { " } & \text { " }\end{array}$

Nuclear Science \& Engineering Corp.

(Pittsburgh, Pa.)

Radi ochemistry, Inc. (Louisville, Ky.)

Tracerlab, A Div. of LFE (Richmond, Calif.)

Hazleton-Nuclear Science Corp.

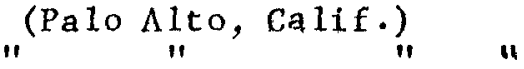

Fopd, Chemical \& Research Labs., Inc.

(Seatlle, Wash.)

Health and Safety Laboratory, USAEC, NYO

The radionuclide measurements in monthly precipitalion expressed as millicuries per square kilometer are given in the tables starting on p. B-3. A separate listing has been made for each nuclide and the avaflable data are given under the appropriate site heading. The first table consists of monthly precipitation measurements made at each site. Data for the following radionuclides are covered in the tables.

Radionuclides

$$
\begin{aligned}
& \mathrm{Mn}-54 \\
& \mathrm{Fe}-55 \\
& \mathrm{~S} r-89 \\
& \mathrm{Sr}-90 \\
& \mathrm{Zr}-95 \\
& \mathrm{Rh}-102 \\
& \mathrm{Ru}-103 \\
& \mathrm{Ru}-106 \\
& \mathrm{Cd}-109 \\
& \mathrm{Sb}-124 \\
& \mathrm{Sb}-125 \\
& \mathrm{Cs}-137 \\
& \mathrm{Ba}-140 \\
& \mathrm{Ce}-141 \\
& \mathrm{Ce}-144 \\
& \mathrm{Pu}-238 \\
& \mathrm{Pu}-239,240
\end{aligned}
$$

Type

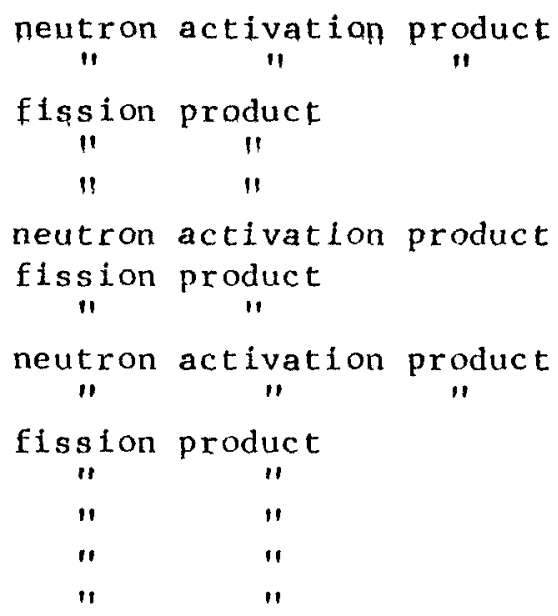


Data for other radionuclides not reported here, were presented in the July 1, 1966 Quarterly, HASL-146. The Identification and data page for each of these radionuclides are as follows:

\begin{tabular}{|c|c|c|c|c|c|}
\hline Radionuc 1ide & Type & Site & $\begin{array}{l}\text { Samp } \\
\text { Per }\end{array}$ & $\begin{array}{l}\text { ling } \\
\text { lod }\end{array}$ & $\begin{array}{c}\text { (HASL-146) } \\
\text { Page } \\
\end{array}$ \\
\hline$C s-136$ & fission product & Richmond, Calif. & $9 / 61$ & $-6 / 62$ & 17 \\
\hline \multirow[t]{2}{*}{$P m-147$} & . " & Richmond, Calif. & $7 / 61$ & $-8 / 61$ & 17 \\
\hline & & $\begin{array}{l}\text { Westwood, N. J. } \\
\text { Pittsburgh, } \mathrm{Pa} \text {. }\end{array}$ & $\begin{array}{l}7 / 61 \\
7 / 61\end{array}$ & $\begin{array}{l}-\quad 8 / 61 \\
-\quad 8 / 61\end{array}$ & $\begin{array}{l}34 \\
45\end{array}$ \\
\hline$W-185$ & $\begin{array}{c}\text { neutron activa- } \\
\text { tion product }\end{array}$ & Richmond, Calif. & $6 / 58$ & $-6 / 60$ & 16 \\
\hline T $1-204$ & $"$ & Westwood, N. J. & $7 / 62$ & $-6 / 63$ & 168 \\
\hline $\begin{array}{l}P u-238 \\
P u-239\end{array}$ & & $\begin{array}{l}\text { Richmond, Ca lif. } \\
\text { " } \\
\text { Loulsville, Ky. } \\
\text { Westwood, N. J. } \\
\text { Pittsburgh, Pa. } \\
\text { Houston, Texas }\end{array}$ & $\begin{array}{l}7 / 61 \\
7 / 61 \\
2 / 60 \\
2 / 60 \\
2 / 60 \\
1 / 60\end{array}$ & $\begin{array}{l}-6 / 63 \\
-\quad 6 / 63 \\
-\quad 6 / 63 \\
-\quad 6 / 63 \\
-\quad 6 / 63 \\
-\quad 6 / 63\end{array}$ & $\begin{array}{lll}17 & \& & 18 \\
" & & \\
& 28 \\
34 & \& & 35 \\
44 & \& & 46 \\
55 & \& & 56\end{array}$ \\
\hline
\end{tabular}


RADIONUCLIDES IN MONTHLX PRECIPITATION

Preclpltation Amount

in $\mathrm{cm}$

\begin{tabular}{|c|c|c|c|c|c|c|c|}
\hline Year & Eleme!!! & 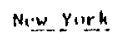 & Weystwingl & Pistefous sh & L, nul ovol tes. & Hersat on & Hellynound \\
\hline \multirow[t]{13}{*}{1057} & IAM. & 1.21 & & & & & \\
\hline & Fels. & 6.75 & & & & & \\
\hline & Mar. & 5.21 & & & & & \\
\hline & Alי. & 1140 & & & & & \\
\hline & Miny & 17.12 & & & & & \\
\hline & hur & 1.22 & & & & & \\
\hline & lulv & 4.12 & & 11.1 .6 & & & \\
\hline & Anx. & 7.20 & & 1.24 & & & \\
\hline & sept. & $7.6,1$ & & 11.73 & & & \\
\hline & iki. & A. 30 & & 4.93 & & & \\
\hline & Nov . & 11.10 & & 5.51 & & & \\
\hline & nase. & 13.36 & & 1752 & & & \\
\hline & $\operatorname{Tet} n 1$ & 92.64 & & & & & \\
\hline \multirow[t]{13}{*}{$195 \pi$} & Jan. & $9.6)$ & 16.31 & H. Kh & & & \\
\hline & Felb. & 157 & - & 254 & & & \\
\hline & Mar. & a. 10 & 12.50 & 8.53 & & & $16 . \mathrm{A6}$ \\
\hline & $\mathrm{APM}$. & 15.601 & 1620 & $98 x$ & & & -- \\
\hline & May & A.26 & 10.10 & 983 & & $7.9 \%$ & $\star$ \\
\hline & June" & 6.48 & $4 \cdot A 3$ & 1.62 & & 5.17 & - \\
\hline & JuIy & is is & 7.06 & 2151 & & 6.91 & * \\
\hline & AnR. & 5.97 & 10.95 & 13.51 & & 16.36 & $\star$ \\
\hline & sipe. & 11.28 & 6.76 & 11.112 & $7 \cdot 19$ & 22.61 & 0.13 \\
\hline & net. & $11 \mathrm{HI}$ & 1199 & $2 \cdot 13$ & 4.19 & 14.99 & 0.53 \\
\hline & Nov. & 4.70 & 4.19 & 7.75 & 1.04 & 4.49 & $\leftarrow$ \\
\hline & Bre. & 3.11 & 2.74 & 2.00 & 302 & 1.17 & 4.50 \\
\hline & Tot 1 & $10 / 4.01$ & 104.21 & $10 x \cdot 1 / 4$ & & & \\
\hline \multirow[t]{13}{*}{1959} & fann. & 5.94 & 6.76 & 7.11 & 14.00 & 14.11 & 11.20 \\
\hline & lieb. & 4.29 & 5.54 & III. IA & 7.01 & 15.52 & 15.54 \\
\hline & Mar. & $9.5 \mathrm{~A}$ & 9.55 & 6.35 & 5.41 & 2.11 & 2.06 \\
\hline & Apr. & 4.85 & H. 29 & 9.27 & 4.21 & 17.59 & d.d? \\
\hline & Mny & 3.34 & 2.74 & 7.42 & 11.23 & 19.05 & $\star$ \\
\hline & Jitne & 10.67 & 13.81 & 914 & 2.H. & 16.56 & $\star$ \\
\hline & Inty & 10.87 & 15.71 & A. 41 & 6.61 & 20.75 & $\star$ \\
\hline & Aug. & 11.30 & 14.78 & $H .94$ & 13.21 & 41.15 & \# \\
\hline & Sopt. & 2.82 & 6.15 & .3 .58 & 1.22 & 10.7 & 1.20 \\
\hline & Act. & 12.26 & 16.15 & A1 66 & A. 29 & 22.42 & $\star$ \\
\hline & Nnv. & 10.72 & 10.40 & 1.17 & 10.54 & 3.68 & $\star$ \\
\hline & ore. & $11.7 \mathrm{~A}$ & 11.76 & 729 & 9.58 & 14.145 & 4.11 \\
\hline & Tintal & 98.146 & 122.04 & 97.71 & $9 / 6.7 .9$ & 1.64 . . 1 & 41.45 \\
\hline \multirow[t]{13}{*}{1960} & Jan. & 6.10 & 1.95 & 1.34 & 6.48 & 4.95 & 13.97 \\
\hline & Finto. & 11.25 & 12.115 & 5.26 & 10.95 & 10. .11 & 11.76 \\
\hline & Mar. & 1.52 & $5.14 / 4$ & 4.014 & 6.15 & -. & 6.22 \\
\hline & Apr. & 7.75 & 10.13 & 4.04 & 2.91 & 2.16 & 2.67 \\
\hline & May & 7.54 & 6.AB & 11.96 & 9. 94 & 2.24 & 0.84 \\
\hline & $\operatorname{sen} \theta$ & 4.42 & $3 . R \mathrm{I}$ & A. 92 & 1568 & 36.52 & $\star$ \\
\hline & Judy & 21.06 & $21 \cdot 1.6$ & 7.34 & 5.05 & 13.92 & $\star$ \\
\hline & Aus. & 19.90 & 47.04 & 6.35 & 4.55 & 18.85 & $\star$ \\
\hline & Sept & 13.66 & 17.65 & 7.42 & 7.62 & 4.72 & $\star$ \\
\hline & nct. & 7.16 & $6.5 \mathrm{H}$ & 4.90 & 4.0 .1 & 27.56 & 0.11 \\
\hline & Nov. & 7.15 & 7.52 & 3.50 & 8. 54 & 12.01 & 12.85 \\
\hline & Bre. & 7.12 & 5.23 & 7.01 & $5 .+1.8$ & 17.01 & 1.96 \\
\hline & $T e+1$ & 117.93 & $121.8 /$ & 79.53 & 96.09 & +500.13 & 50.98 \\
\hline \multirow[t]{13}{*}{$19 \mathrm{bil}$} & Jan. & $4.7 H$ & 7.52 & 2.29 & 6.12 & 11.28 & 7.57 \\
\hline & ti,enh. & 100.06 & A.97 & 7.64 & $\mid 8.31$ & 16.41 & 3.00 \\
\hline & Mns. & 10.74 & $12 \cdot 36$ & 9.35 & $19.3 \mathrm{~A}$ & 4.67 & 7.09 \\
\hline & Apr. & 12.90 & $15,6,7$ & 9.74 & 12.27 & 6.15 & $2 \cdot 5,1$ \\
\hline & May & 9.14 & 7.09 & B. 6,1 & 22.86 & 9.12 & 0.99 \\
\hline & Jumer & 1.26 & 4.95 & $10.7 \%$ & $9.1,2$ & 24.77 & $\star$ \\
\hline & duIV & $4 R .50$ & 17.35 & 10.36 & $\$ 4.73$ & 71.83 & $\star$ \\
\hline & Amp. & 1.85 & 13.89 & 8.33 & $2 \cdot 34$ & 0.20 & 0.20 \\
\hline & sepht: & 4.32 & 9.35 & 4.27 & 3.76 & $20.0 \%$ & 0.76 \\
\hline & act. & 5.61 & 5.27 & 7.85 & 5.08 & 0.53 & 0.23 \\
\hline & Nov. & 6.48 & $1.1,9$ & 8.18 & 10.74 & 14.168 & 12.65 \\
\hline & Aser. & 7.17 & 8.79 & 5.18 & 9.55 & $2 \cdot 24$ & 3.05 \\
\hline & Tatal & 99.86 & $118 \quad 614$ & $91.5 \%$ & 129.26 & 136.16 & 38.05 \\
\hline
\end{tabular}

- data not avallable

* zeró or irace 
in $\mathrm{cm}$.

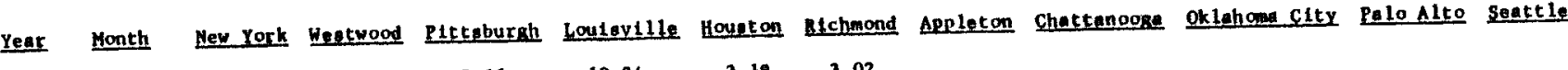

1962

$\begin{array}{lr}\text { Jan. } & 6.65 \\ \text { Feb. } & 9.50 \\ \text { Mar. } & 7.54 \\ \text { Apr. } & 7.62 \\ \text { May } & 3.20 \\ \text { June } & 9.47 \\ \text { Ju1y } & 4.24 \\ \text { Aug. } & 14.50 \\ \text { Bept. } & 7.87 \\ \text { Oct. } & 8.00 \\ \text { Nov. } & 10.01 \\ \text { Dec. } & 5.74 \\ & \end{array}$

Tota $1 \quad 94.34$

1963

Jan. $\quad 4.90 \quad 5.31$

$\begin{array}{lll} & 4.90 & 5.31 \\ \text { Feb. } & 6.48 & 3.05\end{array}$

Mar. $\quad 9.17 \quad 10.21$

Apr. $\quad 3.22$

May $\quad 5.48$

June $\quad 6.91$

$\begin{array}{lrr}\text { July } & 3.96 & 9.02\end{array}$

Aug. $\quad 8.15 \quad 4.57$

Sept. $\quad 10.03 \quad 11.91$

$\begin{array}{lll}0 \mathrm{ct} & 0.36 & 2.34\end{array}$

Nov. $20.93 \quad 11.38$

Dec. $\quad 5.87$

Tota1 87.06

1964

$$
\text { Ja }
$$

$11.73-8.84$

$7.44 \quad 4.80$

$\begin{array}{lll} & 6.53 & 5.69\end{array}$

Apr. $\quad 12.93 \quad 15.60$

$\begin{array}{lll}\text { May } & 1.45 & 2.67\end{array}$

June $\quad 6.78 \quad 6.25$

July $\quad 10.59 \quad 12.93$

$\begin{array}{lll}\text { Aug. } & 0.61 & 2.54\end{array}$

Sept. $\quad 4.29 \quad 3.25$

Oct. $\quad 4.39 \quad 3.45$

$\begin{array}{rrr}\text { Nov. } & 6.48 & 6.10 \\ & 10.57 & 10.97\end{array}$

Total 83.79

63.09

1963

Jan. $\quad 7.85 \quad 6.40$

Feb. $\quad 9.30 \quad 7.72$

$\begin{array}{lll}\text { Mar. } & 6.32 & 3.96\end{array}$

Apr. $\quad 7.37 \quad 4.60$

May $\quad 4.01 \quad 3.40$

June $\quad 3.22 \quad 4.98$

July $\quad 3.38 \quad 8.00$

Aug. $\quad 6.93 \quad 11.61$

Sept. $4.32 \quad 7.72$

Det. $\quad 5.49 \quad 6.12$

Nov. $\quad 3.71 \quad 4.78$

Dec. $4.37 \quad 4.34$

$\begin{array}{lll}\text { Total } & 66.27 \quad 73.63\end{array}$

1966

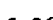

$\begin{array}{rrr} & 6.68 & 5.26 \\ \text { reb. } & 12.69 & 9.65\end{array}$

Mar. $\quad 2.39 \quad 3.38$

Apr. $\quad 6.83 \quad 6.98$

$\begin{array}{lll}\text { May } & 10.82 & 10.29\end{array}$

June $\quad 2.97 \quad 2.11$

July $\quad 3.18 \quad 4.34$

Aug. $\quad 4.80 \quad 3.89$

Sept . $\quad 22.40 \quad 23.93$

Oct. $11.78 \quad 6.83$

Nov. $\quad 8.81 \quad 11.00$

Total $\quad 101.43 \quad 94.98$

1967

$\begin{array}{lr}\text { Jan. } & 3.53 \\ \text { Feb. } & 6.81 \\ \text { Mar. } & 15.16 \\ \text { Apr. } & 8.76 \\ \text { May } & 10.36 \\ \text { June } & 11.78 \\ \text { July } & 17.75 \\ \text { Aug. } & 15.09 \\ \text { Sept. } & 4.67 \\ \text { Oct. } & 8.81 \\ \text { Sov. } & \end{array}$

Mec.

\begin{tabular}{|c|c|c|c|}
\hline 5.16 & 10.24 & $\begin{array}{l}3.18 \\
1.52\end{array}$ & $\begin{array}{r}3.02 \\
27.56\end{array}$ \\
\hline $\begin{array}{l}9.04 \\
7.67\end{array}$ & $\begin{array}{r}16.71 \\
9.09\end{array}$ & $\begin{array}{l}1.32 \\
1.32\end{array}$ & $\begin{array}{r}22.56 \\
2.16\end{array}$ \\
\hline $\begin{array}{r}7.67 \\
11.58\end{array}$ & 3.66 & 12.22 & 0.79 \\
\hline 6.60 & 7.37 & 2.92 & $\star$ \\
\hline 4.09 & 15.09 & 18.80 & $\star$ \\
\hline 8.05 & 1.65 & 7.04 & $\star^{\prime}$ \\
\hline 6.35 & 5.28 & 7.04 & 0.25 \\
\hline 17.42 & 9.35 & 10.08 & 0.25 \\
\hline 5.46 & 11.94 & 7.92 & 16.59 \\
\hline 3.53 & 4.06 & 14.43 & 2.29 \\
\hline 5.94 & 6.91 & 12.01 & 8.38 \\
\hline 91.09 & 101.35 & 98.68 & 36.29 \\
\hline $\begin{array}{l}5.00 \\
6.48\end{array}$ & 3.00 & 7.85 & 11.30 \\
\hline 17.40 & 22.96 & 1.40 & 10.08 \\
\hline 7.72 & 4.75 & 2.34 & 10.92 \\
\hline 4.22 & 11.58 & 1.37 & 1.14 \\
\hline & 10.62 & -- & * \\
\hline
\end{tabular}
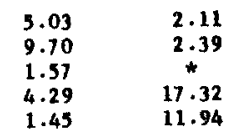

15.09
6.22

6.22
4.60

4.60
0.51

3.05
1.70

6.04

$1.43 \quad 11.94$

1.70

2.64

0.41

4.22

6.60
13.51

13.51
3.84

3.84
14.05

6.17

9.98
0.79

5.41
1.40

14.30
13.97

26.96

24.97

12.55

8.43

14.17

15.72

7.57

9.73

8.66
7.57

69.02

166.60

8.28

1.73

1.93

8.00

9.55

5.99

8.15

6.35

9.42

19.99

4.75
4.72

6.70

87.28

8.28
11.94
25.40

25.40
9.86

7.11

11.46

11.53

11.76

2.26

10.13

2.13

122.95

\subsection{3}

3.40

3.33

9.30

9.73
1.74

2.44
9.50

9.89
2.90

8.89
2.90

15.61

1.70

67.79

5.05

2.87

3.10

6.63

8.61

4.98

4.83
12.75

10.64

3.35

0.10
6.86

69.77

8.48

4.80

11. 35

10.21

14.83

0.02

$\begin{array}{cr}\star & 4.60 \\ \star & 3.99 \\ 0.46 & 2.39 \\ 2.91 & 10.64 \\ 6.96 & 20.12 \\ \star & 12.06\end{array}$

59.62

$8.66 \quad 18.77$

$\begin{array}{ll}0.64 & 3.18 \\ 3.53 & 7.54\end{array}$

$0.10 \quad 3.38$

$1.32 \quad 3.53$

$1.37 \quad 7.80$

$\begin{array}{ll}\star 46 & 2.49\end{array}$

$\star \quad 4.72$

$2.21 \quad 1.65$

$\begin{array}{rr}8.15 & 11.38 \\ 13.11 & 11.38\end{array}$

$39.55 \quad 75.82$

$7.42 \quad 14.81$

$1.93 \quad 11.05$

$3.89 \quad 1.96$

$2.79 \quad 9.04$

* 1.27

* 0.96

1.19

3.91

2.11

$\begin{array}{rr}* & 4.57 \\ 12.29 \quad 12.06\end{array}$

$\begin{array}{rr}12.29 & 14.43\end{array}$

36.45

$77 \cdot 36$

12.45

6.25

12.09

5.13

3.40

1.90

2.77

0.23

3.66

6.63

15.52

90.22

-- data not aval lable

18.72 4.62
7.87 


\section{RADIONUCLIDES IN MONTHLY PRECIP ITATION \\ Manganese -54 in $\mathrm{mCl}$ per $\mathrm{km}^{2}$ \\ decay corrected to $10 / 15 / 61$}

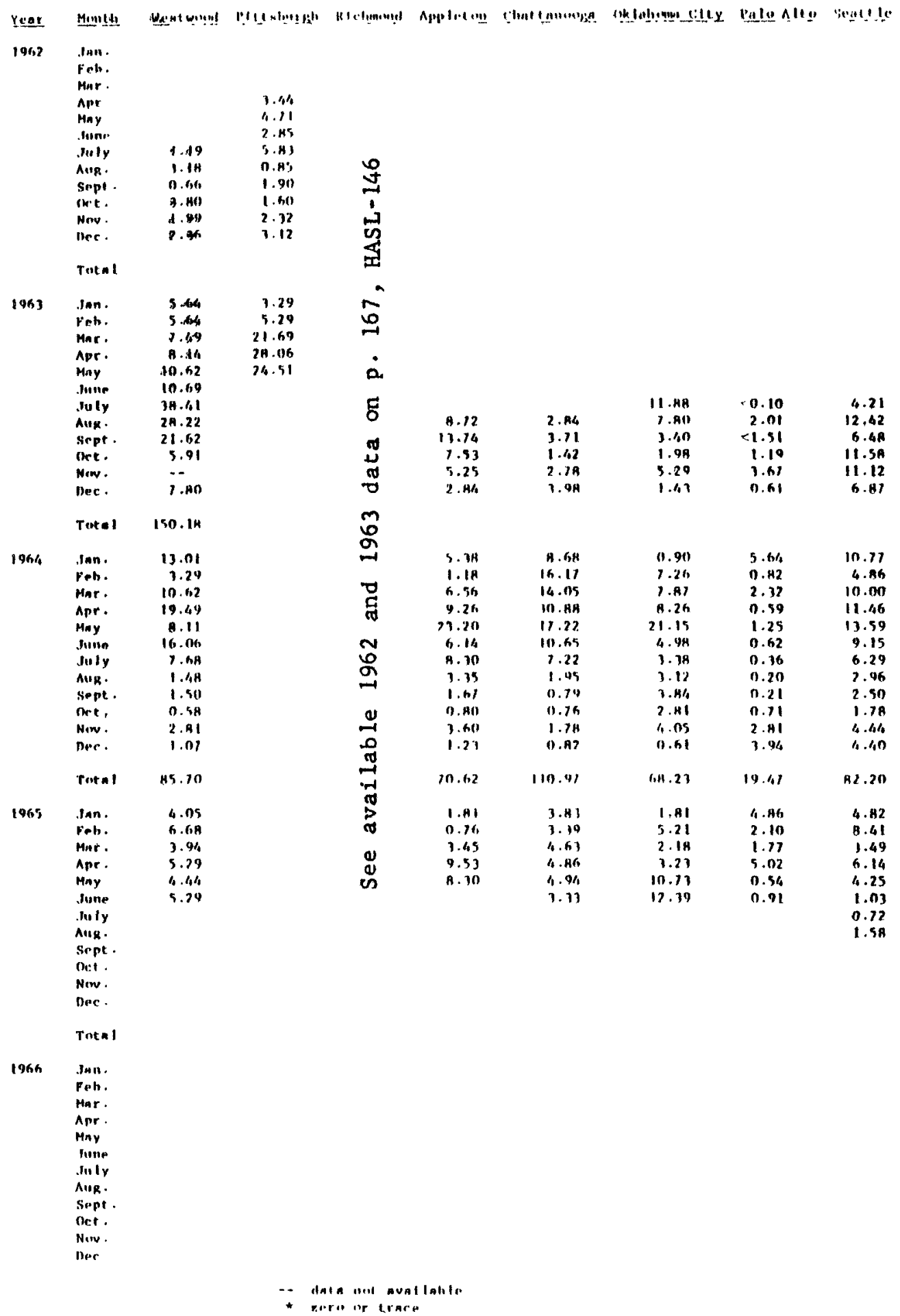




\section{RADIONUCLIDES IN MONTHLY PRECIPITATION \\ Iron-55 in $\mathrm{mCi}$ per $\mathrm{km}^{2}$ \\ decay corrected to $10 / 15 / 61$}

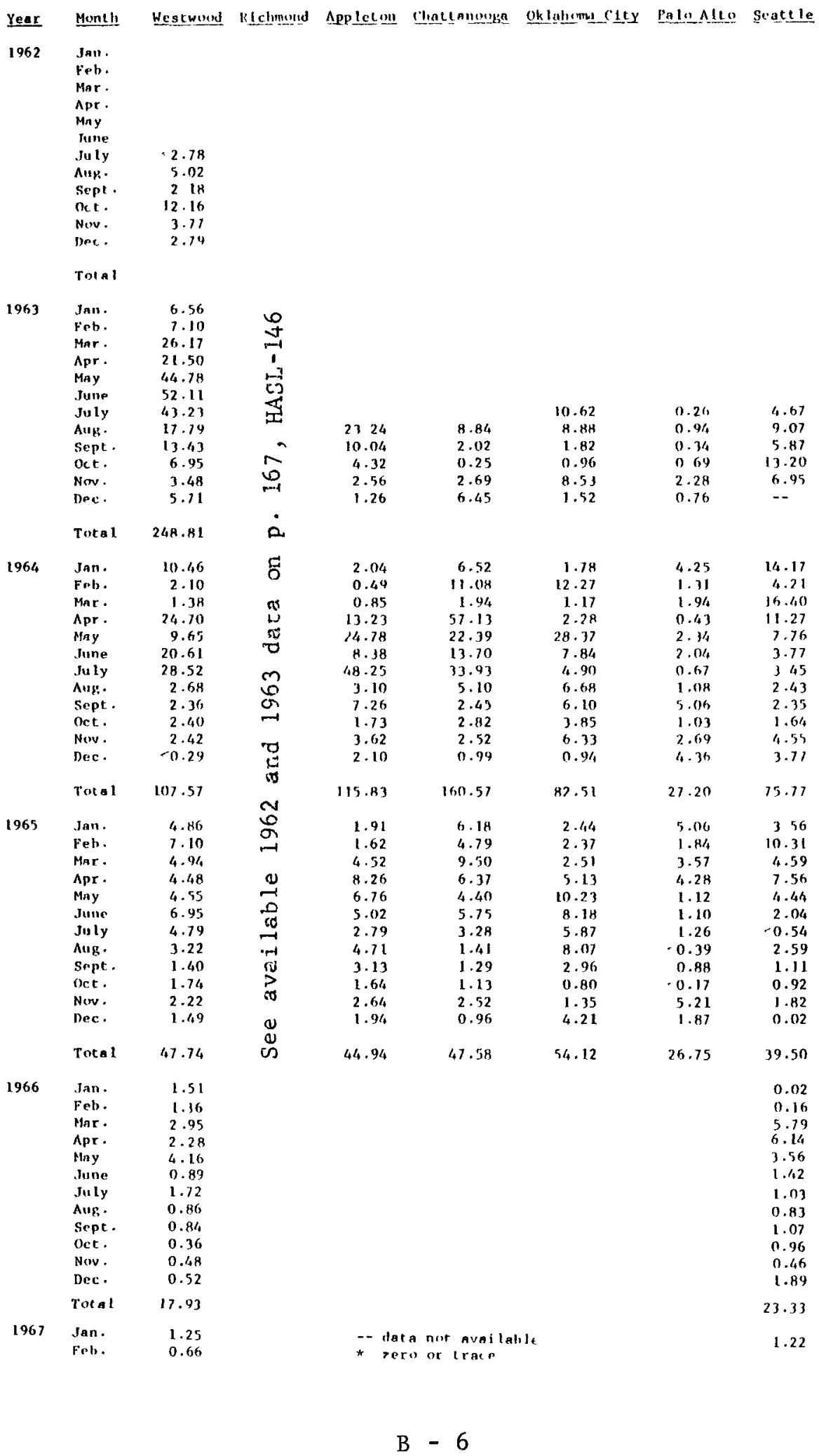


RADIONUCI.IDES IN MONTILY PRECIPITATION

Stront lum-89 in $\mathrm{mCl}$ per $\mathrm{km}^{2}$

decay corrected to the midpoint of ampling month

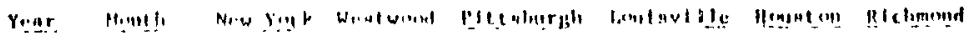

(n)

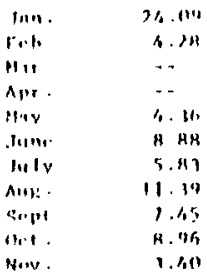

Nave. 1.100

Tol H. H I

1954

$\begin{array}{ll}\text { Inn } & 11.66 \\ \text { Fol } & 1.60 \\ \text { Alir } & 1.87 \\ \text { Apr } & 1.02\end{array}$

Apl 1.02

Hoy 11.16

IIII! A 1/4

Sily $\quad$ 1. 72

$\begin{array}{ll}\text { Aure. } & 4.61 \\ \text { sione. } & 7.01\end{array}$

ON. $\quad 71.69$

Nive. II). 10

bre. 11.47

Int 112.65

1959

Iin. $\quad 16,7$

intin.$$
\sin
$$$$
\text { Arr. }
$$

Mite 2.61

linie. $\quad+65$

Iifly 0.61

Ank. 0.26

inet 0.05

O.e. 0.015

lae.

ronal 91.12

196,

Inn. 0.0

Inn

Finth

Mar.

ing

bionen

livI Iy

Ain!:

Sepl

int.

Nove.

funn

$|46$,

$\begin{array}{rr} & 1.64 \\ & 1.47 \\ 14.94 & 2.01 \\ 12.19 & 3.25 \\ 14.94 & 13.51 \\ 11.02 & 15.90 \\ 9.71 & 15.83 \\ 1.30 & 6.60 \\ 10.14 & 7.94 \\ 20.57 & 9.51 \\ 9.11 & 7.41\end{array}$

2.24

1.11

1. $5 n$

1.17

\begin{tabular}{|c|c|c|c|c|}
\hline 15.63 & 13.20 & 37.31 & $15 . A 6$ & 21.13 \\
\hline 13.97 & 15.76 & 19.42 & 19.11 & 28.02 \\
\hline 23.70 & 23.47 & 21.56 & 9.11 & $4.2 \mathrm{~A}$ \\
\hline 24.96 & 22.12 & 16.75 & 16.31 & 1.89 \\
\hline h.nf & 7.26 & 11.A2 & 13.20 & 0.56 \\
\hline 5.11 & 3.50 & 2.32 & 1.92 & 0.05 \\
\hline ก.A2 & 0.81 & 1.01 & 1.15 & 0.10 \\
\hline 0.11 & 0.10 & (1).OB & 0.23 & 0.02 \\
\hline$\cdot 0.01)$ & 0.01 & 0.06 & 0.15 & n.ns \\
\hline 0.15 & 0.03 & 0.15 & 0.02 & 0.01 \\
\hline 0.12 & $0.0 \mathrm{~h}$ & 0.12 & 0.10 & 0.01 \\
\hline 0.21 & 0.05 & 0.140 & 0.07 & 0.02 \\
\hline$\$ 1.05$ & $10 \mathrm{H} .5 \mathrm{H}$ & 119.02 & 71.40 & 36.73 \\
\hline 0.02 & 0.02 & $(1,)^{4}$ & 0.01 & 0.13 \\
\hline 0.45 & 0.21 & 0.614 & 0.12 & 0.11 \\
\hline 0.10 & 0.23 & 0.50 & - & $0.9 \%$ \\
\hline 0.01 & 0.018 & $0.0 \mathrm{H}$ & 0.22 & $0 . n 2$ \\
\hline 0.102 & 0.014 & 0.nn & 0.01 & 0.01 \\
\hline 0.01 & 0.01 & 007 & .0 .01 & 0.01 \\
\hline$=$ & $\sim$ & $0.0 \%$ & - & -. \\
\hline$\cdot$ & $\therefore$ & - & $m$ & $\cdots$ \\
\hline- & $\cdots$ & -- & - & $\cdots$ \\
\hline$\cdots$ & $\cdots$ & $\cdots$ & - & $\cdots$ \\
\hline$\cdots$ & $\cdots$ & $\cdots$ & $\cdots$ & $\cdots$ \\
\hline - & -. & -. & -. & $\cdots$ \\
\hline
\end{tabular}

$\begin{array}{rrr} & & 11.54 \\ & & 2.62 \\ & 1.35 & 4.09 \\ & 6.60 & 0.83 \\ 17.97 & 0.54 \\ & 13.12 & 0.32 \\ 1.47 & 5.44 & 0.50 \\ 4.11 & 8.10 & 0.89 \\ 4.44 & 1.01 & 0.07 \\ 9.26 & 7.45 & 5.44\end{array}$

I. A9

1.00

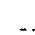

-

-

n $\quad \ldots$

$\rightarrow$

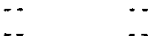

-- $\quad-$

$0.24 \quad 0.14$

0.64 2.30

H. $92 \quad 37.67$

6. $3111.0 \%$

7.16

24.22

11.16

$|\cdot n a|$

G.R.1.1

8. 22

(I) $($ )

0.52
10.012

$20 \cdot 6.9$

16.17

51.15

- daia nore avallablo

* prear or crace 


\section{RADIONUCLIDES IN MONTHLY PRECIPITATION \\ Strontium-89 - cont'd \\ in $\mathrm{mCi}$ per $\mathrm{Km}^{2}$ \\ decay corrected to midpoint of sampling month}

Year Month New York Westwood Plttgburgh Loulsville Hougton Richinond Appleton Chattanuoga Oklahoma_C1ty Palo Alto Seattle

1962

$\begin{array}{lr}\text { Jan. } & 24.32 \\ \text { Feb. } & 29.30 \\ \text { Mar. } & 7.49 \\ \text { Apr. } & 19.61 \\ \text { May } & 10.81\end{array}$

May 10.81

Aug. $\quad 6.52$

Sept. $\quad 8.07$

Oct. 2351

Nov. 16.88

Dec. $\quad 34.51$

Total 195.21

1963

Jan. $\quad 27.68 \quad 28.45$

Jeb. $\quad 38.99 \quad 19.53$

Mar. $\quad 36.79 \quad 27.98$

Apr. $\quad$...

May 16.79

June $\quad 8.45$

Ju ly $\quad 7.64$

$\quad 5.67$

Sept. 2.11

sept

2.11
0.08
1.07

0.07
0.42

23.31
19.99

8.80
12.42

12.42

4.01

2.58
0.60

0.59

Total 145.68

148.44

1964

\begin{tabular}{|c|c|c|}
\hline $\begin{array}{l}\text { Jen. } \\
\text { Feb. }\end{array}$ & $\begin{array}{c}0.31 \\
-\end{array}$ & $\begin{array}{l}0.26 \\
0.07 \\
0.08\end{array}$ \\
\hline Mex. & $\cdots$ & 0.08 \\
\hline Apr. & $\cdots$ & 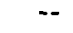 \\
\hline May & $\cdots$ & $\cdots$ \\
\hline June & $=$ & $\cdots$ \\
\hline Ju1y & $\cdots$ & $\cdots$ \\
\hline Aug. & -- & $\rightarrow$ \\
\hline Sept. & - & + \\
\hline Oct. & 0.37 & -0.15 \\
\hline Now. & -- & 0.25 \\
\hline Dec. & $\cdots$ & $<0.01$ \\
\hline Tota 1 & 0.68 & $<0.82$ \\
\hline
\end{tabular}

1965

Jen.

Feb.

Mar.

Apr.

May

June

July

Sept

Oct.

Nov

Dec.

Total

Jan.

Feb.

Mar.

Apr.

May

July

Aug.

sept

Oct.

Nov.

Dec.

Total

Jan.

Feb.

Mar.

$\begin{array}{rrr}36.40 & 18.18 & 12.04 \\ 25.24 & 6.83 & 42.85 \\ 28.60 & 7.10 & 5.56 \\ 7.49 & 24.09 & 3.20 \\ 24.59 & 3.36 & 0.11 \\ 14.86 & 13.82 & 0.20 \\ 5.44 & 15.36 & 0.10 \\ 4.86 & 1.36 & \\ 1.22 & 1.63 & 0.48 \\ 11.46 & 10.04 & 6.87 \\ 23.85 & 73.34 & 6.06 \\ 12.51 & 21.96 & 14.36 \\ & & \\ 202.52 & 185.71 & 91.91 \\ & & \\ 16.79 & 28.95 & 28.06 \\ 16.98 & 23.16 & 21.50 \\ 34.86 & 3.36 & 35.12 \\ 21.69 & 4.55 & 23.43 \\ 40.92 & 6.29 & 2.54 \\ 21.77 & 0.46 & 0.20\end{array}$

$\begin{array}{lr}2.81 & 1.00 \\ 2.08 & 0.37 \\ 0.67 & 0.05 \\ 0.54 & 0.45 \\ 0.10 & 0.38\end{array}$

\subsection{8}

1.67

0.60

0.18

1.21

29.64

28.52

19.92

18.06

4.98

0.47
0.69

0.92

0.15

0.14

0.30

1.37

1.49

0.21

0.99

0.21

0.41

103.81

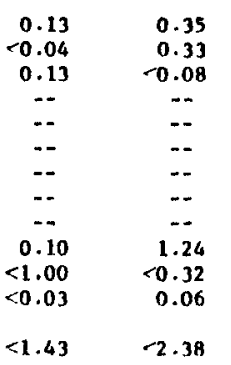

$<0.03$

0.17

$<0.14$

$<0.67$

$<0.10$

0.05

-.

0

0.69

1.59

$<0.16$

0.03

$<0.05$

$<0.01$

$<0.05$

$<0.02$

-.

-

0.20

0.65

0.08

$<1.25$

$<3.45$

$<0.01$

0.02

0.02
0.01
0.25

0.09

$<0.04$

$r 0.03$

0.04

0.04

$<0.05$

$<0.02$

0.15

1.43

$-0.02$

$-2.17$

$-$

-

$+$

$-0.05$

0.44
0.07

0.07

- data not available

* zero or trace 
RADIONUCLIDES IN MONTHLY PRECIPITATION

strontium-90

in $\mathrm{mCl}$ per $\mathrm{km}^{2}$

decay corrected to midpoint of sampling month

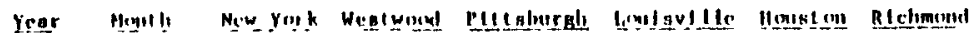

\begin{tabular}{|c|c|c|c|c|}
\hline \multirow[t]{12}{*}{ |गऽ। } & $\operatorname{lnn}$. & 0.10 & & \\
\hline & Pelb. & 0.20 & & \\
\hline & Mar & 0.10 & & \\
\hline & Apr. & I.45 & & \\
\hline & May & 0.16 & & \\
\hline & Inיטי & 0.12 & & \\
\hline & $m \mid y$ & 0.32 & & 013 \\
\hline & AIIR. & 0.19 & $0 \quad 42$ & 0.06 \\
\hline & sept. & 0.16 & 0.46 & 0.06 \\
\hline & ore & 0.15 & 0.31 & 0.12 \\
\hline & Hov. & 0.16 & 0.21 & 0.11 \\
\hline & nere & 0.21 & n.14 & 0.24 \\
\hline
\end{tabular}

Fint $\quad 0.48 \quad 0.28$

Mar. $\quad 0.34 \quad 0.39 \quad 0.18$

Apr. $\quad 0.59 \quad 0.67$

May $1.02 \quad 10 \mathrm{OH}$

Jine $0.68 \quad 0.40 \quad 0.99$

fiuly $\quad 0.61 \quad 0.42 \quad 0.89$

Aitg. $0.23 \quad 0.43 \quad 0.64$

sapt. $0.25 \quad 0.21 \quad 026$

ont. $\quad 0.410 .63 \quad 0.2$

Nive. $\quad 0.3$ A $\quad 0.53 \quad 0.28$

Dec. $0.68 \quad 0.30 \quad 0.29$

4.85

1959

Total

6. 17

599

Ian.

0.46

0.54

0.56

0.67

1. 1.1

0.56

0.56
0.71

1.13

2. OA

Apr. $\quad 2.42 \quad 2.45$

Any $\quad 0.50 \quad 1.02$

lune $\quad 1.71 \quad 1.46$

Jilly $0.19 \quad 0.68$

$\begin{array}{lll}\text { AIR. } & 0.26 & 0.39\end{array}$

Sept. 0.0550 .01

ore 0.14 o.16

Mering

0.10

1. 12

D.An

0.26

0.17

0.02

0.14

n. 14

0.11

$\begin{array}{lll} & & 1.44 \\ & & 0.22 \\ & 0.14 & 0.22 \\ & 0.27 & 0.10 \\ & 0.21 & 0.03 \\ & 0.24 & 0.01 \\ 0.10 & 0.19 & 0.03 \\ 0.19 & 0.25 & 0.03 \\ 0.26 & 0.09 & 0.01 \\ 0.21 & 0.19 & 0.15\end{array}$

pre

9.6B

9.13

7.52

0.10

Ia11. 0.17 n.10

ieh. 0.1100 .26

ก. 15

0.13

0.20

0.31

Apr. $\quad 0.16 \quad 0.10$

$\begin{array}{lll}\text { Mny } & 0.28 & 0.11\end{array}$

line 0.16 0.18

mly $0.10 \quad 0.22$

A11R. D.OR 0.11

sept. 0.nk 0.11

met.

Nen. $\quad$ n.05

0.05

0.05

0.18

0.09

0.06

0.05
0.05

0.05

0.84

0.61

0.61
1.22

1.29

I. 15

0. 36

0.26

0.11

0.03

0.14

0.09

(1)

0.15

$\operatorname{Ton} 1 \quad 1.51$

1.10

0.47

0.11

0.95

1. 58

2. 32

0.31

o. 34

0.13

ก 15

0.23

0.07
0.08

0.72

1.07

0.30

o. IA

0.01

*

0.04

0.01

*.05

$\begin{array}{lll}6.27 & 6.95 \quad 2.41\end{array}$

0.35

0.06

0.24

2.4

0.47

0.14

n.22

0.46

0.50

0.07

0.05

0.03

0.06
0.05

0.07

$$
0 \text { in }
$$

0.10

0.03
0.22

0.04

0.12

n.03

0.09

0.04

0.14

0.16

0.01

0.03

0.01

0.01

*

0.01

0.05

2.43

1.07

0.61

1961

\begin{tabular}{|c|c|}
\hline inn. & 0.04 \\
\hline rah. & 0.06 \\
\hline Mar. & 0.26 \\
\hline Anr. & 0.17 \\
\hline May & ก. 32 \\
\hline lune: & 0.16 \\
\hline mly & 0.01 \\
\hline Ang. & 0.05 \\
\hline Sept. & 0.07 \\
\hline$|k| 1$. & 0.12 \\
\hline Nov & 0.11 \\
\hline
\end{tabular}

0.07

n.05

0.22

0.05

0.10

0.05

0.10

0.31

0.6100 .11

0.11
0.36

0.12

0.13

0.18

0.02

$0.09 \quad 0.07$

$0.26 \quad 0.05$

0.06 0.10

$0.16 \quad 0.01$

$\begin{array}{llll}0.16 & 0.16 & 0.10 & 0.01 \\ 0.08 & 0.06 & 0.08 & 0.02\end{array}$

$\begin{array}{llll}0.16 & 0.16 & 0.10 & 0.01 \\ 0.08 & 0.06 & 0.08 & 0.02\end{array}$

$\begin{array}{llll}0.08 & 0.06 & 0.08 & 0.02 \\ 0.03 & 0.04 & 0.03 & 0.01\end{array}$

$\begin{array}{llll}0.03 & 0.04 & 0.03 & 0.01 \\ 0.15 & 0.10 & 0.01 & 0.03\end{array}$

$0.270 .29 \quad 0.15$

0.15

$0.5 n$
0.25

Totat

2.1 .1

2.95

2.74

2.40

1.36

1.12

- data nor avillable

* cero or trace 


\section{RADTONUCLIDES TN MONTHLY PRECIPTTATION \\ Strontium $\circ 90$ in $\mathrm{mCi}$ per $\mathrm{km}^{2}$ \\ decay corrected to midpoint of sampling month}

Year Month New York Hestwood Pittaburgh Loulgville Houston Richmond Appleton Chattanooga Oklahome City Palo Alto Seattle

1962

$\begin{array}{lll}\text { Jan. } & 0.44 & 0.57 \\ \text { Feb. } & 1.80 & 1.18 \\ \text { Mar. } & 0.86 & 0.81 \\ \text { Apr. } & 1.29 & 2.13 \\ \text { May } & 1.54 & 1.02 \\ \text { June } & 1.56 & 1.84 \\ \text { July } & 1.10 & 1.01 \\ \text { Aug. } & 1.29 & 1.33 \\ \text { Sept. } & 0.44 & 0.47 \\ \text { Oct. } & 0.64 & 1.20 \\ \text { Nov. } & 0.75 & 0.96 \\ \text { Dec. } & 0.62 & 1.14 \\ \text { Tota1 } & 12.33123313 .66\end{array}$

$\begin{array}{llll}0.51 & 0.79 & 0.31 & 0.32 \\ 0.80 & 0.92 & 0.25 & 1.64 \\ 0.87 & 1.16 & 0.39 & 0.26 \\ 1.49 & 0.51 & 1.86 & 0.29 \\ 1.35 & 1.52 & 0.17 & 0.02 \\ 0.60 & 1.55 & 1.59 & 0.04 \\ 1.46 & 0.40 & 50.53 & 0.03 \\ 1.24 & 0.33 & 1.39 & 0.02 \\ 0.59 & 0.21 & 0.20 & 0.03 \\ 0.53 & 0.66 & 0.45 & 0.41 \\ 0.61 & 1.07 & 0.95 & 0.12 \\ 0.73 & 0.46 & 0.72 & 0.39 \\ & & & \\ 10.78 & 9.38 & 7.42 & 3.57 \\ & & & \\ 0.79 & 0.66 & 0.94 & 0.98 \\ 0.90 & 0.84 & 1.11 & 0.97 \\ 2.83 & 2.70 & 0.37 & 2.19 \\ 3.04 & 1.53 & 0.83 & 2.60 \\ 2.20 & 5.80 & 0.96 & 0.40 \\ & 4.02 & 2.16 & 0.10\end{array}$

Jan. $\quad 0.92 \quad 1.03$

$\begin{array}{lll}\text { Feb. } & 2.05 & 1.04 \\ \text { Max. } & 0.98 & 2.43\end{array}$

Apr.

May $\quad 1.90$

June $\quad 2.00$

Aus. $\quad 3.78 \quad 2.21$

Sept. $\quad 1.62 \quad 2.06$

Nov, $\quad 1.79$

0.60

0.98

Dec .

23.79

25.29

1964

Total 1.04

$\begin{array}{ll}\text { Jan. } & 1.04 \\ \text { Feb. } & 2.10\end{array}$

Mar. 1.83

Apr. $\quad 4.74$

May 0.52

June $\quad 2.55$

July 1.28

Aug. $\quad 0.39$

Sept. 0.23

Dct. $\quad 0.29$

$\begin{array}{ll}\text { Nov . } & 0.32 \\ \text { Dec . } & 0.56\end{array}$

1.04

0.42

1.35

2.58
0.86

2.39

1.07

0.43

0.11

0.31

0.34

10.98

1965

Tota $1 \quad 15.85$

10.98

$\begin{array}{ccc}\text { Jan. } & 0.38 & 0.42 \\ \text { Feb. } & 0.76 & 0.66\end{array}$

$\begin{array}{lll}\text { Mar. } & 0.82 & 0.54\end{array}$

$\begin{array}{lll}\text { Apr. } & 1.01 & 0.87\end{array}$

$\begin{array}{lll}\text { May } & 0.43 & 0.51\end{array}$

June $\quad 0.63 \quad 1.10$

$\begin{array}{lll}\mathrm{Ju} 1 \mathrm{y} & 0.55 & 0.62\end{array}$

$\begin{array}{lll}\text { Aug. } & 0.39 & 0.34\end{array}$

Sept. $\quad 0.11 \quad 0.15$

Oct. $0.18 \quad 0.18$

Now. $\quad 0.15 \quad 0.18$

$\begin{array}{lll}\text { Total } \quad 5.52 & 5.70\end{array}$

1966

$\begin{array}{lll} & 0.12 & 0.14\end{array}$

Feb. $\quad 0.17 \quad 0.11$

Mar. $\quad 0.28 \quad 0.40$

Apr. $\quad 0.45 \quad 0.43$

May $\quad 0.56 \quad 0.6$

$\begin{array}{lll}\text { June } & 0.16 & 0.19\end{array}$

$\begin{array}{lll}\text { July } & 0.09 & 0.15\end{array}$

Aug. $\quad 0.12 \quad 0.05$

$\begin{array}{lll}\text { Sept. } & 0.22 & 0.12\end{array}$

oct. $\quad 0.08 \quad 0.03$

Nov. $\quad 0.08 \quad 0.08$

Dec. $\quad 0.10 \quad 0.06$

$\begin{array}{lll}\text { Tota } 1 & 2.43 & 2.45\end{array}$

1967

$\begin{array}{lll}\text { Jan. } & 0.16 & 0.12 \\ \text { Feb. } & 0.11 & 0.06\end{array}$

0.12
0.06

- data not avaflable

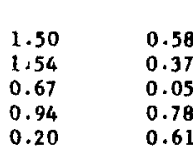

0.58
0.37
0.05
0.78
0.61

1.67

0.81

0.17
0.45

0.16

0.87

1.30

1.85

0.83

0.83
0.11

$0.03 \quad 0.49$

$0.09 \quad 0.69$

$0.14 \quad 0.93$

$\begin{array}{ll}0.24 & 1.04 \\ 0.48 & 1.49 \\ 0.06 & 1.07\end{array}$

7.38

0.5

1.29
1.85

1.88

4.40

2.21

1.35

2.46

0.77

1.19

0.71

0.65

0.38
0.45

0.16

0.86

0.72

0.19

0.43

0.21

9.65

15.42

0.16

0.38

0.14
0.54

0.54

0.82

1.47

1.24

0.89

0.66

0.64

$0.32 \quad 0.39$

$\begin{array}{ll}0.32 & 0.39 \\ 0.51 & 0.16\end{array}$

$\begin{array}{ll}0.27 & 0.12\end{array}$

$0.17 \quad 0.08$

$0.20 \quad 0.25$

$0.17 \quad 0.06$

5.97

4.99

0.18

1.03

1.41

1.33

2.54

0.73

0.46

0.54

0.42

0.36

0.46

0.74

0.10

0.58

0.05

0.22

0.12

0.08

0.01

0.10

0.33

9.50

2.85

0.14

0.3

0.13

0.34

0.13

0.55

1.22

1.10

0.63

0.71

0.17

0.06

0.07
0.44

0.44
0.02

0.04

0.02

0.02

0.02

* 55

0.18

1.51

0.57

1.41

1.54

1.87

1.15
0.68

0.68

0.33

0.30

0.18
0.80

0.62

10.96

0.50

1.23

0.32

0.71

0.43

0.21

0.10

0.25

0.09

0.11

0.22

5.78

2.13

4.42

0.22

0.24

0.46
0.28

0.38

0.15

0.12

0.02

0.08

0.05

0.16
0.14

2.30

0.11

0.13

Mar. 0.17

Apr. $\quad 0.24$

May $\quad 0.31$

June $\quad 0.13$

July 0.18

Aug. $\quad 0.12$

Sept. $\quad 0.04$

Oct.

Nov.

Dec.

Tota 1 


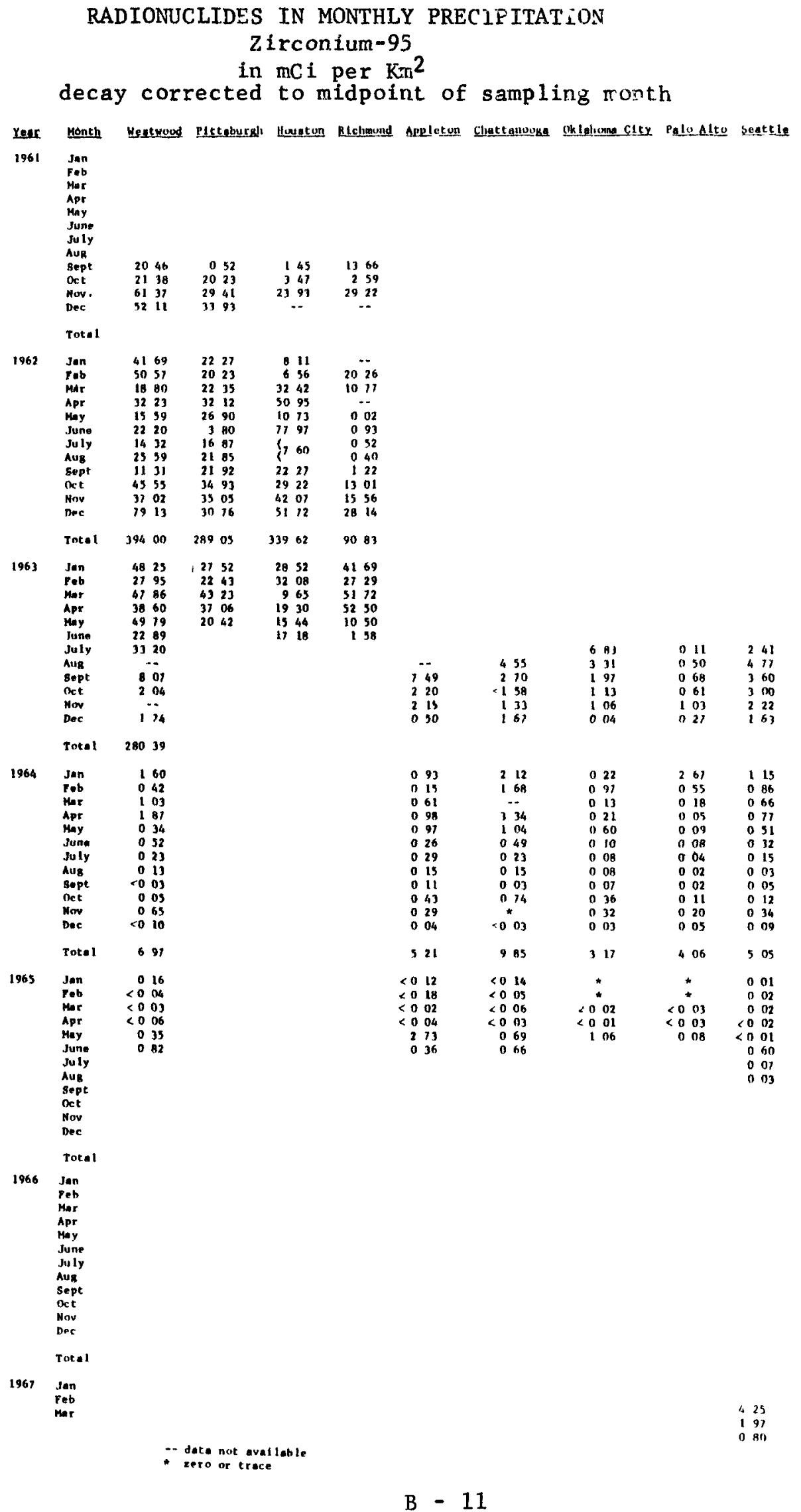




\section{RADIONUCLIDES IN MONTHLY PRECIPITATION}

Rhodium-102

In $\mathrm{mCl}$ per $\mathrm{Km}^{2}$

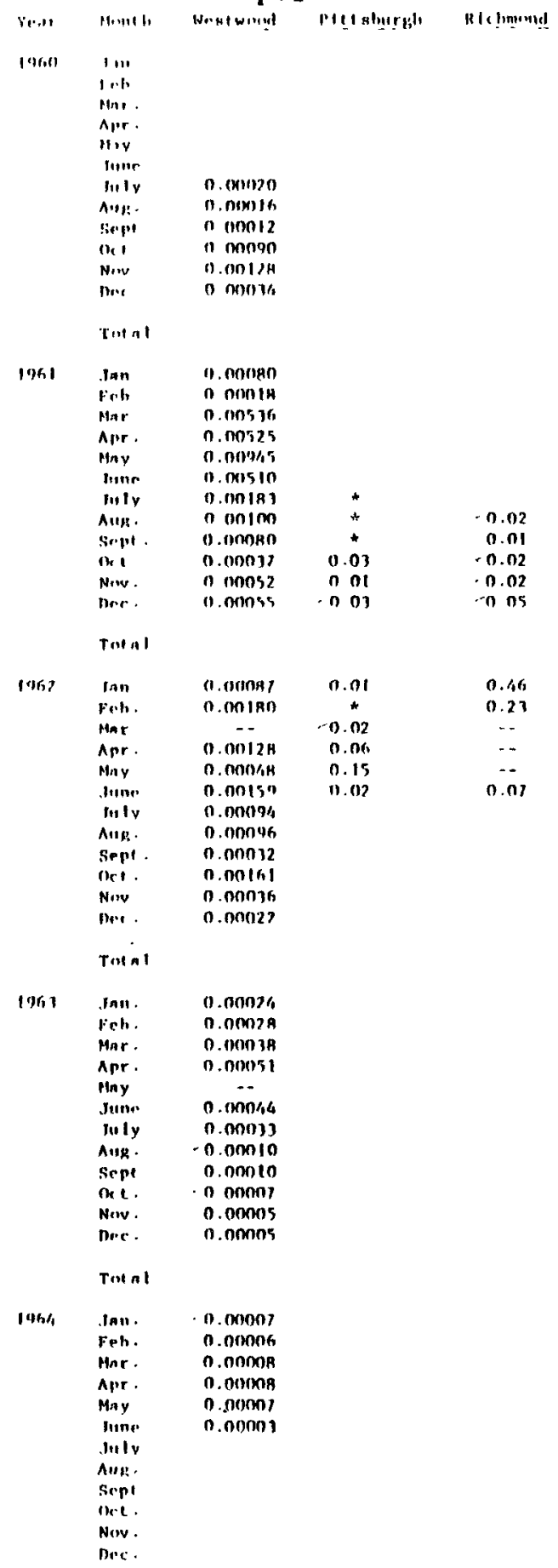

- data not avaltable

* tero ur Irace 


\section{RADIONUCLIDES IN MONTHLY PRECIPITATION}

Ruthenium-103

in $\mathrm{mCi}$ per $\mathrm{Km}^{2}$

Decay corrected to midpoint of sampling month

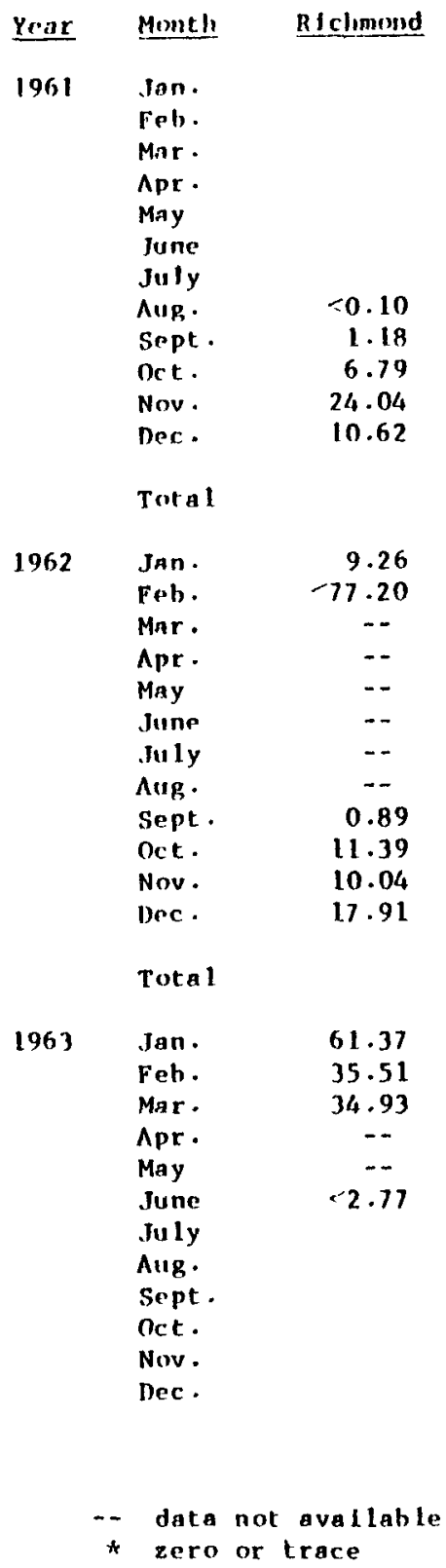




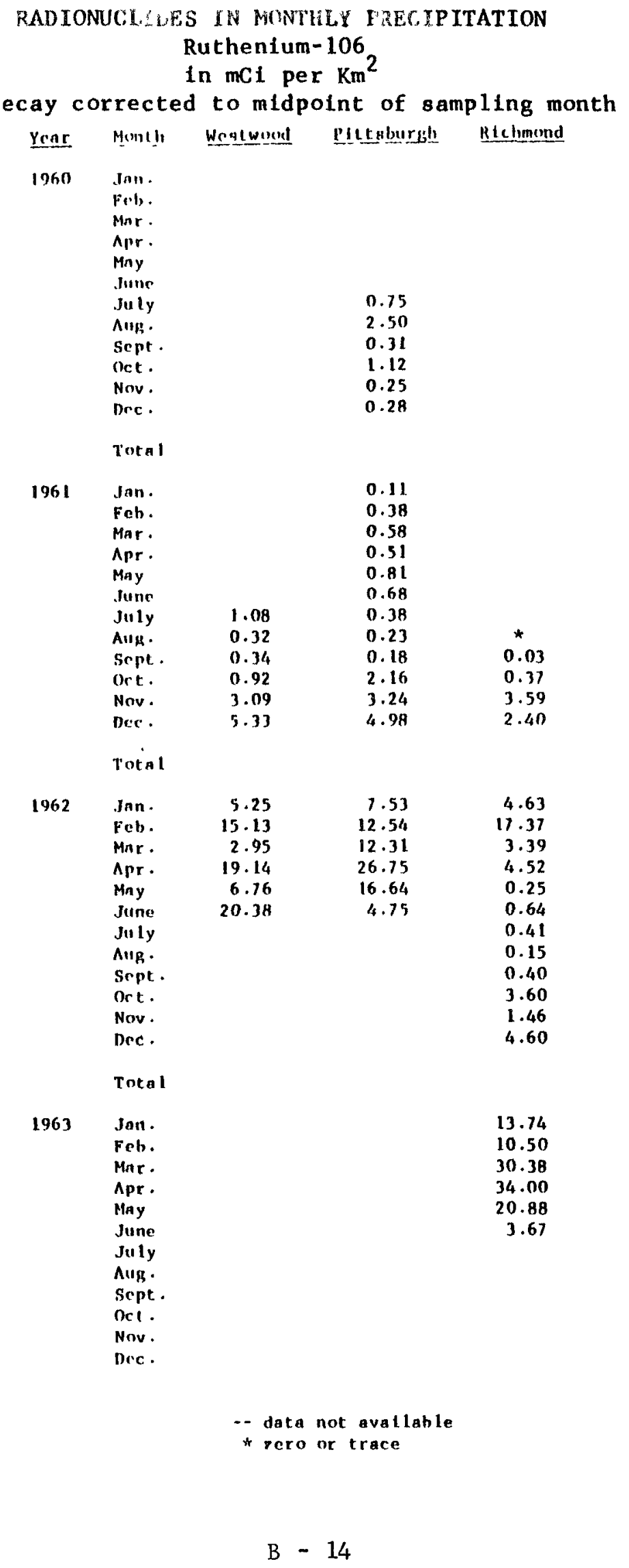




\section{RADIONUCLIDES IN MONTHLY PRECIPITATION \\ Cadmium-109 in mCi per $\mathrm{km}^{2}$ \\ decay corrected to $7 / 9 / 62$}

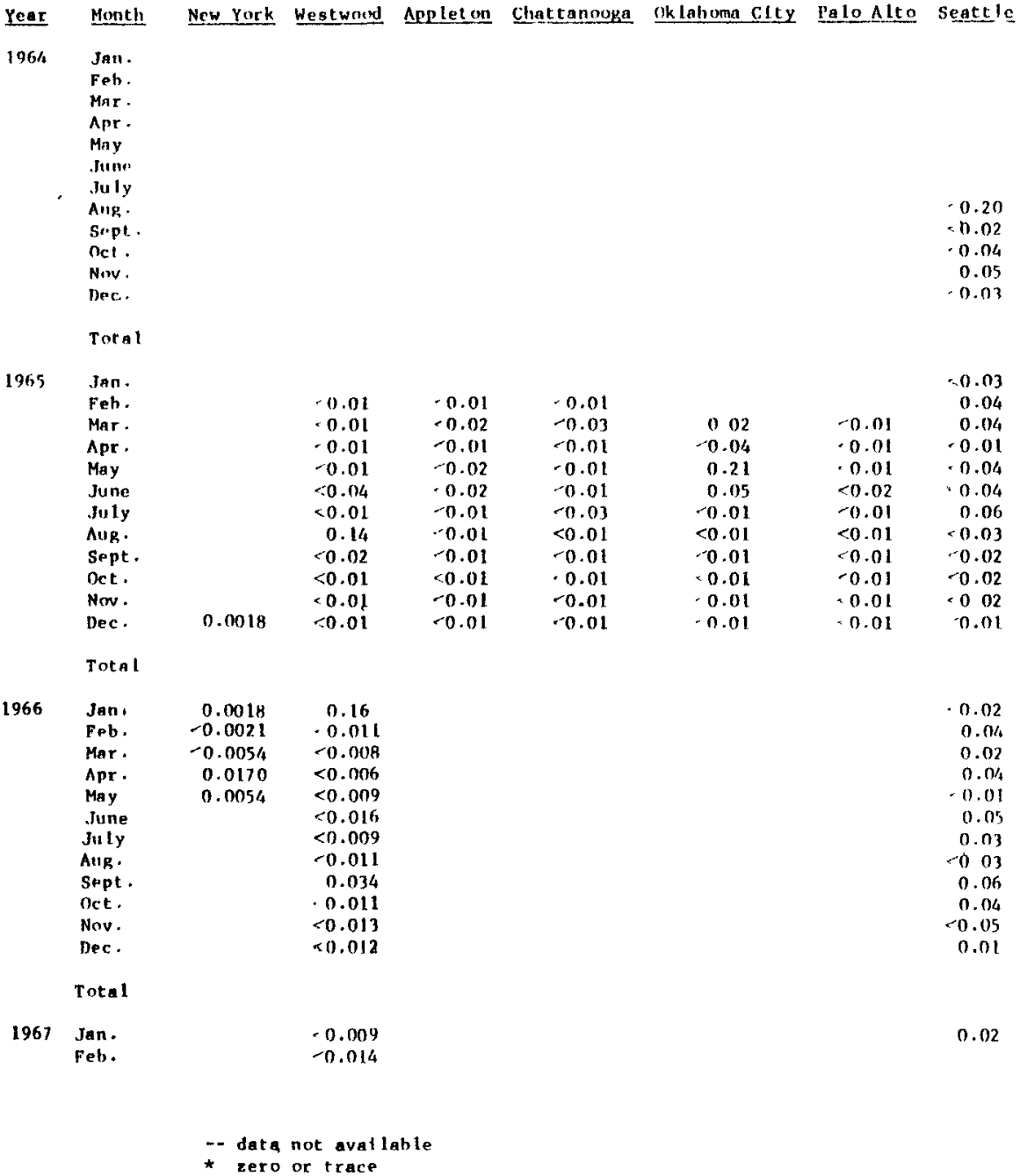


RADIONUCLIDES IN MONTHLY PRECIPITATION

Antimony -124

in $\mathrm{mCl}$ per $\mathrm{Km}^{2}$

decay corrected to midpoint of sampling month

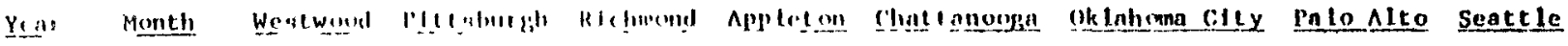

1963 ran.

reb.

Mar.

Apr.

Mny

June

Inly

Anp.

Srpt .

net.

Nov.

Dee.

1.194

0.619

$-\infty$

0.17

0.10

0.72

0.12

0.06

0.07

0.06

O. . OR

0.06

先

Total

1963

Jan. $\quad 0.15$

Feb. $\quad 0.17$

Mar. O.06

Apr $\quad 0.06$

May 40.03

June 0.0 ?

July 0.32

Aug. $\quad<0.49$

sept. $\quad \leq 0.10$

Ort . $<0.10$

Nov. $<0.57$

Dere.

Total

$190 \%$.Jan. $<0.17$

Yeb. $\quad<0.82$

Mar. en. Is

Apr

Mny

Junce

July

Aup.

sepl.

()e-1

0.12

(1). 12

0. II)

(1. 12

0.05

Nov

Dre

- dat" bul svaf lablo

* Per in Irace 


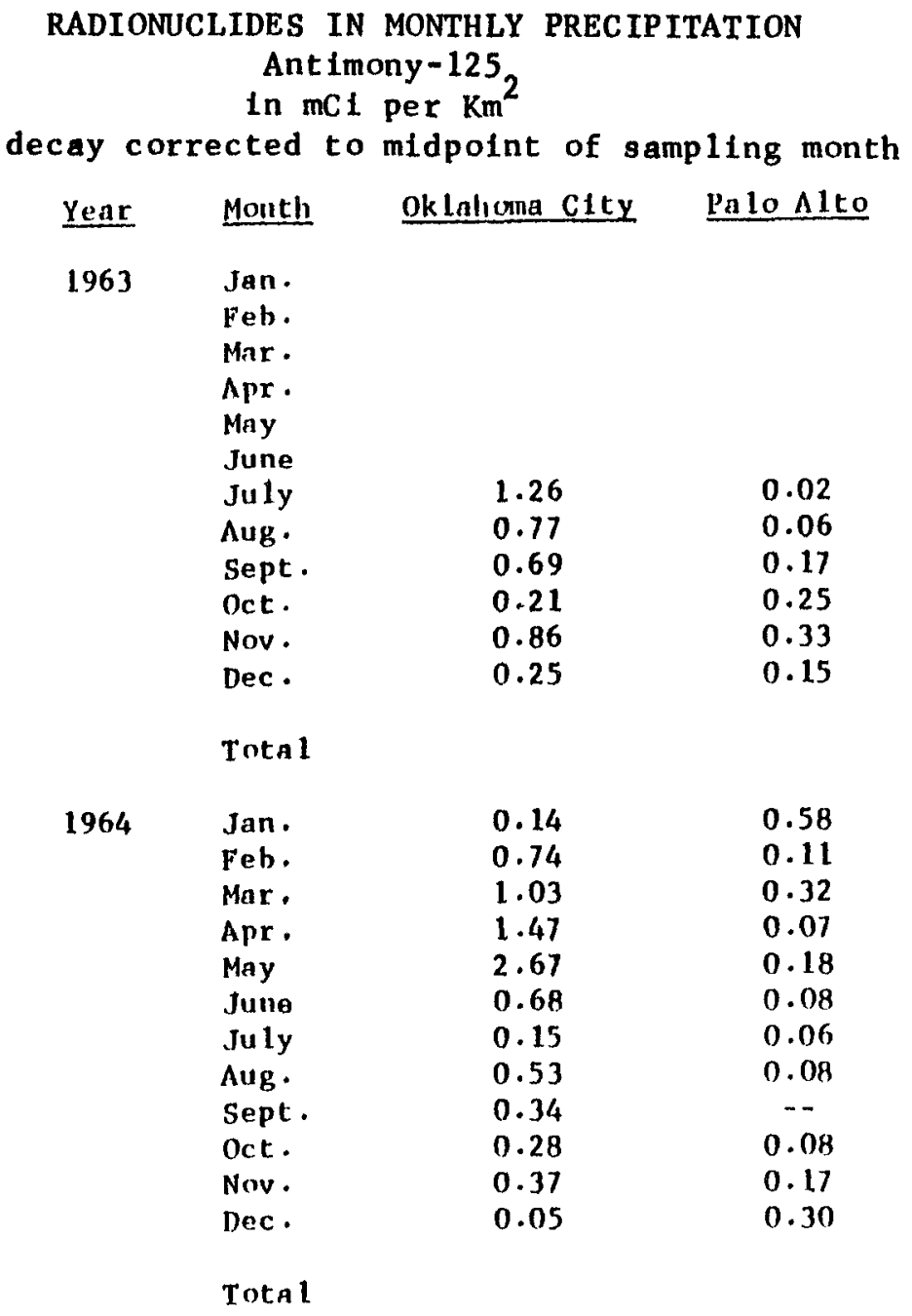

\footnotetext{
- data not avallable

* zero or trace
} 


\section{RADIONUCLIDES IN MONTHLY PRECIPITATION \\ Cesium-137 In mCl per $\mathrm{km}^{2}$ \\ decay corrected to midpoint. of sampling month}

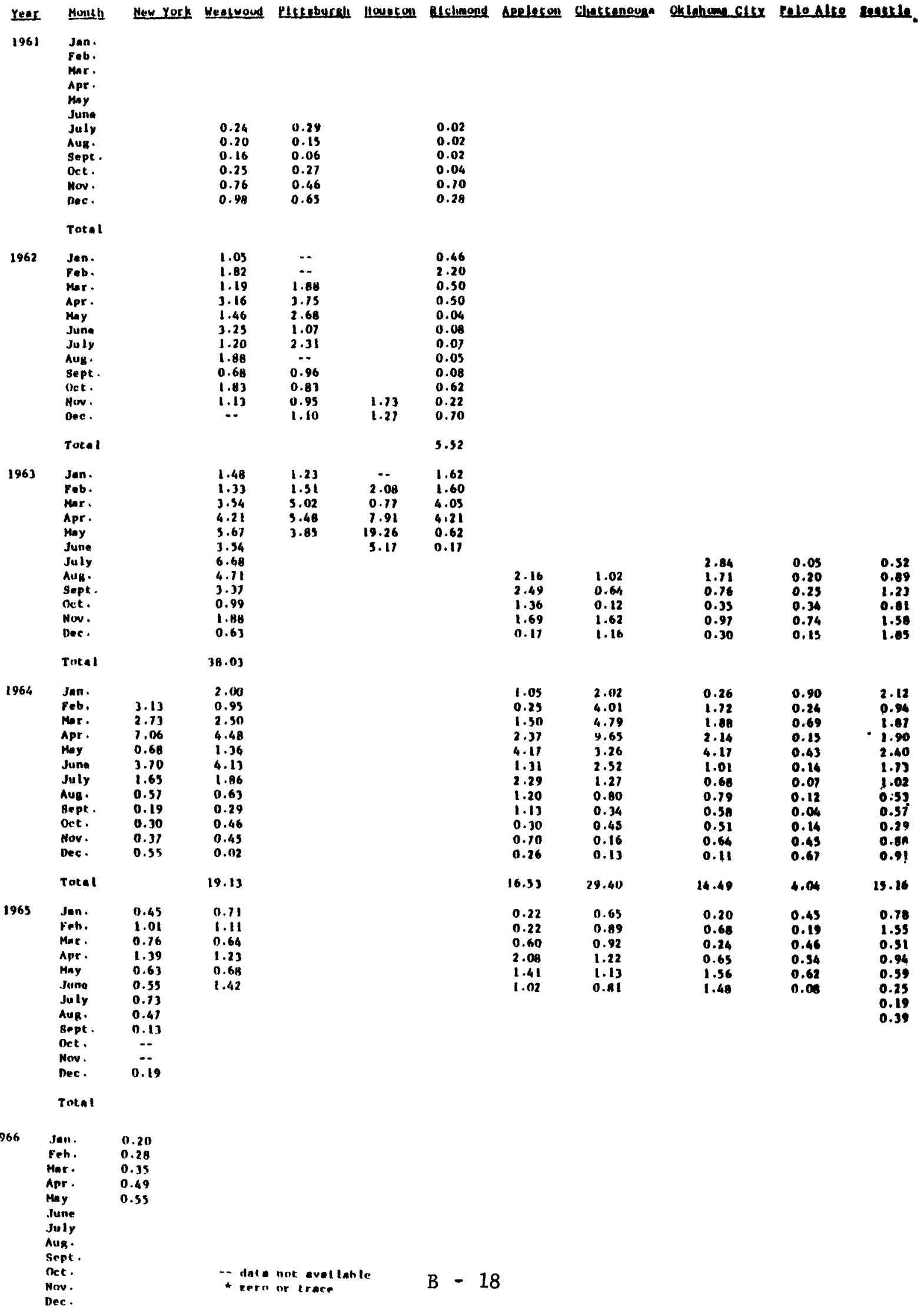




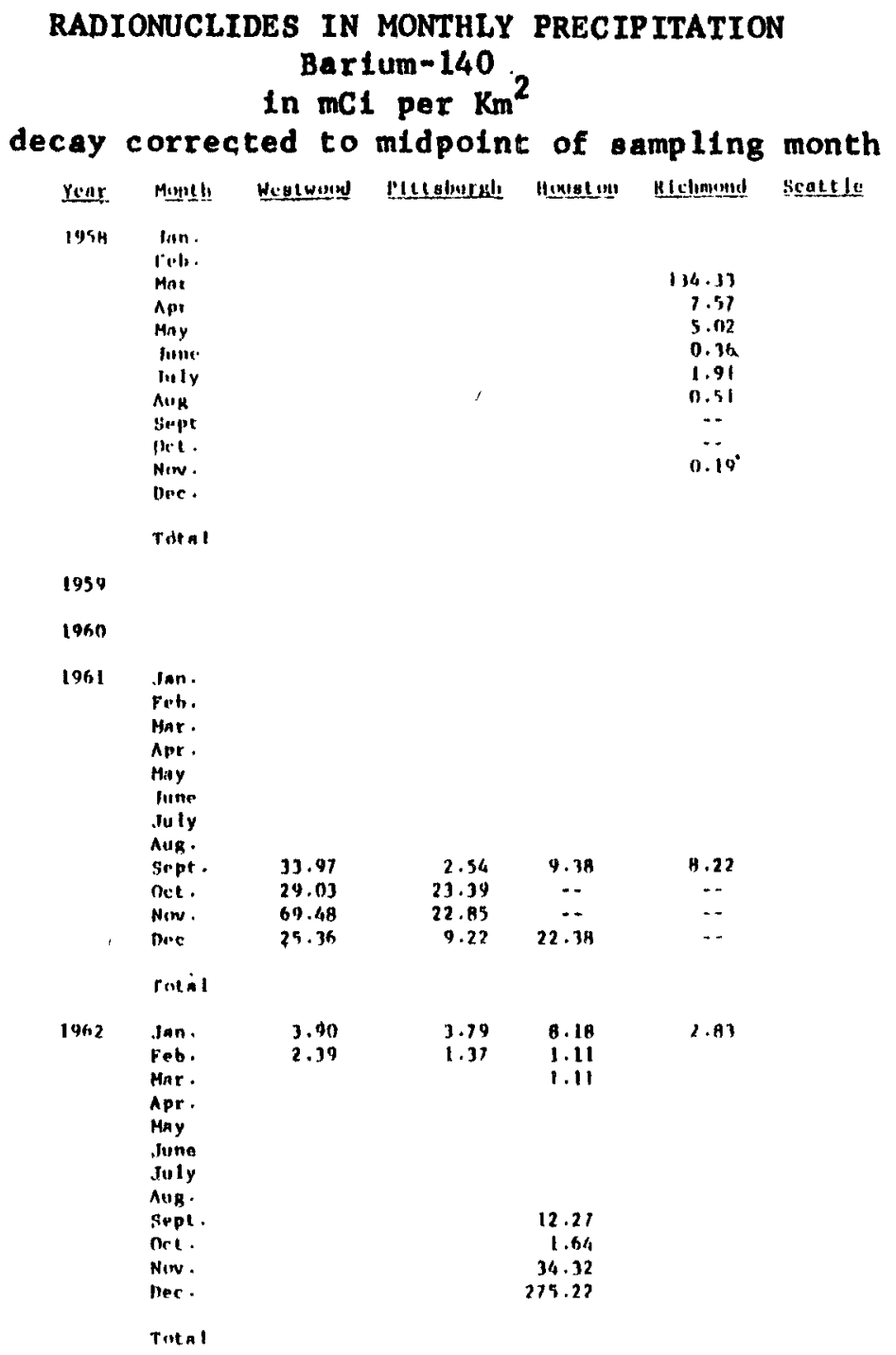

196)

1964

lan.

Mab.

Apr

May

lune

Aisk.

sepe.

Ort.

Nerv. 


\section{RADIONUCLIDES IN MONTLHLY PRECIPITATION \\ Ceriutn- 141 \\ in $\mathrm{mCl}$ per $\mathrm{km}^{2}$ \\ decay corrected to midpoint of sampling month}

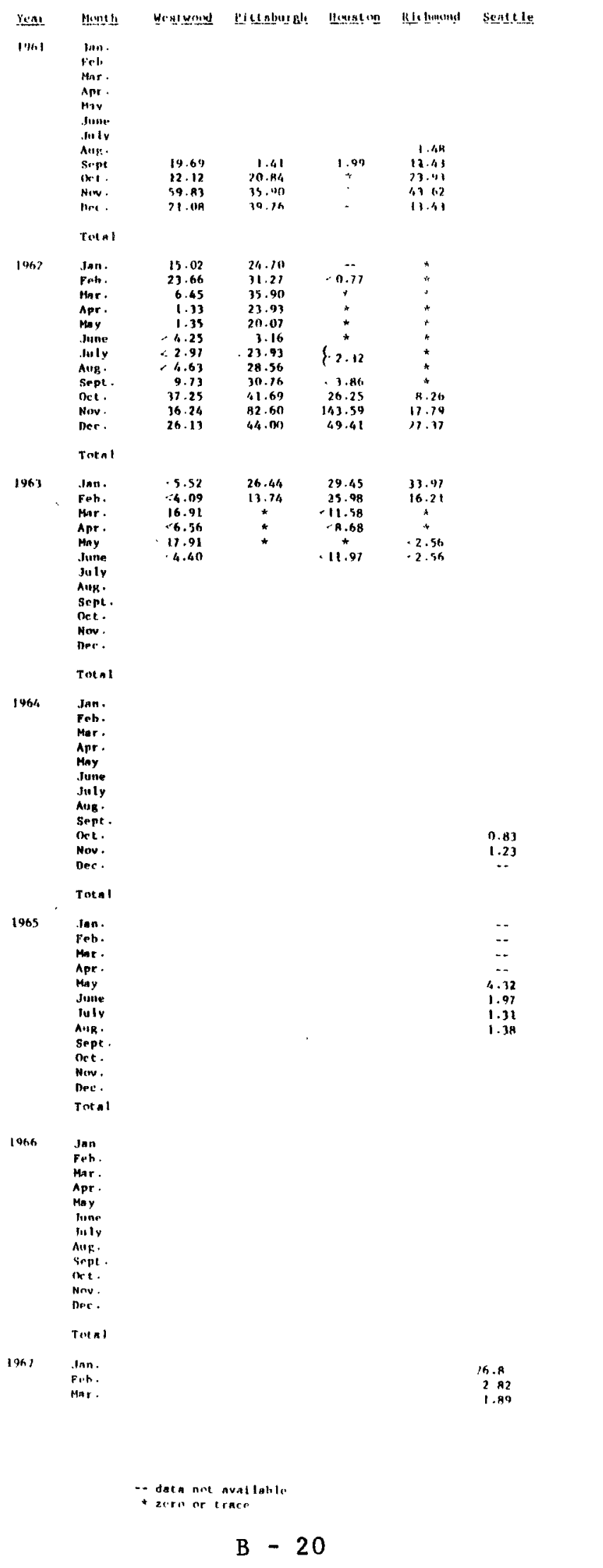




\section{Cerium- 144}

in $\mathrm{mC} i$ per $\mathrm{Km}^{2}$

decay corrected to midpoint of sampling month

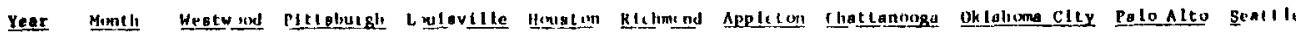

1960

\begin{tabular}{|c|c|c|c|c|c|}
\hline Jan & 080 & & & 091 & 238 \\
\hline Feb & 051 & 105 & 009 & 212 & 11 \\
\hline linr & $\left.\begin{array}{lll}0 & 4\end{array}\right]$ & 086 & 025 & & 135 \\
\hline Apr & 112 & $10 B$ & $\begin{array}{lll}0 & 83\end{array}$ & 110 & \\
\hline May & 137 & 142 & 009 & $04 \mathrm{~A}$ & \\
\hline lune & 076 & 141 & 022 & 121 & 0 \\
\hline Puly & . & 062 & 110 & o 55 & \\
\hline Aug & 049 & 040 & 069 & 103 & 0 \\
\hline sept & 044 & 028 & 026 & (1) 34 & \\
\hline bet & 020 & 022 & 021 & 0 is & 0 \\
\hline Nov & 0114 & $\begin{array}{lll}0 & 17\end{array}$ & 028 & $\begin{array}{lll}0 & 23\end{array}$ & \\
\hline Dec & i) 14 & $\begin{array}{lll}0 & 21\end{array}$ & 013 & 022 & 0 \\
\hline
\end{tabular}

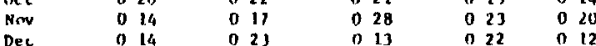

Total $\quad 640 \quad 774 \quad 416 \quad$ B $38 \quad 597$

1961

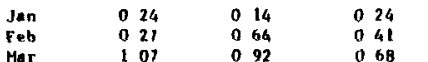

Mar

Apr

June

July

$\begin{array}{lll}1 & 20 \\ 0 & 83\end{array}$

$\begin{array}{ll}0 & 83 \\ 0 & 68\end{array}$

808

086

$\begin{array}{lll}0 & 92 \\ 1 & 32\end{array}$

0 85

$\begin{array}{ll}0 & 80 \\ 0 & 37\end{array}$

068

$\begin{array}{lll}1 & 41 \\ 1 & 53 \\ 0 & 54\end{array}$

(3) 020

$\begin{array}{llll}0 & 46 & 0 & 09 \\ 0 & 35 & 0 & 0\end{array}$

$\begin{array}{llll}0 & 35 & 0 & 30 \\ 0 & 72 & 0 & 18\end{array}$

$\begin{array}{llll}0 & 72 & 0 & 18 \\ 0 & 33 & 0 & 48\end{array}$

$\begin{array}{llll}0 & 33 & 0 & 48 \\ 0 & 66 & 0 & 08\end{array}$

$\begin{array}{llll}0 & 66 & 0 & 08 \\ 0 & 28 & 0 & 17\end{array}$

$\begin{array}{llll}0 & 28 & 0 & 17 \\ 0 & 39 & 0 & 04\end{array}$

$\begin{array}{lll}0 & 55 & 118 \\ 0 & 10 & 1\end{array}$

070
784

$\begin{array}{rrr}1522 & 321 \\ \text { Non } & 1559 & 814\end{array}$

27 5H

1962

Jan 1918

$\begin{array}{lll}\text { Feb } & 31 & 50 \\ \text { Mar } & 18 & 61 \\ \text { Apr } & 42 & 07\end{array}$

$\begin{array}{ll}\text { Apr } & 4207 \\ \text { May } & 1911\end{array}$

June
Juty

Juty $1911 \quad 307$

Aug $2548 \quad 1930$

$\begin{array}{lrl}\text { Sopt } & 884 & 1359 \\ \text { Oct } & 2795 & 1297\end{array}$

Nov $1676 \quad 2331$

Dec 2856

2123

1258

3568

Totel 30465

29139

B $99 \quad 1426$

$2984 \quad 1004$

$\begin{array}{rrrr}69 & 87 & 7 & 33 \\ 7 & 91 & 0 & 67\end{array}$

50 (1)

$\int \begin{array}{ll}8 & 49\end{array}$

113

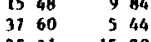

$2521 \quad 1529$

196]

$\begin{array}{llll}\text { Jan } & 2320 & 2262 \\ \text { Feb } & 2791 & 2470 \\ \text { Mar } & 5713 & 7861 \\ \text { Apr } & 7450 & 6678 \\ \text { May } & 7840 & 4864\end{array}$

21244

7811

$\begin{array}{llll}27 & 91 & 34 & 08 \\ 35 & 56 & 27 & 52\end{array}$

$1467 \quad 6022$

$\begin{array}{rrrr}27 & 40 & 13 & 12 \\ 32 & 04 & 2 & 19\end{array}$

7840
Mane

4964

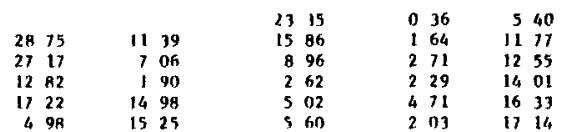

1964

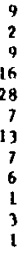

$992 \quad 19$

$209 \quad 2799$

1679

2899

737

110

363

$\begin{array}{ll}3 & 63 \\ 1 & 85 \\ 0 & 50\end{array}$

(1)

$108 \quad 13$

21527

$\begin{array}{ll}1 & 28 \\ 0 & 80 \\ 2 & 49\end{array}$

066
440

317
131

173

0 ค 3

$\begin{array}{ll}0 & 47 \\ 0 & 42\end{array}$

$\begin{array}{ll}2 & 39 \\ 2 & 94 \\ 3 & 61 \\ 2 & 57 \\ 2 & 83 \\ 1 & 55 \\ 0 & 56 \\ 0 & 32 \\ 0 & 27 \\ 0 & 55 \\ 0 & 20\end{array}$

1843

1779

560

$\begin{array}{ll}16 & 33 \\ 17 & 14\end{array}$

Jan 034

Feb $\quad 036$

$\begin{array}{lll}\text { Mar } & 0 & 59 \\ \text { Apr } & 0 & 59\end{array}$

May $\quad 126$

June $\quad 040$

$\begin{array}{lll}\text { July } & 0 & 18 \\ \text { Aug } & 0 & 10\end{array}$

Sept

Nov

Total

$\begin{array}{ll}2 & 94 \\ 3 & 61 \\ -5 \\ 2 & 57 \\ 2 & 83 \\ 1 & 55 \\ 0 & 56 \\ 0 & 32 \\ 0 & 27 \\ 0 & 55 \\ 0 & 20 \\ 17 & 79\end{array}$

$$
\begin{array}{ll}
0 & 67 \\
1 & 93 \\
0 & 84 \\
1 & 79 \\
3 & 89 \\
4 & 28 \\
2 & 58 \\
2 & 71 \\
0 & 53 \\
0 & 21 \\
0 & 24 \\
1 & 74 \\
21 & 07
\end{array}
$$

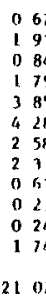

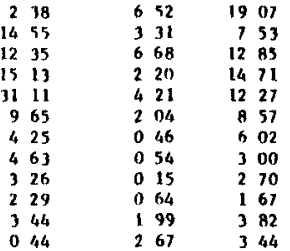

$10748-314129565$

$\begin{array}{lllll}1 & 71 & & 3 & 01 \\ 0 & 59 & & 5 & 52 \\ 1 & 31 & & 1 & 81 \\ 1 & 52 & & 2 & 59 \\ 0 & 17 & & 1 & 69 \\ 0 & 24 & & 0 & 90 \\ 0 & 11 & & 0 & 54 \\ 0 & 05 & & 1 & 20 \\ 0 & 10 & & 0 & 37 \\ 0 & 03 & & 0 & 38 \\ 1 & 24 & & 1 & 02 \\ 0 & 66 & & 0 & 50\end{array}$

$2107 \quad 773 \quad 1953$ 
RADIONUCLIDES IN MONTHLY PRECIPITATION

Plutonium.

in $\mathrm{mCi}$ per $\mathrm{Km}^{2}$

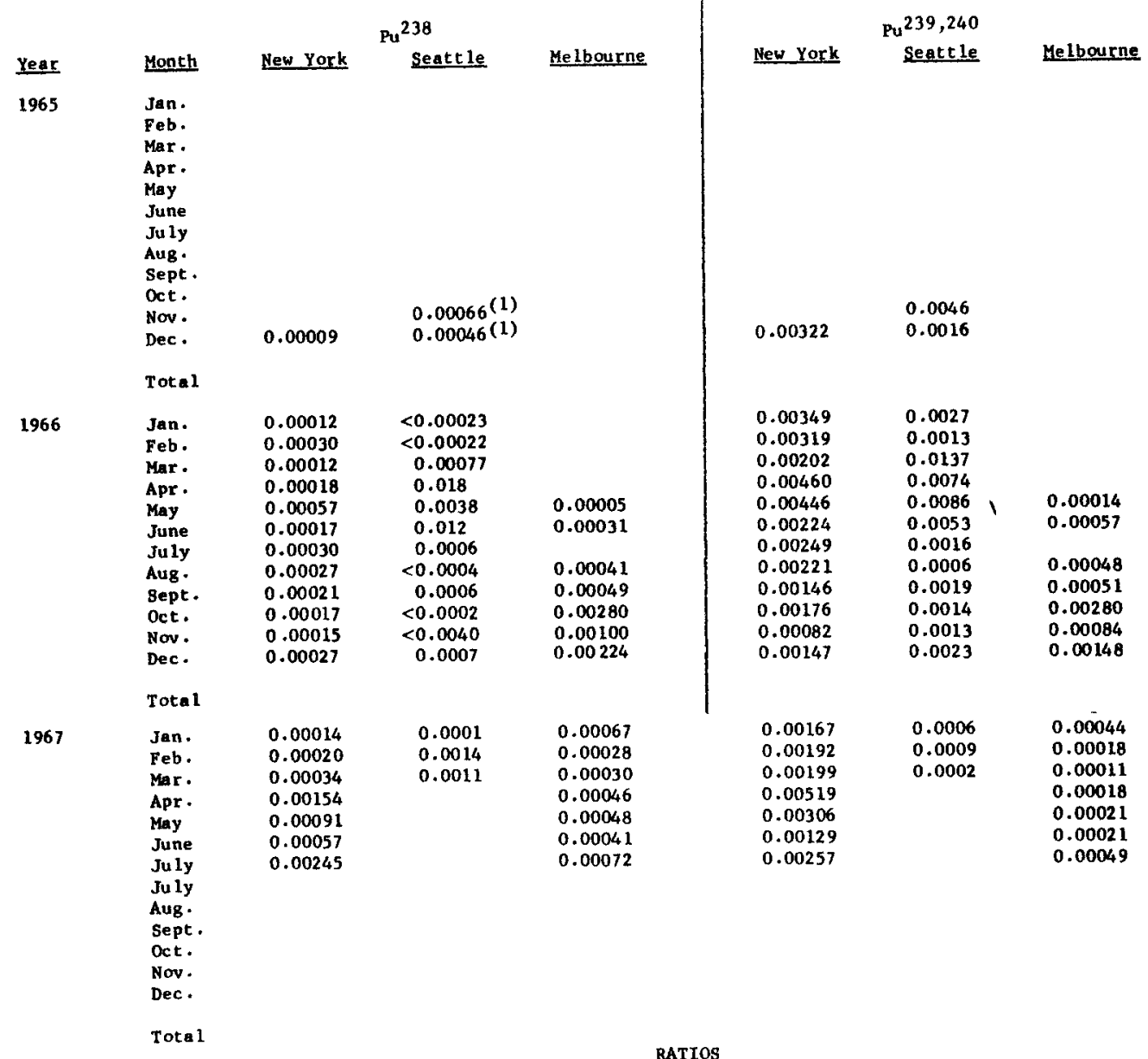

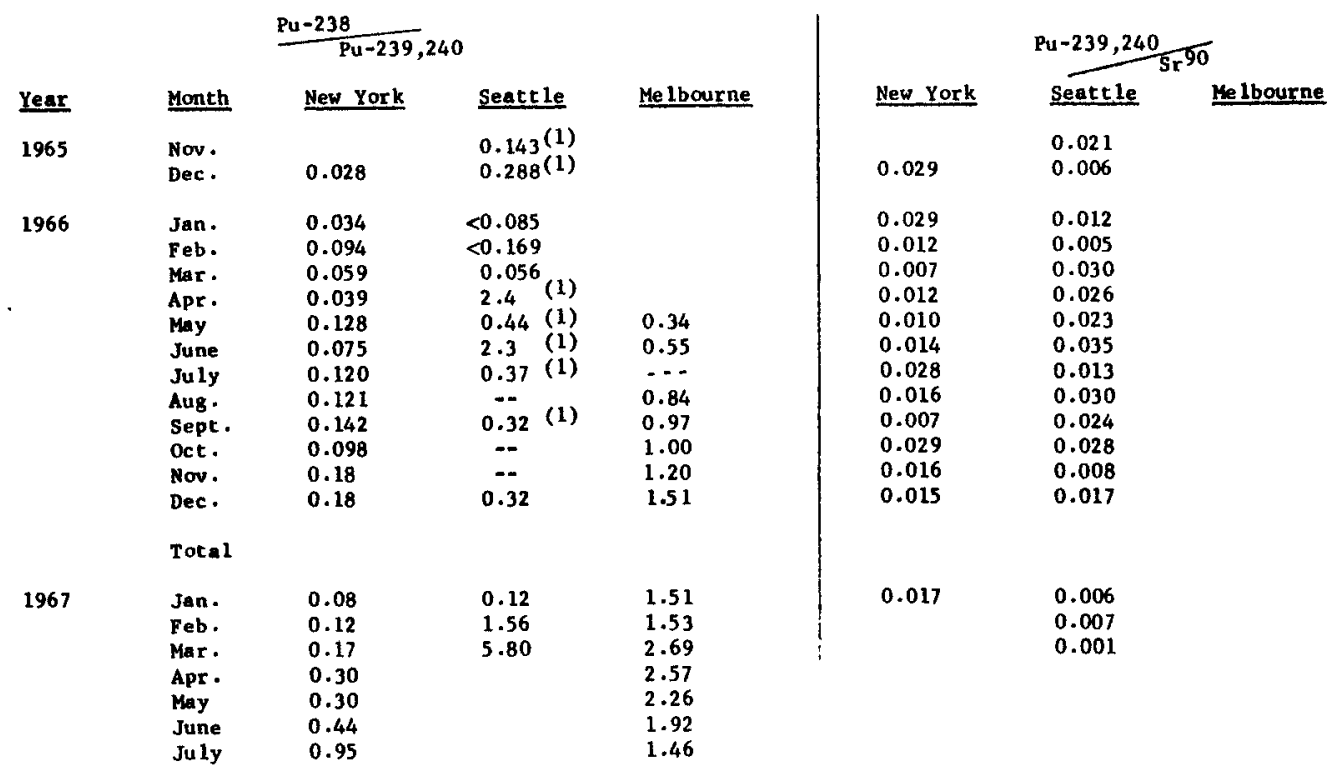

(1) values suspect

- data not aval lable

* zero or crace 
Append 1x C

Radiostrontium Deposition at At lantic Ocean Weather Stations

Since July 1963, U. S. Coast Guard Vessels on location at Station ECHO in the Atlantic Ocean have measured precipitation amount and collected fallout using the funne 1 and ton-exchange column unit supplied by HASL.

A larger sampling program was inftiated in the summer of 1965 for the purpose of extending our knowledge of $\mathrm{Sr}-90$ fallout and preclpitation over the sea. Ion-exchange column fallout collectors and rain gauges have been placed on the 23 Coast Guard vessels assigned to Ocean Station duties under Commender, Eastern area. As a scheduled part of their regular duties, these vessels maintain continuous weather observation stations at four locations in the Atlantic Ocean. These are:

$\begin{array}{lcc}\text { Station } & \text { Latitude } & \text { Longitude } \\ \text { B "BRAVO" } & 56^{\circ} 30^{\prime} \mathrm{N} & 51^{\circ} 00^{\prime} \mathrm{W} \\ \text { C "CHARLIE" } & 52^{\circ} 45^{\prime} \mathrm{N} & 35^{\circ} 30^{\prime} \mathrm{W} \\ \text { D "DELTA" } & 44^{\circ} 00^{\prime} \mathrm{N} & 41^{\circ} 00^{\prime} \mathrm{W} \\ \text { E "ECHO" } & 35^{\circ} 00^{\prime} \mathrm{N} & 48^{\circ} 00^{\prime} \mathrm{W}\end{array}$

Normal scheduling of the ships results in "on station" periods of about 21 days, thus the deposition samples are not monthly as is usual for land sampling.

The designations of the Home Ports and Vesse1s, which appear in the data tables, are as follows:

\begin{tabular}{llll} 
Port & Designation & Vessel & Designation \\
\hline Port land, Maine & PO & $\begin{array}{l}\text { Barataria } \\
\text { Cook Inlet } \\
\end{array}$ & BA \\
& & Coos Bay \\
& BO & Bibb & CB \\
\hline Boston, Mass. & Casco & BI \\
& Castle Rock & CA \\
& Duane & DU \\
& Humboldt & HU \\
& McCulloch & MC \\
\hline New Bedford, Mass. & NB & Escanaba & ES \\
& & Yakutat & YA \\
\hline
\end{tabular}




\begin{tabular}{|c|c|c|c|}
\hline Port & Designation & Vesse 1 & Deslgnation \\
\hline New London, Conn. & $\mathrm{NL}$ & Owasco & OW \\
\hline New York, N, Y. & NY & $\begin{array}{l}\text { Campbel1 } \\
\text { Half Moon } \\
\text { Mackinac } \\
\text { Rockaway } \\
\text { Spencer }\end{array}$ & $\begin{array}{l}\text { CM } \\
\text { HM } \\
\text { MA } \\
\text { RO } \\
\text { SP }\end{array}$ \\
\hline Norfolk, Va. & No & $\begin{array}{l}\text { Absec on } \\
\text { Chinc oteague } \\
\text { Ingham }\end{array}$ & $\begin{array}{l}\mathrm{AB} \\
\mathrm{CH} \\
\text { IN }\end{array}$ \\
\hline Wilmington, N. C. & W I & Mendota & $\mathrm{ME}$ \\
\hline Miami, Florida & MI & Androscoggin & AN \\
\hline Pensacola, Florida & $\mathrm{PE}$ & Sebago & $\mathrm{SE}$ \\
\hline
\end{tabular}

The actual sampling is being carried out by Shipboard Rawinsonde specialists of the U. S. Weather Bureau, Environmental Sciences Service Administration, in addition to their normal duties on the cruise. We acknowledge with gratitude the very fine effort of these people and their supervisors, as we 11 as the Coast Guard personnel who schedule and man the vessels.

The tables on pages $C-3$ through $G-7$ summarize the $5 r-90$ deposition, precipitation and concentrations measured at the stations, to date. 
PRECIPITATION IN THE ATLANTIC OCEAN

U. 8. Conet Guard station BRAVO

1at. $56^{\circ} 30^{\prime} \mathrm{w}$, long. $31^{\circ} 00 \mathrm{w}$

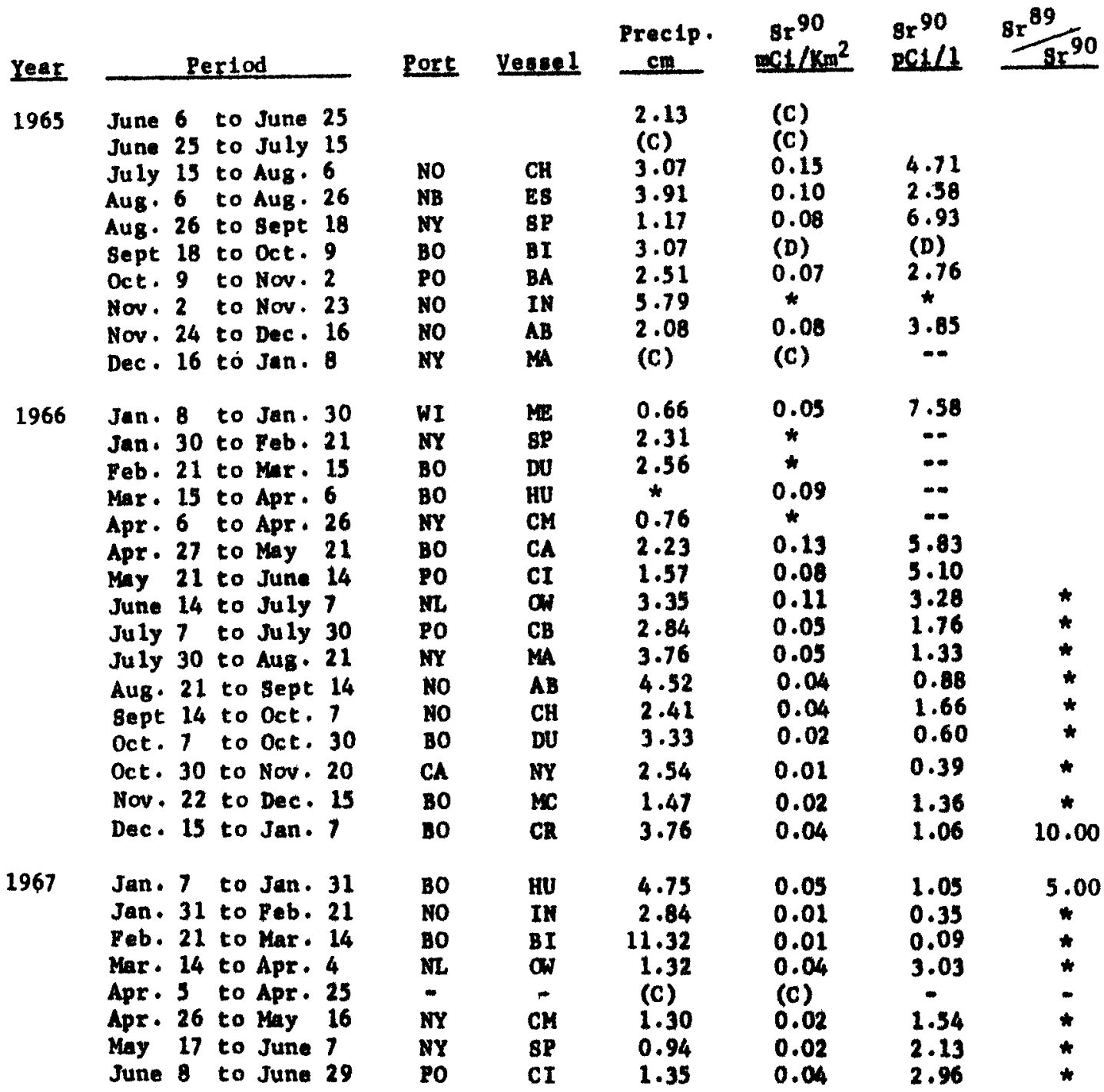

\footnotetext{
NOTE S: B - Suspect

C - No collection

D - Sample lost

* - Zero or trace
} 
PRECIPITATION IN THE ATLANTIC OCEAN

U. s. Conat Guard station CHAMLIE

let. $52^{\circ} 45^{\prime} \mathrm{N}, 10 \mathrm{ng} \cdot 35^{\circ} 30^{\prime} \mathrm{W}$

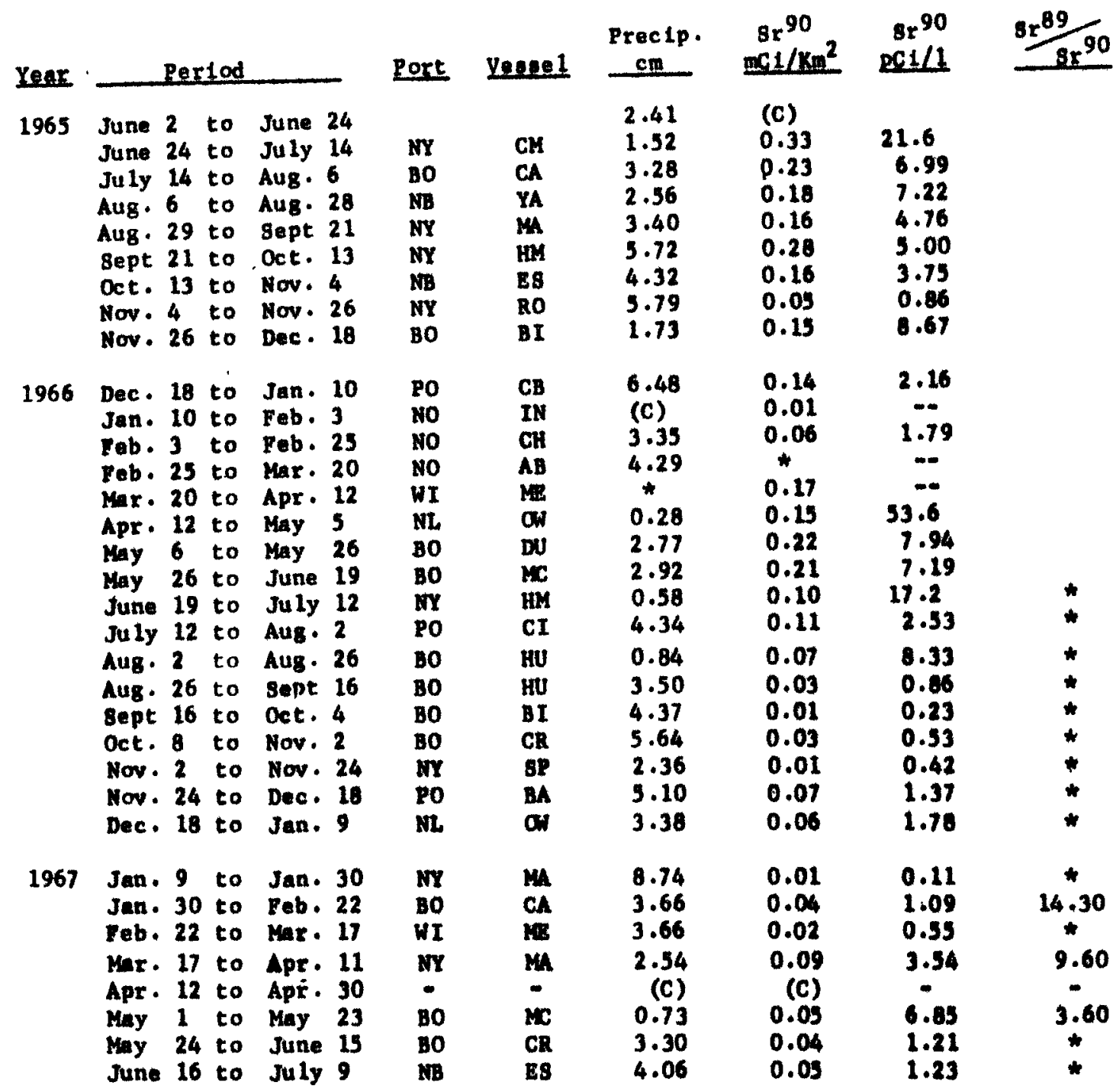

NOTE8, B - Buspect

C - No collection

D - Sample loat

* - Zero or trace 
PRECIPITATION In The ATLANIC ocsal

v. 8. Coast ourd station Diht

lat. $44^{\circ} 00^{\prime} \mathrm{w}, 1 \mathrm{ong} \cdot 41^{\circ} 00 \mathrm{w}$

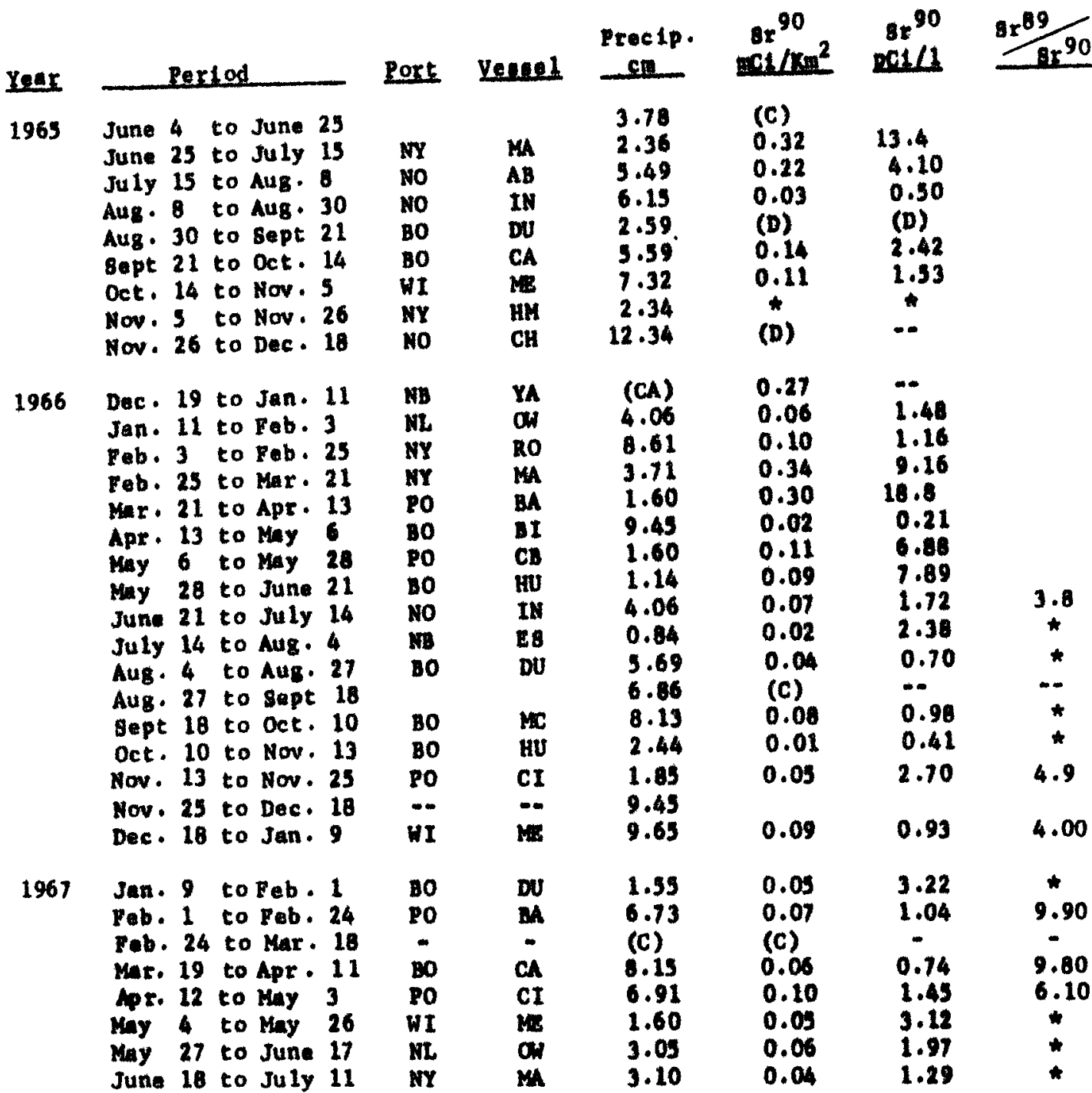

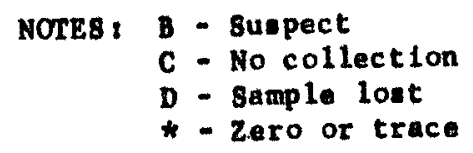


PRECIPITATION IN THE ATLANTIC OCEAN

U. 8. Coest Guard station ECHO

Lat. $35^{\circ} 00^{\prime} \mathrm{H}$, Long . $48^{\circ} 00^{\prime} \mathrm{w}$

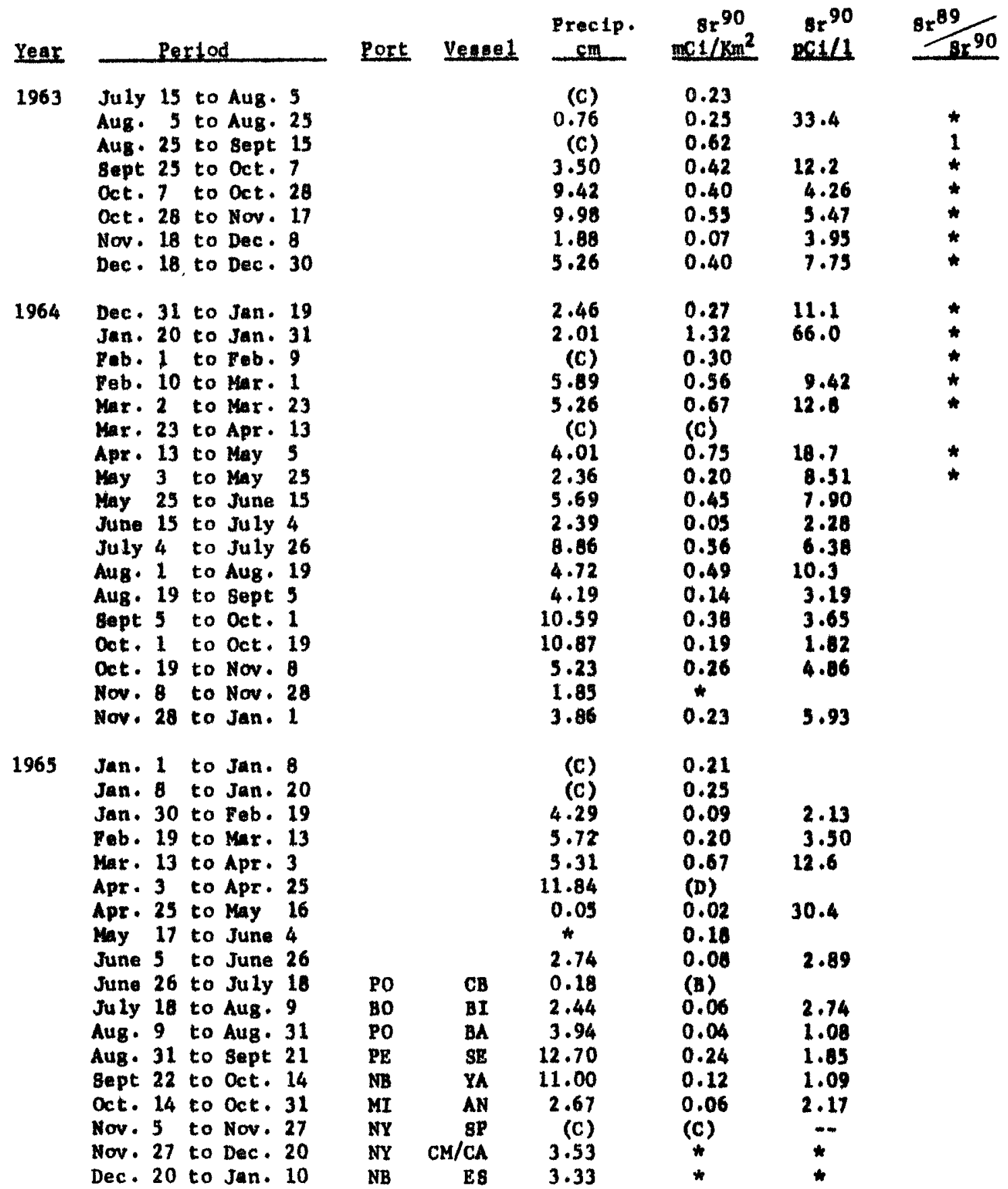

NOTEs: B - suspect

C - No collaction

D - Sample loat

* Zero or trace 
PRECIPITATION IN THE ATLANTIC OCEAN

U.S. Coast Guard station ECHO (cont'd)

Lat. $35^{\circ} 00^{\prime} \mathrm{N}$, long. $48^{\circ} 00 \mathrm{w}$

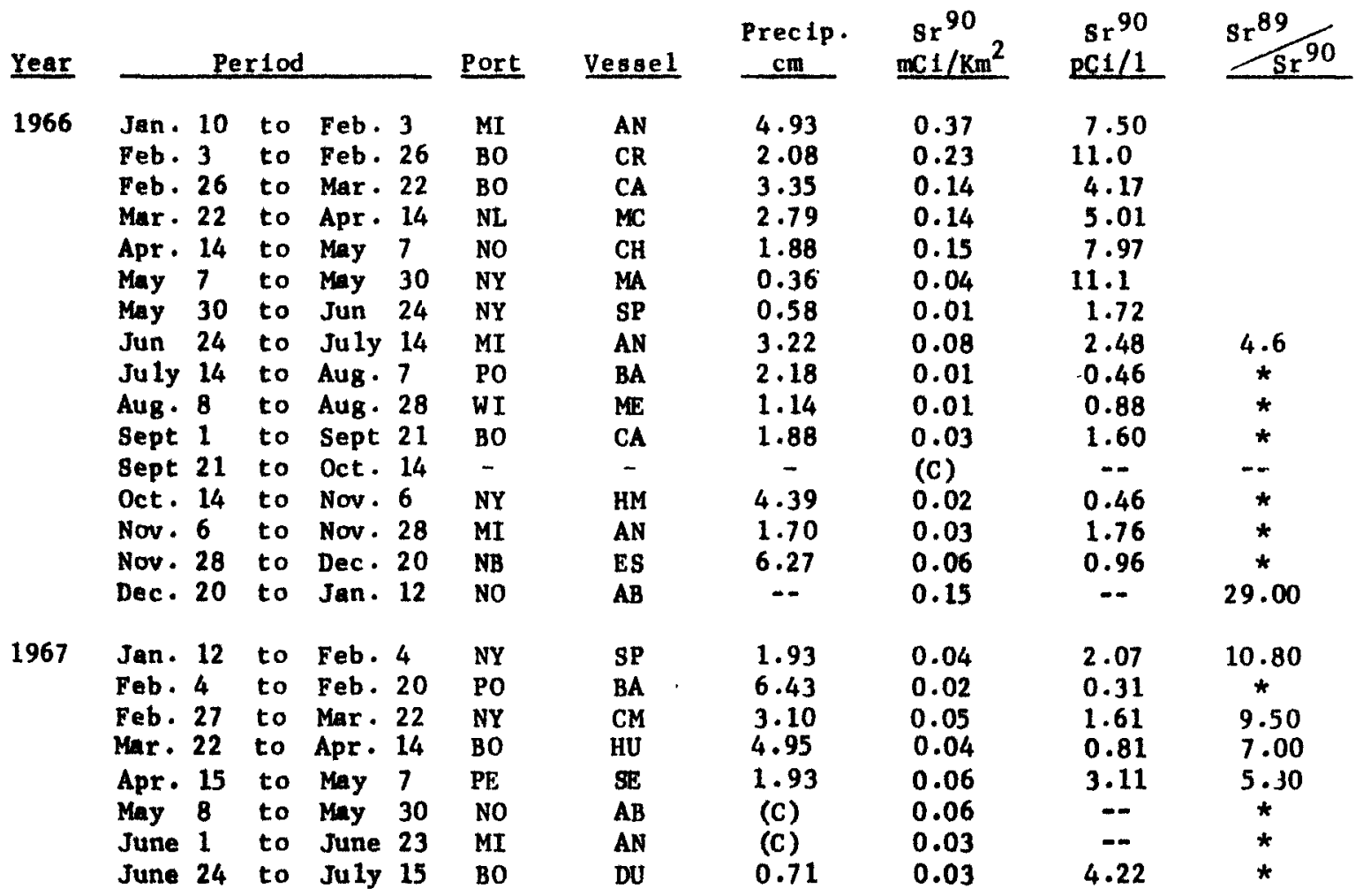

NOTES: $\begin{aligned} & \text { B - Suspect } \\ & \text { C - No collection } \\ & \text { D - Sample lost }\end{aligned}$

* - Zero or trace 
Appendix D

Radiostrontium in Milk and Tap Water

In 1954 the Health and Safety Laboratory began monitoring 1iquid whole milk in New York City for strontium-90 in order to estimate the dietary contribution from the ingestion of this radionuclide from this source. During the same year, tap water sampling was begun on a routine basis at the laboratory which receives its supply from one of the main reservoirs servicing New York City.

Powdered milk monitoring was initiated at a main processing plant in Perry, New York, in 1954, and in 1955, sampling of powdered butteirmilk from the Mandan North Dakota area was begun. The powdered buttermilk is used as cattle feed and it was of interest to have a continuous record of the strontium-90 levels in this animal dietary supplement. Liquid milk from two large dairies serving Honolulu was monitored since the summer of 1959. On the is land of Oahu the dairy cows are on pasture throughout the year and it was of interest to know how well the Sr-90 levels in milk in this area reflect changes in deposition rates.

Milk sampling at Mandan, North Dakota and Oahu, Hawail was terminated at the end of June 1965. The USPHS maintains pasteurized milk sampling stations at Minot, 'North Dakota and Honolulu and it was felt that since the HASL and PHS data parallel one another at these sites, continuance of the HASL stations was no longer necessary. The data for these two milk sampling stations was summarized in tabular and graphical form in HASL-171.

Although a more complete study of the strontium-90 content of the diets in three major $U$. S. cities has been in progress since March 1960, milk and tap water analyses at the above-mentioned sites have been continued in order to provide a detalled and continuous history of the contamination levels of these staples.

$\underline{M i 1 k}$

The New York City sample is a monthly composite of pasteurized milk purchased dally at retail stores. Five main dairies are represented in the sample. The Perry samples are monthly composites of powdered milk collected in weekly fivepound lots. During appropriate periods strontium-89, as we 11 as strontium-90, has been analyzed in these milk samples. These data have been given in previous quarterly reports.

The monthly strontium-90 to calcium ratios for Perry, N. Y. and New York City since the inception of the sampling programs are presented in Tables on page D-2. These data are presented graphically in figures on $p .0-3$.

Strontium-90 and Cesium-137 in New York City Tap Water

Samples of New York City tap water are taken daily at HASL so that by the end of the month, approximately 100 liters have been collected. The strontium-90 data since the inception of the program are presented in the table on p. D-4. The available Cs-137 data expressed as the Cs-137 to $\mathrm{Sr}-90$ ratio are given in the table at the bottom of p. D-4. Tap water sampling and analyses were carried out at Richmond, California from 1959 through the first half of 1963 . These data have been reported in previous quarterlies. A graphical presentation of the New York City Sr-90 data is shown in the figure on $p . D-5$.

$$
\mathrm{D}-1
$$


The strontiun-90 to Gelelun watio in milk

New York C1ty - 11quid milk

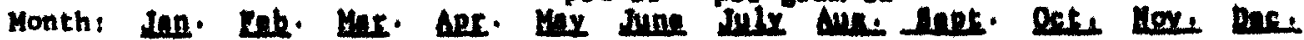

Annue 1

Ave.

\begin{tabular}{|c|c|c|c|c|c|c|c|c|c|c|c|c|c|}
\hline ar! 1954 & & & & & & 0.5 & 0.9 & 0.6 & 2.3 & 1.8 & 1.7 & 1.8 & - \\
\hline 1953 & 1.3 & 0.9 & 1.1 & 0.9 & 2.4 & 3.7 & 3.2 & 2.4 & 4.0 & 3.9 & 3.6 & 3.0 & 2.6 \\
\hline 1956 & 4.2 & 3.7 & 3.7 & 3.5 & 4.2 & & & & & & & & - \\
\hline 1957 & 3.2 & 4.1 & 3.8 & 3.7 & 4.0 & 3.5 & 6.6 & 4.6 & 4.9 & 3.7 & 4.7 & 2.9 & 4.5 \\
\hline 1938 & 3.6 & 4.0 & 3.2 & 4.1 & 3.9 & 10.2 & 11.3 & 10.9 & 10.6 & 9.9 & 11.7 & 0.2 & 7.6 \\
\hline 1939 & 0.2 & 8.4 & 0.0 & 7.7 & 13.4 & 24.2 & 14.1 & 10.7 & 9.0 & 0.3 & 10.2 & 9.2 & 11.0 \\
\hline 1960 & 8.1 & 0.9 & 9.1 & 8.7 & 8.8 & 10.1 & 9.8 & 8.6 & 6.1 & 0.1 & 3.9 & 6.3 & 0.0 \\
\hline 1961 & 6.7 & 1.3 & 0.1 & 6.3 & B.1 & 0.4 & 7.0 & 5.4 & 3.2 & 6.0 & 6.1 & 5.7 & 6.7 \\
\hline 1962 & 3.7 & 6.1 & 4.0 & 9.1 & 14.0 & 14.3 & 23.2 & 17.4 & 15.3 & 13.6 & 10.3 & 10.9 & 18.2 \\
\hline 1963 & 0.3 & $\mathbf{1 1 . 2}$ & 13.4 & 12,1 & 40.6 & 42.4 & 33.8 & 40.5 & 27.1 & 29.6 & 23.9 & 20.7 & 23.6 \\
\hline 1964 & 21.1 & 23.9 & 27.2 & 26.1 & 30.8 & 30.9 & 22.0 & & 17.1 & & & 19.3 & 23.2 \\
\hline 1965 & 20.0 & 18.0 & 21.1 & 19.0 & 22.9 & 18.0 & 18.0 & 22.2 & 16.5 & 17.6 & 17.6 & 17.1 & 19.1 \\
\hline 1966 & 13.9 & 14.3 & 23.0 & 11.5 & 13.8 & 17.0 & $12 \cdot 4$ & 13.6 & $13 \cdot 2$ & 1.4 & 9.0 & 7.2 & 12.1 \\
\hline 1967 & 13.5 & 9.6 & 11.1 & & & & & & & & & & \\
\hline
\end{tabular}

Perry, W. Y. - powdered mole milk

\begin{tabular}{|c|c|c|c|c|c|c|c|c|c|c|c|c|c|}
\hline 1934 & & & & 0.5 & 1.2 & 1.3 & 2.3 & 1.2 & 1.3 & 2.4 & 1.1 & 0.6 & - \\
\hline 1993 & 2.5 & 0.8 & 0.8 & 0.3 & 1.9 & 2.5 & 1.9 & 2.0 & 1.5 & 2.8 & 2.5 & 3.3 & 1.9 \\
\hline 1936 & 2.3 & 2.0 & 2.0 & 2.9 & 2.8 & 3.0 & 2.7 & 3.1 & 4.9 & 2.4 & 3.6 & 3.2 & 3.3 \\
\hline 1937 & 3.8 & 4.0 & 3.0 & 3.1 & 3.9 & 4.6 & 4.7 & 4.2 & 4.3 & 3.0 & 4.4 & 2.0 & .8 \\
\hline 1958 & 3.4 & 3.6 & 3.0 & 3.0 & 3.0 & 3.4 & 8.6 & 1.2 & 6.6 & 7.0 & 10.0 & 6.9 & 3.9 \\
\hline 1959 & 8.1 & 7.6 & 6.6 & 7.2 & 0.9 & 8.9 & 10.0 & 7.8 & 6.1 & 10.5 & 7.0 & 7.7 & 0.0 \\
\hline 1960 & 8.7 & 8.6 & 7.3 & 7.7 & 7.3 & 7.1 & 7.7 & 3.5 & 3.0 & 3.1 & 4.2 & 4.0 & 6.6 \\
\hline 1961 & 6.9 & 7.0 & 6.5 & 7.2 & 6.6 & 3.9 & 6.3 & 3.8 & 6.3 & 3.0 & 3.1 & 3.9 & 6.2 \\
\hline 1962 & 5.2 & 3.0 & 3.6 & 5.6 & 8.8 & 13.0 & 21.7 & 15.6 & 14.2 & 13.6 & 12. & 12.3 & \\
\hline 1963 & 12.5 & 11.4 & 11.9 & 11.8 & 18.3 & 30.9 & 30.1 & 27.1 & 30.6 & 30.1 & 22.2 & 22.1 & \\
\hline 1964 & 20.1 & 21.7. & 20.9 & 21.0 & 19.3 & 24.2 & 28.0 & 22.1 & 18.0 & 16.0 & 14.7 & 10.0 & \\
\hline 1965 & 17.7 & 14.2 & 19.0 & 18.3 & 16.9 & 13.3 & 19.1 & 12.9 & 11.6 & 10.9 & 11.7 & 11.7 & 14.4 \\
\hline 1966 & 22.4 & 11.2 & 11.0 & 10.8 & 11.8 & 13.9 & 13.4 & 13.6 & 12.2 & 11.2 & 9.7 & 8.5 & 11.7 \\
\hline 1967 & 9.5 & 8.9 & 9.8 & & & & & & & & & & \\
\hline
\end{tabular}


STRONTIUM-90 IN POWDERED WHOLE MILK - PERRY, N. Y.

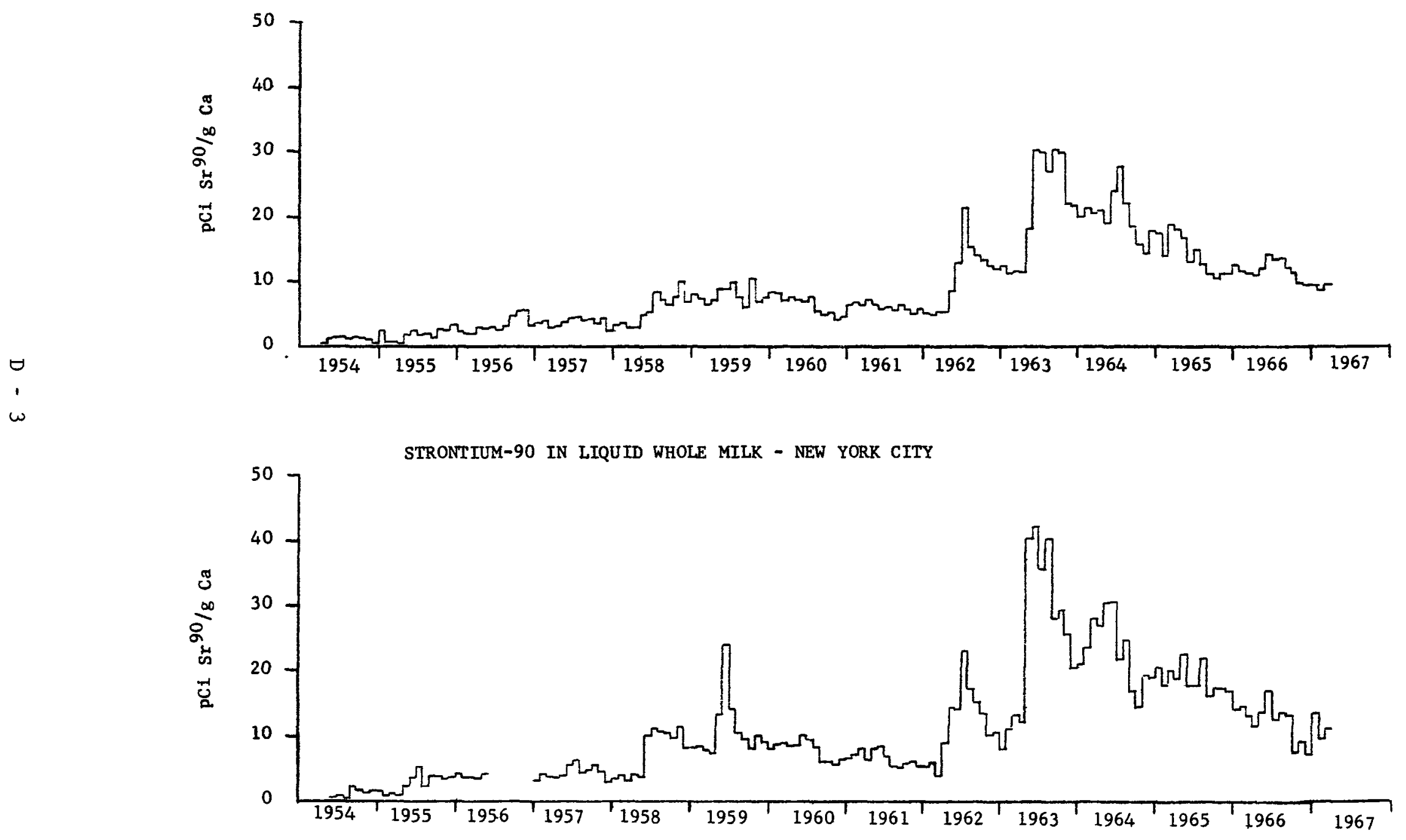


Strontium-90 in New York City Tap Water

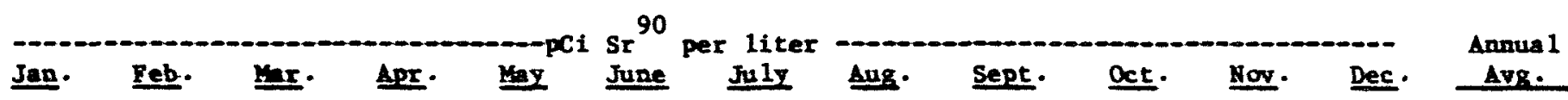

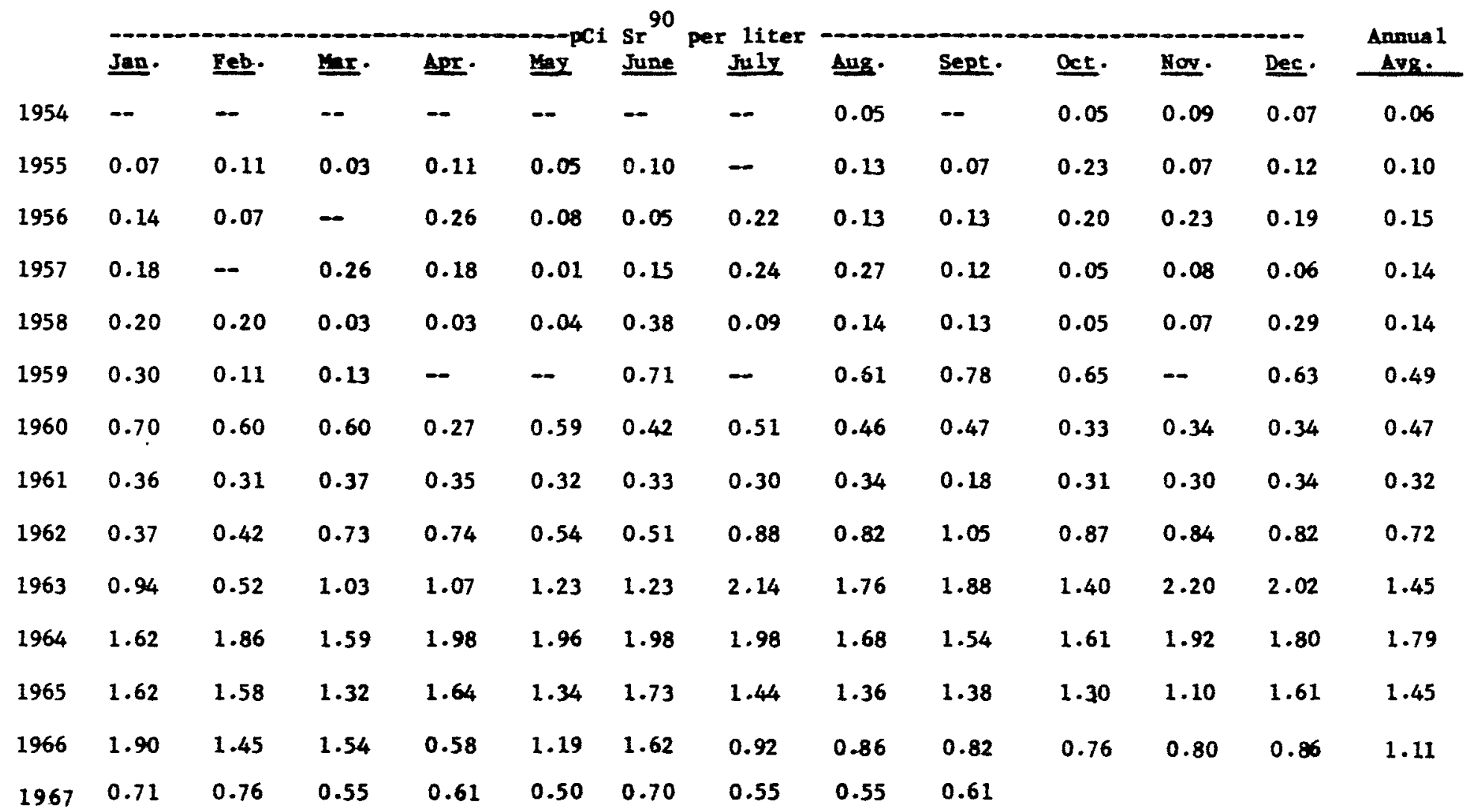

\section{The $C_{8}^{137}$ to $5^{90}$ Bntio in How Tork City Tap Nater}

Jan. Feb. Mar. Apr. Hy Jime July Aug. Sept. Qct. Hor. Dec.

$\begin{array}{lllllllllllll}1965 & 0.08 & 0.10 & 0.09 & 0.13 & 0.18 & 0.12 & 0.19 & 0.15 & 0.12 & - & 0.18 & 0.11 \\ 1966 & 0.23 & -- & 0.13 & 0.14 & 0.18 & 0.17 & 0.24 & 0.21 & 0.12 & 0.09 & 0.14 & 0.07 \\ 1967 & 0.06 & 0.05 & 0.07 & 0.10 & 0.12 & 0.08 & 0.09 & 0.09 & 0.06 & & & \end{array}$.




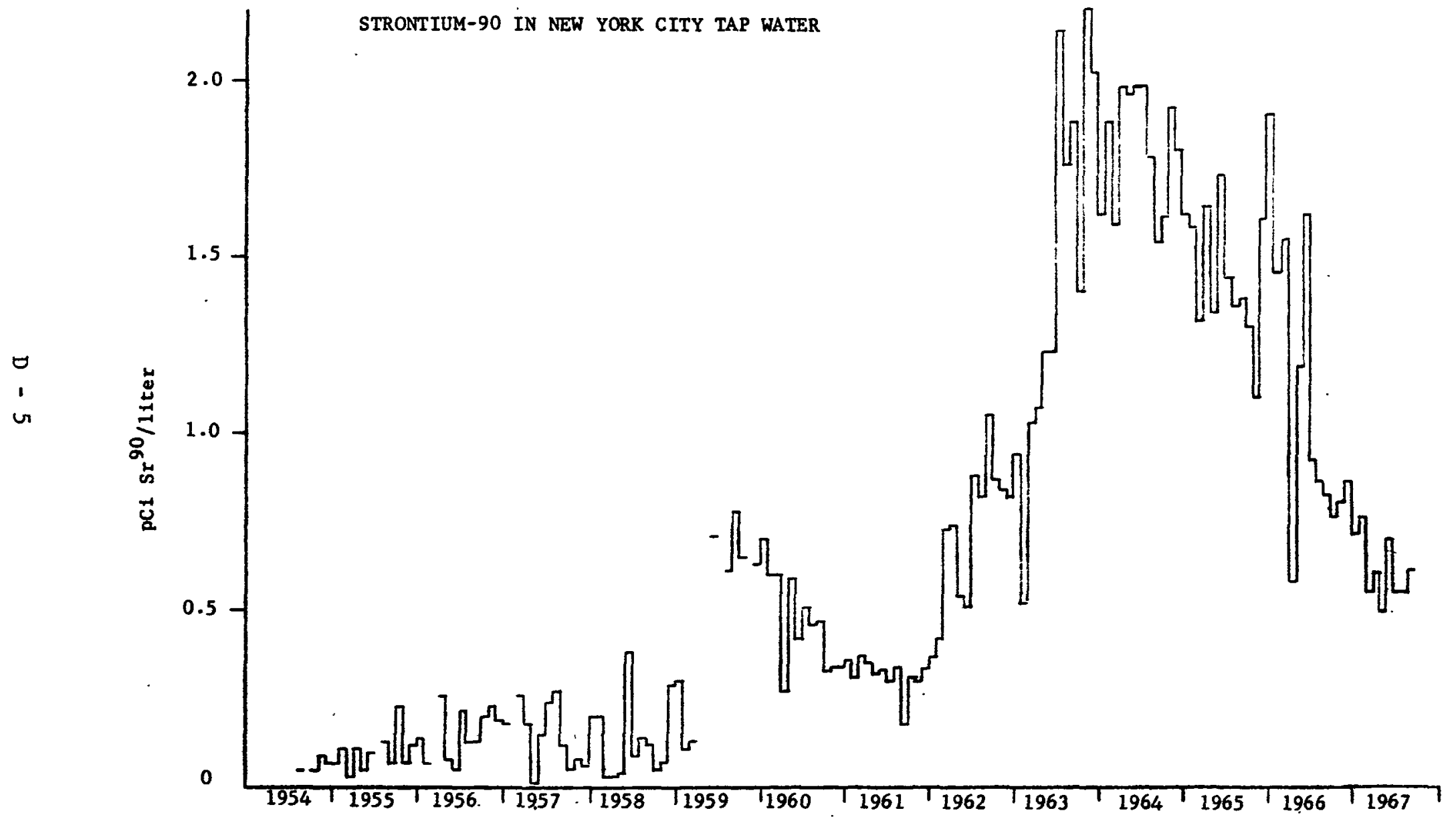


The following bbreviation 11ating and converuion table Involve undte of mawuranent ueed in the Hath tallout and alr wampling prosrams.

\section{ARRFytation:}

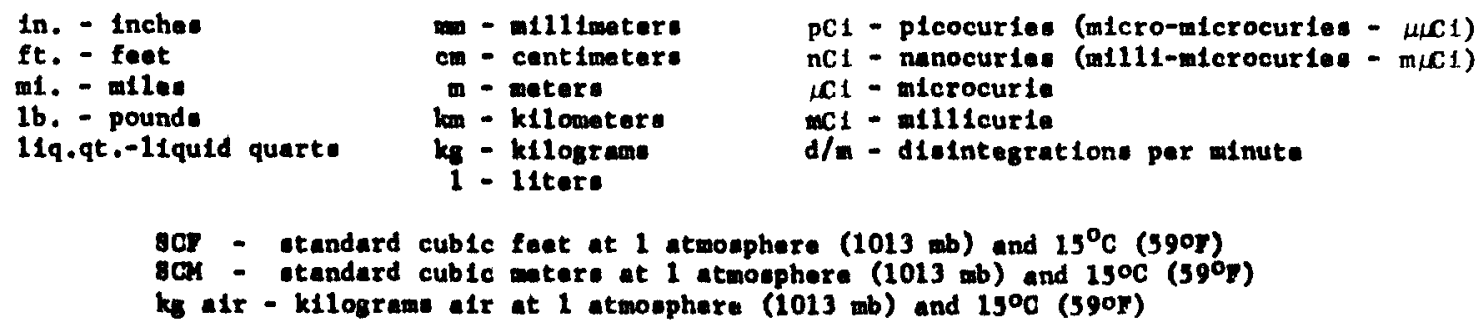

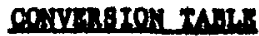

\begin{tabular}{|c|c|c|c|c|c|}
\hline Elltelply & bx & to obtaln & yiletelx & by & to obtrain \\
\hline $\begin{array}{l}\text { In. } \\
\text { in. } \\
\text { ft. } \\
\text { al. }\end{array}$ & $\begin{array}{c}25.4 \\
2.34 \\
0.305 \\
1.61\end{array}$ & $\begin{array}{l}\operatorname{mon} \\
\mathrm{cm} \\
\mathrm{m}\end{array}$ & $\operatorname{com}_{n=m}$ & $\begin{array}{l}0.0394 \\
0.394 \\
3.28 \\
0.621\end{array}$ & $\begin{array}{l}\ln . \\
\ln . \\
\mathrm{ft} . \\
\mathrm{L} .\end{array}$ \\
\hline $\begin{array}{l}1 \mathrm{~b} . \\
11 \mathrm{q} . q \mathrm{q} .-\mathrm{U} .8 .\end{array}$ & $\begin{array}{l}0.4536 \\
0.946\end{array}$ & $\begin{array}{l}k \\
1\end{array}$ & 1 & $\begin{array}{l}2.205 \\
1.057\end{array}$ & $\begin{array}{l}1 b . \\
11 q \cdot q t,-t .8 .\end{array}$ \\
\hline $\mathrm{mL} .2$ & 2.59 & $\operatorname{lnm} 2$ & $m^{2}$ & 0.386 & $m 1.2$ \\
\hline $\mathrm{mCl} / \mathrm{mL} \cdot .^{2}$ & $\begin{array}{l}0.386 \\
15.2\end{array}$ & $\begin{array}{l}\mathrm{mC} 1 / \mathrm{km}^{2}\left(\mathrm{nCl} / \mathrm{m}^{2}\right) \\
\mathrm{pCl} 1 / 1\end{array}$ & $\begin{array}{l}\mathrm{mCl} / \mathrm{km}^{2} \\
\mathrm{pCl} 1 / 1\end{array}$ & $\begin{array}{l}2.59 \\
0.0557\end{array}$ & 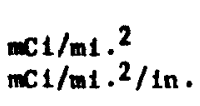 \\
\hline $\mathrm{pC} 1 / 1$ & 0.02 & $\mathrm{mCl} 1 / \mathrm{km}^{2} / \mathrm{cm}$ & $\mathrm{mC1} 1 / \mathrm{km}^{2} / \mathrm{cm}$ & 100 & $\mathrm{pCt} / 1$ \\
\hline $\begin{array}{l}d / m \\
n C 1\end{array}$ & $\begin{array}{l}0.450 \\
1 \times 10^{3}\end{array}$ & $\begin{array}{l}\mathrm{pC} 1 \\
\mathrm{pC} 1\end{array}$ & $\begin{array}{l}p C 1 \\
p C 1\end{array}$ & 2.22 & $\mathrm{nC1}$ \\
\hline$d / m / 1$ & $0.45 \times 10^{-9}$ & $\mu \mathrm{C} 1 / \mathrm{cc}$ & $\mu \mathrm{C} 1 / \mathrm{cc}$ & $2.22 \times 10^{9}$ & $d / a / 1$ \\
\hline $\mathrm{d} / \mathrm{m} / \mathrm{ft} .{ }^{2}$ & 0.01256 & $\mathrm{mCl} / \mathrm{mL} .^{2}$ & $\mathrm{mC1} / \mathrm{mL} .^{2}$ & 79.6 & $d / m / t t .^{2}$ \\
\hline $\begin{array}{l}10^{3} 80 \\
10^{3} 8 \mathrm{Cr}\end{array}$ & $\begin{array}{l}28.3 \\
34.7\end{array}$ & $\begin{array}{l}\text { sox } \\
\text { kg air }\end{array}$ & $\begin{array}{l}\text { 8CH } \\
\text { kg atr }\end{array}$ & $\begin{array}{l}0.0353 \\
0.0288\end{array}$ & $\begin{array}{l}10^{3} \mathrm{gcF} \\
10^{3} \mathrm{BCF}\end{array}$ \\
\hline $80 x$ & 1.226 & $k g a 1 r$ & $k$ alx & 0.816 & $\operatorname{sax}$ \\
\hline
\end{tabular}

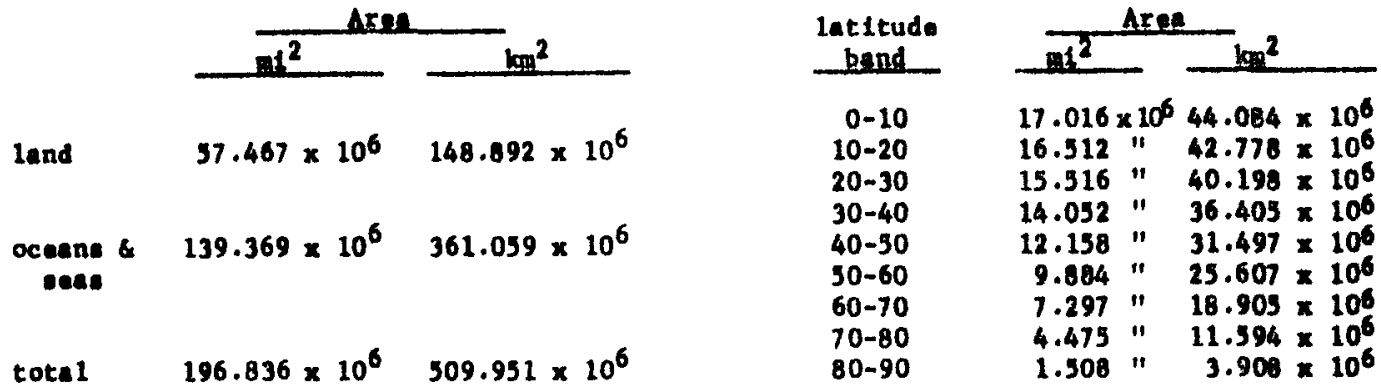


TABLE OF RADIONUCLIDES

The following table is a listing of radionuclide of Interest in the hasi fallout program. The half-11fe velues are thowe currently in uee at HAgL and are wubject to change a new data becoue available.

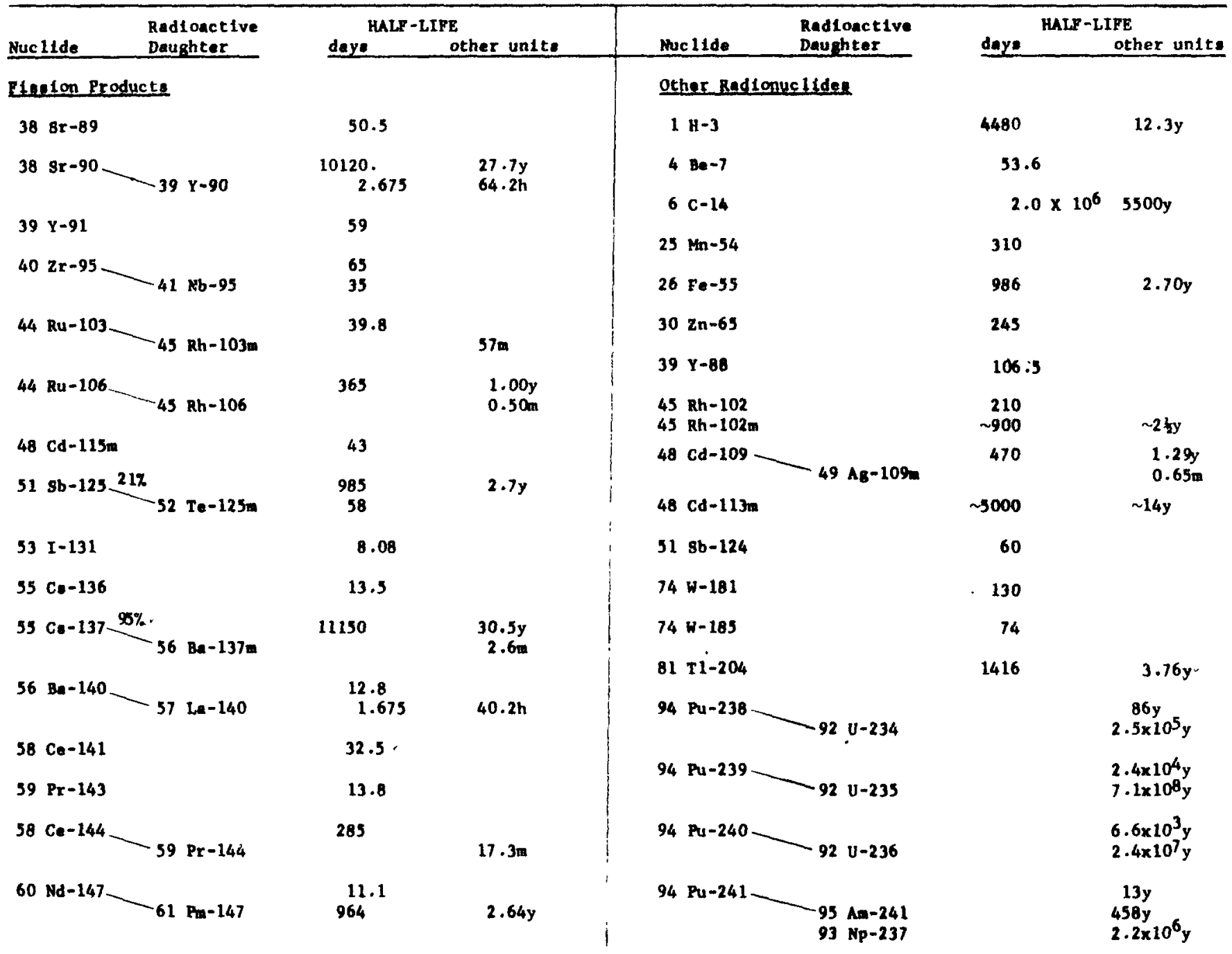

